\title{
Experimental energy levels of the water molecule
}

\author{
Jonathan Tennyson*, Nikolai F. Zobov ${ }^{\dagger}$, Ross Williamson, Oleg L Polyansky ${ }^{\dagger}$ \\ Department of Physics \& Astronomy, University College London, Gower Street, London WC1E \\ $6 B T, U K$. \\ Peter F. Bernath \\ Department of Chemistry, University of Waterloo, Waterloo, ON Canada N2L 3G1 and \\ Department of Chemistry, University of Arizona, Tucson, AZ, 85721
}

\begin{abstract}
Experimentally derived energy levels are presented for 12248 vibrationrotation states of the $\mathrm{H}_{2}{ }^{16} \mathrm{O}$ isotopomer of water, more than doubling the number in previous, disparate, compilations. For each level an error and reference to source data is given. The levels have been checked using energy levels derived from sophisticated variational calculations. These levels span 107 vibrational states including members of all polyads up to and including $8 \nu$. Band origins, in some cases estimates, are presented for 101 vibrational modes.
\end{abstract}

Key words: energy levels, water, vibration, rotation, band origins

\section{CONTENTS}

\section{Introduction}

\footnotetext{
*Email: j.tennyson@ucl.ac.uk

${ }^{\dagger}$ Permanent address: Institute of Applied Physics, Russian Academy of Science, Uljanov Street 46, Nizhnii Novgorod, Russia 603024
} 
2. Notation

3. Method

4. Errors and Tables

5. Summary

6. Acknowledgements

7. References

\section{LIST OF TABLES}

1. Summary of results and band origins.

2. Term values for the ground and first excited state of $\mathrm{H}_{2}{ }^{16} \mathrm{O}$.

3. Term values for the first polyad, $1 \nu$, of $\mathrm{H}_{2}{ }^{16} \mathrm{O}$.

4. Term values for the $1 \nu+\delta$ polyad of $\mathrm{H}_{2}{ }^{16} \mathrm{O}$.

5. Term values for the $2 \nu$ polyad of $\mathrm{H}_{2}{ }^{16} \mathrm{O}$.

6. Term values for the $2 \nu+\delta$ polyad of $\mathrm{H}_{2}{ }^{16} \mathrm{O}$.

7. Term values for the $3 \nu$ polyad of $\mathrm{H}_{2}{ }^{16} \mathrm{O}$.

8. Term values for the $3 \nu+\delta$ polyad of $\mathrm{H}_{2}{ }^{16} \mathrm{O}$.

9. Term values for the $4 \nu$ polyad of $\mathrm{H}_{2}{ }^{16} \mathrm{O}$.

10. Term values for the $4 \nu+\delta$ polyad of $\mathrm{H}_{2}{ }^{16} \mathrm{O}$.

11. Term values for the $5 \nu$ polyad of $\mathrm{H}_{2}{ }^{16} \mathrm{O}$.

12. Term values for the $5 \nu+\delta$ polyad of $\mathrm{H}_{2}{ }^{16} \mathrm{O}$. 
13. Term values for the $6 \nu$ polyad of $\mathrm{H}_{2}{ }^{16} \mathrm{O}$.

14. Term values for the $6 \nu+\delta$ polyad of $\mathrm{H}_{2}{ }^{16} \mathrm{O}$.

15. Term values for the $7 \nu$ polyad of $\mathrm{H}_{2}{ }^{16} \mathrm{O}$.

16. Term values for the $7 \nu+\delta$ polyad of $\mathrm{H}_{2}{ }^{16} \mathrm{O}$.

17. Term values for the $8 \nu$ polyad of $\mathrm{H}_{2}{ }^{16} \mathrm{O}$.

\section{LIST OF FIGURES}

1. Coverage of vibrational bands by polyad.

\section{INTRODUCTION}

Water is arguably the single most important molecule. It is considered fundamental to life, ubiquitous in the Universe, is the dominant greenhouse gas, responsible for about $70 \%$ of the known absorption of sunlight in the Earth's atmosphere, and is the major product of nearly all combustion processes. It is therefore no surprise that the high resolution spectrum of water has been the subject of decades of intensive study. However the rotation-vibration spectrum of water is both dense and complicated. This means that although there have been a great many successes in the spectral analysis of water, there remains much to be done. For example, after nearly thirty years of trying a particularly dense absorption spectrum of water recorded in sunspots has now been successfully analysed (Polyansky et al. 1997a). But this work only assigned about the strongest $15 \%$ of the observed transitions.

A compact way of representing successful spectral assignments is via energy levels. Unlike other parameterizations, this method does not rely on any underlying model or effective Hamiltonian. Tables of vibration-rotation energy levels are built by setting the (000) $0_{00}$ ground energy level as the zero of energy and using transition frequencies to define the relative energies of the excited states. Cleary measurements of new transitions connecting 
particular states allows the precision with which the energies of these states are determined to be systematically improved. There are many partial tabulation of water rotation-vibration energy levels in the literature, see citations below, but there has been no comprehensive one for a considerable time, if ever.

Spectral analysis of water has taken on a new vigor with the use of variational calculations to perform the theoretical analysis replacing traditional perturbation based methods (Partridge and Schwenke 1997, Schwenke 1998, Polyansky et al. 1997a, 1997b, 1997c, 1997d, 1997e, 1998, Carleer et al. 1999, Zobov et al. 1999, 2000a, 2000b, Bykov et al. 2000, Haus et al. 2001, Schermaul et al. 2001). Studies using variational calculations have greatly extended the range, both in vibrational and rotational quanta, of energy levels that have been determined. The result has been an almost three-fold increase in the number of energy levels of the main isotopomer of water, $\mathrm{H}_{2}{ }^{16} \mathrm{O}$, that can be determined directly from experimental data.

Tabulations of experimental rotation-vibration energy levels are important for a number of purposes. They are necessary for spectral analysis, where the use of combination differences provides the most secure way of making assignments. They can be used to compute partition functions and other thermodynamic quantities (eg Vidler and Tennyson 2000). Energy levels are important for developing theoretical models, either by fits to effective Hamiltonians (Polyansky 1985, Tyuterev et al. 1995, Coudert 1997) or, in conjunction with variational calculations, by fits which yield spectroscopically determined (effective) potential energy surfaces (Carter and Handy 1987, Jensen 1989, Halonen and Carrington 1988, Kauppi and Halonen 1990, Jensen et al. 1994, Paulse and Tennyson 1994, Polyansky et al. 1994, 1996a Partridge and Schwenke 1997, Kain et al. 2001). Furthermore the tabulated energy levels can be used to test ideas on the underlying level structure (Child et al. 1999) or as the starting point for further theoretical investigations, such as the search for ortho-para switching transitions (Chapovsky 1999). Probably the most important use for detailed tabulations of energy rules is that, given a knowledge of selection rules, they allow for detailed and accurate spectra to be predicted for a whole variaty of situations which have yet to be 
probed. See Nela et al. (2000) for an example.

In this paper we present a tabulation of 12248 experimentally determined energy levels of water belonging to 107 different vibrational states. These data are derived using published energy levels supplemented by data from analysing 24000 newly assigned transitions. Only the main and most important isotopomer of water, $\mathrm{H}_{2}{ }^{16} \mathrm{O}$, is considered since this is the only one for which sufficient data is available to justify the comprehensive treatment given here. For each energy level derived, we also present an error based on stated measurement errors, the number of transitions used to obtain the level, which gives a measure of certainty, and reference(s) to data used in obtaining the level. As a check on the levels they have been compared with independent determinations made using variational calculations and both the best available spectroscopically determined potential (Partridge and Schwenke 1997) and the best available ab initio procedure (Polyansky et al. 1999, Zobov et al. 2000b).

\section{NOTATION}

As a triatomic asymmetric top molecule, water has three vibrational modes and three rotational modes. The vibrational states can be labelled by both normal mode and local mode notation. In the more standard normal mode notation which is valid for low levels of the stretching excitation, $\left(v_{1} v_{2} v_{3}\right)$ represents vibrational quanta in normal modes $\nu_{1}$ (symmetric stretch), $\nu_{2}$ (bend) and $\nu_{3}$ (asymmetric stretch) respectively. In local mode notation (Halonen 1998) $(m n)^{ \pm} v_{2}, m$ and $n$ represent quanta of local stretch in symmetric, + , or asymmetric, -, symmetry combinations. Note if $m=n$ then the combination has to be symmetric and by convention the superscript is dropped. Previous spectral studies (Carleer et al. 1999, Zobov et al. 2000b) have demonstrated that local modes give a much better description of the higher states. This situation is complicated by the prediction (Rose and Kellman 1996) that it is only the predominantly stretching states for which this is true. However such highly excited bending states have yet to be observed.

In water there is a near coincidence of the frequencies of the symmetric stretch and asym- 
metric stretch modes, which in turn lie close to two quanta excitations of the bending mode. This means that the infrared and optical spectrum of water has a pronounced structure due to the closeness of several interacting vibrational states. It is conventional to label groups of interacting vibrations by a polyad number $n$ where $n=v_{1}+\frac{v_{2}}{2}+v_{3}$ for even $v_{2}$. Polyads with even $v_{2}$ are labelled $n \nu$ and those with an extra quantum of $v_{2}$ bend are labelled $n \nu+\delta$. Polyads $n \nu$ and $n \nu+\delta$ each contain $(n+1)(n+2) / 2$ vibrational states.

Rotational levels of water are labelled using standard asymmetric top notation as $J_{K_{a} K_{c}}$, where $J$ is the rotational angular momentum, $K_{a}$ is the projection of $J$ along the A-axis and $K_{c}$ is its projection along the C-axis. Since the A moment inertia is small $K_{a}$ is a key rotational quantum number. High $K_{a}$ states have in the past proved difficult to analyze as standard expansion techniques diverge for even low values of $K_{a}$ (Polyansky 1985). The use of variational calculations, discussed below, has largely resolved the problems with high $K_{a}$ states (Polyansky et al. 1997a) and similar ones with the higher bending states (Polyansky et al. 1997d).

Most of the labels used to characterize vibration-rotation energy levels are approximate. The only rigorous quantum numbers for water are the rotational angular momentum, $J$, the

(rotational) parity which is given by $p=(-1)^{\left(J+K_{a}+K_{c}\right)}$ and the symmetry of interchanging the two $\mathrm{H}$ atoms. This latter quantum number splits a spectrum into two, essentially distinct, series based on nuclear spin statistics. Ortho states have a nuclear spin statistical weight three times that of the para states $K_{a}+K_{c}+v_{3}$ is odd is for ortho states and even for para states.

\section{METHOD}

The starting point for this study was the many published spectra of both hot (CamyPeyret and Flaud 1975, 1976, Flaud and Camy-Peyret 1976, Flaud et al.1976, 1977, 1979, Camy-Peyret et al. 1977, 1980, Pine et al. 1983, Polyansky et al. 1996b, Esplin et al. 1998, Lanquetin et al. 1999) and cold (Camy-Peyret et al. 1985, Johns 1985, Mandin et al. 1986, 
1988, Guelachvili and Rao 1986, Belov et al. 1987, Nakano et al. 1988, Chevillard et al. 1989, Toth 1991, 1993a, 1993b, 1994a, 1994b, 1998, 1999, Pearson et al. 1991, Matsushima et al. 1995, Paso and Horneman 1995, Flaud et al. 1997, Mikailenko et al. 1997, Harder and Brault 1997, Chen et al. 2000) $\mathrm{H}_{2}{ }^{16} \mathrm{O}$ vapor. In particular we used those which gave reliable tabulations of energy levels (Camy-Peyret and Flaud 1975, 1976, Flaud and Camy-Peyret 1976, Chevillard et al. 1989 Flaud et al. 1977, 1997, Toth 1994a, 1994b, 1997, 1998, 1999, Lanquetin 1997, Lanquetin et al. 1999). A compilation of the earlier work is given by Flaud et al. (1981).

In selecting which previous tabulations of energy levels to start from, only those which also estimate statistical errors were considered. To these previous energy level studies, we added the transitions assigned by us using variational calculations (Polyansky et al. 1997b, 1997c, 1997d, 1997e, 1998, Carleer et al. 1999, Zobov et al. 1999, 2000a, 2000b) Energy levels (Bykov et al. 2000) and newly assigned transitions (Giver 2000, Haus et al. 2001, Schermaul et al. 2001) which became available during the course of the work were also included.

The most rigorous method of obtaining experimental energy levels from transition data is to use the method of over-determined linear equations as described by Flaud et al. (1976). This method may well not be practicable for the large dataset considered here; however since the method requires all the transition data, plus errors, as input, and since much of transition data recorded in earlier studies was not available to us, an alternative approach was in any case necessary.

Gas phase water spectra were divided into catagories depending on whether the water was 'hot' $(T \geq 1000 \mathrm{~K})$ or 'cold', approximately room temperature. The cold spectra were processed first. For the cold spectra, which covered the frequency region above $13,200 \mathrm{~cm}^{-1}$, all transition data was re-processed. The transitions of Mandin et al. (1986), with extra assignments by Polyansky et al. (1998), and Camy-Peyret et al. (1985) were taken electronically from Hitran (Rothman et al. 1998) and merged with more recent data of Carleer et al. (1999) and Zobov et al. (2000) to obtain reduced errors for individual frequencies. Energy levels were then generated starting from the recent low-lying energy levels of Lanquetin et 
al. (1999) whose data essentially reproduces those of Toth (1998). These low-lying levels have been determined to such high accuracy that the error in the upper state energy levels is almost entirely determined by the residual errors in the transition frequencies used. There were few problems with this step of the work, which covered data for the polyads $3 \nu+\delta$, $4 \nu, 4 \nu+\delta, 5 \nu, 5 \nu+\delta, 6 \nu, 7 \nu, 7 \nu+\delta$ and $8 \nu$

To this data was added the energy levels determined by Bykov et al. (2000) and some newly determined transitions due to Haus et al. (2001) and Schermaul et al. (2001). Finally the new assignments to the transitions of Mandin et al. (1986) due to Giver (2000), who interpretted Schwenke's (1998) work, were added. This last dataset has to be treated carefully as the new assignments do not all satisfy combination differences. Tables 8 to 17 presents our energy levels derived from the cold spectra using 7955 assigned transitions of which 4044 were used to obtain energy levels in the original studies (Camy-Peyret et al. 1985, Mandin et al. 1986) once allowance is made for misassignments.

For the 'hot' spectra, transition data was taken from our analysis of spectra of hot water recorded both in the laboratory (Polyansky et al. 1997c, 1997d, 1997e, Zobov et al. 1999, 2000a) and sunspots (Polyansky et al. 1997b, 1997c, Zobov et al. 2000a), note that the correctly calibrated laboratory data of Zobov et al. (2000a) was used. These data were merged to give an improved linelist containing over 21,000 transitions. It should be noted that the laboratory data where available is of much higher accuracy than that obtained from sunspots. Energy levels were taken from the cited compilations. These were augmented by the extensive tabulations in the thesis of Lanquetin (1997). Lanquetin's data, when confirmed by us, is of higher accuracy than ours and therefore contains important information. However the data could not be used uncritically as it contains a significant fraction of incorrect levels presumably arising from mis-assigned transitions. As the levels published by Lanquetin et al. (1999), all of which are correct, have larger errors than the corresponding errors in the thesis, we increased the errors stated in the thesis by $50 \%$. Some other levels taken from previous compilations were also found to be incorrect and were removed during the course of processing the data. 
Determining energy levels from the hot spectra was altogether more complicated as, in particular, some of these spectra are very rich in combination transitions (Zobov et al. 1999) which lead to complicated cross-linkages between the energy levels of different states. We therefore adopted a bottom up approach to the problem: vibrational states were treated from the lowest upwards in order of their band origins which are given in Table 2 . Thus the high lying rotational states of (000), for which of course only pure rotational transitions are relevant, were determined first. Next the levels (010) were determined, first by considering transitions from (000) and then by treating pure rotational transitions within (010). Thirdly the levels of (020) were determined, first from vibrational transitions from (000) and (010), then by treating pure rotational transitions within (020) and finally by considering difference bands involving levels of the (100) and (001) states. As, of course, many of these had yet to be determined at this stage of the calculation, it was necessary to iterate on the difference transitions step of the calculations. This was a common feature of all states up to and including those in the $2 \nu$ polyad and final levels could only be obtained after several iterations. After these iterations were complete we were left with a number of transitions which still had not been processed. A check was performed to see which of these transitions could be coupled into the energy levels that had been determined. After this step we still had a dataset of 194 transitions for which the energy of neither the lower nor the upper level had been determined. These 'unattached' transitions contain information on further water levels which hopefully will be used when further data becomes available.

In principle the hot and cold spectra do not link distinct sets of energy levels. In practice, besides the previously well determined low-lying rotational levels of (000) and (010), the only vibrational state present in both datasets was the state (061). Even for this vibrational state, the sets of spectra were distinct and no individual rotational level was involved in both hot and cold spectra.

After this work had been substantially completed the energy levels for the lowest 5 vibrational states due to Lanquetin et al. (2001) became available. These were used to resolve some discrepencies in the previous data. In general our results give excellent agreement with 
those of Lanquetin et al. (2001). The one notable exception is for high $J$ states with $J=K_{a}$. For many of these states our energies are systematically slightly lower. We note that our energies agree better with previous studies by the same group (Lanquetin 1997, Lanquetin et al. 1999).

Once a complete, initial set of energy levels had been constructed various consistency checks were performed. The most severe of these was to test the levels against the completely independent results of variational calculations. For reasons well documented elsewhere (Polyansky et al. 1997b, Polyansky et al. 1999, Zobov et al. 2000) comparisons were made with energy levels calculated using both the best available spectroscopically determined potential energy surface (Partridge and Schwenke 1997) and the best available ab initio procedure. In practice for low-lying levels, which in any case were well constrained by Partridge and Schwenke's levels, the ZVPT ab initio results (Polyansky et al. 1999) were used while at higher energies, where Partridge and Schwenke's levels become erratic, the more recent calculations of Zobov et al. (2000) were employed. Nearly all our levels gave energies which agreed well with those from the variational calculations; the few which lay outside the reasonable error limits of the variational calculations were removed from the compilation. Some levels, both from this study and previous ones, for which there is only marginal agreement with the variational calculations have been flagged using ? in the tables.

Table 1 summarizes our results. It presents the number of rotational energy levels (term values) obtained for each vibrational state. Figure 1 shows the proportion of vibrational states, as a function of polyad number, for which this term value data are available.

Band origins, $\omega_{0}$, for each vibrational state are given in Table 1 . In cases where transitions to the $0_{00}$ level have not been observed an estimate of the band origin is given where possible. These estimates were obtained by estimating a systematic band error for the variationally calculated energy levels and then using this to correct the $0_{00}$ from the variational calculation. This procedure has proved very reliable (Polyansky et al. 1998, Carleer et al. 1999). It is difficult to give precise errors, but the estimates given in Table 1 should be accurate to approximately plus or minus one unit in the final digit. 
Table 1 gives the source of the literature energy levels used to seed our compilation. In practice for most of the lower states, some levels were also taken from other sources. Information on this is given in the full tabulations. Table 1 provides a key to the papers used to provide energy levels retained in our final compilation. This compilation is given in Tables 2 to 17, with each table presenting the results for one polyad.

\section{ERRORS AND TABLES}

There are two types of errors which affect the energy levels compiled here: statistical and systematic. The main source of systematic errors is incorrect data, primarily misassigned transitions. Although comparisons with the variational calculation removed the glaring systematic errors this method is not guaranteed to remove all such problems.

One indicator of the reliability of a particular assignment is the number of transitions used to determine that level, $N$. Levels for which $N=1$ have not been confirmed by combination differences and therefore must be treated with more caution than those levels for which $N>1$ which have therefore been confirmed using combination differences. However two points should be noted. First not all authors provide values for $N$, in this case a value of -1 is recorded in our tables, the true value of $N$ is fairly large in nearly all of these cases. Secondly our definition of $N$ differs from that used by some other workers. We have use $N$ to record only transitions going to a particular level, i.e. where the level is the upper one. Literature $N$ 's often represent all transitions to and from a particular level. There are many levels, particular high lying rotational states, whose energies rely on a series of single transitions linking states. The assignment of a single transition to a particular level is not confirmed because further transitions are assigned from that level. Hence in such cases we believe that a value $N=1$ is appropriate. Where our data is combined with previous estimates, the value $N$ may well be a hybrid between two definitions. The values of $N$ taken from the literature can be found in the original reference if required. Finally it should be noted that even levels confirmed by combination differences may have incorrect (vibrational) 
labels. Although we believe such cases are rare, the possibility of a few such levels cannot be completely ruled out. We note that vibrational labels are actually not crucial for most applictions.

Statistical errors are altogether easier to deal with. Errors were propagated through the calculations using standard statistical procedures. In all cases when processing the data, the error of the lower level was kept fixed and used to determine the error of the upper level. The over-determined linear equation method (Flaud et al. 1976) is more even handed than this although, in practice, the upper levels have the dominant errors in nearly all cases we considered.

It should be noted that in the course of processing the data we found a small number of seemingly correctly assigned transitions which gave energy levels outside the range one would expect from the error analysis. These transitions were simply dropped from the analysis. The most likely explanation for this anomaly is that for studies which only give an average error, instead of a line by line error, the error for some lines, particularly blended ones, is significantly larger than the average value.

It is a well known property of asymmetric top molecules such as water that as the rotational angular momentum $J$ increases, neighbouring levels with either $K_{a} \sim J$ or $K_{c} \sim J$ appear as quasi-degenerate pairs. This means that in many cases it is impossible to separate between these pairs at the accuracy of the experimental data analysed here. The ab initio variational calculations used here show this degeneracy structure to a high degree of accuracy. The calculations due to Partridge and Schwenke (1997) do not show this structure due to problems with convergence (Polyansky et al. 1997c). The ab initio calculations were therefore used as a guide to which levels should be considered degenerate. Data for levels which were assumed to be degenerate were processed together. In such cases the lower statistical weight para levels, which are given by $K_{a}+K_{c}+v_{3}$ even, are flagged as 'd' for degenerate in the tables.

Tables 2 to 17 present our final results tabulated by vibrational state. The tables give the rotational term values relative to the ground state are given in $\mathrm{cm}^{-1}$. Note $J_{K_{a} K_{c}}$ combi- 
nations for which no levels were determined have been omitted from the tables to save space. For each entry, we also give the statistical error with which the level was determined, in units of $0.001 \mathrm{~cm}^{-1}$, the number of transitions to the level, $N$ defined above, and reference(s) to the source data. Vibrational state labels are given at the head of each column in normal mode and local mode notation in that order. These tables can be downloaded in electronic form via web page www.tampa.phys.ucl.ac.uk/jonny/waterlevels.html or by anonymous ftp from ftp.tampa.phys.ucl.ac.uk and looking in directory pub/astrodata/water/levels.

The tables present all available energy level data with one exception. Bykov et al. (2000) give a value of $14585.1745 \pm 0.0049 \mathrm{~cm}^{-1}$ for the $7_{07}$ level of the bending overtone state with $v_{2}=10$ (00 10 in local mode notation) based on 3 transitions. Although we have no particular reason to suspect this result is incorrect, we were not able to confirm it with the available variational linelists and are cautious since there is a history of incorrect results for high lying bending states obtained from the analysis of perturbations (see Polyansky et al. 1996a).

The highest energy levels considered here contain over $21000 \mathrm{~cm}^{-1}$ in rotational energy or over $25000 \mathrm{~cm}^{-1}$ in vibrational energy. These values represent approximately $47 \%$ and $56 \%$ of the dissociation energy of water. They are significantly above $11100 \mathrm{~cm}^{-1}$, the energy at which the water molecule can go linear (Tarczay et al. 1999, Kain et al. 2000). However, despite the high energies probed in this study, we had little difficulty assigning vibrational quantum number to all the states considered. Vibrational quantum numbers can be somewhat indeterminate in regions where rotational levels from two vibrational states interact, usually called perturbations (see Polyansky et al. 1997d for example), but in no case did we find any general loss of quantum numbers, behaviour which is often associated with the onset of chaos in a quantum mechanical system. 


\section{SUMMARY}

We have taken advantage of recent advances in the spectroscopy of water to determine 12248 rotation-vibration energy levels of $\mathrm{H}_{2}{ }^{16} \mathrm{O}$. These levels belong to 107 different vibrational states of water. Accurate values or reliable estimates of the vibrational band origins for 101 vibrational states have also been determined. These energy levels give a consolidated tabulation of energy levels as well as a significant increase in their number. For the states up to and including the $3 \nu+\delta$ polyad we give 9335 levels, of which 4933 are new, and above this we give 2913 levels, of which 1667 levels are new. This represents a more than doubling of the energy levels in both regions. We believe this dataset of energy levels will be useful for many applications.

The levels tabulated here have already been used to determine the partition function of water as a function of temperature, and related thermodynamic quantities, to much higher accuracy than any previous determination (Vidler and Tennyson 2000). The levels have also been used to help fix the height of the barrier to linearity in water (Kain et al. 2000) and are presently being used to characterize a full, spectroscopically determined potential for the water molecule. The successful determination of this potential will undoubtedly lead to the assignment of further water transitions and hence the determination of yet more rotation-vibration energy levels. It is our intention to maintain and distribute an updated list of these levels as further data becomes available.

\section{ACKNOWLEDGMENTS}

We thank Sophie Kain and Matthew Hall for their help in compiling the previous data, and Marcus Vidler for help with error checking. We are grateful to Jean-Marie Flaud, Laurence Giver, Per Jensen and Robert Toth for supplying information about their own

work. This work was supported by a number of agencies including the Royal Society, NATO, the UK EPSRC and NERC, the Russian Fund for Fundamental Studies and the Natural 
Sciences and Engineering Research Council of Canada (NSERC).

\section{REFERENCES}

Belov S.P., I.N. Kozin, O.L. Polyansky, M.Y. Tretyakov and N.F. Zobov, J. Molec. Spec. 126(1), 113-117 (1987).

Bykov A., O. Naumenko, L. Sinitsa, B. Voronin, J.-M. Flaud, C. Camy-Peyret, and R. Lanquetin, J. Molec. Spec. 205(1), 1-8 (2001).

Camy-Peyret, C. and J.-M. Flaud, Thèse de doctorat de sciences, Université de Pierre et Marie Curie, Paris (1975).

Camy-Peyret, C. and J.-M. Flaud, J. Molec. Spec. 59(3), 327-337 (1976).

Camy-Peyret, C., J.-M. Flaud, J.-P. Maillard and G. Guelachvili, Molec. Phys. 33(6), 1641-1650 (1977).

Camy-Peyret, C., J.-M. Flaud and J.-P. Maillard, J. Phys. Lett. 41(2), L23-L26 (1980).

Camy-Peyret, C., J.-M. Flaud, J.-Y. Mandin, J.-P. Chevillard, J. Brault, D.A. Ramsay, M. Vervloet and J. Chauville, J. Molec. Spec. 113(1), 208-228 (1985).

Carleer, M., A. Jenouvrier, A.-C. Vandaele, P.F. Bernath, M.F. Mérienne, R. Colin, N.F. Zobov, O.L. Polyansky, J. Tennyson and V.A. Savin, J. Chem. Phys. 111(6), 2444$2450(1999)$.

Carter, S. and N.C. Handy, J. Chem. Phys. 87, 4294-4301 (1987).

Chapovsky P.L., Ann. Rev. Phys. Chem. 50, 315-345 (1999)

Chen P., J.C. Pearson, H.M. Pickett, S. Matsuura and G.A. Blake, Astrophys. J. Suppl. 128(1), 371-385 (2000).

Chevillard, J.P., J.Y. Mandin, J.-M. Flaud and C. Camy-Peyret, Can. J. Phys. 67(11), 1065-1084 (1989).

Child, M.S., T. Weston and J. Tennyson, Mol. Phys. 96, 371-379 (1999).

Coudert L.H., J. Molec. Spec. 181(2), 246-273 (1997). 
Esplin, M.P., R.B. Wattson, M.L. Hoke and L.S. Rothman, J. Quant. Spec. Radiat. Transfer 60(5), 771-738 (1998).

Flaud, J.-M., C. Camy-Peyret and J.-P. Maillard, Molec. Phys. 32(2), 499-521 (1976).

Flaud, J.-M., C. Camy-Peyret, J.-P. Maillard, G. Guelachvili, J. Molec. Spec. 65, 219-228 (1977).

Flaud J.-M., C. Camy-Peyret, N.K. Rao, D.W. Chen, Y.S. Hoh and J.-P. Maillard, J. Molec. Spec. 75(3), 339-362 (1979).

Flaud, J.-M., C. Camy-Peyret and R.A. Toth, 'Water vapour line parameters from microwave to medium infrared' (Pergamon, Oxford, UK, 1981).

Flaud, J.-M., C. Camy-Peyret, A. Bykov, O. Naumenko, T. Petrova, A. Scherbakov and L. Sinitsa, J. Molec. Spec. 183(2), 300-309 (1997).

Giver L.P., personnal communication (2000), based on Schwenke (1998).

Guelachvili, G. and N.K. Rao, (eds.) Handbook of IR standards (Academic Press, Orlando, 1986).

Jensen P., J. Molec. Spec. 133, 438-460 (1989).

Johns J.W.C., J. Opt. Soc. Am B 2(8), 1340-1354 (1985).

Kauppi E. and L. Halonen, J. Phys. Chem. 94, 5779-5785 (1990).

Halonen, L., Adv. Chem. Phys. 104, 41-179 (1998).

Halonen, L. and T. Carrington Jr, J. Chem. Phys. 88, 4171-4185 (1988).

Harder, J.W. and J.W. Brault, J. Geophys. Res. 102(D5) 6245-6252 (1997).

Haus, N., W. Ubachs, P.F. Levert, O.L. Polyansky, N.F. Zobov and J. Tennyson, J. Molec. Spec. 205(1), 117-121 (2001).

Kain, J.S., O.L. Polyansky and J. Tennyson, Chem. Phys. Letts. 317(3-5), 365-371 (2000).

Kain, J.S., O.L. Polyansky and J. Tennyson, to be published (2001).

Lanquetin, R., Ph.D. Thesis, Université de Paris Nord (1997).

Lanquetin, R., L.H. Coudert and C. Camy-Peyret, J Molec. Spec. 195(1), 54-67 (1999).

Lanquetin, R., L.H. Coudert and C. Camy-Peyret, J Molec. Spec. 206(1), 83-103 (2001). 
Mandin, J.Y., J.P. Chevillard, C. Camy-Peyret and J.-M. Flaud, J. Molec. Spec. 116(1), $167-190$ (1986).

Mandin, J.Y., J.P. Chevillard, J.-M. Flaud and C. Camy-Peyret, Can. J. Phys. 66(11), 997-1011 (1988).

Matsushima P., H. Odashima, T. Iwasaki, S. Tsunekawa and K. Tagaki, J. Molec. Struct. 352, 371-378 (1995).

Mikhailenko S.N.,V.G. Tyuterev, K.A. Keppler, B.P. Winnewisser, M. Winnewisser, G. Mellau, S. Klee and K.N. Rao, J. Molec. Spec. 184(2), 330-349 (1997).

Nakano K., A. Saito and N. Ohashi, J. Molec. Spec. 131, 405-406 (1988).

Nela M., D. Permogorov, D. Miani and L. Halonen, J. Chem. Phys., 113(5), 1795-1801 (2000).

Partridge H. and D. W. Schwenke, J. Chem. Phys. 106(11), 4618-4639 (1997).

Paso R. and V.M Horneman, J. Opt. Soc. Am. B, 12(10), 1813-1838 (1995).

Paulse C.D. and J. Tennyson, J. Molec. Spec. 168, 313-322 (1994).

Pearson J.C., F.C. de Lucia, T. Anderson, E. Herbst and P. Helminger, Astrophys. J. 379(2), L41-L43 (1991).

Pine A.S., M.J. Coulombe, C. Camy-Peyret and J.-M. Flaud, J. Phys. Chem. Ref. Data 12(3), 413-465 (1983).

Polyansky O.L., J. Molec. Spec., 12(1), 79-87 (1985).

Polyansky O.L., P. Jensen and J. Tennyson, J. Chem. Phys., 101, 7651-7657 (1994).

Polyansky O.L., P. Jensen and J. Tennyson, J. Chem. Phys., 105(15), 6490-6497 (1996a).

Polyansky O.L., J.R. Busler, B.J. Guo, K. Zhang and P. Bernath, J. Molec. Spec., 176(2), 305-315 (1996b).

Polyansky O.L., N.F. Zobov, S. Viti, J. Tennyson, P.F. Bernath and L. Wallace, Science 277(3324), 346-349 (1997a).

Polyansky O.L., N.F. Zobov, S. Viti, J. Tennyson, P.F. Bernath and L. Wallace, Astrophys. J., 489(2), L205-L208 (1997b). 
Polyansky O.L., N.F. Zobov, S. Viti, J. Tennyson, P.F. Bernath and L. Wallace, J. Molec. Spec., 186(2), 422-447 (1997c).

Polyansky O.L., J. Tennyson and P.F. Bernath, J. Molec. Spec., 186(2), 213-221 (1997d)

Polyansky O.L., N.F. Zobov, J. Tennyson, J.A. Lotoski and P.F. Bernath, J. Molec. Spec., 184(1), 35-50 (1997e).

Polyansky O.L., N.F. Zobov, S. Viti and J. Tennyson, J. Molec. Spec. 189(2), 291-300 (1998).

Polyansky O.L., J. Tennyson and N. F. Zobov, Spectrochemica Acta, 55A(3), 659-693 (1999).

Rose J.P. and M.E. Kellman, J. Chem. Phys. 105(17), 7348-7363 (1996).

Schermaul R., A.A.D. Canas, R.C.M. Learner, J.W. Brault, O.L. Polyansky, N.F. Zobov, D. Belmiloud and J. Tennyson, J. Molec. Spec. to be submitted (2001).

Schwenke D.W., J Molec Spec. 190, 397-402 (1998).

Tarczay G., A.G. Császár, W. Klopper, V. Szalay, W.D. Allen and H.F. Schaefer III, J. Chem. Phys., 110(24), 11971-11981 (1999).

Toth, R.A., J. Optical Soc. Am. B 8(11), 2236-2255 (1991).

Toth, R.A., Optical Soc. Am. B 10(9), 1526-1544 (1993).

Toth, R.A., Optical Soc. Am. B 10(11), 2006-2030 (1993).

Toth, R.A., J. Molec. Spec. 166(1), 176-183 (1994a)

Toth, R.A., Appl. Optics 33(21), 4851-4867 (1994b).

Toth, R.A., J. Molec. Spec. 190(2), 379-396 (1998).

Toth, R.A., J. Molec. Spec. 194(1), 28-42 (1999).

Tyuterev, V.G., V.I. Starikov, S.A. Tahkun and S.N. Mikhailenko, J. Molec. Spec. 170(1), 38-58 (1995).

Vidler, M. and J. Tennyson, J. Chem. Phys. 113, 9766-9771 (2000).

Zobov, N.F., O.L. Polyansky, J. Tennyson, J.A. Lotoski, P. Colarusso, K.-Q. Zhang and P.F. Bernath, J. Molec. Spec. 193(1), 118-136 (1999). 
Zobov, N.F., O.L. Polyansky, J. Tennyson, S.V. Shirin, R. Nassar, T. Hirao, T. Imajo, P.F. Bernath and L. Wallace, Astrophys. J. 530(2), 994-998 (2000a).

Zobov, N.F., D. Belmiloud, O.L. Polyansky, J. Tennyson, S.V. Shirin, M. Carleer, A. Jenouvrier, A.-C. Vandaele, P.F. Bernath, M.F. Marienne and R. Colin, J. Chem. Phys., 113(4) 1546-1552 (2000b).

\section{FIGURE CAPTIONS}

Figure 1 Coverage of vibrational bands by polyad: possible (dots) and observed (triangles) vibrational bands in each polyad. The integer $n$ on the x-axis denotes the polyad $n \nu$ and $n+0.5$ represents the polyad $n \nu+\delta$. 
TABLE I.: Summary of results. Given are vibrational band origin, $\omega_{0}$, number of rotational term values determined and reference for the starting energies for each vibrational state. The key lists references used in all tables.

\begin{tabular}{|c|c|c|c|}
\hline$v_{1} v_{2} v_{3}$ & $m n^{ \pm} v_{2}$ & $\omega_{0} / \mathrm{cm}^{-1}$ & levels \\
\hline 000 & 000 & 0.0 & $1026 \mathrm{~b}$ \\
\hline 010 & 001 & 1594.746 & $916 \mathrm{~b}, \mathrm{~g}$ \\
\hline 020 & 002 & 3151.630 & $750 \mathrm{c}$ \\
\hline 100 & $10^{+} 0$ & 3657.053 & $757 \mathrm{c}, \mathrm{f}$ \\
\hline 001 & $10^{-} 0$ & 3755.929 & $829 \mathrm{c}, \mathrm{f}$ \\
\hline 030 & 003 & 4666.790 & $489 \mathrm{~h}$ \\
\hline 110 & $10^{+} 1$ & 5234.978 & $445 \mathrm{i}$ \\
\hline 011 & $10^{-} 1$ & 5331.265 & $582 \mathrm{f}$ \\
\hline 040 & 004 & 6134.015 & $250 \mathrm{j}$ \\
\hline 120 & $10^{+} 2$ & 6775.093 & $166 \mathrm{j}$ \\
\hline 021 & $10^{-} 1$ & 6871.520 & $346 \mathrm{j}, \mathrm{g}$ \\
\hline 200 & $20^{+} 0$ & 7201.540 & $203 \mathrm{j}$ \\
\hline 101 & $20^{-} 0$ & 7249.818 & $299 \mathrm{j}$ \\
\hline 002 & 110 & 7445.045 & $237 \mathrm{j}, \mathrm{g}$ \\
\hline 050 & 005 & 7542.437 & 105 \\
\hline 130 & $10^{+} 3$ & 8273.976 & $55 \mathrm{k}$ \\
\hline 031 & $10^{-} 3$ & 8373.853 & $229 \mathrm{k}, \mathrm{g}$ \\
\hline 210 & $20^{+} 1$ & 8761.582 & $58 \mathrm{k}$ \\
\hline 111 & $20^{-} 1$ & 8806.999 & $209 \mathrm{k}, \mathrm{g}$ \\
\hline 060 & 006 & 8869.954 & 37 \\
\hline
\end{tabular}




\begin{tabular}{|c|c|c|c|}
\hline 012 & 111 & 9000.136 & $148 \mathrm{k}$ \\
\hline 041 & $10^{-4}$ & 9833.585 & $144 \mathrm{~m}$ \\
\hline 220 & $20^{+} 2$ & 10284.367 & $33 \mathrm{~m}$ \\
\hline 121 & $20^{-} 2$ & 10328.731 & $86 \mathrm{~m}$ \\
\hline 022 & 112 & 10521.8 & $47 \mathrm{~m}$ \\
\hline 300 & $30^{+} 0$ & 10599.686 & $83 \mathrm{~m}$ \\
\hline 201 & $30^{-} 0$ & 10613.355 & $120 \mathrm{~m}$ \\
\hline 102 & $21^{+} 0$ & 10868.876 & $73 \mathrm{~m}$ \\
\hline 003 & $21^{-} 0$ & 11032.406 & $95 \mathrm{~m}$ \\
\hline 070 & 007 & & 3 \\
\hline 051 & $10^{-} 5$ & 11242.8 & $64 \mathrm{n}$ \\
\hline 230 & $20^{+} 3$ & 11767.390 & $37 \mathrm{n}$ \\
\hline 131 & $20^{-} 3$ & 11813.207 & $85 \mathrm{n}$ \\
\hline 032 & 113 & 12007.776 & $42 \mathrm{n}$ \\
\hline 310 & $30^{+} 1$ & 12139.315 & $73 \mathrm{n}$ \\
\hline 211 & $30^{-} 1$ & 12151.255 & $122 \mathrm{n}, \mathrm{o}$ \\
\hline 112 & $21^{+} 1$ & 12407.662 & $72 \mathrm{n}, \mathrm{o}$ \\
\hline 013 & $21^{-} 1$ & 12565.007 & $88 \mathrm{n}, \mathrm{o}$ \\
\hline 080 & 008 & & 11 \\
\hline 160 & $10^{+} 6$ & & 4 \\
\hline 061 & $10^{-} 6$ & 12586. & 26 \\
\hline 240 & $20^{+} 4$ & 13205.1 & 44 \\
\hline 141 & $20^{-} 4$ & 13256.2 & 49 \\
\hline 042 & 114 & 13453.6 & 39 \\
\hline 320 & $30^{+} 2$ & 13640.7 & 77 \\
\hline 221 & $30^{-} 2$ & 13652.656 & 96 \\
\hline 122 & $21^{+} 2$ & 13910.896 & 66 \\
\hline
\end{tabular}




\begin{tabular}{|c|c|c|c|}
\hline 023 & $21^{-2}$ & 14066.194 & 83 \\
\hline 400 & $40^{+} 0$ & 13828.277 & 100 \\
\hline 301 & $40^{-} 0$ & 13830.938 & 121 \\
\hline 202 & $31^{+} 0$ & 14221.161 & 102 \\
\hline 103 & $31^{-} 0$ & 14318.812 & 114 \\
\hline 004 & 220 & 14537.504 & 72 \\
\hline 170 & $10^{+} 7$ & 13661.1 & 6 \\
\hline 071 & $10^{-7}$ & 13835.372 & 12 \\
\hline 250 & $20^{+} 5$ & & 1 \\
\hline 151 & $20^{-} 5$ & 14648.2 & 23 \\
\hline 052 & 115 & & 1 \\
\hline 330 & $30^{+} 3$ & 15108.239 & 30 \\
\hline 231 & $30^{-} 3$ & 15119.028 & 72 \\
\hline 132 & $21^{+} 3$ & 15377.7 & 19 \\
\hline 033 & $21^{-} 3$ & 15534.709 & 64 \\
\hline 410 & $40^{+} 1$ & 15344.503 & 74 \\
\hline 311 & $40^{-} 1$ & 15347.956 & 93 \\
\hline 212 & $31^{+} 1$ & 15742.795 & 58 \\
\hline 113 & $31^{-1}$ & 15832.765 & 85 \\
\hline 340 & $30^{+} 4$ & 16534.3 & 30 \\
\hline 241 & $30^{-} 4$ & 16546.3 & 35 \\
\hline 142 & $21^{+} 4$ & 16796.0 & 40 \\
\hline 043 & $21^{-4}$ & 16967.5 & 18 \\
\hline 420 & $40^{+} 2$ & 16823. & 51 \\
\hline 321 & $40^{-} 2$ & 16821.635 & 62 \\
\hline 222 & $31^{+} 2$ & 17227.3 & 40 \\
\hline 123 & $31^{-2}$ & 17312.539 & 45 \\
\hline
\end{tabular}




\begin{tabular}{|c|c|c|}
\hline 500 & $50^{+} 0$ & 16898.4 \\
\hline 401 & $50^{-} 0$ & 16898.842 \\
\hline 302 & $41^{+} 0$ & 17458.354 \\
\hline 203 & $41^{-} 0$ & 17495.528 \\
\hline 104 & $32^{+} 0$ & 17748.1 \\
\hline 053 & $21^{-} 5$ & 18350.3 \\
\hline 430 & $40^{+} 3$ & 18271. \\
\hline 331 & $40^{-} 3$ & 18265.820 \\
\hline 133 & $31^{-} 3$ & 18758.6 \\
\hline 034 & 223 & 18977.2 \\
\hline 510 & $50^{+} 1$ & 18392.974 \\
\hline 411 & $50^{-} 1$ & 18393.314 \\
\hline 213 & $41^{-} 1$ & 18989.960 \\
\hline 063 & $21^{-5}$ & 19721. \\
\hline 341 & $40^{-} 4$ & 19679.1 \\
\hline 520 & $50^{+} 2$ & 19864. \\
\hline 421 & $50^{-} 2$ & 19863.3 \\
\hline 223 & $41^{-} 2$ & 20442.3 \\
\hline 600 & $60^{+} 0$ & 19781. \\
\hline 501 & $60^{-} 0$ & 19781.105 \\
\hline 402 & $51^{+} 0$ & 20533.6 \\
\hline 303 & $51^{-} 0$ & 20543.137 \\
\hline 431 & $50^{-} 3$ & 21312 . \\
\hline 610 & $60^{+} 1$ & 21221.569 \\
\hline 511 & $60^{-} 1$ & 21221.828 \\
\hline 115 & $42^{-} 1$ & 22513 . \\
\hline 50 & $60^{+} 2$ & 22631.390 \\
\hline
\end{tabular}




\begin{tabular}{cccc}
521 & $60^{-} 2$ & 22629.288 & 10 \\
700 & $70^{+} 0$ & 22529.296 & 42 \\
601 & $70^{-} 0$ & 22529.441 & 37 \\
611 & $70^{-} 1$ & 23940. & 9 \\
800 & $80^{+} 0$ & & 20 \\
701 & $80^{-} 0$ & 25120.278 & 24 \\
\hline & & & \\
Total & & & \\
\hline
\end{tabular}

Key for all tables

a This work

b Lanquetin et al. (1999)

c Toth (1999)

d level fixed as degenerate

e Lanquetin (1997)

f Flaud and Camy ${ }^{-}$Peyret (1976)

g Camy-Peyret et al. (1977)

h Flaud et al. (1977)

i Camy-Peyret and Flaud (1975)

j Toth (1994b)

k Mandin et al. (1988)

1 Bykov et al. (2001)

m Chevillard et al. (1989)

n Flaud et al. (1997)

o Toth (1994a)

p Schermaul et al. (2001)

q Giver (2000) 
r Haus et al. (2001)

s Lanquetin et al. (2001)

? doubtful level 
TABLE II.: Term values for the ground and first excited state of $\mathrm{H}_{2}{ }^{16} \mathrm{O}$.

\begin{tabular}{|c|c|c|c|c|c|c|}
\hline \multicolumn{3}{|c|}{$J K_{a} K_{c}$} & \multirow[t]{2}{*}{000 or } & \multirow[t]{2}{*}{000} & \multirow{2}{*}{$\begin{array}{r}010 \text { or } \\
1594.74633\end{array}$} & \multirow{2}{*}{$\frac{001}{0.24-1 \mathrm{~b}}$} \\
\hline 0 & 0 & 0 & & & & \\
\hline 1 & 0 & 1 & 23.79436 & $0.01-1 \mathrm{~b}$ & 1618.55709 & $0.09-1 \mathrm{~b}$ \\
\hline 1 & 1 & 1 & 37.13711 & $0.03-1 \mathrm{~b}$ & 1634.96710 & $0.12-1 \mathrm{~b}$ \\
\hline 1 & 1 & 0 & 42.37174 & $0.02-1 \mathrm{~b}$ & 1640.50582 & $0.09-1 \mathrm{~b}$ \\
\hline 2 & 0 & 2 & 70.09079 & $0.03-1 \mathrm{~b}$ & 1664.96469 & $0.11-1 \mathrm{~b}$ \\
\hline 2 & 1 & 2 & 79.49639 & $0.02-1 \mathrm{~b}$ & 1677.06139 & $0.10-1 \mathrm{~b}$ \\
\hline 2 & 1 & 1 & 95.17591 & $0.03-1 \mathrm{~b}$ & 1693.64982 & $0.12-1 \mathrm{~b}$ \\
\hline 2 & 2 & 1 & 134.90163 & $0.02-1 \mathrm{~b}$ & 1742.30564 & $0.09-1 \mathrm{~b}$ \\
\hline 2 & 2 & 0 & 136.16390 & $0.03-1 \mathrm{~b}$ & 1743.48622 & $0.07-1 \mathrm{~b}$ \\
\hline 3 & 0 & 3 & 136.76166 & $0.03-1 \mathrm{~b}$ & 1731.89667 & $0.09-1 \mathrm{~b}$ \\
\hline 3 & 1 & 3 & 142.27846 & $0.03-1 \mathrm{~b}$ & 1739.48358 & $0.07-1 \mathrm{~b}$ \\
\hline 3 & 1 & 2 & 173.36580 & $0.03-1 \mathrm{~b}$ & 1772.41346 & $0.08-1 \mathrm{~b}$ \\
\hline 3 & 2 & 2 & 206.30140 & $0.04-1 \mathrm{~b}$ & 1813.78749 & $0.09-1 \mathrm{~b}$ \\
\hline 3 & 2 & 1 & 212.15636 & $0.03-1 \mathrm{~b}$ & 1819.33514 & $0.06-1 \mathrm{~b}$ \\
\hline 3 & 3 & 1 & 285.21933 & $0.04-1 \mathrm{~b}$ & 1907.45143 & $0.06-1 \mathrm{~b}$ \\
\hline 3 & 3 & 0 & 285.41858 & $0.03-1 \mathrm{~b}$ & 1907.61580 & $0.03-1 \mathrm{~b}$ \\
\hline 4 & 0 & 4 & 222.05274 & $0.04-1 \mathrm{~b}$ & 1817.45101 & $0.09-1 \mathrm{~b}$ \\
\hline 4 & 1 & 4 & 224.83838 & $0.03-1 \mathrm{~b}$ & 1821.59684 & $0.06-1 \mathrm{~b}$ \\
\hline 4 & 1 & 3 & 275.49702 & $0.04-1 \mathrm{~b}$ & 1875.46972 & $0.04-1 \mathrm{~b}$ \\
\hline 4 & 2 & 3 & 300.36228 & $0.03-1 \mathrm{~b}$ & 1908.01637 & $0.03-1 \mathrm{~b}$ \\
\hline 4 & 2 & 2 & 315.77952 & $0.04-1 \mathrm{~b}$ & 1922.90113 & $0.05-1 \mathrm{~b}, \mathrm{a}$ \\
\hline 4 & 3 & 2 & 382.51690 & $0.03-1 \mathrm{~b}$ & 2004.81575 & $0.08-1 \mathrm{~b}$ \\
\hline 4 & 3 & 1 & 383.84251 & $0.04-1 \mathrm{~b}$ & 2005.91716 & $0.07-1 \mathrm{~b}$ \\
\hline
\end{tabular}




\begin{tabular}{|c|c|c|c|c|c|c|}
\hline 4 & 4 & 1 & 488.10771 & $0.04-1 \mathrm{~b}$ & 2129.59934 & $0.06-1 \mathrm{~b}$ \\
\hline 4 & 4 & 0 & 488.13417 & $0.04-1 \mathrm{~b}$ & 2129.61867 & $0.07-1 \mathrm{~b}, \mathrm{a}$ \\
\hline 5 & 0 & 5 & 325.34790 & $0.03-1 \mathrm{~b}$ & 1920.76653 & $0.08-1 \mathrm{~b}, \mathrm{a}$ \\
\hline 5 & 1 & 5 & 326.62546 & $0.04-1 \mathrm{~b}$ & 1922.82908 & $0.05-1 \mathrm{~b}$ \\
\hline 5 & 1 & 4 & 399.45753 & $0.03-1 \mathrm{~b}$ & 2000.86307 & $0.08-1 \mathrm{~b}$ \\
\hline 5 & 2 & 4 & 416.20873 & $0.04-1 \mathrm{~b}$ & 2024.15276 & $0.07-1 \mathrm{~b}, \mathrm{a}$ \\
\hline 5 & 2 & 3 & 446.51069 & $0.03-1 \mathrm{~b}$ & 2053.96866 & $0.06-1 \mathrm{~b}$ \\
\hline 5 & 3 & 3 & 503.96812 & $0.04-1 \mathrm{~b}$ & 2126.40773 & $0.07-1 \mathrm{~b}, \mathrm{a}$ \\
\hline 5 & 3 & 2 & 508.81210 & $0.03-1 \mathrm{~b}$ & 2130.49443 & $0.06-1 \mathrm{~b}$ \\
\hline 5 & 4 & 2 & 610.11448 & $0.04-1 \mathrm{~b}$ & 2251.69528 & $0.05-1 \mathrm{~b}, \mathrm{a}$ \\
\hline 5 & 4 & 1 & 610.34125 & $0.04-1 \mathrm{~b}$ & 2251.86254 & $0.07-1 \mathrm{~b}, \mathrm{a}$ \\
\hline 5 & 5 & 1 & 742.07308 & $0.06-1 \mathrm{~b}$ & 2406.14097 & $0.08-1 \mathrm{~b}, \mathrm{a}$ \\
\hline 5 & 5 & 0 & 742.07635 & $0.05-1 \mathrm{~b}$ & 2406.14318 & $0.06-1 \mathrm{~b}, \mathrm{a}$ \\
\hline 6 & 0 & 6 & 446.69659 & $0.04-1 \mathrm{~b}$ & 2041.78048 & $0.10-1 \mathrm{~b}$ \\
\hline 6 & 1 & 6 & 447.25237 & $0.03-1 \mathrm{~b}$ & 2042.75332 & $0.06-1 \mathrm{~b}, \mathrm{a}$ \\
\hline 6 & 1 & 5 & 542.90577 & $0.04-1 \mathrm{~b}$ & 2146.26375 & $0.10-1 \mathrm{~b}, \mathrm{a}$ \\
\hline 6 & 2 & 5 & 552.91143 & $0.03-1 \mathrm{~b}$ & 2161.28604 & $0.08-1 \mathrm{~b}, \mathrm{a}$ \\
\hline 6 & 2 & 4 & 602.77351 & $0.04-1 \mathrm{~b}$ & 2211.19064 & $0.09-1 \mathrm{~b}, \mathrm{a}$ \\
\hline 6 & 3 & 4 & 648.97876 & $0.04-1 \mathrm{~b}$ & 2271.71231 & $0.07-1 \mathrm{~b}, \mathrm{a}$ \\
\hline 6 & 3 & 3 & 661.54894 & $0.04-1 \mathrm{~b}$ & 2282.58958 & $0.09-1 \mathrm{~b}, \mathrm{a}$ \\
\hline 6 & 4 & 3 & 756.72487 & $0.04-1 \mathrm{~b}$ & 2398.38159 & $0.06-1 \mathrm{~b}, \mathrm{a}$ \\
\hline 6 & 4 & 2 & 757.78023 & $0.05-1 \mathrm{~b}$ & 2399.16554 & $0.08-1 \mathrm{~b}, \mathrm{a}$ \\
\hline 6 & 5 & 2 & 888.59890 & $0.04-1 \mathrm{~b}$ & 2552.85743 & $0.07-1 \mathrm{~b}, \mathrm{a}$ \\
\hline 6 & 5 & 1 & 888.63271 & $0.05-1 \mathrm{~b}$ & 2552.87979 & $0.08-1 \mathrm{~b}, \mathrm{a}$ \\
\hline 6 & 6 & 1 & 1045.05811 & $0.04-1 \mathrm{~b}$ & 2733.96290 & $0.08-1 \mathrm{~b}, \mathrm{a}$ \\
\hline 6 & 6 & 0 & 1045.05842 & $0.05-1 \mathrm{~b}$ & 2733.96305 & $0.09-1 \mathrm{~b}, \mathrm{a}$ \\
\hline & 0 & 7 & 586.24358 & $0.04-1 \mathrm{~b}$ & 2180.64294 & $0.09-1 \mathrm{~b}, \mathrm{a}$ \\
\hline
\end{tabular}




\begin{tabular}{|c|c|c|c|c|c|c|}
\hline & 1 & 7 & 586.47920 & $0.04-1 \mathrm{~b}$ & 2181.08989 & $0.09-1 \mathrm{~b}, \mathrm{a}$ \\
\hline & 1 & ( & 704.21410 & $0.04-1 \mathrm{~b}$ & 2309.73025 & $0.09-1 \mathrm{~b}, \mathrm{a}$ \\
\hline & 2 & ( & 709.60823 & $0.05-1 \mathrm{~b}$ & 2318.53989 & $0.09-1 \mathrm{~b}, \mathrm{a}$ \\
\hline & 2 & 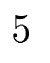 & 782.40990 & $0.04-1 \mathrm{~b}$ & 2392.59258 & $0.08-1 \mathrm{~b}, \mathrm{a}$ \\
\hline & 3 & 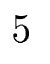 & 816.69428 & $0.05-1 \mathrm{~b}$ & 2439.95449 & $0.09-1 \mathrm{~b}, \mathrm{a}$ \\
\hline & 3 & 4 & 842.35669 & $0.04-1 \mathrm{~b}$ & 2462.87535 & $0.08-1 \mathrm{~b}, \mathrm{a}$ \\
\hline & 4 & 4 & 927.74395 & $0.05-1 \mathrm{~b}$ & 2569.50804 & $0.08-1 \mathrm{~b}, \mathrm{a}$ \\
\hline & 4 & 3 & 931.23724 & $0.04-1 \mathrm{~b}$ & 2572.13934 & $0.07-1 \mathrm{~b}, \mathrm{a}$ \\
\hline 7 & 5 & 3 & 1059.64676 & $0.06-1 \mathrm{~b}$ & 2724.04156 & $0.09-1 \mathrm{~b}, \mathrm{a}$ \\
\hline & 5 & 2 & 1059.83560 & $0.04-1 \mathrm{~b}$ & 2724.16734 & $0.08-1 \mathrm{~b}, \mathrm{a}$ \\
\hline 7 & 6 & 2 & 1216.18988 & $0.06-1 \mathrm{~b}$ & 2905.43078 & $0.09-1 \mathrm{~b}, \mathrm{a}$ \\
\hline 7 & 6 & 1 & 1216.19465 & $0.05-1 \mathrm{~b}, \mathrm{a}$ & 2905.43370 & $0.08-1 \mathrm{~b}, \mathrm{a}$ \\
\hline 7 & 7 & 1 & 1394.81424 & $0.07-1 \mathrm{~b}$ & 3109.91156 & $0.11-1 \mathrm{~b}$ \\
\hline 7 & 7 & 0 & 1394.81437 & $0.06-1 \mathrm{~b}$ & 3109 & $0.09-1 \mathrm{~b}, \mathrm{a}$ \\
\hline 8 & 0 & 8 & 744.06370 & $0.05-1 \mathrm{~b}$ & 2337.46345 & $0.11-1 \mathrm{~b}, \mathrm{a}$ \\
\hline & 1 & 8 & 744.16273 & $0.04-1 \mathrm{~b}$ & 2337.66694 & $0.09-1 \mathrm{~b}, \mathrm{a}$ \\
\hline & 1 & 7 & 882.89037 & $0.05-1 \mathrm{~b}$ & 2490.35413 & $0.10-1 \mathrm{~b}, \mathrm{a}$ \\
\hline & 2 & 7 & 885.60032 & $0.04-1 \mathrm{~b}$ & 2495.16587 & $0.08-1 \mathrm{~b}, \mathrm{a}$ \\
\hline & 2 & 6 & 982.91176 & $0.05-1 \mathrm{~b}$ & 2595.81299 & $0.10-1 \mathrm{~b}, \mathrm{a}$ \\
\hline & 3 & 6 & 1006.11607 & $0.04-1 \mathrm{~b}$ & 2630.19268 & $0.08-1 \mathrm{~b}, \mathrm{a}$ \\
\hline & 3 & 5 & 1050.15772 & $0.05-1 \mathrm{~b}$ & 2670.78971 & $0.08-1 \mathrm{~b}, \mathrm{a}$ \\
\hline & 4 & 5 & 1122.70869 & $0.04-1 \mathrm{~b}$ & 2764.69760 & $0.09-1 \mathrm{~b}$ \\
\hline & 4 & 4 & 1131.77566 & $0.05-1 \mathrm{~b}$ & 2771.69021 & $0.10-1 \mathrm{~b}, \mathrm{a}$ \\
\hline 8 & 5 & 4 & 1255.16692 & $0.04-1 \mathrm{~b}$ & 2919.63318 & $0.08-1 \mathrm{~b}$ \\
\hline 8 & 5 & 3 & 1255.91165 & $0.05-1 \mathrm{~b}, \mathrm{a}$ & 2920.13216 & $0.09-1 \mathrm{~b}, \mathrm{a}$ \\
\hline & 6 & 3 & 1411.61161 & $0.05-1 \mathrm{~b}, \mathrm{a}$ & 3101.12390 & $0.09-1 \mathrm{~b}$ \\
\hline & 6 & 2 & 1411.64199 & $0.06-1 k$ & 3101.14225 & $0.11-1$ \\
\hline
\end{tabular}




\begin{tabular}{|c|c|c|c|c|c|c|}
\hline 8 & 7 & 2 & 90.69028 & $.08-1 \mathrm{~b}, \mathrm{a}$ & 330 & $0.09-1$ \\
\hline 8 & 7 & 1 & 066 & $.09-1 \mathrm{~b}, \mathrm{a}$ & 3306 & $0.10-1$ \\
\hline 8 & 8 & 1 & 89.04301 & $.08-1 \mathrm{~b}$ & 3530.95767 & $0.24-1 \mathrm{~b}$ \\
\hline 8 & 8 & 0 & 89.04303 & $.10-1 \mathrm{~b}$ & 330 & $0.30-1 \mathrm{~b}$ \\
\hline 9 & 0 & 9 & 46 & $05-1 \mathrm{~b}$ & 304 & $0.10-1 \mathrm{~b}, \mathrm{a}$ \\
\hline 9 & 1 & 9 & 06 & $06-1 \mathrm{~b}$ & 578 & $0.10-1 \mathrm{~b}, \mathrm{a}$ \\
\hline 9 & 1 & $\varepsilon$ & 969 & $5-1 b$ & 005 & $0.10-1 \mathrm{~b}, \mathrm{a}$ \\
\hline 9 & 2 & $\xi$ & 52 & $6-1 b$ & 26 & $0.08-1 \mathrm{~b}, \mathrm{a}$ \\
\hline 9 & 2 & 7 & 1201 & $.05-1 \mathrm{~b}$ & 9819 & $0.09-1 \mathrm{~b}, \mathrm{a}$ \\
\hline 9 & 3 & $r$ & 121 & $.05-1 \mathrm{~b}$ & 092 & $0.10-1 \mathrm{~b}, \mathrm{a}$ \\
\hline 9 & 3 & 6 & 128 & $.04-1 \mathrm{~b}$ & & $.09-1$ \\
\hline 9 & 4 & 6 & 98 & $.06-1 \mathrm{~b}$ & 18 & $0.10-1$ \\
\hline 9 & 4 & 5 & 1 & $.04-1$ & 50 & $0.09-1 \mathrm{~b}, \mathrm{a}$ \\
\hline 9 & 5 & 5 & 89 & $.06-1 \mathrm{~b}$ & 596 & $0.11-1$ \\
\hline 9 & 5 & 4 & 147 & $.05-1 \mathrm{~b}, \mathrm{a}$ & 619 & $0.10-1 \mathrm{~b}, \mathrm{a}$ \\
\hline 9 & 6 & 4 & 1631 & $.06-1 \mathrm{~b}, \mathrm{a}$ & 990 & $0.10-1 \mathrm{~b}$ \\
\hline 0 & 6 & 3 & 1631 & $.05-1 \mathrm{~b}, \mathrm{a}$ & 355 & $0.09-1$ \\
\hline 0 & 7 & 3 & 181 & $.09-1 \mathrm{~b}, \mathrm{a}$ & 20 & $0.14-1$ \\
\hline 0 & 7 & 2 & 181 & $.07-1$ & 35 & $0.13-1$ \\
\hline 0 & 8 & 2 & 532 & $.20-1$ & 625 & $0.22-1$ \\
\hline 9 & 8 & 1 & 521 & $.10-1 \mathrm{~b}, \mathrm{a}$ & 648 & $0.11-1$ \\
\hline 9 & 9 & 1 & 6907 & $0.24-1$ & 6043 & $0.36-1$ \\
\hline 9 & 9 & 0 & 931 & $.20-1$ & 39 & $0.23-1$ \\
\hline & 0 & 10 & 231 & $0.09-1$ & 27 & $0.12-1$ \\
\hline & 1 & 10 & 008 & $0.07-$ & 2 & $0.11-1$ \\
\hline & 1 & 9 & 826 & $0.07-$ & 619 & 0.11 \\
\hline & 2 & 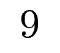 & .63421 & $0.05-1 \mathrm{~b}$ & 2904.42853 & c \\
\hline
\end{tabular}




\begin{tabular}{|c|c|c|c|c|c|c|}
\hline & 2 & 8 & TI & $\mathrm{b}$ & & 1 \\
\hline & 3 & 8 & 2 & $05-1 \mathrm{~b}$ & 654 & $0.09-1$ \\
\hline & 3 & 7 & 60 & $.07-1 \mathrm{~b}, \mathrm{a}$ & 900 & $.11-1$ \\
\hline & 4 & 1 & 8 & $.05-1 \mathrm{~b}, \mathrm{a}$ & 680 & $0.10-1 \mathrm{~b}, \mathrm{a}$ \\
\hline & 4 & 6 & 161 & $.07-1 \mathrm{~b}, \mathrm{a}$ & 16 & $12-1 \mathrm{~b}, \mathrm{a}$ \\
\hline & 5 & 6 & 3 & $.05-1 \mathrm{~b}$ & 63 & $0-1$ \\
\hline & 5 & 5 & 5 & $.07-1 \mathrm{~b}, \mathrm{a}$ & & $1-1$ \\
\hline & 6 & 5 & 0 & $.05-1 \mathrm{~b}, \mathrm{a}$ & & $0-1$ \\
\hline & 6 & 4 & 8 & $.07-1 \mathrm{~b}, \mathrm{a}$ & & $1-1$ \\
\hline & 7 & 4 & 1 & $0.08-1 \mathrm{~b}, \mathrm{a}$ & 68 & $0.11-1 \mathrm{~b}, \mathrm{a}$ \\
\hline & 7 & 3 & 4 & $0.10-1 \mathrm{~b}, \mathrm{a}$ & 67 & 0.14 \\
\hline & 8 & 3 & & $0.12-1 \mathrm{~b}, \mathrm{a}$ & & 0.16 \\
\hline & 8 & 2 & & $0.14-1 \mathrm{~b}, \mathrm{a}$ & & $9-1 \mathrm{~b}$ \\
\hline & 9 & 2 & & $0.17-1 \mathrm{~b}, \mathrm{a}$ & & $0.21-1 \mathrm{~b}, \mathrm{a}$ \\
\hline & 9 & 1 & 77 & $0.23-1 \mathrm{~b}$ & & $3-1 \mathrm{~b}$ \\
\hline & 10 & 1 & 270 & $.21-1 \mathrm{~b}, \mathrm{a}$ & 11 & $0.40-1 \mathrm{~b}, \mathrm{a}$ \\
\hline & 10 & 0 & 270 & $.55-1 \mathrm{~b}$ & 93 & $0.86-1 \mathrm{~b}$ \\
\hline & 0 & 11 & 132 & $.08-1 \mathrm{~b}$ & 451 & $0.12-1 \mathrm{~b}, \mathrm{a}$ \\
\hline & 1 & 11 & 132 & $.09-1$ & 143 & $.13-1$ \\
\hline & 1 & 10 & 152 & $.08-$ & 31 & $1-1 \mathrm{~b}, \mathrm{a}$ \\
\hline & 2 & 10 & 1525 & $.07-$ & 31 & $.12-1$ \\
\hline & 2 & 9 & 1690 & $.06-$ & & $.10-$ \\
\hline & 3 & 9 & 16 & 7 - & 3 & $2-$ \\
\hline & 3 & 8 & $181:$ & $0.06-1$ & 344 & $0.11-1 \mathrm{~b}$ \\
\hline & 4 & 8 & 184 & $0.07-$ & 941 & $0.11-1 \mathrm{~b}$ \\
\hline & 4 & 7 & 1899.00837 & $0.06-1$ & 3535.87070 & $0.11-1 \mathrm{~b}$ \\
\hline & 5 & 7 & 985.78508 & $07-1 \mathrm{~b}, \mathrm{a}$ & 3650.50930 & $.11-1$ \\
\hline
\end{tabular}




\begin{tabular}{|c|c|c|c|c|c|c|}
\hline & 5 & U & & $\mathrm{b}, \mathrm{a}$ & & \\
\hline & 6 & 6 & & $10-1 \mathrm{~b}, \mathrm{a}$ & 202 & $5-1 \mathrm{~b}$, \\
\hline & 6 & 5 & 649 & $06-1 \mathrm{~b}, \mathrm{a}$ & 99 & $1-1 \mathrm{~b}, \mathrm{c}$ \\
\hline & 7 & 5 & 1 & $14-1 \mathrm{~b}, \mathrm{a}$ & & $2-1 \mathrm{~b}, \mathrm{a}$ \\
\hline & 7 & 4 & 7 & $1-1 \mathrm{~b}, \mathrm{a}$ & 63 & $3-1 \mathrm{~b}, \mathrm{a}$ \\
\hline & 8 & 4 & 5 & $5-1 \mathrm{~b}$ & & $0-1$ \\
\hline & 8 & 3 & 37 & $3-1 \mathrm{~b}, \mathrm{a}$ & 66 & $7-$ \\
\hline & 9 & 3 & & $4-1 \mathrm{~b}$ & & $4-1$ \\
\hline & 9 & 2 & & $.18-1 \mathrm{~b}, \mathrm{a}$ & & $2-1$ \\
\hline & 1 & 2 & & $0.25-1 \mathrm{~b}, \mathrm{a}$ & & $\mathrm{b}$ \\
\hline & 1 & 1 & & $0.20-1 \mathrm{~b}, \mathrm{a}$ & & ,a \\
\hline & 1 & 1 & & $71-1 \mathrm{~b}$ & & - \\
\hline & 11 & 0 & & $-1 \mathrm{~b}$ & & , a \\
\hline & 0 & 12 & & $-1 \mathrm{~b}$ & 43 & $7-1 \mathrm{~b}, \mathrm{a}$ \\
\hline & 1 & 12 & 98 & $09-1 \mathrm{~b}$ & 01 & $0.14-1 \mathrm{~b}, \mathrm{a}$ \\
\hline & 1 & 11 & 42 & $10-1 b$ & 60 & $4-1 \mathrm{~b}, \mathrm{a}$ \\
\hline & 2 & 11 & & $8-1 b$ & & $2-1$ \\
\hline & 2 & 10 & 19 & $9-1 \mathrm{~b}, \mathrm{a}$ & & $2-1$ \\
\hline & 3 & 10 & 6 & $3-1 \mathrm{~b}, \mathrm{a}$ & & $2-1$ \\
\hline & 3 & 9 & & $-1 \mathrm{~b}, \mathrm{a}$ & & $3-$ \\
\hline & 4 & 9 & & $-1 b, a$ & & $1-$ \\
\hline & 4 & 8 & 97 & $0.09-1 \mathrm{~b}, \mathrm{a}$ & 68 & $2-$ \\
\hline & 5 & 8 & & -1 & & a \\
\hline & 5 & 7 & 24 & $0.10-1 \mathrm{~b}, \mathrm{a}$ & & 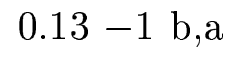 \\
\hline & 6 & 7 & 74 & $0.08-1 \mathrm{~b}, \mathrm{a}$ & & . \\
\hline & 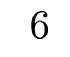 & 6 & 00 & $1 \mathrm{~b}, \mathrm{a}$ & 9933 & 0.13 \\
\hline & & 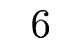 & & $\mathrm{D}, \mathrm{a}$ & 73 & 12 \\
\hline
\end{tabular}




\begin{tabular}{|c|c|c|c|c|c|c|}
\hline & & 5 & & a & & \\
\hline & 8 & 5 & & $3-1 \mathrm{~b}, \mathrm{a}$ & 664 & $5-1 \mathrm{~b}$, \\
\hline & 8 & 4 & 7 & $16-1 \mathrm{~b}, \mathrm{a}$ & 13 & $2-1 \mathrm{~b}$ \\
\hline & 0 & $t$ & 01 & $0.21-1 \mathrm{~b}, \mathrm{a}$ & 480 & $31-1 \mathrm{~b}, \mathrm{a}$ \\
\hline & 9 & 3 & & $.26-1 \mathrm{~b}, \mathrm{a}$ & 98 & $3-1 \mathrm{~b}, \mathrm{a}$ \\
\hline & 1 & 3 & & $1-1 \mathrm{~b}, \mathrm{a}$ & 45 & $\mathrm{~b}, \mathrm{a}$ \\
\hline & 1 & 2 & & $0-1 \mathrm{~b}$ & 77 & $6-1 \mathrm{~b}$ \\
\hline & 1 & 2 & & $9-1 \mathrm{~b}$ & & $2-1 b, a$ \\
\hline & 1 & 1 & & $.80-1 b$ & & .52 \\
\hline & 12 & 1 & & $.24-1 \mathrm{~b}$ & & $8-1 b, a$ \\
\hline & 12 & 0 & & $.11-1 \mathrm{~b}, \mathrm{a}$ & & \\
\hline & 0 & & & $0.11-1 \mathrm{~b}$ & & 9 \\
\hline & 1 & & & $.18-1 \mathrm{~b}$ & & $0.17-1 \mathrm{~b}, \mathrm{a}$ \\
\hline & 1 & 1 & & $10-1 \mathrm{~b}$ & & $0.13-1 \mathrm{~b}, \mathrm{a}$ \\
\hline & 2 & 12 & & $.12-1 \mathrm{~b}$ & & $0.14-1 \mathrm{~b}, \mathrm{a}$ \\
\hline & 2 & 11 & & $.10-1 \mathrm{~b}, \mathrm{a}$ & & $0.12-1 \mathrm{~b}, \mathrm{a}$ \\
\hline & 3 & 11 & 1 & $0.12-1 \mathrm{~b}, \mathrm{a}$ & 38 & $.13-1 \mathrm{~b}, \mathrm{a}$ \\
\hline & 3 & 10 & 3 & $0.08-1 \mathrm{~b}, \mathrm{a}$ & & $3-1$ \\
\hline & 4 & 10 & 3 & $.13-1 \mathrm{~b}, \mathrm{a}$ & & ,a \\
\hline & 4 & 9 & 8 & $-1 \mathrm{~b}, \mathrm{a}$ & $41^{\prime}$ & D,a \\
\hline & 5 & 9 & & $0.12-1 \mathrm{~b}, \mathrm{a}$ & & ,a \\
\hline & 5 & 8 & 80 & $0.10-1 \mathrm{~b}, \mathrm{a}$ & $42 \varepsilon$ & 0 \\
\hline & 6 & 8 & & -1 & & $0.18-1$ \\
\hline & 0 & 7 & $27:$ & $0.12-1 \mathrm{~b}, \mathrm{a}$ & 444 & $0.13-1 \mathrm{~b}, \mathrm{a}$ \\
\hline & 7 & 7 & 292 & $0.16-1 \mathrm{~b}, \mathrm{a}$ & 464 & $0.30-1 \mathrm{~b}, \mathrm{a}$ \\
\hline & 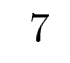 & 6 & 2927 & $0.14-1 \mathrm{~b}, \mathrm{a}$ & 4643 & $0.16-1 \mathrm{~b}, \mathrm{a}$ \\
\hline & & 6 & 4 & $16-1 \mathrm{~b}, \mathrm{a}$ & 34 & $35-$ \\
\hline
\end{tabular}




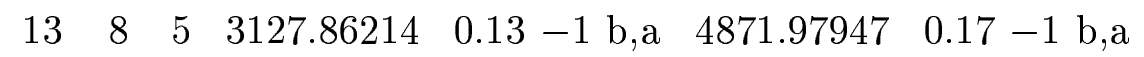

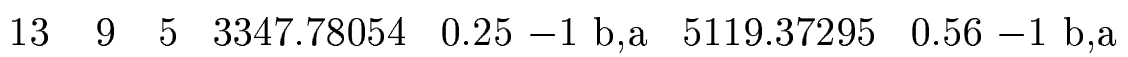

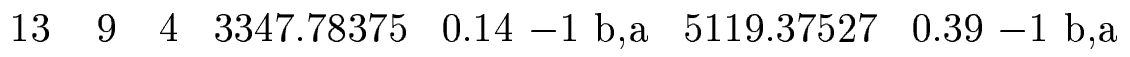

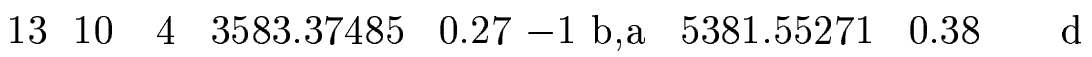

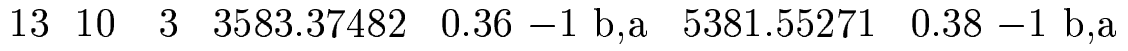

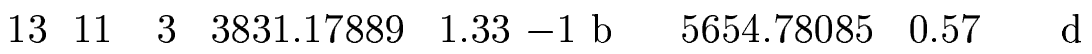

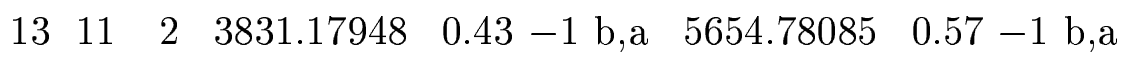

$\begin{array}{lllllllll}13 & 12 & 2 & 4087.98148 & 1.43 & \text { d } & 5935.60006 & 0.53 & \text { d }\end{array}$

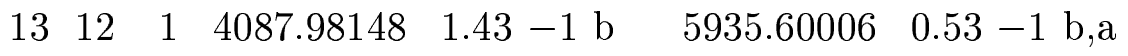

$\begin{array}{lllllllll}13 & 13 & 1 & 4350.59138 & 1.17 & \text { d } & 6220.62745 & 0.89 & \text { d }\end{array}$

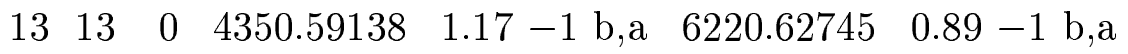

$14 \quad 0 \quad 14 \quad 2073.51524 \quad 0.17-1$ b $\quad 3655.48373 \quad 0.26-1$ b,a

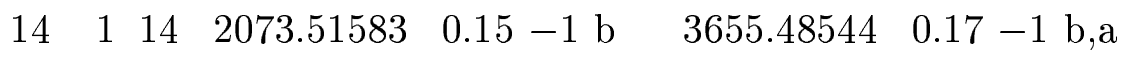

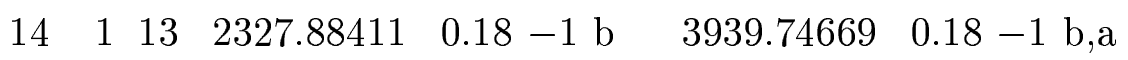

$14 \quad 2 \quad 13 \quad 2327.91443 \quad 0.12-1$ b $\quad 3939.83227 \quad 0.15-1$ b,a

$14 \quad 2 \quad 12 \quad 2550.88259 \quad 0.16-1$ b,a $4183.38928 \quad 0.16-1$ b,a

$14 \quad 3 \quad 12 \quad 2551.48381 \quad 0.10$ - 1 b,a $4184.83164 \quad 0.11$ - 1 b,a

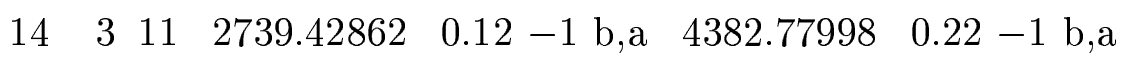

$144112746.02378 \quad 0.12-1$ b,a $4396.05175 \quad 0.15-1$ b,a

$14410 \quad 2880.83438 \quad 0.16-1$ b,a $4525.23956 \quad 0.34-1$ b,a

$14 \quad 5 \quad 10 \quad 2918.24526 \quad 0.12-1$ b,a $4585.34952 \quad 0.17-1$ b,a

$14 \quad 5 \quad 9 \quad 2983.39662 \quad 0.25-1$ b,a $4638.35105 \quad 0.36-1$ b,a

$14 \quad 6 \quad 9 \quad 3084.83610 \quad 0.12-1$ b,a $4774.04454 \quad 0.15-1 \quad b, a$

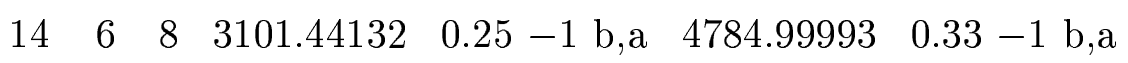

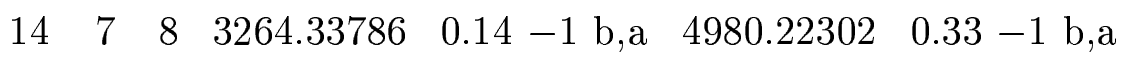

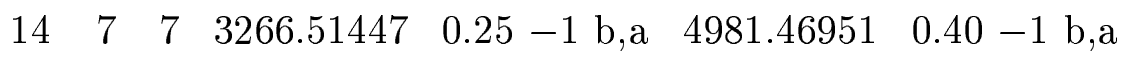

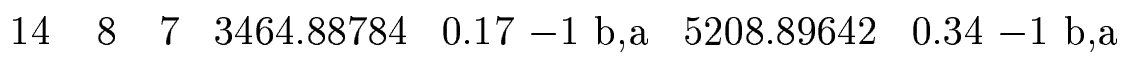




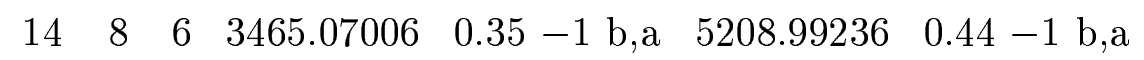

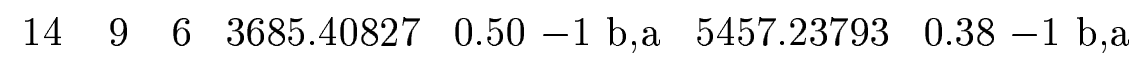
$14 \quad 9 \quad 5 \quad 3685.41921 \quad 0.27-1$ b,a $5457.24342 \quad 0.59-1$ b,a $14 \quad 10 \quad 5 \quad 3922.33059 \quad 0.46-1$ b,a $5721.09870 \quad 0.40 \quad-1$ b,a $\begin{array}{llllllllll}14 & 10 & 4 & 3922.33098 & 0.31 & -1 & \mathrm{~b} & 5721.09870 & 0.40 & \mathrm{~d}\end{array}$ $141144172.15275 \quad 0.65-1$ b,a $5996.69076 \quad 0.47-1$ b,a $\begin{array}{lllllllll}14 & 11 & 3 & 4172.15275 & 0.65 & \mathrm{~d} & 5996.69076 & 0.47 & \mathrm{~d}\end{array}$ $14 \quad 12 \quad 3 \quad 4431.63846 \quad 0.56-1$ b,a $6280.56345 \quad 0.52-1$ b,a

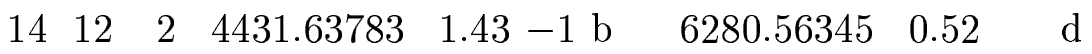
$141324697.66249 \quad 1.15$ - 1 b,a $6569.41205 \quad 0.87-1$ b,a $\begin{array}{lllllllll}14 & 13 & 1 & 4697.66249 & 1.15 & \mathrm{~d} & 6569.41205 & 0.87 & \mathrm{~d}\end{array}$ $14 \quad 14 \quad 1 \quad 4967.04228 \quad 1.19-1$ b,a $6859.88558 \quad 0.95-1$ b,a $\begin{array}{lllllllll}14 & 14 & 0 & 4967.04228 & 1.19 & \text { d } & 6859.88558 & 0.95 & \mathrm{~d}\end{array}$

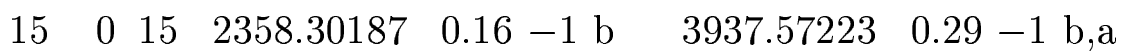

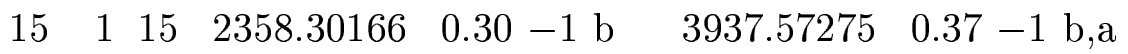
$\begin{array}{lllllllll}15 & 1 & 14 & 2631.26931 & 0.17-1 & \mathrm{~b} & 4243.11011 & 0.18 & -1 \\ \mathrm{~b}, \mathrm{a}\end{array}$

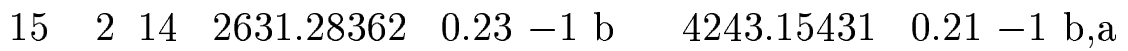

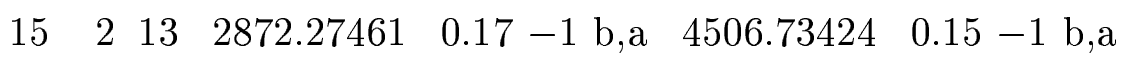
$15 \quad 3 \quad 13 \quad 2872.58063 \quad 0.16-1$ b,a $4507.52284 \quad 0.21-1$ b,a

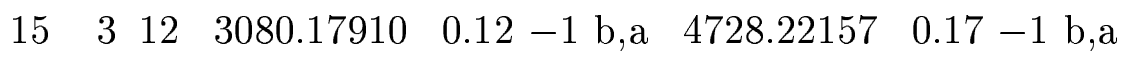
$15412 \quad 3083.86557 \quad 0.27-1$ b,a $4736.24179 \quad 0.24-1$ b,a $154113244.60100 \quad 0.15-1 \mathrm{~b}, \mathrm{a} \quad 4894.58616 \quad 0.21-1 \mathrm{~b}, \mathrm{a}$ $15 \quad 5 \quad 11 \quad 3269.53978 \quad 0.21-1$ b,a $4938.25431 \quad 0.33-1$ b,a

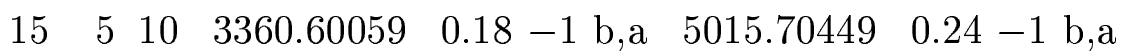
$15 \quad 6 \quad 10 \quad 3443.19219 \quad 0.21-1$ b,a $5132.52219 \quad 0.41-1$ b,a

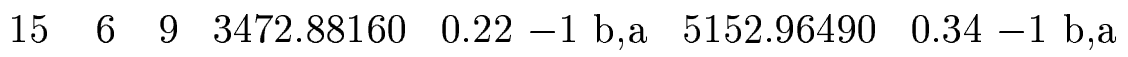

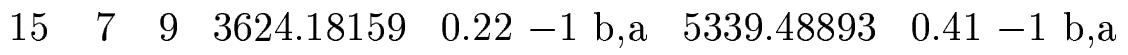




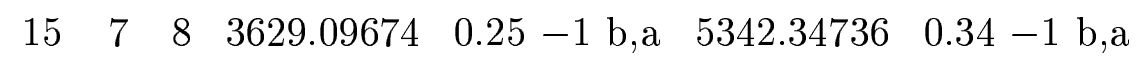

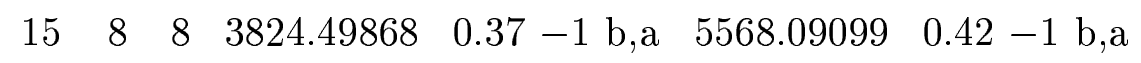

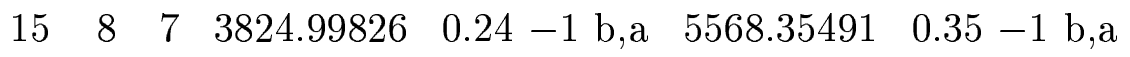

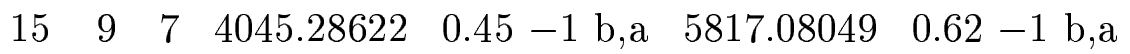

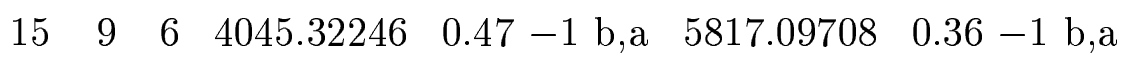

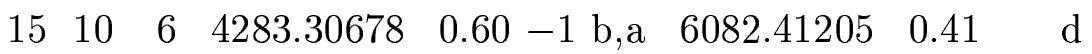

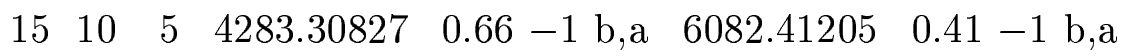

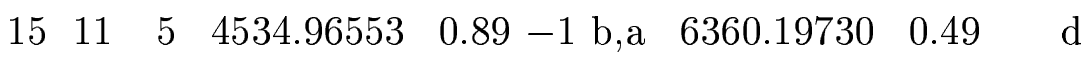

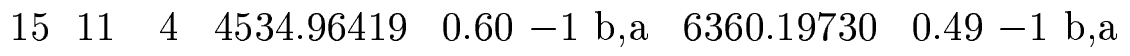

$\begin{array}{lllllllll}15 & 12 & 4 & 4796.97154 & 0.65 & \mathrm{~d} & 6646.95446 & 0.53 & \mathrm{~d}\end{array}$

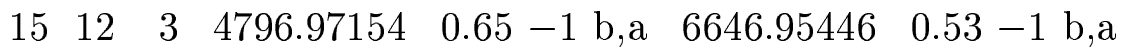

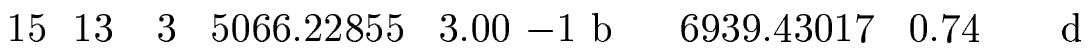

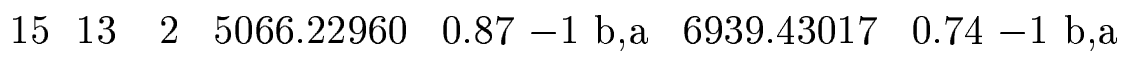

$\begin{array}{lllllllll}15 & 14 & 2 & 5339.67281 & 1.23 & \mathrm{~d} & 7234.42102 & 1.17 & \mathrm{~d}\end{array}$

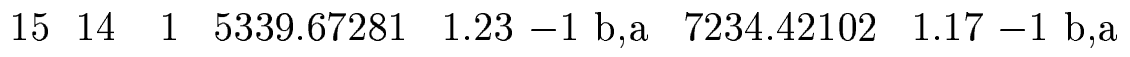

$\begin{array}{llllllllll}15 & 15 & 1 & 5614.08846 & 1.31 & \text { d } & 7528.56244 & 1.01 & \text { d }\end{array}$

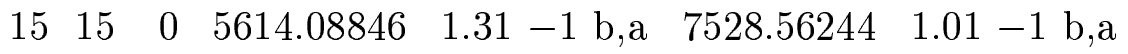

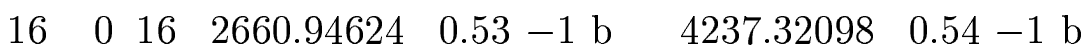

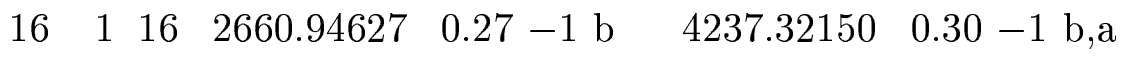

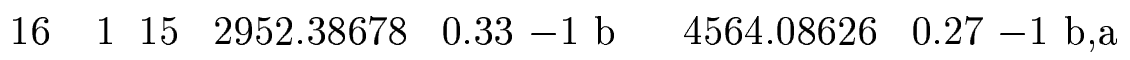

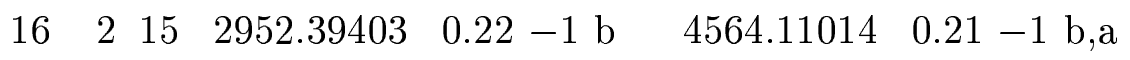

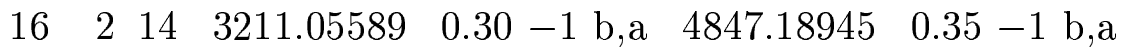

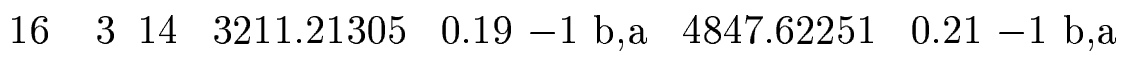

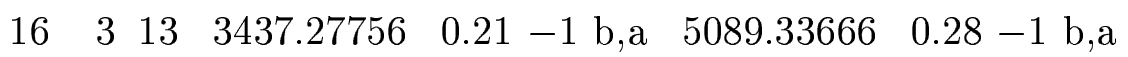

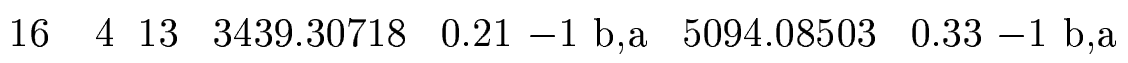

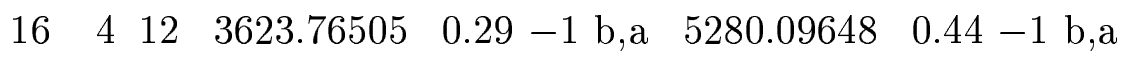

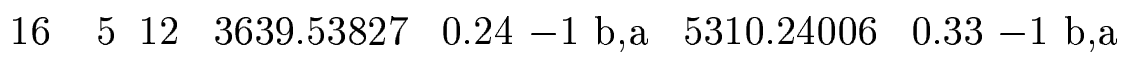




\begin{tabular}{|c|c|c|c|c|c|c|}
\hline & 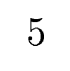 & 11 & 3758.39730 & $\mathrm{a}$ & & 10 \\
\hline & 6 & 11 & 2 & $18-1 \mathrm{~b}, \mathrm{a}$ & 0 & $.33-1 \mathrm{~b}, \mathrm{a}$ \\
\hline & 6 & 10 & 0 & $36-1 \mathrm{~b}, \mathrm{a}$ & 66 & $46-1 \mathrm{~b}, \mathrm{a}$ \\
\hline & 7 & 10 & 400 & $.20-1 \mathrm{~b}, \mathrm{a}$ & 37 & $.38-1 \mathrm{~b}, \mathrm{a}$ \\
\hline & 7 & ) & 81 & $.33-1 \mathrm{~b}, \mathrm{a}$ & & $.40-1 \mathrm{~b}, \mathrm{a}$ \\
\hline & 8 & 9 & 0 & $.25-1 \mathrm{~b}, \mathrm{a}$ & 10 & $38-1 \mathrm{~b}, \mathrm{a}$ \\
\hline & 8 & 0 & 2 & $.40-1 \mathrm{~b}, \mathrm{a}$ & & $3-1 \mathrm{~b}, \mathrm{a}$ \\
\hline & 9 & 8 & & $.41-1 \mathrm{~b}, \mathrm{a}$ & & $41-1 \mathrm{~b}, \mathrm{a}$ \\
\hline & 9 & $r$ & & $.42-1 \mathrm{~b}, \mathrm{a}$ & & $54-1 \mathrm{~b}, \mathrm{a}$ \\
\hline & 1 & 7 & 9 & $0.48-1 \mathrm{~b}, \mathrm{a}$ & & $0.47-1 \mathrm{~b}, \mathrm{a}$ \\
\hline & 1 & 6 & & $0.71-1 \mathrm{~b}, \mathrm{a}$ & & $0.77-1 \mathrm{~b}, \mathrm{a}$ \\
\hline & 1 & 6 & & $0.57-1 \mathrm{~b}, \mathrm{a}$ & & $0.47-$ \\
\hline & 11 & 5 & & .57 & & .47 \\
\hline & 12 & 5 & & $.71-1 \mathrm{~b}, \mathrm{a}$ & & $0.52-1 \mathrm{~b}, \mathrm{a}$ \\
\hline & 12 & 4 & & .71 & & 2 \\
\hline & 13 & 4 & 009 & $.04-1 \mathrm{~b}, \mathrm{a}$ & & $0.75-1 \mathrm{~b}, \mathrm{a}$ \\
\hline & 13 & 3 & 54 & .04 & 73 & 75 \\
\hline & 14 & 3 & 573 & $.12-1 \mathrm{~b}, \mathrm{a}$ & 605 & $0.90-1 \mathrm{~b}, \mathrm{a}$ \\
\hline & 14 & 2 & 57 & .12 & & 0.90 \\
\hline & 15 & 2 & 646 & $.35-1 \mathrm{~b}, \mathrm{a}$ & 79 & $1.09-1 \mathrm{~b}, \mathrm{a}$ \\
\hline & 15 & 1 & 346 & 1.35 & & 1.09 \\
\hline & 16 & 1 & 6290 & $.42-1 \mathrm{~b}, \mathrm{a}$ & 82 & $\mathrm{~b}, \mathrm{a}$ \\
\hline & 16 & 0 & 62 & 1.42 & & 1000 \\
\hline & 0 & 17 & 2981.35975 & $0.41-1 \mathrm{~b}$ & 455 & $0.57-1 \mathrm{~b}, \mathrm{a}$ \\
\hline & 1 & 17 & 2981. & $0.68-1 \mathrm{~b}$ & 234 & $1.24-1 \mathrm{~b}$ \\
\hline & 1 & 16 & 3291.14910 & $0.76-1 \mathrm{~b}$ & 4902.61440 & $0.26-1 \mathrm{~b}, \mathrm{a}$ \\
\hline & 2 & 1 & 3291.15252 & $1.61-1 \mathrm{~b}$ & 4902.62701 & $35-$ \\
\hline
\end{tabular}


$17 \quad 2 \quad 15 \quad 3567.17413 \quad 0.28-1$ b,a $\quad 5204.74894 \quad 0.32-1$ b,a

$17 \quad 3 \quad 15 \quad 3567.25537 \quad 0.50-1$ b,a $5204.98858 \quad 0.43-1$ b,a

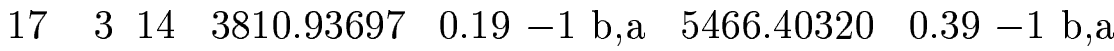

$17414 \quad 3812.04716 \quad 0.35-1$ b,a $5469.18519 \quad 0.40-1$ b,a

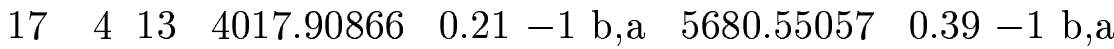

$17 \quad 5 \quad 13 \quad 4027.50637 \quad 0.59-1$ b,a $\quad 5700.48262 \quad 0.57-1$ b,a

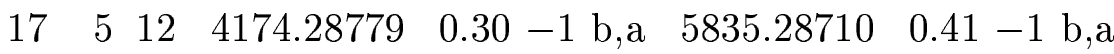

$17 \quad 6 \quad 12 \quad 4221.03999 \quad 0.42 \quad-1$ b,a $5911.62893 \quad 0.44-1$ b,a

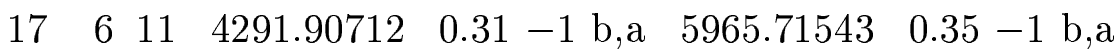

$17 \quad 7 \quad 11 \quad 4409.34441 \quad 0.65-1$ b,a $6123.35892 \quad 0.47-1$ b,a

$17 \quad 7 \quad 10 \quad 4428.11435 \quad 0.43-1$ b,a $6134.89913 \quad 0.36 \quad-1$ b,a

$17 \quad 8 \quad 10 \quad 4610.02320 \quad 0.58-1$ b,a $6351.91737 \quad 0.50 \quad-1$ b,a

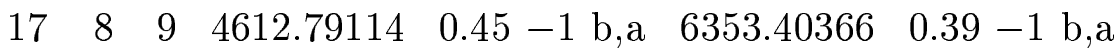

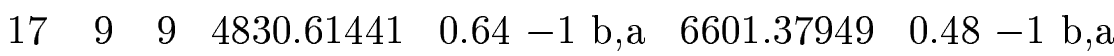

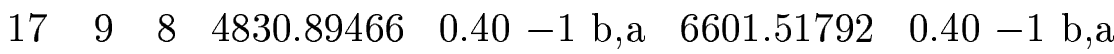

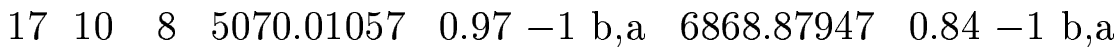

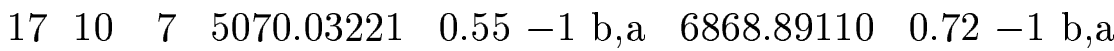

$\begin{array}{lllllllll}17 & 11 & 7 & 5324.66530 & 0.69 & \text { d } & 7150.40813 & 5.94 & -1\end{array}$

$17 \quad 11 \quad 6 \quad 5324.66530 \quad 0.69-1 \quad b, a \quad 7150.40865 \quad 0.52-1$ b,a

$\begin{array}{lllllllll}17 & 12 & 6 & 5591.12082 & 0.98 & \text { d } & 7442.34190 & 0.60 & \text { d }\end{array}$

$17 \quad 12 \quad 5 \quad 5591.12082 \quad 0.98-1 \quad b, a \quad 7442.34190 \quad 0.60 \quad-1$ b,a

$\begin{array}{lllllllll}17 & 13 & 5 & 5866.24176 & 0.77 & \text { d } & 7741.43559 & 0.75 & \text { d }\end{array}$

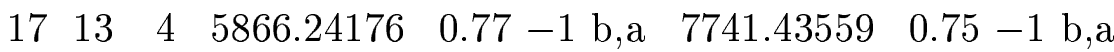

$\begin{array}{lllllllll}17 & 14 & 4 & 6147.08292 & 1.28 & \text { d } & 8044.65063 & 0.96 & \text { d }\end{array}$

$17 \quad 14 \quad 3 \quad 6147.08292 \quad 1.28-1$ b,a $8044.65063 \quad 0.96-1$ b,a

$\begin{array}{lllllllll}17 & 15 & 3 & 6430.72415 & 1.26 & \text { d } & 8348.98056 & 1.10 & \text { d }\end{array}$

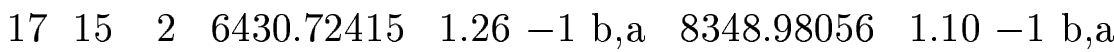


$\begin{array}{lllllllll}17 & 16 & 2 & 6714.12254 & 1.49 & \text { d } & 8651.27772 & 1.10 & \text { d }\end{array}$

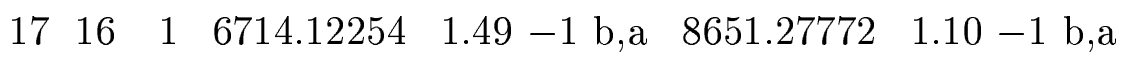

$\begin{array}{lllllllll}17 & 17 & 1 & 6993.88390 & 1.51 & \text { d } & 8948.00249 & 1.08 & \text { d }\end{array}$

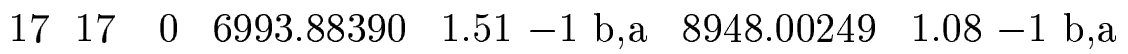

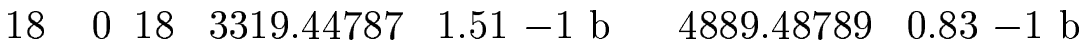

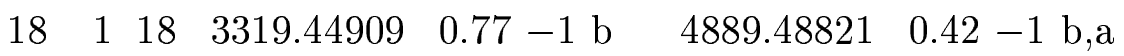

$\begin{array}{llllllll}18 & 1 & 17 & 3647.46058 & 1.05-1 & \mathrm{~b} & 5258.63110 & 1.06\end{array}-1 \mathrm{~b}, \mathrm{a}$

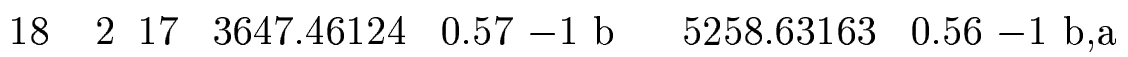

$18216 \quad 3940.54496 \quad 0.61-1$ b,a $5579.35856 \quad 0.50-1$ b,a

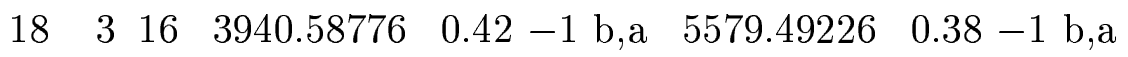

$18 \quad 3 \quad 15 \quad 4201.25211 \quad 0.80-1$ b,a $5859.61612 \quad 0.53-1$ b,a

$18415 \quad 4201.85895 \quad 0.63-1$ b,a $5861.23943 \quad 0.38 \quad-1$ b,a

$18414 \quad 4427.16549 \quad 0.49-1$ b,a $6095.51466 \quad 0.55-1$ b,a

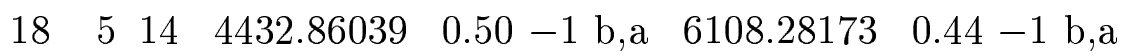

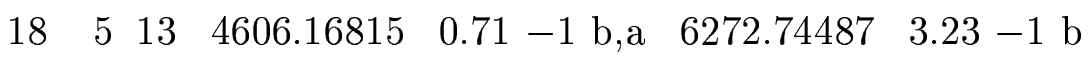

$18 \quad 6 \quad 13 \quad 4638.64543 \quad 0.48$ - 1 b,a $6330.45102 \quad 0.38-1$ b,a

$18 \quad 6 \quad 12 \quad 4735.84590 \quad 1.00-1$ b,a $6408.45890 \quad 0.80-1 \quad b, a$

$18 \quad 7 \quad 12 \quad 4833.20900 \quad 0.49-1$ b,a $6546.72205 \quad 0.39-1$ b,a

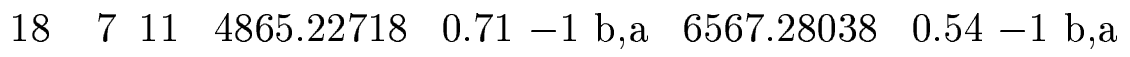

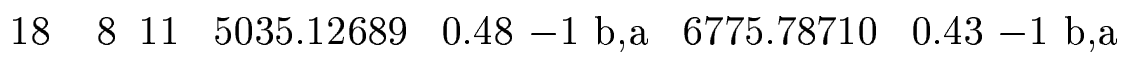

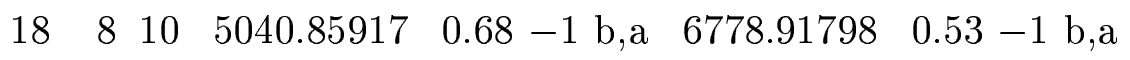

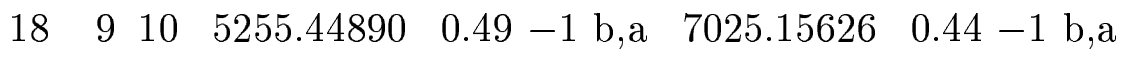

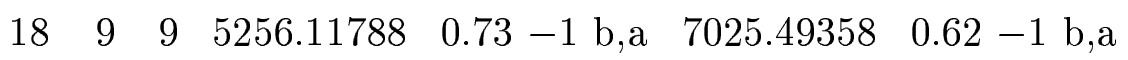

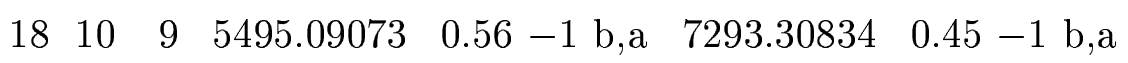

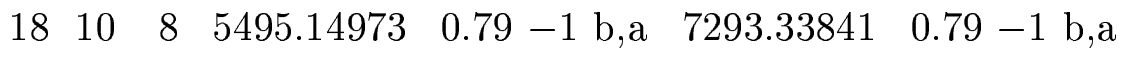

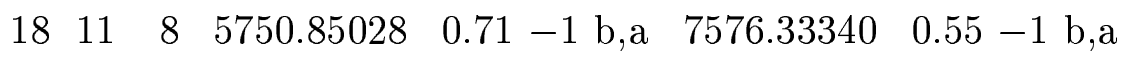

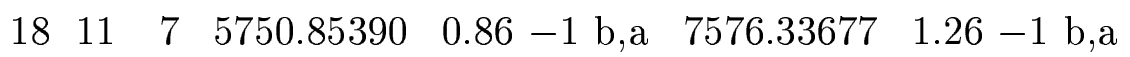




\begin{tabular}{|c|c|c|c|c|c|c|}
\hline & 2 & & & $\mathrm{~b}, \mathrm{a}$ & & \\
\hline & 1 & 6 & 6019.18161 & .72 & 7870.50014 & $7.95-1 \mathrm{~b}$ \\
\hline & 13 & 6 & 6296.89734 & $.19-1 \mathrm{~b}, \mathrm{a}$ & 8172.56423 & $0.77-1 \mathrm{~b}, \mathrm{a}$ \\
\hline & 13 & 5 & 6296 & 19 & 3 & .77 \\
\hline & 14 & 5 & 658 & $.08-1 \mathrm{~b}, \mathrm{a}$ & 847 & $0.97-1 \mathrm{~b}, \mathrm{a}$ \\
\hline & & 4 & 6 & 08 & 1 & .97 \\
\hline & & 4 & 367 & $1.43-1 \mathrm{~b}, \mathrm{a}$ & 97 & $1.05-1 \mathrm{~b}, \mathrm{a}$ \\
\hline & & 3 & & .43 & 97 & 5 \\
\hline & 1 & 3 & 379 & $2.41-1 \mathrm{~b}, \mathrm{a}$ & 08 & $1.16-1 \mathrm{~b}, \mathrm{a}$ \\
\hline & 16 & 2 & & 41 & & 16 \\
\hline & 17 & 2 & 17 & $1.60-1 \mathrm{~b}, \mathrm{a}$ & & $1.14-1 \mathrm{~b}, \mathrm{a}$ \\
\hline & 17 & 1 & 17 & 60 & & .14 \\
\hline & 18 & 1 & 106 & $.61-1 \mathrm{~b}, \mathrm{a}$ & 483 & $1.40-1 \mathrm{~b}, \mathrm{a}$ \\
\hline & 18 & 0 & 06 & .61 & & .40 \\
\hline & 0 & 19 & 377 & $.84-1 \mathrm{~b}$ & 308 & $0.64-1 \mathrm{~b}, \mathrm{a}$ \\
\hline & 1 & 19 & 340 & $.15-1 \mathrm{~b}$ & 52 & $1.71-1 \mathrm{~b}$ \\
\hline & 1 & 18 & 402 & $.35-1 \mathrm{~b}$ & 390 & $0.49-1 \mathrm{~b}, \mathrm{a}$ \\
\hline & 2 & 18 & 4021 & $.39-1$ & 563 & $1.00-1 \mathrm{~b}, \mathrm{a}$ \\
\hline & 2 & 17 & 4331 & $.57-1$ & 005 & $0.45-1 \mathrm{~b}, \mathrm{a}$ \\
\hline & 3 & 17 & 433 & $.83-1$ & 59 & $1.06-1 \mathrm{~b}, \mathrm{a}$ \\
\hline & 3 & 16 & 4608 & $.65-1$ & 344 & $0.49-1 \mathrm{~b}, \mathrm{a}$ \\
\hline & 4 & 16 & 4608.55753 & $0.82-1 \mathrm{~b}, \mathrm{a}$ & 208 & $0.51-1 \mathrm{~b}, \mathrm{a}$ \\
\hline & 4 & 15 & 2112 & $0.43-1$ & 65 & $0.44-$ \\
\hline & 5 & 15 & 5286 & $0.69-1$ & 65 & $0.56-$ \\
\hline & 5 & 14 & 6915 & $0.58-1$ & 67 & $5-1$ \\
\hline & 0 & 4 & 507 & $0.79-1$ & 1 & $8-$ \\
\hline & & & & $0.72-1 \mathrm{~b}, \mathrm{a}$ & 9 & $0.1+\perp 10,0$ \\
\hline
\end{tabular}




$$
\begin{aligned}
& 19 \quad 7 \quad 13 \quad 5276.80340 \quad 0.76-1 \text { b,a } 6990.05604 \quad 0.62 \quad-1 \text { b,a }
\end{aligned}
$$

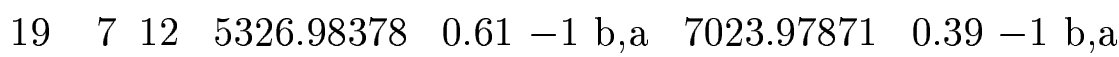

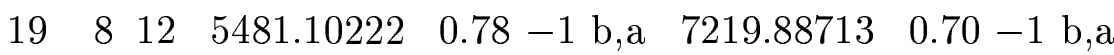

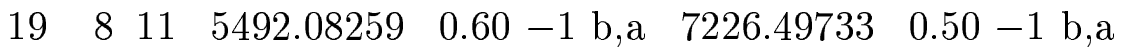

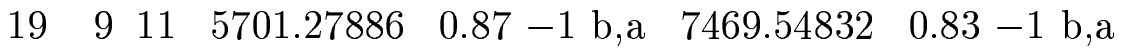

$$
\begin{aligned}
& 19910 \quad 5702.78184 \quad 0.65-1 \text { b,a } 7470.30485 \quad 0.51-1 \quad b, a \\
& 19 \quad 10 \quad 10 \quad 5940.89024 \quad 0.84-1 \text { b,a } 7738.05349 \quad 1.15-1 \text { b,a }
\end{aligned}
$$

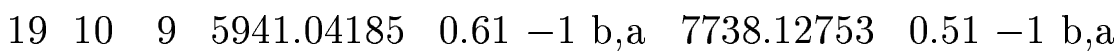

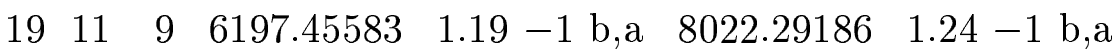

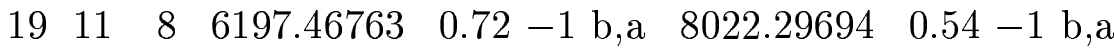

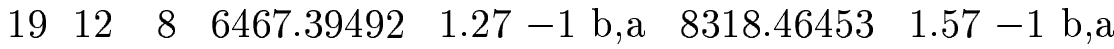

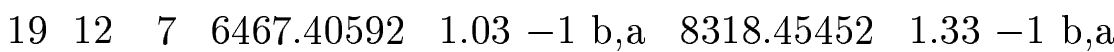

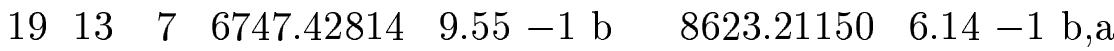

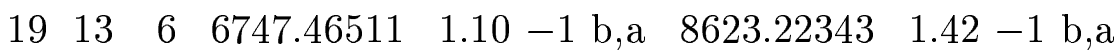

$$
\begin{aligned}
& \begin{array}{lllllllll}
19 & 14 & 6 & 7034.68781 & 1.34 & \text { d } & 8933.56762 & 0.98 & \text { d }
\end{array}
\end{aligned}
$$

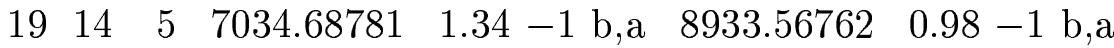

$$
\begin{aligned}
& \begin{array}{lllllllll}
19 & 15 & 5 & 7326.28927 & 1.27 & \mathrm{~d} & 9246.68983 & 1.04 & \mathrm{~d}
\end{array}
\end{aligned}
$$

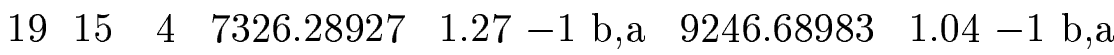

$$
\begin{aligned}
& \begin{array}{lllllllll}
19 & 16 & 4 & 7619.52807 & 1.52 & \text { d } & 9559.78220 & 1.75 & \text { d }
\end{array}
\end{aligned}
$$

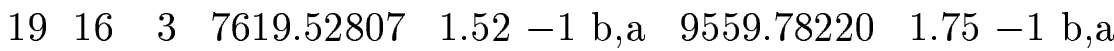

$$
\begin{aligned}
& \begin{array}{lllllllll}
19 & 17 & 3 & 7911.55421 & 2.02 & \text { d } & 9869.93576 & 1.19 & \text { d }
\end{array}
\end{aligned}
$$

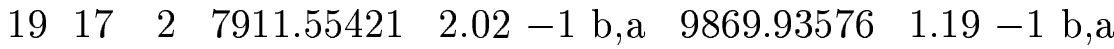

$$
\begin{aligned}
& \begin{array}{lllllllll}
19 & 18 & 2 & 8199.22084 & 1.72 & \text { d } & 10173.89737 & 1.16 & \text { d }
\end{array}
\end{aligned}
$$

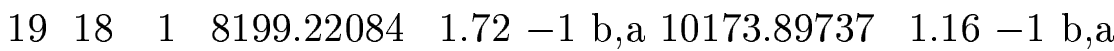

$$
\begin{aligned}
& \begin{array}{lllllllll}
19 & 19 & 1 & 8478.75428 & 1.69 & \text { d } & 10467.71828 & 1.27 & \text { d }
\end{array}
\end{aligned}
$$

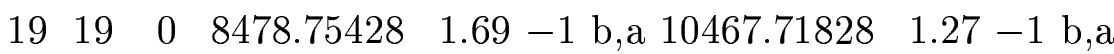

$$
\begin{aligned}
& \begin{array}{lllllllll}
20 & 0 & 20 & 4048.25008 & 1.67 & \text { d } & 5611.33160 & 7.22 & -1 \\
\text { b }
\end{array}
\end{aligned}
$$




$$
\begin{aligned}
& 20 \quad 1 \quad 20 \quad 4048.25008 \quad 1.67-1 \mathrm{~b} \quad 5611.33065 \quad 0.76-1 \mathrm{~b}, \mathrm{a}
\end{aligned}
$$

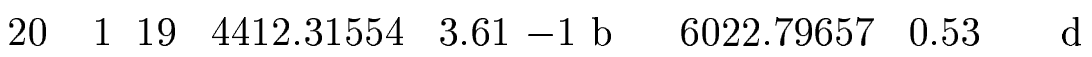

$$
\begin{aligned}
& 20 \quad 2 \quad 19 \quad 4412.31755 \quad 1.16-1 \text { b,a } 6022.79657 \quad 0.53-1 \text { b,a } \\
& 20 \quad 2 \quad 18 \quad 4738.62182 \quad 0.94-1 \text { b,a } 6379.34335 \quad 0.80-1 \text { b,a }
\end{aligned}
$$

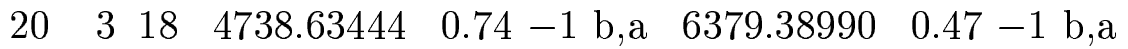

$$
\begin{aligned}
& \begin{array}{lllllllll}
20 & 3 & 17 & 5031.79418 & 0.97-1 & \mathrm{~b}, \mathrm{a} & 6694.74467 & 0.65 & -1 \mathrm{~b}, \mathrm{a}
\end{array} \\
& 20 \quad 4 \quad 17 \quad 5031.97867 \quad 0.78-1 \text { b,a } 6695.29973 \quad 0.50-1 \text { b,a } \\
& 20416 \quad 5292.10256 \quad 0.78-1 \text { b,a } 6969.40281 \quad 0.62-1 \text { b,a } \\
& 20 \quad 5 \quad 16 \quad 5294.03791 \quad 0.65-1 \text { b,a } 6974.36507 \quad 0.42-1 \text { b,a }
\end{aligned}
$$

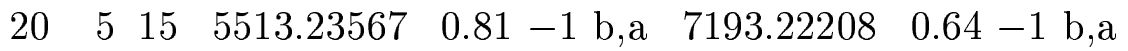

$$
\begin{aligned}
& \begin{array}{lllllllll}
20 & 6 & 15 & 5527.04593 & 0.69-1 & \mathrm{~b}, \mathrm{a} & 7222.25685 & 0.45 & -1 \\
\mathrm{~b}, \mathrm{a}
\end{array} \\
& 20 \quad 6 \quad 14 \quad 5680.78823 \quad 1.01 \text { - } 1 \text { b,a } 7357.05014 \quad 0.63-1 \text { b,a } \\
& 20 \quad 7 \quad 14 \quad 5739.22983 \quad 0.59 \text { - } 1 \text { b,a } 7452.52983 \quad 0.42 \quad-1 \text { b,a } \\
& 20 \quad 7 \quad 13 \quad 5812.07351 \quad 1.12 \text { - } 1 \text { b,a } 7504.57630 \quad 0.73-1 \text { b,a }
\end{aligned}
$$

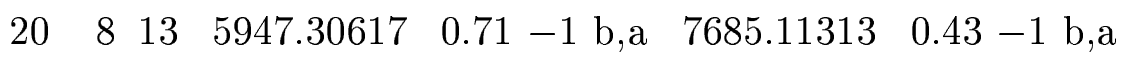

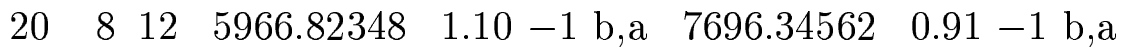

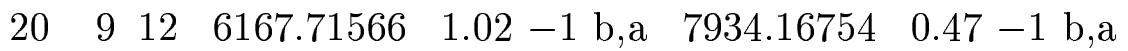

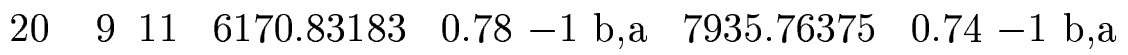

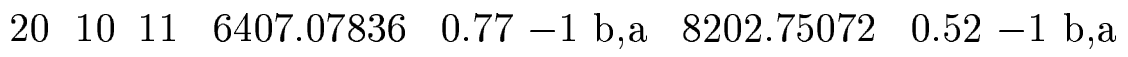

$$
\begin{aligned}
& 20 \quad 10 \quad 10 \quad 6407.44259 \quad 0.89 \text { - } 1 \text { b,a } 8202.92960 \quad 1.26 \quad-1 \text { b,a } \\
& 2011 \quad 10 \quad 6664.14154 \quad 0.76-1 \text { b,a } 8487.90142 \quad 0.60-1 \text { b,a }
\end{aligned}
$$

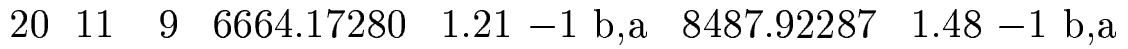

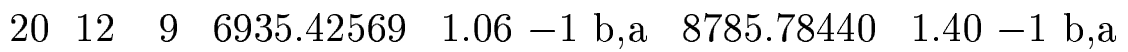

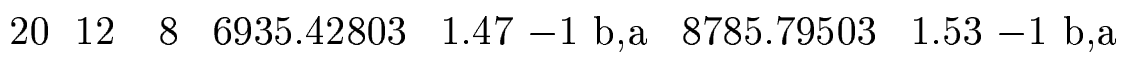

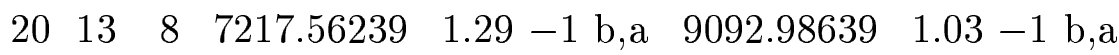

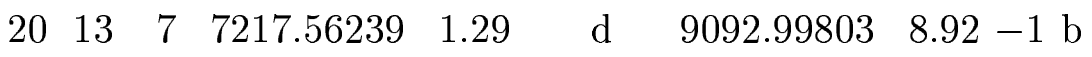

$$
\begin{aligned}
& 2014 \quad 7 \quad 7507.58026 \quad 1.26-1 \text { b,a } 9406.51529 \quad 1.13-1 \text { b,a }
\end{aligned}
$$




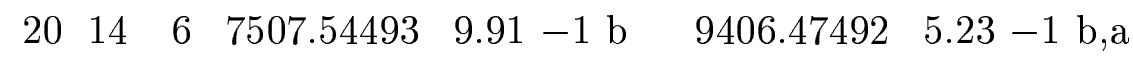

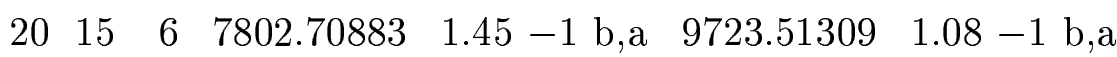

$\begin{array}{lllllllll}20 & 15 & 5 & 7802.70883 & 1.45 & \text { d } & 9723.51309 & 1.08 & \text { d }\end{array}$

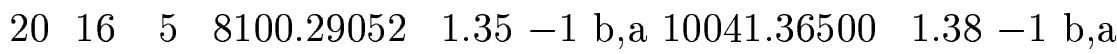

$\begin{array}{lllllllll}20 & 16 & 4 & 8100.29052 & 1.35 & \text { d } & 10041.36500 & 1.38 & \text { d }\end{array}$

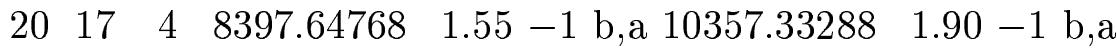

$\begin{array}{lllllllll}20 & 17 & 3 & 8397.64768 & 1.55 & \text { d } & 10357.33288 & 1.90 & \text { d }\end{array}$

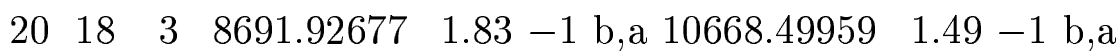

$\begin{array}{lllllllll}20 & 18 & 2 & 8691.92677 & 1.83 & \text { d } & 10668.49959 & 1.49 & \text { d }\end{array}$

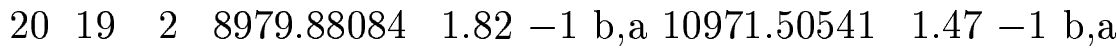

$\begin{array}{lllllllll}20 & 19 & 1 & 8979.88084 & 1.82 & \text { d } & 10971.50541 & 1.47 & \text { d }\end{array}$

$2020 \quad 1 \quad 9257.45868 \quad 1.76-1$ b,a $11262.09231 \quad 1.55-1$ b,a

$\begin{array}{lllllllll}20 & 20 & 0 & 9257.45868 & 1.76 & \mathrm{~d} & 11262.09231 & 1.55 & \mathrm{~d}\end{array}$

$21 \quad 0 \quad 21 \quad 4438.74920 \quad 1.37-1$ b,a $5998.16475 \quad 0.69-1$ b,a

$\begin{array}{llllllllll}21 & 1 & 21 & 4438.74920 & 1.37 & \text { d } & 5998.16475 & 0.69 & \text { d }\end{array}$

$21 \quad 120 \quad 4820.64363 \quad 1.01-1 \quad b, a \quad 6430.79589 \quad 0.52-1$ b,a

$21220 \quad 4820.64370 \quad 5.07-1$ b $\quad 6430.69607 \quad 1.10-1$ b,a

$21 \quad 2 \quad 19 \quad 5163.08236 \quad 0.87-1$ b,a $6804.47421 \quad 0.49-1$ b,a

$21 \quad 3 \quad 19 \quad 5163.08878 \quad 1.28-1$ b,a $6804.50918 \quad 5.62-1$ b

$21 \quad 3 \quad 18 \quad 5471.86495 \quad 0.81 \quad-1$ b,a $\quad 7136.57957 \quad 0.52-1$ b,a

$21418 \quad 5471.96618 \quad 1.21-1$ b,a $7136.91704 \quad 0.75-1$ b,a

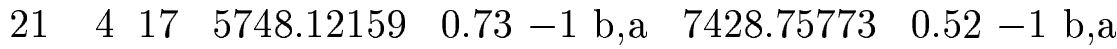

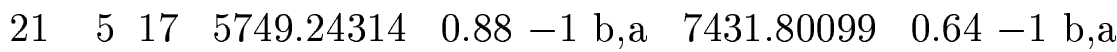

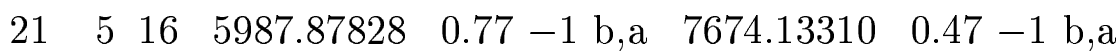

$21 \quad 6 \quad 16 \quad 5996.51847 \quad 0.89-1$ b,a $7693.71982 \quad 0.93-1$ b,a

$21 \quad 6 \quad 15 \quad 6177.35260 \quad 0.63-1$ b,a $7858.38076 \quad 0.47-1$ b,a

$21 \quad 7 \quad 15 \quad 6219.62260 \quad 0.86-1$ b,a $7933.29681 \quad 0.69-1$ b,a 
$21 \quad 7 \quad 14 \quad 6318.55503 \quad 0.79-1$ b,a $8007.87960 \quad 0.56-1$ b,a

$21 \quad 8 \quad 14 \quad 6433.11158 \quad 0.86-1$ b,a $8169.37060 \quad 0.87 \quad-1$ b,a

$21 \quad 8 \quad 13 \quad 6465.23065 \quad 0.64-1$ b,a $8188.71345 \quad 0.54-1$ b,a

$21913 \quad 6654.29805 \quad 0.95-1$ b,a $8418.26209 \quad 1.33-1$ b,a

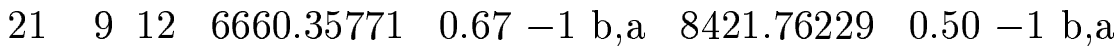

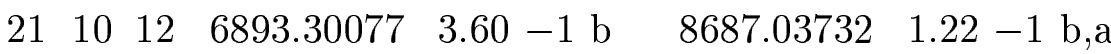

$21 \quad 1011 \quad 6894.11323 \quad 0.69-1$ b,a $8687.43094 \quad 0.80-1$ b,a

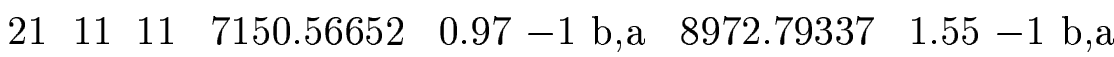

$21 \quad 11 \quad 10 \quad 7150.65491 \quad 0.83-1$ b,a $8972.83675 \quad 1.08-1$ b,a

$21 \quad 12 \quad 10 \quad 7422.88503 \quad 1.49-1$ b,a $9272.11139 \quad 1.73-1$ b,a

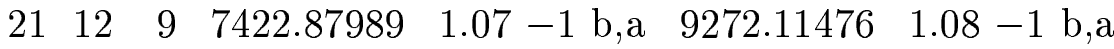

$\begin{array}{lllllllll}21 & 13 & 9 & 7706.83421 & 1.20 & \text { d } & 9581.47925 & 1.76 & -1 \\ \text { b,a }\end{array}$

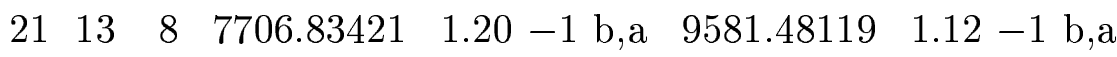

$\begin{array}{llllllllll}21 & 14 & 8 & 7999.35610 & 1.34 & \text { d } & 9897.86534 & 8.98 & 1 & \text { a }\end{array}$

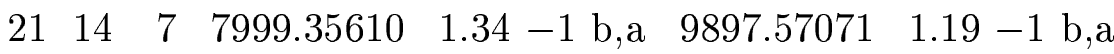

$21 \quad 15 \quad 7 \quad 8297.67293 \quad 14.50-1$ b $\quad 10218.4547110 .00-1$ b

$21 \quad 15 \quad 6 \quad 8297.70726 \quad 1.40-1$ b,a $10218.44110 \quad 1.62-1$ b,a

$\begin{array}{lllllllll}21 & 16 & 6 & 8599.28031 & 1.61 & \text { d } & 10540.66970 & 1.38 & \text { d }\end{array}$

$21 \quad 16 \quad 5 \quad 8599.28031 \quad 1.61-1$ b,a $10540.66970 \quad 1.38$-1 b,a

$\begin{array}{lllllllll}21 & 17 & 5 & 8901.51026 & 1.48 & \mathrm{~d} & 10861.94801 & 1.61 & \mathrm{~d}\end{array}$

$21 \quad 17 \quad 4 \quad 8901.51026 \quad 1.48$-1 b,a $10861.94801 \quad 1.61-1$ b,a

$\begin{array}{lllllllll}21 & 18 & 4 & 9201.76096 & 1.67 & \text { d } & 11179.59730 & 2.01 & \text { d }\end{array}$

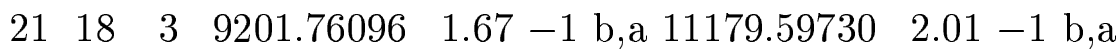

$\begin{array}{lllllllll}21 & 19 & 3 & 9497.15137 & 1.84 & \text { d } & 11490.68187 & 1.70 & \text { d }\end{array}$

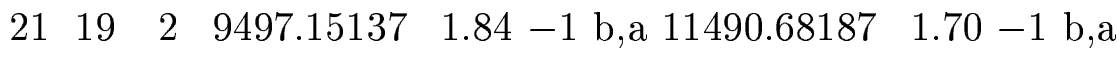

$\begin{array}{lllllllll}21 & 20 & 2 & 9784.30368 & 1.90 & \mathrm{~d} & 11791.69537 & 1.69 & \mathrm{~d}\end{array}$

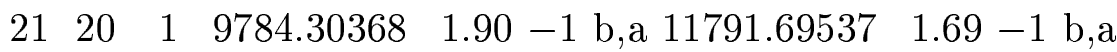


$\begin{array}{lllllllll}21 & 21 & 1 & 10058.81926 & 1.83 & \text { d } & 12077.98915 & 1.76 & \text { d }\end{array}$ $2121 \quad 010058.81926 \quad 1.83-1$ b,a $12077.98915 \quad 1.76-1$ b,a $\begin{array}{lllllllll}22 & 0 & 22 & 4846.49605 & 1.32 & \mathrm{~d} & 6402.15464 & 0.67 & \mathrm{~d}\end{array}$ $\begin{array}{llllllllll}22 & 1 & 22 & 4846.49605 & 1.32 & -1 & \mathrm{~b}, \mathrm{a} & 6402.15464 & 0.67 & 18 \mathrm{e}, \mathrm{a}\end{array}$ $\begin{array}{llllllllll}22 & 1 & 21 & 5246.07089 & 5.40 & -1 & \mathrm{~b} & 6855.98985 & 1.29 & 2 \mathrm{a}\end{array}$ $\begin{array}{llllllllll}22 & 2 & 21 & 5246.08010 & 1.01 & -1 & \mathrm{~b}, \mathrm{a} & 6855.95279 & 1.65 & 17 \mathrm{e}\end{array}$ $22 \quad 2 \quad 20 \quad 5604.30780 \quad 1.52-1$ b,a $7246.15476 \quad 1.50-1$ g,a $22 \quad 3 \quad 20 \quad 5604.31045 \quad 0.90-1$ b,a $7246.17575 \quad 0.53 \quad 16$ e,a

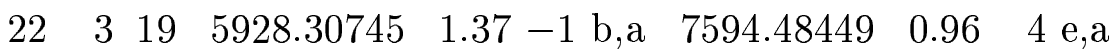
$\begin{array}{llllllllll}22 & 4 & 19 & 5928.36583 & 0.82 & -1 & \mathrm{~b}, \mathrm{a} & 7594.68529 & 0.49 & 16 \\ \mathrm{e}, \mathrm{a}\end{array}$ $\begin{array}{llllllllll}22 & 4 & 18 & 6219.88905 & 0.99 & -1 & \mathrm{~b}, \mathrm{a} & 7902.99121 & 0.74 & 4 \mathrm{a}\end{array}$

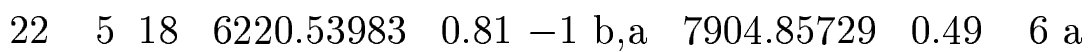

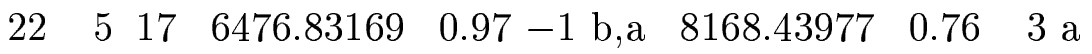
$\begin{array}{llllllllll}22 & 6 & 17 & 6482.15881 & 0.70 & -1 & \mathrm{~b}, \mathrm{a} & 8181.35704 & 0.65 & 9\end{array}$

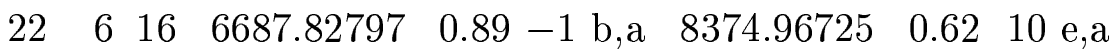

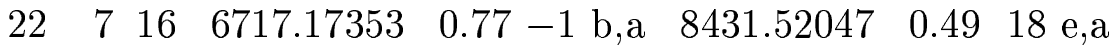

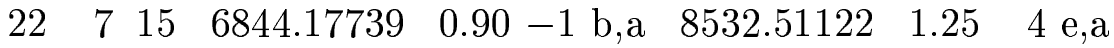

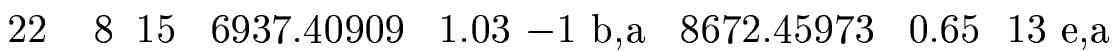
$\begin{array}{llllllllll}22 & 8 & 14 & 6986.94763 & 1.20 & -1 & \text { b,a } & 8703.73959 & 4.80 & 2 \text { e }\end{array}$

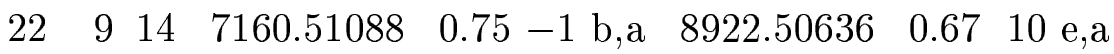

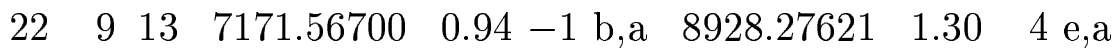
$\begin{array}{llllllllll}22 & 10 & 13 & 7399.23604 & 0.80 & -1 & \mathrm{~b}, \mathrm{a} & 9190.50865 & 0.89 & 3 \mathrm{a}\end{array}$

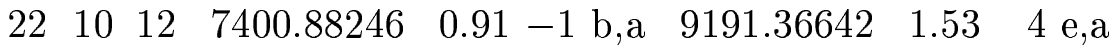
$\begin{array}{llllllllll}22 & 11 & 12 & 7656.38886 & 0.78 & -1 & \text { b,a } & 9476.57392 & 2.43 & 7 \text { e,a }\end{array}$

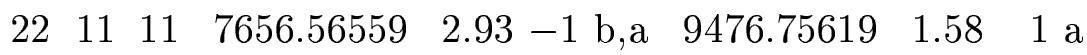
$\begin{array}{llllllllll}22 & 12 & 11 & 7929.41769 & 2.80 & -1 & \mathrm{~b} & 9777.02811 & 1.39 & 10 \text { e,a }\end{array}$ $\begin{array}{llllllllll}22 & 12 & 10 & 7929.45284 & 1.34 & -1 & \mathrm{~b}, \mathrm{a} & 9777.06645 & 1.84 & 1 \mathrm{a}\end{array}$ 


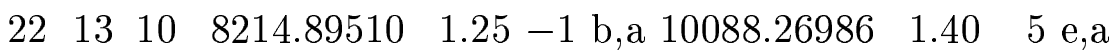

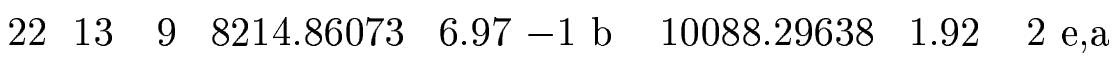

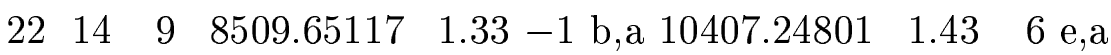

$\begin{array}{llllllllll}22 & 14 & 8 & 8509.65117 & 1.33 & \text { d } & 10407.55682 & 5.30 & 1 & \mathrm{a}\end{array}$

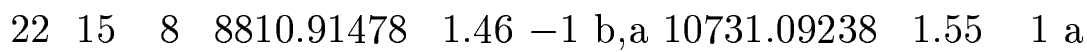

$\begin{array}{lllllllll}22 & 15 & 7 & 8810.91478 & 1.46 & \mathrm{~d} & 10731.09910 & 9.03 & 1 \mathrm{a}\end{array}$

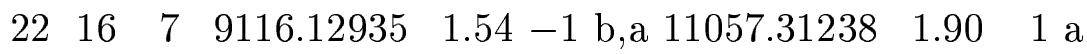

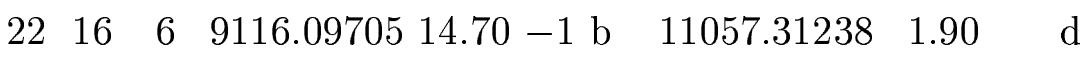

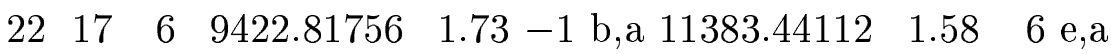

$\begin{array}{lllllllll}22 & 17 & 5 & 9422.81756 & 1.73 & \text { d } & 11383.44112 & 1.58 & \text { d }\end{array}$

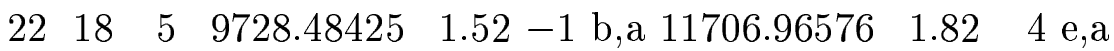

$\begin{array}{lllllllll}22 & 18 & 4 & 9728.48425 & 1.52 & \text { d } & 11706.96576 & 1.82 & \text { d }\end{array}$

$2219 \quad 410030.51630 \quad 1.67-1$ b,a $12025.23286 \quad 2.24 \quad 1$ a

$\begin{array}{lllllllll}22 & 19 & 3 & 10030.51630 & 1.67 & \text { d } & 12025.23286 & 2.24 & \text { d }\end{array}$

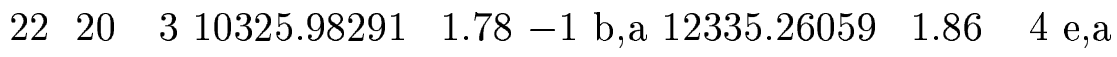

$\begin{array}{lllllllll}22 & 20 & 2 & 10325.98291 & 1.78 & \text { d } & 12335.26059 & 1.86 & \text { d }\end{array}$

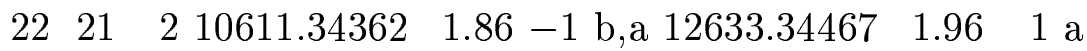

$\begin{array}{lllllllll}22 & 21 & 1 & 10611.34362 & 1.86 & \mathrm{~d} & 12633.34467 & 1.96 & \mathrm{~d}\end{array}$

$\begin{array}{lllllll}22 & 22 & 1 & 10881.77457 & 1.90 & -1 & \mathrm{~b}, \mathrm{a} \\ 12914.37782 & 2.02 & 1 \mathrm{a}\end{array}$

$\begin{array}{lllllllll}22 & 22 & 0 & 10881.77457 & 1.90 & \text { d } & 12914.37782 & 2.02 & \text { d }\end{array}$

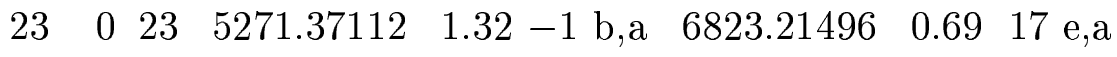

$\begin{array}{llllllllll}23 & 1 & 23 & 5271.36142 & 9.22 & -1 & \mathrm{~b} & 6823.21496 & 0.69 & \mathrm{~d}\end{array}$

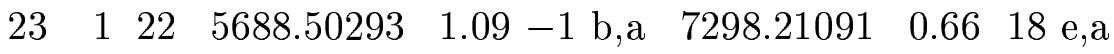

$\begin{array}{lllllllll}23 & 2 & 22 & 5688.50293 & 1.09 & \mathrm{~d} & 7298.21091 & 0.66 & \mathrm{~d}\end{array}$

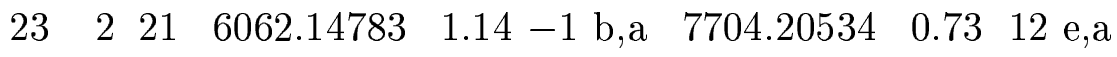

$\begin{array}{llllllllll}23 & 3 & 21 & 6062.14734 & 1.77 & -1 & \mathrm{~b}, \mathrm{a} & 7704.26605 & 1.82 & 1 \mathrm{a}\end{array}$

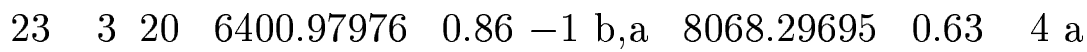


$23420 \quad 6401.01004 \quad 1.54-1$ b,a $8068.42201 \quad 1.07 \quad 2$ a

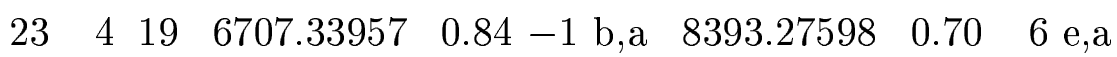

$\begin{array}{llllllllll}23 & 5 & 19 & 6707.72015 & 1.07 & -1 & \mathrm{~b}, \mathrm{a} & 8394.43453 & 0.83 & 3 \mathrm{a}\end{array}$

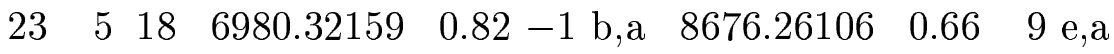

$\begin{array}{llllllllll}23 & 6 & 18 & 6983.57449 & 1.04 & -1 & \mathrm{~b}, \mathrm{a} & 8684.66211 & 0.77 & 6 \text { e,a }\end{array}$

$\begin{array}{llllllllll}23 & 6 & 17 & 7211.46005 & 1.11 & -1 & \mathrm{~b}, \mathrm{a} & 8905.22261 & 0.81 & 6\end{array}$

$\begin{array}{llllllllll}23 & 7 & 17 & 7231.17154 & 0.94 & -1 & \mathrm{~b}, \mathrm{a} & 8946.40805 & 0.88 & 2 \mathrm{a}\end{array}$

$\begin{array}{llllllllll}23 & 7 & 16 & 7386.77781 & 0.85 & -1 & \mathrm{~b}, \mathrm{a} & 9076.10312 & 0.71 & 7 \text { e,a }\end{array}$

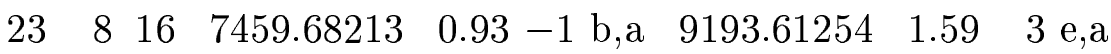

$\begin{array}{llllllllll}23 & 8 & 15 & 7530.92498 & 0.87 & -1 & \mathrm{~b}, \mathrm{a} & 9241.17747 & 0.81 & 7 \text { e,a }\end{array}$

$\begin{array}{llllllllll}23 & 9 & 15 & 7685.71725 & 0.97 & -1 & \mathrm{~b}, \mathrm{a} & 9445.15042 & 1.14 & 2 \mathrm{a}\end{array}$

$\begin{array}{llllllllll}23 & 9 & 14 & 7704.71749 & 0.85 & -1 & \mathrm{~b}, \mathrm{a} & 9455.40275 & 0.74 & 8 \\ \mathrm{e}, \mathrm{a}\end{array}$

$\begin{array}{llllllllll}23 & 10 & 14 & 7924.44967 & 0.93 & -1 & \mathrm{~b}, \mathrm{a} & 9714.75425 & 5.17 & 1 \mathrm{a}\end{array}$

$\begin{array}{llllllllll}23 & 10 & 13 & 7927.65884 & 0.81 & -1 & \mathrm{~b}, \mathrm{a} & 9714.48555 & 0.84 & 9\end{array}$

$\begin{array}{llllllllll}23 & 11 & 13 & 8181.26668 & 1.27 & -1 & \mathrm{~b}, \mathrm{a} & 9998.88967 & 1.83 & 1 \mathrm{a}\end{array}$

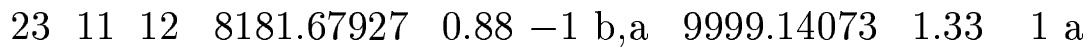

$\begin{array}{llllllllll}23 & 12 & 12 & 8454.68820 & 2.64 & -1 & \text { b,a } & 10300.15371 & 1.87 & 1 \text { a }\end{array}$

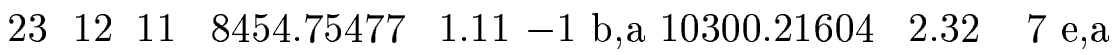

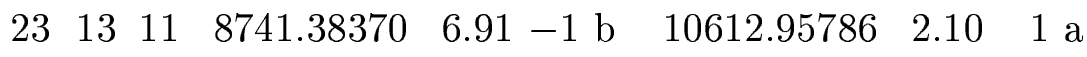

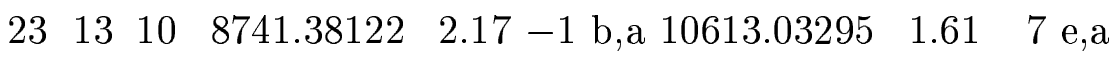

$\begin{array}{lllllllll}23 & 14 & 10 & 9038.08371 & 1.39 & \text { d } & 10934.28277 & 2.16 & 1\end{array}$

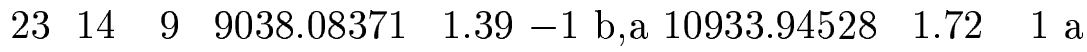

$\begin{array}{lllllllll}23 & 15 & 9 & 9341.97062 & 1.44 & \text { d } & 11261.06903 & 9.65 & 2 \text { e }\end{array}$

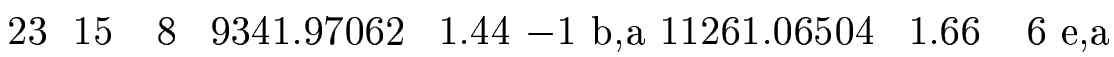

$\begin{array}{lllllllll}23 & 16 & 8 & 9650.48636 & 1.57 & \text { d } & 11590.93106 & 1.85 & \text { d }\end{array}$

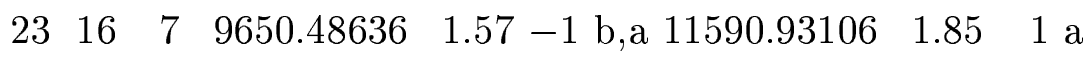

$\begin{array}{lllllllll}23 & 17 & 7 & 9961.22807 & 1.67 & \text { d } & 11921.46753 & 2.15 & \text { d }\end{array}$ 


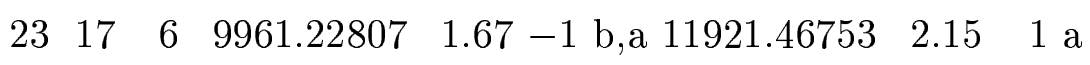

$\begin{array}{lllllllll}23 & 18 & 6 & 10271.82239 & 1.84 & \text { d } & 12250.32082 & 1.87 & \text { d }\end{array}$

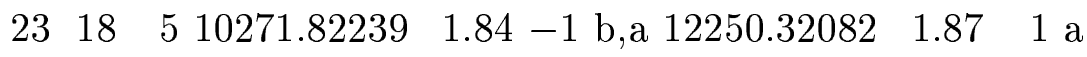

$\begin{array}{lllllllll}23 & 19 & 5 & 10579.82683 & 1.65 & \mathrm{~d} & 12575.06646 & 2.00 & \mathrm{~d}\end{array}$

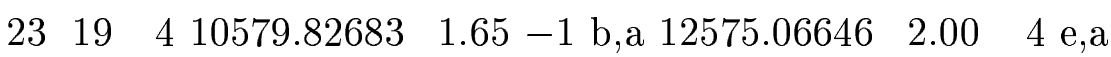

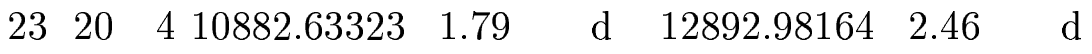

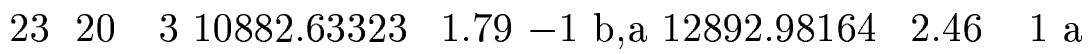

$\begin{array}{lllllllll}23 & 21 & 3 & 11177.24118 & 1.88 & \text { d } & 13201.06198 & 2.11 & \text { d }\end{array}$

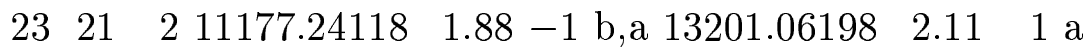

$\begin{array}{lllllllll}23 & 22 & 2 & 11459.91647 & 1.96 & \text { d } & 13495.39392 & 2.20 & \text { d }\end{array}$

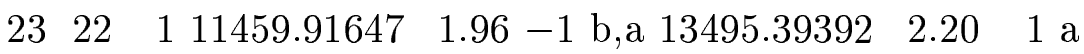

$\begin{array}{lllllllll}23 & 23 & 1 & 11725.32088 & 1.98 & \text { d } & 13770.28395 & 2.25 & \text { d }\end{array}$

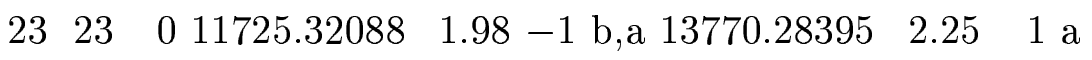

$\begin{array}{lllllllll}24 & 0 & 24 & 5713.25040 & 1.41 & \text { d } & 7261.28902 & 0.78 & \text { d }\end{array}$

$\begin{array}{llllllllll}24 & 1 & 24 & 5713.25040 & 1.41 & -1 & \mathrm{~b}, \mathrm{a} & 7261.28902 & 0.78 & 12 \mathrm{e}, \mathrm{a}\end{array}$

$\begin{array}{lllllllll}24 & 1 & 23 & 6147.78505 & 1.12 & \text { d } & 7757.46841 & 0.66 & \text { d }\end{array}$

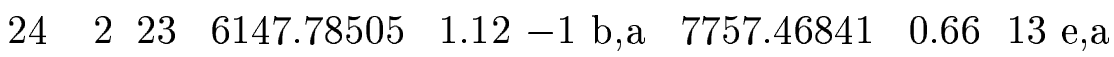

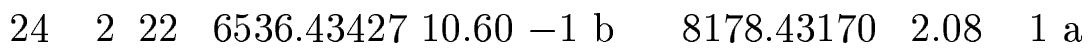

$\begin{array}{lllllllll}24 & 3 & 22 & 6536.44409 & 0.92 & -1 & \mathrm{~b}, \mathrm{a} & 8178.38995 & 0.63-1 \mathrm{~g}, \mathrm{a}\end{array}$

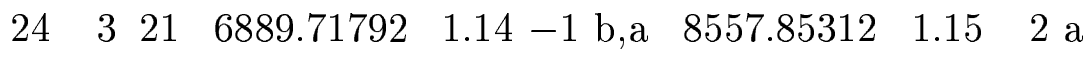

$\begin{array}{llllllllll}24 & 4 & 21 & 6889.73736 & 0.92 & -1 & \mathrm{~b}, \mathrm{a} & 8557.94156 & 0.70 & 6 \mathrm{e}, \mathrm{a}\end{array}$

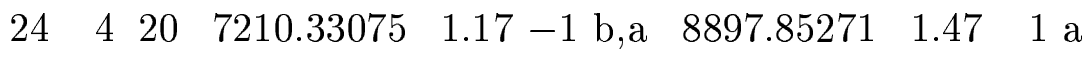

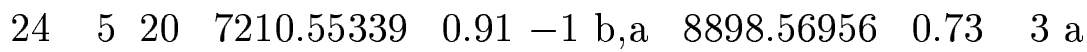

$\begin{array}{lllllllll}24 & 5 & 19 & 7498.46778 & 1.78 & -1 \text { b,a } & 9197.75908 & 1.03 & 2 \mathrm{a}\end{array}$

$\begin{array}{llllllllll}24 & 6 & 19 & 7500.45468 & 0.87 & -1 & \mathrm{~b}, \mathrm{a} & 9203.17602 & 0.87 & 2 \mathrm{a}\end{array}$

$\begin{array}{lllllllll}24 & 6 & 18 & 7748.08136 & 0.99 & -1 \mathrm{~b}, \mathrm{a} & 9448.10077 & 1.33 & 1 \mathrm{a}\end{array}$

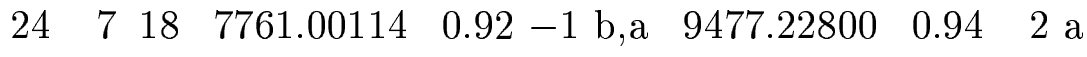




\begin{tabular}{|c|c|c|c|c|c|c|c|}
\hline & 7 & 17 & 194 & $0.96-1 \mathrm{~b}, \mathrm{a}$ & & & $a$ \\
\hline & 8 & 17 & 7999.01829 & $0.87-1 \mathrm{~b}, \mathrm{a}$ & 9030 & 0.83 & $7 \mathrm{e}, \mathrm{a}$ \\
\hline & 8 & 16 & 8095.50869 & $1.32-1 \mathrm{~b}, \mathrm{a}$ & 9801 & 5.08 & $1 \mathrm{a}$ \\
\hline & 9 & 16 & 8229.39309 & $0.92-1 \mathrm{~b}, \mathrm{a}$ & 998 & 0.87 & $7 \mathrm{e}, \mathrm{a}$ \\
\hline & 9 & 15 & 6278 & $1.28-1 \mathrm{~b}, \mathrm{a}$ & 100 & 1.88 & $\mathrm{a}$ \\
\hline & 10 & 15 & 8468 & $0.87-1 \mathrm{~b}, \mathrm{a}$ & 102 & 1.20 & $\mathrm{e}, \mathrm{a}$ \\
\hline & 10 & 14 & 847 & $1.31-1 \mathrm{~b}, \mathrm{a}$ & 102 & 1.50 & $\mathrm{e}, \mathrm{a}$ \\
\hline & 11 & 14 & 872 & $1.13-1 \mathrm{~b}, \mathrm{a}$ & 105 & 1.26 & $5 \mathrm{e}, \mathrm{a}$ \\
\hline & 11 & 13 & 252 & $1.30-1 \mathrm{~b}, \mathrm{a}$ & 65 & 5.26 & $1 \mathrm{a}$ \\
\hline & 12 & 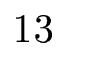 & 899 & $1.20-1 \mathrm{~b}, \mathrm{a}$ & & 1.59 & $2 \mathrm{a}$ \\
\hline & 12 & 12 & 899 & $1.49-1 \mathrm{~b}, \mathrm{a}$ & & 2.08 & $1 \mathrm{a}$ \\
\hline & 13 & 12 & 04 & $1.31-1 \mathrm{~b}, \mathrm{a}$ & 81 & 2.32 & $5 \mathrm{e}, \mathrm{a}$ \\
\hline & 13 & 11 & 928 & $2.51-1 \mathrm{~b}, \mathrm{a}$ & 24 & 2.12 & $1 \mathrm{a}$ \\
\hline & 14 & 11 & 958 & $1.99-1 \mathrm{~b}, \mathrm{a}$ & 19 & 1.79 & $6 \mathrm{e}, \mathrm{a}$ \\
\hline & 14 & 10 & 958 & $5.08-1 \mathrm{~b}, \mathrm{a}$ & & 2.32 & $1 \mathrm{a}$ \\
\hline & 15 & 10 & 9890.49531 & $1.56-1 \mathrm{~b}, \mathrm{a}$ & 70 & 1.99 & $1 \mathrm{a}$ \\
\hline & 15 & 9 & 989 & 1.56 & 025 & 5.47 & $1 \mathrm{a}$ \\
\hline & 16 & & 70 & $1.60-1 \mathrm{~b}, \mathrm{a}$ & & 1.85 & $4 \mathrm{e}, \mathrm{a}$ \\
\hline & 16 & & 1020 & 1.60 & 666 & 1.85 & $\mathrm{~d}$ \\
\hline & 17 & & 10516.41584 & $1.66-1 \mathrm{~b}, \mathrm{a}$ & 9095 & 2.10 & $1 \mathrm{a}$ \\
\hline & 17 & & 10516 & 1.66 & $\$ 9095$ & 2.10 & d \\
\hline & 18 & & 10831.47174 & $1.80-1 \mathrm{~b}, \mathrm{a}$ & 35083 & 2.37 & $1 \mathrm{a}$ \\
\hline & 18 & & 10831 & 1.80 & 083 & 2.37 & 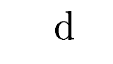 \\
\hline & 19 & & 11144.87570 & $1.96-1 \mathrm{~b}, \mathrm{a}$ & 769 & 2.12 & $1 \mathrm{a}$ \\
\hline & 19 & & 11144.87570 & 1.96 & 13139.91769 & 2.12 & $\mathrm{u}$ \\
\hline & 2 & & 11454.22415 & $1.67-1 \mathrm{~b}, \mathrm{a}$ & 13464.88632 & 2.16 & $3 \mathrm{e}, \mathrm{a}$ \\
\hline & & & 11454.22415 & L.67 & 8632 & .16 & \\
\hline
\end{tabular}


$2421 \quad 411756.89651 \quad 1.89-1$ b,a $13781.63575 \quad 2.65 \quad 1$ a

$\begin{array}{lllllllll}24 & 21 & 3 & 11756.89651 & 1.89 & \text { d } & 13781.63575 & 2.65 & \text { d }\end{array}$

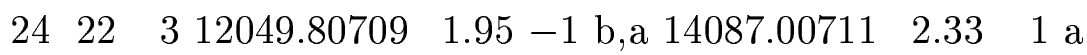

$\begin{array}{lllllllll}24 & 22 & 2 & 12049.80709 & 1.95 & \mathrm{~d} & 14087.00711 & 2.33 & \mathrm{~d}\end{array}$

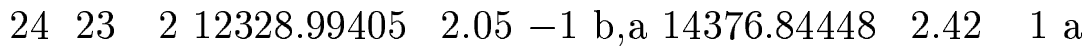

$\begin{array}{lllllllll}24 & 23 & 1 & 12328.99405 & 2.05 & \text { d } & 14376.84448 & 2.42 & \text { d }\end{array}$

$\begin{array}{lllllll}24 & 24 & 1 & 12588.50657 & 2.06 & -1 & \mathrm{~b}, \mathrm{a} \\ 14644.78688 & 2.47 & 1 \mathrm{a}\end{array}$

$\begin{array}{lllllllll}24 & 24 & 0 & 12588.50657 & 2.06 & \text { d } & 14644.78688 & 2.47 & \text { d }\end{array}$

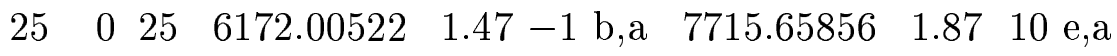

$\begin{array}{lllllllll}25 & 1 & 25 & 6172.00522 & 1.47 & \mathrm{~d} & 7715.65856 & 1.87 & \mathrm{~d}\end{array}$

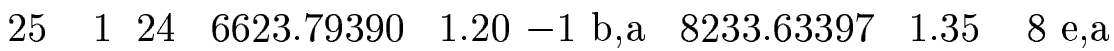

$\begin{array}{lllllllll}25 & 2 & 24 & 6623.79390 & 1.20 & \text { d } & 8233.63397 & 1.35 & \text { d }\end{array}$

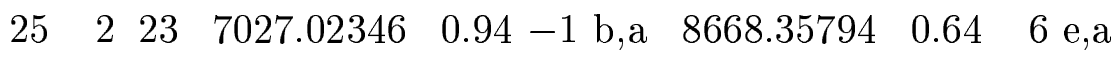

$\begin{array}{lllllllll}25 & 3 & 23 & 7027.02346 & 0.94 & \text { d } & 8668.52859 & 2.25 & 2\end{array}$

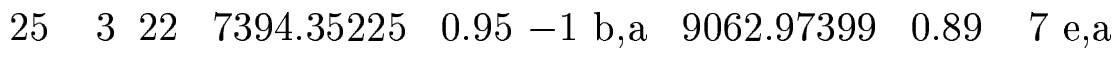

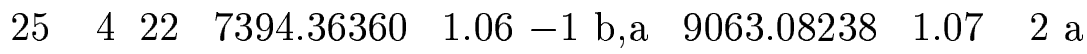

$25 \quad 421 \quad 7729.34050 \quad 0.80-1$ b,a $9417.47391 \quad 0.66 \quad 4$ a

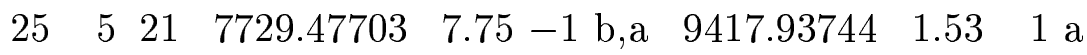

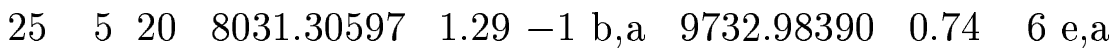

$\begin{array}{llllllllll}25 & 6 & 20 & 8032.51166 & 1.20 & -1 & \mathrm{~b}, \mathrm{a} & 9736.40354 & 2.04 & 1 \mathrm{a}\end{array}$

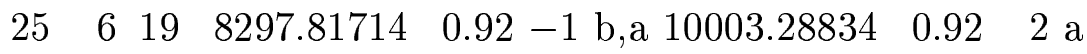

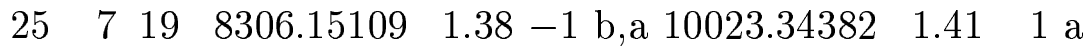

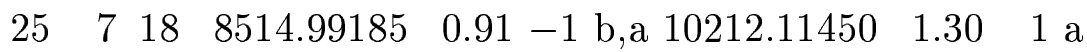

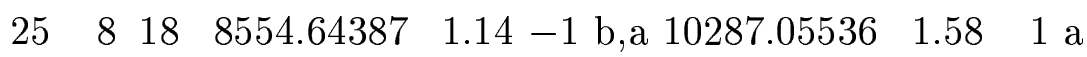

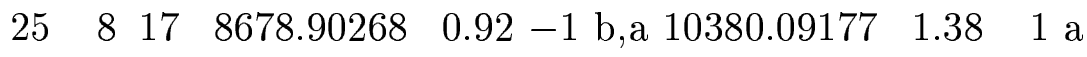

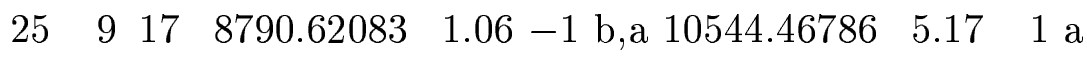

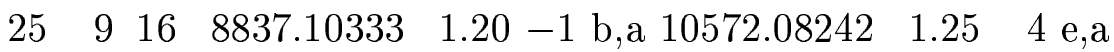


$\begin{array}{llllllllll}25 & 10 & 16 & 9030.87488 & 1.02 & -1 & \mathrm{~b}, \mathrm{a} & 10812.60669 & 2.13 & 1\end{array}$

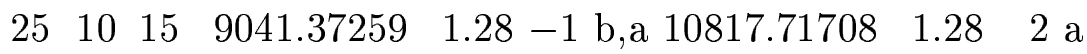

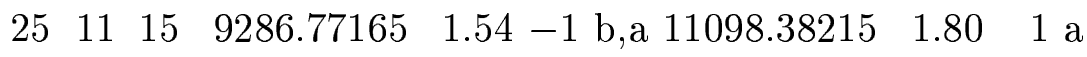

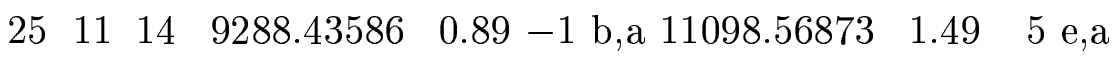

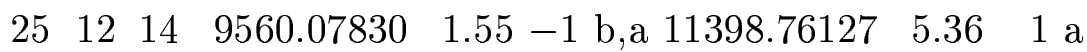

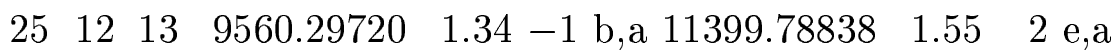

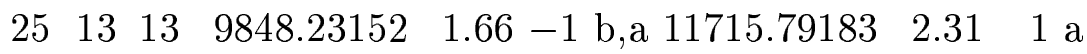

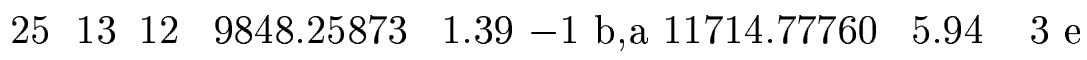

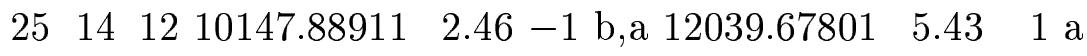
$25141110147.91371 \quad 1.48$-1 b,a $12039.46426 \quad 2.35 \quad 4$ e,a $\begin{array}{llllll}25 & 15 & 11 & 10456.11796 & 1.95 & d\end{array}$

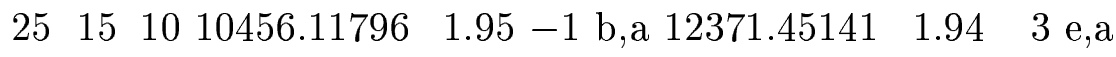

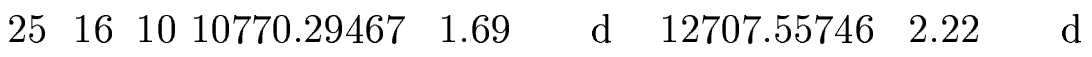

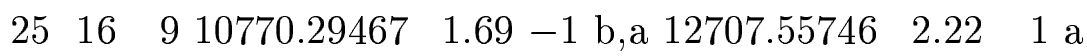

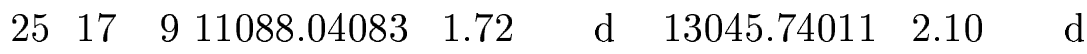

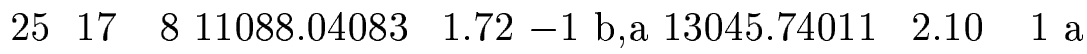

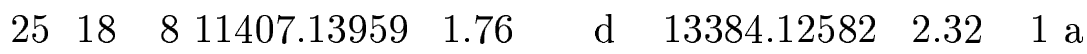

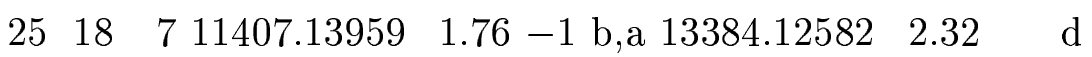

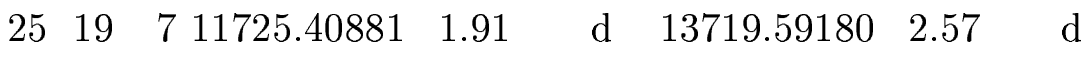

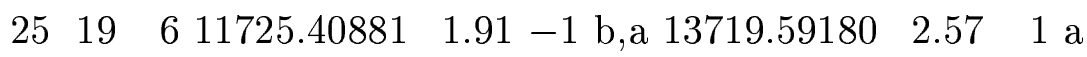
$\begin{array}{lllllllll}25 & 20 & 6 & 12040.64069 & 2.06 & \text { d } & 14050.85659 & 2.34 & \text { d }\end{array}$ $\begin{array}{lllllll}25 & 20 & 5 & 12040.64069 & 2.06 & -1 & \mathrm{~b}, \mathrm{a} \\ 14050.85659 & 2.34 & 1 \mathrm{a}\end{array}$

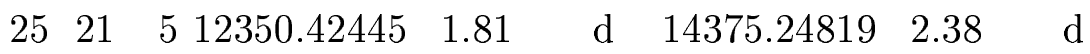

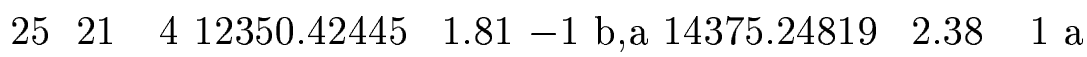

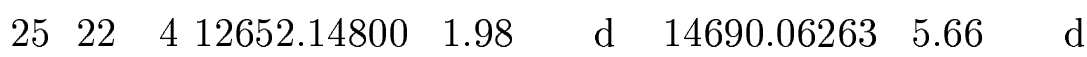
$\begin{array}{llllll}25 & 22 & 3 & 12652.14800 & 1.98 & -1 \\ \mathrm{~b}, \mathrm{a} & 14690.06263 & 5.66 & 1 \mathrm{a}\end{array}$

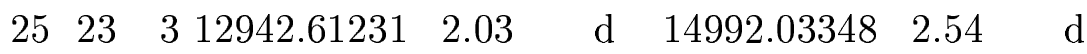




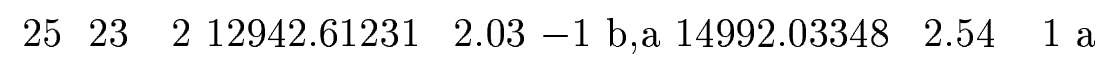

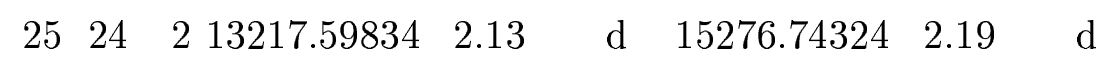

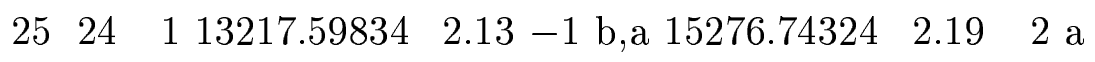

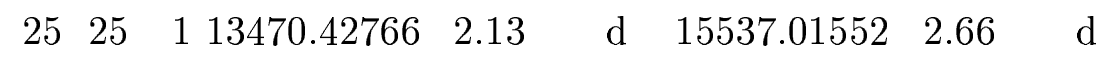
$\begin{array}{lllllll}25 & 25 & 0 & 13470.42766 & 2.13 & -1 & \mathrm{~b}, \mathrm{a} \\ 15537.01552 & 2.66 & 1 \mathrm{a}\end{array}$ $\begin{array}{lllllllll}26 & 0 & 26 & 6647.49310 & 2.64 & \mathrm{~d} & 8187.68007 & 1.14 & \mathrm{~d}\end{array}$ $\begin{array}{llllllllll}26 & 1 & 26 & 6647.49310 & 2.64 & 12 \mathrm{e} & 8187.68007 & 1.14 & 11 \mathrm{e}, \mathrm{a}\end{array}$ $\begin{array}{lllllllll}26 & 1 & 25 & 7116.38279 & 1.87 & \text { d } & 8726.65106 & 0.88 & \text { d }\end{array}$ $\begin{array}{llllllllll}26 & 2 & 25 & 7116.38279 & 1.87 & 16 & \text { e,a } & 8726.65106 & 0.88 & 9 \text { e,a }\end{array}$ $\begin{array}{lllllllll}26 & 2 & 24 & 7533.69254 & 2.70 & \mathrm{~d} & 9174.27166 & 2.47 & 1 \mathrm{a}\end{array}$ $\begin{array}{lllllllll}26 & 3 & 24 & 7533.69254 & 2.70 & 1 \mathrm{a} & 9174.27631 & 1.32 & 3 \mathrm{e}, \mathrm{a}\end{array}$ $\begin{array}{lllllllll}26 & 3 & 23 & 7914.69801 & 1.46 & 1 \mathrm{a} & 9583.45438 & 1.46 & 1 \mathrm{a}\end{array}$

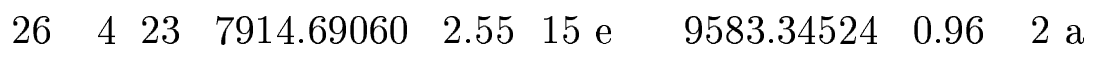
$\begin{array}{lllllllll}26 & 4 & 22 & 8262.76581 & 1.35 & 2 \mathrm{a} & 9951.89993 & 1.78 & 2 \mathrm{a}\end{array}$ $\begin{array}{lllllllll}26 & 5 & 22 & 8262.84502 & 0.94 & 2 \mathrm{a} & 9952.21737 & 0.87 & 2 \mathrm{a}\end{array}$ $\begin{array}{lllllllll}26 & 5 & 21 & 8578.76660 & 1.07 & 2 \mathrm{a} & 10281.59851 & 1.56 & 1 \mathrm{a}\end{array}$ $\begin{array}{lllllllll}26 & 6 & 21 & 8579.50182 & 1.05 & 2 \mathrm{a} & 10283.80226 & 0.80 & 3 \mathrm{a}\end{array}$ $\begin{array}{lllllllll}26 & 6 & 20 & 8860.90030 & 1.71 & 1 \mathrm{a} & 10570.56708 & 1.72 & 1 \mathrm{a}\end{array}$ $\begin{array}{lllllllll}26 & 7 & 20 & 8866.18424 & 0.90 & 8 \mathrm{e}, \mathrm{a} & 10584.15122 & 0.90 & 2 \mathrm{a}\end{array}$ $\begin{array}{llllll}26 & 7 & 19 & 9098.06732 & 1.09 & 2 \mathrm{a}\end{array}$ $\begin{array}{lllllllll}26 & 8 & 19 & 9125.86354 & 1.04 & 2 \text { a } & 10857.75605 & 0.80 & 3 \text { a }\end{array}$ $\begin{array}{lllllllll}26 & 8 & 18 & 9278.47311 & 1.71 & 1 \mathrm{a} & 10978.19153 & 5.19 & 1 \mathrm{a}\end{array}$ $\begin{array}{lllllllll}26 & 9 & 18 & 9368.73378 & 0.96 & 2 \text { a } & 11120.18888 & 1.06 & 2 \text { a }\end{array}$ $\begin{array}{llllll}26 & 9 & 17 & 9435.38362 & 1.51 & 1 \mathrm{a}\end{array}$ $\begin{array}{lllllllll}26 & 10 & 17 & 9611.33220 & 4.17 & 6 \mathrm{e} & 11388.86771 & 1.55 & 3 \mathrm{e}, \mathrm{a}\end{array}$ $\begin{array}{lllllllll}26 & 10 & 16 & 9628.60752 & 1.45 & 1 \mathrm{a} & 11397.69400 & 5.27 & 1 \mathrm{a}\end{array}$ $\begin{array}{lllllllll}26 & 11 & 16 & 9866.64129 & 1.04 & 2 \mathrm{a} & 11673.78669 & 1.49 & 3 \mathrm{a}\end{array}$ 
$\begin{array}{lllllllll}26 & 11 & 15 & 9869.68079 & 1.43 & 1 \mathrm{a} & 11674.84307 & 2.35 & 1 \mathrm{a}\end{array}$

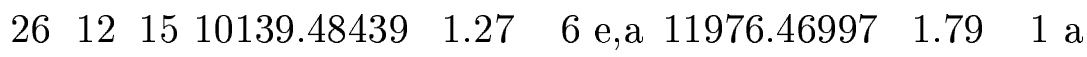

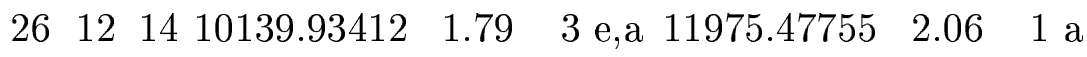

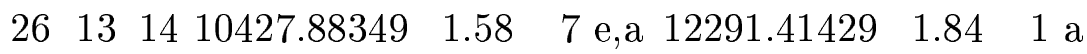
$\begin{array}{lllllllll}26 & 13 & 13 & 10427.95141 & 1.84 & 1 \text { a } & 12290.95969 & 5.45 & 1 \text { a }\end{array}$

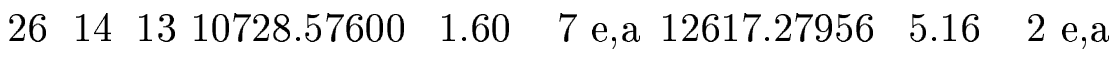

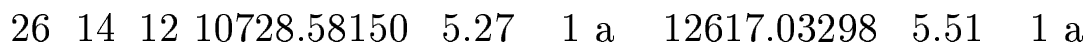

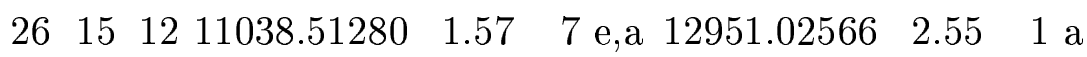

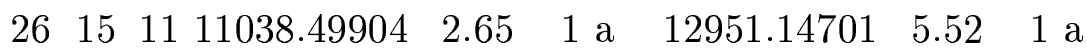

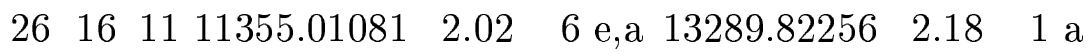

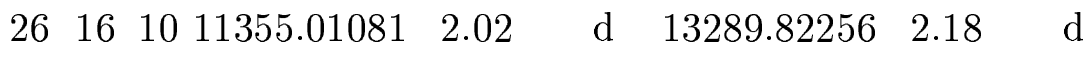

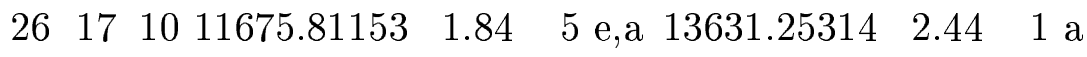
$\begin{array}{lllllllll}26 & 17 & 9 & 11675.87915 & 1.97 & 1 \mathrm{a} & 13631.25314 & 2.44 & \mathrm{~d}\end{array}$

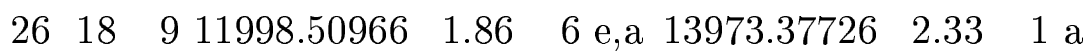
$\begin{array}{lllllllll}26 & 18 & 8 & 11998.50966 & 1.86 & \text { d } & 13973.37726 & 2.33 & \text { d }\end{array}$

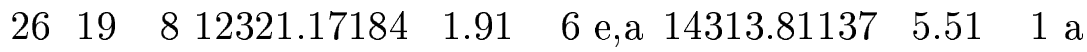
$\begin{array}{lllllllll}26 & 19 & 7 & 12321.17184 & 1.91 & \text { d } & 14313.81137 & 5.51 & \text { d }\end{array}$

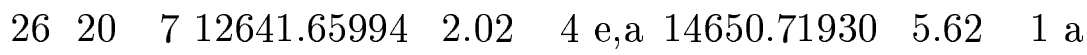
$\begin{array}{lllllllll}26 & 20 & 6 & 12641.65994 & 2.02 & \text { d } & 14650.71930 & 5.62 & \text { d }\end{array}$ $\begin{array}{lllllllll}26 & 21 & 6 & 12957.80670 & 2.29 & 1 \mathrm{a} & 14981.89217 & 5.52 & 1 \mathrm{a}\end{array}$

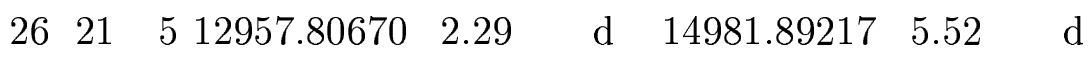

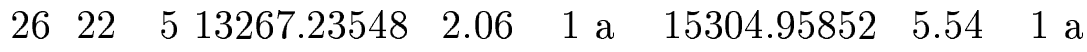

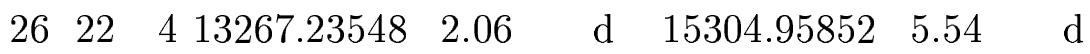

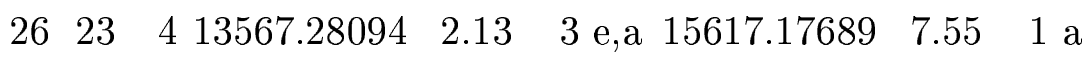
$\begin{array}{lllllllll}26 & 23 & 3 & 13567.28094 & 2.13 & \text { d } & 15617.17689 & 7.55 & \text { d }\end{array}$

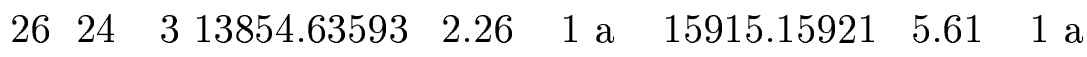
$\begin{array}{lllllllll}26 & 24 & 2 & 13854.63593 & 2.26 & \text { d } & 15915.15921 & 5.61 & \text { d }\end{array}$ 


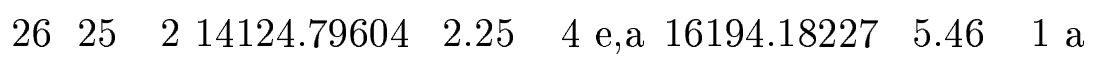
$\begin{array}{lllllllll}26 & 25 & 1 & 14124.79604 & 2.25 & \text { d } & 16194.18227 & 5.46 & \text { d }\end{array}$

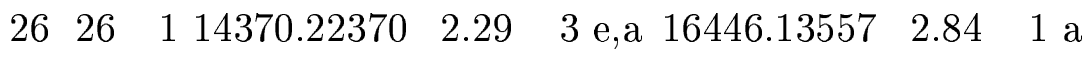
$\begin{array}{lllllllll}26 & 26 & 0 & 14370.22370 & 2.29 & \text { d } & 16446.13557 & 2.84 & \text { d }\end{array}$ $\begin{array}{lllllllll}27 & 0 & 27 & 7139.59254 & 1.99 & 14 \text { e,a } & 8676.10299 & 1.02 & 9\end{array}$ $\begin{array}{llllllllll}27 & 1 & 27 & 7139.59254 & 1.99 & \text { d } & 8676.10299 & 1.02 & \text { d }\end{array}$ $\begin{array}{lllllllll}27 & 1 & 26 & 7625.42754 & 1.52 & 14 \text { e,a } & 9236.46260 & 0.80 & 7 \text { e,a }\end{array}$ $\begin{array}{lllllllll}27 & 2 & 26 & 7625.42754 & 1.52 & \text { d } & 9236.46260 & 0.80 & \text { d }\end{array}$ $\begin{array}{lllllllll}27 & 2 & 25 & 8056.27630 & 1.73 & 3 \mathrm{a} & 9695.33495 & 1.10 & 3 \mathrm{a}\end{array}$ $\begin{array}{llllllllll}27 & 3 & 25 & 8056.27630 & 1.73 & \text { d } & 9695.35436 & 2.66 & 1 & \text { a }\end{array}$ $\begin{array}{llllllllll}27 & 3 & 24 & 8450.54780 & 1.48 & 12 \text { e,a } & 10119.12126 & 2.74 & 1 \text { a }\end{array}$ $\begin{array}{lllllllll}27 & 4 & 24 & 8450.56331 & 1.77 & 1 \text { a } & 10119.02359 & 1.77 & 1 \text { a }\end{array}$ $\begin{array}{lllllllll}27 & 4 & 23 & 8811.58617 & 1.37 & 1 \mathrm{a} & 10500.88622 & 2.37 & 1 \mathrm{a}\end{array}$ $\begin{array}{lllllllll}27 & 5 & 23 & 8811.63196 & 1.68 & 1 \mathrm{a} & 10501.20468 & 2.04 & 1 \mathrm{a}\end{array}$ $\begin{array}{lllllllll}27 & 5 & 22 & 9140.68761 & 2.15 & 7 \text { e,a } & 10837.77858 & 1.28 & 1 \text { a }\end{array}$

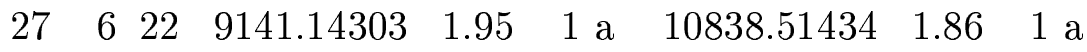
$\begin{array}{llllllllll}27 & 6 & 21 & 9437.36460 & 1.34 & 1 \mathrm{a} & 11149.99198 & 1.34 & 1 \mathrm{a}\end{array}$

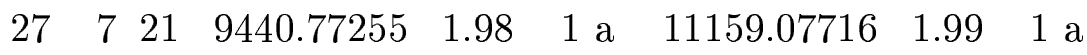

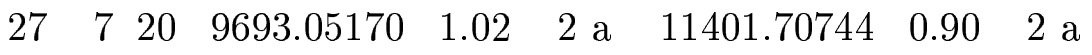
$\begin{array}{lllllll}27 & 8 & 20 & 9712.04365 & 1.04 & 2 \mathrm{a}\end{array}$ $\begin{array}{llllll}27 & 8 & 19 & 9892.70425 & 1.30 & 4 \mathrm{e}, \mathrm{a}\end{array}$ $\begin{array}{lllllllll}27 & 9 & 19 & 9962.97353 & 1.98 & 1 \text { a } & 11711.88599 & 5.29 & 1 \text { a }\end{array}$

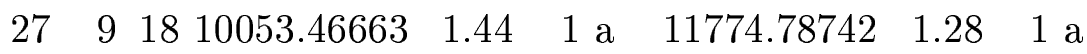
$\begin{array}{llllll}27 & 10 & 18 & 10208.67538 & 1.15 \quad 2 \mathrm{a}\end{array}$

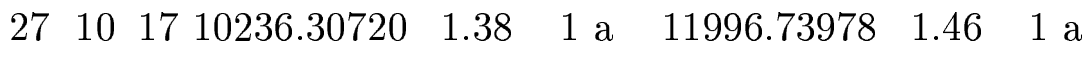

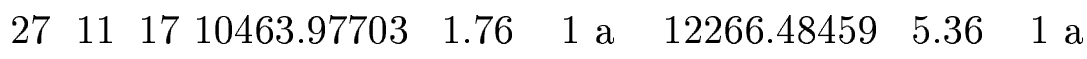

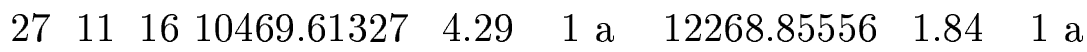




\begin{tabular}{|c|c|c|c|c|c|c|c|}
\hline 27 & 12 & 1610736.17236 & 1.74 & $1 \mathrm{a}$ & 12568.14068 & 2.55 & $1 \mathrm{a}$ \\
\hline 27 & 12 & 1510737.11681 & 1.44 & $1 \mathrm{a}$ & 12568.00037 & 1.79 & $1 \mathrm{a}$ \\
\hline 27 & 13 & 1511024.56818 & 1.99 & $2 \mathrm{e}, \mathrm{a}$ & 12884.12983 & 5.41 & $1 \mathrm{a}$ \\
\hline 27 & 13 & 1411024.70057 & 1.54 & $5 \mathrm{e}, \mathrm{a}$ & & & \\
\hline 27 & 14 & 1411325.96099 & 2.10 & $1 \mathrm{a}$ & 13211.16617 & 5.54 & $1 \mathrm{a}$ \\
\hline 27 & 14 & 1311325.98765 & 1.78 & $5 \mathrm{e}, \mathrm{a}$ & 13210.76970 & 2.10 & \\
\hline 27 & 15 & 1311637.27352 & 5.36 & $1 \mathrm{a}$ & & & \\
\hline 27 & 15 & 1211637.29475 & 1.78 & $6 \mathrm{e}, \mathrm{a}$ & & & \\
\hline 27 & 16 & 1211955.80916 & 2.83 & $1 \mathrm{a}$ & 13887.56787 & 7.45 & $1 \mathrm{a}$ \\
\hline 27 & 16 & 1111955.83542 & 1.77 & $4 \mathrm{e}, \mathrm{a}$ & 13887.49545 & 5.62 & $1 \mathrm{a}$ \\
\hline 27 & 17 & 1112279.23222 & 2.10 & d & 14231.90688 & 2.40 & $1 \mathrm{a}$ \\
\hline 27 & 17 & 1012279.23222 & 2.10 & $5 \mathrm{e}, \mathrm{a}$ & 14231.90688 & 2.40 & d \\
\hline 27 & 18 & 1012605.54615 & 3.98 & d & 14577.57449 & 5.56 & d \\
\hline 27 & 18 & 912605.54615 & 3.98 & $1 \mathrm{a}$ & 14577.57449 & 5.56 & $\mathrm{a}$ \\
\hline 27 & 19 & 912931.87672 & 1.99 & d & 14922.27868 & 5.51 & d \\
\hline 27 & 19 & 812931.87672 & 1.99 & $1 \mathrm{a}$ & 14922.27868 & 5.51 & $1 \mathrm{a}$ \\
\hline 27 & 20 & 813257.10311 & 2.04 & d & 15264.25320 & 7.44 & d \\
\hline 27 & 20 & 713257.10311 & 2.04 & $4 \mathrm{e}, \mathrm{a}$ & 15264.25320 & 7.44 & $\mathrm{a}$ \\
\hline 27 & 21 & 713578.90874 & 2.15 & $\mathrm{~d}$ & 15601.47143 & 7.52 & d \\
\hline 27 & 21 & 613578.90874 & 2.15 & $2 \mathrm{e}, \mathrm{a}$ & 15601.47143 & 7.52 & \\
\hline 27 & 22 & 613895.17236 & 2.49 & d & 15931.80770 & 4.48 & d \\
\hline 27 & 22 & 513895.17236 & 2.49 & $1 \mathrm{a}$ & 15931.80770 & 4.48 & \\
\hline 27 & 23 & 514203.51514 & 2.29 & d & 16252.89138 & 7.46 & d \\
\hline 27 & 23 & 414203.51514 & 2.29 & $1 \mathrm{a}$ & 16252.89138 & 7.46 & \\
\hline 27 & 24 & 414501.23669 & 2.27 & d & 16561.93898 & 9.06 & $\mu$ \\
\hline & 24 & 314501.23669 & 2.27 & 3 e,a & 16561.93898 & 9.06 & \\
\hline & 25 & 314784.89777 & 5.49 & d & 16855.41391 & 7.51 & d \\
\hline
\end{tabular}




\begin{tabular}{|c|c|c|c|c|c|c|c|c|}
\hline 27 & 25 & & 14784.89777 & 5.49 & $1 \mathrm{a}$ & 16855.41391 & 7.51 & $1 \mathrm{a}$ \\
\hline 27 & 26 & & 15049.69158 & 2.36 & $\mathrm{~d}$ & 17128.28932 & 7.40 & d \\
\hline 27 & 26 & & 15049.69158 & 2.36 & 3 e,a & 17128.28932 & 7.40 & $1 \mathrm{a}$ \\
\hline 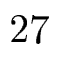 & 27 & & 15287.07544 & 2.50 & $d$ & 17371.35145 & 5.75 & d \\
\hline 1 & 27 & & 15287.07544 & 2.50 & $1 \mathrm{a}$ & 17371.35145 & 5.75 & $1 \mathrm{a}$ \\
\hline 28 & 0 & 28 & 7648.15125 & 1.90 & d & 9181.13639 & 0.96 & $d$ \\
\hline 28 & 1 & 28 & 7648.15125 & 1.90 & $12 \mathrm{e}, \mathrm{a}$ & 9181.13639 & 0.96 & $11 \mathrm{e}, \mathrm{a}$ \\
\hline 28 & 1 & 27 & 8150.77169 & 1.51 & d & 9763.18388 & 0.71 & $d$ \\
\hline 28 & 2 & 27 & 8150.77169 & 1.51 & $12 \mathrm{e}, \mathrm{a}$ & 9763.18388 & 0.71 & 5 a \\
\hline 28 & 2 & 26 & 8594.55013 & 1.79 & d & 10231.28755 & 1.81 & $1 \mathrm{a}$ \\
\hline 28 & 3 & 26 & 8594.55013 & 1.79 & $2 \mathrm{a}$ & 10231.34539 & 2.00 & $1 \mathrm{a}$ \\
\hline 2 & 3 & 25 & 9001.72235 & 0.93 & d & & & \\
\hline 2 & 4 & 25 & 9001.72235 & 0.93 & $11 \mathrm{e}, \mathrm{a}$ & 10669.59036 & 1.40 & $2 \mathrm{a}$ \\
\hline 28 & 4 & 24 & 9375.31030 & 1.96 & $1 \mathrm{a}$ & 11064.22348 & 2.28 & $1 \mathrm{a}$ \\
\hline 28 & 5 & 24 & 9375.33989 & 1.70 & $1 \mathrm{a}$ & 11063.79320 & 1.70 & $1 \mathrm{a}$ \\
\hline 28 & 5 & 23 & 9716.75173 & 1.33 & $2 \mathrm{a}$ & 11424.87078 & 2.11 & $1 \mathrm{a}$ \\
\hline 28 & 6 & 23 & 9717.02087 & 1.43 & $2 \mathrm{a}$ & 11425.83117 & 1.67 & $1 \mathrm{a}$ \\
\hline 28 & 6 & 22 & 10027.38485 & 2.22 & $1 \mathrm{a}$ & & & \\
\hline 28 & 7 & 22 & 10029.51187 & 1.68 & $1 \mathrm{a}$ & 11747.31363 & 1.68 & $1 \mathrm{a}$ \\
\hline 28 & 7 & 21 & 10299.90534 & 1.44 & $1 \mathrm{a}$ & & & \\
\hline 28 & 8 & 21 & 10312.64405 & 1.43 & $1 \mathrm{a}$ & 12043.54558 & 1.35 & $1 \mathrm{a}$ \\
\hline 28 & 8 & 20 & 10519.74111 & 2.22 & $1 \mathrm{a}$ & & & \\
\hline 2 & 9 & 20 & 10572.59107 & 1.17 & $2 \mathrm{a}$ & & & \\
\hline 28 & 10 & 19 & 10822.54060 & 1.43 & $1 \mathrm{a}$ & & & \\
\hline 28 & 10 & 18 & 10864.39736 & 2.22 & $1 \mathrm{a}$ & 12614.68645 & 5.15 & a \\
\hline 28 & 11 & 18 & 11079.21352 & 1.58 & $5 \mathrm{e}, \mathrm{a}$ & 12876.17563 & 1.77 & \\
\hline & 11 & 17 & 11087.99270 & 1.52 & $1 \mathrm{a}$ & & & \\
\hline
\end{tabular}




\begin{tabular}{|c|c|c|c|c|c|c|}
\hline & 12 & 1711349.984 & 40 & $1 \mathrm{a}$ & 1 & \\
\hline & 12 & 1611351.62162 & 2.03 & $1 \mathrm{a}$ & 13176.99032 & 5.46 \\
\hline & 13 & 1611637.94054 & 1.75 & $1 \mathrm{a}$ & 3492.75861 & 2.05 \\
\hline & 13 & 1511638.13385 & 2.01 & $1 \mathrm{a}$ & 184 & $\$ 1$ \\
\hline & 14 & 1511939.69754 & 1.75 & $4 \mathrm{e}, \mathrm{a}$ & & \\
\hline & 14 & 1411939.75855 & 2.23 & $1 \mathrm{a}$ & 13820.12568 & 7.36 \\
\hline & 15 & 1412252.10280 & 1.95 & 3 e,a & 14157.37707 & 2.14 \\
\hline & 15 & 1312252.13250 & 2.32 & $1 \mathrm{a}$ & 8973 & 7.46 \\
\hline & 16 & 1312572.33205 & 1.93 & $4 \mathrm{e}, \mathrm{a}$ & & \\
\hline & 16 & 1212572.35872 & 7.33 & $1 \mathrm{a}$ & & \\
\hline 2 & 17 & 121289 & 1.94 & $3 \mathrm{e}, \mathrm{a}$ & 2698 & 7.52 \\
\hline & 17 & 1112898.10402 & 5.75 & $1 \mathrm{a}$ & 14847.18506 & 8.97 \\
\hline & 18 & 1113227.0 & 2.20 & $4 \mathrm{e}, \mathrm{a}$ & 229 & 5.55 \\
\hline & 18 & 1013227.02320 & 2.20 & d & 0229 & 5.55 \\
\hline & 19 & 1013557.51142 & 4.10 & $1 \mathrm{a}$ & 7105 & 7.48 \\
\hline & 19 & 913557.51142 & 4.10 & d & 4.67105 & 7.48 \\
\hline 2 & 20 & 913886.70595 & 2.22 & $1 \mathrm{a}$ & 15891.19177 & 7.44 \\
\hline & 20 & 813886.70595 & 2.22 & d & 15891.19177 & 7.44 \\
\hline 2 & 21 & 814213.58668 & 2.18 & $2 \mathrm{e}, \mathrm{a}$ & 16233.80789 & 8.97 \\
\hline 2 & 21 & 714213.58668 & 2.18 & d & 16233.80789 & 8.97 \\
\hline & 22 & 714535.89800 & 2.36 & $1 \mathrm{a}$ & 8649 & 9.03 \\
\hline & 22 & 614535.89800 & 2.36 & d & 58649 & 9.03 \\
\hline & 23 & 614851.56256 & 5.58 & $1 \mathrm{a}$ & 16899.43740 & 6.71 \\
\hline & 23 & 514851.56256 & 5.58 & $d$ & 16899.43740 & 6.7 \\
\hline & 24 & 515158.16498 & 5.50 & $1 \mathrm{a}$ & 17217.96456 & 8.98 \\
\hline & 24 & 415158.16498 & 5.50 & d & 17217.96456 & 8.98 \\
\hline & & 415452.99194 & 2.42 & $\mathrm{e}, \mathrm{a}$ & 23.3 & 10.35 \\
\hline
\end{tabular}




\begin{tabular}{|c|c|c|c|c|c|c|c|c|}
\hline 8 & 25 & & 15452.99194 & 2.42 & $\mathrm{~d}$ & 17523.37409 & 10.35 & $1 \mathrm{a}$ \\
\hline 28 & 26 & & 15732.45423 & 7.42 & $1 \mathrm{a}$ & 17811.86941 & 9.03 & $1 \mathrm{a}$ \\
\hline & 26 & & 15732.45423 & 7.42 & $d$ & 17811.86941 & 9.03 & $d$ \\
\hline 28 & 27 & & 15991.42817 & 5.00 & $2 \mathrm{e}, \mathrm{a}$ & 18078.22408 & 8.93 & $1 \mathrm{a}$ \\
\hline 2 & 27 & & 15991.42817 & 5.00 & $d$ & 18078.22408 & 8.93 & d \\
\hline 28 & 28 & & 16220.19037 & 5.59 & $1 \mathrm{a}$ & 18311.89961 & 7.62 & $1 \mathrm{a}$ \\
\hline 28 & 28 & & 16220.19037 & 5.59 & d & 18311.89961 & 7.62 & d \\
\hline 2 & 0 & 29 & 8173.02249 & 1.89 & 12 e,a & 9702.72221 & 1.12 & $6 \mathrm{e}, \mathrm{a}$ \\
\hline 29 & 1 & 29 & 8173.02249 & 1.89 & d & 9702.72221 & 1.12 & d \\
\hline 29 & 1 & 28 & 8692.26398 & 1.57 & $9 \mathrm{e}, \mathrm{a}$ & 10312.82326 & 1.02 & $2 \mathrm{a}$ \\
\hline 29 & 2 & 28 & 8692.26398 & 1.57 & d & 10312.93194 & 0.99 & $2 \mathrm{a}$ \\
\hline 29 & 2 & 27 & 9148.28433 & 1.82 & $-1 \mathrm{f}, \mathrm{a}$ & 10781.89282 & 1.51 & $2 \mathrm{a}$ \\
\hline 29 & 3 & 27 & 9148.28433 & 1.82 & d & 10781.89282 & 1.51 & d \\
\hline 29 & 3 & 26 & 9567.99354 & 1.28 & $10 \mathrm{e}, \mathrm{a}$ & 11234.83216 & 1.07 & $2 \mathrm{a}$ \\
\hline 29 & 4 & 26 & 9567.99354 & 1.28 & d & 11234.91541 & 1.91 & $1 \mathrm{a}$ \\
\hline 29 & 4 & 25 & 9953.71416 & 1.97 & $1 \mathrm{a}$ & 11641.55919 & 1.97 & $1 \mathrm{a}$ \\
\hline 29 & 5 & 25 & 9953.73017 & 2.20 & $1 \mathrm{a}$ & 11641.40036 & 2.49 & $1 \mathrm{a}$ \\
\hline 29 & 5 & 24 & 10300.64016 & 1.04 & $2 \mathrm{a}$ & & & \\
\hline 29 & 6 & 24 & 10300.71255 & 1.05 & $2 \mathrm{a}$ & 12015.49023 & 2.33 & $1 \mathrm{a}$ \\
\hline 29 & 6 & 23 & 10630.78550 & 1.95 & $1 \mathrm{a}$ & & & \\
\hline 29 & 7 & 23 & 10632.13888 & 2.43 & $1 \mathrm{a}$ & & & \\
\hline 29 & 7 & 22 & 10918.75574 & 1.75 & $1 \mathrm{a}$ & & & \\
\hline 29 & 8 & 22 & 10927.18818 & 1.76 & $1 \mathrm{a}$ & & & \\
\hline 29 & 8 & 21 & 11158.44709 & 1.54 & $1 \mathrm{a}$ & & & \\
\hline 29 & 9 & 21 & 11196.87490 & 2.43 & $1 \mathrm{a}$ & & & \\
\hline 29 & 9 & 20 & 11342.27943 & 1.75 & $1 \mathrm{a}$ & & & \\
\hline 2 & 10 & 19 & 11512.44959 & 1.53 & $1 \mathrm{a}$ & & & \\
\hline
\end{tabular}


$\begin{array}{lllllll}29 & 11 & 18 & 11724.97706 & 1.74 & 1 \mathrm{a}\end{array}$

$\begin{array}{llllll}29 & 12 & 18 & 11980.27957 & 1.82 \quad 1 \mathrm{a}\end{array}$

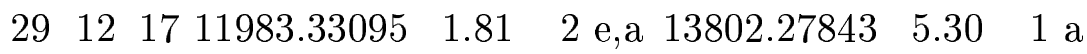

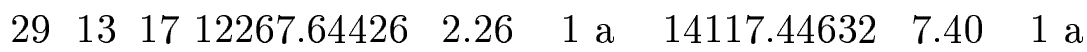

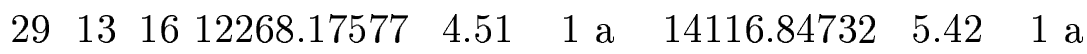

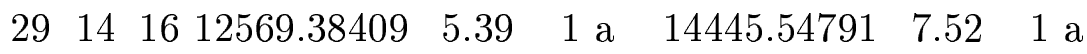

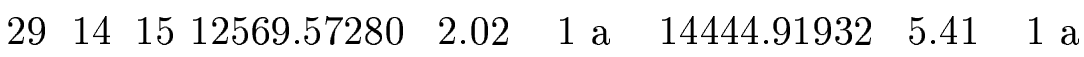

$\begin{array}{llllllllll}29 & 15 & 15 & 12882.61536 & 2.44 & 1 \mathrm{a} & 14783.39851 & 8.90 & 1 \mathrm{a}\end{array}$

$29 \quad 15 \quad 1412882.67254 \quad 1.93 \quad 2$ e,a

$\begin{array}{lllllllll}29 & 16 & 14 & 13204.16644 & 2.52 & 1 \text { a } & 15127.88248 & 8.98 & 1 \text { a }\end{array}$

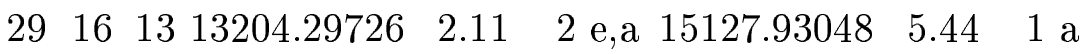

$\begin{array}{llllll}29 & 17 & 13 & 13532.04293 & 8.87 & 1 \mathrm{a}\end{array}$

$29 \quad 17 \quad 12 \quad 13532.06163 \quad 4.75 \quad 2$ e,a

$\begin{array}{lllllllll}29 & 18 & 12 & 13863.54177 & 5.36 & \text { d } & 15828.79550 & 9.03 & \text { d }\end{array}$

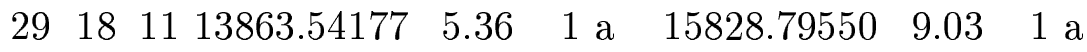

$\begin{array}{lllllllll}29 & 19 & 11 & 14196.87669 & 2.31 & \text { d } & 16180.71780 & 7.47 & \text { d }\end{array}$

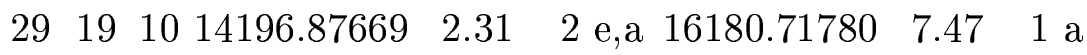

$\begin{array}{lllllllll}29 & 20 & 10 & 14530.48920 & 4.22 & \text { d } & 16531.21737 & 9.00 & \text { d }\end{array}$

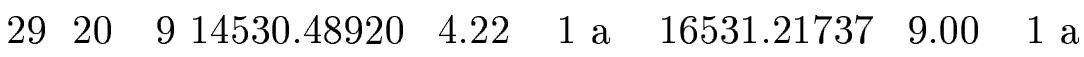

$\begin{array}{lllllllll}29 & 21 & 9 & 14861.62532 & 2.43 & \text { d } & 16878.60565 & 8.97 & \text { d }\end{array}$

$\begin{array}{lllllllll}29 & 21 & 8 & 14861.62532 & 2.43 & 1 \text { a } & 16878.60565 & 8.97 & 1 \text { a }\end{array}$

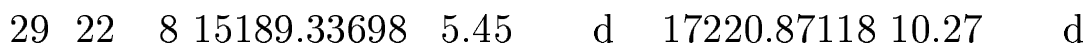

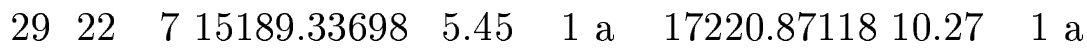

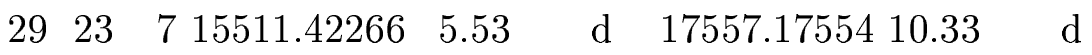

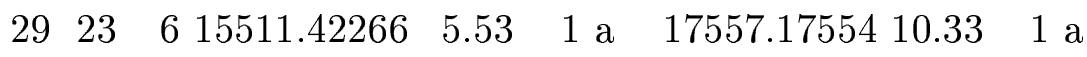

$\begin{array}{lllllllll}29 & 24 & 6 & 15825.76850 & 7.50 & \text { d } & 17883.69814 & 8.37 & \text { d }\end{array}$ 


\begin{tabular}{|c|c|c|c|c|c|c|}
\hline 29 & 24 & 515825.76850 & 7.50 & $1 \mathrm{a}$ & 17883.69814 & 8.37 \\
\hline 29 & 25 & 516130.12885 & 7.43 & d & 18199.13906 & 10.28 \\
\hline 9 & 25 & 416130.12885 & 7.43 & $1 \mathrm{a}$ & 18199.13906 & 10.28 \\
\hline 29 & 26 & 416421.56158 & 5.55 & $\mathrm{~d}$ & 18500.37007 & 11.49 \\
\hline$y$ & 26 & 316421.56158 & 5.55 & $1 \mathrm{a}$ & 18500.37007 & 11.49 \\
\hline$y$ & 27 & 316696.39277 & 8.95 & $\mathrm{~d}$ & 18783.62186 & 10.32 \\
\hline 9 & 27 & 216696.39277 & 8.95 & $1 \mathrm{a}$ & 18783.62186 & 10.32 \\
\hline 29 & 28 & 216949.17494 & 7.07 & $d$ & 19043.16970 & 10.24 \\
\hline 29 & 28 & 116949.17494 & 7.07 & $1 \mathrm{a}$ & 19043.16970 & 10.24 \\
\hline 29 & 29 & 117168.81788 & 7.50 & $\mathrm{~d}$ & 19267.04640 & 9.11 \\
\hline 29 & 29 & 017168.81788 & 7.50 & $1 \mathrm{a}$ & 19267.04640 & 9.11 \\
\hline 30 & 0 & $30 \quad 8714.05583$ & 1.96 & d & 10240.80422 & 1.20 \\
\hline 30 & 1 & $30 \quad 8714.05583$ & 1.96 & $11 \mathrm{e}, \mathrm{a}$ & 10240.80422 & 1.20 \\
\hline 30 & 1 & $29 \quad 9249.74957$ & 1.66 & $\mathrm{~d}$ & 10863.88979 & 0.85 \\
\hline 30 & 2 & $29 \quad 9249.74957$ & 1.66 & $8 \mathrm{e}, \mathrm{a}$ & 10863.88979 & 0.85 \\
\hline 30 & 2 & $28 \quad 9717.22916$ & 1.94 & $\mathrm{~d}$ & 11346.62153 & 1.36 \\
\hline 30 & 3 & $28 \quad 9717.22916$ & 1.94 & $2 \mathrm{a}$ & 11346.62153 & 1.36 \\
\hline 30 & 3 & 2710149.14283 & 1.54 & $\mathrm{~d}$ & 11814.71698 & 1.09 \\
\hline 30 & 4 & 2710149.14283 & 1.54 & $6 \mathrm{e}, \mathrm{a}$ & 11814.71698 & 1.09 \\
\hline 30 & 4 & 2610546.56317 & 2.42 & $1 \mathrm{a}$ & 12232.86510 & 2.21 \\
\hline 30 & 5 & 2610546.57445 & 2.21 & $1 \mathrm{a}$ & 12232.86510 & 2.21 \\
\hline 30 & 6 & 2510913.68294 & 1.02 & $2 \mathrm{a}$ & & \\
\hline 30 & 6 & 2411247.41183 & 2.63 & $1 \mathrm{a}$ & & \\
\hline 30 & 7 & 2411248.25376 & 2.19 & $1 \mathrm{a}$ & & \\
\hline 30 & 7 & 2311549.77265 & 2.02 & $1 \mathrm{a}$ & & \\
\hline 30 & 8 & 2311555.26215 & 2.01 & $1 \mathrm{a}$ & & \\
\hline & 9 & 2211835.28406 & 1.84 & $1 \mathrm{a}$ & & \\
\hline
\end{tabular}


$\begin{array}{llllll}30 & 10 & 21 & 12097.46204 & 2.01 & 1 \mathrm{a}\end{array}$

$\begin{array}{llllll}30 & 11 & 20 & 12357.01343 & 1.83 & 1 \mathrm{a}\end{array}$

$\begin{array}{lllllll}30 & 12 & 18 & 14443.18584 & 7.24 & 1 \mathrm{a}\end{array}$

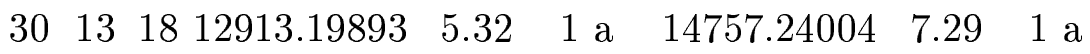

$\begin{array}{llllll}30 & 13 & 17 & 12914.37313 & 5.32 & 1 \mathrm{a}\end{array}$

$\begin{array}{llllll}30 & 14 & 17 & 13214.88329 & 4.62 & 1 \text { a }\end{array}$

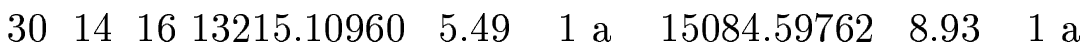

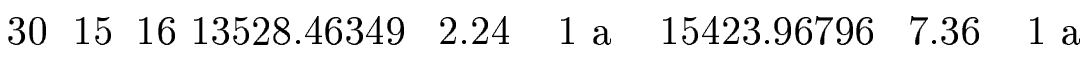

$\begin{array}{lllllllll}30 & 15 & 15 & 13528.51843 & 7.35 & 1 \mathrm{a} & 15424.13543 & 9.03 & 1 \mathrm{a}\end{array}$

$\begin{array}{llllll}30 & 16 & 15 & 13850.79168 & 5.36 & 1 \mathrm{a}\end{array}$

$\begin{array}{llllll}30 & 16 & 14 & 13851.27320 & 5.56 & 1 \mathrm{a}\end{array}$

$\begin{array}{lllllllll}30 & 17 & 14 & 14180.73135 & 2.32 & 1 \text { a } & 16120.48944 & 7.39 & 1 \text { a }\end{array}$

$\begin{array}{llllll}30 & 17 & 13 & 14180.77264 & 5.60 \quad 1 \mathrm{a}\end{array}$

$\begin{array}{llllll}30 & 18 & 13 & 14514.43214 & 5.71 & 2 \mathrm{a}\end{array}$

$\begin{array}{llllll}30 & 18 & 12 & 14514.43214 & 5.71 & \text { d }\end{array}$

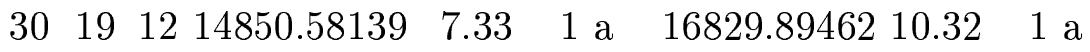

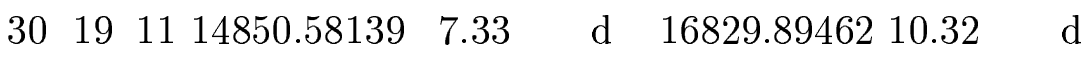

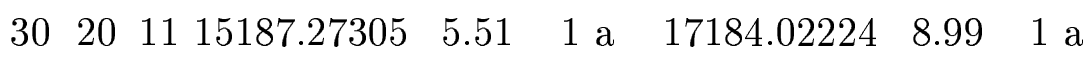

$\begin{array}{lllllllll}30 & 20 & 10 & 15187.27305 & 5.51 & \text { d } & 17184.02224 & 8.99 & \text { d }\end{array}$

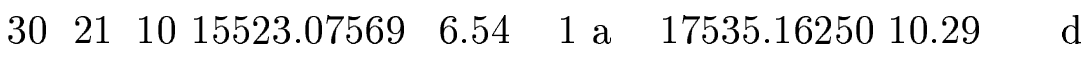

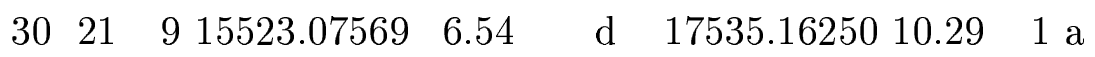

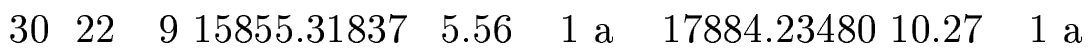

$\begin{array}{lllllllll}30 & 22 & 8 & 15855.31837 & 5.56 & \text { d } & 17884.23480 & 10.27 & \text { d }\end{array}$

$\begin{array}{llllll}30 & 23 & 8 & 16183.12256 & 7.40 & 1 \mathrm{a}\end{array}$

$\begin{array}{llllll}30 & 23 & 7 & 16183.12256 & 7.40 \quad \mathrm{~d}\end{array}$

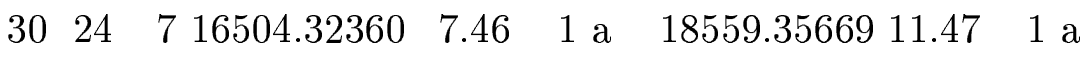

$\begin{array}{lllllllll}30 & 24 & 6 & 16504.32360 & 7.46 & \text { d } & 18559.35669 & 11.47 & \text { d }\end{array}$ 


\begin{tabular}{|c|c|c|c|c|c|c|c|}
\hline 30 & 25 & 616816.85425 & 9.01 & $1 \mathrm{a}$ & 18883.43508 & 9.75 & $1 \mathrm{a}$ \\
\hline 30 & 25 & 516816.85425 & 9.01 & $\mathrm{~d}$ & 18883.43508 & 9.75 & d \\
\hline 30 & 26 & 517118.34980 & 8.96 & $1 \mathrm{a}$ & & & \\
\hline 30 & 26 & 417118.34980 & 8.96 & $\mathrm{~d}$ & & & \\
\hline 0 & 27 & 417405.98907 & 7.47 & $1 \mathrm{a}$ & 19492.32106 & 12.53 & $1 \mathrm{a}$ \\
\hline 0 & 27 & 317405.98907 & 7.47 & $d$ & 19492.32106 & 12.53 & $\mathrm{~d}$ \\
\hline U & 28 & 317675.82703 & 10.25 & $1 \mathrm{a}$ & 19769.73459 & 11.46 & $1 \mathrm{a}$ \\
\hline 30 & 28 & 217675.82703 & 10.25 & d & 19769.73459 & 11.46 & $d$ \\
\hline 30 & 29 & 217922.13026 & 8.66 & $1 \mathrm{a}$ & 20022.33431 & 11.39 & $1 \mathrm{a}$ \\
\hline 30 & 29 & 117922.13026 & 8.66 & d & 20022.33431 & 11.39 & $d$ \\
\hline 30 & 30 & 118132.22760 & 9.01 & $1 \mathrm{a}$ & & & \\
\hline 30 & 30 & 018132.22760 & 9.01 & $\mathrm{~d}$ & & & \\
\hline 31 & 0 & $31 \quad 9271.09431$ & 2.05 & $8 \mathrm{e}, \mathrm{a}$ & 10795.33973 & 1.23 & $5 \mathrm{e}, \mathrm{a}$ \\
\hline 31 & 1 & 319271.09431 & 2.05 & $d$ & 10795.33973 & 1.23 & $d$ \\
\hline 31 & 1 & $30 \quad 9823.06898$ & 1.76 & 9 e,a & 11439.90083 & 1.31 & d \\
\hline 31 & 2 & $30 \quad 9823.06898$ & 1.76 & d & 11439.90083 & 1.31 & $1 \mathrm{a}$ \\
\hline 31 & 2 & 2910301.11362 & 2.18 & $1 \mathrm{a}$ & 11925.57718 & 1.34 & $\mathrm{~d}$ \\
\hline 31 & 3 & 2910301.11362 & 2.18 & d & 11925.57718 & 1.34 & $2 \mathrm{a}$ \\
\hline 31 & 3 & 2810744.94457 & 1.46 & $2 \mathrm{a}$ & 12408.87860 & 1.48 & $1 \mathrm{a}$ \\
\hline 3. & 4 & 2810744.94457 & 1.46 & d & & & \\
\hline 31 & 4 & 2711153.62102 & 2.42 & $1 \mathrm{a}$ & 12836.65642 & 2.42 & $1 \mathrm{a}$ \\
\hline 31 & 5 & 2711153.62763 & 2.61 & $1 \mathrm{a}$ & & & \\
\hline 31 & 5 & 2611531.72164 & 1.43 & $1 \mathrm{a}$ & & & \\
\hline 31 & 6 & 2511876.51865 & 2.41 & $1 \mathrm{a}$ & & & \\
\hline 21 & 7 & 2511877.04330 & 2.81 & $1 \mathrm{a}$ & & & \\
\hline 31 & 7 & 2412192.87434 & 2.25 & $1 \mathrm{a}$ & & & \\
\hline & & 19 & & & 15411.99718 & 8.80 & $1 \mathrm{a}$ \\
\hline
\end{tabular}




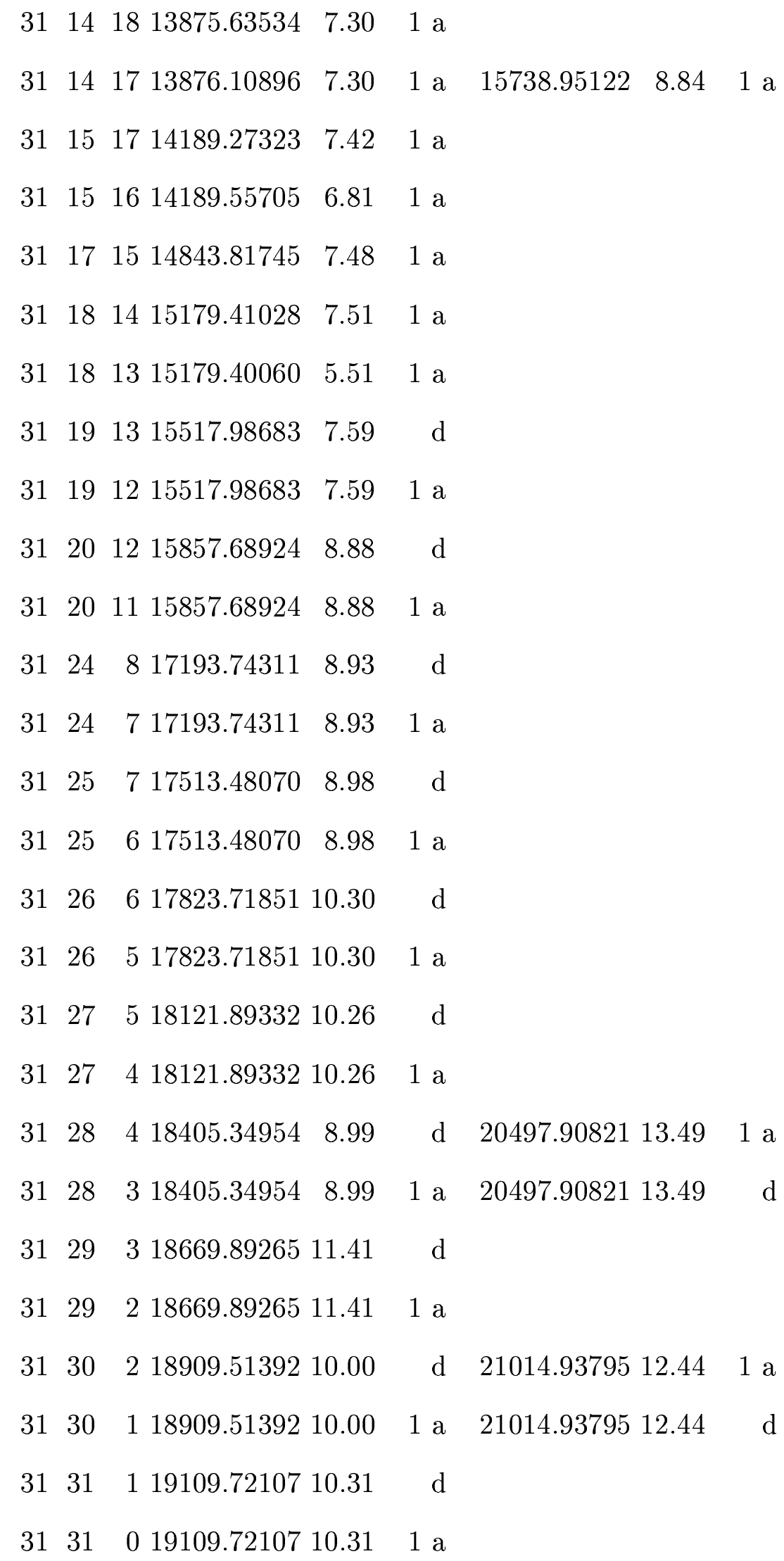




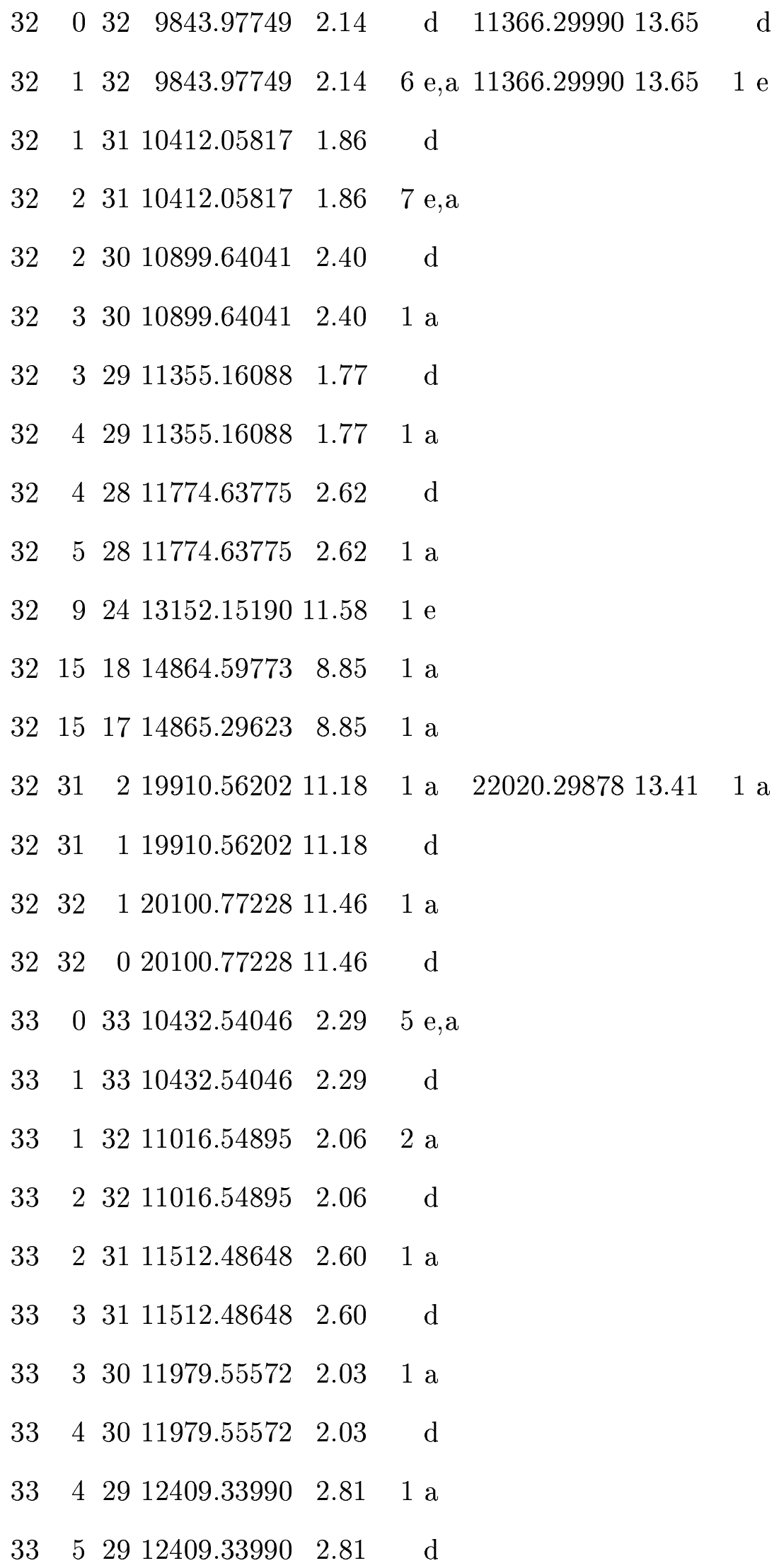


$\begin{array}{llllll}33 & 33 & 1 & 21105.38800 & 12.50 \quad \mathrm{~d}\end{array}$

$\begin{array}{llllll}33 & 33 & 0 & 21105.38800 & 12.50 \quad 1 \mathrm{a}\end{array}$

$\begin{array}{llllll}34 & 0 & 34 & 11036.61378 & 2.42 & \text { d }\end{array}$

$\begin{array}{lllll}34 & 1 & 34 & 11036.61378 & 2.42 \quad 4 \mathrm{e}, \mathrm{a}\end{array}$

$\begin{array}{llllll}34 & 1 & 33 & 11636.36835 & 2.28 & \text { d }\end{array}$

$34 \quad 2 \quad 3311636.36835 \quad 2.28-1 \mathrm{f}, \mathrm{a}$

$\begin{array}{lllllll}35 & 0 & 35 & 11656.02503 & 2.62 & 1 \mathrm{a}\end{array}$

$\begin{array}{llllll}35 & 1 & 35 & 11656.02503 & 2.62 & \mathrm{~d}\end{array}$ 
TABLE III.: Term values for the first polyad, $1 \nu$, of $\mathrm{H}_{2}{ }^{16} \mathrm{O}$.

\begin{tabular}{|c|c|c|c|c|c|c|c|c|}
\hline \multicolumn{3}{|c|}{$J K_{a} K_{c}$} & \multirow{2}{*}{$\begin{array}{r}020 \text { or } \\
3151.63007\end{array}$} & \multirow{2}{*}{$\frac{002}{0.08-1 \mathrm{c}, \mathrm{a}}$} & \multicolumn{2}{|c|}{100 or $10^{+} 0$} & \multicolumn{2}{|c|}{001 or $10^{-} 0$} \\
\hline 0 & 0 & 0 & & & 3657 & $4-1 c$ & 2868 & $0.03-1 \mathrm{c}, \mathrm{a}$ \\
\hline 1 & 0 & 1 & 2 & $3-1 c, a$ & & $06-1 \mathrm{c}, \mathrm{a}$ & & $05-1 \mathrm{c}$ \\
\hline 1 & 1 & 1 & 33 & $02-1 \mathrm{c}, \mathrm{a}$ & & $-1 \mathrm{c}$ & & $0.02-1 \mathrm{c}, \mathrm{a}$ \\
\hline 1 & 1 & 0 & 2 & $03-1 \mathrm{c}$ & & $06-1$ & & $0.05-1 \mathrm{c}, \mathrm{a}$ \\
\hline 2 & 0 & 2 & 7 & $02-1 \mathrm{c}, \mathrm{a}$ & & $03-1$ c,a & & $.06-1 \mathrm{c}, \mathrm{a}$ \\
\hline 2 & 1 & 2 & 22 & $.04-1 \mathrm{c}, \mathrm{a}$ & & $19-1 \mathrm{c}, \mathrm{a}$ & & $.03-1 \mathrm{c}, \mathrm{a}$ \\
\hline 2 & 1 & 1 & 5 & $.04-1 \mathrm{c}, \mathrm{a}$ & & $.04-1 \mathrm{c}, \mathrm{a}$ & & $0.04-1 \mathrm{c}, \mathrm{a}$ \\
\hline 2 & 2 & 1 & 34 & $.04-1 \mathrm{c}, \mathrm{a}$ & & $0.07-1 \mathrm{c}, \mathrm{a}$ & & $0.06-1 \mathrm{c}, \mathrm{a}$ \\
\hline 2 & 2 & 0 & 317.21064 & $0.05-1 \mathrm{c}, \mathrm{a}$ & & $0.05-1 \mathrm{c}, \mathrm{a}$ & & $0.03-1 \mathrm{c}, \mathrm{a}$ \\
\hline 3 & 0 & 3 & 253 & $.03-1 \mathrm{c}, \mathrm{a}$ & 7207 & $0.06-1 \mathrm{c}, \mathrm{a}$ & & $0.07-1 \mathrm{c}, \mathrm{a}$ \\
\hline 3 & 1 & 3 & 106 & $.05-1 \mathrm{c}, \mathrm{a}$ & 3796.53970 & $0.04-1 \mathrm{c}, \mathrm{a}$ & & $0.02-1 \mathrm{c}, \mathrm{a}$ \\
\hline 3 & 1 & 2 & 654 & $.03-1 \mathrm{c}, \mathrm{a}$ & & $0.02-1 \mathrm{c}, \mathrm{a}$ & & $0.07-1 \mathrm{c}, \mathrm{a}$ \\
\hline 3 & 2 & 2 & 065 & $0.05-1 \mathrm{c}, \mathrm{a}$ & & $0.04-1 \mathrm{c}, \mathrm{a}$ & & $0.07-1 \mathrm{c}, \mathrm{a}$ \\
\hline 3 & 2 & 1 & 34 & $0.04-1 \mathrm{c}, \mathrm{a}$ & 864.76372 & $0.05-1 \mathrm{c}, \mathrm{a}$ & 3962 & $0.03-1 \mathrm{c}, \mathrm{a}$ \\
\hline 3 & 3 & 1 & 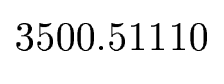 & $.04-1 \mathrm{c}, \mathrm{a}$ & 126 & $0.08-1 \mathrm{c}, \mathrm{a}$ & 4030.06989 & $0.06-1 \mathrm{c}, \mathrm{a}$ \\
\hline 3 & 3 & 0 & 869 & $0.05-1 \mathrm{c}, \mathrm{a}$ & 4470 & $0.04-1 \mathrm{c}, \mathrm{a}$ & 4030.30616 & $0.04-1 \mathrm{c}, \mathrm{a}$ \\
\hline 4 & 0 & 4 & 782 & $0.07-1 \mathrm{c}, \mathrm{a}$ & 5.01704 & $0.06-1 \mathrm{c}, \mathrm{a}$ & 3974.63090 & $0.05-1 \mathrm{c}, \mathrm{a}$ \\
\hline 4 & 1 & 4 & 424 & $0.04-1 \mathrm{c}, \mathrm{a}$ & 57516 & $0.05-1 \mathrm{c}, \mathrm{a}$ & 3977.26146 & $0.03-1 \mathrm{c}, \mathrm{a}$ \\
\hline 4 & 1 & 3 & & $0.05-1 \mathrm{c}, \mathrm{a}$ & 275 & $0.12-1 \mathrm{c}, \mathrm{a}$ & 7.80399 & $0.03-1 \mathrm{c}, \mathrm{a}$ \\
\hline 4 & 2 & 3 & & $0.04-1 \mathrm{c}, \mathrm{a}$ & 505 & $0.04-1 \mathrm{c}, \mathrm{a}$ & 405 & $0.04-1 \mathrm{c}, \mathrm{a}$ \\
\hline 4 & 2 & 2 & 349 & $0.05-1 \mathrm{c}, \mathrm{a}$ & 932 & $0.07-1 \mathrm{c}, \mathrm{a}$ & 4066 & $0.02-1 \mathrm{c}, \mathrm{a}$ \\
\hline 4 & 3 & 2 & 602 & $0.04-1 \mathrm{c}, \mathrm{a}$ & 4030.83892 & $0.06-1 \mathrm{c}, \mathrm{a}$ & 4125.14862 & $0.06-1 \mathrm{c}, \mathrm{a}$ \\
\hline 4 & 3 & 1 & 02 & $04-1 \mathrm{c}, \mathrm{a}$ & 8 & $.04-1 \mathrm{c}$ & 336 & $0.04-1 \mathrm{c}, \mathrm{a}$ \\
\hline
\end{tabular}


$4 \quad 4 \quad 1 \quad 3746.76262 \quad 0.04-1 \quad c, a \quad 4135.01762 \quad 0.03-1$ c,a $4224.81686 \quad 0.05-1$ c

$44 \quad 0 \quad 3746.77595 \quad 0.04-1 \quad c, a \quad 4134.79845 \quad 0.06-1 \quad c, a \quad 4224.85096 \quad 0.08-1 \quad c, a$

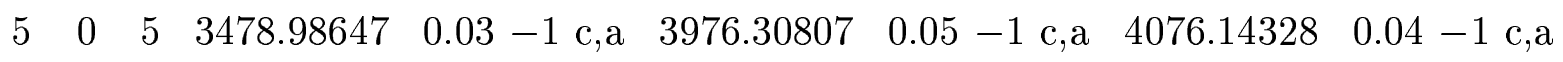

$5 \quad 1 \quad 5 \quad 3482.48019 \quad 0.05-1$ c,a $3977.45644 \quad 0.06-1$ c,a $4076.89581 \quad 0.03-1$ c,a

$5 \quad 143565.45461 \quad 0.03-1$ c,a $4049.53611 \quad 0.07-1$ c,a $4149.89926 \quad 0.04-1$ c,a

$5243598.51596 \quad 0.05-1 \mathrm{c}, \mathrm{a} \quad 4065.13186 \quad 0.06-1 \mathrm{c} \quad 4165.47381 \quad 0.06-1 \mathrm{c}, \mathrm{a}$

$523 \quad 3626.92216 \quad 0.03-1$ c,a $4095.91994 \quad 0.06-1$ c,a $4195.97092 \quad 0.04-1$ c,a

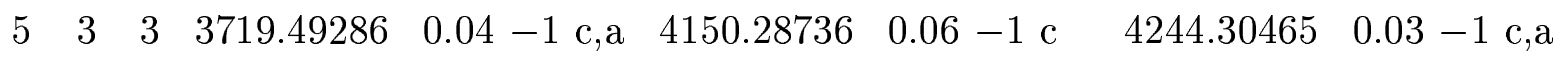

$5 \quad 3 \quad 2 \quad 3722.73097 \quad 0.05-1 \mathrm{c}, \mathrm{a} \quad 4153.93806 \quad 0.04-1 \mathrm{c}, \mathrm{a} \quad 4248.15245 \quad 0.04-1 \mathrm{c}, \mathrm{a}$

$5423868.87286 \quad 0.04-1$ c,a $4257.78674 \quad 0.05-1 \quad$ c,a $4345.27203 \quad 0.07-1$ c,a

$5 \quad 4 \quad 1 \quad 3868.98687 \quad 0.04-1$ c,a $4256.24128 \quad 0.05-1$ c,a $4345.55908 \quad 0.06-1$ c,a

$5 \quad 5 \quad 1 \quad 4050.50371 \quad 0.06-1 \mathrm{c}, \mathrm{a} \quad 4381.90416 \quad 0.07-1 \mathrm{c} \quad 4468.69324 \quad 0.08-1 \mathrm{c}, \mathrm{a}$

$5 \quad 5 \quad 0 \quad 4050.51269 \quad 0.08$ - 1 c,a $4381.90399 \quad 0.03-1$ c,a $4468.69775 \quad 0.09$-1 c

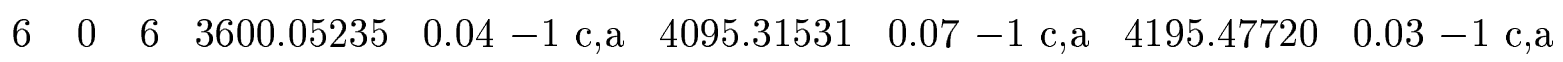

$6 \quad 1 \quad 6 \quad 3601.85888 \quad 0.03-1 \quad c, a \quad 4095.80318 \quad 0.06-1 \quad c, a \quad 4195.81803 \quad 0.04-1$ c,a

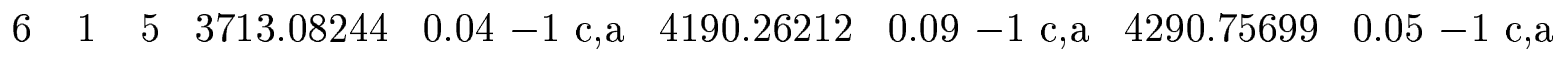

$625 \quad 3736.17076 \quad 0.05-1 \mathrm{c}, \mathrm{a} \quad 4199.39098 \quad 0.05-1 \mathrm{c}, \mathrm{a} \quad 4296.56345 \quad 0.04-1 \mathrm{c}, \mathrm{a}$

$6243784.67912 \quad 0.05-1$ c,a $4249.52442 \quad 0.05-1$ c,a $4350.69931 \quad 0.03-1$ c,a

$6 \quad 3 \quad 4 \quad 3864.96604 \quad 0.03-1 \quad \mathrm{c}, \mathrm{a} \quad 4292.90989 \quad 0.06-1 \quad \mathrm{c}, \mathrm{a} \quad 4387.23468 \quad 0.03-1 \mathrm{c}, \mathrm{a}$

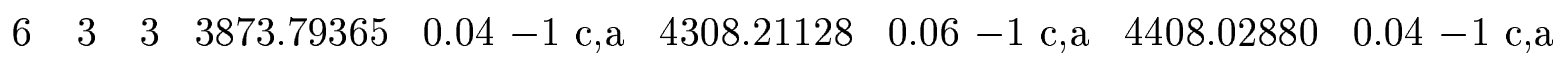

$6434015.51500 \quad 0.05-1 \mathrm{c}, \mathrm{a} \quad 4394.46433 \quad 0.03-1 \mathrm{c}, \mathrm{a} \quad 4490.06386 \quad 0.05-1 \mathrm{c}, \mathrm{a}$

$6424016.05274 \quad 0.05-1 \mathrm{c}, \mathrm{a} \quad 4401.94198 \quad 0.06-1 \mathrm{c}, \mathrm{a} \quad 4491.36969 \quad 0.10-1 \mathrm{c}, \mathrm{a}$

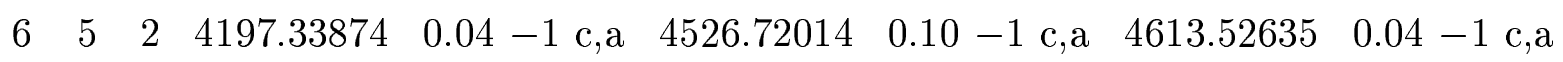

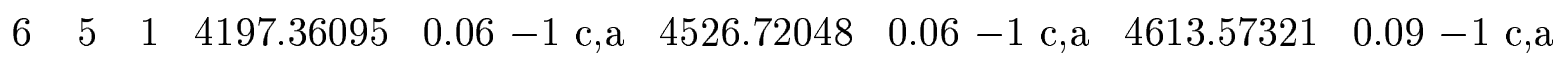

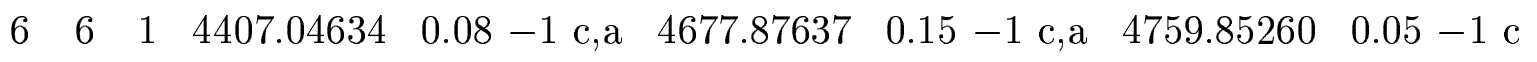

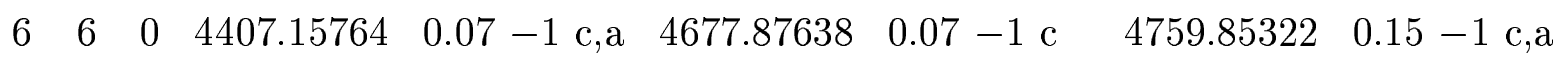

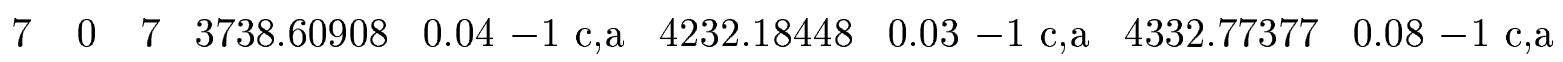




\begin{tabular}{|c|c|c|c|c|c|c|c|c|}
\hline & & & & & & & & \\
\hline & 1 & 6 & 17 & $04-1 \mathrm{c}, \mathrm{a}$ & 66 & $.03-$ & 39 & 05 \\
\hline & & & 68 & $4-1 \mathrm{c}, \mathrm{a}$ & 353.23140 & $8-1 \mathrm{c}, \mathrm{a}$ & 4452.35271 & $06-1 \mathrm{c}$ \\
\hline & & & 3967.48843 & $05-1 \mathrm{c}, \mathrm{a}$ & 426.06635 & $0.08-1 \mathrm{c}, \mathrm{a}$ & 4527.94930 & 07 \\
\hline & & & 4033 & $5-$ & 70 & 7 & 455 & 94 \\
\hline & 3 & & 36 & $55-$ & 12 & $06-$ & 25 & 4 \\
\hline & 4 & 4 & 4186 & $08-1 \mathrm{c}, \mathrm{a}$ & 65 & $3-$ & 471 & 05 \\
\hline & & 3 & 426 & $04-1 \mathrm{c}, \mathrm{a}$ & 629 & $0.03-1$ & 4663.15064 & $.08-1 \mathrm{c}$ \\
\hline & & 3 & 589 & $04-1 \mathrm{c}, \mathrm{a}$ & 638 & $0.10-$ & 211 & 0.06 \\
\hline & & 2 & 436 & $06-1 \mathrm{c}, \mathrm{a}$ & 25 & $0.04-$ & 2006 & 0.10 \\
\hline & 6 & & 4578 & $.07-1 \mathrm{c}, \mathrm{a}$ & 846.77361 & $0.04-$ & 4929 & $0.08-1 c$ \\
\hline & 6 & & 3 & $.06-1 \mathrm{c}, \mathrm{a}$ & 94 & $0.10-1$ & 00 & $0.25-1 \mathrm{c}$ \\
\hline & & & 77 & .05 & 502 & $0.10-1 \mathrm{c}$ & 50 & 0.10 \\
\hline & & & 7 & -1 & 5 & 0. & & 0 \\
\hline & 0 & 8 & 5 & $5-1>1$ & 23 & 0 & 50 & 07 \\
\hline & 1 & 8 & 87 & $04-1$ & 285 & $0.06-$ & 448 & $0.15-1$ \\
\hline & 1 & 7 & 406 & $05-1 \mathrm{c}$ & 890 & $0.05-1$ & 46 & $0.05-1$ \\
\hline & 2 & 7 & 4071 & $03-1 \mathrm{c}, \mathrm{a}$ & 452 & $0.05-1 \mathrm{c}, \mathrm{a}$ & 462 & $0.05-1$ \\
\hline & 2 & c & 50 & $8-$ & 462 & $0.06-$ & 472 & 0.04 \\
\hline & 3 & 6 & & $0.05-1 \mathrm{c}, \mathrm{a}$ & 9 & 0 & 4 & 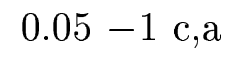 \\
\hline & 3 & 5 & 4259 & $04-$ & 4689 & $0.12-$ & & 0.0 \\
\hline & 4 & 5 & 4 & a & 3 & $0.04-$ & & 0.12 \\
\hline & 4 & 4 & 4 & $06-1,9$ & 476 & $0.07-1 \mathrm{c}$ & $4 \varepsilon$ & $0.08-1$ \\
\hline & 5 & 4 & 71 & $6-1 \mathrm{c}, \mathrm{a}$ & 488 & $0.08-1$ & 49 & $0.06-$ \\
\hline & 5 & 3 & 456 & $0.05-1 \mathrm{c}, \mathrm{a}$ & 488 & $0.04-1$ & 497 & 0.06 \\
\hline & 6 & 3 & 477 & $.05-1 \mathrm{c}, \mathrm{a}$ & 503 & $0.15-$ & 5122.34742 & $0.14-1$ \\
\hline & 6 & & & & & & 5122.3 & $0.14-1$ \\
\hline
\end{tabular}



$8 \quad 7 \quad 2 \quad 5008.96271 \quad 0.08-1 \quad c, a \quad 5213.26941 \quad 0.14-1$ c,a $5289.95805 \quad 0.20-1$ c
$8 \quad 7 \quad 1 \quad 5008.96276 \quad 0.10-1 \mathrm{c}, \mathrm{a} \quad 5213.26885 \quad 0.06-1 \mathrm{c} \quad 5289.95903 \quad 0.14-1 \mathrm{c}, \mathrm{a}$

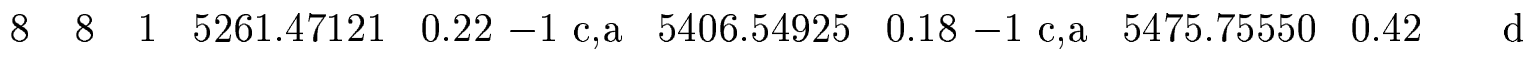

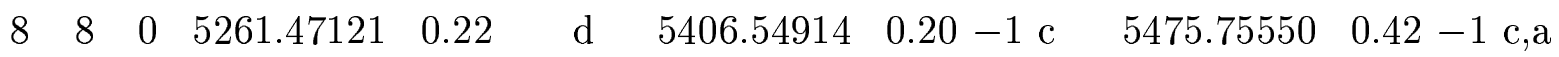

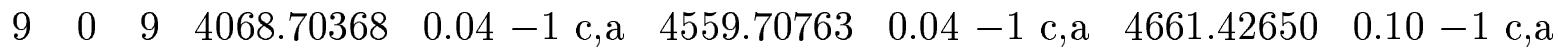
$9 \quad 1 \quad 9 \quad 4068.93093 \quad 0.04-1 \mathrm{c}, \mathrm{a} \quad 4559.75209 \quad 0.08-1 \mathrm{c}, \mathrm{a} \quad 4661.44856 \quad 0.07-1 \mathrm{c}, \mathrm{a}$
$9 \quad 1 \quad 8 \quad 4263.15032 \quad 0.04-1 \quad c, a \quad 4715.96693 \quad 0.05-1 \quad c, a \quad 4816.99088 \quad 0.07$ - 1 c,a
$92 \quad 8 \quad 4268.24075 \quad 0.04-1$ c,a $4717.10442 \quad 0.07-1$ c,a $4817.73572 \quad 0.07$-1 c,a
$922 \quad 7 \quad 4399.54208 \quad 0.03-1$ c,a $4837.69963 \quad 0.06-1$ c,a $4939.79427 \quad 0.04-1$ c,a
$9 \quad 3 \quad 7 \quad 4436.94025 \quad 0.08-1 \quad c, a \quad 4850.44135 \quad 0.05-1 \quad c, a \quad 4949.00296 \quad 0.07-1$ c,a
$9 \quad 3 \quad 6 \quad 4493.80421 \quad 0.05-1 \quad c, a \quad 4918.23479 \quad 0.09-1 \quad c, a \quad 5022.28125 \quad 0.08$ - 1 c,a
$94 \quad 6 \quad 4600.49724 \quad 0.05-1 \mathrm{c}, \mathrm{a} \quad 4971.26072 \quad 0.04-1 \mathrm{c}, \mathrm{a} \quad 5067.07667 \quad 0.05-1 \mathrm{c}, \mathrm{a}$
$9455 \quad 4611.79473 \quad 0.07-1 \quad c, a \quad 4992.12154 \quad 0.11$-1 c,a $5087.01712 \quad 0.04-1$ c,a
$9 \quad 5 \quad 5 \quad 4783.64081 \quad 0.10-1$ c,a $5108.34934 \quad 0.05-1$ c,a $5193.45764 \quad 0.06-1$ c,a
$9 \quad 5 \quad 4 \quad 4784.66206 \quad 0.08-1 \mathrm{c}, \mathrm{a} \quad 5107.72912 \quad 0.05-1 \mathrm{c}, \mathrm{a} \quad 5196.50048 \quad 0.06-1 \mathrm{c}, \mathrm{a}$
$9 \quad 6 \quad 4 \quad 4994.70285 \quad 0.31 \quad 25$ e,a $5256.38144 \quad 0.05-1$ c,a $5339.64133 \quad 0.05$-1 c,a
$9 \quad 6 \quad 3 \quad 4996.33144 \quad 0.06-1$ c,a $5256.44870 \quad 0.08-1$ c,a $5339.84331 \quad 0.05-1$ c,a
$9 \quad 7 \quad 3 \quad 5229.57675 \quad 0.14-1 \mathrm{c}, \mathrm{a} \quad 5430.18080 \quad 0.15-1 \mathrm{c} \quad 5507.47498 \quad 0.07-1 \mathrm{c}, \mathrm{a}$

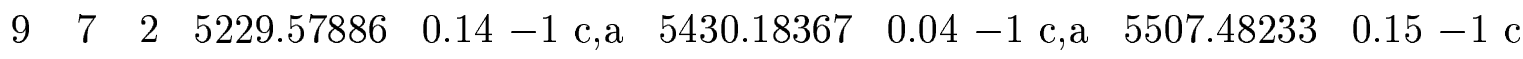

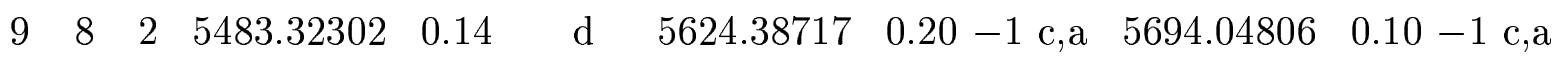

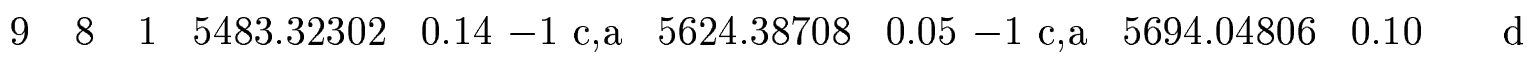

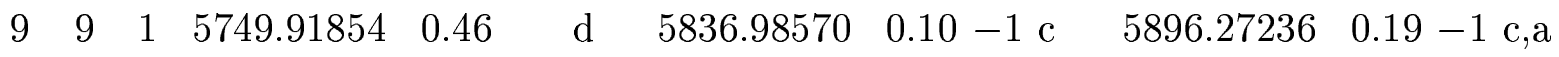

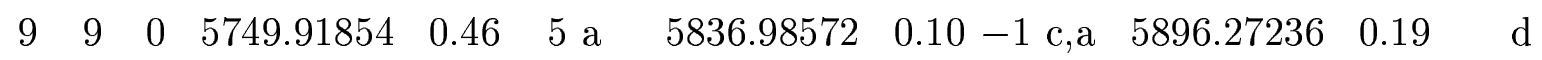

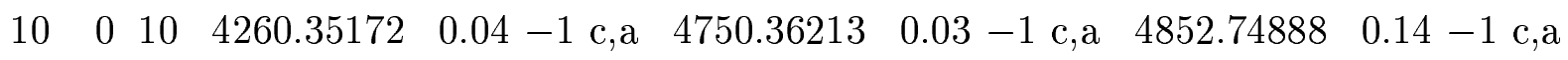
$\begin{array}{llllllllllll}10 & 1 & 10 & 4260.46683 & 0.04-1 & \mathrm{c}, \mathrm{a} & 4750.38769 & 0.05 & -1 & \mathrm{c}, \mathrm{a} & 4852.75521 & 0.06-1 \mathrm{c}, \mathrm{a}\end{array}$

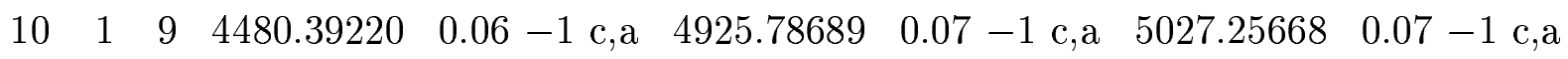

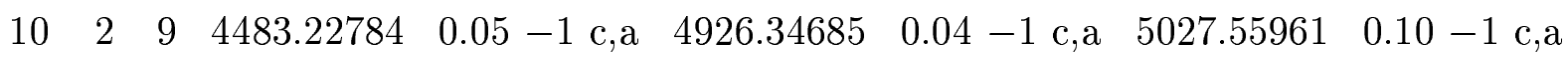




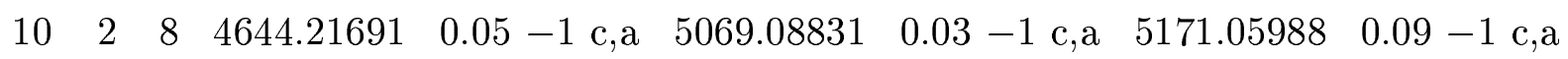

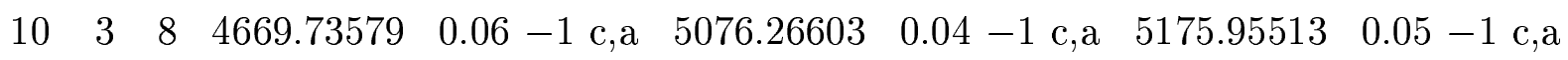

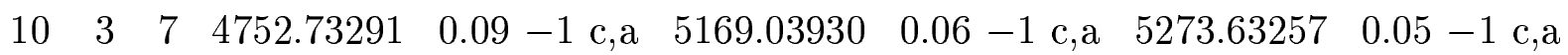

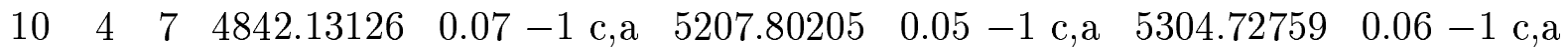

$10 \quad 4 \quad 6 \quad 4864.37331 \quad 0.10-1 \mathrm{c}, \mathrm{a} \quad 5246.80023 \quad 0.07-1 \mathrm{c}, \mathrm{a} \quad 5355.26292 \quad 0.10-1 \mathrm{c}, \mathrm{a}$

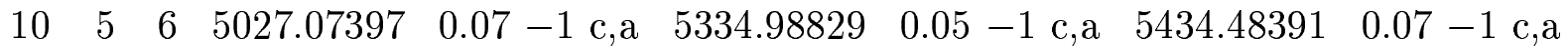

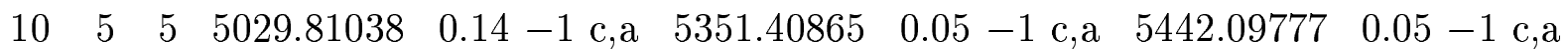

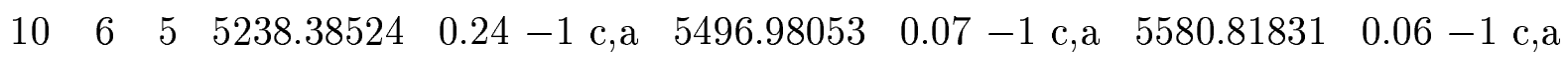

$10 \quad 6 \quad 4 \quad 5237.42021 \quad 0.10-1 \quad c, a \quad 5497.21532 \quad 0.05-1$ c,a $5581.52478 \quad 0.05-1$ c,a

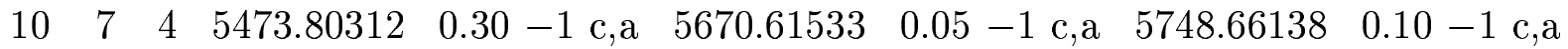

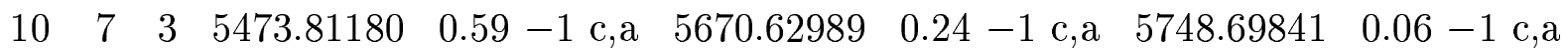

$\begin{array}{llllllllllll}10 & 8 & 3 & 5728.66075 & 0.41 & 7 \mathrm{a} & 5865.60408 & 0.18 & -1 & \mathrm{c}, \mathrm{a} & 5935.83130 & 0.15\end{array}$

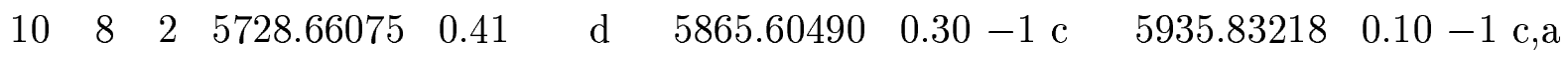

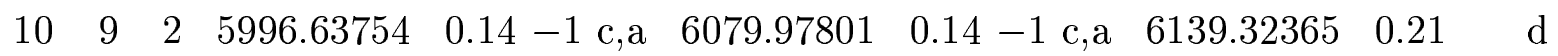

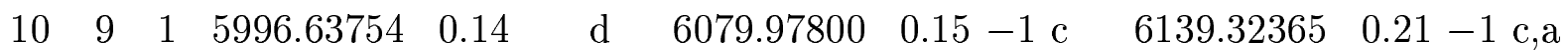

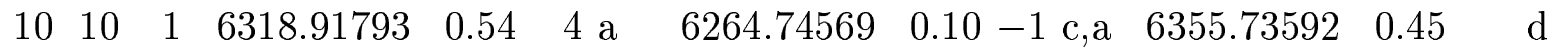

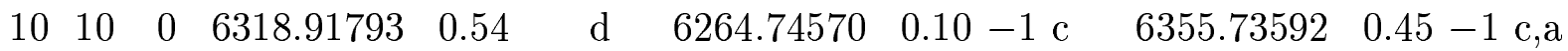

$11 \quad 0 \quad 11 \quad 4469.73722 \quad 0.07-1$ c,a $4958.90119 \quad 0.05-1$ c,a $5062.01060 \quad 0.20$-1 c,a

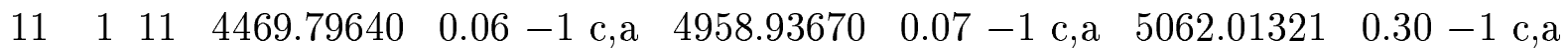

$11 \quad 1 \quad 10 \quad 4714.81865 \quad 0.05-1 \mathrm{c}, \mathrm{a} \quad 5153.18846 \quad 0.03-1 \mathrm{c}, \mathrm{a} \quad 5255.20532 \quad 0.07$ - 1 c,a

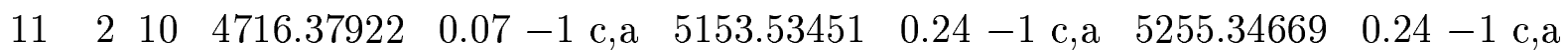

$1125 \quad 9 \quad 4905.65344 \quad 0.06-1 \mathrm{c}, \mathrm{a} \quad 5316.80421 \quad 0.07-1 \mathrm{c}, \mathrm{a} \quad 5418.80340 \quad 0.08$-1 c,a

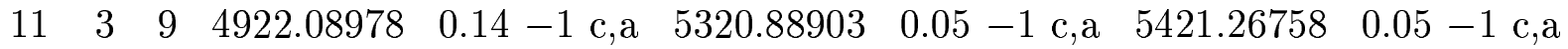

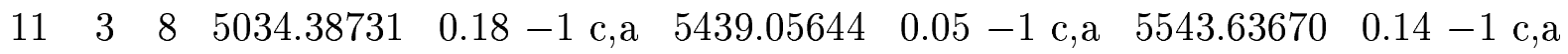

$11 \quad 4 \quad 8 \quad 5105.72967 \quad 0.07-1 \mathrm{c}, \mathrm{a} \quad 5465.05349 \quad 0.10-1 \mathrm{c}, \mathrm{a} \quad 5563.39973 \quad 0.10-1 \mathrm{c}, \mathrm{a}$

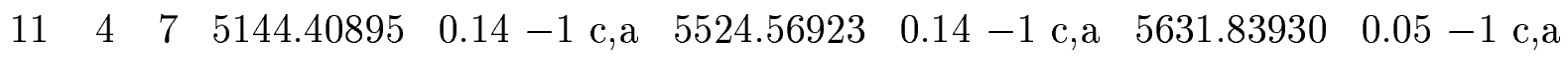

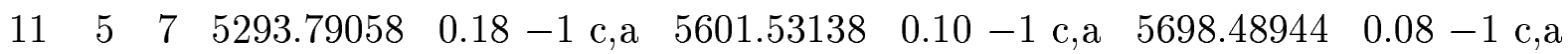


$115 \quad 6 \quad 5300.17803 \quad 0.07-1 \mathrm{c}, \mathrm{a} \quad 5621.33440 \quad 0.10-1 \mathrm{c}, \mathrm{a} \quad 5714.53190 \quad 0.10-1 \mathrm{c}, \mathrm{a}$

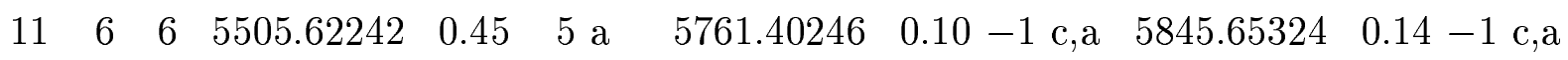

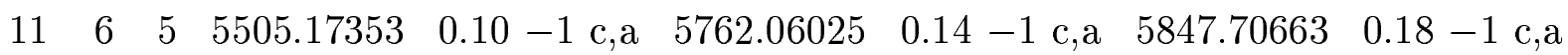

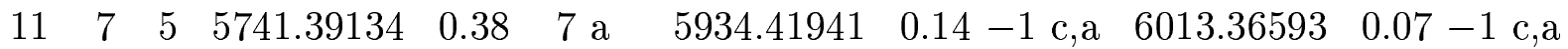

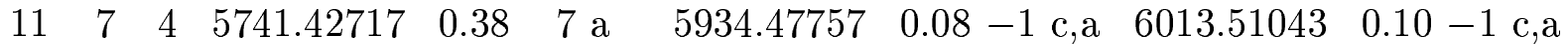

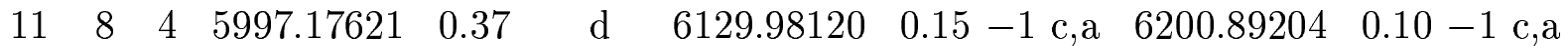

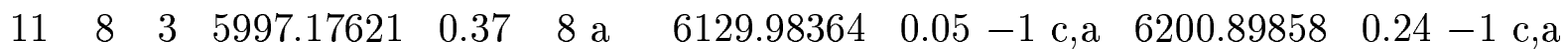

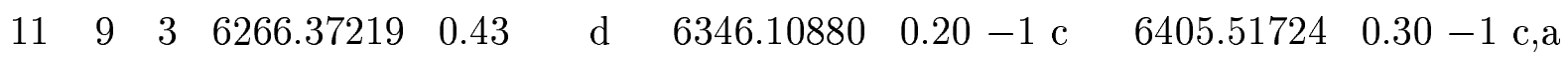

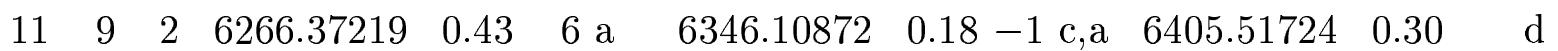

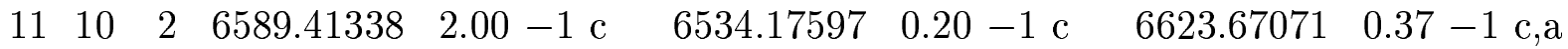

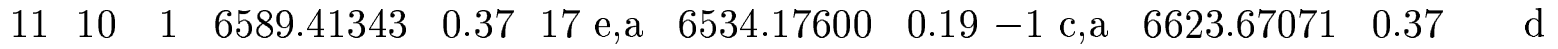

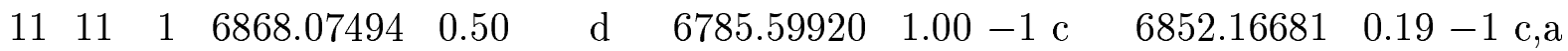

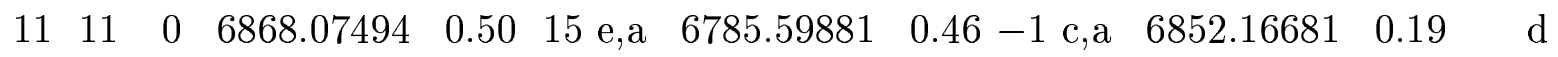

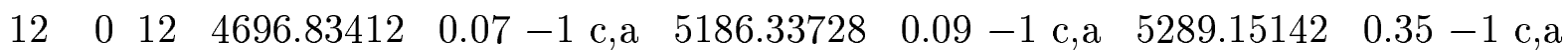

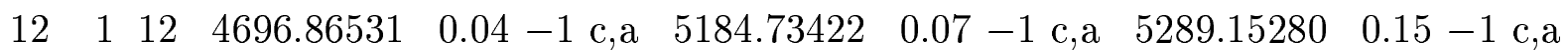

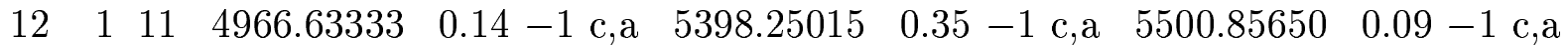

$12211 \quad 4967.49062 \quad 0.09-1 \mathrm{c}, \mathrm{a} \quad 5399.33072 \quad 0.32-1 \mathrm{c}, \mathrm{a} \quad 5500.91570 \quad 0.23-1 \mathrm{c}, \mathrm{a}$

$122 \quad 10 \quad 5182.09490 \quad 0.06-1$ c,a $5581.10939 \quad 0.14-1$ c,a $5683.33260 \quad 0.07$-1 c,a

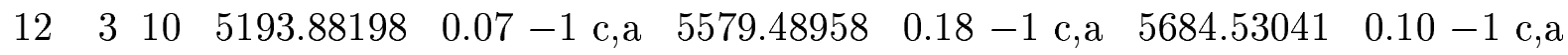

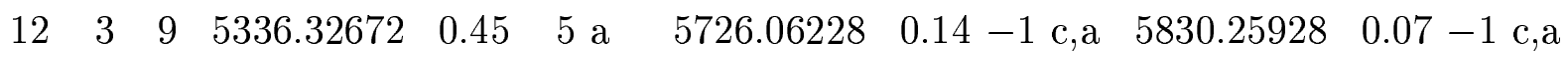

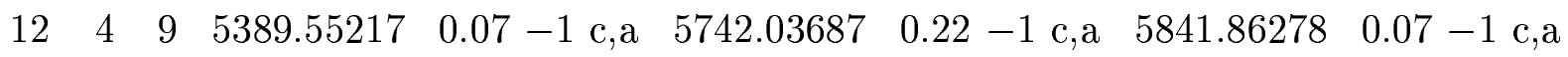

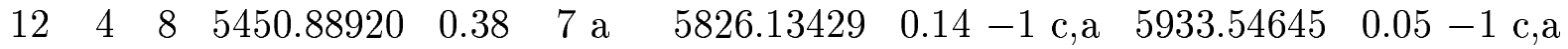

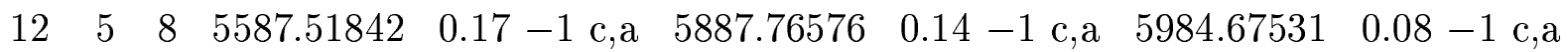

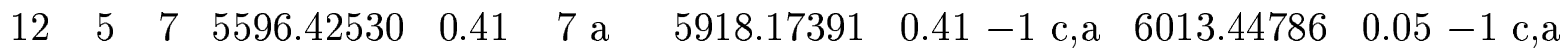

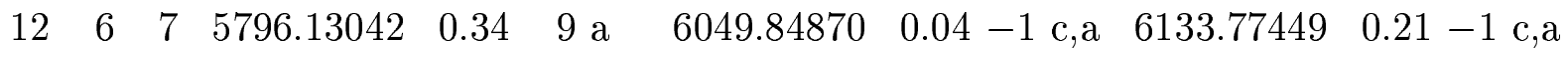

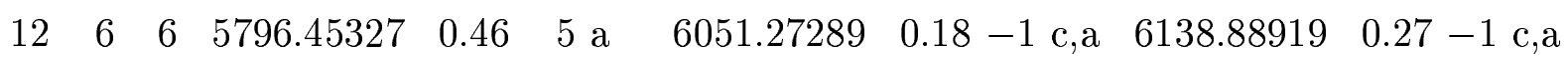

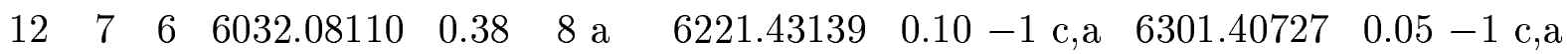


$\begin{array}{lllllllllll}12 & 7 & 5 & 6032.19792 & 0.46 & 6 \mathrm{a} & 6221.62313 & 0.18-1 \mathrm{c}, \mathrm{a} & 6301.87330 & 0.14-1 \mathrm{c}, \mathrm{a}\end{array}$

$\begin{array}{lllllllllll}12 & 8 & 5 & 6288.55100 & 0.34 & 9 \mathrm{a} & 6417.29612 & 0.10-1 \mathrm{c}, \mathrm{a} & 6489.01097 & 0.19-1 \mathrm{c}, \mathrm{a}\end{array}$

$\begin{array}{llllllllllll}12 & 8 & 4 & 6288.55100 & 0.34 & \mathrm{~d} & 6417.30575 & 0.38 & -1 \mathrm{c}, \mathrm{a} & 6489.03897 & 0.32 & -1 \mathrm{c}, \mathrm{a}\end{array}$

$\begin{array}{lllllllllllll}12 & 9 & 4 & 6558.75177 & 0.39 & 7 \mathrm{a} & 6635.10921 & 0.18 & -1 \mathrm{c}, \mathrm{a} & 6694.57877 & 0.10 & \mathrm{~d}\end{array}$

$\begin{array}{llllllllllll}12 & 9 & 3 & 6558.75177 & 0.39 & \mathrm{~d} & 6635.10920 & 0.20-1 \mathrm{c} & 6694.57877 & 0.10-1 \mathrm{c}, \mathrm{a}\end{array}$

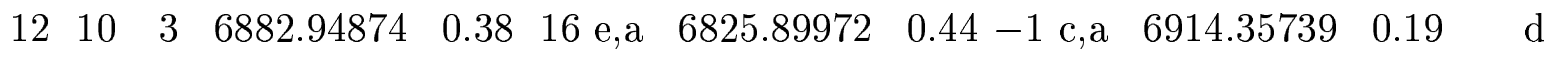

$\begin{array}{llllllllll}12 & 10 & 2 & 6882.94805 & 2.00-1 \mathrm{c} & 6825.89900 & 0.80-1 \mathrm{c} & 6914.35739 & 0.19-1 \mathrm{c}, \mathrm{a}\end{array}$

$\begin{array}{llllllllllll}12 & 11 & 2 & 7166.44114 & 0.59 & 14 \mathrm{e}, \mathrm{a} & 7077.57868 & 0.51 & 5 \mathrm{a} & 7145.10804 & 0.19 & \mathrm{~d}\end{array}$

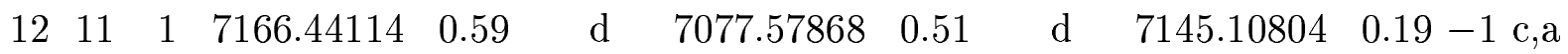

$\begin{array}{llllllllllll}12 & 12 & 1 & 7464.58662 & 0.57 & 8 \mathrm{e}, \mathrm{a} & 7328.06863 & 0.57 & 9 \mathrm{e}, \mathrm{a} & 7383.68027 & 0.19 & \mathrm{~d}\end{array}$

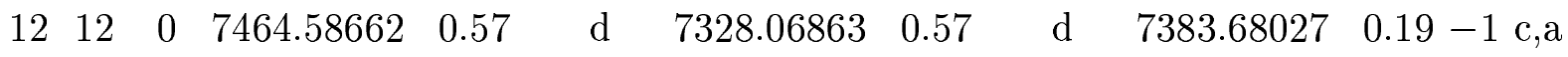

$\begin{array}{lllllllllll}13 & 0 & 13 & 4941.60586 & 0.10-1 & \mathrm{c}, \mathrm{a} & 5429.11842 & 0.10-1 & \mathrm{c}, \mathrm{a} & 5534.11115 & 0.28-1 \mathrm{c}, \mathrm{a}\end{array}$

$\begin{array}{llllllllll}13 & 1 & 13 & 4941.62242 & 0.10-1 \mathrm{c}, \mathrm{a} & 5429.12800 & 0.10-1 \mathrm{c} & 5534.11070 & 0.18-1 \mathrm{c}, \mathrm{a}\end{array}$

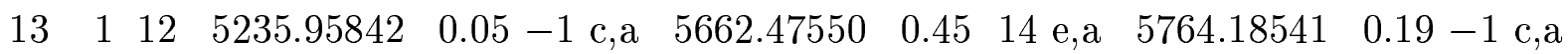

$\begin{array}{lllllllllll}13 & 2 & 12 & 5236.43191 & 0.45 & 5 \mathrm{a} & 5660.40395 & 0.19-1 \mathrm{c}, \mathrm{a} & 5764.20438 & 0.14-1 \mathrm{c}, \mathrm{a}\end{array}$

$\begin{array}{llllllllllll}13 & 2 & 11 & 5477.00574 & 0.07-1 & \mathrm{c}, \mathrm{a} & 5862.33890 & 0.10-1 \mathrm{c}, \mathrm{a} & 5964.91268 & 0.10-1 \mathrm{c}, \mathrm{a}\end{array}$

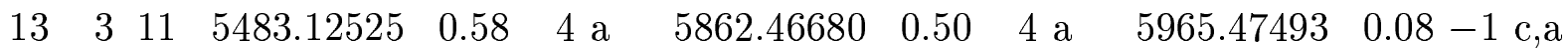

$\begin{array}{lllllllllll}13 & 3 & 10 & 5654.76092 & 0.14-1 \mathrm{c}, \mathrm{a} & 6028.85836 & 0.23-1 \mathrm{c}, \mathrm{a} & 6132.64413 & 0.06 & -1 \mathrm{c}, \mathrm{a}\end{array}$

$\begin{array}{lllllllllll}13 & 4 & 10 & 5695.88317 & 0.41 & 6 \mathrm{a} & 6037.87430 & 0.36-1 \mathrm{c}, \mathrm{a} & 6139.02954 & 0.10-1 \mathrm{c}, \mathrm{a}\end{array}$

$\begin{array}{lllllllllll}13 & 4 & 9 & 5781.95860 & 0.34 & 9 \mathrm{a} & 6148.68278 & 0.38 & 7 \mathrm{a} & 6256.02107 & 0.09-1 \mathrm{c}, \mathrm{a}\end{array}$

$\begin{array}{lllllllllll}13 & 5 & 9 & 5896.77364 & 0.39 & 7 \mathrm{a} & 6194.30410 & 0.50-1 \mathrm{c}, \mathrm{a} & 6292.11953 & 0.14-1 \mathrm{c}, \mathrm{a}\end{array}$

$\begin{array}{llllllllllll}13 & 5 & 8 & 5919.00864 & 0.32 & 11 \mathrm{a} & 6241.53013 & 0.32 & -1 \mathrm{c}, \mathrm{a} & 6336.03636 & 0.19 & -1 \mathrm{c}, \mathrm{a}\end{array}$

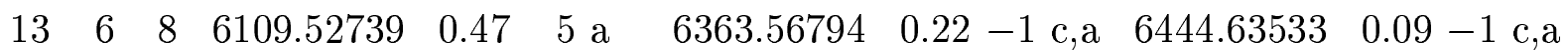

$\begin{array}{lllllllllll}13 & 6 & 7 & 6111.46780 & 0.34 & 9 \mathrm{a} & 6365.37933 & 0.10-1 \mathrm{c}, \mathrm{a} & 6455.74205 & 0.14-1 \mathrm{c}, \mathrm{a}\end{array}$

$\begin{array}{lllllllllll}13 & 7 & 7 & 6345.58740 & 0.43 & 6 \mathrm{a} & 6531.47838 & 0.18-1 & \mathrm{c}, \mathrm{a} & 6612.54826 & 0.10-1 \mathrm{c}, \mathrm{a}\end{array}$

$\begin{array}{llllllllllll}13 & 7 & 6 & 6345.92557 & 0.42 & 6 \mathrm{a} & 6532.02024 & 0.18-1 & \mathrm{c}, \mathrm{a} & 6613.83518 & 0.14-1 \mathrm{c}, \mathrm{a}\end{array}$

$\begin{array}{llllllllllll}13 & 8 & 6 & 6602.46815 & 0.76 & 2 \mathrm{a} & 6727.31854 & 0.38-1 \mathrm{c}, \mathrm{a} & 6799.95707 & 0.08-1 \mathrm{c}, \mathrm{a}\end{array}$ 
$\begin{array}{lllllllllll}13 & 8 & 5 & 6602.46148 & 0.40 & 7 \mathrm{a} & 6727.35786 & 0.36-1 \mathrm{c}, \mathrm{a} & 6800.05515 & 0.29-1 \mathrm{c}, \mathrm{a}\end{array}$

$\begin{array}{lllllllllllll}13 & 9 & 5 & 6873.41227 & 0.35 & \mathrm{~d} & 6946.69930 & 0.88-1 & \mathrm{c}, \mathrm{a} & 7006.22991 & 0.48 & 6 \mathrm{a}\end{array}$

$\begin{array}{llllllllllll}13 & 9 & 4 & 6873.41227 & 0.35 & 24 \mathrm{e}, \mathrm{a} & 6946.70135 & 0.36 & -1 \mathrm{c}, \mathrm{a} & 7006.23508 & 1.00 & 2 \mathrm{a}\end{array}$

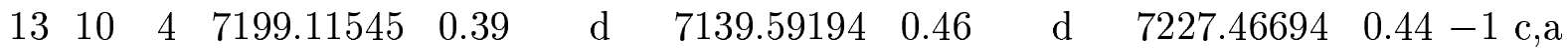

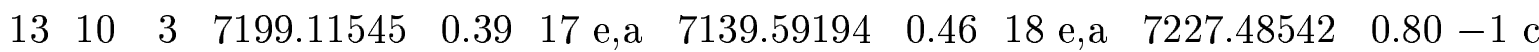

$\begin{array}{llllllllllll}13 & 11 & 3 & 7487.08429 & 0.42 & \mathrm{~d} & 7391.67623 & 0.44 & \mathrm{~d} & 7460.35668 & 0.58 & 4 \mathrm{a}\end{array}$

$\begin{array}{llllllllllll}13 & 11 & 2 & 7487.08429 & 0.42 & 16 \text { e,a } & 7391.67623 & 0.44 & 6 \mathrm{a} & 7460.35668 & 0.58 & \mathrm{~d}\end{array}$

$\begin{array}{llllllllllll}13 & 12 & 2 & 7789.13708 & 0.75 & \mathrm{~d} & 7644.44342 & 0.62 & \mathrm{~d} & 7701.74421 & 0.64 & 4 \mathrm{a}\end{array}$

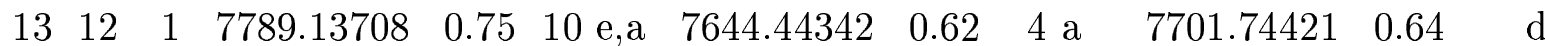

$\begin{array}{llllllllllll}13 & 13 & 1 & 8095.49183 & 0.91 & \mathrm{~d} & 7901.19557 & 0.79 & \mathrm{~d} & 7948.49768 & 0.74 & 3 \mathrm{a}\end{array}$

$\begin{array}{llllllllllll}13 & 13 & 0 & 8095.49183 & 0.91 & 2 \text { a } & 7901.19557 & 0.79 & 8 \text { e,a } & 7948.49768 & 0.74 & \text { d }\end{array}$

$\begin{array}{llllllllllll}14 & 0 & 14 & 5204.00841 & 0.29-1 & \mathrm{c}, \mathrm{a} & 5690.87848 & 0.45-1 \mathrm{c}, \mathrm{a} & 5796.94256 & 0.10-1 \mathrm{c}, \mathrm{a}\end{array}$

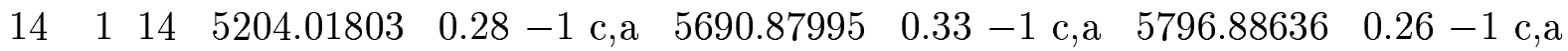

$\begin{array}{lllllllllll}14 & 1 & 13 & 5522.85389 & 0.51 & 4 \mathrm{a} & 5940.54202 & 0.72-1 & \mathrm{c}, \mathrm{a} & 6045.17181 & 0.14-1 \mathrm{c}, \mathrm{a}\end{array}$

$\begin{array}{lllllllllll}14 & 2 & 13 & 5523.11748 & 0.41 & 6 \mathrm{a} & 5940.63739 & 0.23-1 \mathrm{c}, \mathrm{a} & 6045.14240 & 0.10 & -1 \mathrm{c}, \mathrm{a}\end{array}$

$\begin{array}{lllllllllll}14 & 2 & 12 & 5786.85252 & 0.41 & 6 \mathrm{a} & 6161.13296 & 0.69 & 5 \mathrm{e}, \mathrm{a} & 6263.69895 & 0.14-1 \mathrm{c}, \mathrm{a}\end{array}$

$\begin{array}{llllllllllll}14 & 3 & 12 & 5790.43293 & 0.38 & 7 \mathrm{a} & 6160.37241 & 0.34-1 \mathrm{c}, \mathrm{a} & 6263.91987 & 0.28 & -1 \mathrm{c}, \mathrm{a}\end{array}$

$\begin{array}{lllllllllll}14 & 3 & 11 & 5993.19862 & 0.47 & 5 \mathrm{a} & 6347.29250 & 0.62 & 3 \mathrm{e}, \mathrm{a} & 6451.08137 & 0.17-1 \mathrm{c}, \mathrm{a}\end{array}$

$\begin{array}{lllllllllll}14 & 4 & 11 & 6019.83227 & 0.38 & 8 \mathrm{a} & 6351.85497 & 0.10-1 & \mathrm{c}, \mathrm{a} & 6454.13381 & 0.14-1 \mathrm{c}, \mathrm{a}\end{array}$

$\begin{array}{llllllllll}14 & 4 & 10 & 6134.91802 & 0.43 & 6 \mathrm{a} & 6489.62170 & 0.59-1 \mathrm{c}, \mathrm{a} & 6596.22226 & 0.10-1 \mathrm{c}, \mathrm{a}\end{array}$

$\begin{array}{llllllllllll}14 & 5 & 10 & 6229.89508 & 0.36 & 8 \mathrm{a} & 6520.60691 & 0.22-1 \mathrm{c}, \mathrm{a} & 6619.78888 & 0.14-1 \mathrm{c}, \mathrm{a}\end{array}$

$\begin{array}{llllllllllll}14 & 5 & 9 & 6267.88245 & 0.42 & 6 \mathrm{a} & 6589.74135 & 0.98 & 2 \mathrm{e}, \mathrm{a} & 6705.59373 & 0.20 & -1 \mathrm{c}, \mathrm{a}\end{array}$

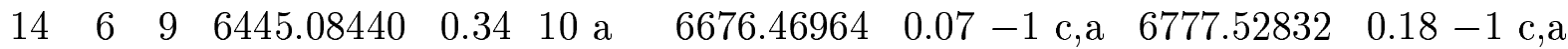

$\begin{array}{llllllllllll}14 & 6 & 8 & 6450.41585 & 0.44 & 10 \mathrm{e}, \mathrm{a} & 6705.04574 & 0.35-1 \mathrm{c}, \mathrm{a} & 6798.71897 & 0.23 & -1 \mathrm{c}, \mathrm{a}\end{array}$

$\begin{array}{llllllllllll}14 & 7 & 8 & 6681.59268 & 0.37 & 8 \mathrm{a} & 6864.39488 & 0.25-1 & \mathrm{c}, \mathrm{a} & 6946.46370 & 0.12-1 \mathrm{c}, \mathrm{a}\end{array}$

$\begin{array}{llllllllllll}14 & 7 & 7 & 6682.45789 & 0.48 & 5 \mathrm{a} & 6865.72728 & 0.25-1 \mathrm{c}, \mathrm{a} & 6949.57994 & 0.17-1 \mathrm{c}, \mathrm{a}\end{array}$

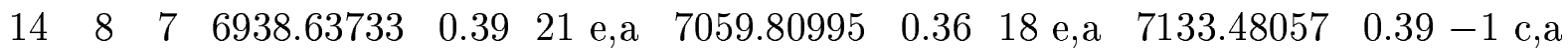




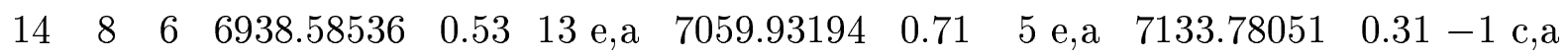

$\begin{array}{llllllllllll}14 & 9 & 6 & 7209.99158 & 0.36 & 18 \mathrm{e}, \mathrm{a} & 7280.59514 & 0.42 & 14 \mathrm{e}, \mathrm{a} & 7340.17479 & 1.03 & 1 \mathrm{a}\end{array}$

$\begin{array}{llllllllllll}14 & 9 & 5 & 7209.99564 & 0.72 & 3 \mathrm{a} & 7280.59926 & 0.73 & 3 \mathrm{a} & 7340.20445 & 0.41 & 17 \mathrm{e}, \mathrm{a}\end{array}$

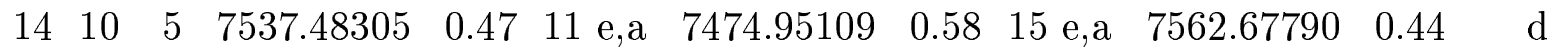

$\begin{array}{llllllllllll}14 & 10 & 4 & 7537.48305 & 0.47 & \mathrm{~d} & 7474.94957 & 5.00 & 1 \mathrm{a} & 7562.67790 & 0.44 & 16 \mathrm{e}, \mathrm{a}\end{array}$

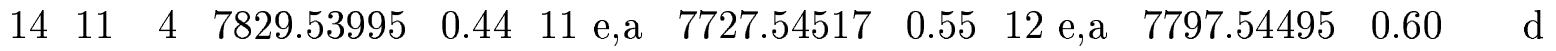

$\begin{array}{llllllllllll}14 & 11 & 3 & 7829.53995 & 0.44 & \mathrm{~d} & 7727.54517 & 0.55 & \mathrm{~d} & 7797.54495 & 0.60 & 4 \mathrm{a}\end{array}$

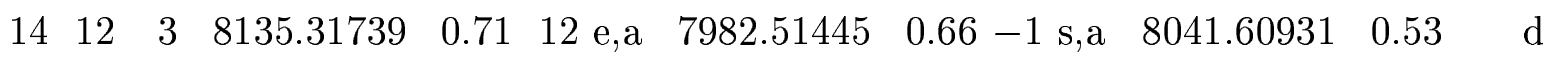

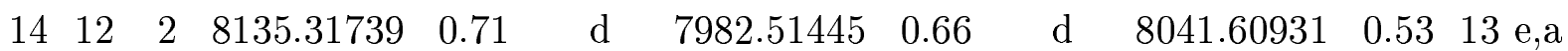

$\begin{array}{llllllllllll}14 & 13 & 2 & 8445.99308 & 0.92 & 10 \text { e,a } & 8242.31596 & 0.83 & 6 \text { e,a } & 8291.78909 & 0.59 & \mathrm{~d}\end{array}$

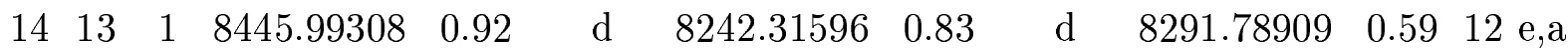

$\begin{array}{lllllllllllll}14 & 14 & 1 & 8756.97469 & 0.98 & 2 \text { a } & 8505.19881 & 0.82 & -1 & \text { s,a } & 8544.94550 & 0.76 & \text { d }\end{array}$

$\begin{array}{llllllllllll}14 & 14 & 0 & 8756.97469 & 0.98 & \mathrm{~d} & 8505.19881 & 0.82 & \mathrm{~d} & 8544.94550 & 0.76 & 8 \mathrm{e}, \mathrm{a}\end{array}$

$\begin{array}{llllllllllll}15 & 0 & 15 & 5483.99636 & 0.14-1 \mathrm{c}, \mathrm{a} & 5970.20254 & 0.68 & 9 \mathrm{e}, \mathrm{a} & 6077.10437 & 0.31 & \mathrm{~d}\end{array}$

$\begin{array}{llllllllllll}15 & 1 & 15 & 5483.99636 & 0.14 & \mathrm{~d} & 5970.19982 & 3.00 & -1 \mathrm{c} & 6077.10437 & 0.31 & -1 \mathrm{c}, \mathrm{a}\end{array}$

$\begin{array}{llllllllllll}15 & 1 & 14 & 5827.33862 & 0.42 & 6 \mathrm{a} & 6238.21410 & 0.51-1 \mathrm{c}, \mathrm{a} & 6343.43380 & 0.23-1 \mathrm{c}, \mathrm{a}\end{array}$

$\begin{array}{llllllllll}15 & 2 & 14 & 5827.48679 & 0.52 & 4 \mathrm{a} & 6238.24178 & 0.99-1 \mathrm{~s}, \mathrm{a} & 6342.52748 & 0.18-1 \mathrm{c}, \mathrm{a}\end{array}$

$\begin{array}{llllllllllll}15 & 2 & 13 & 6113.51123 & 0.39 & 7 \mathrm{a} & 6474.70427 & 0.26-1 \mathrm{c}, \mathrm{a} & 6578.86996 & 0.14-1 \mathrm{c}, \mathrm{a}\end{array}$

$\begin{array}{lllllllllll}15 & 3 & 13 & 6115.65653 & 0.51 & 5 \mathrm{a} & 6475.44705 & 0.69 & 5 \mathrm{e}, \mathrm{a} & 6579.74014 & 0.10-1 \mathrm{c}, \mathrm{a}\end{array}$

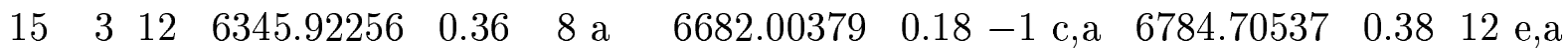

$\begin{array}{lllllllllll}15 & 4 & 12 & 6362.64104 & 0.60 & 3 \mathrm{a} & 6683.43527 & 0.70 & 5 \mathrm{e}, \mathrm{a} & 6786.68808 & 0.07-1 \mathrm{c}, \mathrm{a}\end{array}$

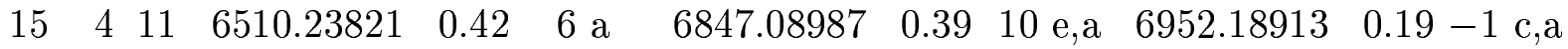

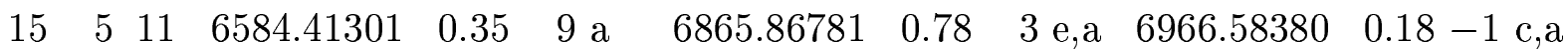

$\begin{array}{llllllllllll}15 & 5 & 10 & 6641.88818 & 0.43 & 6 \mathrm{a} & 6960.38605 & 0.44 & 14 \mathrm{e}, \mathrm{a} & 7074.48314 & 0.51 & 4 \mathrm{a}\end{array}$

$\begin{array}{llllllllllll}15 & 6 & 10 & 6803.40263 & 0.48 & 5 \mathrm{a} & 7032.73679 & 0.63 & 3 \mathrm{a} & 7131.62961 & 0.38 & 7 \mathrm{a}\end{array}$

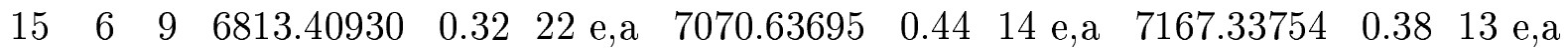

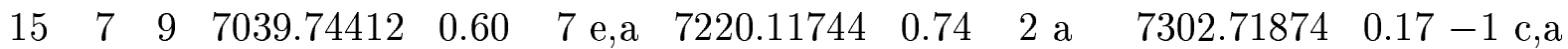




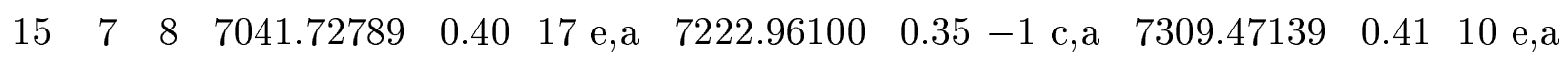

$\begin{array}{lllllllllll}15 & 8 & 8 & 7296.77493 & 0.54-1 \mathrm{~s}, \mathrm{a} & 7414.52296 & 0.59 & 6 \mathrm{e}, \mathrm{a} & 7489.30523 & 0.33-1 \mathrm{c}, \mathrm{a}\end{array}$

$\begin{array}{llllllllllll}15 & 8 & 7 & 7296.60483 & 0.35 & 19 \mathrm{e}, \mathrm{a} & 7414.85934 & 0.42 & 6 \mathrm{a} & 7490.10899 & 0.54 & 5 \mathrm{a}\end{array}$

$\begin{array}{llllllllllll}15 & 9 & 7 & 7568.14240 & 0.82 & 4 \mathrm{e}, \mathrm{a} & 7636.48452 & 0.57 & -1 \mathrm{~s}, \mathrm{a} & 7696.16462 & 0.41 & 8 \mathrm{a}\end{array}$

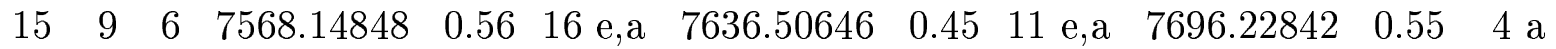

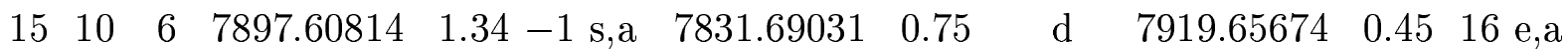

$\begin{array}{llllllllllll}15 & 10 & 5 & 7897.60408 & 0.38 & 14 \mathrm{e}, \mathrm{a} & 7831.69031 & 0.75 & 8 \mathrm{e}, \mathrm{a} & 7919.65050 & 1.44 & 1 \mathrm{a}\end{array}$

$\begin{array}{llllllllllll}15 & 11 & 5 & 8193.35811 & 0.48 & \mathrm{~d} & 8084.84151 & 0.55 & \mathrm{~d} & 8156.30877 & 0.53 & 14 \mathrm{e}, \mathrm{a}\end{array}$

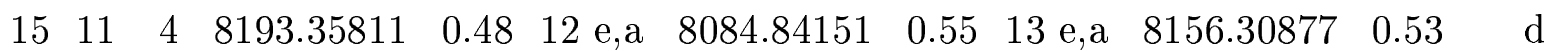

$\begin{array}{llllllllllll}15 & 12 & 4 & 8502.64699 & 0.55 & \mathrm{~d} & 8341.89666 & 0.60 & \mathrm{~d} & 8402.87876 & 0.61 & 12 \mathrm{e}, \mathrm{a}\end{array}$

$\begin{array}{lllllllllllll}15 & 12 & 3 & 8502.64699 & 0.55 & 11 \mathrm{e}, \mathrm{a} & 8341.89666 & 0.60 & -1 \mathrm{~s}, \mathrm{a} & 8402.87876 & 0.61 & \mathrm{~d}\end{array}$

$\begin{array}{llllllllllll}15 & 13 & 3 & 8817.43629 & 0.80 & \mathrm{~d} & 8604.60271 & 0.71 & \mathrm{~d} & 8656.30755 & 0.60 & 12 \mathrm{e}, \mathrm{a}\end{array}$

$\begin{array}{llllllllllll}15 & 13 & 2 & 8817.43629 & 0.80 & 11 \mathrm{e}, \mathrm{a} & 8604.60271 & 0.71 & -1 \mathrm{~s}, \mathrm{a} & 8656.30755 & 0.60 & \mathrm{~d}\end{array}$

$\begin{array}{llllllllllll}15 & 14 & 2 & 9133.40999 & 0.92 & \mathrm{~d} & 8871.13216 & 0.82 & \mathrm{~d} & 8913.56293 & 1.16 & 1 \mathrm{a}\end{array}$

$\begin{array}{llllllllllll}15 & 14 & 1 & 9133.40999 & 0.92 & 8 \mathrm{e}, \mathrm{a} & 8871.13216 & 0.82 & 9 \mathrm{e}, \mathrm{a} & 8913.56293 & 1.16 & \mathrm{~d}\end{array}$

$\begin{array}{llllllllllll}15 & 15 & 1 & 9446.84063 & 1.01 & \mathrm{~d} & 9138.96061 & 0.85 & \mathrm{~d} & 9171.47141 & 0.81 & 6 \mathrm{e}, \mathrm{a}\end{array}$

$\begin{array}{lllllllllllll}15 & 15 & 0 & 9446.84063 & 1.01 & 2 \mathrm{a} & 9138.96061 & 0.85 & -1 \mathrm{~s}, \mathrm{a} & 9171.47141 & 0.81 & \mathrm{~d}\end{array}$

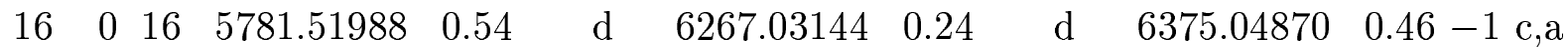

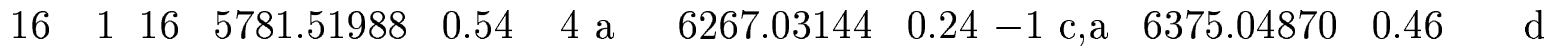

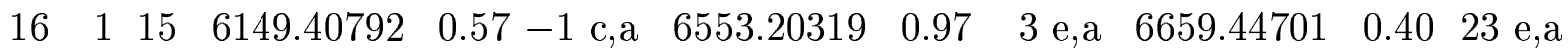

$\begin{array}{llllllllllll}16 & 2 & 15 & 6149.49399 & 0.54 & 4 \mathrm{a} & 6553.21913 & 0.66 & 9 \mathrm{e}, \mathrm{a} & 6659.44701 & 0.40 & \mathrm{~d}\end{array}$

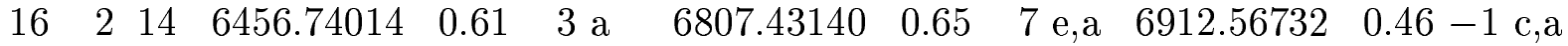

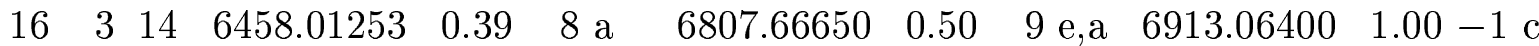

$\begin{array}{llllllllllll}16 & 3 & 13 & 6711.70399 & 0.54 & 4 \mathrm{a} & 7027.33908 & 1.31-1 \mathrm{~s}, \mathrm{a} & 7135.32098 & 0.24-1 \mathrm{c}, \mathrm{a}\end{array}$

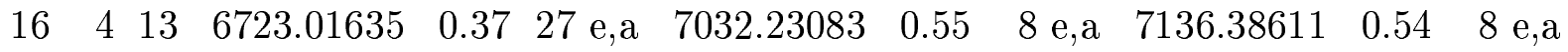

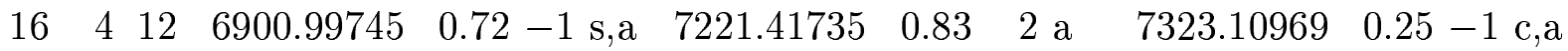

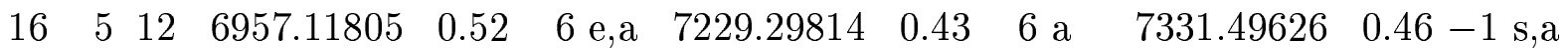




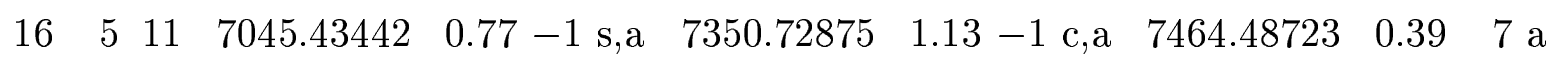

$\begin{array}{lllllllllll}16 & 6 & 11 & 7182.25097 & 0.41 & 14 \mathrm{e}, \mathrm{a} & 7406.95201 & 0.47 & 5 \mathrm{a} & 7506.03975 & 0.64-1 \mathrm{c}, \mathrm{a}\end{array}$

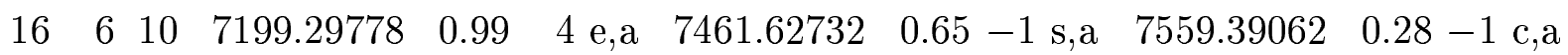

$\begin{array}{lllllllllllll}16 & 7 & 10 & 7419.60375 & 0.34 & 21 \text { e,a } & 7599.09269 & 0.38 & 15 & \text { e,a } & 7680.81567 & 0.41 & 7 \text { a a }\end{array}$

$\begin{array}{llllllllllll}16 & 7 & 9 & 7423.75069 & 0.48 & -1 \mathrm{~s}, \mathrm{a} & 7604.12052 & 0.98 & 3 \mathrm{e}, \mathrm{a} & 7694.02684 & 0.33 & 10 \mathrm{a}\end{array}$

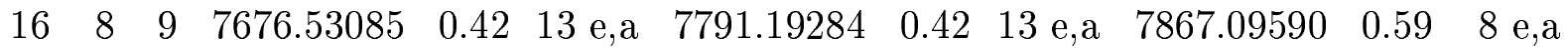

$\begin{array}{llllllllllll}16 & 8 & 8 & 7676.16025 & 0.49 & 8 \mathrm{e}, \mathrm{a} & 7792.01810 & 0.64 & -1 \mathrm{~s}, \mathrm{a} & 7869.03583 & 0.40 & 8 \mathrm{a}\end{array}$

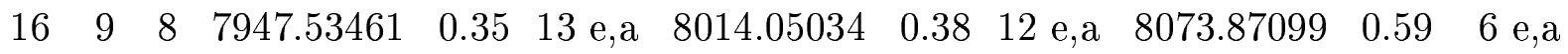

$\begin{array}{lllllllllllll}16 & 9 & 7 & 7947.57398 & 0.85 & -1 \mathrm{~s}, \mathrm{a} & 8014.11342 & 0.64 & -1 \mathrm{~s}, \mathrm{a} & 8074.05541 & 0.49 & 6 \mathrm{a}\end{array}$

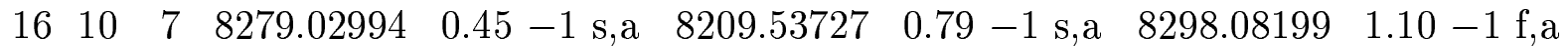

$\begin{array}{lllllllllllll}16 & 10 & 6 & 8279.03386 & 0.83 & -1 \mathrm{~s}, \mathrm{a} & 8209.54219 & 0.87 & -1 \mathrm{f}, \mathrm{a} & 8298.09480 & 0.50 & 15 \mathrm{e}, \mathrm{a}\end{array}$

$\begin{array}{lllllllllllll}16 & 11 & 6 & 8578.07652 & 0.63 & 6 \text { e,a } & 8463.22394 & 0.71 & -1 & \text { s,a } & 8536.28603 & 0.49 & \text { d }\end{array}$

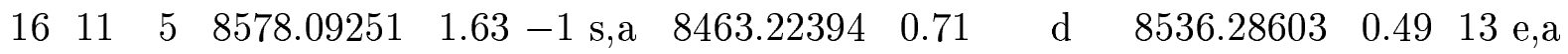

$\begin{array}{llllllllllll}16 & 12 & 5 & 8890.66536 & 0.80 & 7 \text { e,a } & 8722.21449 & 0.67 & 9 \text { e,a } & 8785.15987 & 0.60 & \text { d }\end{array}$

$\begin{array}{lllllllllllll}16 & 12 & 4 & 8890.66536 & 0.80 & \text { d } & 8722.21449 & 0.67 & \text { d } & 8785.15987 & 0.60 & 12 & \text { e,a }\end{array}$

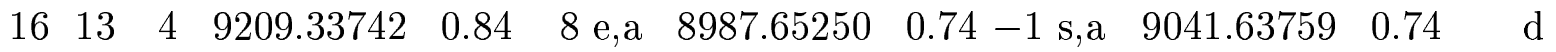

$\begin{array}{llllllllllll}16 & 13 & 3 & 9209.33742 & 0.84 & \text { d } & 8987.65250 & 0.74 & \text { d } & 9041.63759 & 0.74 & 9 \text { e,a }\end{array}$

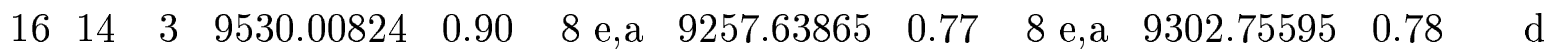

$\begin{array}{llllllllllll}16 & 14 & 2 & 9530.00824 & 0.90 & \mathrm{~d} & 9257.63865 & 0.77 & \mathrm{~d} & 9302.75595 & 0.78 & 9 \mathrm{e}, \mathrm{a}\end{array}$

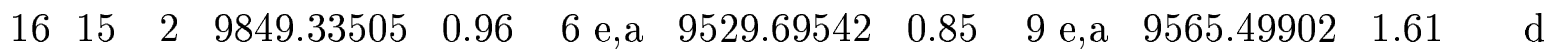

$\begin{array}{lllllllllllll}16 & 15 & 1 & 9849.33505 & 0.96 & \mathrm{~d} & 9529.69542 & 0.85 & \mathrm{~d} & 9565.49902 & 1.61 & 1 & \mathrm{a}\end{array}$

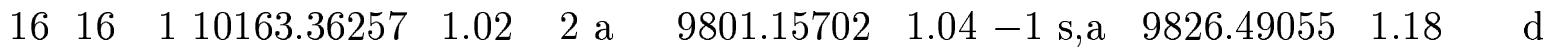

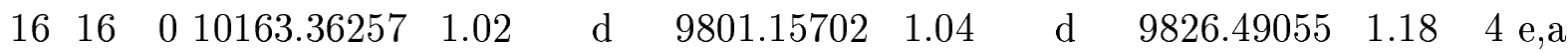

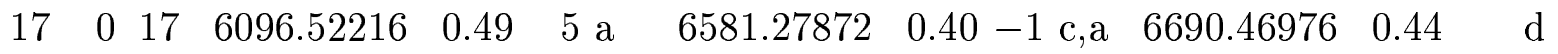

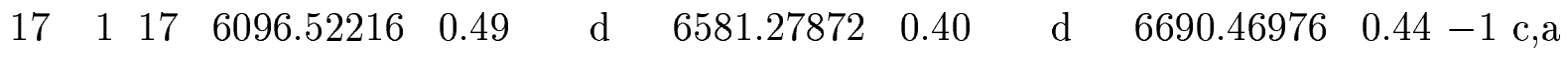

$\begin{array}{llllllllllll}17 & 1 & 16 & 6489.04977 & 0.48 & 5 \mathrm{a} & 6885.48676 & 0.61 & 9 \mathrm{e}, \mathrm{a} & 6992.76200 & 1.50 & -1 \mathrm{c}\end{array}$

$\begin{array}{llllllllllll}17 & 2 & 16 & 6489.10129 & 0.75 & 2 \mathrm{a} & 6885.48029 & 1.24 & -1 \mathrm{~s}, \mathrm{a} & 6992.76138 & 0.43 & 17 \mathrm{e}, \mathrm{a}\end{array}$ 


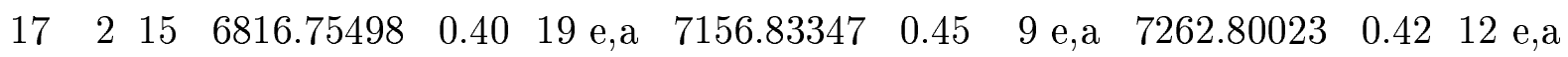

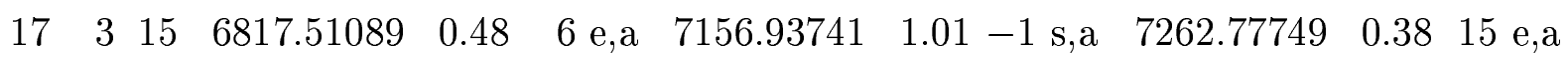

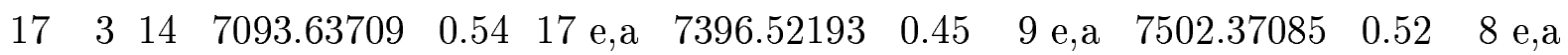
$\begin{array}{lllllllllll}17 & 4 & 14 & 7100.78661 & 0.75 & -1 \mathrm{~s}, \mathrm{a} & 7397.95961 & 0.87 & 3 \mathrm{e}, \mathrm{a} & 7503.07234 & 0.37-1 \mathrm{c}, \mathrm{a}\end{array}$

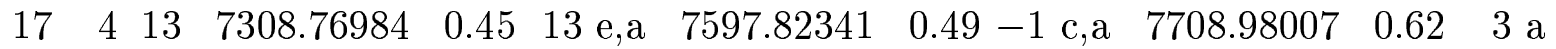
$\begin{array}{llllllllllll}17 & 5 & 13 & 7349.02764 & 0.63 & -1 \mathrm{~s}, \mathrm{a} & 7610.22218 & 0.59 & -1 \mathrm{~s}, \mathrm{a} & 7713.75385 & 0.46 & 11 \mathrm{e}, \mathrm{a}\end{array}$ $\begin{array}{llllllllllll}17 & 5 & 12 & 7463.39700 & 0.50 & 10 \mathrm{e}, \mathrm{a} & 7758.39693 & 0.48 & 8 \mathrm{e}, \mathrm{a} & 7871.73703 & 0.65 & 3 \mathrm{a}\end{array}$ $\begin{array}{llllllllll}17 & 6 & 12 & 7581.53770 & 0.50-1 \mathrm{~s}, \mathrm{a} & 7799.62089 & 0.93-1 \mathrm{~s}, \mathrm{a} & 7899.80795 & 0.34-1 \mathrm{c}, \mathrm{a}\end{array}$

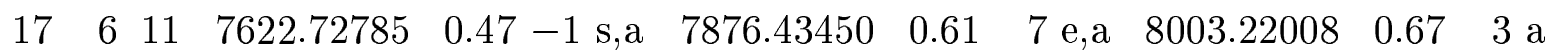
$\begin{array}{lllllllllllll}17 & 7 & 11 & 7820.67857 & 0.77-1 \mathrm{~s}, \mathrm{a} & 7970.18658 & 0.75-1 \mathrm{~s}, \mathrm{a} & 8076.57411 & 0.37 & 15 & \mathrm{e}, \mathrm{a}\end{array}$

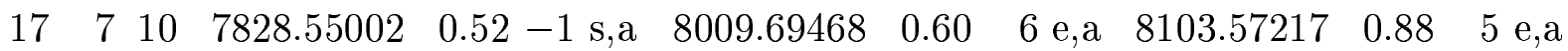
$\begin{array}{llllllllllll}17 & 8 & 10 & 8077.54589 & 0.63 & -1 \mathrm{~s}, \mathrm{a} & 8189.54832 & 0.68 & -1 \mathrm{~s}, \mathrm{a} & 8266.44577 & 0.44 & 11 \mathrm{e}, \mathrm{a}\end{array}$

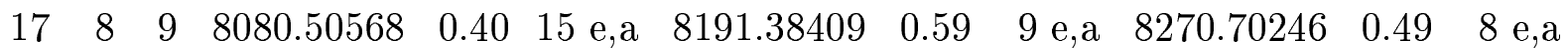
$\begin{array}{lllllllllll}17 & 9 & 9 & 8347.84765 & 0.64-1 \mathrm{~s}, \mathrm{a} & 8412.97480 & 1.13 & -1 \mathrm{~s}, \mathrm{a} & 8472.99999 & 0.42 & 14 \mathrm{e}, \mathrm{a}\end{array}$ $\begin{array}{llllllllllll}17 & 9 & 8 & 8347.95880 & 0.73 & 8 \mathrm{e}, \mathrm{a} & 8413.11581 & 0.44 & 10 \mathrm{e}, \mathrm{a} & 8473.47617 & 0.61 & 5 \mathrm{e}, \mathrm{a}\end{array}$ $\begin{array}{llllllllllll}17 & 10 & 8 & 8681.30924 & 0.86 & -1 \mathrm{~s}, \mathrm{a} & 8608.20340 & 1.08 & -1 \mathrm{f}, \mathrm{a} & 8697.62774 & 0.49 & 14 \mathrm{e}, \mathrm{a}\end{array}$ $\begin{array}{lllllllllll}17 & 10 & 7 & 8681.32555 & 0.41-1 \mathrm{~s}, \mathrm{a} & 8608.22435 & 0.99 & 6 & \mathrm{e}, \mathrm{a} & 8697.66700 & 0.83-1 \mathrm{~s}, \mathrm{a}\end{array}$

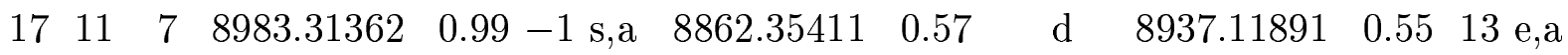
$\begin{array}{lllllllllll}17 & 11 & 6 & 8983.29001 & 0.66-1 \mathrm{~s}, \mathrm{a} & 8862.35411 & 0.57-1 \mathrm{~s}, \mathrm{a} & 8937.12188 & 1.40-1 \mathrm{f}, \mathrm{a}\end{array}$

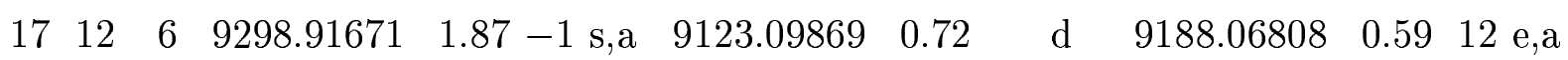
$\begin{array}{llllllllllll}17 & 12 & 5 & 9298.91219 & 0.85 & -1 \mathrm{~s}, \mathrm{a} & 9123.09869 & 0.72 & -1 \mathrm{~s}, \mathrm{a} & 9188.06808 & 0.59 & \mathrm{~d}\end{array}$ $\begin{array}{llllllllllll}17 & 13 & 5 & 9621.23041 & 1.15 & \mathrm{~d} & 9391.07256 & 0.72 & \mathrm{~d} & 9447.36858 & 0.79 & 8 \mathrm{e}, \mathrm{a}\end{array}$ $\begin{array}{llllllllllll}17 & 13 & 4 & 9621.23041 & 1.15 & 6 \mathrm{e}, \mathrm{a} & 9391.07256 & 0.72 & 8 \mathrm{e}, \mathrm{a} & 9447.36858 & 0.79 & \mathrm{~d}\end{array}$ $\begin{array}{llllllllllll}17 & 14 & 4 & 9946.29779 & 1.27 & \mathrm{~d} & 9664.30568 & 0.81 & \mathrm{~d} & 9712.09881 & 0.80 & 9 \mathrm{e}, \mathrm{a}\end{array}$ $\begin{array}{llllllllllll}17 & 14 & 3 & 9946.29779 & 1.27 & -1 \mathrm{~s}, \mathrm{a} & 9664.30568 & 0.81 & -1 \mathrm{~s}, \mathrm{a} & 9712.09881 & 0.80 & \mathrm{~d}\end{array}$

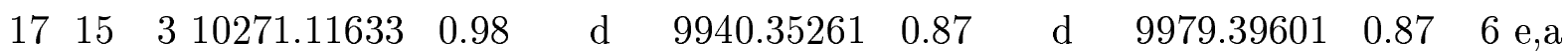

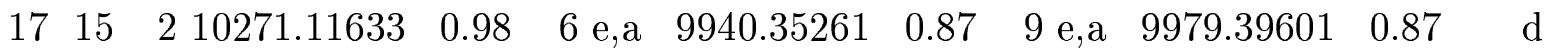




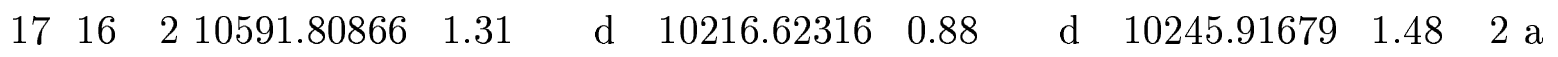

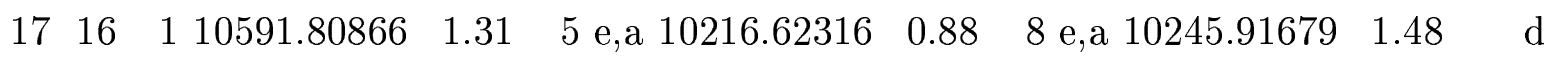

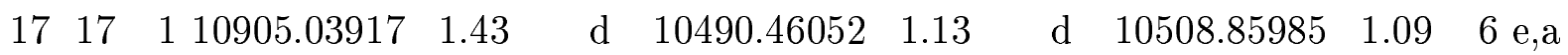

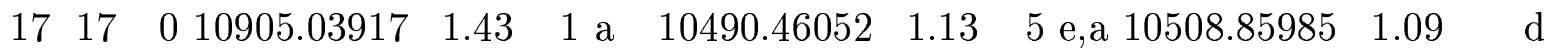
$\begin{array}{llllllllllll}18 & 0 & 18 & 6428.96531 & 0.60 & \mathrm{~d} & 6912.85414 & 0.77 & \mathrm{~d} & 7023.28252 & 0.38 & -1 \\ \mathrm{c}, \mathrm{a}\end{array}$

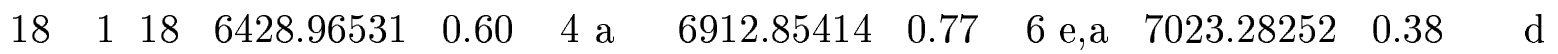
$\begin{array}{lllllllllll}18 & 1 & 17 & 6846.24114 & 0.78 & 3 \mathrm{a} & 7234.96500 & 1.20-1 \mathrm{f}, \mathrm{a} & 7343.34106 & 0.47-1 \mathrm{c}, \mathrm{a}\end{array}$

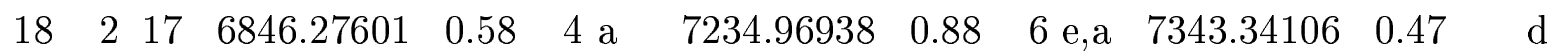

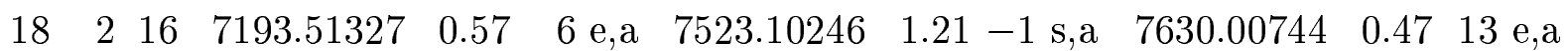

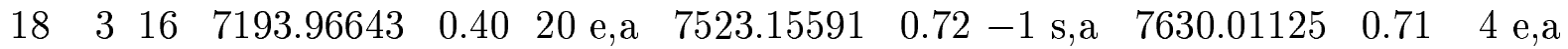

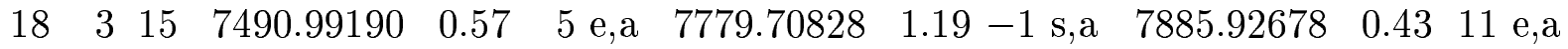

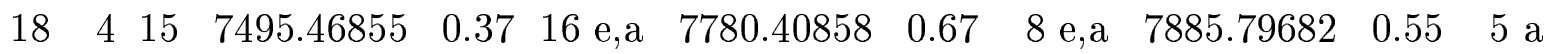

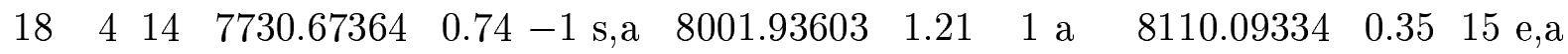
$\begin{array}{llllllllllll}18 & 5 & 14 & 7758.78588 & 0.43 & 10 \mathrm{e}, \mathrm{a} & 8008.11452 & 0.56 & -1 \mathrm{~s}, \mathrm{a} & 8112.86188 & 0.68 & 3 \mathrm{a}\end{array}$ $\begin{array}{llllllllllll}18 & 5 & 13 & 7904.22388 & 0.68 & -1 \mathrm{~s}, \mathrm{a} & 8181.44296 & 1.24 & -1 \mathrm{~s}, \mathrm{a} & 8294.08979 & 0.56 & 11 \mathrm{e}, \mathrm{a}\end{array}$

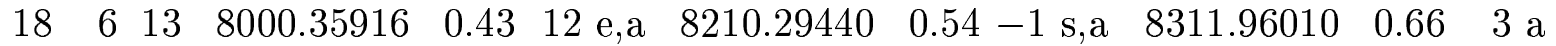
$\begin{array}{llllllllllll}18 & 6 & 12 & 8057.70535 & 1.14 & -1 \mathrm{~s}, \mathrm{a} & 8312.77911 & 1.12 & -1 \mathrm{~s}, \mathrm{a} & 8433.96572 & 0.54 & 9 \mathrm{e}, \mathrm{a}\end{array}$ $\begin{array}{llllllllllll}18 & 7 & 12 & 8242.39180 & 0.36 & -1 \mathrm{~s}, \mathrm{a} & 8394.96006 & 0.42 & -1 \mathrm{~s}, \mathrm{a} & 8496.42227 & 0.72 & 3 \mathrm{a}\end{array}$ $\begin{array}{lllllllllllll}18 & 7 & 11 & 8256.09117 & 1.09 & -1 \mathrm{~s}, \mathrm{a} & 8439.89736 & 0.83 & -1 \mathrm{~s}, \mathrm{a} & 8537.65160 & 0.87 & 6 \mathrm{e}, \mathrm{a}\end{array}$ $\begin{array}{lllllllllllll}18 & 8 & 11 & 8499.29572 & 0.42 & 11 & \mathrm{e}, \mathrm{a} & 8609.36456 & 0.57 & -1 \mathrm{~s}, \mathrm{a} & 8686.85089 & 0.71 & 6 \mathrm{e}, \mathrm{a}\end{array}$ $\begin{array}{llllllllllll}18 & 8 & 10 & 8502.27199 & 0.83 & -1 \mathrm{~s}, \mathrm{a} & 8613.05745 & 5.04-1 \mathrm{~s} & 8695.40754 & 0.46 & 11 \mathrm{e}, \mathrm{a}\end{array}$ $\begin{array}{llllllllllll}18 & 9 & 10 & 8768.73470 & 1.13 & 10 \mathrm{e}, \mathrm{a} & 8832.84415 & 0.53 & 11 \mathrm{e}, \mathrm{a} & 8893.21911 & 0.90 & 3 \mathrm{a}\end{array}$

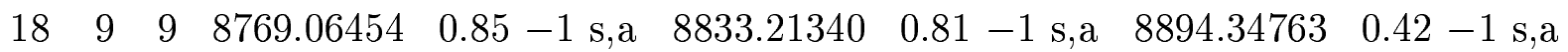
$\begin{array}{llllllllllll}18 & 10 & 9 & 9103.44473 & 0.76 & -1 \mathrm{~s}, \mathrm{a} & 9027.43378 & 0.79 & -1 \mathrm{~s}, \mathrm{a} & 9117.97285 & 0.77 & 3 \mathrm{a}\end{array}$ $\begin{array}{llllllllllll}18 & 10 & 8 & 9103.95773 & 1.29 & 1 \mathrm{a} & 9027.47543 & 0.90 & 4 \mathrm{e}, \mathrm{a} & 9118.08037 & 0.42 & 16 \mathrm{e}, \mathrm{a}\end{array}$ $\begin{array}{llllllllllll}18 & 11 & 8 & 9408.54490 & 1.02 & -1 \mathrm{~s}, \mathrm{a} & 9281.89384 & 0.95 & 8 \mathrm{e}, \mathrm{a} & 9358.45730 & 1.21 & 2 \mathrm{e}, \mathrm{a}\end{array}$ $\begin{array}{llllllllllll}18 & 11 & 7 & 9408.52195 & 1.15 & -1 \mathrm{~s}, \mathrm{a} & 9281.89269 & 1.41 & -1 \mathrm{~s}, \mathrm{a} & 9358.46186 & 0.56 & 11 \mathrm{e}, \mathrm{a}\end{array}$ 
$\begin{array}{llllllllllll}18 & 12 & 7 & 9726.94238 & 0.99 & -1 \mathrm{~s}, \mathrm{a} & 9544.18189 & 0.72 & -1 \mathrm{~s}, \mathrm{a} & 9611.22367 & 0.68 & \mathrm{~d}\end{array}$

$\begin{array}{lllllllllll}18 & 12 & 6 & 9726.94561 & 1.74-1 \mathrm{~s}, \mathrm{a} & 9544.18189 & 0.72 & \mathrm{~d} & 9611.22367 & 0.68 & 11 \mathrm{e}, \mathrm{a}\end{array}$

$\begin{array}{lllllllllll}18 & 13 & 6 & 10052.65206 & 0.94-1 \mathrm{~s}, \mathrm{a} & 9814.47855 & 0.79-1 \mathrm{~s}, \mathrm{a} & 9873.10470 & 0.67 & \mathrm{~d}\end{array}$

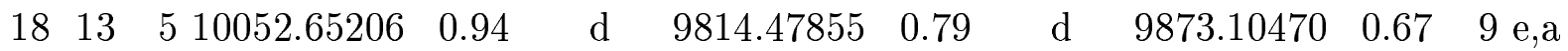

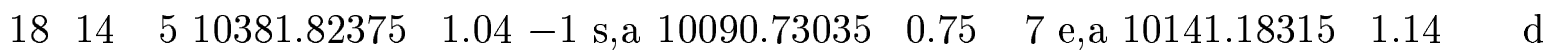

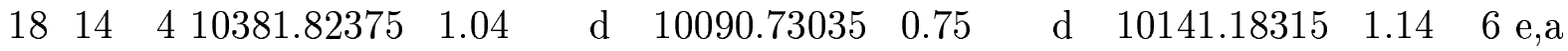

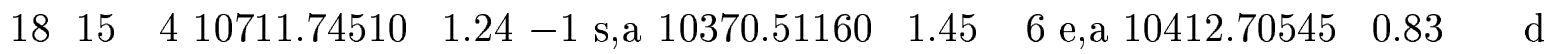

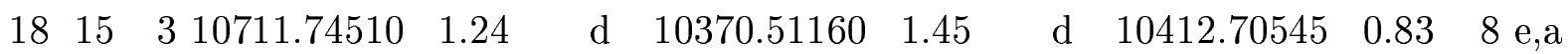

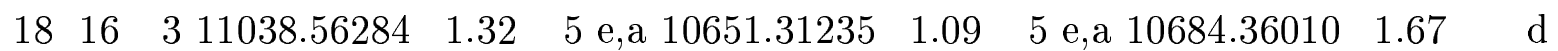

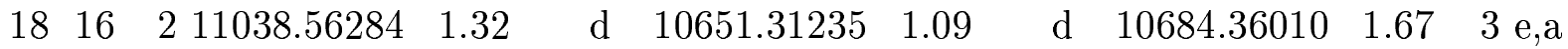

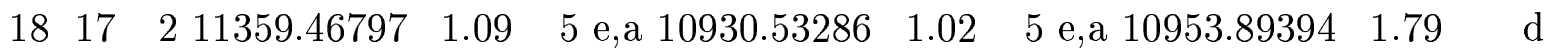

$\begin{array}{llllllllllll}18 & 17 & 1 & 11359.46797 & 1.09 & \mathrm{~d} & 10930.53286 & 1.02 & \mathrm{~d} & 10953.89394 & 1.79 & 1 \mathrm{a}\end{array}$

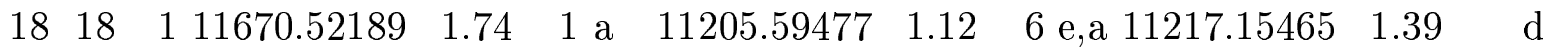

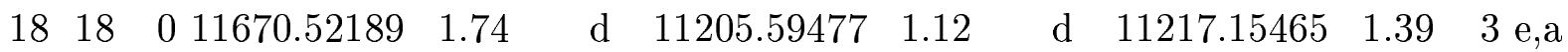

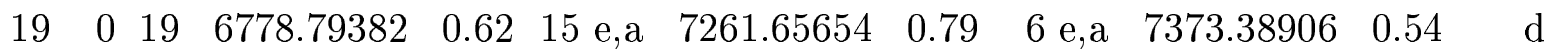

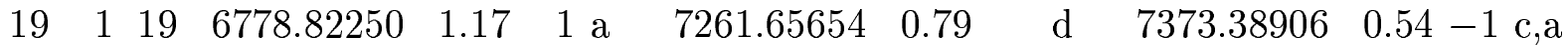

$\begin{array}{llllllllllll}19 & 1 & 18 & 7220.96223 & 0.49 & 12 \mathrm{e}, \mathrm{a} & 7601.54855 & 0.72 & 5 \mathrm{e}, \mathrm{a} & 7711.08862 & 0.50 & \mathrm{~d}\end{array}$

$\begin{array}{llllllllllll}19 & 2 & 18 & 7221.45790 & 0.60 & 5 \mathrm{a} & 7601.54855 & 0.72 & \mathrm{~d} & 7711.08862 & 0.50 & 13 \mathrm{e}, \mathrm{a}\end{array}$

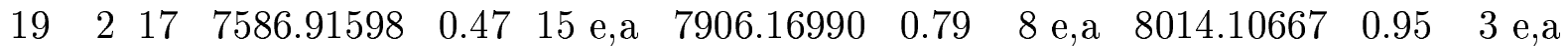

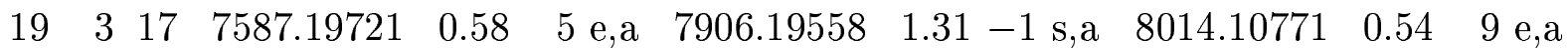

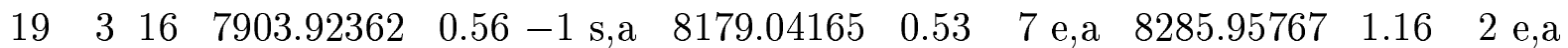

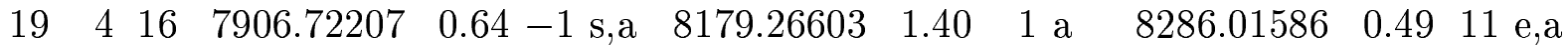

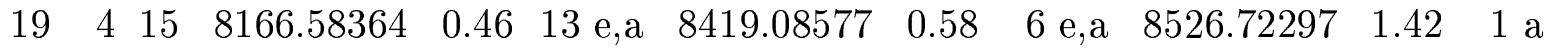

$\begin{array}{llllllllllll}19 & 5 & 15 & 8185.64603 & 1.12 & -1 \mathrm{~s}, \mathrm{a} & 8422.55882 & 0.78 & -1 \mathrm{~s}, \mathrm{a} & 8529.17063 & 0.44 & 11 \mathrm{e}, \mathrm{a}\end{array}$

$\begin{array}{llllllllllll}19 & 5 & 14 & 8361.94728 & 0.71 & 5 \mathrm{e}, \mathrm{a} & 8618.88404 & 0.66 & 6 \mathrm{e}, \mathrm{a} & 8730.62598 & 0.64 & 6 \mathrm{e}, \mathrm{a}\end{array}$

$\begin{array}{llllllllllll}19 & 6 & 14 & 8437.83977 & 1.20 & -1 \mathrm{~s}, \mathrm{a} & 8638.31309 & 1.39 & -1 \mathrm{~s}, \mathrm{a} & 8741.60333 & 0.78 & 6 \\ \mathrm{ee,a}\end{array}$

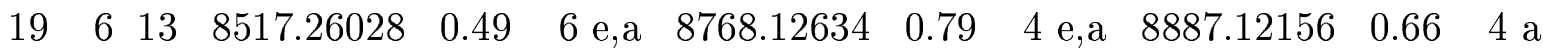




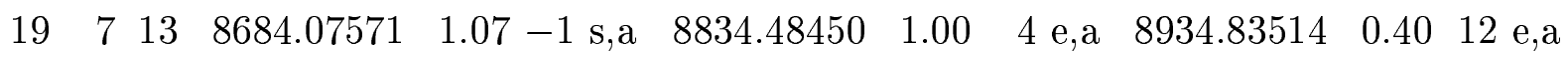

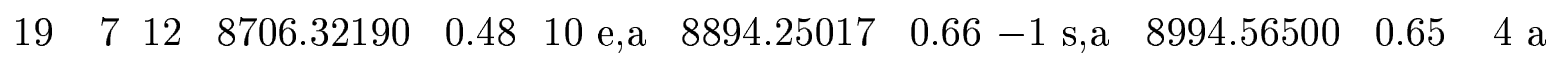

$\begin{array}{llllllllllll}19 & 8 & 12 & 8941.39970 & 1.48 & 1 \mathrm{a} & 9050.62548 & 0.87 & -1 \mathrm{~s}, \mathrm{a} & 9127.71483 & 0.62 & 8 \mathrm{e}, \mathrm{a}\end{array}$

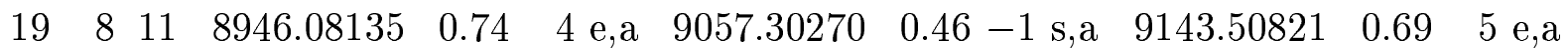

$\begin{array}{llllllllllll}19 & 9 & 11 & 9210.12865 & 1.29 & -1 \mathrm{~s}, \mathrm{a} & 9273.32683 & 1.14 & -1 \mathrm{~s}, \mathrm{a} & 9334.15335 & 0.47 & 6 \mathrm{a}\end{array}$

$\begin{array}{lllllllllllll}19 & 9 & 10 & 9210.69630 & 0.65 & 6 \mathrm{e}, \mathrm{a} & 9274.13587 & 0.61 & -1 \mathrm{~s}, \mathrm{a} & 9336.61438 & 0.65 & 4 \mathrm{a}\end{array}$

$\begin{array}{llllllllllll}19 & 10 & 10 & 9546.78531 & 0.90 & -1 \mathrm{~s}, \mathrm{a} & 9466.92399 & 0.75 & -1 \mathrm{~s}, \mathrm{a} & 9558.78593 & 0.56 & 8 \mathrm{e}, \mathrm{a}\end{array}$

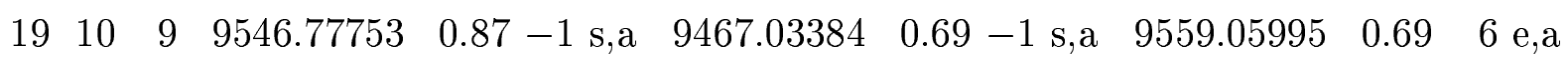

$\begin{array}{lllllllllllll}19 & 11 & 9 & 9853.31638 & 1.63 & 1 \mathrm{a} & 9721.51834 & 1.02 & -1 \mathrm{f}, \mathrm{a} & 9799.94838 & 0.80 & 7 \mathrm{e}, \mathrm{a}\end{array}$

$\begin{array}{llllllllllll}19 & 11 & 8 & 9853.38266 & 1.42 & -1 \mathrm{~s}, \mathrm{a} & 9721.52345 & 1.33 & 5 \mathrm{e}, \mathrm{a} & 9799.97641 & 1.26 & 1 \mathrm{a}\end{array}$

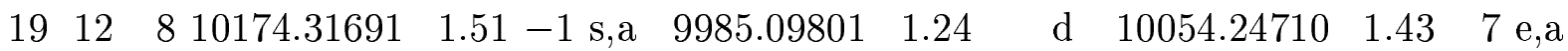

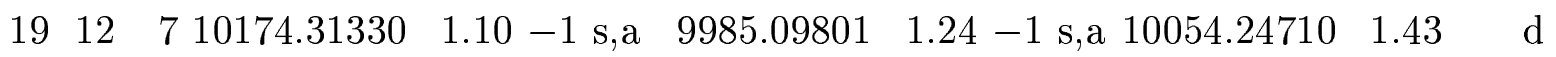

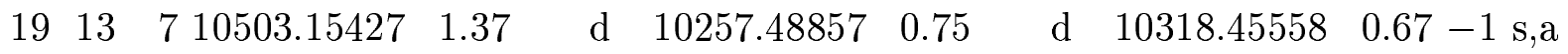

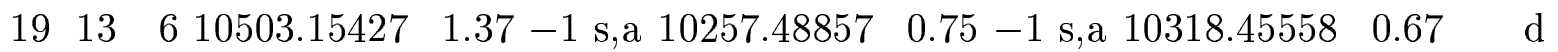

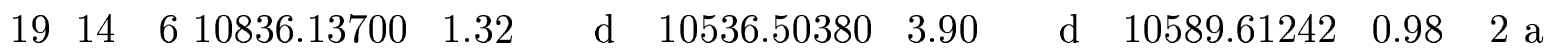

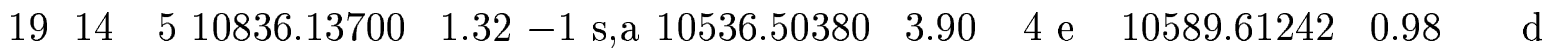

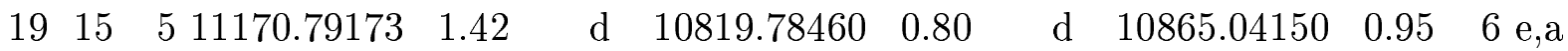

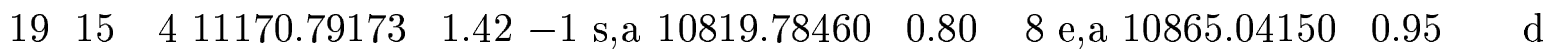

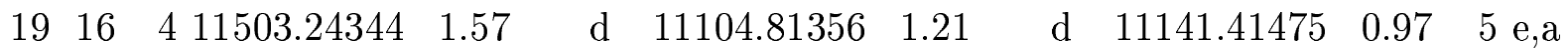

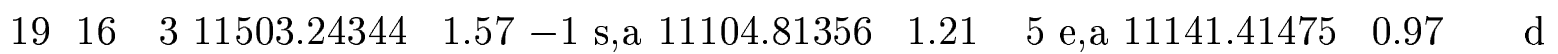

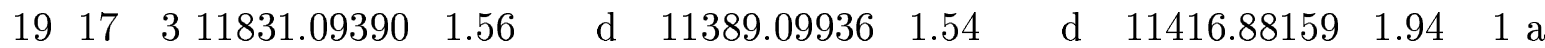

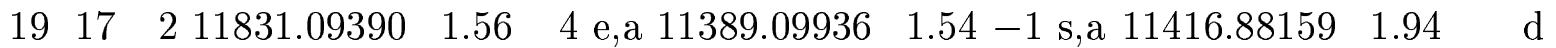

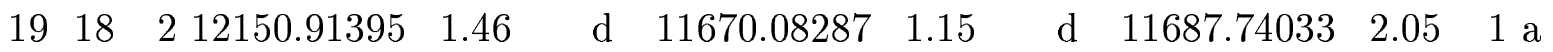

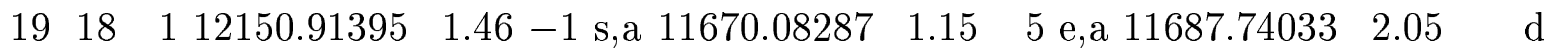

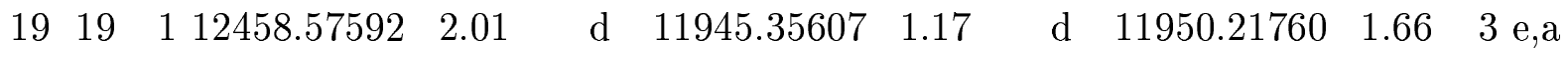

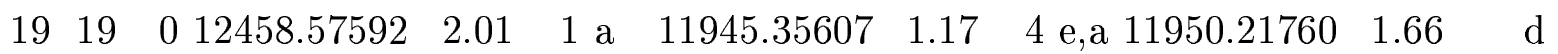

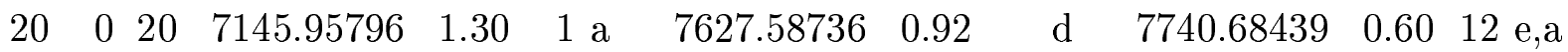




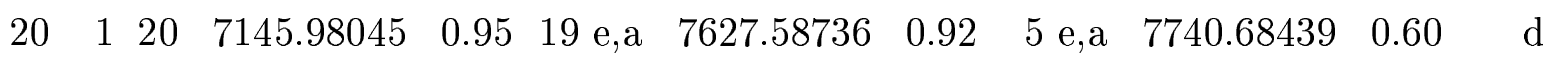

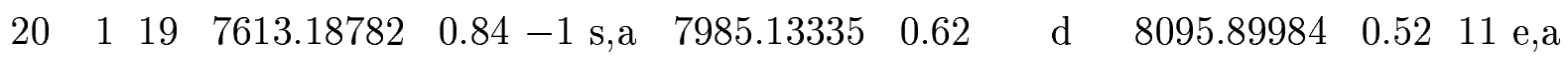

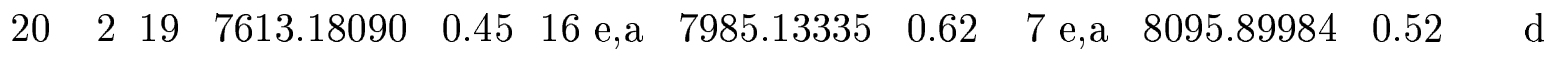

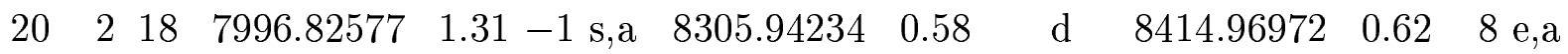

$\begin{array}{llllllllllll}20 & 3 & 18 & 7997.00058 & 0.44 & 14 \text { e,a } & 8305.94234 & 0.58 & 9 \text { e,a } & 8414.97545 & 1.28 & 2 \text { e,a }\end{array}$

$\begin{array}{llllllllllll}20 & 3 & 17 & 8332.50886 & 0.65 & -1 \mathrm{~s}, \mathrm{a} & 8594.60643 & 1.51 & -1 \mathrm{~s}, \mathrm{a} & 8702.37858 & 0.44 & 12 \mathrm{e}, \mathrm{a}\end{array}$

$\begin{array}{llllllllllll}20 & 4 & 17 & 8334.26287 & 0.53 & 8 \mathrm{e}, \mathrm{a} & 8594.81064 & 0.64 & -1 \mathrm{~s}, \mathrm{a} & 8702.41701 & 1.53 & 1 \mathrm{a}\end{array}$

$\begin{array}{llllllllllll}20 & 4 & 16 & 8616.11137 & 0.80 & -1 \mathrm{~s}, \mathrm{a} & 8851.23379 & 1.23 & -1 \mathrm{~s}, \mathrm{a} & 8958.97986 & 0.46 & 11 \mathrm{e}, \mathrm{a}\end{array}$

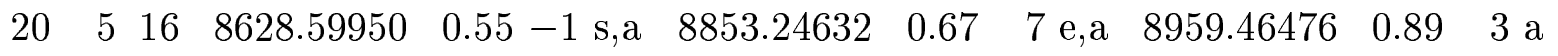

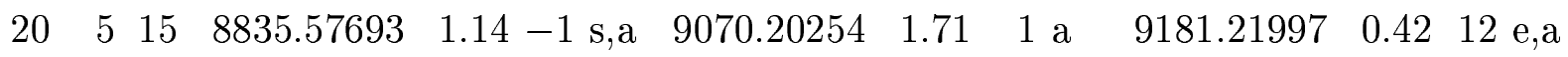

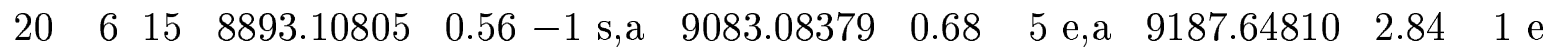

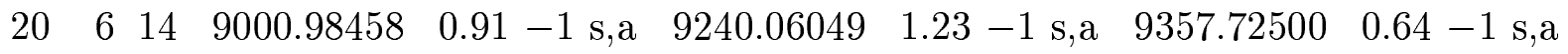

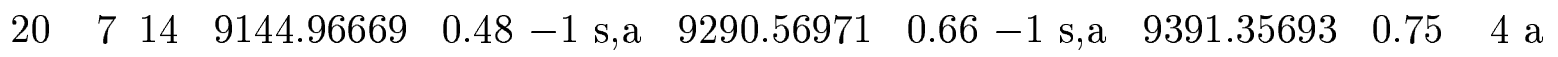

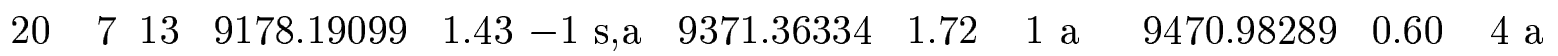

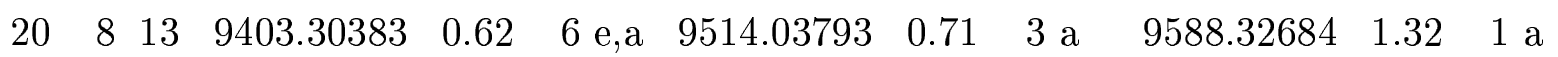

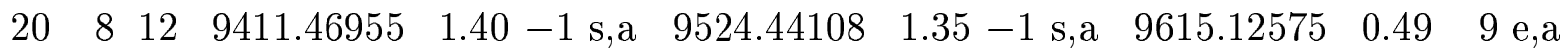

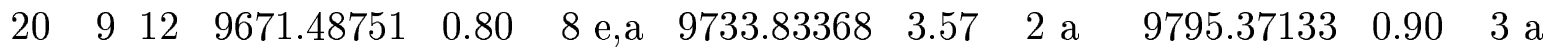

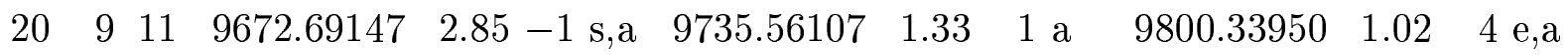

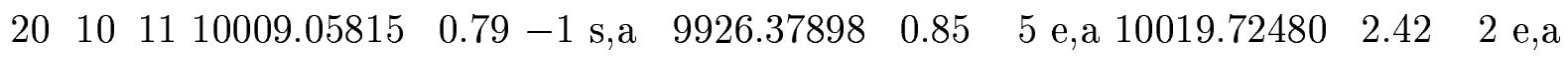

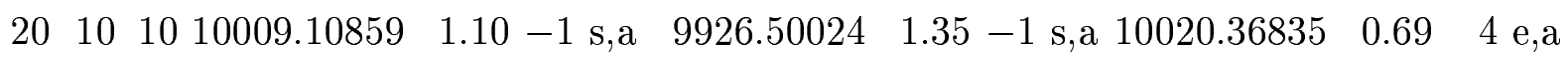

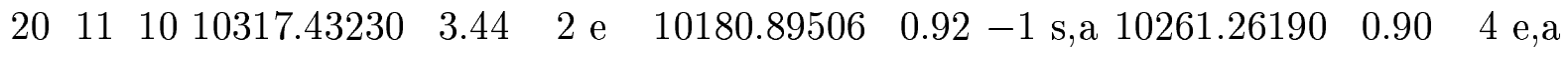

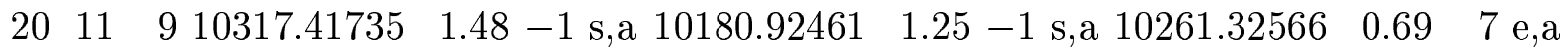

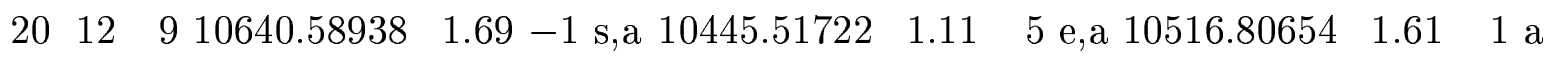

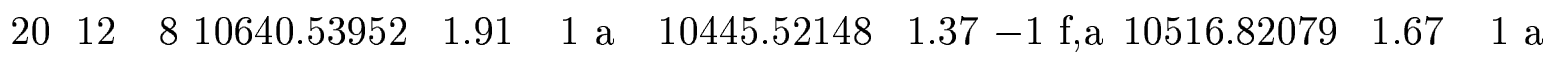

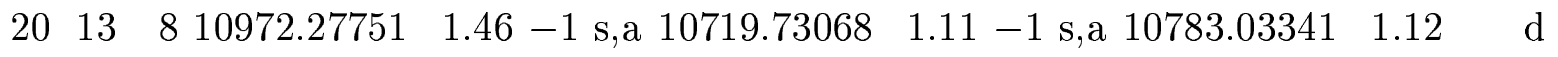

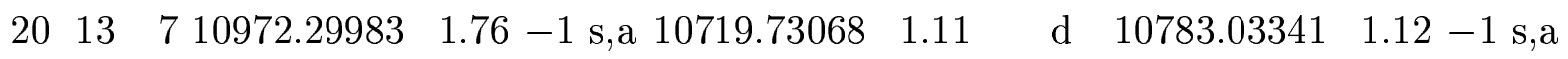

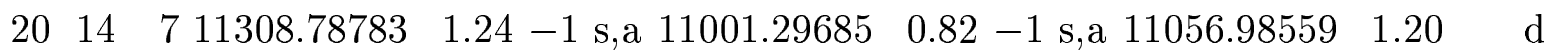




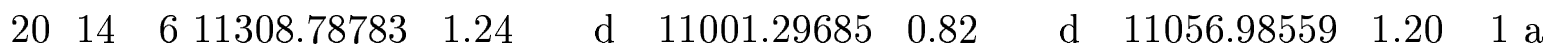

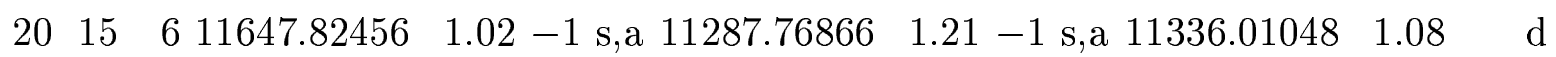

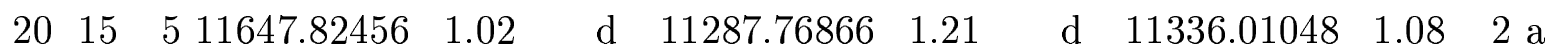

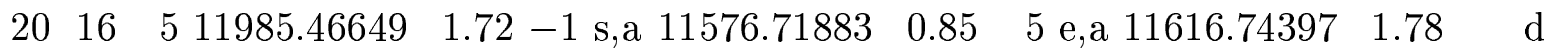

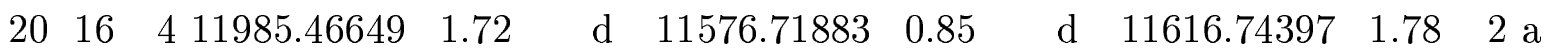

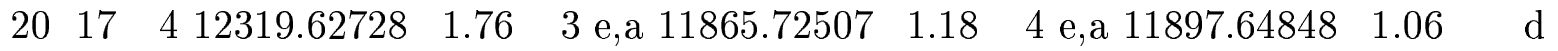

$\begin{array}{llllllllllll}20 & 17 & 3 & 12319.62728 & 1.76 & \mathrm{~d} & 11865.72507 & 1.18 & \mathrm{~d} & 11897.64848 & 1.06 & 4 \mathrm{e}, \mathrm{a}\end{array}$

$\begin{array}{lllllllllll}20 & 18 & 3 & 12647.26051 & 1.82 & -1 & \mathrm{~s}, \mathrm{a} & 12152.31441 & 1.15 & -1 & \mathrm{~s}, \mathrm{a} \\ 12175.22961 & 2.19 & \mathrm{~d}\end{array}$

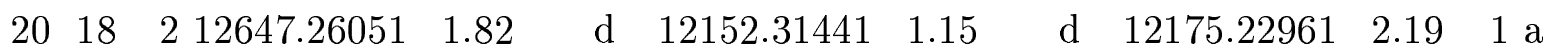

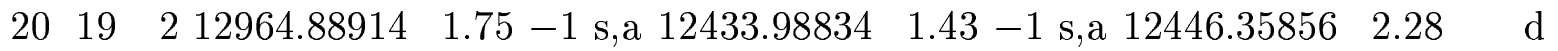

$\begin{array}{llllllllllll}20 & 19 & 1 & 12964.88914 & 1.75 & \mathrm{~d} & 12433.98834 & 1.43 & \mathrm{~d} & 12446.35856 & 2.28 & 1 \mathrm{a}\end{array}$

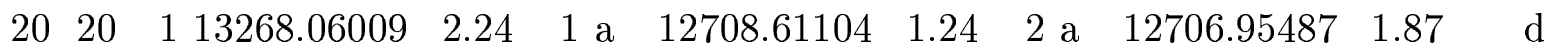

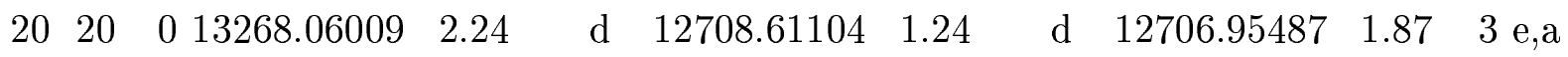

$\begin{array}{lllllllllllll}21 & 0 & 21 & 7530.50899 & 0.53 & 15 & \text { e,a } & 8010.53273 & 0.85 & 6 \text { e,a } & 8125.05940 & 0.65 & \mathrm{~d}\end{array}$

$\begin{array}{llllllllllll}21 & 1 & 21 & 7530.50899 & 0.53 & \mathrm{~d} & 8010.53273 & 0.85 & \mathrm{~d} & 8125.05940 & 0.65 & 10 \mathrm{e}, \mathrm{a}\end{array}$

$\begin{array}{lllllllllllll}21 & 1 & 20 & 8022.98862 & 0.57 & 11 \mathrm{e}, \mathrm{a} & 8385.60306 & 0.88 & 6 \mathrm{e}, \mathrm{a} & 8497.66150 & 0.53 & \mathrm{~d}\end{array}$

$\begin{array}{llllllllllll}21 & 2 & 20 & 8022.88760 & 1.13 & 1 \mathrm{a} & 8385.60306 & 0.88 & \mathrm{~d} & 8497.66150 & 0.53 & 10 \mathrm{e}, \mathrm{a}\end{array}$

$\begin{array}{lllllllllllll}21 & 2 & 19 & 8423.00580 & 0.55 & 9 \text { e,a } & 8722.25096 & 0.73 & 5 \text { e,a } & 8832.47619 & 0.61 & \mathrm{~d}\end{array}$

$\begin{array}{llllllllllll}21 & 3 & 19 & 8423.48166 & 1.28 & 1 \mathrm{a} & 8722.27195 & 1.21 & 1 \mathrm{a} & 8832.47619 & 0.61 & 10 \mathrm{e}, \mathrm{a}\end{array}$

$\begin{array}{llllllllllll}21 & 3 & 18 & 8776.73869 & 0.45 & 8 \mathrm{e}, \mathrm{a} & 9026.34296 & 1.14 & 4 \mathrm{e}, \mathrm{a} & 9135.07484 & 0.85 & 7 \mathrm{e}, \mathrm{a}\end{array}$

$\begin{array}{llllllllllll}21 & 4 & 18 & 8777.85162 & 1.19 & 1 \mathrm{a} & 9026.45222 & 1.70 & 1 \mathrm{a} & 9135.09343 & 0.54 & 8 \mathrm{e}, \mathrm{a}\end{array}$

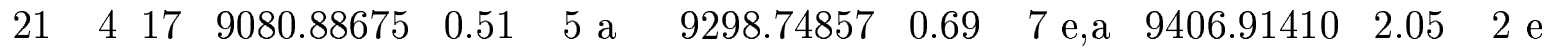

$\begin{array}{llllllllllll}21 & 5 & 17 & 9089.15022 & 0.87 & 2 \mathrm{a} & 9299.92464 & 1.33 & 1 \mathrm{a} & 9407.22853 & 0.60 & 8 \mathrm{e}, \mathrm{a}\end{array}$

$\begin{array}{llllllllllll}21 & 5 & 16 & 9323.24911 & 0.57 & 4 \mathrm{a} & 9535.84965 & 0.74 & 3 \mathrm{a} & 9646.10544 & 1.35 & 2 \mathrm{a}\end{array}$

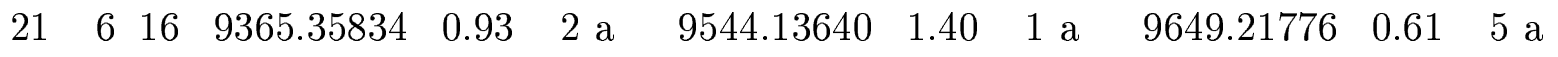

$\begin{array}{llllllllllll}21 & 6 & 15 & 9502.85181 & 0.81 & 2 \mathrm{a} & 9726.64740 & 0.86 & 3 \mathrm{e}, \mathrm{a} & 9843.08060 & 1.33 & 1 \mathrm{a}\end{array}$

$\begin{array}{llllllllllll}21 & 7 & 15 & 9624.41380 & 6.51 & 1 \mathrm{e} & 9763.44664 & 1.50 & 2 \mathrm{a} & 9865.27805 & 0.48 & 7 \mathrm{e}, \mathrm{a}\end{array}$ 
$\begin{array}{llllllllllll}21 & 7 & 14 & 9676.59623 & 0.79 & 2 \mathrm{a} & 9869.20743 & 0.62 & 5 \text { e,a } 10001.32011 & 1.50 & 1 \mathrm{a}\end{array}$ $\begin{array}{llllllllllll}21 & 8 & 14 & 9884.50272 & 1.74 & 1 \mathrm{a} & 9962.12047 & 1.33 & 2 \mathrm{a} & 10068.01650 & 1.58 & 4 \mathrm{e}\end{array}$ $\begin{array}{llllllllllll}21 & 8 & 13 & 9898.41927 & 1.11 & 3 \mathrm{a} & 10015.05119 & 0.85 & 2 \mathrm{a} & 10109.64699 & 1.08 & 2 \mathrm{a}\end{array}$ $\begin{array}{llllll}21 & 9 & 13 & 10152.81179 & 1.16 & 2 \mathrm{a}\end{array}$ $10276.36230 \quad 0.69 \quad 4 \mathrm{e}, \mathrm{a}$

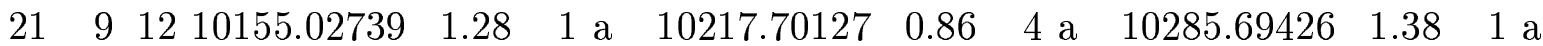

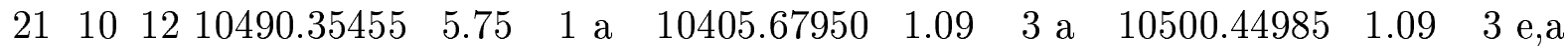

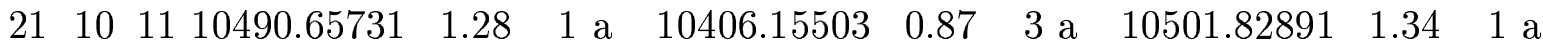

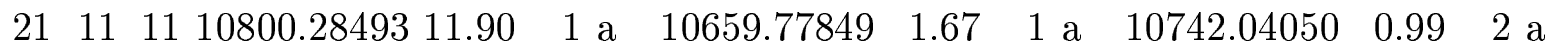

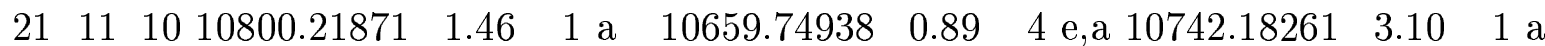

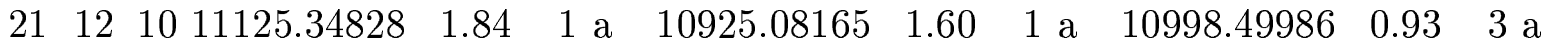

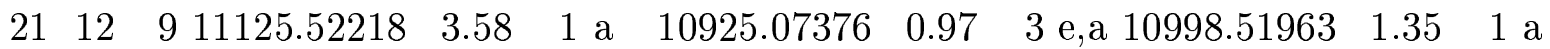

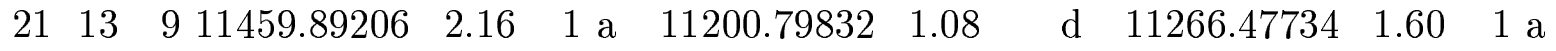

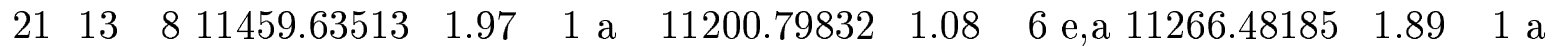

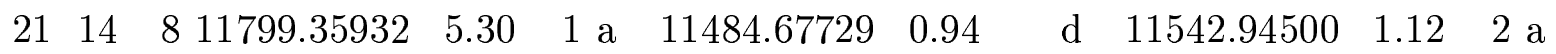

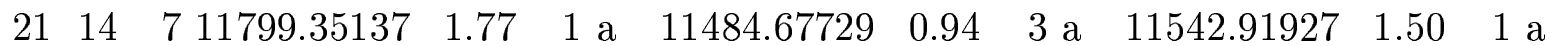
$\begin{array}{lllllllllll}21 & 15 & 712142.41449 & 1.59 & \mathrm{~d} & 11774.08303 & 1.06 & \mathrm{~d} & 11825.21659 & 1.77 & 1 \mathrm{a}\end{array}$

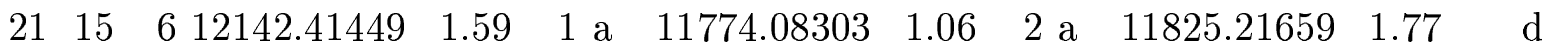

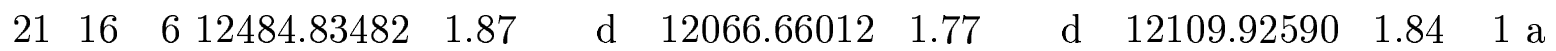

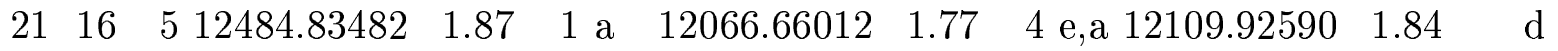

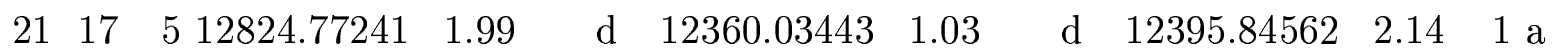

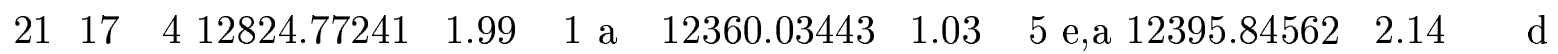

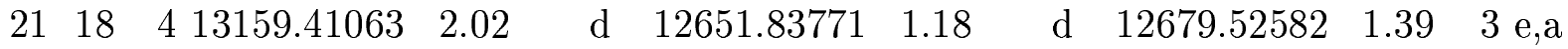

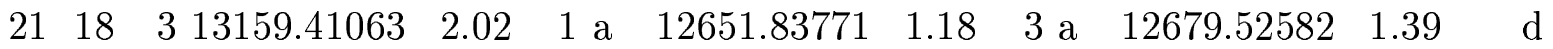

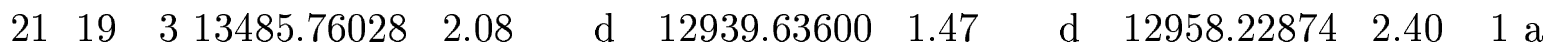
$\begin{array}{llllllllllll}21 & 19 & 2 & 13485.76028 & 2.08 & 1 \text { a } & 12939.63600 & 1.47 & 2 \text { a } & 12958.22874 & 2.40 & \mathrm{~d}\end{array}$

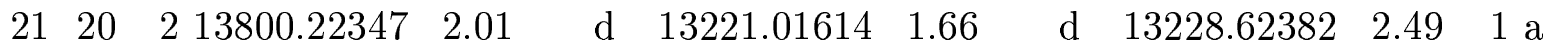

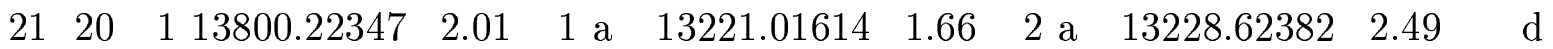


$\begin{array}{llllllllllll}21 & 21 & 1 & 14097.90967 & 2.46 & \mathrm{~d} & 13494.28863 & 1.53 & \mathrm{~d} & 13486.39363 & 2.12 & 1\end{array}$

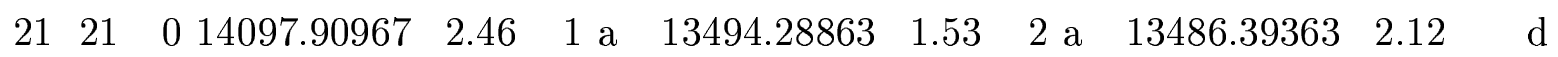

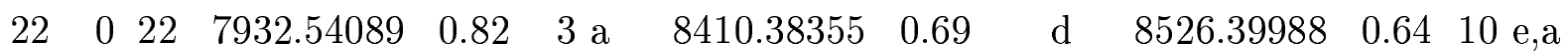

$\begin{array}{lllllllllllll}22 & 1 & 22 & 7932.56081 & 1.20 & 8 \text { e,a } & 8410.38355 & 0.69 & 8 \text { e,a } & 8526.39988 & 0.64 & \text { d }\end{array}$

$\begin{array}{lllllllllllll}22 & 1 & 21 & 8450.03209 & 1.06 & 2 & \text { a } & 8802.85124 & 0.99 & \text { d } & 8916.25320 & 0.70 & 8 \text { e,a }\end{array}$

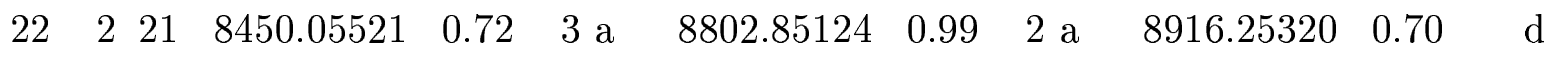

$\begin{array}{llllllllllll}22 & 2 & 20 & 8865.21492 & 0.55 & \mathrm{~d} & 9155.00217 & 1.27 & \mathrm{~d} & 9266.47886 & 0.54 & 9 \mathrm{e}, \mathrm{a}\end{array}$

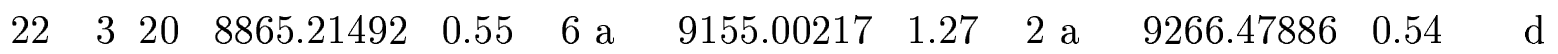

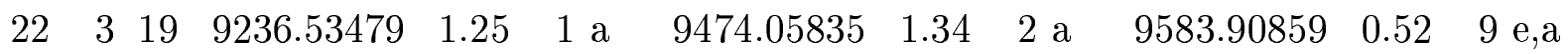

$\begin{array}{llllllllllll}22 & 4 & 19 & 9237.28426 & 0.56 & 4 \mathrm{a} & 9474.19067 & 0.97 & 4 \text { e,a } & 9583.91631 & 1.57 & 1 \mathrm{a}\end{array}$

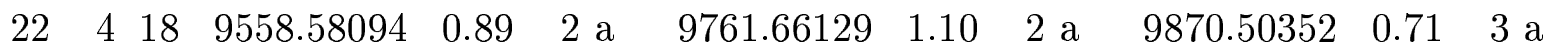

$\begin{array}{llllllllllll}22 & 5 & 18 & 9564.03535 & 0.57 & 4 \mathrm{a} & 9762.38491 & 0.86 & 4 \mathrm{e}, \mathrm{a} & 9870.65533 & 1.02 & 4 \mathrm{e}, \mathrm{a}\end{array}$

$\begin{array}{llllllrll}22 & 5 & 17 & 9823.92169 & 1.36 & 1 \mathrm{a} & 10125.50796 & 0.70 & 4 \mathrm{e}, \mathrm{a}\end{array}$

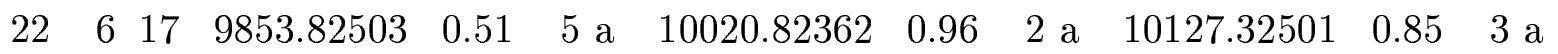

$22 \quad 6 \quad 16$

$10341.84147 \quad 0.68 \quad 5 \mathrm{a}$

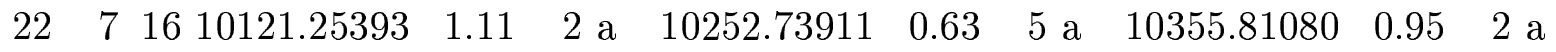

$22 \quad 715$

$10511.96779 \quad 1.28 \quad 1 \mathrm{a}$

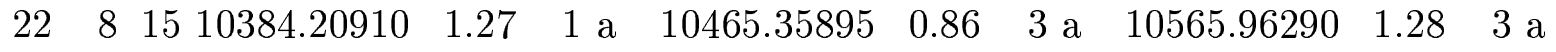

$\begin{array}{llllll}22 & 8 & 14 & 10405.72714 & 6.59 & 1 \text { a }\end{array}$

$10624.96654 \quad 0.59 \quad 7 \mathrm{e}, \mathrm{a}$

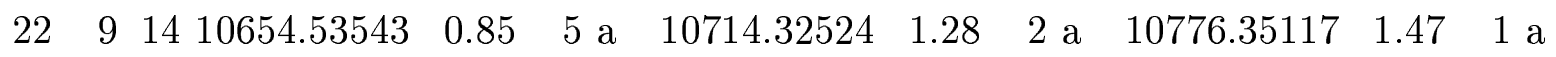

$22 \quad 9 \quad 1310657.65095 \quad 2.01 \quad 1$ a

$10793.09025 \quad 1.13 \quad 4 \mathrm{e}, \mathrm{a}$

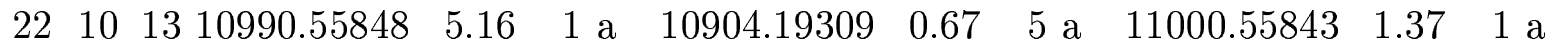

$\begin{array}{llllllllll}22 & 10 & 12 & 10905.34581 & 1.07 & 2 & \mathrm{a} & 11003.33822 & 0.75 & 4 \mathrm{a}\end{array}$

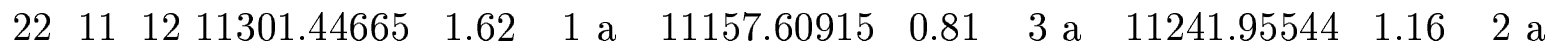

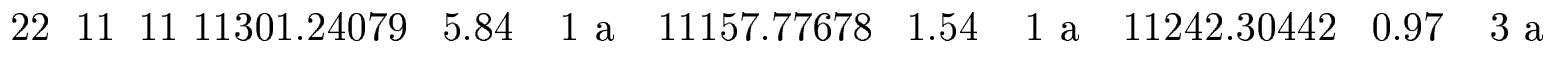

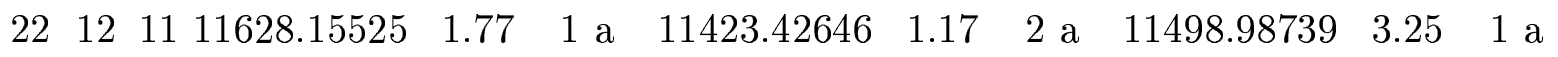

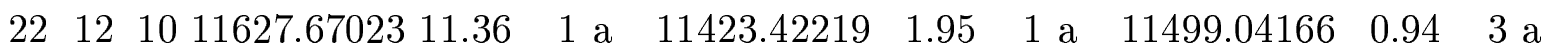




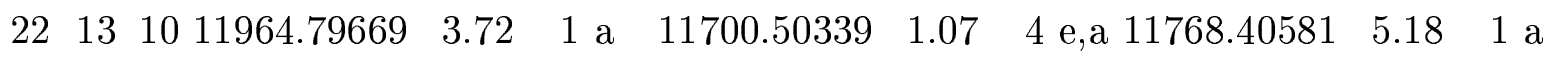

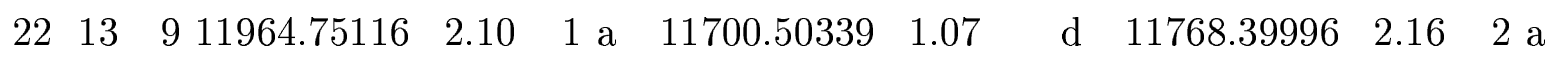

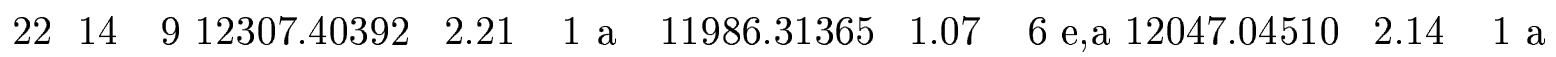

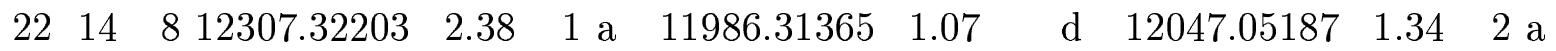

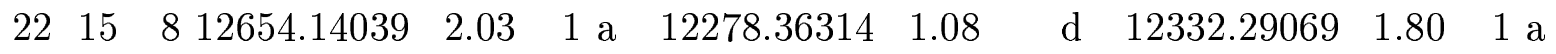

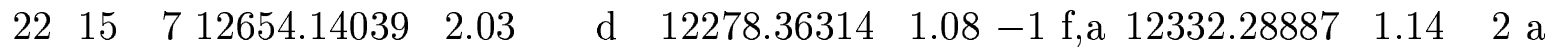

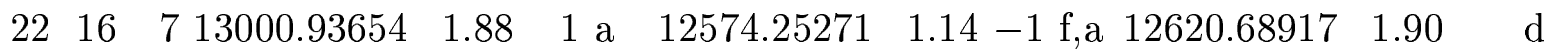

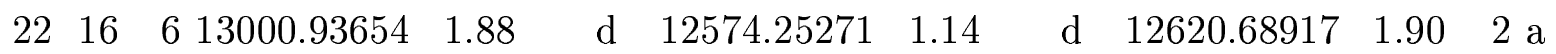

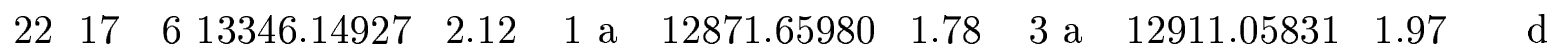

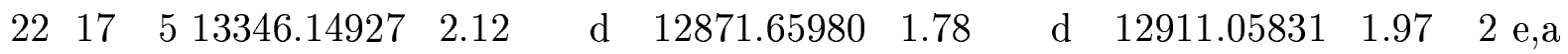

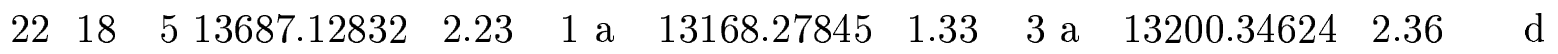

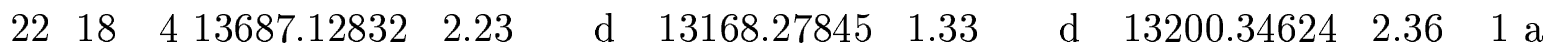

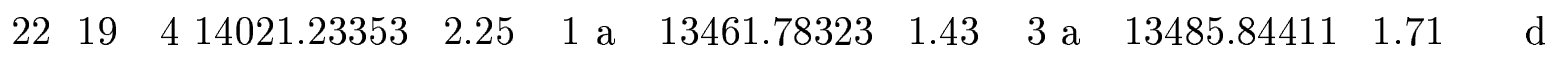

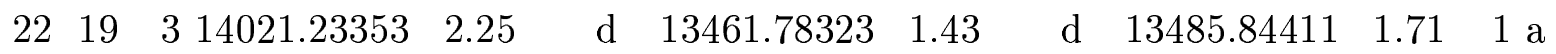

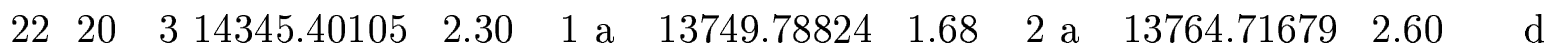

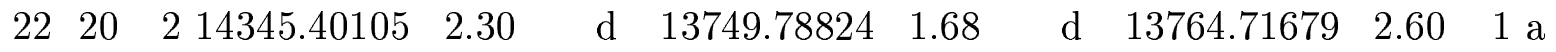

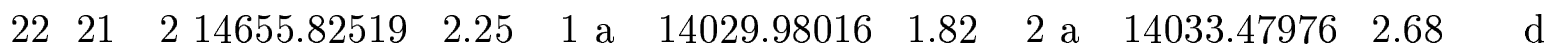

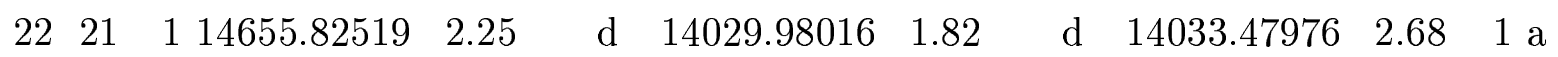

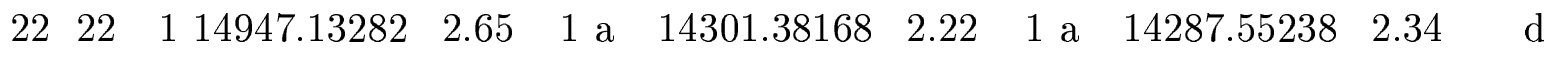

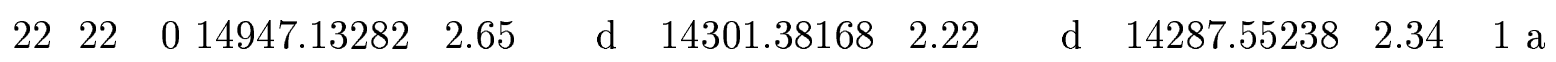
$\begin{array}{llllllllllll}23 & 0 & 23 & 8350.96548 & 0.72 & 8 \mathrm{e}, \mathrm{a} & 8827.02152 & 1.03 & 4 \text { e,a } & 8944.57941 & 1.43 & \mathrm{~d}\end{array}$ $\begin{array}{llllllllllll}23 & 1 & 23 & 8350.97116 & 1.30 & 1 \mathrm{a} & 8827.02152 & 1.03 & \mathrm{~d} & 8944.57941 & 1.43 & 5 \mathrm{e}, \mathrm{a}\end{array}$

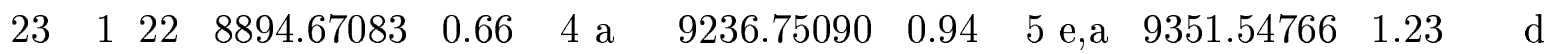

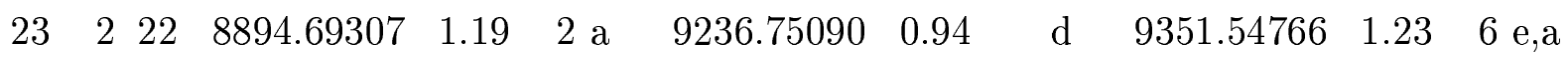
$\begin{array}{llllllllllll}23 & 2 & 21 & 9323.13976 & 0.54 & 5 \mathrm{a} & 9603.99885 & 1.04 & 2 \mathrm{a} & 9716.81820 & 2.89 & \mathrm{~d}\end{array}$ $\begin{array}{llllllllllll}23 & 3 & 21 & 9323.08209 & 0.96 & 2 \mathrm{a} & 9604.01756 & 1.61 & 1 \mathrm{a} & 9716.81820 & 2.89 & 1 \mathrm{e}\end{array}$

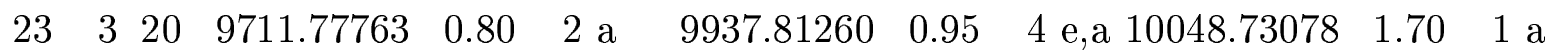


$\begin{array}{llllllllllll}23 & 4 & 20 & 9714.80344 & 1.60 & 1 \mathrm{a} & 9937.85957 & 1.67 & 1 \mathrm{a} & 10048.74048 & 0.94 & 2 \mathrm{a}\end{array}$

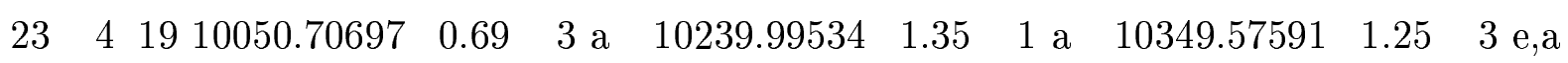

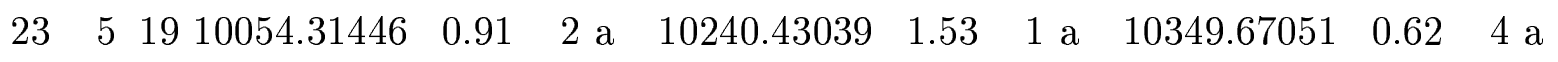

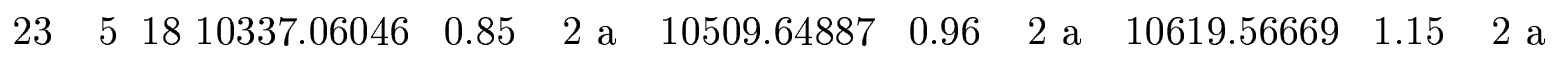

$\begin{array}{lllllllll}23 & 6 & 18 & 10513.07532 & 2.04 & 1 \mathrm{a} & 10620.57806 & 0.72 & 4 \mathrm{a}\end{array}$

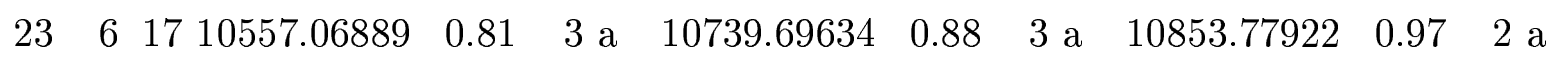

$\begin{array}{lllllll}23 & 7 & 17 & 10862.21872 & 0.84 & 5 \mathrm{a}\end{array}$

$\begin{array}{lllllllll}23 & 7 & 16 & 10733.46529 & 1.12 & 1 \mathrm{a} & 11041.31810 & 1.39 & 1 \mathrm{a}\end{array}$

$\begin{array}{lllllll}23 & 8 & 16 & 11081.34255 & 0.78 & 4 \mathrm{a}\end{array}$

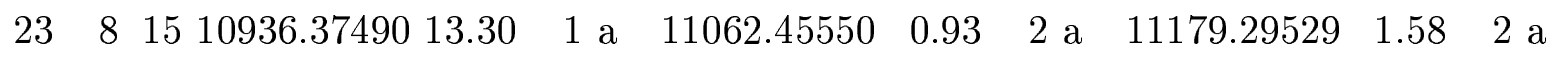

$\begin{array}{lllllll}23 & 9 & 15 & 11295.32745 & 0.73 & 6 \mathrm{e}, \mathrm{a}\end{array}$

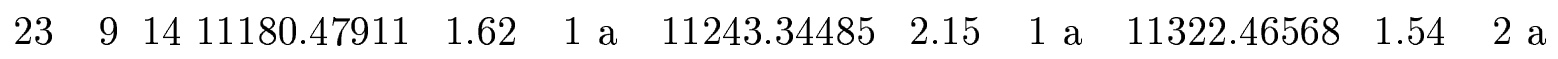

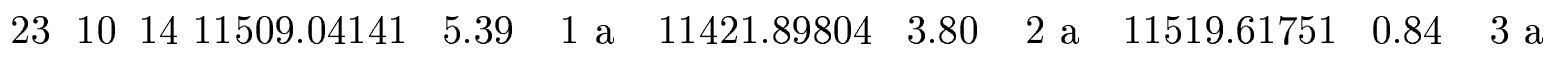

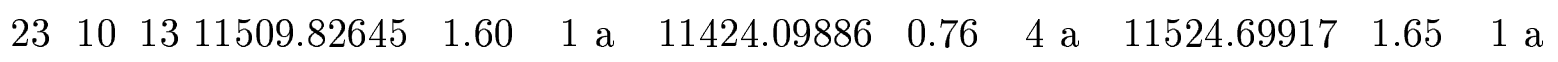

$\begin{array}{lllllllll}23 & 11 & 11674.25604 & 1.80 & 1 \mathrm{a} & 11760.64494 & 0.96 & 2 \mathrm{a}\end{array}$

$\begin{array}{llllllllllll}23 & 11 & 12 & 11820.38089 & 5.26 & 1 \mathrm{a} & 11674.65153 & 0.99 & 2 \text { a } & 11761.40044 & 1.64 & 1 \mathrm{a}\end{array}$

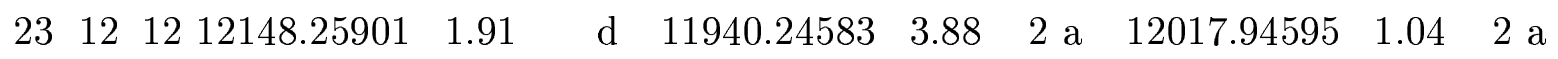

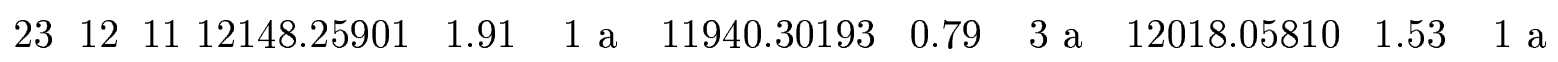

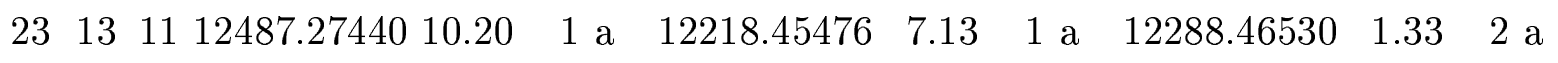

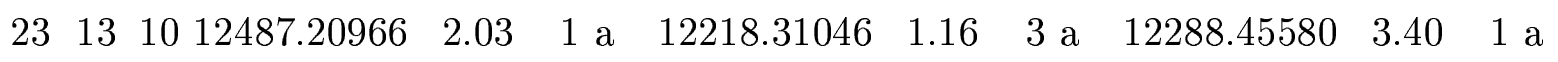

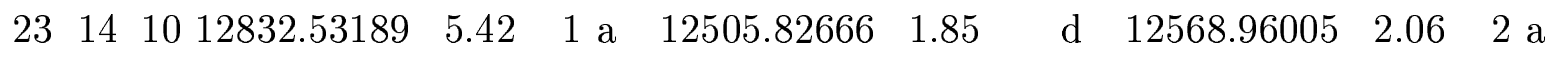

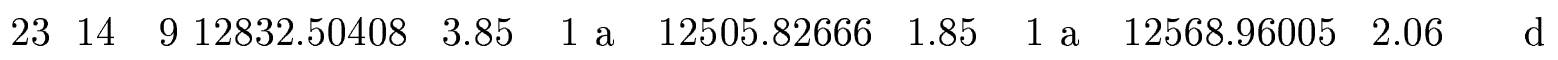

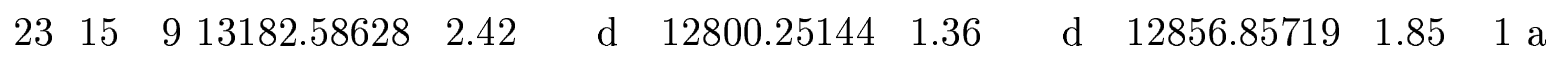

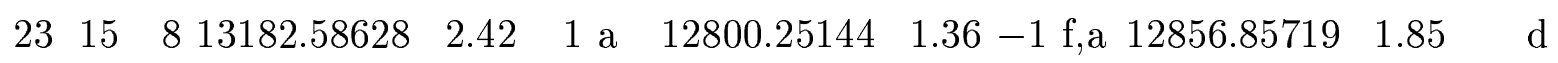

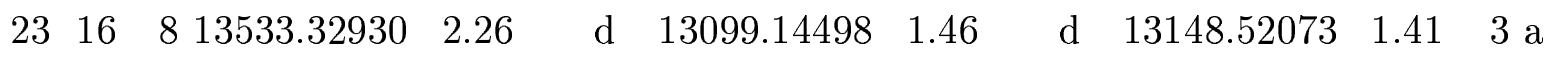

$\begin{array}{llllllllll}23 & 16 & 713533.32930 & 2.26 & 1 \mathrm{a} & 13099.14498 & 1.46-1 \mathrm{f}, \mathrm{a} & 13148.52073 & 1.41 & \mathrm{~d}\end{array}$

$\begin{array}{lllllllllll}23 & 17 & 713883.40943 & 2.13 & \mathrm{~d} & 13400.23273 & 1.40 & \mathrm{~d} & 13443.12748 & 2.27 & 1 \mathrm{a}\end{array}$ 


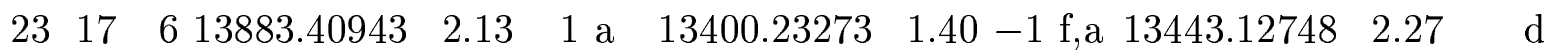

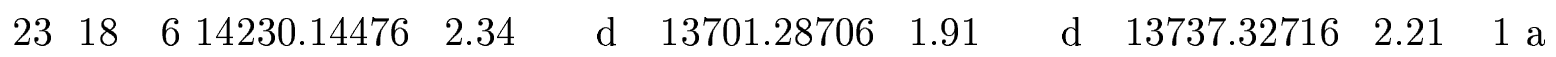
$\begin{array}{llllllllllll}23 & 18 & 5 & 14230.14476 & 2.34 & 1 \text { a } & 13701.28706 & 1.91 & 2 \text { a } & 13737.32716 & 2.21 & \mathrm{~d}\end{array}$

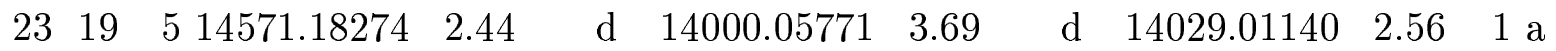

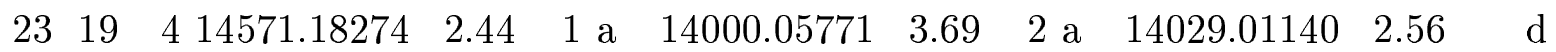

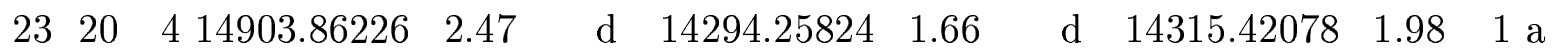

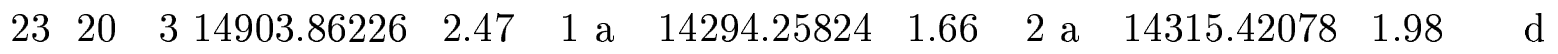

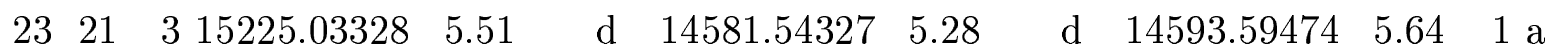

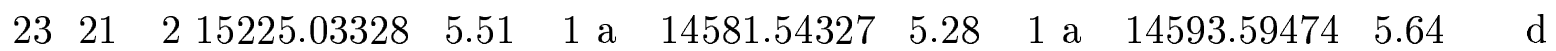

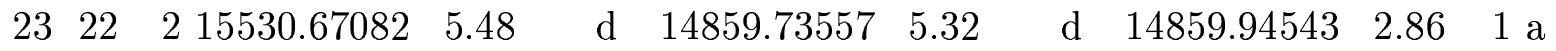

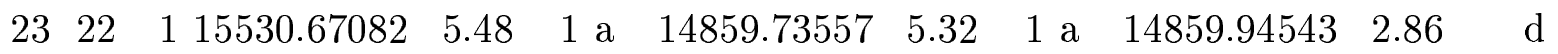

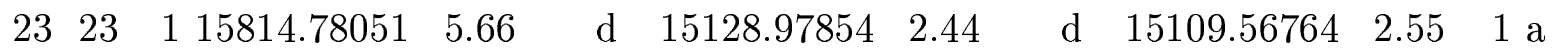

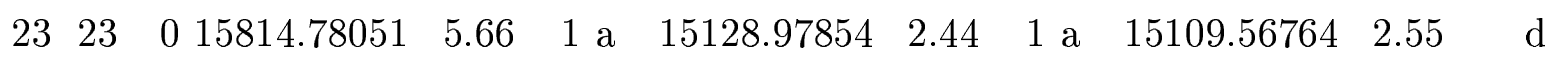
$\begin{array}{llllllllllll}24 & 0 & 24 & 8787.45680 & 2.07 & \mathrm{~d} & 9260.32144 & 1.03 & \mathrm{~d} & 9379.49004 & 0.71 & 8 \mathrm{e}, \mathrm{a}\end{array}$

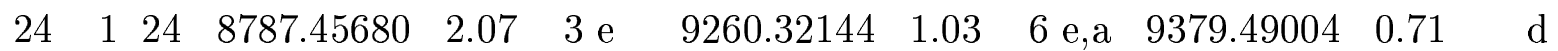
$\begin{array}{llllllllllll}24 & 1 & 23 & 9356.78306 & 1.56 & 1 \mathrm{a} & 9687.17416 & 1.00 & \mathrm{~d} & 9803.42830 & 1.84 & 4 \mathrm{e}\end{array}$

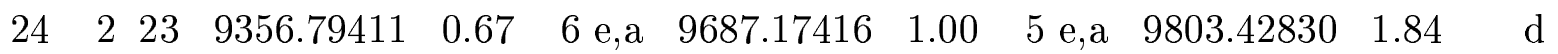
$\begin{array}{llllllllllll}24 & 2 & 22 & 9794.77398 & 2.08 & 1 \mathrm{a} & 10069.10723 & 0.98 & \mathrm{~d} & 10183.35126 & 1.02 & 2 \mathrm{a}\end{array}$

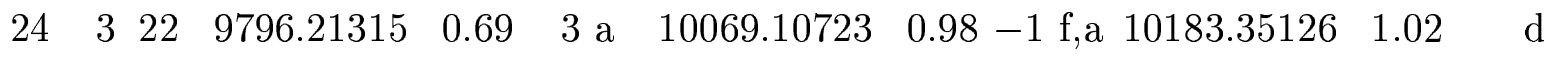

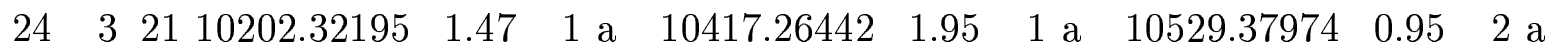

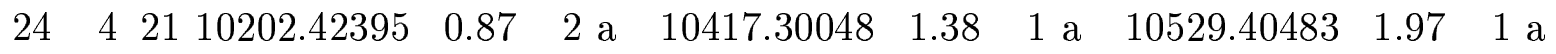
$\begin{array}{lllllllll}24 & 4 & 20 & 10733.57651 & 1.83 & 1 \mathrm{a} & 10844.09194 & 0.72 & 4 \mathrm{e}, \mathrm{a}\end{array}$

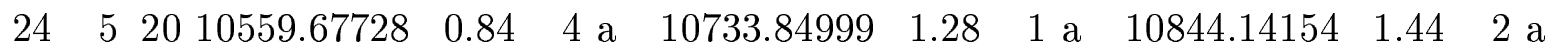
$\begin{array}{llllllll}24 & 5 & 19 & 1128.33047 & 0.78 & 3 \mathrm{a}\end{array}$

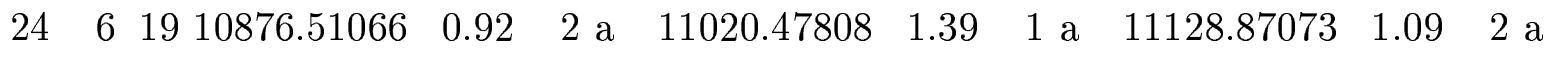
$24 \quad 6 \quad 18$ $11379.07040 \quad 1.00 \quad 2$ a

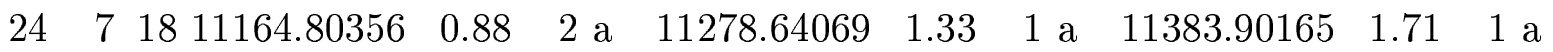




\begin{tabular}{|c|c|c|c|c|c|c|c|c|c|c|}
\hline 24 & 7 & & & & & & & 11585.61731 & 0.91 & $4 \mathrm{a}$ \\
\hline 24 & 8 & 17 & & & & & & 11613.34565 & 1.52 & $1 \mathrm{a}$ \\
\hline 24 & 8 & & & & & & & 11728.51795 & 1.36 & $1 \mathrm{a}$ \\
\hline 24 & 9 & 16 & & & 11697.82740 & 1.61 & $2 \mathrm{a}$ & & & \\
\hline 24 & 9 & 15 & & & & & & 11873.52732 & 0.95 & $4 \mathrm{a}$ \\
\hline 24 & 10 & 1512045.43900 & 1.43 & $3 \mathrm{a}$ & & & & & & \\
\hline 24 & 10 & 14 & & & & & & 12066.66992 & 0.85 & $3 \mathrm{a}$ \\
\hline 24 & 11 & 1412357.08612 & 1.89 & $1 \mathrm{a}$ & 12209.61398 & 1.29 & $1 \mathrm{a}$ & 12297.54361 & 1.93 & $1 \mathrm{a}, ?$ \\
\hline 24 & 11 & 13 & & & 12210.26065 & 5.46 & $1 \mathrm{a}$ & 12299.25779 & 0.93 & $2 \mathrm{a}$ \\
\hline 24 & 12 & 1312686.01496 & 5.35 & $1 \mathrm{a}$ & 12475.20317 & 1.40 & $1 \mathrm{a}$ & 12555.01130 & 1.92 & $1 \mathrm{a}$ \\
\hline 24 & 12 & 12 & & & 12475.30482 & 5.31 & $1 \mathrm{a}$ & 12555.20111 & 1.04 & $3 \mathrm{a}$ \\
\hline 24 & 13 & 1213026.68360 & 2.15 & $1 \mathrm{a}$ & 12753.93790 & 1.78 & $1 \mathrm{a}$ & 12826.26847 & 5.23 & $1 \mathrm{a}$ \\
\hline 24 & 13 & 11 & & & 12753.93790 & 1.78 & $\mathrm{~d}$ & 12826.33117 & 1.10 & $2 \mathrm{a}$ \\
\hline 4 & 14 & 1113374.26161 & 5.40 & $1 \mathrm{a}$ & 13042.87683 & 1.53 & $1 \mathrm{a}$ & 13108.34499 & 6.05 & $1 \mathrm{a}$ \\
\hline 24 & 14 & 1013374.28426 & 8.89 & $1 \mathrm{a}$ & 13043.03389 & 8.71 & $1 \mathrm{a}$ & 13108.37238 & 1.21 & $2 \mathrm{a}$ \\
\hline 24 & 15 & 1013727.31049 & 3.98 & $1 \mathrm{a}$ & 13339.37638 & 1.82 & $-1 \mathrm{f}, \mathrm{a}$ & 13398.56313 & 5.41 & d \\
\hline 24 & 15 & 913727.31049 & 3.98 & d & 13339.37638 & 1.82 & $\mathrm{~d}$ & 13398.56313 & 5.41 & $1 \mathrm{a}$ \\
\hline 24 & 16 & 9 & & & 13640.98587 & 1.60 & $2 \mathrm{a}$ & 13693.27593 & 5.33 & $\mathrm{~d}$ \\
\hline 24 & 16 & 8 & & & 13640.98587 & 1.60 & $\mathrm{~d}$ & 13693.27593 & 5.33 & $1 \mathrm{a}$ \\
\hline 24 & 17 & 814436.15635 & 2.47 & $1 \mathrm{a}$ & 13945.42353 & 1.60 & $3 \mathrm{a}$ & & & \\
\hline 24 & 1 & 714436.15635 & 2.47 & $\mathrm{~d}$ & 13945.42353 & 1.60 & $\mathrm{~d}$ & & & \\
\hline 24 & 18 & 714788.13071 & 2.35 & $1 \mathrm{a}$ & 14250.52172 & 1.56 & $3 \mathrm{a}$ & 14290.32805 & 2.48 & $\mathrm{~d}$ \\
\hline 24 & 18 & 614788.13071 & 2.35 & d & 14250.52172 & 1.56 & $\mathrm{~d}$ & 14290.32805 & 2.48 & $1 \mathrm{a}$ \\
\hline 24 & 19 & 615135.42276 & 2.55 & $1 \mathrm{a}$ & 14554.12180 & 1.99 & $2 \mathrm{a}$ & 14587.43269 & 2.43 & d \\
\hline 24 & 19 & 515135.42276 & 2.55 & d & 14554.12180 & 1.99 & $\mathrm{~d}$ & 14587.43269 & 2.43 & $1 \mathrm{a}$ \\
\hline 24 & 20 & 515475.64109 & 2.64 & $1 \mathrm{a}$ & 14854.03725 & 2.67 & $3 \mathrm{a}$ & 14880.59843 & 2.75 & d \\
\hline 24 & 20 & 415475.64109 & 2.64 & $\mathrm{~d}$ & 14854.03725 & 2.67 & $\mathrm{~d}$ & 14880.59843 & 2.75 & $1 \mathrm{a}$ \\
\hline
\end{tabular}




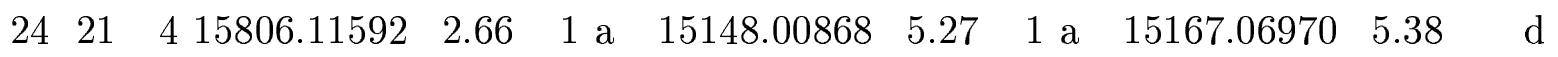

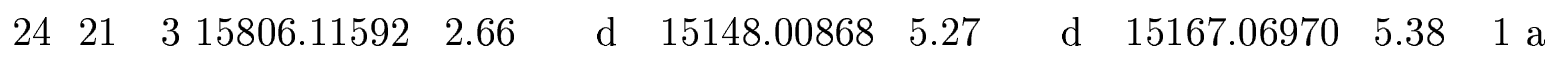

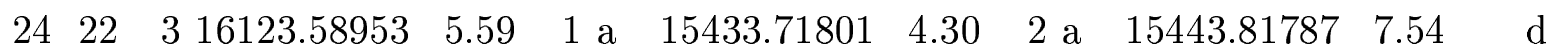

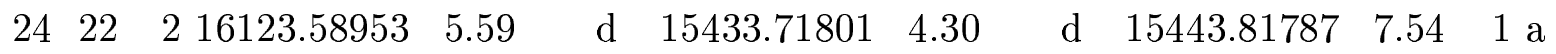

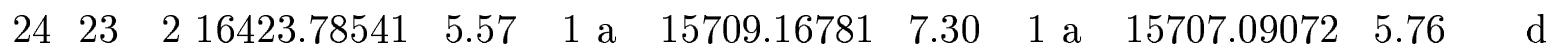

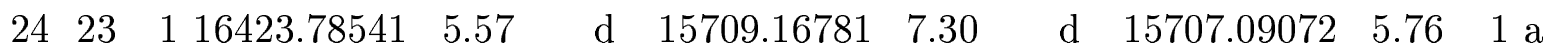

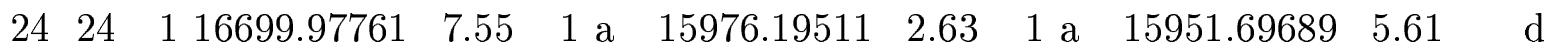

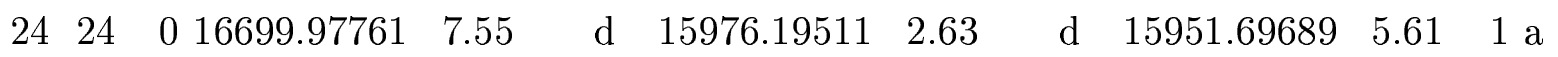

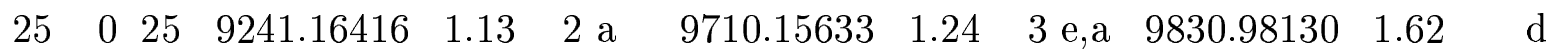
$\begin{array}{llllllllllll}25 & 1 & 25 & 9241.16416 & 1.13 & \mathrm{~d} & 9710.15633 & 1.24 & \mathrm{~d} & 9830.98130 & 1.62 & 5 \mathrm{e}, \mathrm{a}\end{array}$

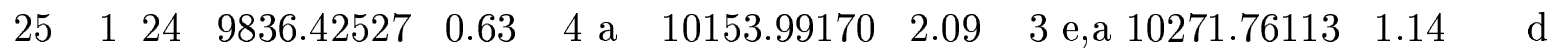
$\begin{array}{llllllllllll}25 & 2 & 24 & 9836.45021 & 1.20 & 1 \mathrm{a} & 10153.99170 & 2.09 & \mathrm{~d} & 10271.76113 & 1.14 & 5 \mathrm{e}, \mathrm{a}\end{array}$

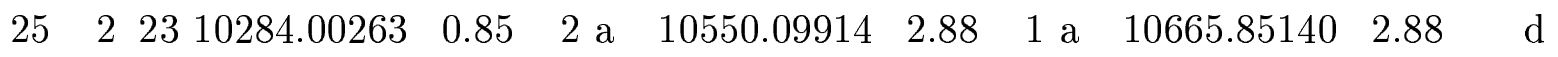

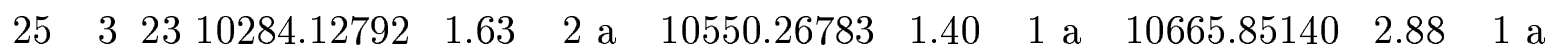

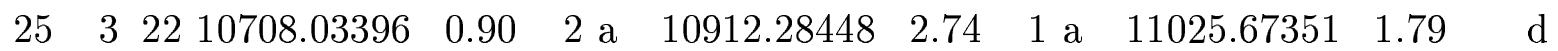

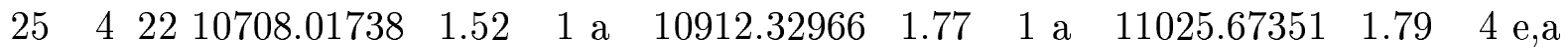

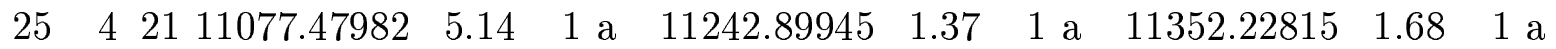
$\begin{array}{lllllllll}25 & 5 & 21 & 11243.10901 & 2.09 & 1 \mathrm{a} & 11352.23815 & 0.76 & 3 \mathrm{a}\end{array}$ $25 \quad 5 \quad 2011399.06611 \quad 1.36 \quad 1 \mathrm{a}$ $11651.73264 \quad 1.46 \quad 1 \mathrm{a}$ $25 \quad 6 \quad 20$ $11652.02962 \quad 1.45 \quad 1 \mathrm{a}$ $25 \quad 6 \quad 19$ $11805.14000 \quad 1.67 \quad 1 \mathrm{a} \quad 11917.59561 \quad 1.98 \quad 1 \mathrm{a}$ $25 \quad 719$ $11920.38222 \quad 5.08 \quad 1 \mathrm{a}$

$\begin{array}{llllll}25 & 7 & 18 & 11866.92451 & 5.08 & 1 \mathrm{a}\end{array}$ $12142.93632 \quad 1.48 \quad 1 \mathrm{a}$ $25 \quad 8 \quad 18$ $12161.14816 \quad 1.44 \quad 1 \mathrm{a}$

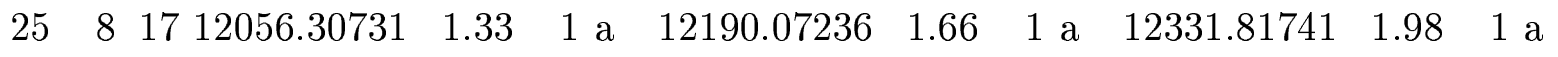
$25 \quad 917$ $12386.17800 \quad 3.56 \quad 1 \mathrm{e}$ $25 \quad 916$ $12445.09224 \quad 1.81 \quad 1 \mathrm{a}$ 
251016

251015

251115

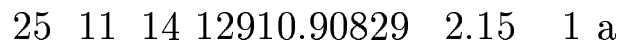

$25 \quad 12 \quad 14$

$\begin{array}{llllll}25 & 12 & 13 & 13240.06962 & 2.14 & 1 \mathrm{a}\end{array}$

$25 \quad 13 \quad 13$

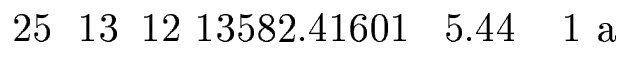

251412

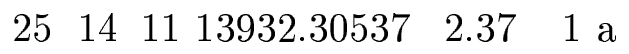

$\begin{array}{llllll}25 & 15 & 11 & 14287.93052 & 7.36 & \mathrm{~d}\end{array}$

$\begin{array}{llllll}25 & 15 & 10 & 14287.93052 & 7.36 & 1 \mathrm{a}\end{array}$

$\begin{array}{llllll}25 & 16 & 10 & 14645.96493 & 6.39 & 1 \mathrm{a}\end{array}$

$\begin{array}{llllll}25 & 16 & 9 & 14645.94849 & 4.10 & 1 \mathrm{a}\end{array}$

$\begin{array}{lll}25 & 17 & 9\end{array}$

$\begin{array}{lll}25 & 17 & 8\end{array}$

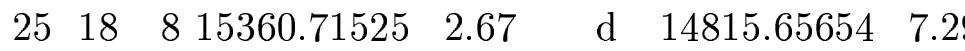

$\begin{array}{lllllllll}25 & 18 & 7 & 15360.71525 & 2.67 & 1 \mathrm{a} & 14815.65654 & 7.29 & 1 \mathrm{a}\end{array}$

$\begin{array}{lllllllllll}25 & 19 & 715713.64376 & 5.53 & \mathrm{~d} & 15123.66418 & 1.75 & \mathrm{~d} & 15161.01692 & 5.58 & 1 \mathrm{a}\end{array}$

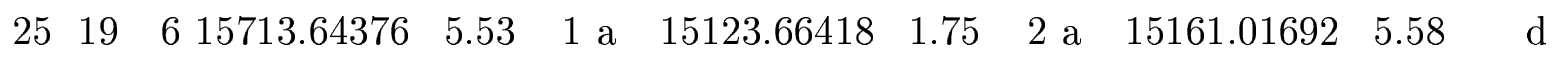

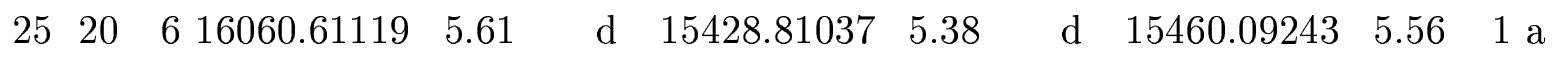

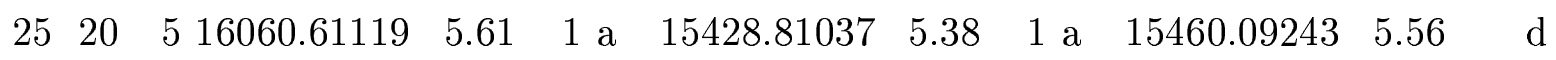

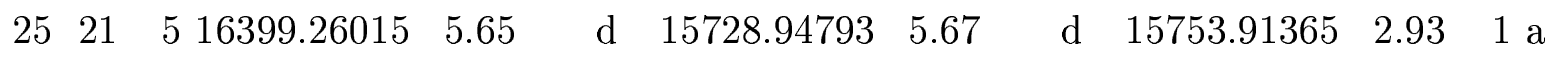

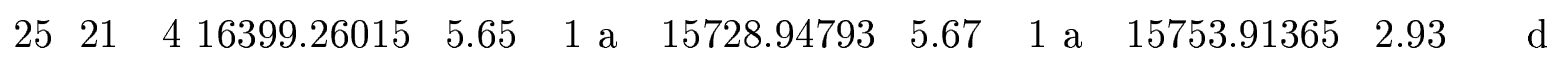

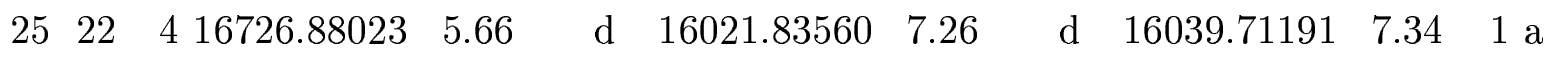

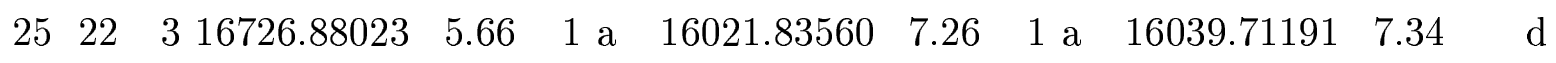

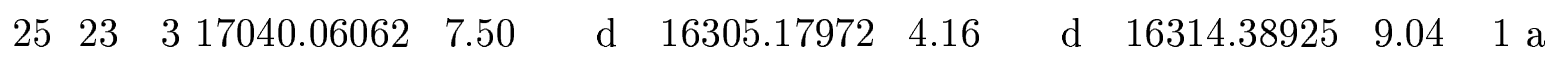




\begin{tabular}{|c|c|c|c|c|c|c|c|c|c|c|}
\hline 5 & 23 & 217040.06062 & 7.50 & $1 \mathrm{a}$ & 16305.17972 & 4.16 & $2 \mathrm{a}$ & 16314.38925 & 9.04 & $\mathrm{~d}$ \\
\hline & 24 & 217334.23694 & 7.49 & $\mathrm{~d}$ & 16577.19152 & 8.85 & $\mathrm{~d}$ & 16574.05503 & 5.85 & $1 \mathrm{a}$ \\
\hline & 24 & 117334.23694 & 7.49 & $1 \mathrm{a}$ & 16577.19152 & 8.85 & $1 \mathrm{a}$ & 16574.05503 & 5.85 & d \\
\hline & 25 & 117601.87994 & 9.06 & $\mathrm{~d}$ & 16842.20405 & 5.65 & $\mathrm{~d}$ & 16813.24862 & 7.52 & $1 \mathrm{a}$ \\
\hline 25 & 25 & 017601.87994 & 9.06 & $1 \mathrm{a}$ & 16842.20405 & 5.65 & $1 \mathrm{a}$ & 16813.24862 & 7.52 & $\mathrm{~d}$ \\
\hline & 0 & $26 \quad 9712.25069$ & 0.90 & $\mathrm{~d}$ & 10176.39630 & 1.24 & $d$ & 10298.93749 & 1.01 & $7 \mathrm{e}, \mathrm{a}$ \\
\hline 6 & 1 & $26 \quad 9712.25069$ & 0.90 & $3 \mathrm{a}$ & 10176.39630 & 1.24 & $5 \mathrm{e}, \mathrm{a}$ & 10298.93749 & 1.01 & d \\
\hline 6 & 1 & 2510333.95393 & 1.68 & $1 \mathrm{a}$ & 10637.08963 & 1.38 & $d$ & 10756.40368 & 1.02 & $7 \mathrm{e}, \mathrm{a}$ \\
\hline 26 & 2 & 2510334.01339 & 1.68 & $1 \mathrm{a}$ & 10637.08963 & 1.38 & $4 \mathrm{e}, \mathrm{a}$ & 10756.40368 & 1.02 & d \\
\hline 26 & 2 & 2410786.19698 & 1.51 & $2 \mathrm{a}$ & 11046.80894 & 1.67 & $\mathrm{~d}$ & 11164.14931 & 1.67 & $2 \mathrm{a}$ \\
\hline 26 & 3 & 2410786.26991 & 1.19 & $1 \mathrm{a}$ & 11046.80894 & 1.67 & $2 \mathrm{a}$ & 11164.14931 & 1.67 & d \\
\hline 26 & 3 & 23 & & & & & & 11537.44195 & 1.25 & $3 \mathrm{e}, \mathrm{a}$ \\
\hline 26 & 4 & 2311228.59288 & 0.95 & $2 \mathrm{a}$ & 11422.78306 & 1.79 & $1 \mathrm{a}$ & 11537.57183 & 2.03 & $1 \mathrm{a}$ \\
\hline 26 & 4 & 22 & & & & & & 11878.78134 & 1.70 & $1 \mathrm{a}$ \\
\hline 26 & 5 & 2211612.35405 & 5.24 & $1 \mathrm{a}$ & 11766.30424 & 1.20 & $2 \mathrm{a}$ & & & \\
\hline 26 & 5 & 21 & & & & & & 12189.63260 & 2.37 & $1 \mathrm{a}$ \\
\hline 6 & 6 & 2111939.58767 & 1.69 & $1 \mathrm{a}$ & & & & & & \\
\hline 26 & 6 & 20 & & & & & & 12469.71380 & 1.68 & $1 \mathrm{a}$ \\
\hline 26 & 7 & 20 & & & 12365.15257 & 1.94 & $1 \mathrm{a}$ & 12471.31789 & 2.22 & $1 \mathrm{a}$ \\
\hline 26 & 7 & 19 & & & & & & 12712.54493 & 1.43 & $1 \mathrm{a}$ \\
\hline 6 & 8 & 19 & & & & & & 12723.98924 & 1.44 & $1 \mathrm{a}$ \\
\hline 26 & 8 & 18 & & & & & & 12911.85883 & 1.64 & $1 \mathrm{a}$ \\
\hline 9 & 9 & 18 & & & & & & 12956.18123 & 2.22 & $1 \mathrm{a}$ \\
\hline 26 & 9 & 17 & & & & & & 13034.76236 & 1.66 & $2 \mathrm{a}$ \\
\hline 26 & 10 & 16 & & & & & & 13211.73255 & 6.14 & $1 \mathrm{a}$ \\
\hline 26 & 11 & 15 & & & & & & 13431.05576 & 3.34 & $2 \mathrm{a}$ \\
\hline 26 & 12 & 1513811.84047 & 2.37 & $1 \mathrm{a}$ & & & & & & \\
\hline
\end{tabular}




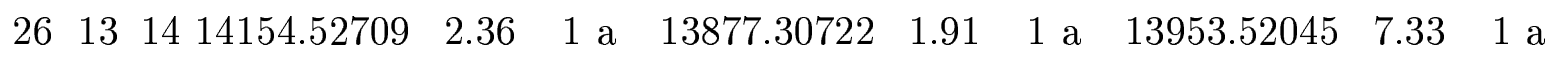
261313 $13877.56213 \quad 7.47 \quad 1 \mathrm{a} \quad 13953.86676 \quad 3.68 \quad 2 \mathrm{a}$

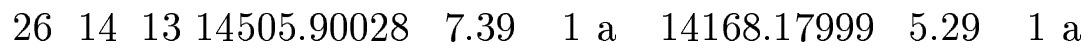
$26 \quad 14 \quad 1214506.0679910 .23 \quad 1 \mathrm{a}$ $14238.06554 \quad 1.75 \quad 1 \mathrm{a}$ $\begin{array}{llllll}26 & 15 & 12 & 14864.01232 & 5.53 \quad 1 \mathrm{a}\end{array}$ $\begin{array}{llllll}14467.99521 & 5.40 & \mathrm{~d} & 14531.60041 & 8.82 & 1 \mathrm{a}\end{array}$ 261511 $\begin{array}{llllll}14467.99521 & 5.40 & 1 \mathrm{a} & 14531.67958 & 7.25 & 1 \mathrm{a}\end{array}$

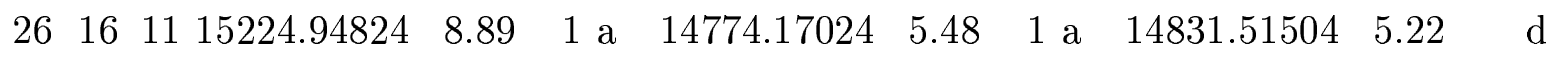
261610 $\begin{array}{llllll}14774.17024 & 5.48 & \mathrm{~d} & 14831.51504 & 5.22 & 1 \mathrm{a}\end{array}$

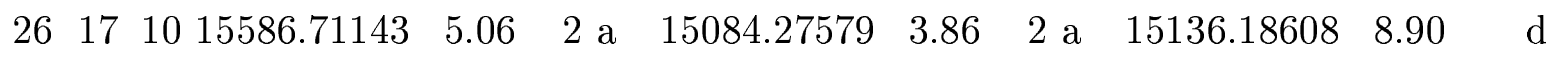

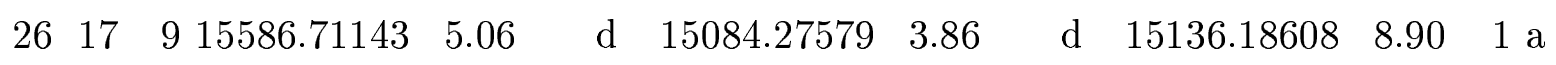
$2618 \quad 9$

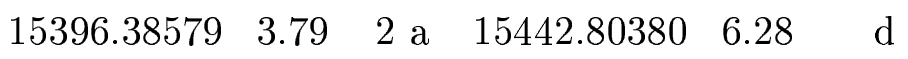
$2618 \quad 8$ $15396.38579 \quad 3.79$ d $\quad \begin{array}{llll}15442.80380 & 6.28 \quad 1 \mathrm{a}\end{array}$

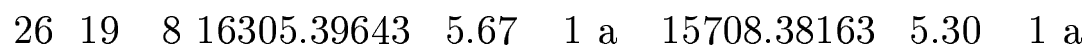
$\begin{array}{llllllllll}26 & 19 & 7 & 16305.39643 & 5.67 & \mathrm{~d} & 15708.38163 & 5.30 & \mathrm{~d}\end{array}$

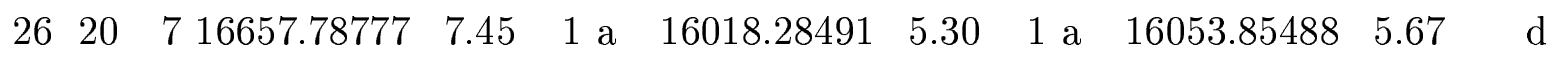

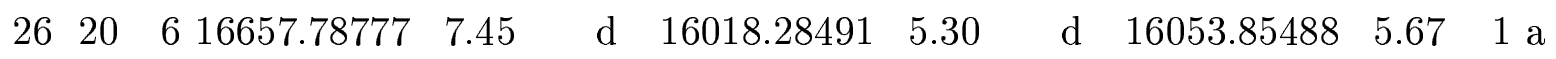

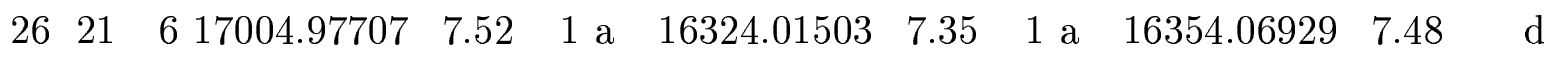
$\begin{array}{llllllllllll}26 & 21 & 5 & 17004.97707 & 7.52 & \mathrm{~d} & 16324.01503 & 7.35 & \mathrm{~d} & 16354.06929 & 7.48 & 1 \mathrm{a}\end{array}$

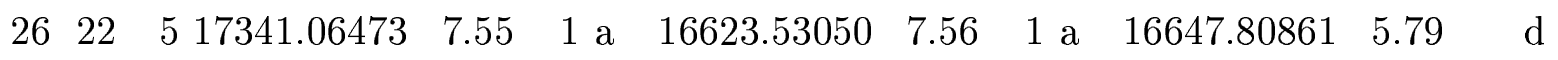

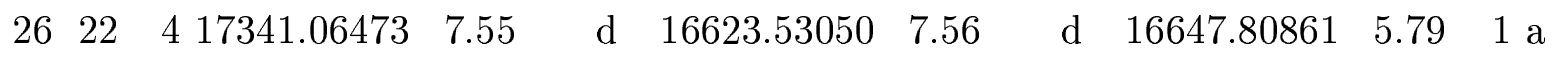

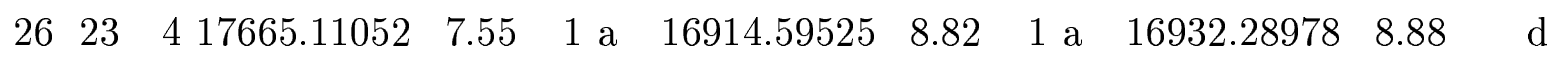

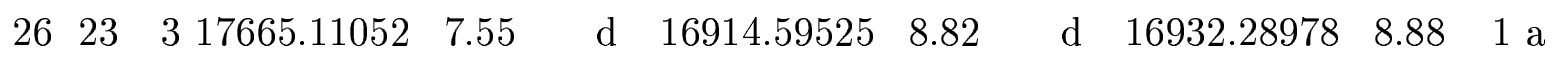

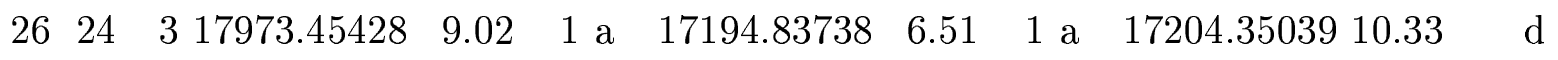

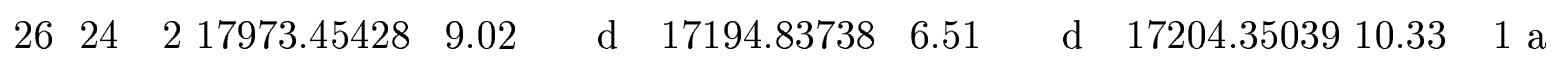

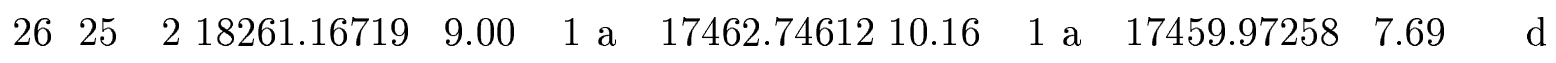

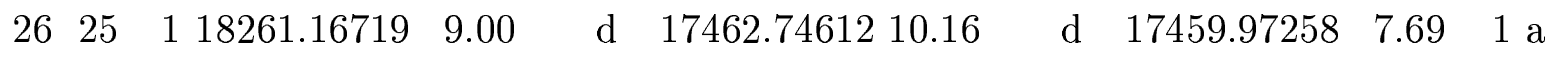


$26 \quad 26 \quad 118519.68215 \quad 10.35 \quad 1 \mathrm{a}$

$\begin{array}{lllll}26 & 26 & 0 & 18519.68215 & 10.35 \quad \mathrm{~d}\end{array}$

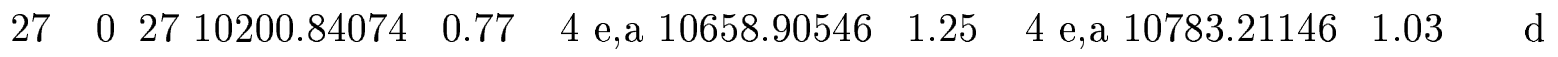

$\begin{array}{llllllllllll}27 & 1 & 27 & 10200.84074 & 0.77 & \mathrm{~d} & 10658.90546 & 1.25 & \mathrm{~d} & 10783.21146 & 1.03 & 7 \mathrm{e}, \mathrm{a}\end{array}$

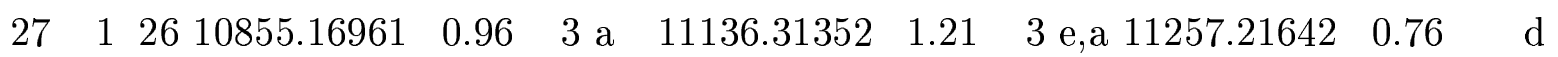

$\begin{array}{lllllllll}27 & 2 & 26 & 11136.31352 & 1.21 & \mathrm{~d} & 11257.21642 & 0.76 & 7 \mathrm{e}, \mathrm{a}\end{array}$

$\begin{array}{llllllllllll}27 & 2 & 25 & 11302.07872 & 2.92 & 1 \mathrm{a} & 11559.01895 & 1.95 & 1 \mathrm{a} & 11677.99964 & 1.41 & \mathrm{~d}\end{array}$

$\begin{array}{llllllllll}27 & 3 & 25 & 11302.07872 & 2.92 & \mathrm{~d} & 11677.99964 & 1.41 & 2 \mathrm{a}\end{array}$

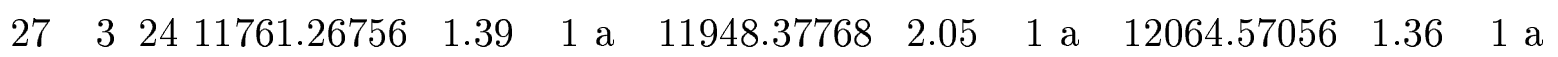

$27 \quad 4 \quad 24$

$12064.48352 \quad 1.11 \quad 4 \mathrm{e}, \mathrm{a}$

$\begin{array}{lll}27 & 4 & 23\end{array}$

$\begin{array}{llllll}12304.68337 & 1.56 & 1 \mathrm{a} & 12418.22570 & 5.37 & 1 \mathrm{a}\end{array}$

$\begin{array}{lll}27 & 5 & 23\end{array}$

$12418.41692 \quad 1.97 \quad 1 \mathrm{a}$

$\begin{array}{lll}27 & 6 & 22\end{array}$

$12742.20642 \quad 1.02 \quad 3 \mathrm{a}$

$\begin{array}{lll}27 & 6 & 21\end{array}$

$13035.50639 \quad 2.43 \quad 1 \mathrm{a}$

$\begin{array}{lll}27 & 7 & 21\end{array}$

$13036.33163 \quad 1.95 \quad 1 \mathrm{a}$

$\begin{array}{lll}27 & 10 & 18\end{array}$

$13774.94268 \quad 5.20 \quad 1 \mathrm{a}$

$27 \quad 1117$

$14015.72414 \quad 5.24 \quad 1 \mathrm{a}$

$27 \quad 12 \quad 16$

$14271.35618 \quad 6.66 \quad 1 \mathrm{a}$

$27 \quad 13 \quad 15$

$14542.79758 \quad 5.30 \quad 1 \mathrm{a}$

$\begin{array}{llllll}27 & 13 & 14 & 14742.43503 & 5.53 & 1 \mathrm{a}\end{array}$

271414

$14827.60784 \quad 4.03 \quad 2$ a

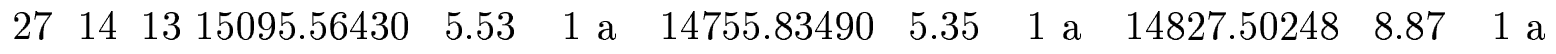

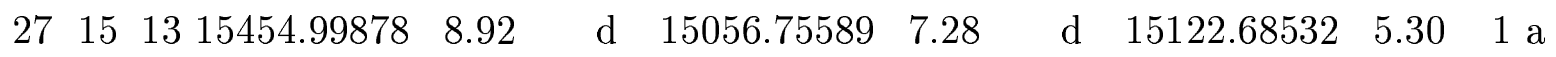

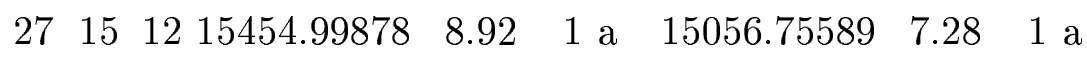

$\begin{array}{lllllllll}27 & 16 & 12 & 15364.73162 & 7.36 & 1 \mathrm{a} & 15424.31355 & 7.32 & 1 \mathrm{a}\end{array}$

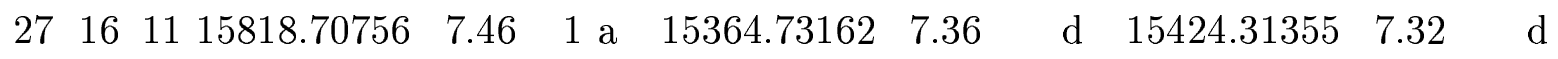

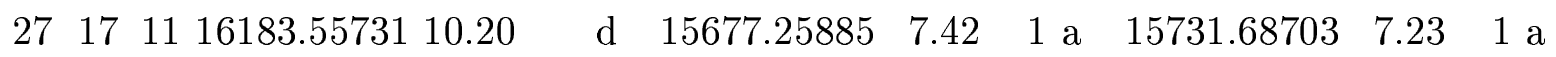




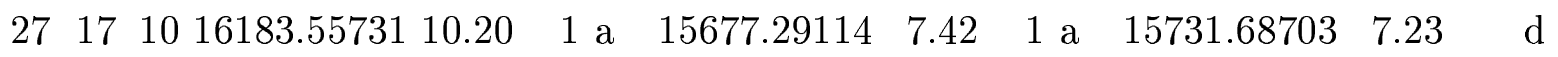

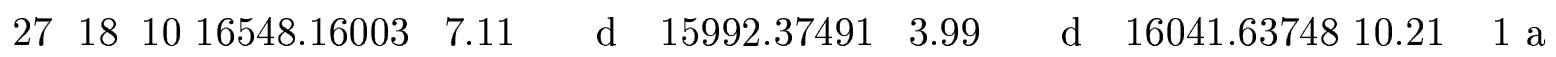

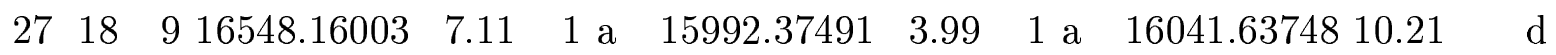
$\begin{array}{llllll}16307.98621 & 3.16 & \mathrm{~d} & 16352.25018 & 8.03 & 1 \mathrm{a}\end{array}$ $27 \quad 19 \quad 8$ $\begin{array}{llllll}16307.98621 & 3.16 & 2 \text { a } & 16352.25018 & 8.03 & \mathrm{~d}\end{array}$ $27 \quad 20 \quad 8$ $16622.20707 \quad 4.31 \quad \mathrm{~d}$

$\begin{array}{lllllllll}27 & 20 & 7 & 17270.57870 & 7.56 & 1 \mathrm{a} & 16622.20707 & 4.31 & 2 \mathrm{a}\end{array}$

$\begin{array}{lll}27 & 21 & 7\end{array}$ $\begin{array}{llllll}16933.08309 & 7.28 & \mathrm{~d} & 16967.56762 & 7.56 & 1 \mathrm{a}\end{array}$ $2721 \quad 6$

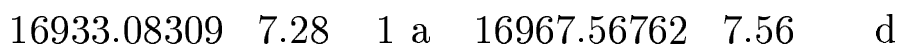

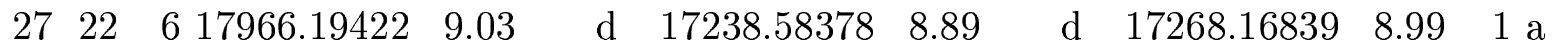

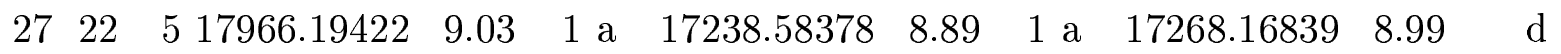

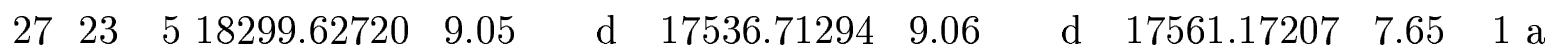

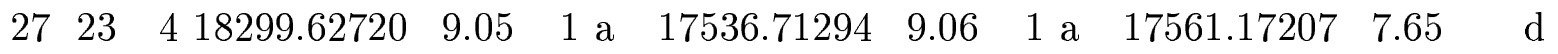

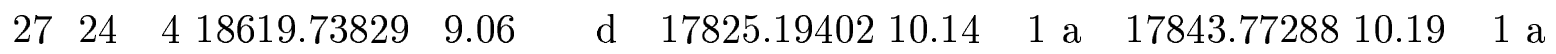

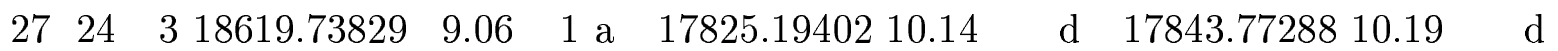

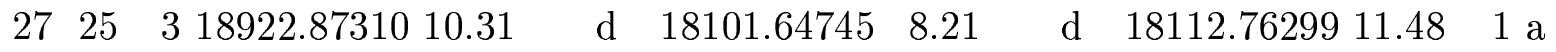

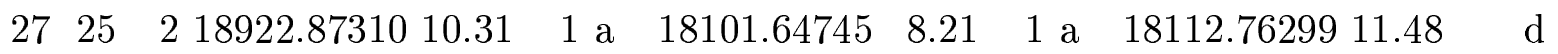

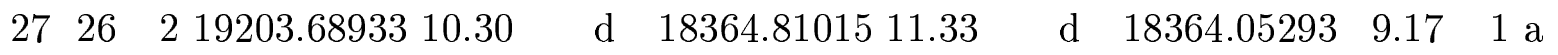

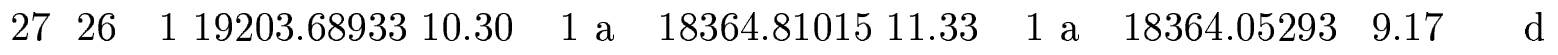
$\begin{array}{lllll}27 & 27 & 1 & 19452.61389 & 11.49 \quad \mathrm{~d}\end{array}$

$\begin{array}{llllll}27 & 27 & 0 & 19452.61389 & 11.49 \quad 1 \mathrm{a}\end{array}$

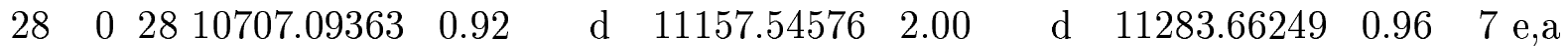

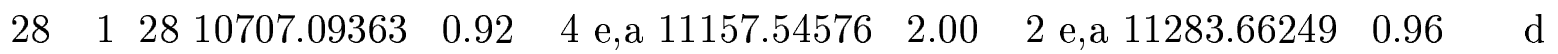

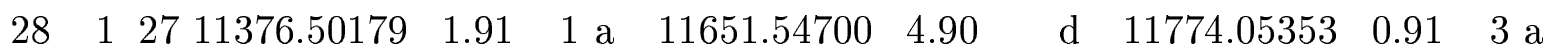
$\begin{array}{llllllllllll}28 & 2 & 27 & 11376.61216 & 1.28 & 1 \mathrm{a} & 11651.54700 & 4.90-1 \mathrm{f} & 11774.05353 & 0.91 & \mathrm{~d}\end{array}$ $28 \quad 2 \quad 26$ $\begin{array}{llllll}12086.59837 & 2.19 & \text { d } & 12207.18357 & 1.44 & 2 \mathrm{a}\end{array}$ $28 \quad 3 \quad 26$ $\begin{array}{llllll}12086.59837 & 2.19 & 1 \mathrm{a} & 12207.18357 & 1.44 & \mathrm{~d}\end{array}$ 


\begin{tabular}{|c|c|c|c|c|c|c|c|c|c|c|}
\hline 28 & 3 & 25 & & & & & & 12606.59721 & 0.89 & $5 \mathrm{e}, \mathrm{a}$ \\
\hline 28 & 4 & 2512314.18770 & 1.71 & $1 \mathrm{a}$ & 12488.99955 & 2.28 & $1 \mathrm{a}$ & 12606.55287 & 1.62 & 1 a \\
\hline 28 & 4 & 24 & & & & & & 12972.72281 & 1.56 & $2 \mathrm{a}$ \\
\hline 28 & 5 & 24 & & & 12857.53415 & 1.85 & $1 \mathrm{a}$ & 12972.72281 & 1.56 & d \\
\hline 28 & 5 & 23 & & & & & & 13308.38709 & 1.37 & $2 \mathrm{a}$ \\
\hline 28 & 6 & 22 & & & & & & 13614.76644 & 2.19 & $1 \mathrm{a}$ \\
\hline 28 & 7 & 21 & & & & & & 13888.30527 & 2.01 & $1 \mathrm{a}$ \\
\hline 2 & 8 & 20 & & & & & & 14120.48658 & 1.84 & $1 \mathrm{a}$ \\
\hline 2 & 10 & 18 & & & & & & 14436.54800 & 5.23 & $1 \mathrm{a}$ \\
\hline 28 & 13 & 16 & & & & & & 15148.69566 & 8.33 & $\mathrm{~d}$ \\
\hline 28 & 13 & 15 & & & & & & 15148.69566 & 8.33 & $1 \mathrm{a}$ \\
\hline 28 & 14 & 1515699.27438 & 7.46 & $1 \mathrm{a}$ & & & & & & \\
\hline 28 & 15 & 14 & & & 15661.32529 & 7.33 & $1 \mathrm{a}$ & 15729.10913 & 10.18 & $1 \mathrm{a}$ \\
\hline 28 & 15 & 13 & & & & & & 15729.25701 & 6.42 & $1 \mathrm{a}$ \\
\hline 28 & 16 & 1316426.78223 & 10.23 & $1 \mathrm{a}$ & & & & & & \\
\hline 28 & 17 & 1216794.30445 & 8.98 & $1 \mathrm{a}$ & 16285.89056 & 5.70 & $2 \mathrm{a}$ & 16342.14767 & 8.86 & d \\
\hline 28 & 17 & 1116794.30445 & 8.98 & $\mathrm{~d}$ & & & & 16342.14767 & 8.86 & $1 \mathrm{a}$ \\
\hline 28 & 18 & 11 & & & 16603.39394 & 8.95 & $1 \mathrm{a}$ & & & \\
\hline 28 & 18 & 10 & & & 16603.39394 & 8.95 & $\mathrm{~d}$ & & & \\
\hline 28 & 19 & 10 & & & 16922.17447 & 6.39 & $1 \mathrm{a}$ & 16969.31746 & 11.37 & d \\
\hline 28 & 19 & 9 & & & 16922.17447 & 6.39 & $\mathrm{~d}$ & 16969.31746 & 11.37 & $1 \mathrm{a}$ \\
\hline 28 & 20 & 9 & & & 17240.27925 & 5.91 & $1 \mathrm{a}$ & 17282.96240 & 9.46 & $\mathrm{~d}$ \\
\hline 28 & 20 & 8 & & & 17240.27925 & 5.91 & $\mathrm{~d}$ & 17282.96240 & 9.46 & $1 \mathrm{a}$ \\
\hline 28 & 21 & 818251.60522 & 9.06 & $1 \mathrm{a}$ & 17555.79404 & 6.60 & $1 \mathrm{a}$ & & & \\
\hline 28 & 21 & 718251.60522 & 9.06 & $\mathrm{~d}$ & 17555.79404 & 6.60 & $\mathrm{~d}$ & & & \\
\hline 28 & 22 & 7 & & & 17866.81055 & 8.84 & $1 \mathrm{a}$ & 17900.91721 & 9.06 & d \\
\hline 28 & 22 & 6 & & & 17866.81055 & 8.84 & $\mathrm{~d}$ & 17900.91721 & 9.06 & $1 \mathrm{a}$ \\
\hline
\end{tabular}




\begin{tabular}{|c|c|c|c|c|c|c|c|c|}
\hline 28 & 23 & 618943.9672010 .32 & $1 \mathrm{a}$ & 18171.2907810 .20 & $\mathrm{~d}$ & 18201.23515 & 10.29 & $\mathrm{~d}$ \\
\hline 28 & 23 & 518943.9672010 .32 & d & 18171.2907810 .20 & $1 \mathrm{a}$ & 18201.23515 & 10.29 & $1 \mathrm{a}$ \\
\hline 28 & 24 & 519274.0664210 .34 & $1 \mathrm{a}$ & 18467.3529910 .35 & $1 \mathrm{a}$ & 18492.93676 & 9.14 & d \\
\hline 28 & 24 & 419274.0664210 .34 & $d$ & 18467.3529910 .35 & $\mathrm{~d}$ & 18492.93676 & 9.14 & $1 \mathrm{a}$ \\
\hline 28 & 25 & 419589.7817910 .35 & $1 \mathrm{a}$ & 18752.5960411 .30 & $1 \mathrm{a}$ & 18773.19396 & 11.36 & d \\
\hline 28 & 25 & 319589.7817910 .35 & d & 18752.5960411 .30 & d & 18773.19396 & 11.36 & $1 \mathrm{a}$ \\
\hline 28 & 26 & 319887.2984811 .46 & $1 \mathrm{a}$ & $19024.62479 \quad 9.61$ & $1 \mathrm{a}$ & 19038.69933 & 12.52 & d \\
\hline 28 & 26 & 219887.2984811 .46 & d & $19024.62479 \quad 9.61$ & d & 19038.69933 & 12.52 & $1 \mathrm{a}$ \\
\hline 28 & 27 & 220160.8967711 .45 & $1 \mathrm{a}$ & 19282.4120012 .38 & $1 \mathrm{a}$ & 19285.50433 & 10.45 & $\mathrm{~d}$ \\
\hline 28 & 27 & 120160.8967711 .45 & d & 19282.4120012 .38 & d & 19285.50433 & 10.45 & $1 \mathrm{a}$ \\
\hline 28 & 28 & 120399.9337112 .53 & $1 \mathrm{a}$ & & & & & \\
\hline 28 & 28 & 020399.9337112 .53 & $\mathrm{~d}$ & & & & & \\
\hline 29 & 0 & $2911231.21765 \quad 0.93$ & $5 \mathrm{e}, \mathrm{a}$ & $11672.25335 \quad 2.09$ & $-1 \mathrm{f}, \mathrm{a}$ & 11800.14248 & 0.83 & d \\
\hline 29 & 1 & $2911231.21765 \quad 0.93$ & d & $11672.25335 \quad 2.09$ & $\mathrm{~d}$ & 11800.14248 & 0.83 & $4 \mathrm{a}$ \\
\hline 29 & 1 & $28 \quad 11926.21358 \quad 1.62$ & $1 \mathrm{a}$ & $12182.58242 \quad 1.94$ & $1 \mathrm{a}$ & 12306.76072 & 1.03 & $\mathrm{~d}$ \\
\hline 29 & 2 & $28 \quad 11926.21358 \quad 1.62$ & d & $12182.58242 \quad 1.94$ & $\mathrm{~d}$ & 12306.76072 & 1.03 & $3 \mathrm{a}$ \\
\hline 29 & 2 & 27 & & $12629.25537 \quad 2.18$ & $1 \mathrm{a}$ & 12751.53794 & 1.36 & $\mathrm{~d}$ \\
\hline 29 & 3 & 27 & & $12629.25537 \quad 2.18$ & d & 12751.53794 & 1.36 & $2 \mathrm{a}$ \\
\hline 29 & 3 & $2612878.74832 \quad 1.98$ & $1 \mathrm{a}$ & & & 13163.56498 & 1.04 & d \\
\hline 29 & 4 & 26 & & & & 13163.56498 & 1.04 & $4 \mathrm{e}, \mathrm{a}$ \\
\hline 29 & 8 & 22 & & & & 14496.89376 & 5.39 & $1 \mathrm{a}$ \\
\hline 29 & 9 & 21 & & & & 14758.31656 & 5.33 & $1 \mathrm{a}$ \\
\hline 29 & 14 & 16 & & & & 16054.39749 & 9.71 & $1 \mathrm{a}$ \\
\hline 29 & 14 & 15 & & & & 16054.39749 & 9.71 & d \\
\hline 29 & 15 & $1416680.82930 \quad 8.98$ & $1 \mathrm{a}$ & & & & & \\
\hline 29 & 16 & 14 & & $16592.27979 \quad 8.87$ & $\mathrm{~d}$ & & & \\
\hline 9 & 16 & 13 & & $16592.27979 \quad 8.87$ & $1 \mathrm{a}$ & & & \\
\hline
\end{tabular}


$29 \quad 17 \quad 12 \quad 17418.34127 \quad 11.38 \quad 1 \mathrm{a}$

$\begin{array}{lll}29 & 18 & 12\end{array}$

$17282.7737110 .18 \quad 1 \mathrm{a}$

$\begin{array}{lll}29 & 18 & 11\end{array}$

$17282.7737110 .18 \quad \mathrm{~d}$

291911

$17550.53296 \quad 6.77 \quad \mathrm{~d}$

291910

$17550.53296 \quad 6.77 \quad 2$ a

$29 \quad 2010$

$\begin{array}{lll}17872.21390 & 8.12 \quad \mathrm{~d}\end{array}$

$\begin{array}{lll}29 & 20 & 9\end{array}$

$\begin{array}{lll}17872.21390 & 8.12 \quad 1 \mathrm{a}\end{array}$

$\begin{array}{llllll}29 & 24 & 6 & 19937.03786 & 11.47 \quad \mathrm{~d}\end{array}$

$\begin{array}{llllll}29 & 24 & 5 & 19937.03786 & 11.47 & 1 \mathrm{a}\end{array}$

$\begin{array}{llllll}29 & 25 & 5 & 20263.32600 & 11.49 \quad \mathrm{~d}\end{array}$

$\begin{array}{lllll}29 & 25 & 4 & 20263.32600 & 11.49 \quad 1 \mathrm{a}\end{array}$

$\begin{array}{lllll}29 & 26 & 4 & 20574.28152 & 11.49 \quad \mathrm{~d}\end{array}$

$19719.4549212 .41 \quad 1 \mathrm{a}$

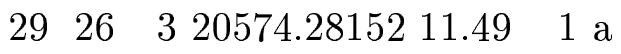
$19719.4549212 .41 \quad \mathrm{~d}$

$\begin{array}{llllll}29 & 27 & 3 & 20865.95704 & 12.50 \quad \mathrm{~d}\end{array}$

$\begin{array}{lllll}29 & 27 & 2 & 20865.95704 & 12.50 \quad 1 \mathrm{a}\end{array}$

$\begin{array}{lllll}29 & 28 & 2 & 21132.76592 & 12.49 \quad \mathrm{~d}\end{array}$

$\begin{array}{llllll}29 & 28 & 1 & 21132.76592 & 12.49 & 1 \mathrm{a}\end{array}$

$\begin{array}{lllll}29 & 29 & 1 & 21360.91376 & 13.49 \quad \mathrm{~d}\end{array}$

$\begin{array}{llllll}29 & 29 & 0 & 21360.91376 & 13.49 & 1 \mathrm{a}\end{array}$

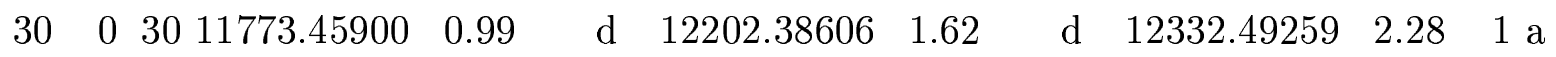

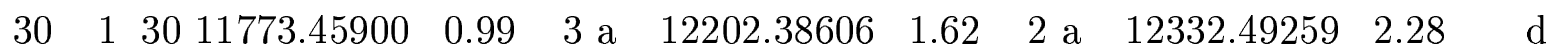

$\begin{array}{lll}30 & 1 & 29\end{array}$

$12855.18472 \quad 1.17 \quad 2$ a

$\begin{array}{lll}30 & 2 & 29\end{array}$

$12855.18472 \quad 1.17 \quad \mathrm{~d}$

$\begin{array}{lll}30 & 2 & 28\end{array}$

$13310.97193 \quad 1.38 \quad 2$ a

$30 \quad 328$

$13310.97193 \quad 1.38 \quad \mathrm{~d}$

$\begin{array}{lll}30 & 3 & 27\end{array}$

$13735.17962 \quad 1.12 \quad 2$ a

$\begin{array}{llllll}30 & 4 & 27 & 13458.65651 & 2.22 & 1 \mathrm{a}\end{array}$

$\begin{array}{lll}13735.17962 & 1.12 \quad \mathrm{~d}\end{array}$ 
$30 \quad 426$

$\begin{array}{lll}30 & 5 & 26\end{array}$

$\begin{array}{lll}30 & 7 & 23\end{array}$

$\begin{array}{lll}30 & 8 & 23\end{array}$

$\begin{array}{lll}30 & 19 & 11\end{array}$

$\begin{array}{lllll}30 & 27 & 3 & 21857.93301 & 13.46\end{array}$

$\begin{array}{llllll}30 & 28 & 3 & 21857.93301 & 13.46 & 1 \mathrm{a}\end{array}$

$\begin{array}{llllll}30 & 30 & 1 & 22334.88021 & 14.39 & 1 \mathrm{a}\end{array}$

$\begin{array}{llllll}30 & 30 & 0 & 22334.88021 & 14.39 \quad \mathrm{~d}\end{array}$

$\begin{array}{llllll}31 & 0 & 31 & 12334.08558 & 1.02 & 3 \mathrm{a}\end{array}$

$31 \quad 131$

$31 \quad 130$

$\begin{array}{lll}31 & 230\end{array}$

$\begin{array}{lll}31 & 2 & 29\end{array}$

$\begin{array}{lll}31 & 3 & 29\end{array}$

$\begin{array}{lll}31 \quad 4 & 28\end{array}$

$\begin{array}{lll}32 & 0 & 32\end{array}$

$\begin{array}{lll}32 & 1 & 32\end{array}$

$\begin{array}{lll}32 & 1 & 31\end{array}$

$32 \quad 2 \quad 31$

$\begin{array}{lll}32 & 4 & 28\end{array}$
$14124.41407 \quad 2.62 \quad 1 \mathrm{a}$

$\begin{array}{lll}14124.41407 & 2.62 \quad \mathrm{~d}\end{array}$

$15113.78031 \quad 5.48 \quad 1 \mathrm{a}$

$15113.78031 \quad 5.48 \quad \mathrm{~d}$

$18244.6312111 .34 \quad 1 \mathrm{a}$

\begin{tabular}{ccc}
12880.58602 & 1.25 & $\mathrm{~d}$ \\
12880.58602 & 1.25 & $5 \mathrm{e}, \mathrm{a}$ \\
13419.16328 & 2.11 & $\mathrm{~d}$ \\
13419.16328 & 2.11 & $1 \mathrm{a}$ \\
13885.33413 & 2.60 & $\mathrm{~d}$ \\
13885.33413 & 2.60 & $1 \mathrm{a}$ \\
14321.04720 & 4.18 & $1 \mathrm{e}$ \\
13444.09735 & 2.20 & $-1 \mathrm{f}, \mathrm{a}$ \\
13444.09735 & 2.20 & $\mathrm{~d}$ \\
13998.54517 & 2.14 & $-1 \mathrm{f}, \mathrm{a}$ \\
13998.54517 & 2.14 & $\mathrm{~d}$ \\
15331.91400 & 5.73 & $1 \mathrm{a}$ \\
\hline
\end{tabular}


TABLE IV .: Term values for the $1 \nu+\delta$ polyad of $\mathrm{H}_{2}{ }^{16} \mathrm{O}$.

\begin{tabular}{|c|c|c|c|c|c|c|c|c|c|c|}
\hline \multicolumn{3}{|c|}{$J K_{a} K_{c}$} & 030 or & \multicolumn{2}{|l|}{003} & \multicolumn{2}{|c|}{110 or $10^{+} 1$} & \multicolumn{3}{|c|}{011 or $10^{-} 1$} \\
\hline 0 & 0 & & 960 & & - & 0 & 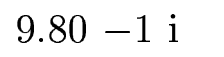 & & & $\mathrm{e}$, \\
\hline 1 & 0 & & 2 & & 16 & 3 & $\mathrm{a}$ & & & $e, a$ \\
\hline 1 & 1 & & 471 & & $8 \mathrm{e}, \mathrm{a}$ & 0 & $-1 \mathrm{i}$ & & & $8 \mathrm{e}$ \\
\hline 1 & 1 & 0 & 4723 & & $13 \mathrm{e}, \mathrm{a}$ & 27 & $4-1$ & 7 & & $3 \epsilon$ \\
\hline 2 & 0 & 2 & 4737 & & $9 \mathrm{e}, \mathrm{a}$ & 5302 & $0-1 \mathrm{i}$ & 540 & & $6 \mathrm{e}$ \\
\hline 2 & 1 & 2 & 475 & & $25 \mathrm{e}, \mathrm{a}$ & 1 & $5-1$ & & & $10 \mathrm{e}, \mathrm{a}$ \\
\hline 2 & 1 & 1 & 4777 & & $12 \mathrm{e}, \mathrm{a}$ & 1 & $3-1$ i,a & 37 & & $14 \mathrm{e}, \mathrm{a}$ \\
\hline 2 & 2 & 1 & 485 & & $14 \mathrm{e}, \mathrm{a}$ & 537 & $0.88-1 \mathrm{i}, \mathrm{a}$ & 9 & & $7 \mathrm{e}, \mathrm{a}$ \\
\hline 2 & 2 & r & 856 & & $8 \mathrm{e}, \mathrm{a}$ & 537 & $0.88-1 \mathrm{i}, \mathrm{a}$ & 9 & & $10 \mathrm{e}, \mathrm{a}$ \\
\hline 3 & 0 & $J$ & 804 & & $26 \mathrm{e}, \mathrm{a}$ & 536 & $42-1$ & 31 & & $5 \mathrm{e}, \mathrm{a}$ \\
\hline 3 & 1 & 3 & 1 & & $17 \mathrm{e}, \mathrm{a}$ & 76 & $55-1 \mathrm{i}, \mathrm{a}$ & & & $6 \mathrm{e}, \mathrm{a}$ \\
\hline 3 & 1 & & 6 & & $24 \mathrm{e}, \mathrm{a}$ & 5 & $1-1$ & & & $\mathrm{e}, \mathrm{a}$ \\
\hline 3 & 2 & & 4926.86303 & & $7 \mathrm{e}, \mathrm{a}$ & 0 & $0-1 \mathrm{i}$ & & & $11 \mathrm{e}, \mathrm{a}$ \\
\hline 3 & 2 & & 93 & & 19 e,a & 0 & $5-1 \mathrm{i}$ & & & 9 e,a \\
\hline 3 & 3 & & $t$ & & $9 \mathrm{e}, \mathrm{a}$ & 70 & $0-1 \mathrm{i}$ & 5 & & $12 \mathrm{e}, \mathrm{a}$ \\
\hline 3 & 3 & 0 & 22 & 0.47 & $16 \mathrm{e}, \mathrm{a}$ & 5 & $6-1 \mathrm{i}, \mathrm{a}$ & 71 & & $8 \mathrm{e}, \mathrm{a}$ \\
\hline 4 & 0 & 4 & 327 & 7 & $14 \mathrm{e}, \mathrm{a}$ & 0 & $0-1 \mathrm{i}$ & 88 & & $16 \mathrm{e}, \mathrm{a}$ \\
\hline 4 & 1 & 4 & 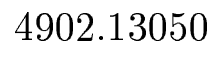 & 5 & $24 \mathrm{e}, \mathrm{a}$ & 9 & $9-1$ & 2 & & 8 \\
\hline 4 & 1 & 3 & 45 & 8 & $17 \mathrm{e}, \mathrm{a}$ & 75 & $8-1$ & 9 & & $1 / \mathrm{e}, \mathrm{a}$ \\
\hline 4 & 2 & 3 & 502 & 2 & $22 \mathrm{e}, \mathrm{a}$ & 004 & $8-1$ & 50 & & $3 \mathrm{e}$ \\
\hline 4 & 2 & 2 & 300 & 8 & $11 \mathrm{e}, \mathrm{a}$ & 20 & $1.60-1 \mathrm{i}$ & 30 & 0.39 & $13 \mathrm{e}, \mathrm{a}$ \\
\hline 4 & 3 & 2 & 5162. & 0.42 & $21 \mathrm{e}, \mathrm{a}$ & 5635. & $0.48-1 \mathrm{i}, \mathrm{a}$ & 5726 & 0.45 & $7 \mathrm{e}, \mathrm{a}$ \\
\hline 4 & 3 & 1 & 5163.26295 & 0.47 & $12 \mathrm{e}, \mathrm{a}$ & 5639.72848 & $0.50 \quad 4 \mathrm{a}$ & 5727.57053 & 0.39 & 13 e,a \\
\hline 4 & 4 & 1 & 5342.18730 & 54 & $13 \mathrm{e}, \mathrm{a}$ & 756. & -1 & 5842.00035 & 0. & $\mathrm{e}$, \\
\hline
\end{tabular}




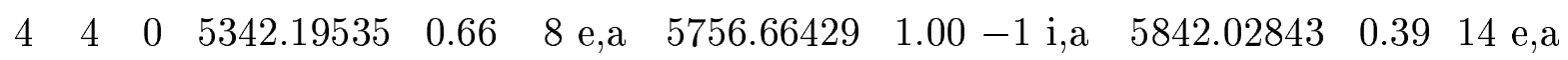

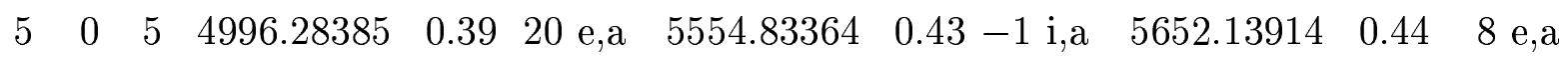

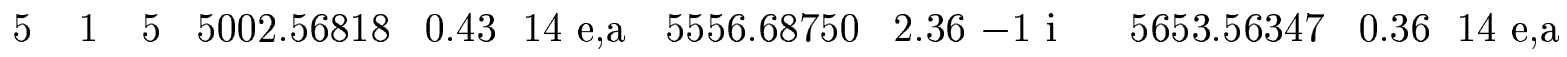

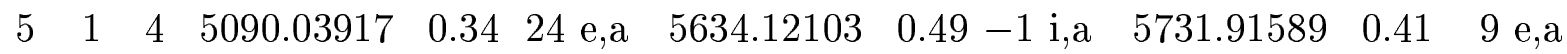

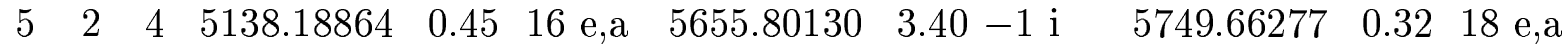

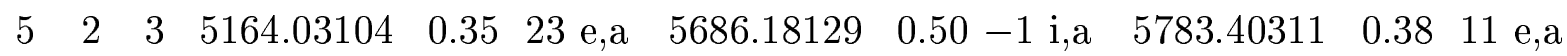

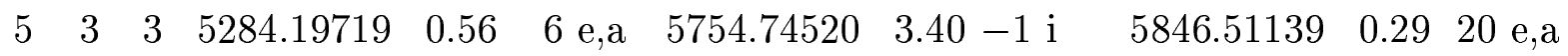

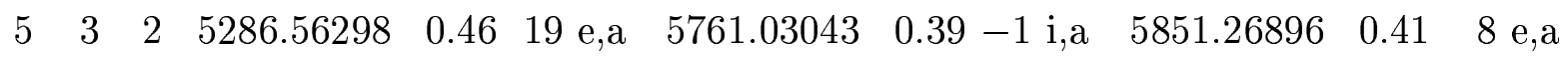

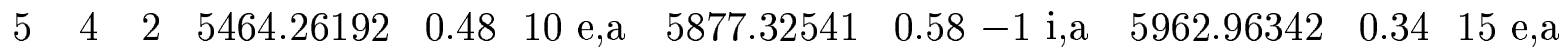

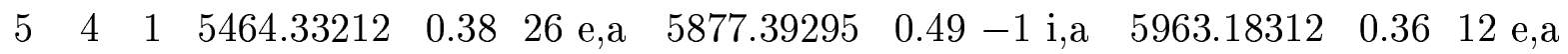

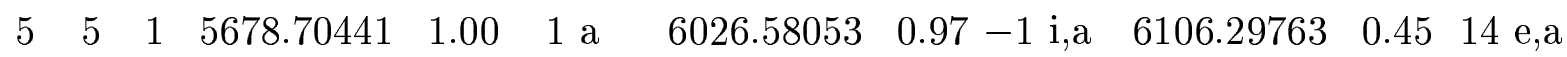

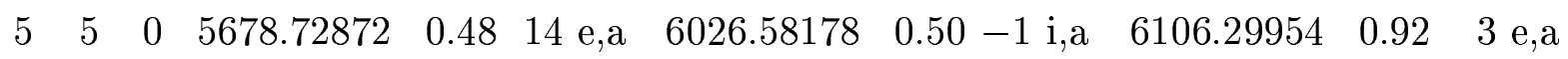

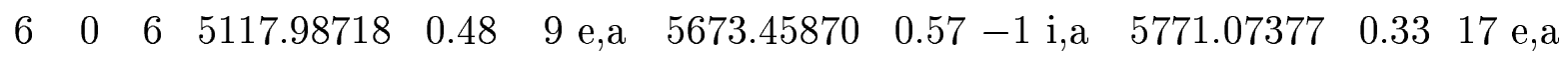

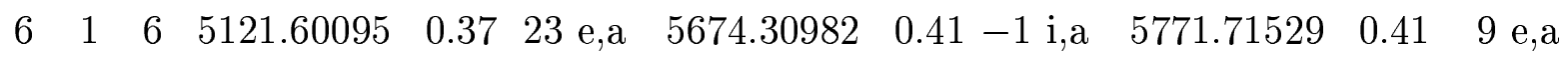

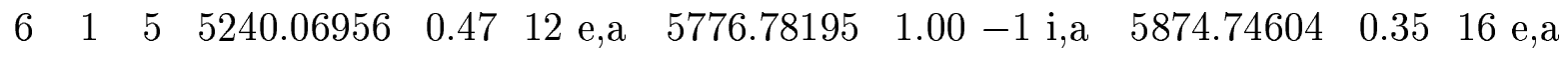

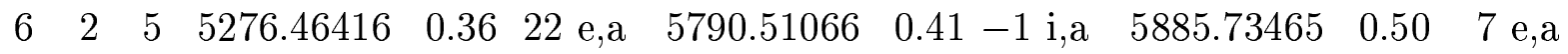

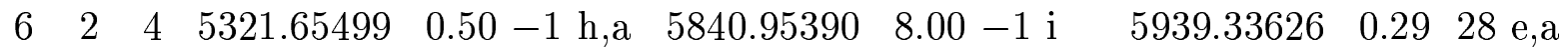

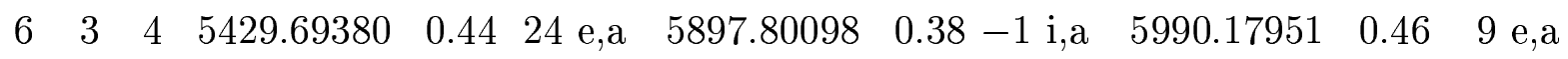

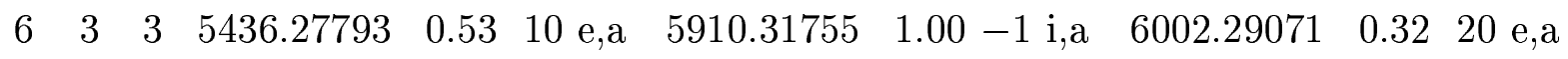

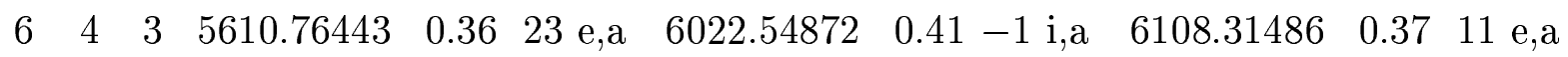

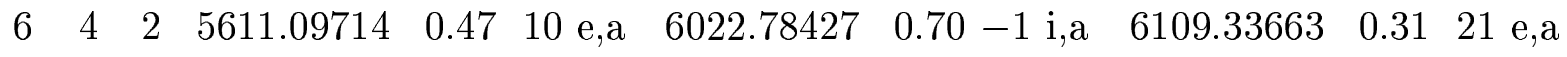

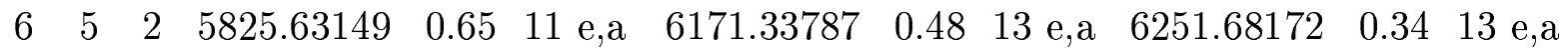

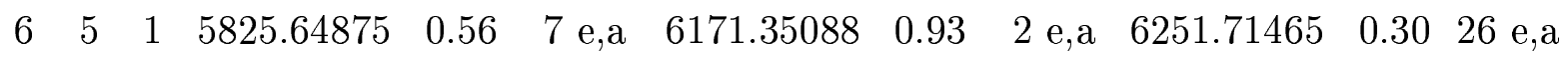

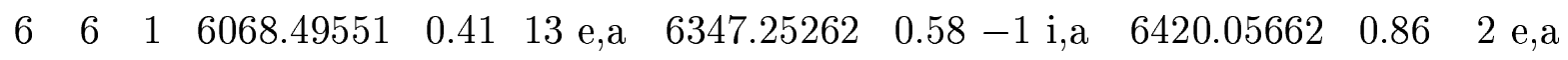

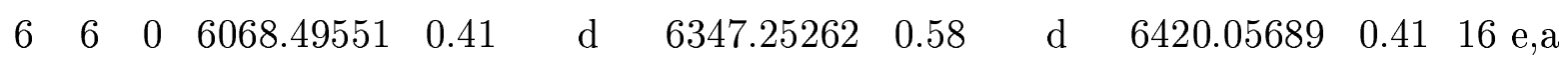

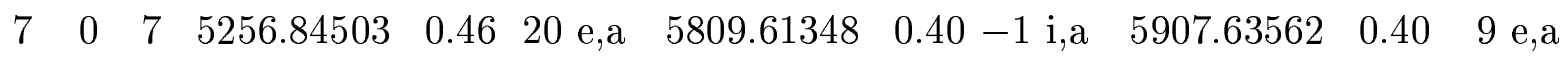

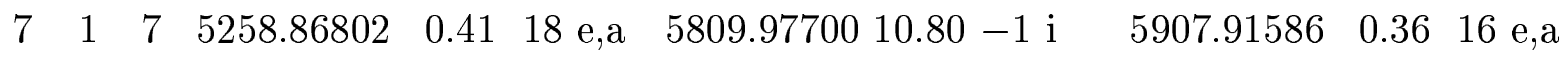


$\begin{array}{llllllllllll}7 & 1 & 6 & 5409.69741 & 0.40 & 24 \mathrm{e}, \mathrm{a} & 5937.03674 & 0.38-1 \mathrm{i}, \mathrm{a} & 6034.98875 & 0.42 & 8 \mathrm{e}, \mathrm{a}\end{array}$ $\begin{array}{llllllllllll}7 & 2 & 6 & 5435.40649 & 0.76 & 11 \mathrm{e}, \mathrm{a} & 5944.92879 & 0.70-1 \mathrm{i}, \mathrm{a} & 6041.07167 & 0.30 & 20 \mathrm{e}, \mathrm{a}\end{array}$ $\begin{array}{llllllllllll}7 & 2 & 5 & 5505.21083 & 0.34 & 26 \mathrm{e}, \mathrm{a} & 6019.38562 & 0.45-1 \mathrm{i}, \mathrm{a} & 6118.63376 & 0.40 & 10 \mathrm{e}, \mathrm{a}\end{array}$ $\begin{array}{llllllllllll}7 & 3 & 5 & 5598.57768 & 0.35 & 15 \mathrm{e}, \mathrm{a} & 6063.38819 & 1.00 & -1 \mathrm{i}, \mathrm{a} & 6156.50259 & 0.26 & 22 \mathrm{e}, \mathrm{a}\end{array}$ $\begin{array}{llllllllllll}7 & 3 & 4 & 5613.36901 & 0.35 & 26 & \mathrm{e}, \mathrm{a} & 6087.85069 & 0.45-1 \mathrm{i}, \mathrm{a} & 6179.83552 & 0.36 & 12 \mathrm{e}, \mathrm{a}\end{array}$ $\begin{array}{llllllllllll}7 & 4 & 4 & 5781.58056 & 0.48 & 11 \mathrm{e}, \mathrm{a} & 6193.36116 & 0.50-1 \mathrm{i}, \mathrm{a} & 6277.86276 & 0.23 & 29 \mathrm{e}, \mathrm{a}\end{array}$ $\begin{array}{llllllllllll}7 & 4 & 3 & 5782.70838 & 0.42 & 19 \mathrm{e}, \mathrm{a} & 6193.36493 & 0.58 & -1 \mathrm{i}, \mathrm{a} & 6281.23448 & 0.31 & 13 \mathrm{e}, \mathrm{a}\end{array}$ $\begin{array}{llllllllllll}7 & 5 & 3 & 5996.76241 & 0.43 & 12 \mathrm{e}, \mathrm{a} & 6340.23678 & 0.84 & 4 \mathrm{e}, \mathrm{a} & 6421.35945 & 0.28 & 22 \mathrm{e}, \mathrm{a}\end{array}$ $\begin{array}{lllllllllllll}7 & 5 & 2 & 5996.84354 & 0.40 & 18 \mathrm{e}, \mathrm{a} & 6340.33788 & 0.41-1 \mathrm{i}, \mathrm{a} & 6421.53948 & 0.31 & 15 \mathrm{e}, \mathrm{a}\end{array}$ $\begin{array}{llllllllllll}7 & 6 & 2 & 6240.47851 & 0.98 & -1 \mathrm{~h}, \mathrm{a} & 6516.40690 & 5.00 & 1 \mathrm{e} & 6589.96992 & 0.36 & 20 \mathrm{e}, \mathrm{a}\end{array}$ $\begin{array}{lllllllllllll}7 & 6 & 1 & 6240.48622 & 0.45 & 13 & \mathrm{e}, \mathrm{a} & 6516.40522 & 0.66 & 10 \mathrm{e}, \mathrm{a} & 6589.97319 & 0.61 & 4 \mathrm{e}, \mathrm{a}\end{array}$ $\begin{array}{lllllllllllll}7 & 7 & 1 & 6505.53517 & 0.38 & \mathrm{~d} & 6716.16742 & 0.50 & \mathrm{~d} & 6780.42043 & 0.39 & 13 \mathrm{e}, \mathrm{a}\end{array}$ $\begin{array}{llllllllllll}7 & 7 & 0 & 6505.53517 & 0.38 & 15 \mathrm{e}, \mathrm{a} & 6716.16742 & 0.50 & 13 \mathrm{e}, \mathrm{a} & 6780.42030 & 1.67 & 1 \mathrm{e}\end{array}$ $\begin{array}{lllllllllllll}8 & 0 & 8 & 5413.00202 & 0.47 & 13 \mathrm{e}, \mathrm{a} & 5963.26087 & 0.70 & -1 \mathrm{i}, \mathrm{a} & 6061.91910 & 0.34 & 16 \mathrm{e}, \mathrm{a}\end{array}$ $\begin{array}{lllllllllllll}8 & 1 & 8 & 5414.12663 & 0.37 & 21 \mathrm{e}, \mathrm{a} & 5963.51900 & 0.48 & 11 \mathrm{e}, \mathrm{a} & 6062.03954 & 0.50 & 4 \mathrm{a}\end{array}$ $\begin{array}{llllllllllllll}8 & 1 & 7 & 5597.18261 & 0.53 & 11 \mathrm{e}, \mathrm{a} & 6114.05454 & 0.99 & -1 \mathrm{i}, \mathrm{a} & 6212.03970 & 0.32 & 15 \mathrm{e}, \mathrm{a}\end{array}$ $\begin{array}{llllllllllll}8 & 2 & 7 & 5614.27003 & 0.36 & 24 \mathrm{e}, \mathrm{a} & 6118.31182 & 0.50-1 \mathrm{i}, \mathrm{a} & 6215.16360 & 0.35 & -1 \mathrm{f}, \mathrm{a}\end{array}$ $\begin{array}{llllllllllll}8 & 2 & 6 & 5712.87666 & 0.55 & 9 \mathrm{e}, \mathrm{a} & 6219.05421 & 0.98 & 3 \mathrm{e}, \mathrm{a} & 6318.71683 & 0.30 & 21 \mathrm{e}, \mathrm{a}\end{array}$ $\begin{array}{llllllllllll}8 & 3 & 6 & 5790.11448 & 0.31 & 28 \mathrm{e}, \mathrm{a} & 6250.55428 & 0.43 & 14 \mathrm{e}, \mathrm{a} & 6344.53798 & 0.36 & 11 \mathrm{e}, \mathrm{a}\end{array}$ $\begin{array}{llllllllllll}8 & 3 & 5 & 5818.33069 & 0.35 & 10 \mathrm{e}, \mathrm{a} & 6292.71123 & 0.71-1 \mathrm{i}, \mathrm{a} & 6394.24398 & 0.35 & 12 \mathrm{e}, \mathrm{a}\end{array}$ $\begin{array}{llllllllllll}8 & 4 & 5 & 5976.45877 & 0.42 & 21 \mathrm{e}, \mathrm{a} & 6378.01846 & 0.32 & 19 \mathrm{e}, \mathrm{a} & 6471.16595 & 0.38 & 10 \mathrm{e}, \mathrm{a}\end{array}$ $\begin{array}{llllllllllll}8 & 4 & 4 & 5979.71917 & 0.42 & 12 \mathrm{e}, \mathrm{a} & 6389.98141 & 0.68 & 3 \mathrm{e}, \mathrm{a} & 6479.89186 & 0.27 & 21 \mathrm{e}, \mathrm{a}\end{array}$ $\begin{array}{llllllllllll}8 & 5 & 4 & 6191.96498 & 0.36 & 24 \mathrm{e}, \mathrm{a} & 6533.26143 & 0.42 & 12 \mathrm{e}, \mathrm{a} & 6615.26478 & 0.31 & 15 \mathrm{e}, \mathrm{a}\end{array}$ $\begin{array}{llllllllllll}8 & 5 & 3 & 6192.24396 & 0.51 & 8 \mathrm{e}, \mathrm{a} & 6533.64433 & 0.68 & 4 \mathrm{e}, \mathrm{a} & 6615.96867 & 0.28 & 23 \mathrm{e}, \mathrm{a}\end{array}$ $\begin{array}{lllllllllllll}8 & 6 & 3 & 6436.51545 & 0.49 & 13 \mathrm{e}, \mathrm{a} & 6709.44775 & 0.54 & 12 \mathrm{e}, \mathrm{a} & 6783.92335 & 0.55 & 5 \mathrm{e}, \mathrm{a}\end{array}$ $\begin{array}{llllllllllll}8 & 6 & 2 & 6436.53812 & 0.56 & 9 \mathrm{e}, \mathrm{a} & 6709.45996 & 0.70 & 3 \mathrm{e}, \mathrm{a} & 6783.95173 & 0.29 & 24 \mathrm{e}, \mathrm{a}\end{array}$ $\begin{array}{llllllllllllll}8 & 7 & 2 & 6702.58293 & 0.38 & 16 \mathrm{e}, \mathrm{a} & 6909.99225 & 0.45 & 14 \mathrm{e}, \mathrm{a} & 6974.98892 & 0.99 & 2 \mathrm{e}, \mathrm{a}\end{array}$ 


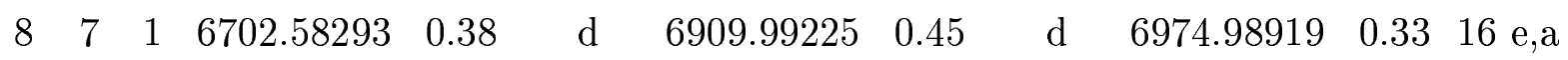

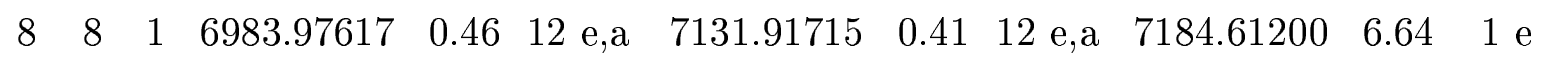
$\begin{array}{lllllllllllll}8 & 8 & 0 & 6983.97617 & 0.46 & \mathrm{~d} & 7131.91715 & 0.41 & \mathrm{~d} & 7184.60167 & 0.41 & 14 \mathrm{e}, \mathrm{a}\end{array}$

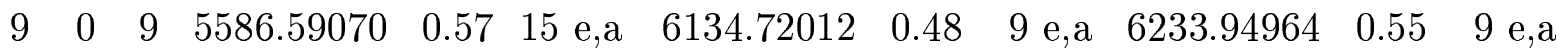

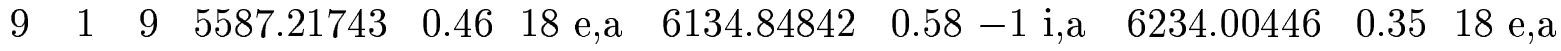
$\begin{array}{lllllllllllll}9 & 1 & 8 & 5801.55276 & 0.39 & 22 & \mathrm{e}, \mathrm{a} & 6307.85367 & 0.88 & 4 \text { e,a } & 6406.02299 & 0.41 & 7 \text { e,a }\end{array}$

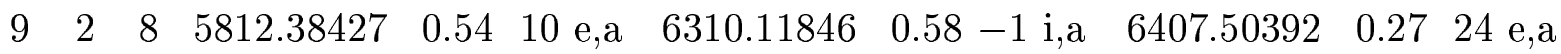

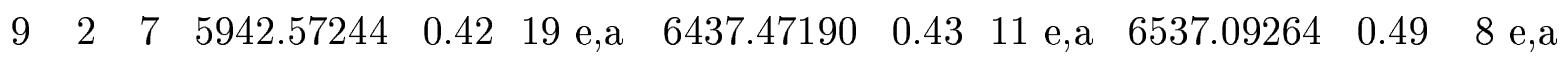

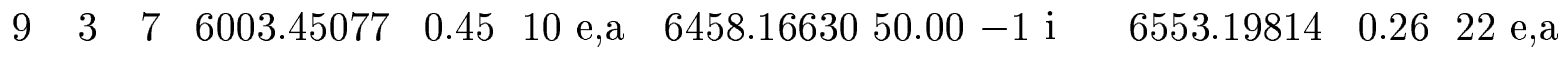

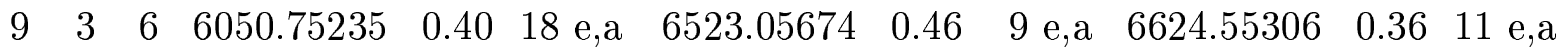
$9 \quad 4 \quad 6 \quad 6194.97491 \quad 0.56 \quad 8$ e,a $\quad 6594.76963 \quad 0.97 \quad 3$ e,a $\quad 6687.53055 \quad 0.25 \quad 24$ e,a

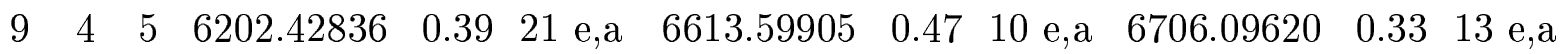

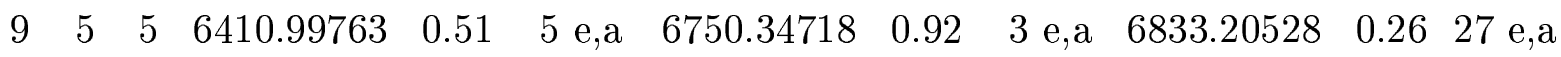

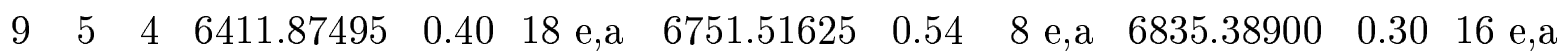

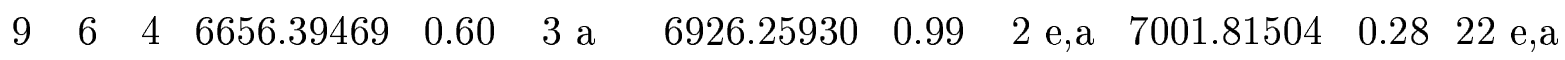

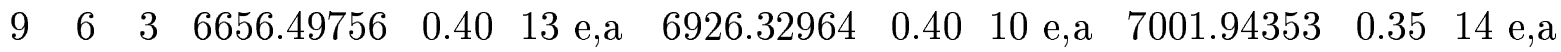
$\begin{array}{lllllllllllll}9 & 7 & 3 & 6923.34613 & 0.38 & \mathrm{~d} & 7127.43304 & 0.41 & \mathrm{~d} & 7193.32597 & 0.30 & 20 & \mathrm{e}, \mathrm{a}\end{array}$

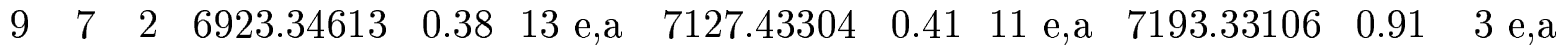

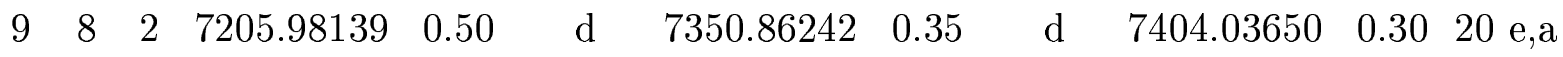
$\begin{array}{llllllllllll}9 & 8 & 1 & 7205.98139 & 0.50 & 10 \mathrm{e}, \mathrm{a} & 7350.86242 & 0.35 & 14 \mathrm{e}, \mathrm{a} & 7404.03580 & 1.67 & 1 \mathrm{e}\end{array}$

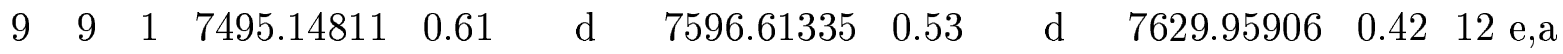

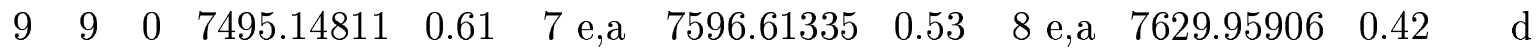

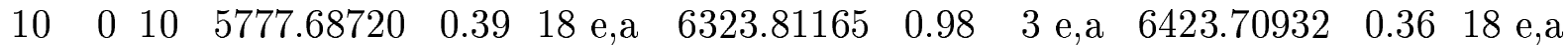

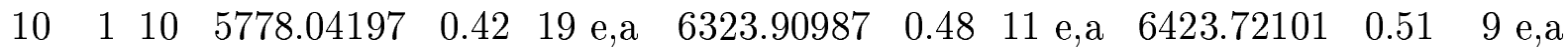

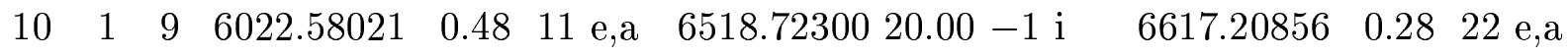

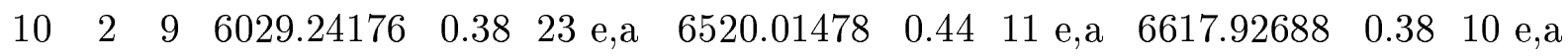

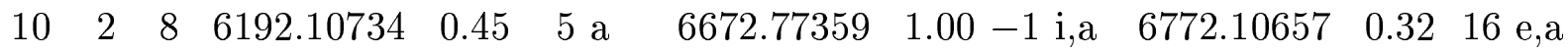




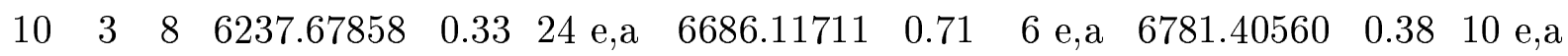

$\begin{array}{llllllllllll}10 & 3 & 7 & 6309.33218 & 0.42 & 6 \mathrm{a} & 6776.75800 & 20.00-1 \mathrm{i} & 6878.47700 & 0.30 & 20 \mathrm{e}, \mathrm{a}\end{array}$

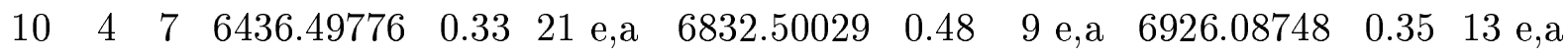

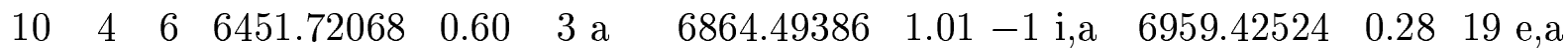

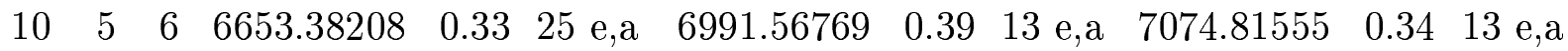

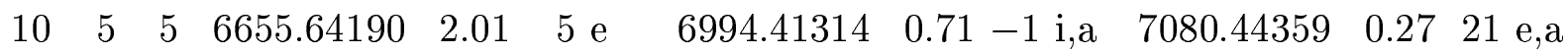

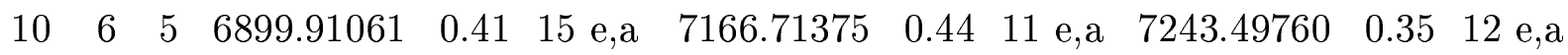

$\begin{array}{lllllllllllll}10 & 6 & 4 & 6900.28548 & 0.48 & 5 \mathrm{a} & 7166.96678 & 0.81 & 2 & \mathrm{a} & 7243.95202 & 0.26 & 22 \mathrm{e}, \mathrm{a}\end{array}$

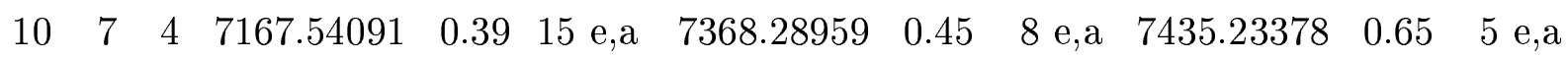

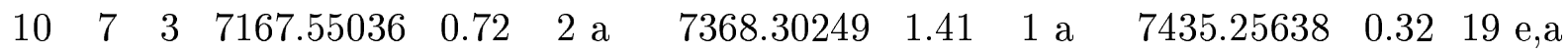

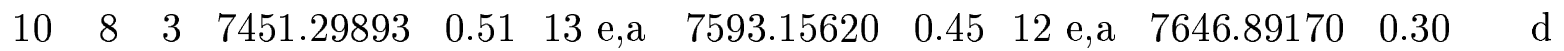

$\begin{array}{llllllllllllll}10 & 8 & 2 & 7451.29893 & 0.51 & \mathrm{~d} & 7593.15620 & 0.45 & \mathrm{~d} & 7646.89170 & 0.30 & 17 & \mathrm{e}, \mathrm{a}\end{array}$

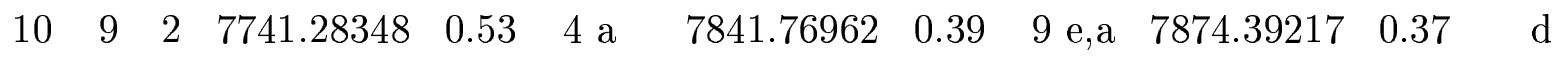

$\begin{array}{lllllllllllll}10 & 9 & 1 & 7741.28348 & 0.53 & \mathrm{~d} & 7841.76962 & 0.39 & \mathrm{~d} & 7874.39217 & 0.37 & 14 \mathrm{e}, \mathrm{a}\end{array}$

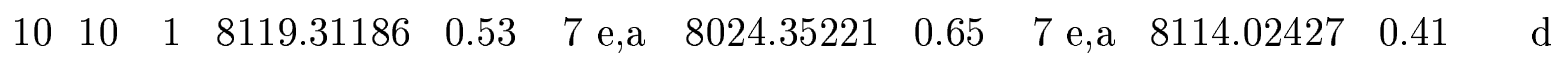

$\begin{array}{lllllllllllll}10 & 10 & 0 & 8119.31186 & 0.53 & \text { d } & 8024.35221 & 0.65 & \text { d } & 8114.02427 & 0.41 & 12 & \text { e,a }\end{array}$

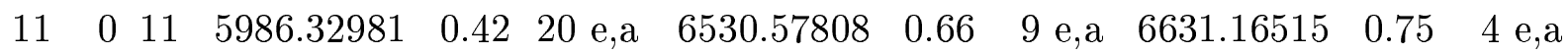

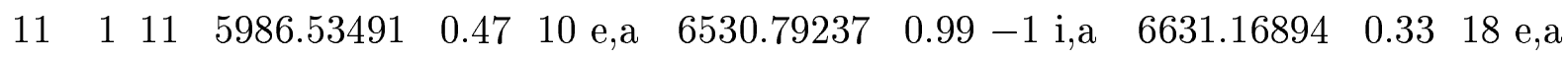

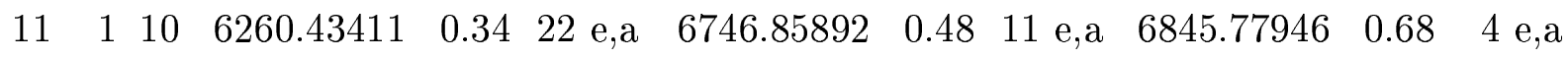

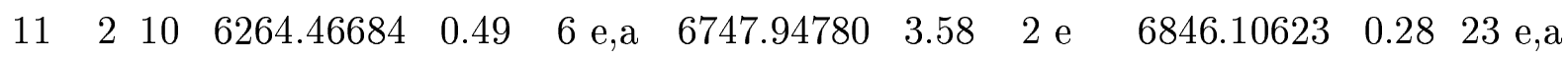

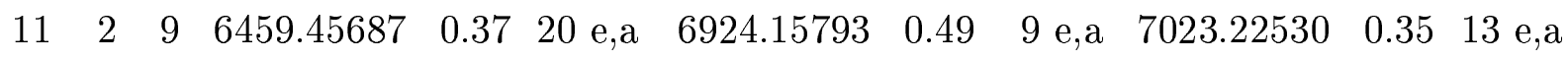

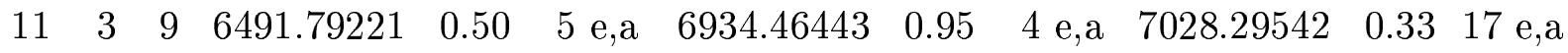

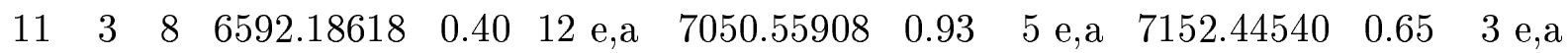

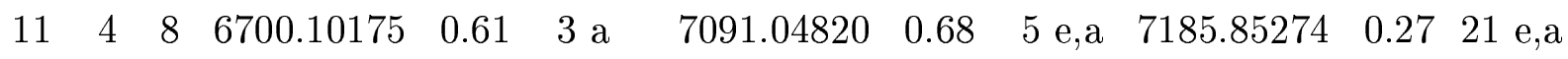

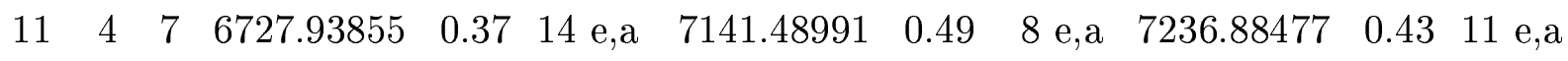

$\begin{array}{llllllllllll}11 & 5 & 7 & 6917.30629 & 0.49 & 6 \mathrm{e}, \mathrm{a} & 7258.05231 & 1.79 & 1 \mathrm{a} & 7339.51705 & 0.28 & 21 \mathrm{e}, \mathrm{a}\end{array}$

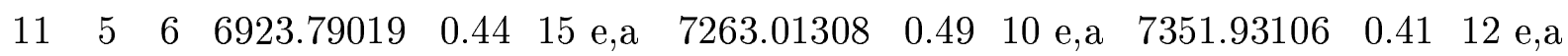


$\begin{array}{lllllllllllll}11 & 6 & 6 & 7166.83076 & 0.47 & 5 \mathrm{a} & 7430.63876 & 0.78 & 2 \mathrm{a} & 7508.75032 & 0.27 & 20 \mathrm{e}, \mathrm{a}\end{array}$

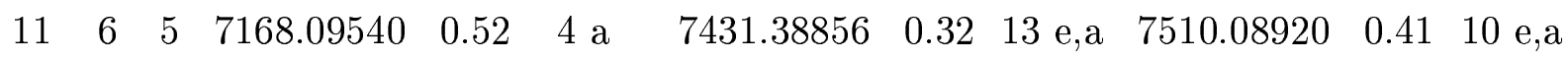

$\begin{array}{llllllllllllll}11 & 7 & 5 & 7434.87577 & 0.47 & 5 \mathrm{a} & 7632.36535 & 0.80 & 2 & \mathrm{a} & 7700.51150 & 0.33 & 17 & \mathrm{e}, \mathrm{a}\end{array}$

$\begin{array}{lllllllllllll}11 & 7 & 4 & 7434.90045 & 0.42 & 12 & \text { e,a } & 7632.40977 & 0.41 & 7 \text { e,a } & 7700.59561 & 0.44 & 9 \text { e,a }\end{array}$

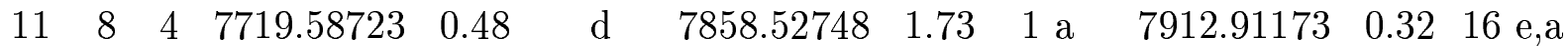

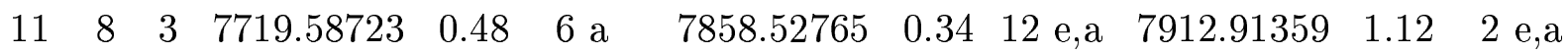

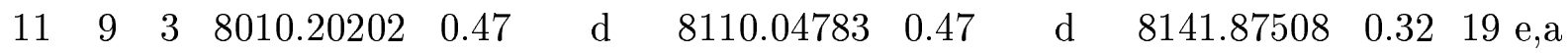

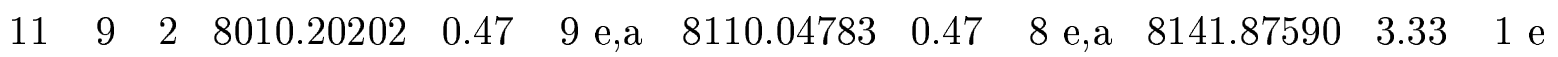

$\begin{array}{lllllllllllll}11 & 10 & 2 & 8392.15589 & 0.40 & \mathrm{~d} & 8293.37281 & 0.57 & \mathrm{~d} & 8383.60257 & 0.45 & 9 & \mathrm{e}, \mathrm{a}\end{array}$

$\begin{array}{llllllllllll}11 & 10 & 1 & 8392.15589 & 0.40 & 14 \text { e,a } & 8293.37281 & 0.57 & 4 \text { a } & 8383.60257 & 0.45 & d\end{array}$

$\begin{array}{lllllllllllll}11 & 11 & 1 & 8698.87125 & 0.84 & \mathrm{~d} & 8567.65689 & 0.59 & \mathrm{~d} & 8634.52025 & 0.52 & 9 & \mathrm{e}, \mathrm{a}\end{array}$

$\begin{array}{llllllllllll}11 & 11 & 0 & 8698.87125 & 0.84 & 5 \mathrm{e}, \mathrm{a} & 8567.65689 & 0.59 & 8 \text { e,a } & 8634.52025 & 0.52 & \mathrm{~d}\end{array}$

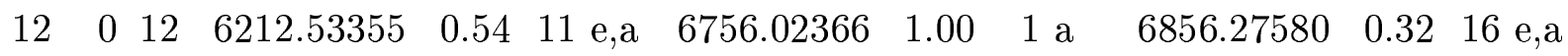

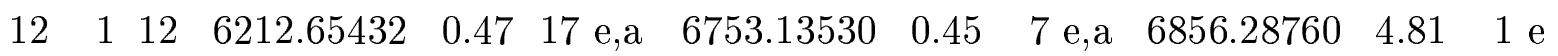

$\begin{array}{lllllllllllll}12 & 1 & 11 & 6515.38299 & 0.46 & 5 \mathrm{a} & 6992.47126 & 1.03 & 1 \mathrm{a} & 7091.82287 & 0.34 & 15 \mathrm{e}, \mathrm{a}\end{array}$

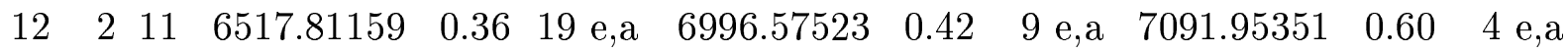

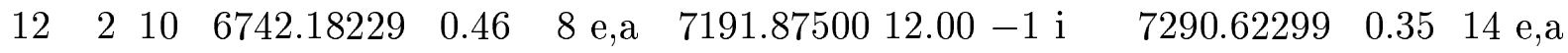

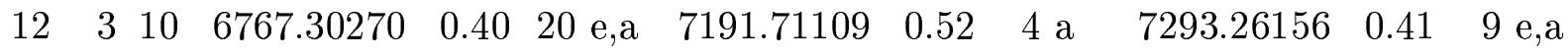

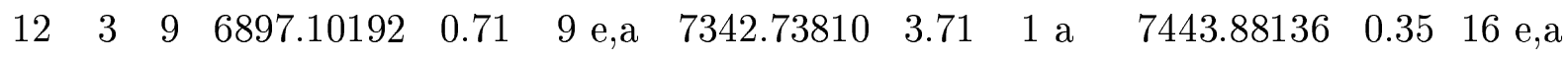

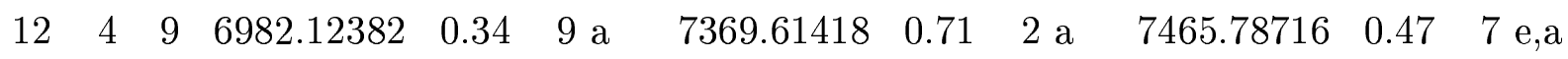

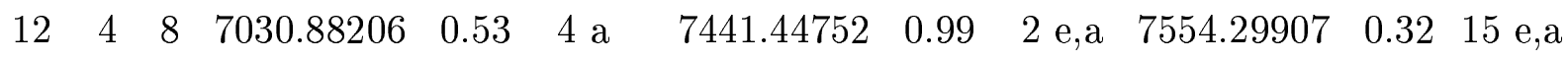

$\begin{array}{llllllllllll}12 & 5 & 8 & 7213.35267 & 0.38 & 14 \mathrm{e}, \mathrm{a} & 7531.13951 & 0.69-1 \mathrm{i}, \mathrm{a} & 7626.54755 & 0.51 & 7 \mathrm{e}, \mathrm{a}\end{array}$

$\begin{array}{lllllllllllll}12 & 5 & 7 & 7216.60538 & 0.48 & 5 \mathrm{a} & 7558.00808 & 1.02 & 1 \mathrm{a} & 7650.34860 & 0.27 & 23 & \mathrm{e}, \mathrm{a}\end{array}$

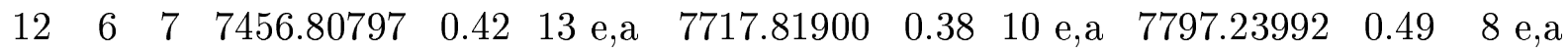

$\begin{array}{lllllllllllll}12 & 6 & 6 & 7461.03574 & 0.55 & 4 \mathrm{a} & 7719.73860 & 1.48 & 1 \mathrm{a} & 7800.64570 & 0.31 & 16 & \mathrm{e}, \mathrm{a}\end{array}$

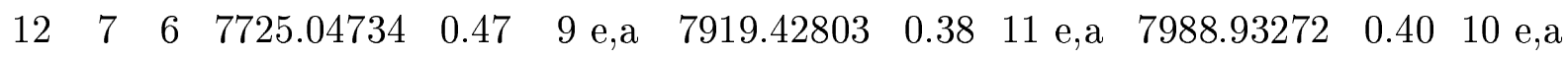

$\begin{array}{llllllllllll}12 & 7 & 5 & 7725.12572 & 0.78 & 2 \mathrm{a} & 7919.57561 & 1.27 & 1 \mathrm{a} & 7989.21265 & 0.32 & 17 \mathrm{e}, \mathrm{a}\end{array}$ 


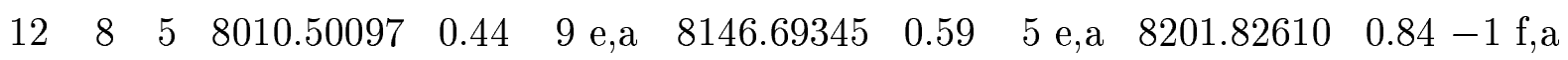

$\begin{array}{lllllllllll}12 & 8 & 4 & 8010.50097 & 0.44 & \mathrm{~d} & 8201.84006 & 0.39 & 13 \mathrm{e}, \mathrm{a}\end{array}$

$\begin{array}{llllllllllll}12 & 9 & 4 & 8301.52566 & 0.53 & 9 \mathrm{e}, \mathrm{a} & 8401.09266 & 0.39 & 11 \mathrm{e}, \mathrm{a} & 8432.08300 & 2.70 & -1 \mathrm{f}\end{array}$

$\begin{array}{llllllllllll}12 & 9 & 3 & 8301.52566 & 0.53 & \mathrm{~d} & 8401.09266 & 0.39 & \mathrm{~d} & 8432.08560 & 0.39 & 15 \mathrm{e}, \mathrm{a}\end{array}$

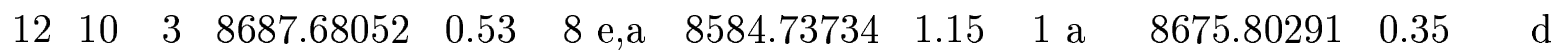

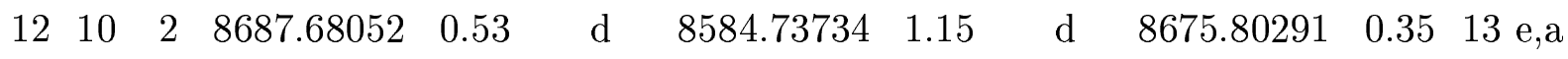

$\begin{array}{llllllllllll}12 & 11 & 2 & 8998.73255 & 0.83 & 2 \text { a } & 8860.41048 & 0.82 & 2 \text { a } & 8929.37846 & 0.49 & \text { d }\end{array}$

$\begin{array}{llllllllllll}12 & 11 & 1 & 8998.73255 & 0.83 & \mathrm{~d} & 8860.41048 & 0.82 & \mathrm{~d} & 8929.37846 & 0.49 & 9 \text { e,a }\end{array}$

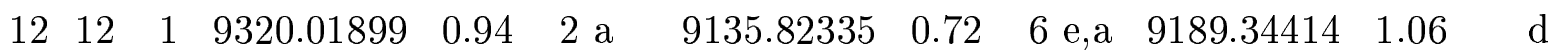

$\begin{array}{llllllllllll}12 & 12 & 0 & 9320.01899 & 0.94 & \mathrm{~d} & 9135.82335 & 0.72 & \mathrm{~d} & 9189.34414 & 1.06 & 4 \mathrm{e}, \mathrm{a}\end{array}$

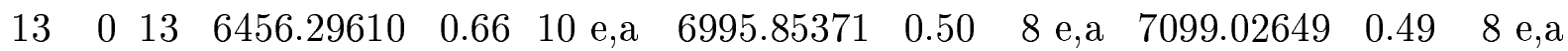

$\begin{array}{llllllllllll}13 & 1 & 13 & 6456.37035 & 0.74 & 2 \mathrm{a} & 6995.95836 & 0.98 & 2 \mathrm{e}, \mathrm{a} & 7098.99587 & 0.38 & 13 \mathrm{e}, \mathrm{a}\end{array}$

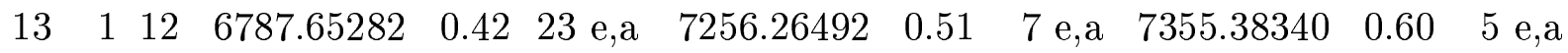

$\begin{array}{llllllllllll}13 & 2 & 12 & 6789.12054 & 0.52 & 8 \mathrm{e}, \mathrm{a} & 7253.61759 & 1.02 & 1 \mathrm{a} & 7355.38081 & 0.35 & 16 \\ \mathrm{e}, \mathrm{a}\end{array}$

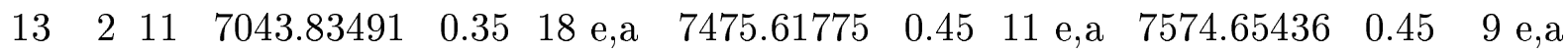

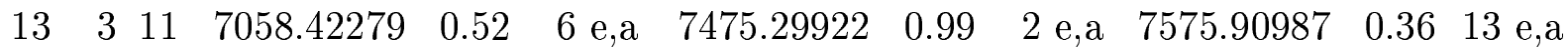

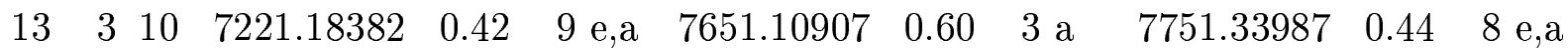

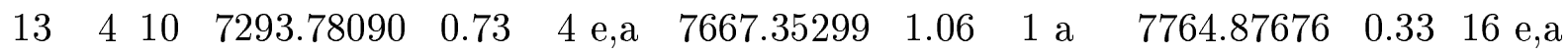

$\begin{array}{llllllllllll}13 & 4 & 9 & 7359.47255 & 0.42 & 12 \mathrm{e}, \mathrm{a} & 7780.82563 & 0.52 & 4 \mathrm{a} & 7878.20970 & 0.40 & 11 \mathrm{e}, \mathrm{a}\end{array}$

$\begin{array}{llllllllllll}13 & 5 & 9 & 7523.00185 & 0.63 & 3 \mathrm{a} & 7840.67358 & 1.03 & 2 \mathrm{e}, \mathrm{a} & 7935.02442 & 0.34 & 14 \mathrm{e}, \mathrm{a}\end{array}$

$\begin{array}{llllllllllll}13 & 5 & 8 & 7534.43178 & 0.39 & 7 \mathrm{a} & 7879.55926 & 0.58 & 3 \mathrm{a} & 7975.18896 & 0.64 & 5 \mathrm{e}, \mathrm{a}\end{array}$

$\begin{array}{llllllllllll}13 & 6 & 8 & 7769.34463 & 1.09 & 1 \mathrm{a} & 8028.06037 & 0.85 & 3 \mathrm{e}, \mathrm{a} & 8108.48930 & 0.34 & 15 \mathrm{e}, \mathrm{a}\end{array}$

$\begin{array}{llllllllllll}13 & 6 & 7 & 7760.13126 & 0.40 & 10 \mathrm{e}, \mathrm{a} & 8032.32200 & 0.71 & 2 \mathrm{a} & 8116.11919 & 0.45 & 8 \mathrm{e}, \mathrm{a}\end{array}$

$\begin{array}{llllllllllll}13 & 7 & 7 & 8037.81719 & 1.13 & 1 \mathrm{a} & 8229.23567 & 1.09 & 1 \mathrm{a} & 8300.23542 & 0.37 & 16 \mathrm{e}, \mathrm{a}\end{array}$

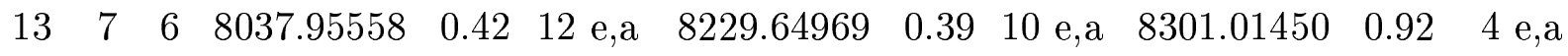

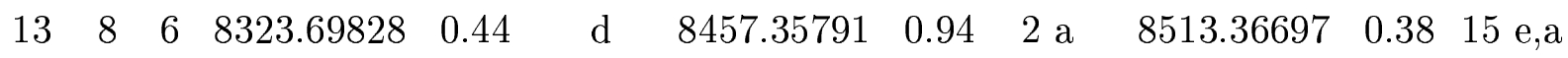

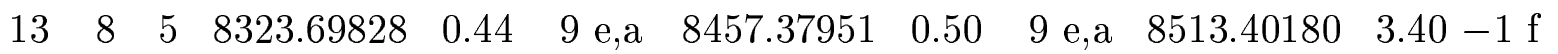




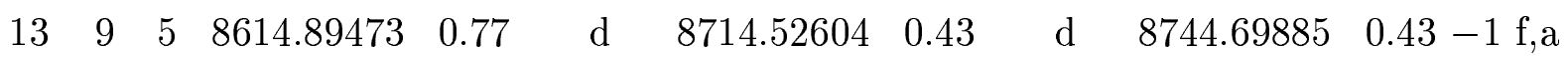

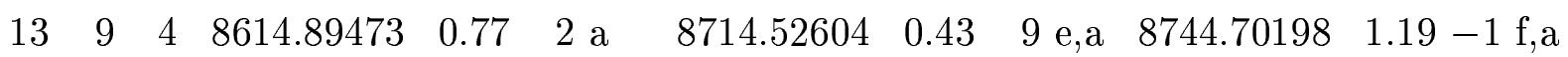
$\begin{array}{llllllllllll}13 & 10 & 4 & 9005.41981 & 0.49 & \mathrm{~d} & 8898.08509 & 0.65 & \mathrm{~d} & 8990.25519 & 0.40 & 13 \mathrm{e}, \mathrm{a}\end{array}$ $\begin{array}{llllllllllll}13 & 10 & 3 & 9005.41981 & 0.49 & 7 \mathrm{e}, \mathrm{a} & 8898.08509 & 0.65 & 3 \mathrm{a} & 8990.25519 & 0.40 & \mathrm{~d}\end{array}$ $\begin{array}{llllllllllll}13 & 11 & 3 & 9320.60954 & 0.73 & \mathrm{~d} & 9175.16172 & 0.72 & \mathrm{~d} & 9246.36842 & 0.54 & 9 \mathrm{e}, \mathrm{a}\end{array}$ $\begin{array}{llllllllllll}13 & 11 & 2 & 9320.60954 & 0.73 & 4 \mathrm{e}, \mathrm{a} & 9175.16172 & 0.72 & 6 \mathrm{e}, \mathrm{a} & 9246.36842 & 0.54 & \mathrm{~d}\end{array}$

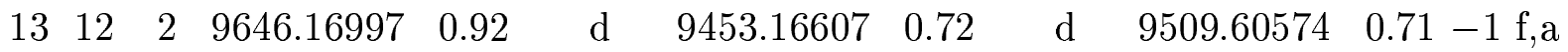
$\begin{array}{llllllllllll}13 & 12 & 1 & 9646.16997 & 0.92 & 3 \mathrm{a} & 9453.16607 & 0.72 & 3 \mathrm{a} & 9509.60574 & 0.71 & \mathrm{~d}\end{array}$ $\begin{array}{llllllllllll}13 & 13 & 1 & 9974.35427 & 0.98 & \mathrm{~d} & 9733.20701 & 0.91 & \mathrm{~d} & 9776.57113 & 0.82 & 5 \text { e,a }\end{array}$

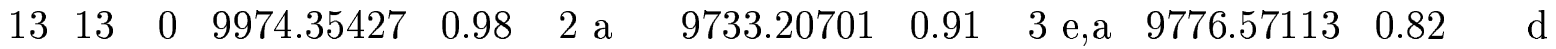
$\begin{array}{llllllllllll}14 & 0 & 14 & 6717.61295 & 0.61 & 3 \mathrm{a} & 7255.21110 & 1.05 & 2 \mathrm{e}, \mathrm{a} & 7358.52426 & 0.34 & 17 \mathrm{e}, \mathrm{a}\end{array}$

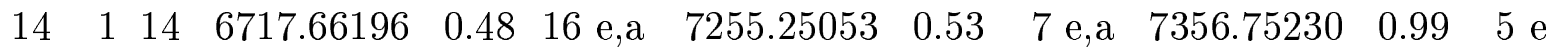
$\begin{array}{llllllllllll}14 & 1 & 13 & 7077.41612 & 0.62 & 3 \mathrm{a} & 7533.21475 & 1.03 & 1 \mathrm{a} & 7636.80001 & 0.36 & 13 \mathrm{e}, \mathrm{a}\end{array}$

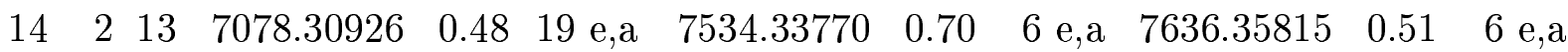
$\begin{array}{llllll}14 & 2 & 12 & 7356.75397 & 0.53 \quad 4 \mathrm{a}\end{array}$ $7875.85597 \quad 0.35 \quad 14 \mathrm{e}, \mathrm{a}$

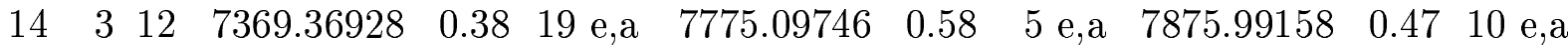
$\begin{array}{llllllllllll}14 & 3 & 11 & 7567.31925 & 0.63 & 5 \mathrm{e}, \mathrm{a} & 7975.00528 & 1.06 & 1 \mathrm{a} & 8074.21064 & 0.39 & 14 \mathrm{e}, \mathrm{a}\end{array}$

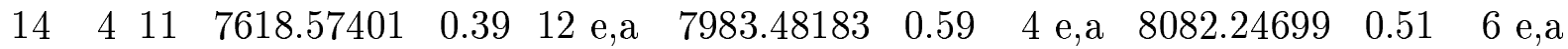
$\begin{array}{llllllllllll}14 & 4 & 10 & 7711.66061 & 1.17 & 1 \mathrm{a} & 8124.78571 & 1.18 & 1 \mathrm{a} & 8222.84133 & 0.37 & 16 \\ \mathrm{e}, \mathrm{a}\end{array}$ $\begin{array}{llllllllllll}14 & 5 & 10 & 7855.03728 & 0.46 & 11 \mathrm{e}, \mathrm{a} & 8168.87912 & 0.47 & 7 \mathrm{e}, \mathrm{a} & 8263.97533 & 0.43 & 9 \text { e,a }\end{array}$ $\begin{array}{llllllllllll}14 & 5 & 9 & 7877.47499 & 0.66 & 3 \mathrm{a} & 8226.81460 & 1.08 & 1 \mathrm{a} & 8324.34944 & 0.32 & 19 \mathrm{e}, \mathrm{a}\end{array}$

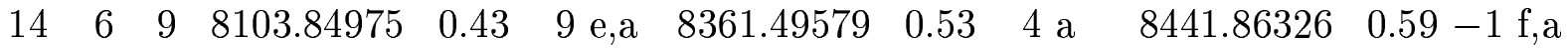
$\begin{array}{llllllllllll}14 & 6 & 8 & 8097.61823 & 1.18 & 1 \mathrm{a} & 8369.64947 & 0.86 & 2 \mathrm{a} & 8457.10171 & 0.39 & 15 \mathrm{e}, \mathrm{a}\end{array}$ $\begin{array}{llllllllllll}14 & 7 & 8 & 8372.56946 & 0.44 & 8 \mathrm{e}, \mathrm{a} & 8561.48912 & 0.98 & 3 \mathrm{e}, \mathrm{a} & 8634.09289 & 0.72 & -1 \mathrm{f}, \mathrm{a}\end{array}$ $\begin{array}{llllllllllll}14 & 7 & 7 & 8373.22863 & 1.48 & 1 \mathrm{a} & 8562.54680 & 1.31 & 1 \mathrm{a} & 8636.05380 & 0.45 & 11 \mathrm{e}, \mathrm{a}\end{array}$

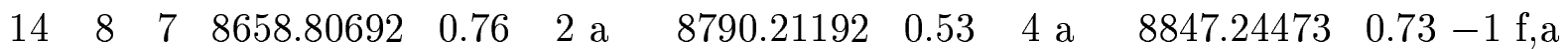
$\begin{array}{llllllllllll}14 & 8 & 6 & 8658.84049 & 1.30 & 1 \mathrm{a} & 8790.28546 & 1.48 & 1 \mathrm{a} & 8847.41243 & 0.31 & 17 \mathrm{e}, \mathrm{a}\end{array}$ 


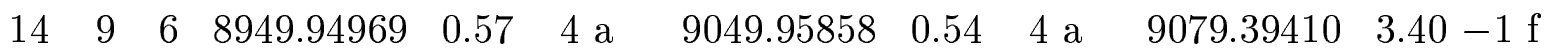

$\begin{array}{llllllllllll}14 & 9 & 5 & 8949.94969 & 0.57 & \mathrm{~d} & 9049.96188 & 1.37 & 1 \mathrm{a} & 9079.40082 & 0.43 & 10 \mathrm{e}, \mathrm{a}\end{array}$

$\begin{array}{llllllllllll}14 & 10 & 5 & 9344.89673 & 0.78 & 3 \mathrm{e}, \mathrm{a} & 9233.07006 & 0.84 & 2 \mathrm{a} & 9326.58818 & 0.50 & \mathrm{~d}\end{array}$

$\begin{array}{llllllllllll}14 & 10 & 4 & 9344.89673 & 0.78 & \mathrm{~d} & 9233.07006 & 0.84 & \mathrm{~d} & 9326.58818 & 0.50 & 11 \mathrm{e}, \mathrm{a}\end{array}$

$\begin{array}{llllllllllll}14 & 11 & 4 & 9663.99448 & 0.81 & 5 \mathrm{e}, \mathrm{a} & 9511.51725 & 0.65 & 3 \mathrm{a} & 9585.08052 & 0.55 & \mathrm{~d}\end{array}$

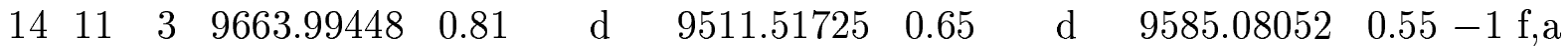

$\begin{array}{lllllllllllll}14 & 12 & 3 & 9993.66008 & 0.90 & 3 \mathrm{a} & 9792.02420 & 0.87 & 6 & \mathrm{e}, \mathrm{a} & 9851.43942 & 0.53 & \mathrm{~d}\end{array}$

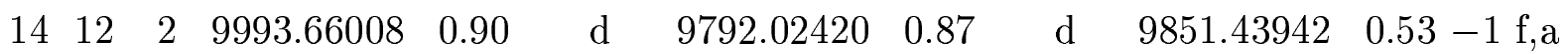

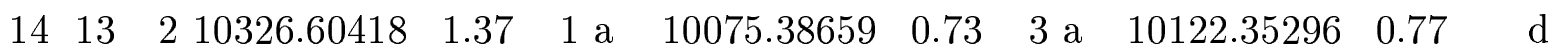

$\begin{array}{llllllllllll}14 & 13 & 1 & 10326.60418 & 1.37 & \mathrm{~d} & 10075.38659 & 0.73 & \mathrm{~d} & 10122.35296 & 0.77 & -1 \mathrm{f}, \mathrm{a}\end{array}$

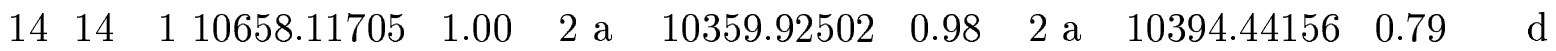

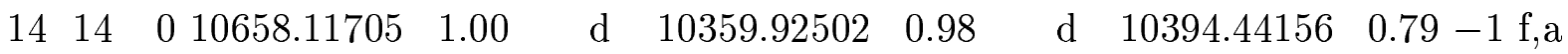

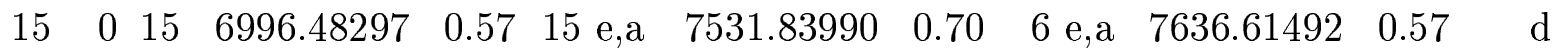

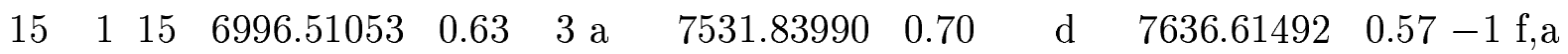

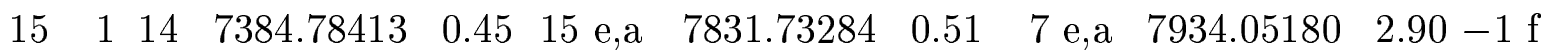

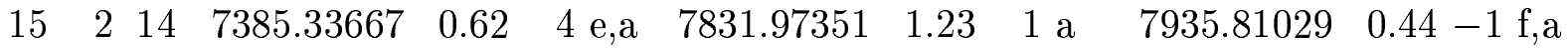

$\begin{array}{llllllllllll}15 & 2 & 13 & 7690.74924 & 0.43 & 8 \mathrm{e}, \mathrm{a} & 8101.16226 & 0.52 & 6 \mathrm{e}, \mathrm{a} & 8191.81170 & 0.51 & -1 \mathrm{f}, \mathrm{a}\end{array}$

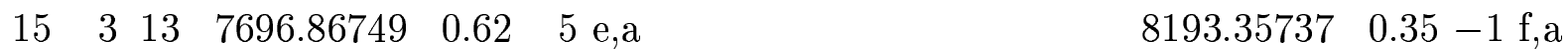

$\begin{array}{llllllllllll}15 & 3 & 12 & 7924.34243 & 0.42 & 12 \mathrm{e}, \mathrm{a} & 8314.70962 & 1.05 & 1 \mathrm{a} & 8412.50483 & 1.26 & 5 \mathrm{e}, \mathrm{a}\end{array}$

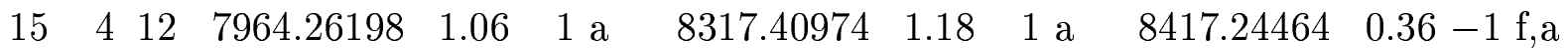

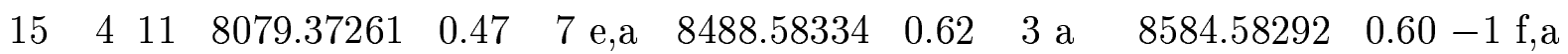

$\begin{array}{llllllllllll}15 & 5 & 11 & 8210.22808 & 0.69 & 4 \mathrm{a} & 8516.05758 & 1.05 & 2 \mathrm{e}, \mathrm{a} & 8612.44753 & 0.41 & -1 \mathrm{f}, \mathrm{a}\end{array}$

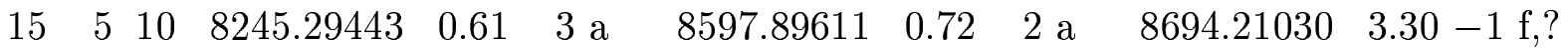

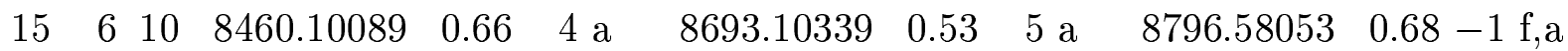

$\begin{array}{llllllllllll}15 & 6 & 9 & 8455.86347 & 0.54 & 5 \mathrm{a} & 8732.18831 & 0.61 & 3 \mathrm{a} & 8823.89813 & 0.47 & -1 \mathrm{f}, \mathrm{a}\end{array}$

$\begin{array}{llllllllllll}15 & 7 & 9 & 8729.19486 & 0.90 & 2 \mathrm{a} & 8915.87845 & 1.32 & 1 \mathrm{a} & 8990.08206 & 0.67 & -1 \mathrm{f}, \mathrm{a}\end{array}$

$\begin{array}{llllllllllll}15 & 7 & 8 & 8730.57214 & 0.53 & 7 \mathrm{e}, \mathrm{a} & 8918.31981 & 0.75 & 6 \mathrm{e}, \mathrm{a} & 8994.48648 & 0.60 & 4 \mathrm{e}, \mathrm{a}\end{array}$ 


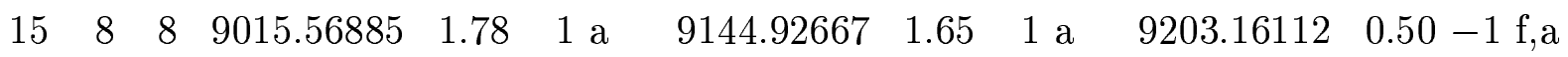

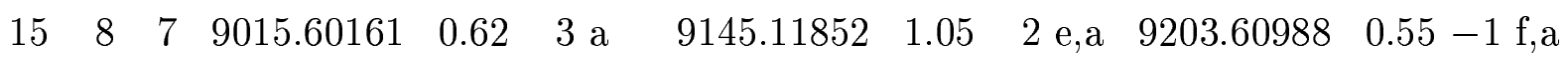

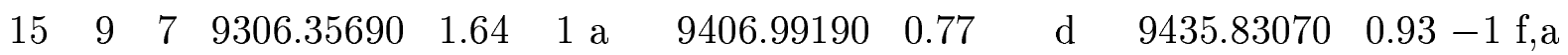
$\begin{array}{llllllllllll}15 & 9 & 6 & 9306.36224 & 0.69 & 3 \mathrm{a} & 9406.99190 & 0.77 & 2 \mathrm{a} & 9435.87359 & 1.24 & 1 \mathrm{a}\end{array}$

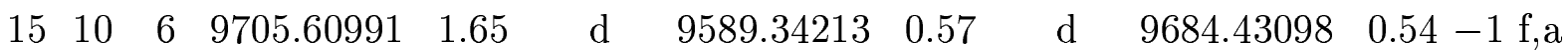
$\begin{array}{llllllllllll}15 & 10 & 5 & 9705.60991 & 1.65 & 1 \mathrm{a} & 9589.34213 & 0.57 & 4 \mathrm{a} & 9684.43098 & 0.54 & \mathrm{~d}\end{array}$

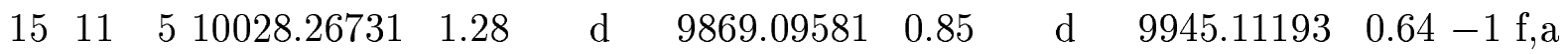

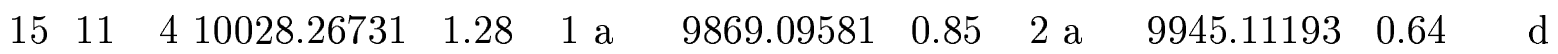

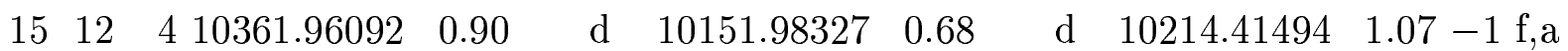

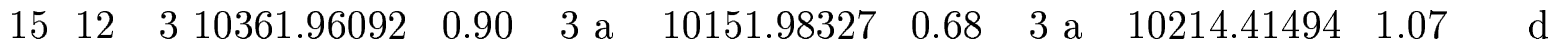

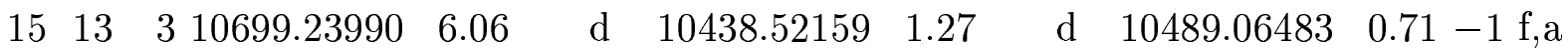

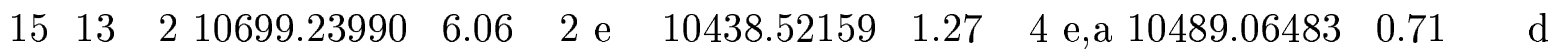

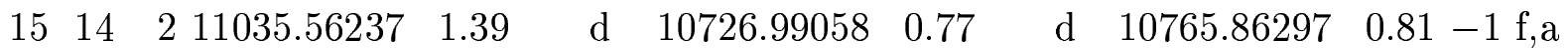

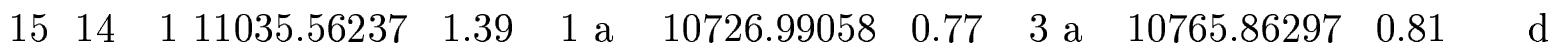

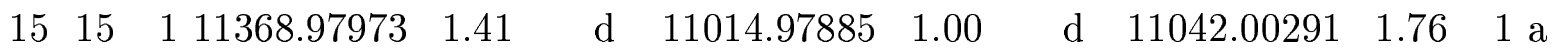

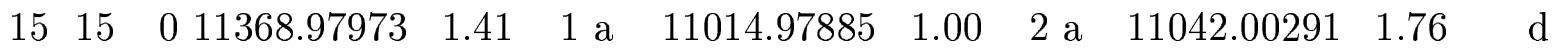

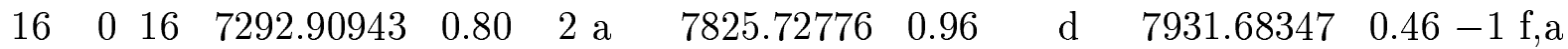

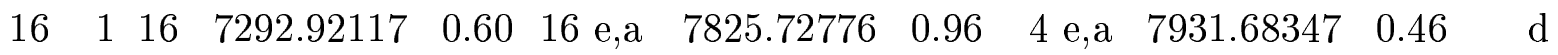
$\begin{array}{llllllllllll}16 & 1 & 15 & 7709.84676 & 0.58 & 5 \mathrm{e}, \mathrm{a} & 8146.69409 & 1.40 & 1 \mathrm{a} & 8249.95492 & 0.43 & -1 \mathrm{f}, \mathrm{a}\end{array}$ $\begin{array}{llllllllllll}16 & 2 & 15 & 7710.19787 & 0.51 & 15 \mathrm{e}, \mathrm{a} & 8146.78611 & 1.04 & 3 \mathrm{e}, \mathrm{a} & 8249.85418 & 0.90 & 2 \mathrm{a}\end{array}$

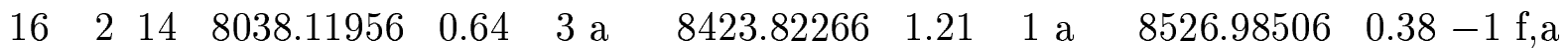

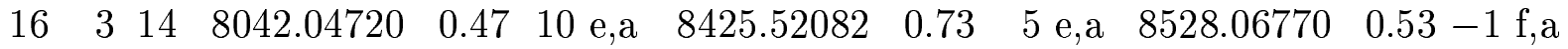
$\begin{array}{lllllllll}16 & 3 & 13 & 8300.04287 & 0.67 & 3 \mathrm{a} & 8766.54070 & 0.36 & -1 \mathrm{f}, \mathrm{a}\end{array}$

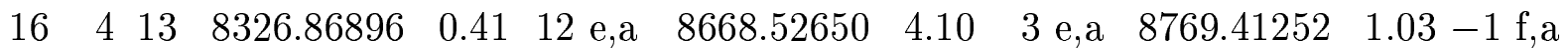

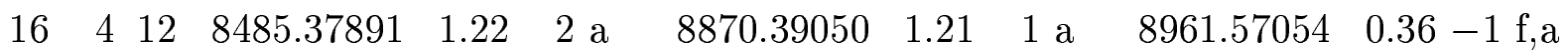

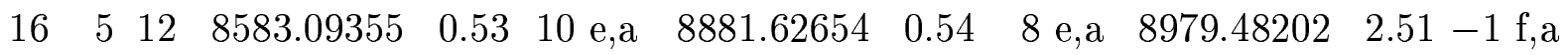

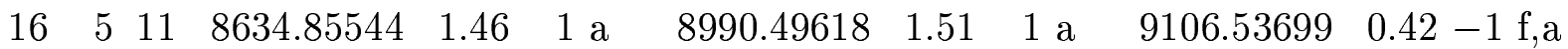




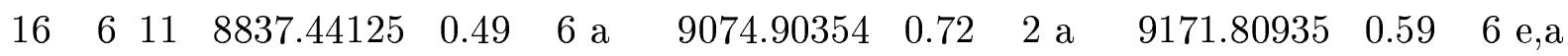
$\begin{array}{llllllllllll}16 & 6 & 10 & 8835.07984 & 1.21 & 1 \mathrm{a} & 9120.01101 & 1.11 & 1 \mathrm{a} & 9216.00983 & 0.39 & -1 \mathrm{f}, \mathrm{a}\end{array}$

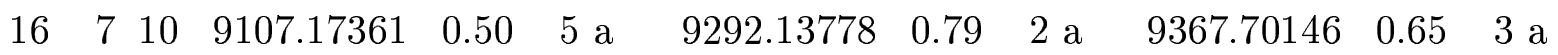
$\begin{array}{llllllllllll}16 & 7 & 9 & 9110.04149 & 1.20 & 1 \mathrm{a} & 9296.94696 & 1.13 & 1 \mathrm{a} & 9376.65305 & 0.39-1 \mathrm{f}, \mathrm{a}\end{array}$

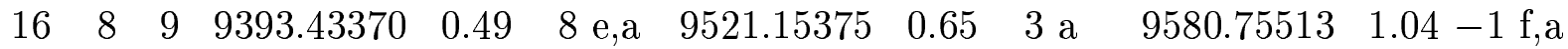
$\begin{array}{llllllllllll}16 & 8 & 8 & 9393.68920 & 1.35 & 1 \mathrm{a} & 9521.60829 & 1.66 & 1 \mathrm{a} & 9581.86913 & 0.47 & -1 \mathrm{f}, \mathrm{a}\end{array}$

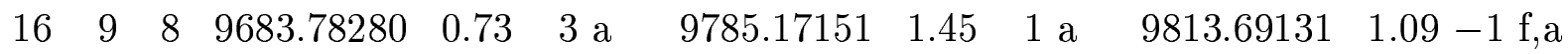
$\begin{array}{llllllllllll}16 & 9 & 7 & 9683.80647 & 1.30 & 1 \mathrm{a} & 9785.18724 & 2.12 & 1 \mathrm{a} & 9813.77624 & 0.71 & -1 \mathrm{f}, \mathrm{a}\end{array}$

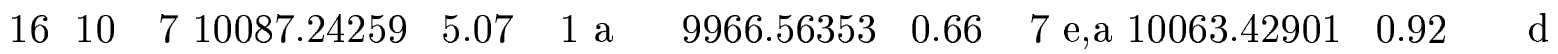

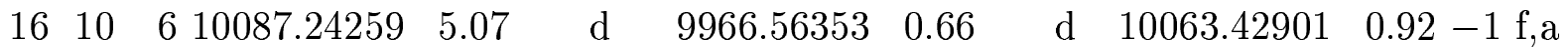
$\begin{array}{llllllllllll}16 & 11 & 6 & 10413.27036 & 1.31 & 1 \text { a } & 10247.51016 & 0.81 & 3 \text { e,a } & 10326.06832 & 0.65 & \mathrm{~d}\end{array}$

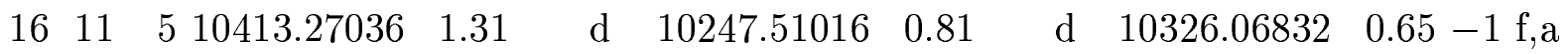

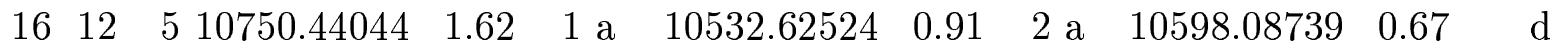

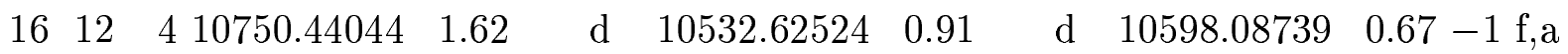
$\begin{array}{llllllllllll}16 & 13 & 4 & 11092.33158 & 1.36 & 1 \text { a } & 10822.17212 & 0.91 & 2 \text { a } & 10876.24460 & 2.90 & \text { d }\end{array}$

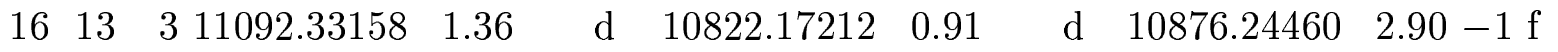
$\begin{array}{lllllllllll}16 & 14 & 3 & 11432.81680 & 6.14 & 1 \text { a } & 11114.38534 & 1.09 & 4 \text { e,a } & 11157.49234 & 1.37\end{array}$

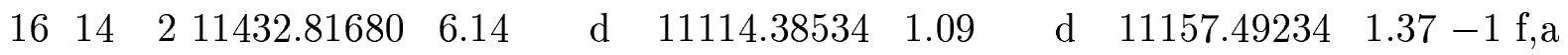

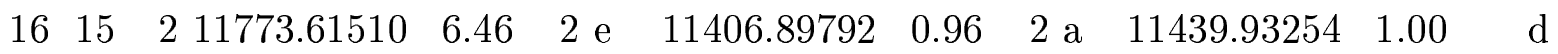

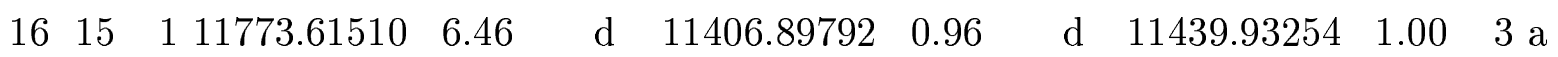

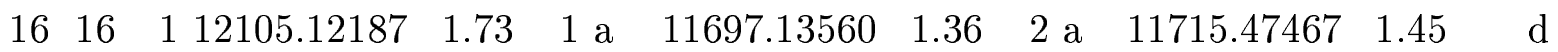

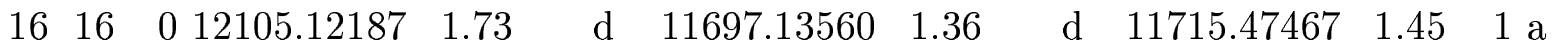

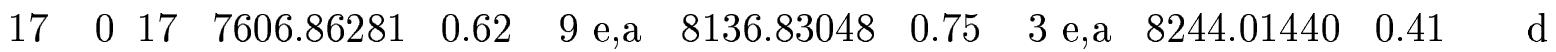
$\begin{array}{lllllllll}17 & 1 & 17 & 7606.89779 & 1.15 & 3 \mathrm{e}, \mathrm{a} & 8244.01440 & 0.41-1 \mathrm{f}, \mathrm{a}\end{array}$

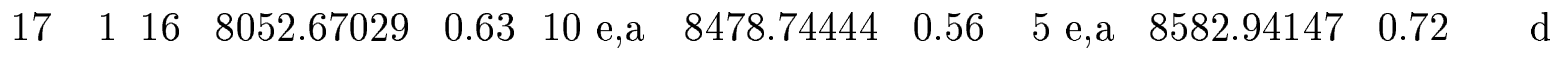

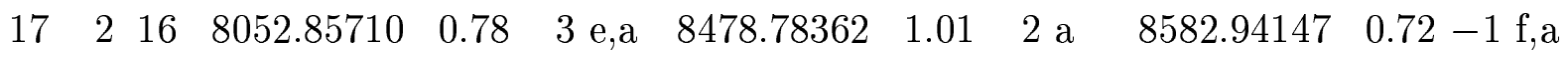

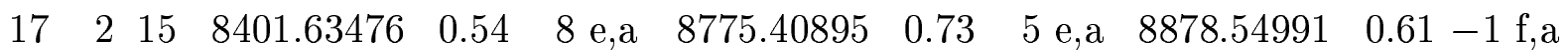


$\begin{array}{llllll}17 & 3 & 15 & 8404.14386 & 0.66 & 3 \mathrm{a}\end{array}$

$\begin{array}{llllllllllll}17 & 3 & 14 & 8688.68380 & 0.48 & 7 \mathrm{e}, \mathrm{a} & 9027.62908 & 1.02 & 3 \mathrm{e}, \mathrm{a} & 9136.54485 & 1.03 & 4 \mathrm{e}, \mathrm{a}\end{array}$

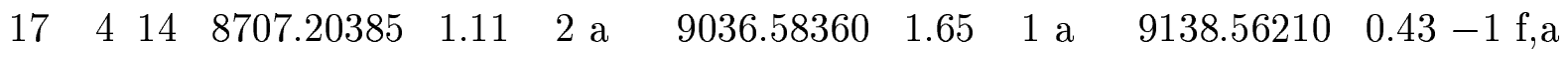

$\begin{array}{llllllllllll}17 & 4 & 13 & 8899.18054 & 0.64 & 3 \mathrm{a} & 9232.80859 & 0.76 & 4 \mathrm{e}, \mathrm{a} & 9353.06641 & 0.49 & -1 \mathrm{f}, \mathrm{a}\end{array}$

$17 \quad 5 \quad 13$

$\begin{array}{llllllllllll}17 & 5 & 12 & 9067.08069 & 0.62 & 5 \mathrm{e}, \mathrm{a} & 9401.97392 & 1.04 & 2 \mathrm{a} & 9516.82335 & 0.76 & 4 \text { e,a }\end{array}$

$\begin{array}{llllllllllll}17 & 6 & 12 & 9235.18868 & 1.01 & 3 \mathrm{a} & 9470.77548 & 1.13 & 1 \mathrm{a} & 9566.66013 & 0.42 & -1 \mathrm{f}, \mathrm{a}\end{array}$

$\begin{array}{llllllllllll}17 & 6 & 11 & 9271.57847 & 0.56 & 7 \mathrm{e}, \mathrm{a} & 9532.51978 & 0.62 & 6 \mathrm{e}, \mathrm{a} & 9631.77520 & 0.76 & 2 \mathrm{a}\end{array}$

$\begin{array}{llllllllllll}17 & 7 & 11 & 9506.01663 & 1.57 & 1 \mathrm{a} & 9689.92800 & 1.49 & 1 \mathrm{a} & 9766.25641 & 0.44 & 6 \mathrm{a}\end{array}$

$\begin{array}{llllllllllll}17 & 7 & 10 & 9511.49152 & 5.03 & 1 \mathrm{a} & 9699.07392 & 1.06 & 2 \mathrm{e}, \mathrm{a} & 9782.86655 & 0.80 & 3 \mathrm{e}, \mathrm{a}\end{array}$

$\begin{array}{llllll}17 & 8 & 10 & 9792.37160 & 7.89 & 1 \mathrm{e}\end{array}$

$\begin{array}{llllllllllll}17 & 8 & 9 & 9792.84160 & 0.79 & 2 \mathrm{a} & 9919.62004 & 0.65 & 4 \mathrm{a} & 9982.26970 & 5.05 & 1 \mathrm{a}\end{array}$

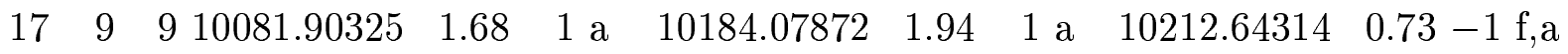

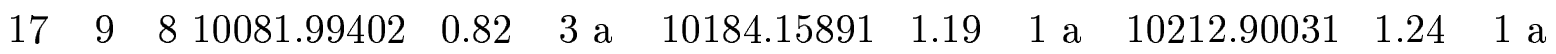

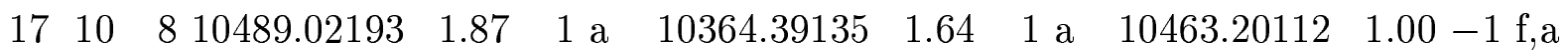

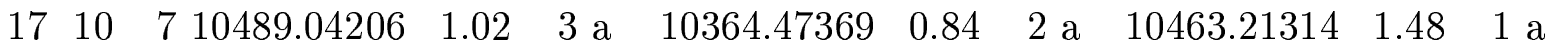

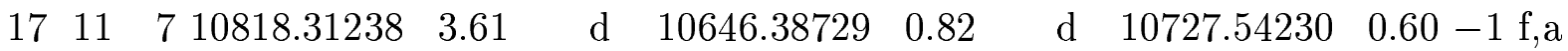

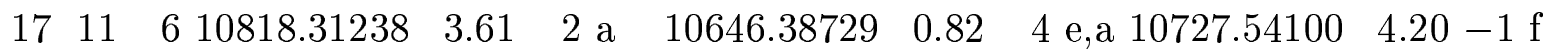

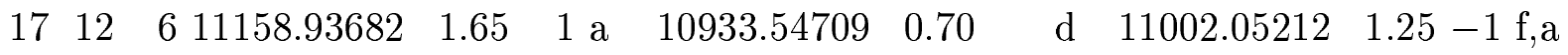

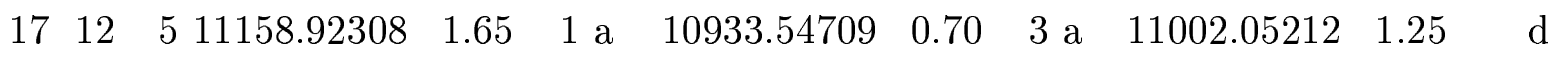

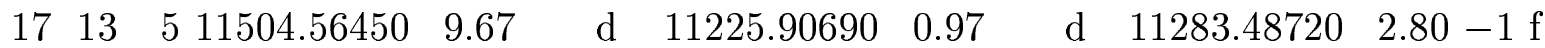

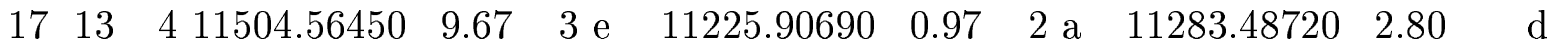

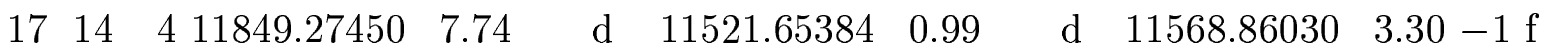

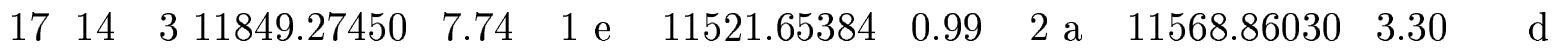

$17 \quad 15 \quad 3$

$17 \quad 15 \quad 2$

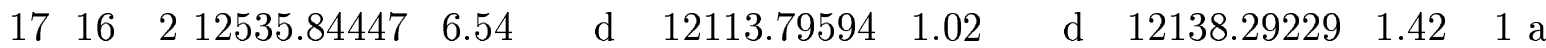

$\begin{array}{llllll}11818.45734 & 1.48 & \mathrm{~d} & 11857.23248 & 1.69 & 1 \mathrm{a}\end{array}$

$11818.45734 \quad 1.48 \quad 1 \mathrm{a} \quad 11857.23248 \quad 1.69 \quad \mathrm{~d}$ 


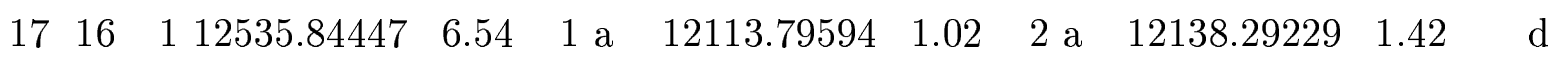
$\begin{array}{llllllllll}17 & 17 & 1 & 12865.13235 & 1.99 & \mathrm{~d} & 12415.71640 & 4.45 & 1 \mathrm{e}\end{array}$

$\begin{array}{lllllllll}17 & 17 & 0 & 12865.13235 & 1.99 & 1 \mathrm{a} & 12415.71640 & 4.45 & \mathrm{~d}\end{array}$

$\begin{array}{llllllllllll}18 & 0 & 18 & 7938.47348 & 1.11 & 1 \mathrm{a} & 8465.07571 & 1.11 & \mathrm{~d} & 8573.55356 & 0.50 & -1 \mathrm{f}, \mathrm{a}\end{array}$

$\begin{array}{llllllllllll}18 & 1 & 18 & 7938.49589 & 0.63 & 12 \mathrm{e}, \mathrm{a} & 8465.07571 & 1.11 & 2 \mathrm{e}, \mathrm{a} & 8573.55356 & 0.50 & \mathrm{~d}\end{array}$

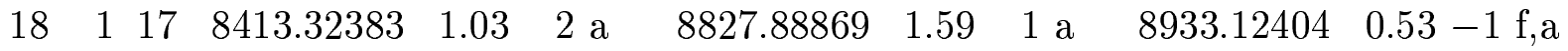

$\begin{array}{llllllllllll}18 & 2 & 17 & 8413.43642 & 0.50 & 5 \mathrm{a} & 8827.92271 & 0.81 & 2 \text { a } & 8933.12404 & 0.53 & \mathrm{~d}\end{array}$

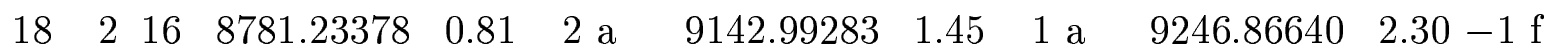

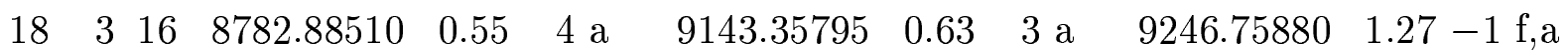

$\begin{array}{llllllllllll}18 & 3 & 15 & 9091.97266 & 1.25 & 1 \mathrm{a} & 9417.65481 & 1.28 & 2 \mathrm{a} & 9522.64715 & 0.49 & -1 \mathrm{f}, \mathrm{a}\end{array}$

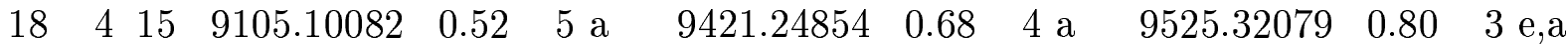

$18 \quad 4 \quad 14$

$9759.05346 \quad 0.60-1 \mathrm{f}, \mathrm{a}$

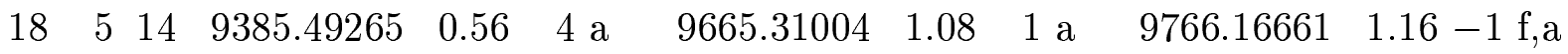

$\begin{array}{llll}18 & 5 & 13\end{array}$

$9944.80509 \quad 0.44-1 \mathrm{f}, \mathrm{a}$

$\begin{array}{llllllllllll}18 & 6 & 13 & 9652.58134 & 1.06 & 1 \mathrm{a} & 9883.58127 & 0.83 & 5 \mathrm{a} & 9980.30973 & 0.78 & 3 \mathrm{a}\end{array}$

$\begin{array}{lll}18 & 6 & 12\end{array}$

$10068.14119 \quad 0.54-1 \mathrm{f}, \mathrm{a}$

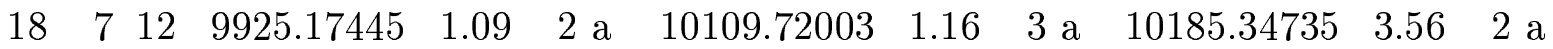

$\begin{array}{llllll}18 & 7 & 11 & 9934.72325 & 1.42 & 1 \mathrm{a}\end{array}$

$10215.10606 \quad 0.53-1 \mathrm{f}, \mathrm{a}$

$\begin{array}{lllllll}18 & 8 & 11 & 10210.04321 & 0.77 & 3 & \mathrm{a}\end{array}$

$10399.52957 \quad 1.26-1 \mathrm{f}, \mathrm{a}$

$18 \quad 8 \quad 10$

$10339.14923 \quad 1.80 \quad 1 \mathrm{a} \quad 10404.52154 \quad 1.04-1 \mathrm{f}, \mathrm{a}$

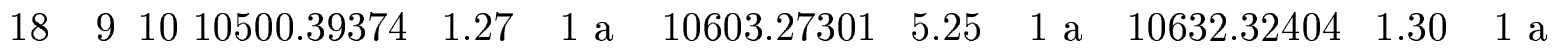

$\begin{array}{llllll}18 & 9 & 9 & 10500.56929 & 7.95 \quad 1 \mathrm{a}\end{array}$

$10632.92770 \quad 0.83-1 \mathrm{f}, \mathrm{a}$

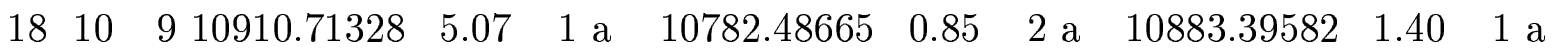

$\begin{array}{llllll}18 & 10 & 8 & 10910.75746 & 1.54 & 2 \mathrm{a}\end{array}$ $10883.46200 \quad 0.80 \quad 2$ a

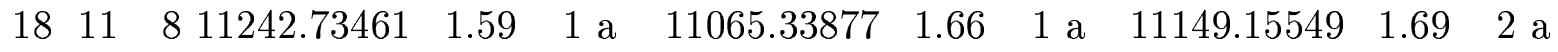
$\begin{array}{lllllllllll}18 & 11 & 711242.73461 & 1.59 & \mathrm{~d} & 11065.35104 & 1.92 & 1 \mathrm{a} & 11149.16524 & 0.89 & 2 \mathrm{a}\end{array}$ $\begin{array}{lllllllllll}18 & 12 & 711586.74736 & 5.25 & 1 \text { a } & 11354.35215 & 0.94 & 2 \text { a } & 11425.99529 & 1.16 & \text { d }\end{array}$ 


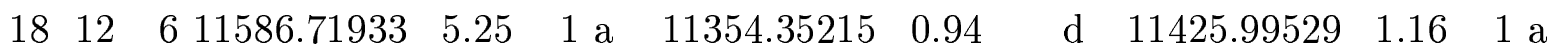

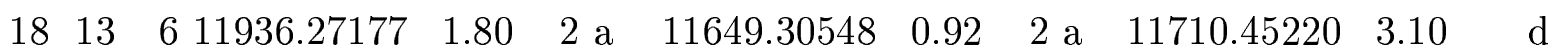

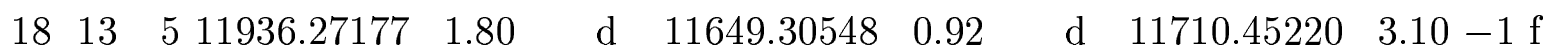

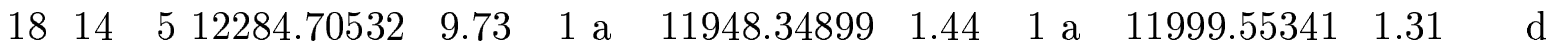

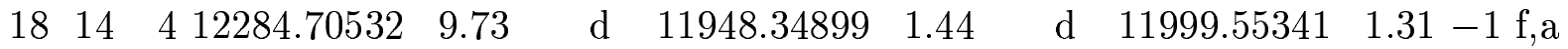

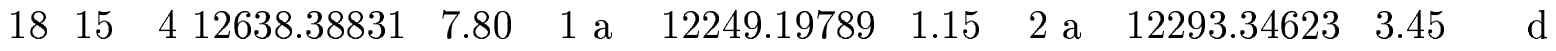

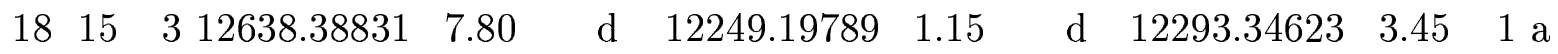

$18 \quad 16 \quad 3$

$12549.37385 \quad 1.17 \quad 2$ a

$18 \quad 16 \quad 2$

$12549.37385 \quad 1.17 \quad \mathrm{~d}$

$\begin{array}{llllllllllll}18 & 17 & 2 & 13321.89639 & 6.61 & 1 \mathrm{a} & 12846.34528 & 1.43 & 1 \mathrm{a} & 12864.67327 & 1.73 & \mathrm{~d}\end{array}$

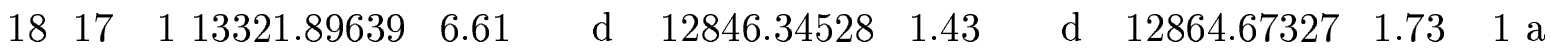

$\begin{array}{llllll}18 & 18 & 1 & 13647.64870 & 2.22 & 1 \mathrm{a}\end{array}$

$13141.04283 \quad 4.56 \quad \mathrm{~d}$

$\begin{array}{llllll}18 & 18 & 0 & 13647.64870 & 2.22 & \mathrm{~d}\end{array}$

$13141.04283 \quad 4.56 \quad 1 \mathrm{a}$

$\begin{array}{llllllllllll}19 & 0 & 19 & 8287.74127 & 0.80 & 5 \mathrm{e}, \mathrm{a} & 8810.40739 & 0.63 & 4 \mathrm{a} & 8920.21768 & 0.49 & \mathrm{~d}\end{array}$

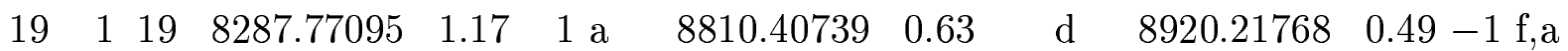

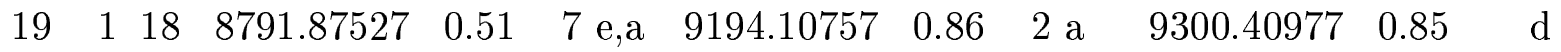

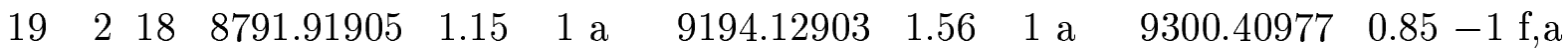

$\begin{array}{llllllllllll}19 & 2 & 17 & 9176.64456 & 0.79 & 2 \mathrm{a} & 9527.07176 & 1.11 & 1 \mathrm{a} & 9631.84807 & 0.86 & 2 \mathrm{a}\end{array}$

$\begin{array}{llllllllllll}19 & 3 & 17 & 9177.63989 & 1.15 & 1 \mathrm{a} & 9527.25161 & 1.28 & 1 \mathrm{a} & 9631.81269 & 1.10 & -1 \mathrm{f}, \mathrm{a}\end{array}$

$\begin{array}{llllllllllll}19 & 3 & 16 & 9509.91976 & 0.63 & 3 \mathrm{a} & 9820.40544 & 0.78 & 4 \mathrm{a} & 9924.88019 & 0.61 & 5 \mathrm{a}\end{array}$

$\begin{array}{lllllllll}19 & 4 & 16 & 9518.29659 & 1.15 & 1 \mathrm{a} & 9924.26327 & 0.56 & -1 \mathrm{f}, \mathrm{a}\end{array}$

$\begin{array}{llllllllllll}19 & 4 & 15 & 9765.52909 & 0.79 & 4 \mathrm{a} & 10069.79637 & 0.78 & 3 \mathrm{a} & 10179.77689 & 0.79 & -1 \mathrm{f}, \mathrm{a}\end{array}$

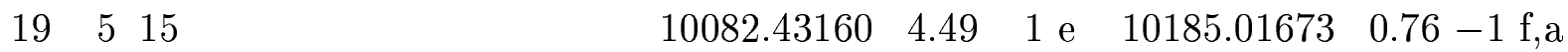

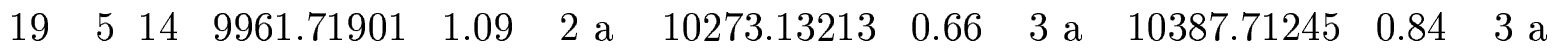

$\begin{array}{lllllll}19 & 6 & 14 & 10412.03251 & 0.62-1 \mathrm{f}, \mathrm{a}\end{array}$

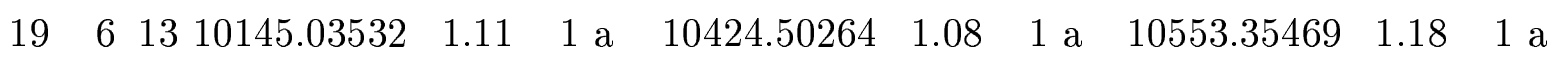

$\begin{array}{lllllll}19 & 7 & 13 & 10623.31139 & 0.55 & 4 \mathrm{a}\end{array}$ 
$\begin{array}{llllll}19 & 7 & 12 & 10380.03431 & 1.46 & 1 \mathrm{a}\end{array}$

$\begin{array}{lll}19 & 8 & 12\end{array}$

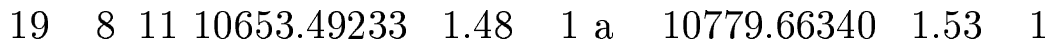

$\begin{array}{lll}19 & 9 & 11\end{array}$

$\begin{array}{lll}19 & 9 & 10\end{array}$

191010

$\begin{array}{lllllllll}19 & 10 & 9 & 11351.89848 & 5.16 & 1 \mathrm{a} & 11220.94010 & 5.83 & 1 \mathrm{e}\end{array}$

$\begin{array}{llllll}19 & 11 & 9 & 11686.24750 & 2.40 & 1 \mathrm{a}\end{array}$

$\begin{array}{lllllllll}19 & 11 & 8 & 11686.28920 & 5.16 & 1 \mathrm{a} & 11504.02864 & 1.31 & 1\end{array}$

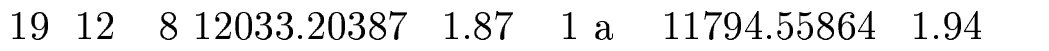

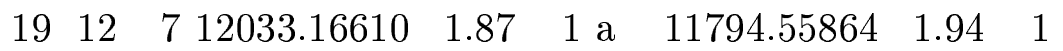

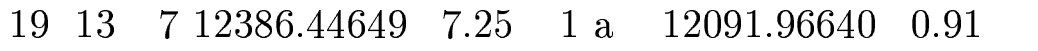

$\begin{array}{lllllllll}19 & 13 & 6 & 12386.44540 & 5.35 & 1 \mathrm{a} & 12091.96640 & 0.91 & 2\end{array}$

$\begin{array}{llllllll}19 & 14 & 6 & 12738.70215 & 5.32 & \text { d } & 12394.08633 & 1.23\end{array}$

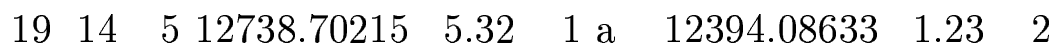

$\begin{array}{llllllll}19 & 15 & 5 & 13098.03429 & 9.78 & \mathrm{~d} & 12698.67695 & 1.76\end{array}$

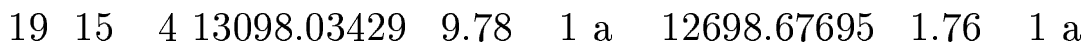

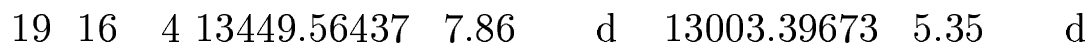

$\begin{array}{lllllllll}19 & 16 & 3 & 13449.56437 & 7.86 & 1 \mathrm{a} & 13003.39673 & 5.35 & 1 \mathrm{a}\end{array}$

$\begin{array}{lll}19 & 17 & 3\end{array}$

$1917 \quad 2$

$\begin{array}{llllllll}19 & 18 & 2 & 14130.40109 & 6.68 & \mathrm{~d} & 13603.23254 & 1.74\end{array}$

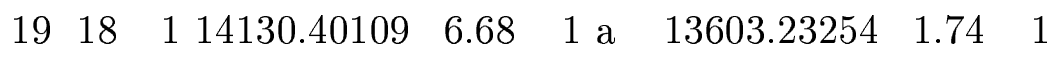

$\begin{array}{llllll}19 & 19 & 1 & 14451.47010 & 2.43 & \mathrm{~d}\end{array}$

$\begin{array}{llllll}19 & 19 & 0 & 14451.47010 & 2.43 \quad 1 \mathrm{a}\end{array}$

$\begin{array}{llllll}20 & 0 & 20 & 8655.01993 & 1.54 & 1 \mathrm{a}\end{array}$

$\begin{array}{llllllllll}20 & 1 & 20 & 8655.20974 & 0.57 & 6 \text { e,a } & 9172.71941 & 0.88 & 2\end{array}$ $13305.73977 \quad 1.54 \quad \mathrm{~d}$ $13305.73977 \quad 1.54 \quad 1 \mathrm{a}$

d

$10669.17373 \quad 1.21 \quad 2 \mathrm{a}$

$10839.611031 .02-1 \mathrm{f}, \mathrm{a}$

$10849.22760 \quad 1.43 \quad 2$ a $11072.36255 \quad 0.64 \quad 5 \mathrm{a}$ $11073.70156 \quad 4.51-1 \mathrm{f}, \mathrm{a}$ $11323.66700 \quad 1.20-1 \mathrm{f}, \mathrm{a}$

$11590.53479 \quad 0.84-1 \mathrm{f}, \mathrm{a}$ $11590.57962 \quad 1.79 \quad 1 \mathrm{a}$ $11869.21090 \quad 2.71-1 \mathrm{f}, \mathrm{a}$ $\begin{array}{lll}11869.21090 & 2.71 \quad \mathrm{~d}\end{array}$ d $\quad 12156.50045 \quad 1.53 \quad 1 \mathrm{a}$ $\begin{array}{lll}12156.50045 & 1.53 \quad \mathrm{~d}\end{array}$ $\begin{array}{ll}12449.32510 & 6.50-1 \mathrm{f}\end{array}$ $\begin{array}{lll}12449.32510 & 6.50 \quad \mathrm{~d}\end{array}$ $\begin{array}{llll}d & 12747.73618 & 1.65 & 1 \mathrm{a}\end{array}$ d

\section{a} $\begin{array}{lll}13890.05575 & 4.67 \quad 1 \mathrm{a}\end{array}$ $\begin{array}{lll}13890.05575 & 4.67 \quad \mathrm{~d}\end{array}$ $9283.92326 \quad 0.50-1 \mathrm{f}, \mathrm{a}$ $\begin{array}{lll}9283.92326 & 0.50 \quad \mathrm{~d}\end{array}$ 


$\begin{array}{lllllllllllll}20 & 1 & 19 & 9189.70484 & 1.17 & 1 \mathrm{a} & 9577.31377 & 1.48 & 1 \mathrm{a} & 9684.73538 & 0.49 & -1 \mathrm{f}, \mathrm{a} \\ 20 & 2 & 19 & 9188.37431 & 0.50 & 5 \mathrm{a} & 9577.32300 & 1.23 & 1 \mathrm{a} & 9684.73538 & 0.49 & \mathrm{~d} \\ 20 & 2 & 18 & 9587.63037 & 1.15 & 1 \mathrm{a} & 9927.65265 & 1.62 & 1 \mathrm{a} & 10033.37631 & 1.22 & -1 \mathrm{f}, \mathrm{a} \\ 20 & 3 & 18 & 9588.25260 & 0.57 & 5 \mathrm{e}, \mathrm{a} & 9927.59591 & 0.89 & 2 \mathrm{a} & 10033.38100 & 5.60 & -1 \mathrm{f} \\ 20 & 3 & 17 & 9942.64727 & 1.19 & 1 \mathrm{a} & & & & 10343.19869 & 0.43 & -1 \mathrm{f}, \mathrm{a} \\ 20 & 4 & 17 & 9948.25360 & 0.66 & 3 \mathrm{a} & 10239.48557 & 1.13 & 1 \mathrm{a} & 10343.09448 & 0.98 & 2 \mathrm{a} \\ 20 & 4 & 16 & 10227.66650 & 1.50 & 1 \mathrm{a} & & & & 10615.46765 & 0.79 & -1 \mathrm{f}, \mathrm{a} \\ 20 & 5 & 16 & 10259.31313 & 0.79 & 2 \mathrm{a} & 10515.26817 & 0.81 & 2 \mathrm{a} & & & \\ 20 & 5 & 15 & & & & & & & 10844.37693 & 0.58 & 6 \mathrm{a} \\ 20 & 6 & 15 & 10542.98437 & 1.08 & 2 \mathrm{a} & 10760.47261 & 1.11 & 4 \mathrm{a} & 10862.07476 & 1.32 & 2 \mathrm{a} \\ 20 & 6 & 14 & 10627.03488 & 1.19 & 1 \mathrm{a} & & & & 11022.10401 & 1.20 & 2 \mathrm{a} \\ 20 & 7 & 14 & 10822.01725 & 0.93 & 2 \mathrm{a} & 10982.95206 & 1.06 & 4 \mathrm{a} & & & \\ 20 & 7 & 13 & 10844.05409 & 1.32 & 2 \mathrm{a} & & & & 11146.45901 & 0.80 & 3 \mathrm{a} \\ 20 & 8 & 13 & & & & & & & & & & \end{array}$




\begin{tabular}{|c|c|c|c|c|c|c|c|c|c|c|}
\hline 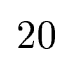 & 15 & 513575.03104 & 7.30 & $\mathrm{~d}$ & 13166.51190 & 1.58 & $\mathrm{~d}$ & 13220.37845 & 6.58 & $1 \mathrm{a}$ \\
\hline & 16 & 513932.23090 & 9.83 & $1 \mathrm{a}$ & 13475.42097 & 1.89 & $2 \mathrm{a}$ & 13515.32059 & 1.93 & $\mathrm{~d}$ \\
\hline & 16 & 413932.23090 & 9.83 & $\mathrm{~d}$ & 13475.42097 & 1.89 & $\mathrm{~d}$ & 13515.32059 & 1.93 & $1 \mathrm{a}$ \\
\hline & 17 & 414284.17858 & 7.92 & $1 \mathrm{a}$ & 13782.86801 & 5.44 & $1 \mathrm{a}$ & & & \\
\hline & 17 & 314284.17858 & 7.92 & $\mathrm{~d}$ & 13782.86801 & 5.44 & $\mathrm{~d}$ & & & \\
\hline & 18 & 3 & & & 14086.20321 & 1.83 & $1 \mathrm{a}$ & & & \\
\hline & 18 & 2 & & & 14086.20321 & 1.83 & $\mathrm{~d}$ & & & \\
\hline & 19 & 214960.13434 & 8.35 & $1 \mathrm{a}$ & 14383.19577 & 2.01 & $1 \mathrm{a}$ & 14390.74535 & 2.24 & d \\
\hline & 19 & 114960.13434 & 8.35 & $\mathrm{~d}$ & 14383.19577 & 2.01 & $d$ & 14390.74535 & 2.24 & $1 \mathrm{a}$ \\
\hline $\mathrm{J}$ & 20 & 115275.49850 & 5.56 & $1 \mathrm{a}$ & & & & 14661.68972 & 6.84 & d \\
\hline J & 20 & 015275.49850 & 5.56 & $\mathrm{~d}$ & & & & 14661.68972 & 6.84 & $1 \mathrm{a}$ \\
\hline & 0 & $21 \quad 9038.83134$ & 0.65 & $4 \mathrm{a}$ & 9551.92577 & 0.90 & $2 \mathrm{a}$ & 9664.57936 & 0.55 & $\mathrm{~d}$ \\
\hline 21 & 1 & $21 \quad 9038.89900$ & 1.64 & $1 \mathrm{a}$ & 9551.56200 & 8.43 & $1 \mathrm{e}$ & 9664.57936 & 0.55 & $-1 \mathrm{f}, \mathrm{a}$ \\
\hline 21 & 1 & $20 \quad 9602.70779$ & 0.61 & $4 \mathrm{a}$ & 9977.43859 & 1.93 & $1 \mathrm{a}$ & 10086.02379 & 0.51 & $\mathrm{~d}$ \\
\hline & 2 & $20 \quad 9602.87233$ & 1.30 & $1 \mathrm{a}$ & & & & 10086.02379 & 0.51 & $-1 \mathrm{f}, \mathrm{a}$ \\
\hline & 2 & 1910013.34041 & 0.78 & $3 \mathrm{a}$ & 10344.12290 & 1.16 & $1 \mathrm{a}$ & 10451.29034 & 0.61 & $\mathrm{~d}$ \\
\hline & 3 & 1910014.07466 & 1.65 & $1 \mathrm{a}$ & & & & 10451.29034 & 0.61 & $-1 \mathrm{f}, \mathrm{a}$ \\
\hline & 3 & 1810390.24767 & 0.81 & $2 \mathrm{a}$ & 10672.15173 & 1.11 & $1 \mathrm{a}$ & 10777.50107 & 1.07 & $2 \mathrm{a}$ \\
\hline & 4 & 1810393.83698 & 1.19 & $1 \mathrm{a}$ & & & & 10777.46108 & 0.50 & $-1 \mathrm{f}, \mathrm{a}$ \\
\hline & 4 & 1710697.27063 & 1.28 & $1 \mathrm{a}$ & 10959.25951 & 0.71 & $3 \mathrm{a}$ & 11066.55986 & 1.25 & $1 \mathrm{a}$ \\
\hline & 5 & 17 & & & & & & 11066.47716 & 0.58 & $5 \mathrm{a}$ \\
\hline & 5 & 1610929.88477 & 1.63 & $1 \mathrm{a}$ & & & & 11314.53080 & 1.26 & $1 \mathrm{a}$ \\
\hline & 6 & 16 & & & & & & 11319.80826 & 1.12 & $4 \mathrm{a}$ \\
\hline & 6 & 1511117.75787 & 1.28 & $1 \mathrm{a}$ & & & & 11511.02992 & 0.83 & $2 \mathrm{a}$ \\
\hline & 7 & 15 & & & & & & 11554.53719 & 0.64 & $5 \mathrm{a}$ \\
\hline & 7 & 1411337.94700 & 1.46 & $1 \mathrm{a}$ & & & & 11644.76125 & 1.53 & $2 \mathrm{a}$ \\
\hline & 8 & 14 & & & & & & 11778.33564 & 0.72 & 5 \\
\hline
\end{tabular}




\begin{tabular}{|c|c|c|c|c|c|c|c|c|c|c|}
\hline 21 & 8 & 13 & & & & & & 11806.54901 & 4.90 & $1 \mathrm{a}$ \\
\hline 21 & 9 & 13 & & & & & & 12011.83602 & 1.33 & $3 \mathrm{a}$ \\
\hline . & 9 & 12 & & & & & & 12017.08585 & 1.64 & $1 \mathrm{a}$ \\
\hline & 10 & 12 & & & & & & 12262.89006 & 1.34 & $1 \mathrm{a}$ \\
\hline & 11 & 11 & & & & & & 12531.02181 & 1.46 & $-1 \mathrm{f}, \mathrm{a}$ \\
\hline & 12 & 10 & & & & & & 12812.67713 & 1.62 & $-1 \mathrm{f}, \mathrm{a}$ \\
\hline & 12 & 912980.39943 & 6.33 & $1 \mathrm{a}$ & & & & 12812.67470 & 11.80 & $-1 \mathrm{f}$ \\
\hline & 13 & 913340.23827 & 5.63 & $1 \mathrm{a}$ & & & & & & \\
\hline 21 & 13 & 813340.25440 & 5.35 & $1 \mathrm{a}$ & 13033.45528 & 1.93 & $1 \mathrm{a}$ & & & \\
\hline 21 & 14 & 813700.02250 & 2.33 & $\mathrm{~d}$ & & & & & & \\
\hline 21 & 14 & 713700.02250 & 2.33 & $1 \mathrm{a}$ & 13340.84640 & 1.94 & $1 \mathrm{a}$ & & & \\
\hline 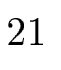 & 15 & 714069.04037 & 8.86 & $\mathrm{~d}$ & 13652.23423 & 1.43 & $\mathrm{~d}$ & & & \\
\hline 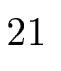 & 15 & 614069.04037 & 8.86 & $1 \mathrm{a}$ & 13652.23423 & 1.43 & $2 \mathrm{a}$ & & & \\
\hline 1 & 16 & 614431.36209 & 8.85 & $\mathrm{~d}$ & & & & 14009.88960 & 8.26 & $1 \mathrm{a}$ \\
\hline 21 & 16 & 514431.36209 & 8.85 & $1 \mathrm{a}$ & & & & 14009.88960 & 8.26 & $\mathrm{~d}$ \\
\hline 21 & 17 & 514789.56904 & 11.02 & $\mathrm{~d}$ & & & & 14313.08891 & 2.17 & $1 \mathrm{a}$ \\
\hline 21 & 17 & 414789.56904 & 11.02 & $1 \mathrm{a}$ & 14277.11401 & 2.13 & $1 \mathrm{a}$ & 14313.08891 & 2.17 & $\mathrm{~d}$ \\
\hline . & 18 & 415140.76413 & 9.37 & $\mathrm{~d}$ & 14586.11549 & 7.39 & $\mathrm{~d}$ & & & \\
\hline 1 & 18 & 315140.76413 & 9.37 & $1 \mathrm{a}$ & 14586.11549 & 7.39 & $1 \mathrm{a}$ & & & \\
\hline 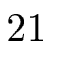 & 19 & 3 & & & 14889.43620 & 5.33 & $\mathrm{~d}$ & & & \\
\hline . & 19 & 2 & & & 14889.43620 & 5.33 & $1 \mathrm{a}$ & & & \\
\hline 21 & 20 & 215809.96632 & 9.73 & $\mathrm{~d}$ & 15185.02377 & 5.39 & $\mathrm{~d}$ & 15188.16991 & 2.45 & $1 \mathrm{a}$ \\
\hline 21 & 20 & 115809.96632 & 9.73 & $1 \mathrm{a}$ & 15185.02377 & 5.39 & $1 \mathrm{a}$ & 15188.16991 & 2.45 & $\mathrm{~d}$ \\
\hline 21 & 21 & 116118.65998 & 7.47 & $\mathrm{~d}$ & & & & 15454.97113 & 8.47 & $1 \mathrm{a}$ \\
\hline 21 & 21 & 016118.65998 & 7.47 & $1 \mathrm{a}$ & & & & 15454.97113 & 8.47 & $\mathrm{~d}$ \\
\hline 22 & 0 & 22 & & & & & & 10062.09289 & 0.52 & $-1 \mathrm{f}, \mathrm{a}$ \\
\hline 22 & 1 & $22 \quad 9441.92379$ & 0.85 & 3 e,a & 9947.93700 & 0.90 & $2 \mathrm{a}$ & 10062.09289 & 0.52 & d \\
\hline
\end{tabular}


$22 \quad 1 \quad 2110035.39367 \quad 1.51 \quad 1 \mathrm{a}$

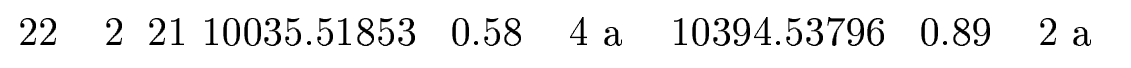

$22 \quad 2 \quad 20$

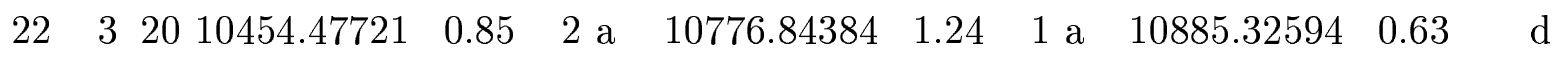

$\begin{array}{lll}22 & 3 & 19\end{array}$

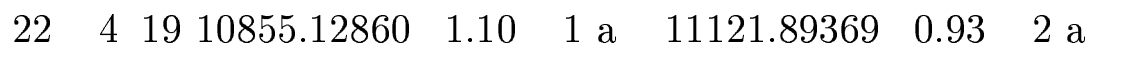

22418

$\begin{array}{llllll}22 & 5 & 18 & 11195.48826 & 1.12 & 1 \mathrm{a}\end{array}$

$\begin{array}{lll}22 & 5 & 17\end{array}$

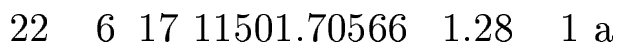

$22 \quad 6 \quad 16$

$22 \quad 7 \quad 15$

$22 \quad 8 \quad 15$

$22 \quad 814$

$\begin{array}{lll}22 & 9 & 13\end{array}$

$22 \quad 1012$

221111

$22 \quad 1210$

$22 \quad 1310$

$22 \quad 13 \quad 9$

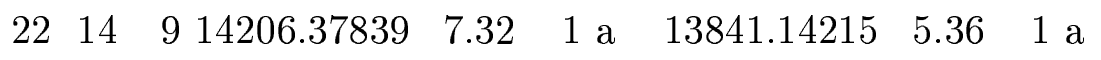

$\begin{array}{llllll}22 & 14 & 8 & 14206.37839 & 7.32 & \mathrm{~d}\end{array}$

\section{$2215 \quad 8$}

$22 \quad 15 \quad 7$

$2217 \quad 6$

$2217 \quad 5$

$2218 \quad 5$
$10504.15926 \quad 0.52-1$ f,a

$10885.32594 \quad 0.63-1$ f,a

$11227.65603 \quad 1.32 \quad 1 \mathrm{a}$

$11227.63380 \quad 1.47 \quad 1 \mathrm{a}$

$11532.08529 \quad 0.87-1 \mathrm{f}, \mathrm{a}$

$11532.20643 \quad 1.30 \quad 1 \mathrm{a}$

$11798.28487 \quad 0.69 \quad 3$ a

$11802.11464 \quad 1.22 \quad 2$ a

$12015.52821 \quad 0.85 \quad 4 \mathrm{a}$

$12160.48988 \quad 0.80 \quad 4 \mathrm{a}$

$12275.48186 \quad 1.88 \quad 1 \mathrm{a}$

$12318.89969 \quad 1.21 \quad 3 \mathrm{a}$

$12519.83844 \quad 2.94 \quad 3$ a

$12762.70414 \quad 5.07 \quad 1 \mathrm{a}$

$13031.51800 \quad 6.21 \quad 1 \mathrm{e}$

$13314.29560 \quad 4.33 \quad 1 \mathrm{e}$

$13605.80588 \quad 5.26 \quad \mathrm{~d}$

$13605.80588 \quad 5.26 \quad 1$ a
$10504.15926 \quad 0.52 \quad \mathrm{~d}$ 


\begin{tabular}{|c|c|c|c|c|c|c|c|c|c|c|}
\hline 22 & 18 & 4 & & & & & & 15135.02799 & 5.45 & $1 \mathrm{a}$ \\
\hline 22 & 19 & 4 & & & 15411.83470 & 8.92 & $1 \mathrm{a}$ & & & \\
\hline & 19 & 3 & & & 15411.83470 & 8.92 & $\mathrm{~d}$ & & & \\
\hline & 20 & 3 & & & 15714.21121 & 7.30 & $1 \mathrm{a}$ & & & \\
\hline & 20 & 2 & & & 15714.21121 & 7.30 & $\mathrm{~d}$ & & & \\
\hline & 21 & 216678.84045 & 10.94 & $1 \mathrm{a}$ & 16007.53275 & 7.35 & $1 \mathrm{a}$ & 16007.14899 & 5.57 & $\mathrm{~d}$ \\
\hline & 21 & 116678.84045 & 10.94 & $\mathrm{~d}$ & 16007.53275 & 7.35 & $\mathrm{~d}$ & 16007.14899 & 5.57 & $1 \mathrm{a}$ \\
\hline 22 & 22 & 116980.11113 & 8.99 & $1 \mathrm{a}$ & & & & 16269.01406 & 9.84 & $\mathrm{~d}$ \\
\hline 22 & 22 & 016980.11113 & 8.99 & $\mathrm{~d}$ & & & & 16269.01406 & 9.84 & $1 \mathrm{a}$ \\
\hline 23 & 0 & $23 \quad 9863.01871$ & 0.75 & $4 \mathrm{e}, \mathrm{a}$ & 10360.70111 & 0.90 & $2 \mathrm{a}$ & 10476.37460 & 0.50 & d \\
\hline 23 & 1 & 23 & & & & & & 10476.37460 & 0.50 & $-1 \mathrm{f}, \mathrm{a}$ \\
\hline 23 & 1 & 2210486.28367 & 0.70 & $3 \mathrm{a}$ & 10828.19129 & 0.75 & $3 \mathrm{a}$ & 10939.14210 & 0.64 & $\mathrm{~d}$ \\
\hline 23 & 2 & 2210486.40925 & 1.46 & $1 \mathrm{a}$ & & & & 10939.14210 & 0.64 & $-1 \mathrm{f}, \mathrm{a}$ \\
\hline 23 & 2 & 2110907.60765 & 1.14 & $1 \mathrm{a}$ & 11225.26852 & 1.59 & $1 \mathrm{a}$ & 11335.15402 & 0.67 & $\mathrm{~d}$ \\
\hline 23 & 3 & 21 & & & & & & 11335.15402 & 0.67 & $-1 \mathrm{f}, \mathrm{a}$ \\
\hline 23 & 3 & 2011330.26030 & 1.05 & $2 \mathrm{a}$ & 11586.33108 & 1.22 & $1 \mathrm{a}$ & 11693.60827 & 1.52 & $1 \mathrm{a}$ \\
\hline 3 & 4 & 20 & & & & & & 11693.50706 & 0.91 & $2 \mathrm{a}$ \\
\hline 3 & 4 & 19 & & & & & & 12013.56337 & 1.68 & $2 \mathrm{a}$ \\
\hline 3 & 5 & 19 & & & & & & 12013.64077 & 1.09 & $2 \mathrm{a}$ \\
\hline 3 & 5 & 18 & & & & & & 12295.81746 & 1.38 & $2 \mathrm{a}$ \\
\hline 3 & 6 & 18 & & & & & & 12298.03414 & 0.86 & $4 \mathrm{a}$ \\
\hline 3 & 6 & 1712167.38602 & 5.12 & $1 \mathrm{a}$ & & & & 12533.21417 & 1.67 & $1 \mathrm{a}$ \\
\hline 3 & 7 & 17 & & & & & & 12553.29387 & 1.33 & $2 \mathrm{a}$ \\
\hline 3 & 8 & 16 & & & & & & 12790.17282 & 0.91 & $5 \mathrm{a}$ \\
\hline 3 & 9 & 15 & & & & & & 13027.25943 & 1.25 & $3 \mathrm{a}$ \\
\hline 2 & 10 & 14 & & & & & & 13278.06325 & 5.07 & $1 \mathrm{a}$ \\
\hline 23 & 12 & 12 & & & & & & 13831.43758 & 7.97 & $1 \mathrm{a}, ?$ \\
\hline
\end{tabular}


$\begin{array}{llllll}23 & 15 & 9 & 15105.63259 & 8.87 & \text { d }\end{array}$

$\begin{array}{llllll}23 & 15 & 8 & 15105.63259 & 8.87 & 1 \mathrm{a}\end{array}$

$23 \quad 18 \quad 6$

$23 \quad 18 \quad 5$

$23 \quad 19 \quad 5$

$23 \quad 19 \quad 4$

$23 \quad 20 \quad 4$

$\begin{array}{lll}23 & 20 & 3\end{array}$

$\begin{array}{llllll}23 & 22 & 2 & 17565.76840 & 12.03 \quad \mathrm{~d}\end{array}$

$\begin{array}{llllll}23 & 22 & 1 & 17565.76840 & 12.03 \quad 1 \mathrm{a}\end{array}$

$\begin{array}{lllll}23 & 23 & 1 & 17858.89783 & 10.29 \quad \mathrm{~d}\end{array}$

$\begin{array}{lllll}23 & 23 & 0 & 17858.89783 & 10.29 \quad 1 \text { a }\end{array}$

$\begin{array}{llllll}24 & 0 & 24 & 10302.69639 & 1.06 & d\end{array}$

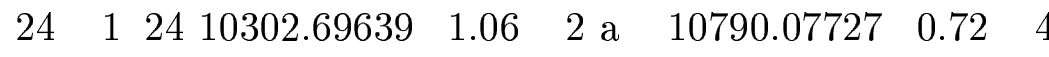

$24 \quad 12310955.70087 \quad 1.56 \quad 1 \mathrm{a}$

$\begin{array}{lllllll}24 & 2 & 23 & 10955.91507 & 0.85 & 2 & \mathrm{a}\end{array}$

$24 \quad 2 \quad 22$

$\begin{array}{lllllll}24 & 3 & 22 & 11376.36304 & 1.14 & 1 \mathrm{a}\end{array}$

$24 \quad 3 \quad 21$

$24 \quad 4 \quad 21$

$24 \quad 420$

$24 \quad 5 \quad 19$

$\begin{array}{lll}24 & 6 & 19\end{array}$

$24 \quad 618$

$24 \quad 7 \quad 17$

24816
$15672.9981910 .87 \quad 1$ a

$15672.9981910 .87 \quad \mathrm{~d}$ $15979.91152 \quad 7.40 \quad 1 \mathrm{a}$ $15979.91152 \quad 7.40 \quad \mathrm{~d}$ $16258.7515110 .23 \quad \mathrm{~d}$ $16258.75151 \quad 10.23 \quad 1 \mathrm{a}$

$\begin{array}{llllll}16849.61612 & 8.89 & d & 16846.64634 & 7.48 & 1 \text { a }\end{array}$ $\begin{array}{llllll}16849.61612 & 8.89 & 1 \text { a } & 16846.64634 & 7.48 & \text { d }\end{array}$ $\begin{array}{lll}17103.01263 & 9.89 & 1 \mathrm{a}\end{array}$ $17103.01263 \quad 9.89 \quad \mathrm{~d}$ $\begin{array}{lllll}10790.07727 & 0.72 \quad d \quad 10907.34605 & 0.87-1 & \mathrm{f}, \mathrm{a}\end{array}$ $10907.34605 \quad 0.87 \quad \mathrm{~d}$ $11390.79706 \quad 0.91-1 \mathrm{f}, \mathrm{a}$ $\begin{array}{lllllll}11278.63578 & 1.01 & 2 & \text { a } & 11390.79706 & 0.91 & \text { d }\end{array}$ $11800.40581 \quad 0.80-1 \mathrm{f}, \mathrm{a}$ $11800.40581 \quad 0.80 \quad \mathrm{~d}$ $12174.85823 \quad 5.09 \quad 1 \mathrm{a}$ $\begin{array}{llllll}12066.94816 & 1.58 & 1 \mathrm{a} & 12174.85694 & 1.47 & 1 \mathrm{a}\end{array}$ $12509.31329 \quad 0.87 \quad 3$ a $12807.20796 \quad 1.24 \quad 1 \mathrm{a}$ $12808.33409 \quad 2.27 \quad 1 \mathrm{a}$ $13063.58830 \quad 5.08 \quad 1 \mathrm{a}$ $13271.08473 \quad 5.08 \quad 1 \mathrm{a}$ $13424.43959 \quad 1.61 \quad 2$ a 


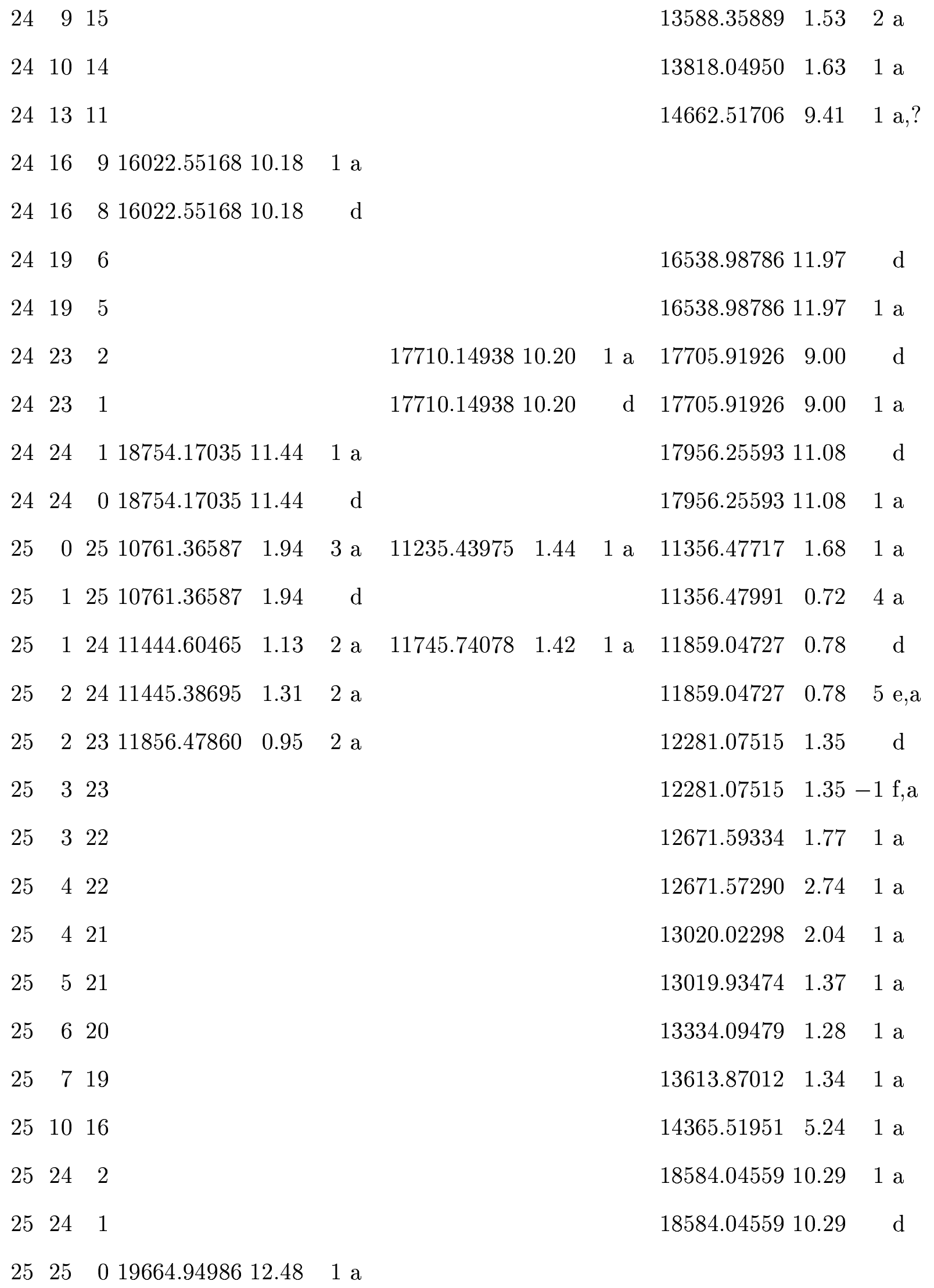




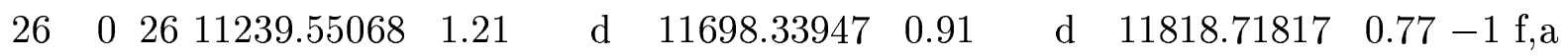

$\begin{array}{llllllllllll}26 & 1 & 26 & 11239.55068 & 1.21 & 3 \mathrm{a} & 11698.33947 & 0.91 & 3 \mathrm{a} & 11818.71817 & 0.77 & \mathrm{~d}\end{array}$ $26 \quad 125$

$12343.92097 \quad 0.89-1 \mathrm{f}, \mathrm{a}$

$26 \quad 2 \quad 25$

$12343.92097 \quad 0.89 \quad \mathrm{~d}$

$26 \quad 2 \quad 24$

$12777.75516 \quad 1.43 \quad 2$ a

$\begin{array}{llllll}26 & 3 & 24 & 12350.22210 & 0.95 \quad 2 & \mathrm{a}\end{array}$

$12777.75516 \quad 1.43 \quad \mathrm{~d}$

$26 \quad 323$

$13183.45127 \quad 2.92 \quad 1 \mathrm{a}$

$26 \quad 422$

$13545.29549 \quad 2.30 \quad 2$ a

$\begin{array}{lll}26 & 5 & 22\end{array}$

$13545.29549 \quad 2.30 \quad \mathrm{~d}$

$\begin{array}{lll}26 & 7 & 19\end{array}$

$14404.76097 \quad 1.35 \quad 1 \mathrm{a}$

$26 \quad 25 \quad 2$

$19480.1960911 .45 \quad \mathrm{~d}$

$26 \quad 25 \quad 1$

$19480.1960911 .45 \quad 1 \mathrm{a}$

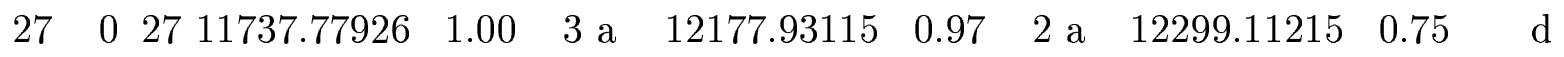

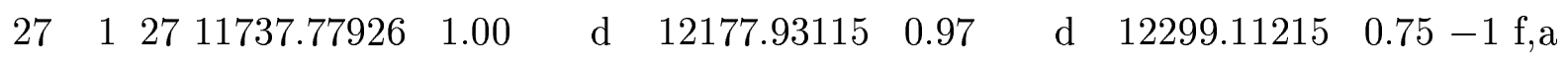

$27 \quad 126$

$\begin{array}{lll}12845.22975 & 0.91 \quad \mathrm{~d}\end{array}$

$\begin{array}{lll}27 & 2 & 26\end{array}$

$12845.22975 \quad 0.91-1 \mathrm{f}, \mathrm{a}$

$\begin{array}{lll}27 & 2 & 25\end{array}$

$13290.57118 \quad 2.06 \quad \mathrm{~d}$

$\begin{array}{lll}27 & 3 & 25\end{array}$

$\begin{array}{lll}13290.57118 & 2.06 \quad 2 \mathrm{a}\end{array}$

$27 \quad 424$

$\begin{array}{lll}13710.07970 & 1.36 & 1 \mathrm{a}\end{array}$

$\begin{array}{lll}27 & 5 & 23\end{array}$

$\begin{array}{lll}14084.87620 & 3.73 \quad 2 \text { a }\end{array}$

$\begin{array}{lll}27 & 26 & 2\end{array}$

$20393.57577 \quad 12.49 \quad 1 \mathrm{a}$

$\begin{array}{lll}27 & 26 & 1\end{array}$

$20393.5757712 .49 \quad \mathrm{~d}$

$\begin{array}{llllll}28 & 0 & 28 & 12256.52101 & 0.93 & \text { d }\end{array}$

$12795.90223 \quad 0.75-1 \mathrm{f}, \mathrm{a}$

$\begin{array}{llllll}28 & 1 & 28 & 12256.52101 & 0.93 & 3\end{array}$

$12795.90223 \quad 0.75 \quad \mathrm{~d}$

$\begin{array}{lll}28 & 127\end{array}$

$13363.07168 \quad 1.35-1 \mathrm{f}, \mathrm{a}$

$\begin{array}{lll}28 & 2 & 27\end{array}$

$\begin{array}{lll}13363.07168 & 1.35 \quad \mathrm{~d}\end{array}$

$\begin{array}{lll}28 & 2 & 26\end{array}$

$13818.60815 \quad 5.32 \quad 1 \mathrm{a}$ 


\begin{tabular}{llllrrrr}
28 & 3 & 26 & 13818.60815 & 5.32 & $\mathrm{~d}$ \\
29 & 0 & 29 & 13309.33058 & 1.41 & $\mathrm{~d}$ \\
29 & 1 & 29 & 13309.33058 & 1.41 & $-1 \mathrm{f}, \mathrm{a}$ \\
29 & 1 & 28 & 13897.13085 & 5.06 & $\mathrm{~d}$ \\
29 & 2 & 28 & 13897.13085 & $5.06-1 \mathrm{f}, \mathrm{a}$ \\
30 & 0 & 30 & 13837.64289 & 2.48 & $-1 \mathrm{f}, \mathrm{a}$ \\
30 & 1 & 30 & 13837.64289 & 2.48 & $\mathrm{~d}$ \\
30 & 1 & 29 & 14445.75560 & 1.65 & $1 \mathrm{a}$ \\
30 & 2 & 29 & 14445.75560 & 1.65 & $\mathrm{~d}$ \\
\hline
\end{tabular}


TABLE V.: Term values for the $2 \nu$ polyad of $\mathrm{H}_{2}{ }^{16} \mathrm{O}$.

\begin{tabular}{|c|c|c|c|c|c|c|c|c|}
\hline \multicolumn{3}{|c|}{$J K_{a} K_{c}$} & \multicolumn{2}{|c|}{040 or 004} & \multicolumn{2}{|c|}{120 or $10^{+} 2$} & \multicolumn{2}{|c|}{021 or $10^{-} 2$} \\
\hline 0 & 0 & 0 & 6134.01482 & $0.40-1 \mathrm{j}$ & 6775.09297 & $0.10-1 \mathrm{j}$ & 6871.52035 & $0.14-1 \mathrm{j}, \mathrm{a}$ \\
\hline 1 & 0 & 1 & 6157.75249 & $0.08-1 \mathrm{j}, \mathrm{a}$ & 6798.51896 & $0.05-1 \mathrm{j}$ & 6895.14778 & $0.15-1 \mathrm{j}, \mathrm{a}$ \\
\hline 1 & 1 & 1 & 6194.79360 & $0.40-1 \mathrm{j}$ & 6818.31085 & $0.40-1 \mathrm{j}$ & 6913.66819 & $0.08-1 \mathrm{j}, \mathrm{a}$ \\
\hline 1 & 1 & 0 & 6201.06682 & $0.19-1 \mathrm{j}, \mathrm{a}$ & 6824.11748 & $0.05-1 \mathrm{j}$ & 6919.57157 & $0.05-1 \mathrm{j}, \mathrm{a}$ \\
\hline 2 & 0 & 2 & 6204.44363 & $0.19-1 \mathrm{j}, \mathrm{a}$ & 6844.23519 & $0.05-1 \mathrm{j}$ & 6941.16296 & $0.04-1 \mathrm{j}, \mathrm{a}$ \\
\hline 2 & 1 & 2 & 6236.05916 & $0.22-1 \mathrm{j}, \mathrm{a}$ & 6859.37401 & $0.10-1 \mathrm{j}$ & 6955.08587 & $0.05-1 \mathrm{j}, \mathrm{a}$ \\
\hline 2 & 1 & 1 & 6254.85487 & $0.05-1 \mathrm{j}, \mathrm{a}$ & 6876.76046 & $0.05-1 \mathrm{j}$ & 6972.73796 & $0.04-1 \mathrm{j}, \mathrm{a}$ \\
\hline 2 & 2 & 1 & 6359.04798 & $0.32-1 \mathrm{j}, \mathrm{a}$ & 6935.00618 & $0.07-1 \mathrm{j}, \mathrm{a}$ & 7027.35021 & $0.10-1 \mathrm{j}, \mathrm{a}$ \\
\hline 2 & 2 & 0 & 6359.78195 & $0.46-1 \mathrm{j}, \mathrm{a}$ & 6936.07676 & $0.06-1 \mathrm{j}$ & 7028.54944 & $0.04-1 \mathrm{j}, \mathrm{a}$ \\
\hline 3 & 0 & 3 & 6272.62857 & $0.09-1 \mathrm{j}, \mathrm{a}$ & 6910.24989 & $0.09-1 \mathrm{j}$ & 7007.42871 & $0.10-1 \mathrm{j}, \mathrm{a}$ \\
\hline 3 & 1 & 3 & 6297.47336 & $0.40-1 \mathrm{j}$ & 6920.29347 & $0.09-1 \mathrm{j}$ & 7016.72574 & $0.04-1 \mathrm{j}, \mathrm{a}$ \\
\hline 3 & 1 & 2 & 6334.89381 & $0.31-1 \mathrm{j}, \mathrm{a}$ & 6954.83343 & $0.05-1 \mathrm{j}, \mathrm{a}$ & 7051.49003 & $0.06-1 \mathrm{j}, \mathrm{a}$ \\
\hline 3 & 2 & 2 & 6430.62122 & $0.20-1 \mathrm{j}, \mathrm{a}$ & 7005.35052 & $0.06-1 \mathrm{j}$ & 7098.06992 & $0.05-1 \mathrm{j}, \mathrm{a}$ \\
\hline 3 & 2 & 1 & 6434.18854 & $0.19-1 \mathrm{j}, \mathrm{a}$ & 7010.23276 & $0.07-1 \mathrm{j}, \mathrm{a}$ & 7103.61322 & $0.10-1 \mathrm{j}, \mathrm{a}$ \\
\hline 3 & 3 & 1 & 6604.31943 & $0.10-1 \mathrm{j}$ & 7114.61172 & $0.07-1 \mathrm{j}$ & 7201.79316 & $0.21-1 \mathrm{j}, \mathrm{a}$ \\
\hline 3 & 3 & 0 & 6604.37842 & $0.39-1 \mathrm{j}, \mathrm{a}$ & 7114.63500 & $0.09-1 \mathrm{j}, \mathrm{a}$ & 7201.95274 & $0.10-1 \mathrm{j}, \mathrm{a}$ \\
\hline 4 & 0 & 4 & 6360.50922 & $0.42-1 \mathrm{j}, \mathrm{a}$ & 6994.57575 & $0.09-1 \mathrm{j}, \mathrm{a}$ & 7091.91860 & $0.09-1 \mathrm{j}, \mathrm{a}$ \\
\hline 4 & 1 & 4 & 6378.56143 & $0.18-1 \mathrm{j}, \mathrm{a}$ & 7000.45122 & $0.08-1 \mathrm{j}, \mathrm{a}$ & 7096.72208 & $0.08-1 \mathrm{j}, \mathrm{a}$ \\
\hline 4 & 1 & 3 & 6440.39401 & $1.11 \quad 1 \mathrm{a}$ & 7057.11848 & $0.12-1 \mathrm{j}$ & 7154.47825 & $0.11-1 \mathrm{j}, \mathrm{a}$ \\
\hline 4 & 2 & 3 & 6525.29910 & $0.21-1 \mathrm{j}, \mathrm{a}$ & 7098.14619 & $0.09-1 \mathrm{j}, \mathrm{a}$ & 7191.37356 & $0.08-1 \mathrm{j}, \mathrm{a}$ \\
\hline 4 & 2 & 2 & 6535.45725 & $0.15-1 \mathrm{j}, \mathrm{a}$ & 7112.63971 & $0.18-1 \mathrm{j}$ & 7205.41955 & $0.07-1 \mathrm{j}, \mathrm{a}$ \\
\hline 4 & 3 & 2 & 6701.47936 & $0.30-1 \mathrm{j}, \mathrm{a}$ & 7211.74696 & $0.20-1 \mathrm{j}, \mathrm{a}$ & 7298.39153 & $0.09-1 \mathrm{j}, \mathrm{a}$ \\
\hline 4 & 3 & 1 & 6701.88407 & $0.23-1 \mathrm{j}, \mathrm{a}$ & 7211.44360 & $0.80-1 \mathrm{j}$ & 7299.46107 & $0.07-1 \mathrm{j}, \mathrm{a}$ \\
\hline 4 & 4 & 1 & 6919.93672 & $0.35-1 \mathrm{j}, \mathrm{a}$ & 7354.09523 & $0.15-1 \mathrm{j}, \mathrm{a}$ & 7435.15543 & $0.15-1 \mathrm{j}$ \\
\hline
\end{tabular}


$\begin{array}{lllllllll}4 & 4 & 0 & 6919.94144 & 0.50-1 \mathrm{j} & 7354.10723 & 0.18-1 \mathrm{j} & 7435.17337 & 0.07-1 \mathrm{j}, \mathrm{a}\end{array}$

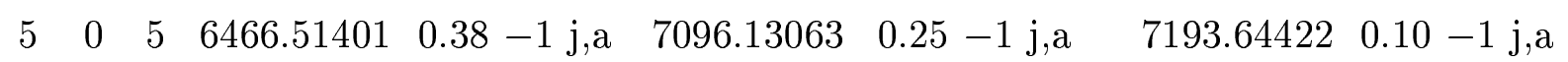

$\begin{array}{lllllllllll}5 & 1 & 5 & 6478.79941 & 0.14 & -1 & \mathrm{j}, \mathrm{a} & 7099.26985 & 0.20-1 \mathrm{j} & 7196.14190 & 0.15-1 \mathrm{j}, \mathrm{a}\end{array}$

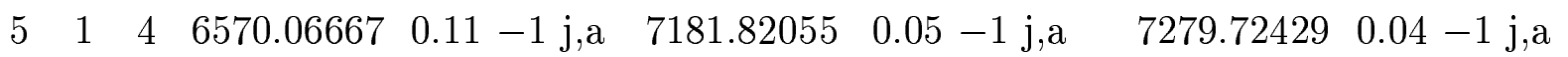

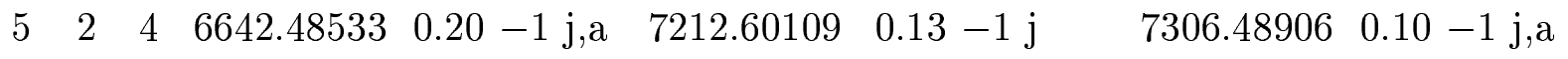

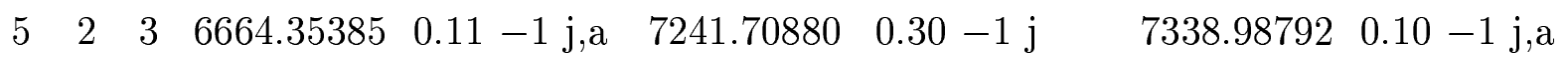

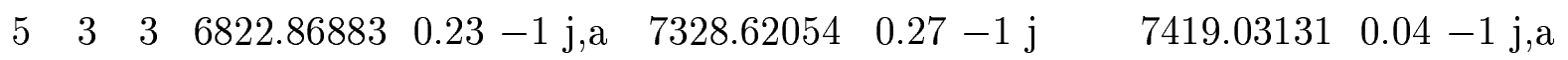

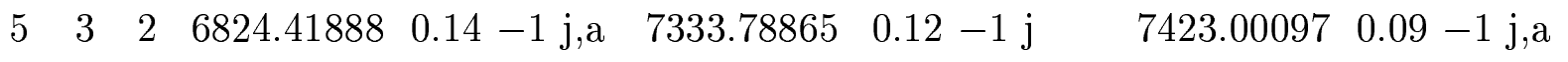

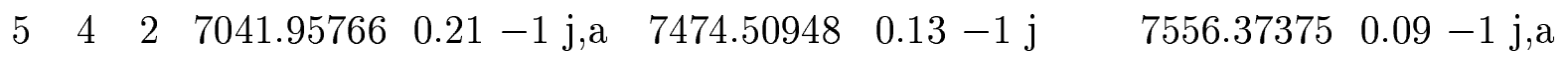

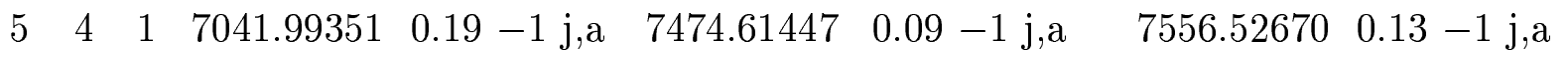

$\begin{array}{lllllllllll}5 & 5 & 1 & 7295.48626 & 0.58 & \mathrm{~d} & 7650.32973 & 0.13-1 \mathrm{j} & 7723.60395 & 0.10-1 \mathrm{j}, \mathrm{a}\end{array}$

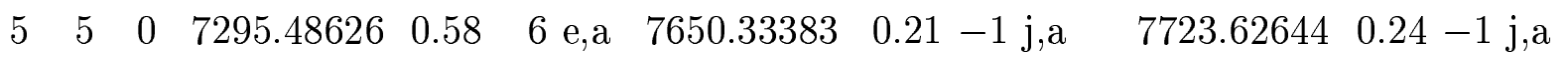

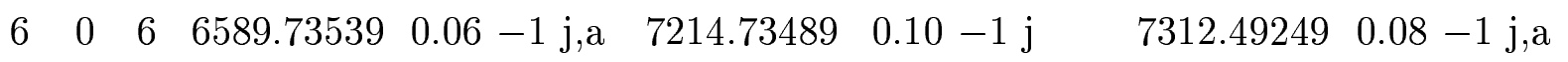

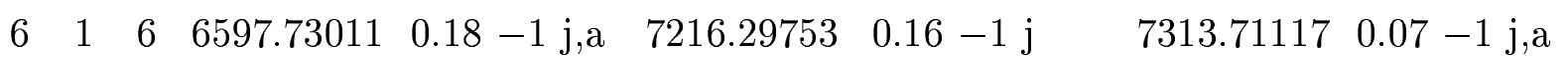

$\begin{array}{llllllllll}6 & 1 & 5 & 6722.49989 & 0.25-1 \mathrm{j} & 7326.70489 & 0.12-1 \mathrm{j} & 7424.84076 & 0.08-1 \mathrm{j}, \mathrm{a}\end{array}$

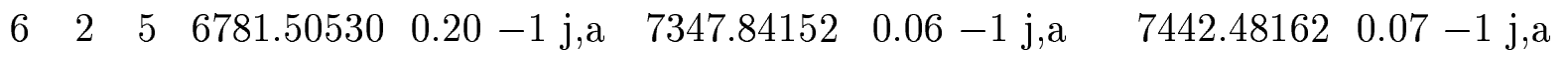

$\begin{array}{lllllllllll}6 & 2 & 4 & 6820.80448 & 0.77 & 2 \mathrm{a} & 7397.14981 & 0.19-1 \mathrm{j} & 7495.31415 & 0.10-1 \mathrm{j}, \mathrm{a}\end{array}$

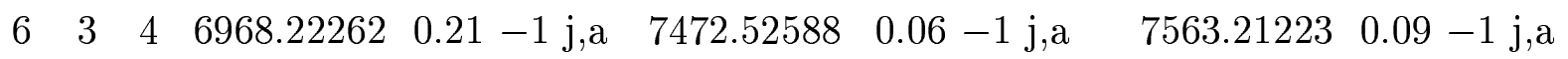

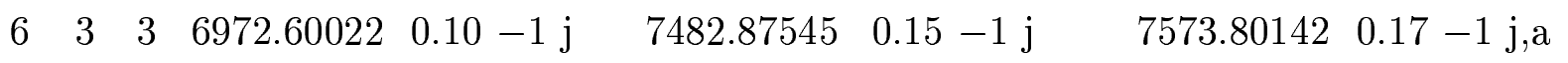

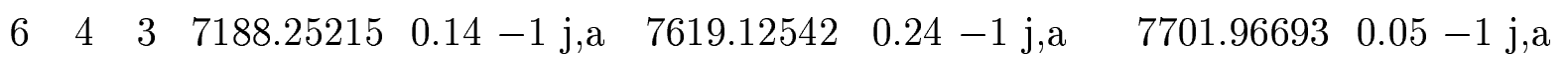

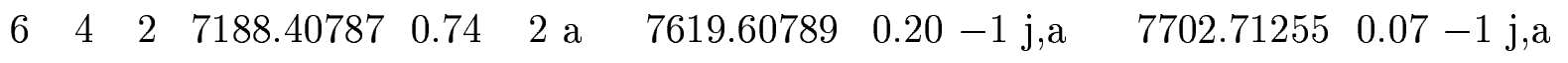

$\begin{array}{lllllllllll}6 & 5 & 2 & 7442.33600 & 0.52 & 4 \mathrm{a} & 7795.06854 & 0.30 & -1 \mathrm{j}, \mathrm{a} & 7868.61994 & 0.14-1 \mathrm{j}\end{array}$

$\begin{array}{lllllllllllll}6 & 5 & 1 & 7442.38137 & 0.81 & 2 \mathrm{a} & 7795.09863 & 0.36-1 \mathrm{j} & 7869.35417 & 0.06-1 \mathrm{j}, \mathrm{a}\end{array}$

$\begin{array}{llllllllllll}6 & 6 & 1 & 7722.60588 & 0.53 & 5 \mathrm{e}, \mathrm{a} & 7999.17504 & 0.36-1 \mathrm{j}, \mathrm{a} & 8063.01241 & 0.20-1 \mathrm{j}\end{array}$

$\begin{array}{llllllllllll}6 & 6 & 0 & 7722.60588 & 0.53 & \mathrm{~d} & 7999.17565 & 0.10 & -1 \mathrm{j} & 8063.01260 & 0.18-1 & \mathrm{j}, \mathrm{a}\end{array}$

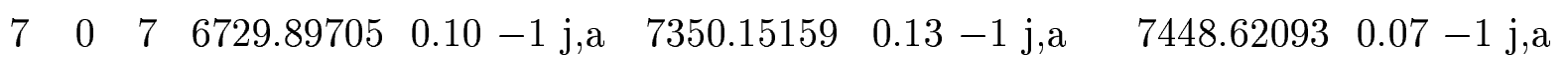

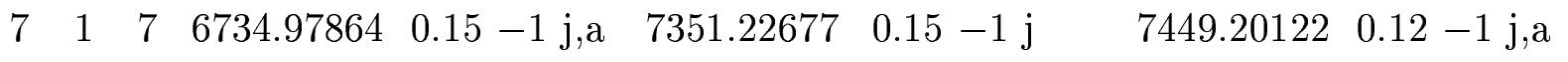


$\begin{array}{lllllllll}7 & 1 & 6 & 6895.86324 & 0.33-1 \mathrm{j}, \mathrm{a} & 7489.65811 & 0.15-1 \mathrm{j} & 7587.74125 & 0.15-1 \mathrm{j}, \mathrm{a}\end{array}$ $\begin{array}{lllllllll}7 & 2 & 6 & 6941.63552 & 0.07-1 \mathrm{j}, \mathrm{a} & 7503.01105 & 0.50-1 \mathrm{j} & 7598.35038 & 0.10-1 \mathrm{j}, \mathrm{a}\end{array}$

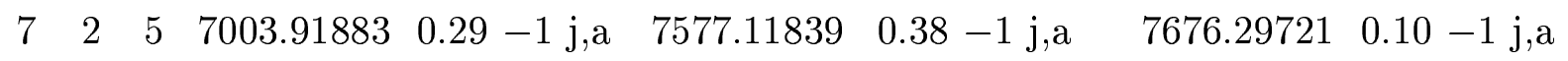
$\begin{array}{llllllllllll}7 & 3 & 5 & 7137.09907 & 0.64 & 3 \mathrm{a} & 7638.99658 & 0.25-1 \mathrm{j} & 7730.18509 & 0.10-1 \mathrm{j}, \mathrm{a}\end{array}$

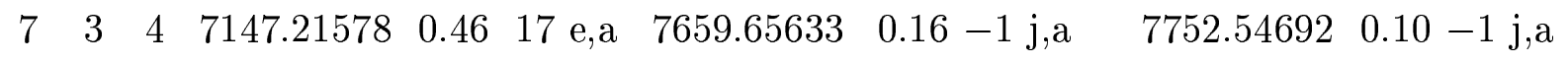
$\begin{array}{llllllllll}7 & 4 & 4 & 7358.67650 & 0.64 & 4 \mathrm{e}, \mathrm{a} & 7787.89031 & 0.15-1 \mathrm{j} & 7871.76011 & 0.14-1 \mathrm{j}, \mathrm{a}\end{array}$ $\begin{array}{lllllllllll}7 & 4 & 3 & 7359.61327 & 0.51 & 10 \mathrm{e}, \mathrm{a} & 7789.54465 & 0.20-1 \mathrm{j} & 7874.25665 & 0.14-1 \mathrm{j}, \mathrm{a}\end{array}$

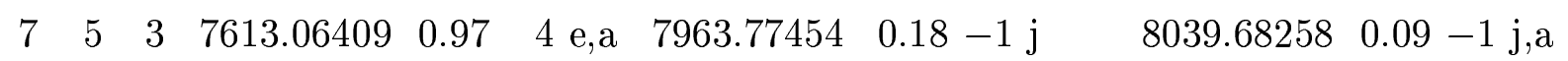

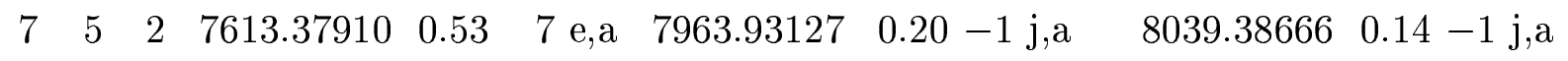

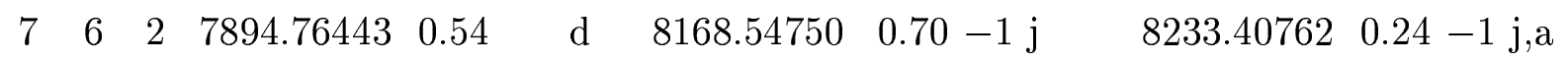
$\begin{array}{lllllllllll}7 & 6 & 1 & 7894.76443 & 0.54 & 4 \mathrm{a} & 8168.54865 & 0.30 & -1 \mathrm{j}, \mathrm{a} & 8233.41177 & 0.10-1 \mathrm{j}\end{array}$

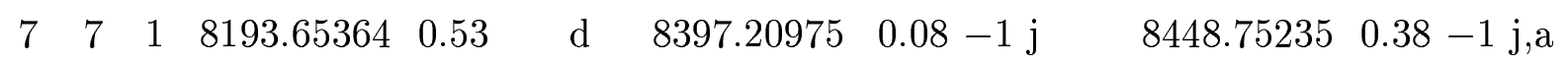

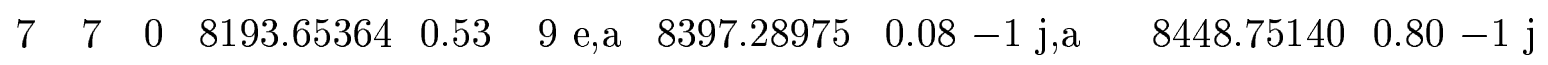

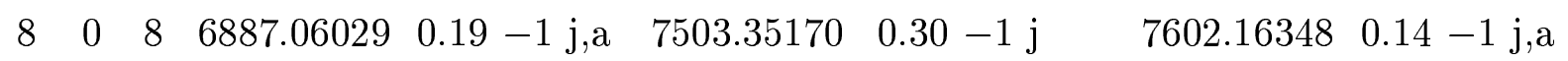

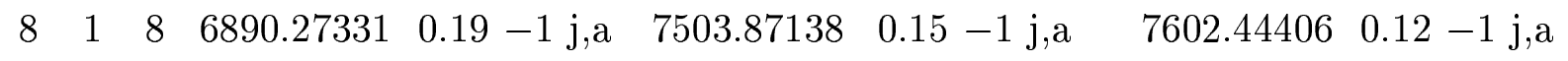
$\begin{array}{llllllllllll}8 & 1 & 7 & 7088.36156 & 0.75 & 2 \mathrm{a} & 7669.39727 & 0.25-1 \mathrm{j} & 7767.34344 & 0.12-1 \mathrm{j}, \mathrm{a}\end{array}$

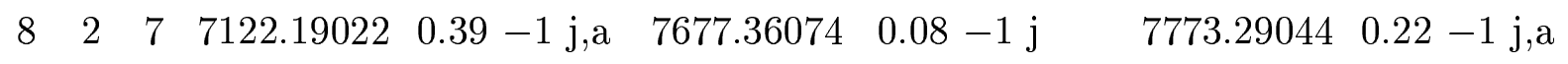
$\begin{array}{llllllllll}8 & 2 & 6 & 7212.27293 & 0.54 & 4 \mathrm{a} & 7779.41329 & 0.29-1 \mathrm{j} & 7879.25124 & 0.10-1 \mathrm{j}, \mathrm{a}\end{array}$

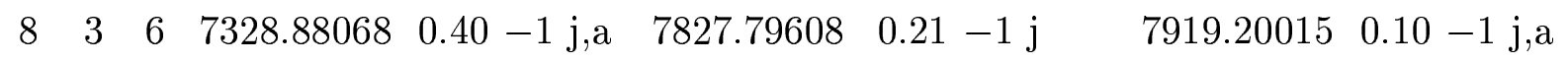
$\begin{array}{lllllllllll}8 & 3 & 5 & 7348.90597 & 1.00 & 1 \mathrm{a} & 7864.05607 & 0.19-1 \mathrm{j} & 7958.42343 & 0.14-1 \mathrm{j}, \mathrm{a}\end{array}$ $\begin{array}{llllllllll}8 & 4 & 5 & 7552.97463 & 0.42 & 10 \mathrm{e}, \mathrm{a} & 7980.85436 & 0.12-1 \mathrm{j}, \mathrm{a} & 8065.43278 & 0.10-1 \mathrm{j}, \mathrm{a}\end{array}$ $\begin{array}{llllllllll}8 & 4 & 4 & 7554.92077 & 0.55 & 4 \mathrm{a} & 7985.02308 & 0.15-1 \mathrm{j} & 8072.00802 & 0.17-1 \mathrm{j}, \mathrm{a}\end{array}$ $\begin{array}{lllllllllll}8 & 5 & 4 & 7807.15083 & 0.95 & 5 \mathrm{e}, \mathrm{a} & 8156.19124 & 0.32-1 \mathrm{j} & 8233.73024 & 0.17-1 \mathrm{j}, \mathrm{a}\end{array}$

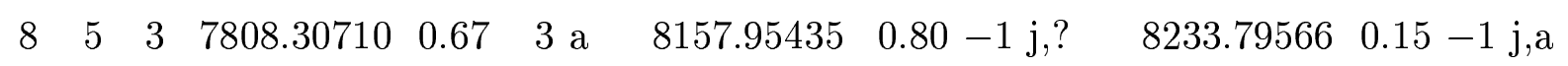
$\begin{array}{llllllllllll}8 & 6 & 3 & 8090.81123 & 0.73 & 5 \mathrm{e}, \mathrm{a} & 8361.71175 & 0.48 & 2 \mathrm{a} & 8427.77119 & 0.10 & -1 \mathrm{j}, \mathrm{a}\end{array}$ $\begin{array}{lllllll}8 & 6 & 2 & 8090.82172 & 1.40 & 1 \mathrm{a} \quad 8427.79355 & 0.06-1 \mathrm{j}, \mathrm{a}\end{array}$ $\begin{array}{llllllllllll}8 & 7 & 2 & 8390.80382 & 0.54 & 5 \mathrm{e}, \mathrm{a} & 8591.35884 & 0.54 & 5 \mathrm{e}, \mathrm{a} & 8643.78675 & 0.20 & -1 \mathrm{j}\end{array}$ 


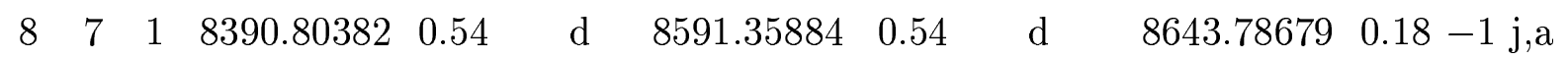

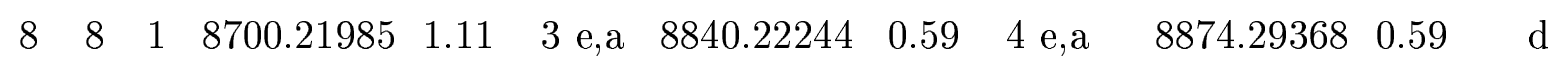
$\begin{array}{lllllllllllll}8 & 8 & 0 & 8700.21985 & 1.11 & d & 8840.22244 & 0.59 & d & 8874.29368 & 0.59 & 3 & \text { a }\end{array}$

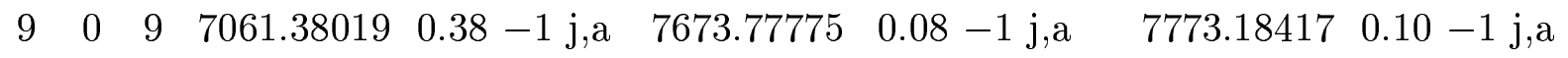
$\begin{array}{llllllllll}9 & 1 & 9 & 7063.42090 & 0.34 & -1 \mathrm{j}, \mathrm{a} & 7674.13782 & 0.65-1 \mathrm{j} & 7772.93111 & 0.08-1 \mathrm{j}, \mathrm{a}\end{array}$ $\begin{array}{llllllllll}9 & 1 & 8 & 7298.57468 & 0.52 & 4 \mathrm{a} & 7865.59837 & 0.26-1 \mathrm{j}, \mathrm{a} & 7963.50243 & 0.27-1 \mathrm{j}, \mathrm{a}\end{array}$ $\begin{array}{lllllllllll}9 & 2 & 8 & 7322.53470 & 0.62 & 3 \mathrm{a} & 7870.33453 & 0.15-1 \mathrm{j} & 7966.67856 & 0.14-1 \mathrm{j}, \mathrm{a}\end{array}$

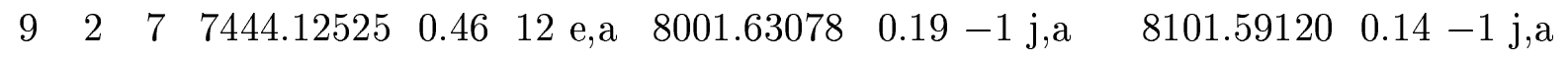
$\begin{array}{llllllll}9 & 3 & 7 & 7542.76335 & 1.17 & 1 \mathrm{a} & 8128.66703 & 0.08-1 \mathrm{j}, \mathrm{a}\end{array}$ $\begin{array}{lllllllllll}9 & 3 & 6 & 7577.86372 & 0.47 & 8 \mathrm{e}, \mathrm{a} & 8094.84371 & 0.33-1 \mathrm{j} & 8200.22526 & 0.59 & 3 \mathrm{a}\end{array}$ $\begin{array}{llllllllllll}9 & 4 & 6 & 7770.74959 & 1.20 & 2 \mathrm{a} & 8187.03942 & 1.01 & 1 \mathrm{a} & 8281.94092 & 0.17-1 \mathrm{j}, \mathrm{a}\end{array}$

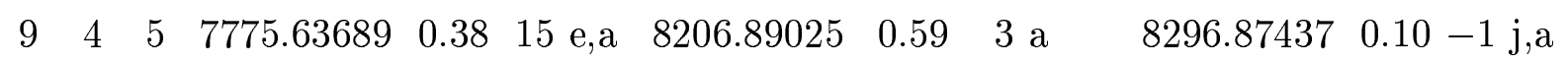
955 $8375.67360 \quad 2.23 \quad 2$ e $\quad 8451.79180 \quad 0.15-1 \mathrm{j}$

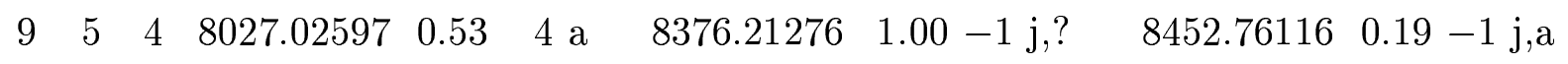

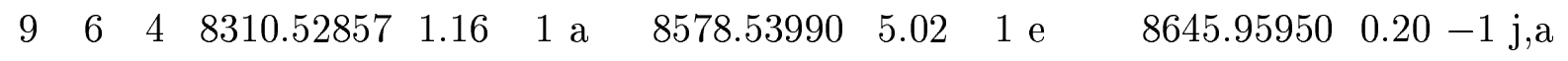
$\begin{array}{lllllllllll}9 & 6 & 3 & 8310.56088 & 0.53 & 4 \mathrm{a} & 8578.57695 & 0.61 & 3 \mathrm{a} & 8646.05346 & 0.24-1 \mathrm{j}, \mathrm{a}\end{array}$ $\begin{array}{rrrrrrrr}9 & 7 & 3 & 8611.49360 & 0.55 \quad \mathrm{~d} & 8862.43001 & 0.32-1 \mathrm{j}, \mathrm{a}\end{array}$

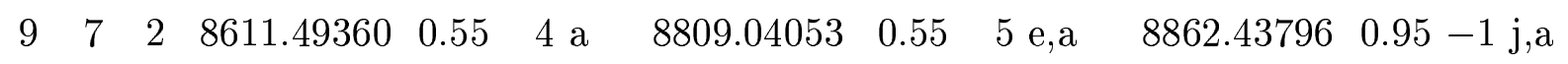

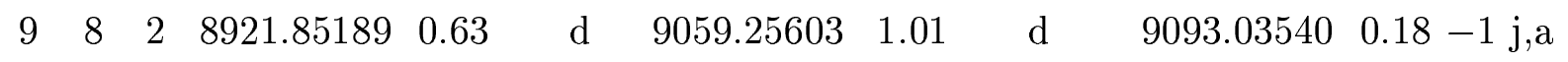

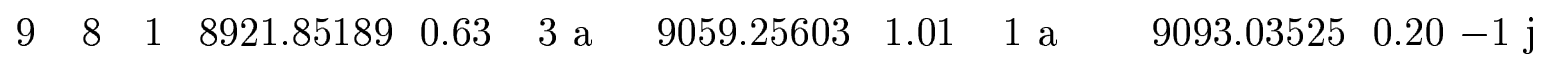
$\begin{array}{llllllllllll}9 & 9 & 1 & 9306.82308 & 1.49 & \text { d } & 9227.58720 & 1.49 & \text { d } & 9375.82640 & 1.03 & 1\end{array}$

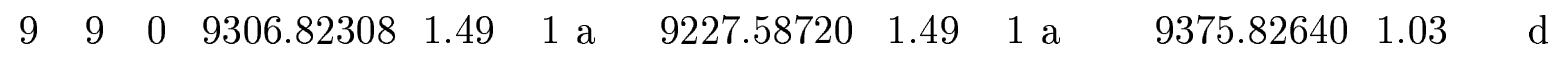

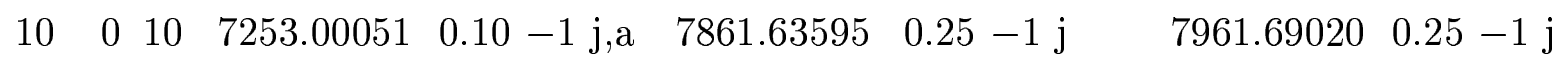

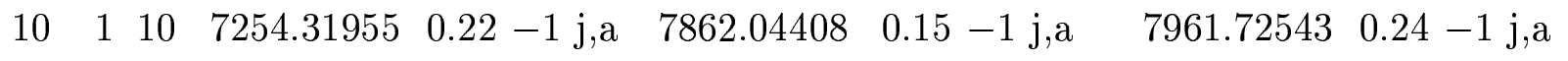
$\begin{array}{lllllllllllll}10 & 1 & 9 & 7525.71151 & 0.78 & 2 \mathrm{a} & 8078.45131 & 0.20-1 \mathrm{j} & 8176.46631 & 0.09-1 \mathrm{j}, \mathrm{a}\end{array}$

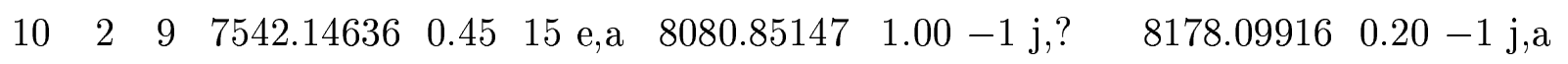

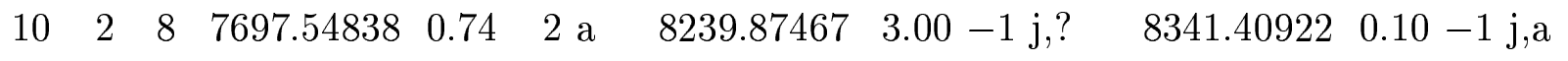




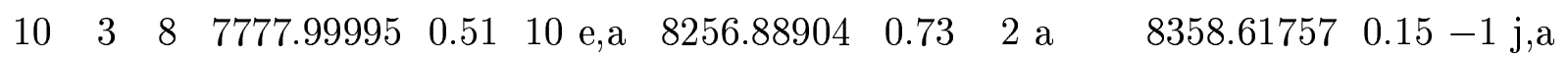

$\begin{array}{lllllll}10 & 3 & 7 & 7833.56479 & 0.83 & 2 \text { a }\end{array}$

$8452.137880 .14-1 \mathrm{j}, \mathrm{a}$

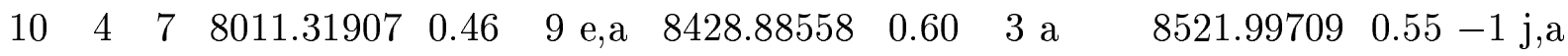

$\begin{array}{llllll}10 & 4 & 6 & 8020.86484 & 0.92 & 2 \text { a }\end{array}$

$8549.24274 \quad 0.14-1 \mathrm{j}, \mathrm{a}$

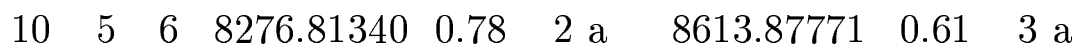

$8693.52239 \quad 0.19-1 \mathrm{j}, \mathrm{a}$

$\begin{array}{llllll}10 & 5 & 5 & 8269.37398 & 1.10 & 1 \mathrm{a}\end{array}$

$8696.61302 \quad 0.21-1 \mathrm{j}, \mathrm{a}$

$\begin{array}{lllllllll}10 & 6 & 5 & 8553.59442 & 0.54 & 4 \mathrm{a} & 8818.74309 & 0.76 & 3 \mathrm{e}, \mathrm{a}\end{array}$

$8887.792110 .37-1 \mathrm{j}, \mathrm{a}$

$\begin{array}{llllll}10 & 6 & 4 & 8553.74000 & 1.07 & 1 \mathrm{a}\end{array}$

$8888.121540 .30-1 \mathrm{j}, \mathrm{a}$

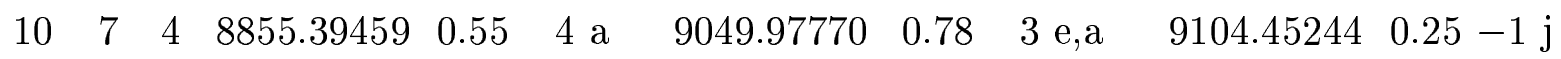

$\begin{array}{llllll}10 & 7 & 3 & 8855.39459 & 0.55 & \mathrm{~d}\end{array}$

$9104.467890 .14-1 \mathrm{j}, \mathrm{a}$

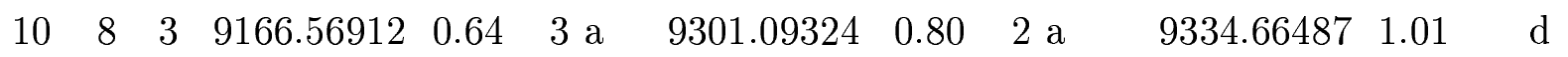

$\begin{array}{lllllllllllll}10 & 8 & 2 & 9166.56912 & 0.64 & \mathrm{~d} & 9301.09324 & 0.80 & \mathrm{~d} & 9334.66487 & 1.01 & 1 \mathrm{a}\end{array}$

$10 \quad 9 \quad 2$

$9470.68619 \quad 1.01 \quad 2$ e,a,? $9620.27473 \quad 0.72 \quad \mathrm{~d}$

$\begin{array}{lll}10 & 9 & 1\end{array}$

$\begin{array}{llllll}9470.68619 & 1.01 & \mathrm{~d} & 9620.27473 & 0.72 & 2 \mathrm{a}\end{array}$

$\begin{array}{llllllllllll}10 & 10 & 1 & 9920.58735 & 1.80 & 1 \mathrm{a} & 9800.94762 & 1.80 & 1 \mathrm{a} & 9876.08128 & 1.08 & \mathrm{~d}\end{array}$

$\begin{array}{llllllllllll}10 & 10 & 0 & 9920.58735 & 1.80 & \text { d } & 9800.94762 & 1.80 & \text { d } & 9876.08128 & 1.08 & 1\end{array}$

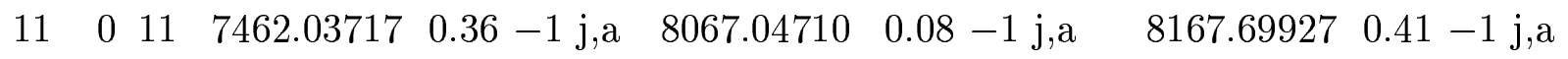

$\begin{array}{lllllll}11 & 1 & 11 & 7462.90921 & 0.75 & 2 \text { a }\end{array}$

$8167.689280 .18-1 \mathrm{j}, \mathrm{a}$

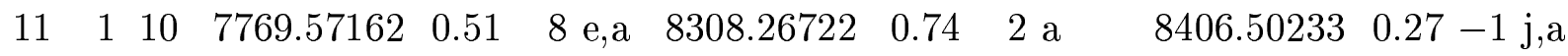

$\begin{array}{lllllll}11 & 2 & 10 & 7780.61578 & 0.75 & 2 & \text { a }\end{array}$

$8407.29036 \quad 0.26-1 \mathrm{j}, \mathrm{a}$

$\begin{array}{llllll}11 & 2 & 9 & 7970.45787 & 0.61 & 4 \mathrm{a}\end{array}$

$8597.053540 .24-1$ j,a

$\begin{array}{llllll}11 & 3 & 9 & 8032.91626 & 1.10 & 1 \text { a }\end{array}$

$8607.32000 \quad 0.25-1 \mathrm{j}, \mathrm{a}$

$\begin{array}{llllll}11 & 3 & 8 & 8114.76786 & 0.43 & 6 \text { a }\end{array}$

$8730.16217 \quad 0.50-1$ j

$\begin{array}{llllll}11 & 4 & 8 & 8273.19378 & 0.64 & 3 \mathrm{a}\end{array}$

$8782.72250 \quad 0.22-1$ j,a

$\begin{array}{llllllllllll}11 & 4 & 7 & 8292.02662 & 0.47 & 9 \mathrm{e}, \mathrm{a} & 8731.32849 & 1.12 & 1 \mathrm{a} & 8827.49564 & 1.01 & 1 \mathrm{a}\end{array}$

$\begin{array}{lllllll}11 & 5 & 7 & 8539.05517 & 1.14 & 1 \mathrm{a}\end{array}$

$8958.396680 .24-1 \mathrm{j}, \mathrm{a}$

$\begin{array}{lllllllllllll}11 & 5 & 6 & 8535.23258 & 0.48 & 5 \mathrm{a} & 8881.89530 & 0.55 & 4 \mathrm{a} & 8965.40364 & 1.01 & 1 \mathrm{a}\end{array}$ 
$\begin{array}{llllll}11 & 6 & 6 & 8819.64945 & 0.89 & 2 \mathrm{a}\end{array}$

$\begin{array}{lllllllll}11 & 6 & 5 & 8820.14291 & 0.49 & 6 \text { e,a } & 9083.39370 & 0.64 & 3 \text { a }\end{array}$

$\begin{array}{llllll}11 & 7 & 5 & 9122.19448 & 1.46 & 1 \mathrm{a}\end{array}$

$\begin{array}{lllllllll}11 & 7 & 4 & 9122.17937 & 0.77 & 2 \text { a } & 9313.93690 & 0.81 & 2 \text { a }\end{array}$

$\begin{array}{llllll}11 & 8 & 4 & 9433.12308 & 1.48 & 1 \mathrm{a}\end{array}$

$\begin{array}{lllllllll}11 & 8 & 3 & 9433.99667 & 0.55 & 4 \mathrm{a} & 9565.72469 & 1.27 & 1 \mathrm{a}\end{array}$

$\begin{array}{lllllllll}11 & 9 & 3 & 9810.82789 & 5.54 & \mathrm{~d} & 9885.17657 & 1.07 & \mathrm{~d}\end{array}$

$\begin{array}{lllllllll}11 & 9 & 2 & 9810.82789 & 5.54 & 1 \mathrm{a} & 9885.17657 & 1.07 & 1 \mathrm{a}\end{array}$

$\begin{array}{llllllll}11 & 10 & 2 & 10193.84100 & 6.30 & \text { d } & 10068.80862 & 1.16\end{array}$

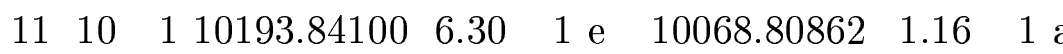

$\begin{array}{llllllll}11 & 11 & 1 & 10532.70160 & 2.06 & \text { d } & 10355.10720 & 2.06\end{array}$

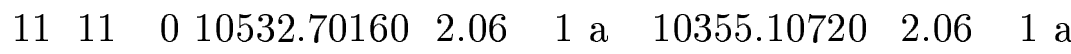

$\begin{array}{lllllll}12 & 0 & 12 & 7688.56000 & 5.00 & -1 & \mathrm{j}\end{array}$

$\begin{array}{lllllllll}12 & 1 & 12 & 7689.16450 & 0.52 & 11 \mathrm{e}, \mathrm{a} & 8286.27111 & 1.20 & 1 \mathrm{a}\end{array}$

$\begin{array}{llllll}12 & 1 & 11 & 8030.31363 & 0.78 & 2 \mathrm{a}\end{array}$

$\begin{array}{lllllllll}12 & 2 & 11 & 8037.66120 & 0.47 & 11 \mathrm{e}, \mathrm{a} & 8546.42100 & 1.12 & 1 \mathrm{a}\end{array}$

$\begin{array}{llllll}12 & 2 & 10 & 8259.83930 & 2.56 & 5 \mathrm{e}\end{array}$

$\begin{array}{lllllllll}12 & 3 & 10 & 8313.00601 & 0.95 & 4 \mathrm{e}, \mathrm{a} & 8774.16083 & 1.05 & 1 \mathrm{a}\end{array}$

$\begin{array}{llllll}12 & 3 & 9 & 8419.67964 & 0.84 & 2 \text { a }\end{array}$

$\begin{array}{lllllllll}12 & 4 & 9 & 8570.13032 & 0.75 & 4 \mathrm{e}, \mathrm{a} & 8970.12380 & 6.43 & 1 \mathrm{e}\end{array}$

$\begin{array}{llllll}12 & 4 & 8 & 8589.01650 & 1.11 & 1 \mathrm{a}\end{array}$

$\begin{array}{lllllllll}12 & 5 & 8 & 8825.76944 & 0.54 & 5 \mathrm{e}, \mathrm{a} & 9164.06652 & 0.61 & 3 \mathrm{a}\end{array}$

$\begin{array}{llllll}12 & 5 & 7 & 8824.53672 & 0.83 & 2 \mathrm{a}\end{array}$

$\begin{array}{lllllllll}12 & 6 & 7 & 9108.59850 & 1.07 & 1 \mathrm{a} & 9368.55148 & 0.80 & 2 \mathrm{a}\end{array}$

$\begin{array}{llllll}12 & 6 & 6 & 9109.77145 & 1.52 & 1 \mathrm{a}\end{array}$

$\begin{array}{lllllllll}12 & 7 & 6 & 9411.49539 & 1.08 & 1 \mathrm{a} & 9600.55122 & 0.80 & 2 \mathrm{a}\end{array}$ $\begin{array}{lll}12 & 7 & 5\end{array}$
$9153.03475 \quad 0.38-1 \mathrm{j}, \mathrm{a}$

$9154.008890 .56-1 \mathrm{j}, \mathrm{a}$

$9369.59893 \quad 0.44-1 \mathrm{~g}, \mathrm{a}$

$9598.77967 \quad 0.46 \quad 6$ a

$9887.89849 \quad 1.46 \quad 1 \mathrm{a}$ $9887.89849 \quad 1.46 \quad \mathrm{~d}$

$10147.07078 \quad 0.80 \quad 2$ a $10147.07078 \quad 0.80 \quad \mathrm{~d}$ $10419.10640 \quad 0.79 \quad 2$ a $\begin{array}{lll}10419.10640 & 0.79 \quad \mathrm{~d}\end{array}$ $8391.193290 .08-1 \mathrm{j}, \mathrm{a}$ $8391.130330 .15-1 \mathrm{j}, \mathrm{a}$ $8653.81241 \quad 0.25-1 \mathrm{j}, \mathrm{a}$ $8654.095290 .27-1 \mathrm{j}, \mathrm{a}$ $8868.717980 .14-1 \mathrm{j}, \mathrm{a}$ $8874.36402 \quad 0.59 \quad 3$ a $9025.955690 .14-1 \mathrm{j}, \mathrm{a}$ $9064.05401 \quad 1.01 \quad 1 \mathrm{a}$ $9131.71985 \quad 0.19-1 \mathrm{j}, \mathrm{a}$ $9245.74566 \quad 0.69-1 \mathrm{~g}, \mathrm{a}$ $9262.47803 \quad 0.46 \quad 9$ e,a $9441.38360 \quad 1.02 \quad 1 \mathrm{a}$ $9443.85390 \quad 0.46 \quad 7$ e,a $9657.59940 \quad 3.20-1 \mathrm{~g}$ $9657.81824 \quad 0.41-1 \mathrm{~g}, \mathrm{a}$ 
$\begin{array}{lllllllll}12 & 8 & 5 & 9723.73565 & 1.06 & 1 \mathrm{a} & 9852.68737 & 1.29 & 1 \mathrm{a}\end{array}$

$12 \quad 8 \quad 4$

$9885.10990 \quad 0.52 \quad 5 \mathrm{e}, \mathrm{a}$

$\begin{array}{lllllllllll}12 & 9 & 4 & 10097.22325 & 1.62 & 1 \text { a } & 10026.72099 & 1.09 & 2 \text { e,a,? } 10178.07617 & 0.63 & \mathrm{~d}\end{array}$

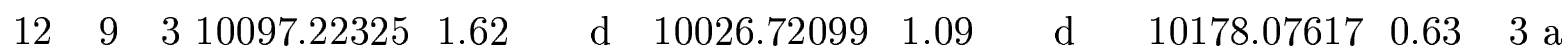

$\begin{array}{llllll}12 & 10 & 3 & 10489.77710 & 5.45 & 2 \mathrm{e}\end{array}$ $10440.57146 \quad 1.06 \quad \mathrm{~d}$

$\begin{array}{llllll}12 & 10 & 2 & 10489.77710 & 5.45 & \mathrm{~d}\end{array}$ $10440.57146 \quad 1.06 \quad 1 \mathrm{a}$

$\begin{array}{llllll}12 & 11 & 2 & 10833.52237 & 6.38 & 1 \mathrm{a}\end{array}$ $10715.87837 \quad 1.13 \quad \mathrm{~d}$

$\begin{array}{llllll}12 & 11 & 1 & 10833.52237 & 6.38 & \mathrm{~d}\end{array}$ $10715.87837 \quad 1.13 \quad 1 \mathrm{a}$

$\begin{array}{llllll}12 & 12 & 1 & 11179.18354 & 2.29 & 1 \mathrm{a}\end{array}$ $10996.92100 \quad 0.90 \quad \mathrm{~d}$

$\begin{array}{llllll}12 & 12 & 0 & 11179.18354 & 2.29 & \mathrm{~d}\end{array}$ $10996.92100 \quad 0.90 \quad 2$ a

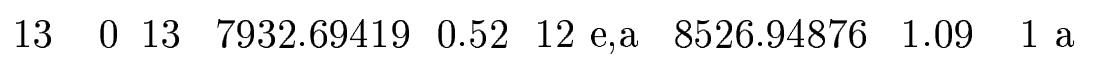
$8632.49491 \quad 0.59 \quad 3 \mathrm{a}$ $\begin{array}{llllll}13 & 1 & 13 & 7933.10053 & 1.03 & 2 \mathrm{e}, \mathrm{a}\end{array}$ $8632.098740 .32-1 \mathrm{j}, \mathrm{a}$ $\begin{array}{lllllllll}13 & 1 & 12 & 8308.23443 & 0.76 & 2 \text { a } & 8820.42729 & 1.02 & 2 \text { e,a }\end{array}$ $\begin{array}{lllllll}13 & 2 & 12 & 8313.09204 & 0.80 & 2 \mathrm{a}\end{array}$ $8918.64956 \quad 0.77 \quad 2$ a

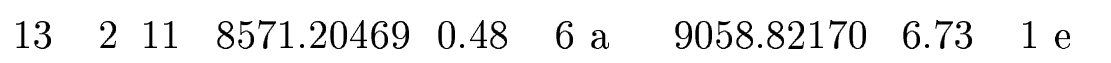
$\begin{array}{llllll}13 & 3 & 11 & 8605.54319 & 1.10 & 1 \mathrm{a}\end{array}$ $8918.43706 \quad 0.08-1 \mathrm{j}, \mathrm{a}$

$\begin{array}{llllll}13 & 3 & 10 & 8745.85618 & 0.76 & 2 \mathrm{a}\end{array}$ $9157.03470 \quad 0.60 \quad 3 \mathrm{a}$

$\begin{array}{llllll}13 & 4 & 10 & 8870.58645 & 1.12 \quad 3 \mathrm{a}\end{array}$ $9159.191890 .99 \quad 2$ a

$\begin{array}{llllll}13 & 4 & 9 & 8911.50949 & 0.54 & 4 \mathrm{a}\end{array}$ $\begin{array}{lll}9339.14617 & 0.72 \quad 2 \text { a }\end{array}$ $\begin{array}{lllllllll}13 & 5 & 9 & 9133.86820 & 7.12 & 1 \mathrm{e} & 9456.31598 & 1.06 & 1 \mathrm{a}\end{array}$ $\begin{array}{llllll}13 & 5 & 8 & 9137.27421 & 1.25 & 1 \mathrm{a}\end{array}$ $9364.93604 \quad 0.42 \quad 11 \mathrm{e}, \mathrm{a}$ $\begin{array}{lll}9476.50830 & 5.20 \quad 1 \mathrm{e}\end{array}$ $\begin{array}{lll}13 & 6 & 8\end{array}$ $9554.75430 \quad 0.46 \quad 8$ e,a $\begin{array}{lllllllll}13 & 6 & 7 & 9422.84021 & 0.63 & 7 \text { e,a } & 9682.73024 & 1.05 & 2 \text { e,a }\end{array}$ $\begin{array}{lll}13 & 7 & 7\end{array}$ $9590.35116 \quad 1.01 \quad 1 \mathrm{a}$ $9752.48802 \quad 0.46 \quad 6 \mathrm{e}, \mathrm{a}$ $\begin{array}{lll}9757.91327 & 0.99 \quad 2 \text { e,a }\end{array}$ $\begin{array}{lll}9968.18929 & 0.62 \quad 3 \mathrm{a}\end{array}$ $\begin{array}{lllllllll}13 & 7 & 6 & 9723.03734 & 1.26 & 1 \mathrm{a} & 9909.83810 & 1.28 & 1 \mathrm{a}\end{array}$ $9968.79442 \quad 1.43 \quad 1 \mathrm{a}$ $13 \quad 8 \quad 6$ $10193.38276 \quad 1.06 \quad 2$ e,a $\begin{array}{llllll}13 & 8 & 5 & 10035.43651 & 1.06 & 1 \mathrm{a}\end{array}$ $10193.39383 \quad 1.41 \quad 2 \mathrm{e}, \mathrm{a}$ 
$13 \quad 9 \quad 5$

$\begin{array}{lll}13 & 9 & 4\end{array}$

$13 \quad 10 \quad 4$

$13 \quad 10 \quad 3$

$\begin{array}{llllll}13 & 11 & 3 & 11157.10594 & 5.54 & \mathrm{~d}\end{array}$

$\begin{array}{llllll}13 & 11 & 2 & 11157.10594 & 5.54 & 1 \mathrm{a}\end{array}$

$\begin{array}{llllll}13 & 12 & 2 & 11506.38331 & 6.46 & \mathrm{~d}\end{array}$

$\begin{array}{llllll}13 & 12 & 1 & 11506.38331 & 6.46 & 1 \mathrm{a}\end{array}$

$\begin{array}{llllll}13 & 13 & 1 & 11857.34222 & 2.50 & \mathrm{~d}\end{array}$

$\begin{array}{llllll}13 & 13 & 0 & 11857.34222 & 2.50 & 1 \mathrm{a}\end{array}$

$\begin{array}{llllll}14 & 0 & 14 & 8194.49389 & 0.57 & 4 \mathrm{a}\end{array}$

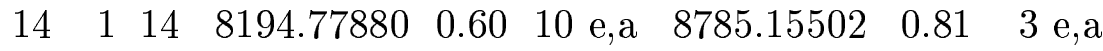

$\begin{array}{llllll}14 & 1 & 13 & 8603.62709 & 1.12 & 1 \mathrm{a}\end{array}$

$\begin{array}{llllll}14 & 2 & 13 & 8606.84956 & 0.49 & 9 \\ \mathrm{e}, \mathrm{a}\end{array}$

$\begin{array}{llllll}14 & 2 & 12 & 8896.83265 & 1.07 & 1 \mathrm{a}\end{array}$

$\begin{array}{llllll}14 & 3 & 12 & 8919.71946 & 0.60 & 8 \mathrm{e}, \mathrm{a}\end{array}$

$14 \quad 311$

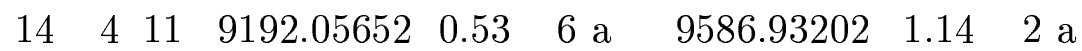

$\begin{array}{lllllll}14 & 4 & 10 & 9258.37187 & 1.21 & 1 \mathrm{a}\end{array}$

$\begin{array}{lllllllll}14 & 5 & 10 & 9470.53238 & 0.48 & 6 \mathrm{a} & 9790.56132 & 1.01 & 1 \mathrm{a}\end{array}$

$14 \quad 5 \quad 9$

$\begin{array}{lllllll}14 & 6 & 9 & 9752.76783 & 0.51 & 6 & \mathrm{e}, \mathrm{a}\end{array}$

$\begin{array}{lll}14 & 6 & 8\end{array}$

$\begin{array}{lllllllll}14 & 7 & 8 & 10056.24873 & 1.18 & 1 \mathrm{a} & 10241.07552 & 1.45 & 1 \mathrm{a}\end{array}$

$\begin{array}{lll}14 & 7 & 7\end{array}$

$\begin{array}{lll}14 & 8 & 7\end{array}$

$\begin{array}{lll}14 & 9 & 6\end{array}$
$10490.57941 \quad 1.13 \quad 1 \mathrm{a}$ $\begin{array}{llllll}10338.18324 & 1.46 & 1 \mathrm{a} & 10490.57941 & 1.13 & \mathrm{~d}\end{array}$ $\begin{array}{llllll}10671.59182 & 1.09 & \mathrm{~d} & 10756.13472 & 0.66 & 3 \mathrm{a}\end{array}$ $\begin{array}{llllll}10671.59182 & 1.09 & 1 \mathrm{a} & 10756.13472 & 0.66 & \mathrm{~d}\end{array}$ $11034.59305 \quad 1.46 \quad 1 \mathrm{a}$ $11034.59305 \quad 1.46 \quad \mathrm{~d}$ $11318.29040 \quad 7.22 \quad 1 \mathrm{e}$ $11318.29040 \quad 7.22 \quad \mathrm{~d}$

\begin{abstract}
$8889.17792 \quad 0.34-1 \mathrm{j}, \mathrm{a}$ $8886.68602 \quad 1.07 \quad 1 \mathrm{a}$ $9204.50246 \quad 0.98 \quad 3 \mathrm{a}$ $9200.32081 \quad 1.00 \quad 2 \mathrm{e}, \mathrm{a}$ $9456.00109 \quad 0.58 \quad 6$ e,a $9461.63674 \quad 0.72 \quad 2$ a $9667.69980 \quad 0.39 \quad 10 \mathrm{e}, \mathrm{a}$ $9684.37859 \quad 0.72 \quad 2$ a $9818.56806 \quad 0.42 \quad 11 \mathrm{e}, \mathrm{a}$ $9884.47214 \quad 0.73-1 \mathrm{~g}, \mathrm{a}$ $9934.93616 \quad 0.52 \quad 6$ e,a $10086.08262 \quad 1.08 \quad 1 \mathrm{a}$ $10096.41951 \quad 0.60 \quad 4$ a $10301.00394 \quad 1.02 \quad 2 \mathrm{a}$ $10302.56004 \quad 1.06 \quad 1 \mathrm{a}$
\end{abstract} $\begin{array}{llllll}10492.57810 & 6.77 & 3 \mathrm{e} & 10523.70790 & 6.28 & 1 \mathrm{e}\end{array}$ $\begin{array}{llllll}10669.95737 & 1.46 & 1 \mathrm{a} & 10825.26960 & 0.76 & \mathrm{~d}\end{array}$ 
$14 \quad 9 \quad 5$

$14 \quad 10 \quad 5$

$14 \quad 10 \quad 4$

$1411 \quad 4$

$1411 \quad 3$

$1413 \quad 2$

$1413 \quad 1$

$\begin{array}{lllllllll}15 & 0 & 15 & 8474.07591 & 0.56 & 7 \mathrm{e}, \mathrm{a} & 9059.40000 & 7.49 & 1 \mathrm{e}\end{array}$

$\begin{array}{llllll}15 & 1 & 15 & 8474.26080 & 0.82 & 2 \mathrm{a}\end{array}$

$\begin{array}{lllllllll}15 & 1 & 14 & 8916.71187 & 0.56 & 5 \mathrm{e}, \mathrm{a} & 9394.64720 & 7.02 & 1 \mathrm{e}\end{array}$

$\begin{array}{llllll}15 & 2 & 14 & 8918.89860 & 1.18 & 1 \mathrm{a}\end{array}$

$\begin{array}{lllllllll}15 & 2 & 13 & 9231.85227 & 0.63 & 3 \mathrm{a} & 9685.76030 & 1.16 & 1 \mathrm{a}\end{array}$

$15 \quad 3 \quad 13$

$\begin{array}{lllllllll}15 & 3 & 12 & 9463.29057 & 0.62 & 4 \mathrm{a} & 9916.68475 & 0.83 & 2 \mathrm{a}\end{array}$

$\begin{array}{llllll}15 & 4 & 12 & 9543.23885 & 1.15 & 2 \mathrm{a}\end{array}$

$\begin{array}{llllll}15 & 4 & 11 & 9626.50565 & 1.07 & 1 \mathrm{a}\end{array}$

$\begin{array}{llllll}15 & 5 & 11 & 9820.01569 & 1.57 & 1 \mathrm{a}\end{array}$

$\begin{array}{lll}15 & 5 & 10\end{array}$

$\begin{array}{lll}15 & 6 & 10\end{array}$

$\begin{array}{llllll}15 & 6 & 9 & 10119.01136 & 1.12 & 1 \mathrm{a}\end{array}$

$\begin{array}{lll}15 & 7 & 9\end{array}$

$\begin{array}{llllll}15 & 7 & 8 & 10411.30494 & 0.78 & 2 \mathrm{a}\end{array}$

$\begin{array}{lll}15 & 8 & 8\end{array}$

$\begin{array}{lll}15 & 9 & 7\end{array}$

$15 \quad 10 \quad 6$

$15 \quad 10 \quad 5$

$1511 \quad 5$
$10825.26960 \quad 0.76 \quad 2$ a

$\begin{array}{lll}11093.67992 & 1.07 \quad \mathrm{~d}\end{array}$

$\begin{array}{lll}11093.67992 & 1.07 \quad 1 \mathrm{a}\end{array}$

$11374.28092 \quad 1.10 \quad d$

$11374.28092 \quad 1.10 \quad 2 \mathrm{e}, \mathrm{a}$

$\begin{array}{lll}11953.72809 & 7.29 \quad \mathrm{~d}\end{array}$

$\begin{array}{lll}11953.72809 & 7.29 & 1 \mathrm{a}\end{array}$

$\begin{array}{lll}9165.03960 & 0.80 & 2 \\ \text { a }\end{array}$

$\begin{array}{lll}9164.94958 & 0.48 \quad 5 \mathrm{a}\end{array}$

$9497.98098 \quad 1.03 \quad 1 \mathrm{a}$

$9500.22166 \quad 0.52 \quad 9$ e,a

$9778.35609 \quad 1.06 \quad 1 \mathrm{a}$

$\begin{array}{lll}9781.06161 & 0.52 \quad 4 \mathrm{a}\end{array}$

$10011.39857 \quad 1.04 \quad 1 \mathrm{a}$

$10022.09592 \quad 0.43 \quad 9$ e,a

$10184.26545 \quad 0.76 \quad 2$ a

$10234.29441 \quad 0.73 \quad 4 \mathrm{a}$

$\begin{array}{lll}10306.97007 & 0.76 \quad 2 & \text { a }\end{array}$

$10439.496510 .97-1 \mathrm{~g}, \mathrm{a}$

$\begin{array}{lll}10459.62786 & 5.02 \quad 1 \mathrm{a}\end{array}$

$10655.63015 \quad 0.74-1 \mathrm{~g}, \mathrm{a}$

$10659.19816 \quad 1.08 \quad 1 \mathrm{a}$

$\begin{array}{lll}10919.68128 & 0.78 \quad 2 \text { a }\end{array}$

$11181.35528 \quad 1.07 \quad 1 \mathrm{a}$

$11451.83411 \quad 1.08 \quad 1 \mathrm{a}$

$\begin{array}{llll}11451.83411 & 1.08 \quad \mathrm{~d}\end{array}$

$11736.32069 \quad 1.46 \quad 1 \mathrm{a}$ 


\begin{tabular}{|c|c|c|c|c|c|c|c|c|c|c|c|}
\hline 15 & 11 & 4 & & & & & & & 11736.32069 & 1.46 & $\mathrm{~d}$ \\
\hline 15 & 14 & 2 & & & & & & & 12619.17511 & 7.36 & $1 \mathrm{a}$ \\
\hline 5 & 14 & 1 & & & & & & & 12619.17511 & 7.36 & d \\
\hline 16 & 0 & 16 & 8771.45993 & 0.83 & $2 \mathrm{a}$ & & & & 9457.78956 & 0.74 & $5 \mathrm{e}, \mathrm{a}$ \\
\hline 16 & 1 & 16 & 8771.67201 & 0.57 & $4 \mathrm{a}$ & 9351.08661 & 1.07 & $1 \mathrm{a}$ & 9457.78956 & 0.74 & $\mathrm{~d}$ \\
\hline 16 & 1 & 15 & & & & & & & 9814.84603 & 0.71 & $6 \mathrm{e}, \mathrm{a}$ \\
\hline 16 & 2 & 15 & 9249.27272 & 0.63 & $4 \mathrm{e}, \mathrm{a}$ & 9712.28778 & 1.11 & $2 \mathrm{e}, \mathrm{a}$ & 9814.45240 & 1.06 & $1 \mathrm{a}$ \\
\hline 16 & 2 & 14 & 9584.49673 & 0.84 & $2 \mathrm{a}$ & & & & 10115.56191 & 0.61 & $4 \mathrm{e}, \mathrm{a}$ \\
\hline 16 & 3 & 14 & 9597.10743 & 0.63 & $3 \mathrm{a}$ & & & & 10117.70941 & 0.74 & $2 \mathrm{a}$ \\
\hline 16 & 3 & 13 & & & & & & & 10369.86354 & 0.74 & $5 \mathrm{e}, \mathrm{a}$ \\
\hline 16 & 4 & 13 & 9907.07240 & 1.11 & $2 \mathrm{a}$ & 10276.08625 & 1.13 & $2 \mathrm{a}$ & 10376.87965 & 0.74 & $2 \mathrm{a}$ \\
\hline 16 & 4 & 12 & & & & & & & 10567.49565 & 0.53 & $6 \mathrm{e}, \mathrm{a}$ \\
\hline 16 & 5 & 12 & 10190.49250 & 1.47 & $1 \mathrm{a}$ & & & & 10602.68520 & 1.01 & $3 \mathrm{a}$ \\
\hline 16 & 5 & 11 & & & & & & & 10700.40264 & 0.61 & $3 \mathrm{a}$ \\
\hline 16 & 6 & 11 & & & & 10736.19263 & 0.64 & $4 \mathrm{a}$ & 10814.36260 & 5.02 & $1 \mathrm{a}$ \\
\hline 16 & 6 & 10 & & & & & & & 10847.71503 & 0.61 & $4 \mathrm{a}$ \\
\hline 16 & 7 & 9 & & & & & & & 11038.94295 & 1.03 & $3 \mathrm{e}, \mathrm{a}$ \\
\hline 16 & 9 & 7 & & & & & & & 11558.75624 & 1.04 & $2 \mathrm{a}$ \\
\hline 17 & 0 & 17 & 9087.02301 & 0.59 & $4 \mathrm{a}$ & 9659.71660 & 7.63 & $1 \mathrm{e}$ & 9767.69044 & 0.54 & $\mathrm{~d}$ \\
\hline 17 & 1 & 17 & & & & & & & 9767.69044 & 0.54 & $9 \mathrm{e}, \mathrm{a}$ \\
\hline 17 & 1 & 16 & 9597.13360 & 0.57 & $5 \mathrm{e}, \mathrm{a}$ & & & & 10148.69113 & 0.96 & $2 \mathrm{a}$ \\
\hline 17 & 2 & 16 & 9598.06523 & 1.16 & $1 \mathrm{a}$ & & & & 10148.49517 & 0.54 & $8 \mathrm{e}, \mathrm{a}$ \\
\hline 17 & 2 & 15 & 9951.89147 & 1.08 & $1 \mathrm{a}$ & & & & 10469.02361 & 1.12 & $1 \mathrm{a}$ \\
\hline 17 & 3 & 15 & 9960.71986 & 1.19 & $1 \mathrm{a}$ & & & & 10472.50198 & 1.05 & $4 \mathrm{e}, \mathrm{a}$ \\
\hline 17 & 3 & 14 & 10242.79814 & 1.07 & $1 \mathrm{a}$ & & & & 10743.63836 & 1.02 & $2 \mathrm{a}$ \\
\hline 17 & 4 & 14 & 10289.11045 & 1.20 & $1 \mathrm{a}$ & & & & 10748.63512 & 0.53 & \\
\hline 17 & 4 & 13 & & & & & & & 10965.14674 & 5.03 & $1 \mathrm{a}$ \\
\hline
\end{tabular}


$\begin{array}{lll}17 & 5 & 13\end{array}$

$\begin{array}{lll}17 \quad 6 & 12\end{array}$

$17 \quad 7 \quad 11$

$\begin{array}{lll}17 & 10 & 8\end{array}$

$\begin{array}{lll}18 & 0 & 18\end{array}$

$\begin{array}{lllllllll}18 & 1 & 18 & 9421.57417 & 0.68 & 3 \mathrm{a} & 9985.45769 & 0.90 & 2 \mathrm{a}\end{array}$

$\begin{array}{llllll}18 & 1 & 17 & 9964.95246 & 1.27 & 1 \mathrm{a}\end{array}$

$\begin{array}{llllll}18 & 2 & 17 & 9965.37994 & 0.80 & 2 \mathrm{a}\end{array}$

$\begin{array}{llllll}18 & 2 & 16 & 10333.40135 & 1.20 & 1 \mathrm{a}\end{array}$

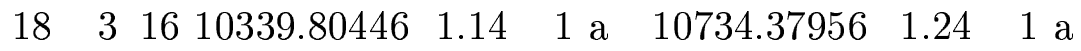

$\begin{array}{lll}18 & 3 & 15\end{array}$

$\begin{array}{llllll}18 & 4 & 15 & 10688.42829 & 0.80 & 2 \mathrm{a}\end{array}$

$18 \quad 414$

$\begin{array}{lll}18 & 5 & 14\end{array}$

$\begin{array}{lll}18 & 5 & 13\end{array}$

$\begin{array}{lll}18 & 6 & 13\end{array}$

$\begin{array}{lll}18 & 6 & 12\end{array}$

$18 \quad 7 \quad 11$

$\begin{array}{lllllll}19 & 0 & 19 & 9777.90532 & 1.33 & 2 \mathrm{a}\end{array}$

$\begin{array}{llllll}19 & 1 & 19 & 9772.38433 & 1.50 & 1 \mathrm{a}\end{array}$

$\begin{array}{llllll}19 & 1 & 18 & 10350.41739 & 1.10 & 1 \mathrm{a}\end{array}$

$\begin{array}{llllll}19 & 2 & 18 & 10351.35459 & 1.43 & 1 \mathrm{a}\end{array}$

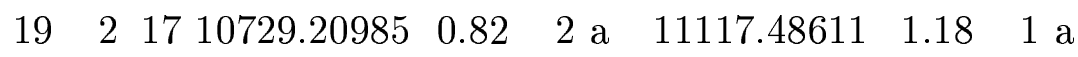

$\begin{array}{lll}19 & 3 & 17\end{array}$

$\begin{array}{llllll}19 & 3 & 16 & 11079.50960 & 1.13 & 1 \mathrm{a}\end{array}$

$19 \quad 416$

$19 \quad 4 \quad 15$
$10989.24998 \quad 1.09 \quad 1 \mathrm{a}$

$11209.08298 \quad 0.61 \quad 4 \mathrm{a}$

$11428.20142 \quad 0.76 \quad 2$ a

$12230.81156 \quad 1.44 \quad 1 \mathrm{a}$

$10094.67991 \quad 0.51 \quad 7 \mathrm{e}, \mathrm{a}$

$10094.679910 .51 \quad \mathrm{~d}$

$10499.72194 \quad 0.48 \quad 7 \mathrm{~L} \mathrm{nc}$

$\begin{array}{lll}10499.22079 & 5.10 \quad 1 \mathrm{a}\end{array}$

$10838.693350 .62-1 \mathrm{~g}, \mathrm{a}$

$\begin{array}{lll}10837.68300 & 1.09 & 1 \mathrm{a}\end{array}$ $\begin{array}{lll}11132.86274 & 0.77 \quad 3 \mathrm{a}\end{array}$

$11137.48933 \quad 1.12 \quad 1 \mathrm{a}$

$\begin{array}{lll}11376.77216 & 1.07 \quad 2 \mathrm{a}\end{array}$

$\begin{array}{lll}11393.33677 & 5.03 \quad 1 \mathrm{a}\end{array}$

$\begin{array}{lll}11562.56056 & 0.58 \quad 4 \mathrm{a}\end{array}$

$\begin{array}{lll}11622.97056 & 5.03 \quad 1 \mathrm{a}\end{array}$

$\begin{array}{lll}11695.91245 & 0.62 \quad 4 \mathrm{a}\end{array}$

$\begin{array}{lll}11868.24286 & 0.76 & 2 \text { a }\end{array}$

$\begin{array}{llll}10438.75558 & 0.51 \quad \mathrm{~d}\end{array}$

$10438.75558 \quad 0.51-1 \mathrm{~g}, \mathrm{a}$

$\begin{array}{lll}10867.91510 & 0.63 \quad \mathrm{~d}\end{array}$

$10867.91510 \quad 0.63-1 \mathrm{~g}, \mathrm{a}$

$\begin{array}{lll}11223.99301 & 5.06 \quad 1 \mathrm{a}\end{array}$

$11223.86943 \quad 0.54-1 \mathrm{~g}, \mathrm{a}$

$11546.61152 \quad 3.57 \quad 2 \mathrm{a}$

$\begin{array}{lll}11802.03524 & 1.12 \quad 2 \mathrm{a}\end{array}$ 
$\begin{array}{lll}19 \quad 5 & 15\end{array}$

$19 \quad 5 \quad 14$

$19 \quad 7 \quad 13$

$\begin{array}{lll}19 & 8 & 12\end{array}$

$\begin{array}{llllll}20 & 0 & 20 & 10144.06800 & 1.54 & 1 \mathrm{a}\end{array}$

$\begin{array}{lllllll}20 & 1 & 20 & 10144.30471 & 0.85 & 2 \mathrm{a}\end{array}$

$\begin{array}{lllllllll}20 & 1 & 19 & 10755.58357 & 1.53 & 1 \mathrm{a} & 11147.29731 & 1.86 & 1 \mathrm{a}\end{array}$

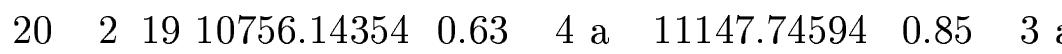

$20 \quad 2 \quad 18$

$\begin{array}{llllll}20 & 3 & 18 & 11141.07013 & 0.86 & 3\end{array}$ a

$\begin{array}{lll}20 & 3 & 17\end{array}$

$20 \quad 416$

$20 \quad 5 \quad 15$

$20 \quad 6 \quad 14$

$\begin{array}{lll}20 & 7 & 13\end{array}$

$\begin{array}{llllll}21 & 0 & 21 & 10535.41594 & 1.12 & 2 \mathrm{a}\end{array}$

$\begin{array}{llllll}21 & 1 & 21 & 10535.54730 & 1.83 & 1 \mathrm{a}\end{array}$

$\begin{array}{llllll}21 & 1 & 20 & 11179.33073 & 1.12 & 1\end{array}$

$\begin{array}{lll}21 & 2 & 20\end{array}$

$\begin{array}{llllll}21 & 2 & 19 & 11558.32663 & 1.15 & 1 \mathrm{a}\end{array}$

$\begin{array}{lll}21 & 3 & 19\end{array}$

$\begin{array}{llllll}21 & 3 & 18 & 11970.69699 & 1.20 & 1 \mathrm{a}\end{array}$

$21 \quad 4 \quad 18$

$21 \quad 6 \quad 16$

$21 \quad 7 \quad 15$

$\begin{array}{lll}22 & 0 & 22\end{array}$

$\begin{array}{llllll}22 & 1 & 22 & 10947.08647 & 0.86 & 2 \mathrm{a}\end{array}$
$11813.29112 \quad 0.76 \quad 3$ a

$12009.27858 \quad 3.38 \quad 1 \mathrm{a}$

$\begin{array}{lll}12280.59548 & 1.01 \quad 4 \mathrm{a}\end{array}$

$12554.97541 \quad 1.04 \quad 3 \mathrm{a}$

$10799.84938 \quad 0.69 \quad 4$ e,a

$\begin{array}{lll}10799.84938 & 0.69 & \mathrm{~d}\end{array}$

$11253.35746 \quad 0.67-1 \mathrm{~g}, \mathrm{a}$

$\begin{array}{lll}11253.35746 & 0.67 \quad \mathrm{~d}\end{array}$

$11623.36057 \quad 1.11 \quad 1 \mathrm{a}$

$11957.95435 \quad 0.77 \quad 3 \mathrm{a}$

$12241.11163 \quad 1.09 \quad 2$ a

$12470.85010 \quad 1.08 \quad 2$ a

$12626.54400 \quad 1.08 \quad 2 \mathrm{a}$

$12789.80263 \quad 5.03 \quad 1 \mathrm{a}$

$\begin{array}{llll}11177.97621 & 0.62 \quad \mathrm{~d}\end{array}$

$11177.97621 \quad 0.62 \quad 5$ e,a

$11655.96493 \quad 1.64 \quad 1 \mathrm{a}$

$11655.88791 \quad 0.62-1 \mathrm{~g}, \mathrm{a}$

$12056.32279 \quad 5.03 \quad 1 \mathrm{a}$

$12392.96375 \quad 5.02 \quad 1 \mathrm{a}$

$\begin{array}{lll}12956.66447 & 1.10 \quad 2 \mathrm{a}\end{array}$

$13206.16099 \quad 1.50 \quad 1 \mathrm{a}$

$11573.13456 \quad 0.60 \quad 5 \mathrm{e}, \mathrm{a}$

$\begin{array}{lll}11573.13456 & 0.60 \quad \mathrm{~d}\end{array}$ 
$22 \quad 121$

$22 \quad 2 \quad 21 \quad 11623.02038 \quad 1.17 \quad 1 \mathrm{a}$

$22 \quad 2 \quad 20$

$\begin{array}{lll}22 & 3 & 19\end{array}$

$22 \quad 418$

$\begin{array}{lll}22 & 5 & 17\end{array}$

$22 \quad 6 \quad 16$

$22 \quad 7 \quad 15$

$\begin{array}{llllll}23 & 0 & 23 & 11379.87957 & 1.32 & 1 \mathrm{a}\end{array}$

$23 \quad 123$

$\begin{array}{llllll}23 & 1 & 22 & 12084.66883 & 1.16 & 1 \mathrm{a}\end{array}$

$\begin{array}{lll}23 & 2 & 22\end{array}$

$23 \quad 321$

$\begin{array}{lll}23 & 5 & 19\end{array}$

$24 \quad 0 \quad 24$

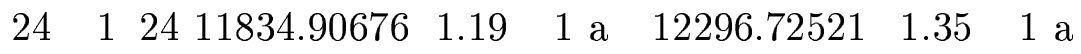

$24 \quad 123$

$\begin{array}{llllll}24 & 2 & 23 & 12569.26819 & 1.22 \quad 1 \mathrm{a}\end{array}$

$24 \quad 321$

$24 \quad 420$

$\begin{array}{llllll}25 & 0 & 25 & 12312.17000 & 1.46 & 1 \mathrm{a}\end{array}$

$25 \quad 125$

$25 \quad 124$

$25 \quad 2 \quad 24$

$25 \quad 3 \quad 23$

$25 \quad 422$

$\begin{array}{lll}26 & 0 & 26\end{array}$
$12075.40177 \quad 1.11-1 \mathrm{~g}, \mathrm{a}$

$12075.60124 \quad 1.20 \quad 1 \mathrm{a}$

$12492.31590 \quad 5.05 \quad 1 \mathrm{a}$

$12844.77339 \quad 1.18 \quad 1 \mathrm{a}$

$13161.04649 \quad 5.05 \quad 1 \mathrm{a}$

$\begin{array}{lll}13430.37036 & 1.27 \quad 2 \mathrm{a}\end{array}$

$13648.12295 \quad 5.07 \quad 1 \mathrm{a}$

$13797.73986 \quad 3.60 \quad 2 \mathrm{a}$

$11984.640130 .88 \quad \mathrm{~d}$

$11984.64013 \quad 0.88-1 \mathrm{~g}, \mathrm{a}$

$12512.40886 \quad 1.20 \quad \mathrm{~d}$

$12512.40886 \quad 1.20 \quad 1 \mathrm{a}$

$12920.49436 \quad 5.04 \quad 1 \mathrm{a}$

$\begin{array}{lll}13639.66840 & 5.05 \quad 1 \mathrm{a}\end{array}$

$12413.77697 \quad 0.76-1 \mathrm{~g}, \mathrm{a}$

$\begin{array}{lll}12413.77697 & 0.76 \quad \mathrm{~d}\end{array}$

$\begin{array}{lll}12966.37456 & 1.19 & 1 \mathrm{a}\end{array}$

$13791.92696 \quad 5.08 \quad 1 \mathrm{a}$

$14133.22428 \quad 5.04 \quad 1 \mathrm{a}$

$\begin{array}{llll}12859.73584 & 0.97 & d\end{array}$

$12859.735840 .97-1 \mathrm{~g}, \mathrm{a}$

$13437.51912 \quad 1.24 \quad \mathrm{~d}$

$13437.519121 .24-1 \mathrm{~g}, \mathrm{a}$

$13871.30654 \quad 1.49 \quad 2 \mathrm{a}$

$\begin{array}{lll}14287.18841 & 5.09 \quad 1 \mathrm{a}\end{array}$

$13322.79392 \quad 1.70-1 \mathrm{~g}, \mathrm{a}$ 
$\begin{array}{llllll}26 & 1 & 26 & 12811.07304 & 1.37 & 2 \mathrm{a}\end{array}$

$26 \quad 125$

$\begin{array}{lll}26 & 2 & 24\end{array}$

$\begin{array}{lll}27 & 0 & 27\end{array}$

$\begin{array}{lll}27 & 1 & 27\end{array}$

$\begin{array}{lll}27 & 3 & 25\end{array}$

$\begin{array}{lll}28 & 0 & 28\end{array}$

$\begin{array}{lll}29 & 1 & 29\end{array}$
$13322.79392 \quad 1.70 \quad \mathrm{~d}$

$\begin{array}{lll}13926.08390 & 1.24 \quad 2 \mathrm{a}\end{array}$

$14367.28459 \quad 5.12 \quad 1 \mathrm{a}$

$\begin{array}{lll}13803.79416 & 1.28 \quad \mathrm{~d}\end{array}$

$13803.79416 \quad 1.28-1 \mathrm{~g}, \mathrm{a}$

$14875.89444 \quad 5.38 \quad 1 \mathrm{a}$

$14298.94621 \quad 3.64 \quad 2$ a

$14814.11468 \quad 1.36 \quad 2 \mathrm{a}$

\begin{tabular}{rrrrrrrrr}
\hline$J$ & $K_{a}$ & $K_{c}$ & 200 & or $20^{+} 0$ & \multicolumn{1}{r}{101 or } & $20^{-} 0$ & 002 or & 110 \\
\hline 0 & 0 & 0 & 7201.54020 & $0.20-1 \mathrm{j}$ & 7249.81837 & $0.10-1 \mathrm{j}$ & 7445.04530 & $0.10-1 \mathrm{j}$ \\
1 & 0 & 1 & 7224.58070 & $0.10-1 \mathrm{j}$ & 7273.00000 & $0.15-1 \mathrm{j}$ & 7468.34147 & $0.05-1 \mathrm{j}, \mathrm{a}$ \\
1 & 1 & 1 & 7236.80764 & $0.04-1 \mathrm{j}$ & 7284.74246 & $0.14-1 \mathrm{j}, \mathrm{a}$ & 7479.63551 & $0.10-1 \mathrm{j}$ \\
1 & 1 & 0 & 7241.99452 & $0.04-1 \mathrm{j}$ & 7289.98113 & $0.10-1 \mathrm{j}$ & 7484.93647 & $0.24-1 \mathrm{j}, \mathrm{a}$ \\
2 & 0 & 2 & 7269.31333 & $0.07-1 \mathrm{j}$ & 7317.91734 & $0.10-1 \mathrm{j}, \mathrm{a}$ & 7513.42981 & $0.04-1 \mathrm{j}, \mathrm{a}$ \\
2 & 1 & 2 & 7277.68819 & $0.08-1 \mathrm{j}$ & 7325.22803 & $0.06-1 \mathrm{j}$ & 7520.93445 & $0.15-1 \mathrm{j}$ \\
2 & 1 & 1 & 7293.20514 & $0.06-1 \mathrm{j}$ & 7341.80267 & $0.05-1 \mathrm{j}$ & 7536.81233 & $0.15-1 \mathrm{j}$ \\
2 & 2 & 1 & 7329.42025 & $0.08-1 \mathrm{j}$ & 7376.57916 & $0.06-1 \mathrm{j}$ & 7570.46362 & $0.15-1 \mathrm{j}$ \\
2 & 2 & 0 & 7331.62289 & $0.03-1 \mathrm{j}$ & 7377.97676 & $0.08-1 \mathrm{j}, \mathrm{a}$ & 7571.93542 & $0.15-1 \mathrm{j}, \mathrm{a}$ \\
3 & 0 & 3 & 7333.55302 & $0.05-1 \mathrm{j}$ & 7382.25822 & $0.06-1 \mathrm{j}$ & 7577.97301 & $0.10-1 \mathrm{j}, \mathrm{a}$ \\
3 & 1 & 3 & 7338.25124 & $0.04-1 \mathrm{j}$ & 7386.24441 & $0.06-1 \mathrm{j}, \mathrm{a}$ & 7582.01625 & $0.07-1 \mathrm{j}, \mathrm{a}$ \\
3 & 1 & 2 & 7368.97355 & $0.11-1 \mathrm{j}$ & 7418.05827 & $0.05-1 \mathrm{j}$ & 7613.42341 & $0.11-1 \mathrm{j}$ \\
3 & 2 & 2 & 7398.40734 & $0.08-1 \mathrm{j}$ & 7445.57945 & $0.08-1 \mathrm{j}, \mathrm{a}$ & 7640.38676 & $0.10-1 \mathrm{j}$ \\
3 & 2 & 1 & 7405.23814 & $0.06-1 \mathrm{j}$ & 7451.87300 & $0.10-1 \mathrm{j}$ & 7647.07771 & $0.10-1 \mathrm{j}, \mathrm{a}$ \\
3 & 3 & 1 & 7472.95248 & $0.12-1 \mathrm{j}$ & 7517.49036 & $0.07-1 \mathrm{j}$ & 7709.66650 & $0.21-1 \mathrm{j}, \mathrm{a}$ \\
3 & 3 & 0 & 7473.06138 & $0.06-1 \mathrm{j}$ & 7517.74505 & $0.11-1 \mathrm{j}, \mathrm{a}$ & 7709.93668 & $0.14-1 \mathrm{j}, \mathrm{a}$ \\
4 & 0 & 4 & 7415.59915 & $0.04-1 \mathrm{j}$ & 7464.43707 & $0.04-1 \mathrm{j}, \mathrm{a}$ & 7660.31994 & $0.10-1 \mathrm{j}, \mathrm{a}$ \\
4 & 1 & 4 & 7417.84285 & $0.05-1 \mathrm{j}$ & 7466.23546 & $0.05-1 \mathrm{j}$ & 7662.18625 & $0.17-1 \mathrm{j}, \mathrm{a}$
\end{tabular}


$\begin{array}{lllllllll}4 & 1 & 3 & 7467.77336 & 0.04-1 \mathrm{j} & 7517.21214 & 0.04-1 \mathrm{j}, \mathrm{a} & 7713.08143 & 0.21-1 \mathrm{j}\end{array}$

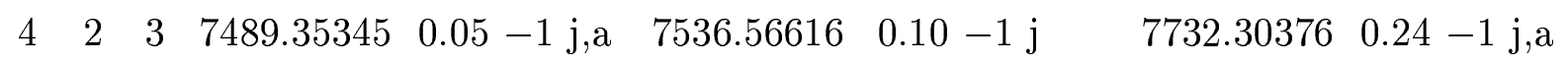

$\begin{array}{lllllllll}4 & 2 & 2 & 7505.81263 & 0.10-1 \mathrm{j} & 7552.36533 & 0.08-1 \mathrm{j}, \mathrm{a} & 7749.46651 & 0.10-1 \mathrm{j}, \mathrm{a}\end{array}$

$\begin{array}{llllllllll}4 & 3 & 2 & 7568.20455 & 0.08-1 \mathrm{j} & 7611.81050 & 0.11-1 \mathrm{j} & 7805.30314 & 0.27-1 \mathrm{j}, \mathrm{a}\end{array}$

$\begin{array}{lllllllllll}4 & 3 & 1 & 7568.89106 & 0.06 & -1 & \mathrm{j} & 7613.43068 & 0.18-1 \mathrm{j}, \mathrm{a} & 7807.07468 & 0.22-1 \mathrm{j}\end{array}$

$\begin{array}{lllllllll}4 & 4 & 1 & 7665.53547 & 0.10-1 \mathrm{j} & 7707.63904 & 0.15-1 \mathrm{j}, \mathrm{a} & 7897.44589 & 0.09-1 \mathrm{j}, \mathrm{a}\end{array}$

$\begin{array}{lllllllll}4 & 4 & 0 & 7665.57165 & 0.06-1 \mathrm{j} & 7707.71847 & 0.15-1 \mathrm{j}, \mathrm{a} & 7897.48763 & 0.15-1 \mathrm{j}\end{array}$

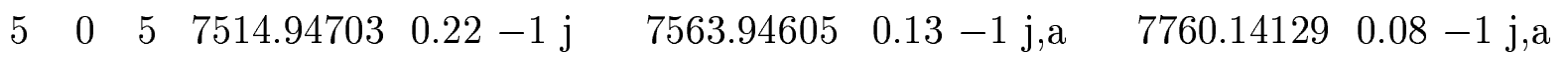

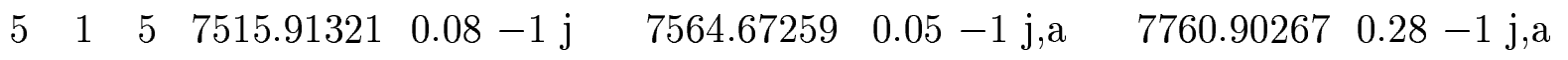

$\begin{array}{lllllllll}5 & 1 & 4 & 7587.38639 & 0.25-1 \mathrm{j} & 7636.97312 & 0.07-1 \mathrm{j}, \mathrm{a} & 7833.39552 & 0.15-1 \mathrm{j}, \mathrm{a}\end{array}$

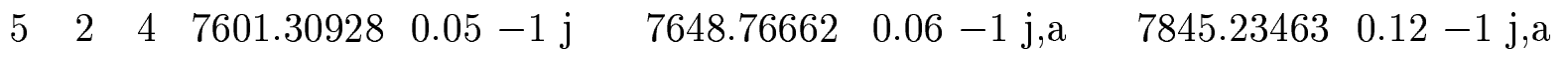

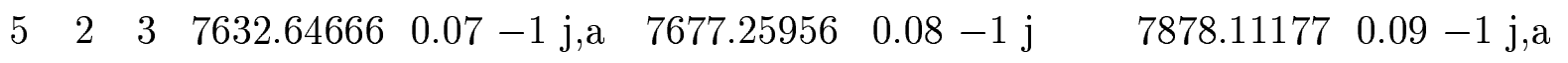

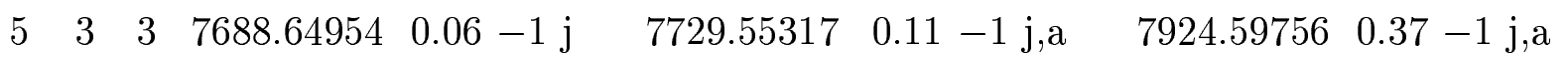

$\begin{array}{lllllllll}5 & 3 & 2 & 7690.43440 & 0.05-1 \mathrm{j} & 7735.32680 & 0.13-1 \mathrm{j} & 7930.85259 & 0.28-1 \mathrm{j}, \mathrm{a}\end{array}$

$\begin{array}{lllllllllll}5 & 4 & 2 & 7784.28334 & 0.04 & -1 & \mathrm{j} & 7825.81951 & 0.08-1 \mathrm{j} & 8017.62015 & 0.30-1 \mathrm{j}\end{array}$

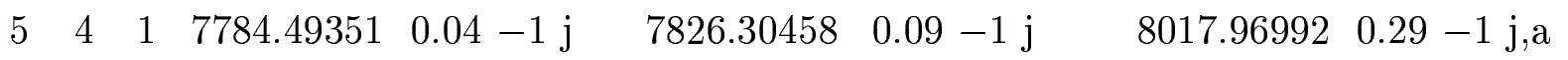

$\begin{array}{lllllllll}5 & 5 & 1 & 7906.96499 & 0.08-1 \mathrm{j} & 7945.96683 & 0.15-1 \mathrm{j}, \mathrm{a} & 8132.69778 & 0.29-1 \mathrm{j}\end{array}$

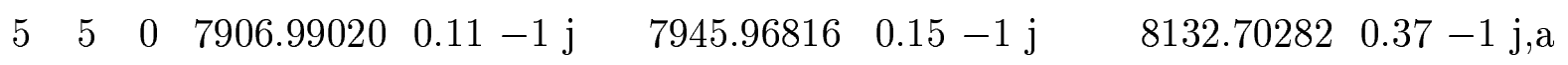

$\begin{array}{llllllllll}6 & 0 & 6 & 7631.70601 & 0.10 & -1 \mathrm{j} & 7680.92649 & 0.17-1 \mathrm{j}, \mathrm{a} & 7877.87210 & 0.37-1 \mathrm{j}, \mathrm{a}\end{array}$

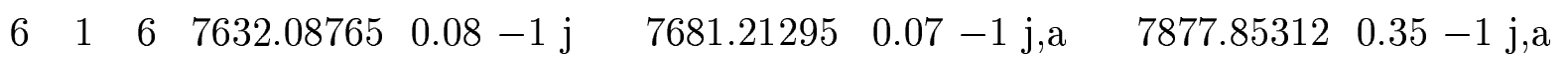

$\begin{array}{lllllllll}6 & 1 & 5 & 7725.44900 & 0.05-1 \mathrm{j} & 7774.99747 & 0.06-1 \mathrm{j}, \mathrm{a} & 7972.56235 & 0.16-1 \mathrm{j}, \mathrm{a}\end{array}$

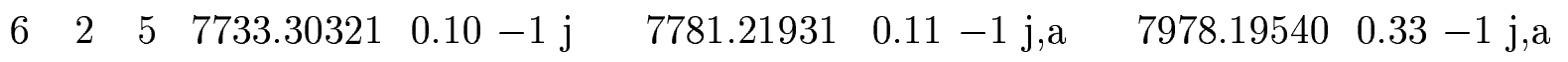

$\begin{array}{llllllllll}6 & 2 & 4 & 7784.02997 & 0.08 & -1 \mathrm{j} & 7836.64253 & 0.07-1 \mathrm{j}, \mathrm{a} & 8031.11063 & 0.23-1 \mathrm{j}\end{array}$

$\begin{array}{lllllllll}6 & 3 & 4 & 7821.68963 & 0.15-1 \mathrm{j} & 7870.45834 & 0.10-1 \mathrm{j}, \mathrm{a} & 8066.81021 & 0.30-1 \mathrm{j}, \mathrm{a}\end{array}$

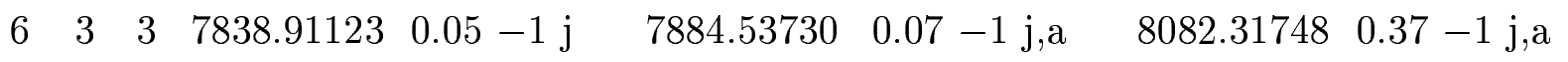

$\begin{array}{lllllllll}6 & 4 & 3 & 7926.99458 & 0.10 & -1 & \mathrm{j} & 7967.29952 & 0.12-1 \mathrm{j}\end{array} \quad 8161.88794 \quad 0.12-1 \mathrm{j}, \mathrm{a}$

$\begin{array}{lllllllll}6 & 4 & 2 & 7927.92019 & 0.07-1 \mathrm{j} & 7969.67463 & 0.14-1 \mathrm{j}, \mathrm{a} & 8163.47664 & 0.20-1 \mathrm{j}\end{array}$ 


\begin{tabular}{|c|c|c|c|c|c|c|c|}
\hline & 5 & $2 \quad 8049.32582$ & $0.07-1 \mathrm{j}$ & 36 & $.15-1 \mathrm{j}$ & 81 & $0.28-1 \mathrm{j}, \mathrm{a}$ \\
\hline & 5 & $1 \quad 8049.49192$ & $0.08-1 \mathrm{j}$ & 087.97649 & $0.10-1 \mathrm{j}, \mathrm{a}$ & 732 & $0.24-1 \mathrm{j}, \mathrm{a}$ \\
\hline & 6 & 8195.79741 & $0.10-1 \mathrm{j}, \mathrm{a}$ & 8230.65477 & $.70-1 \mathrm{j}$ & 322 & $0.20-1 \mathrm{j}, \mathrm{a}$ \\
\hline & 6 & $0 \quad 8195.79962$ & $0.20-1 \mathrm{j}$ & 8230.65535 & $0.57-1 \mathrm{j}, \mathrm{a}$ & 323 & $0.20-1 \mathrm{j}$ \\
\hline & 0 & $7 \quad 7765.97418$ & $0.08-1 \mathrm{j}, \mathrm{a}$ & 7815.54783 & $0.10-1 \mathrm{j}$ & 257 & $0.07-1 \mathrm{j}, \mathrm{a}$ \\
\hline & 1 & $7 \quad 7766.14441$ & $0.08-1 \mathrm{j}$ & 7815.66024 & $0.11-1 \mathrm{j}, \mathrm{a}$ & 008 & $0.06-1 \mathrm{j}$ \\
\hline & 1 & $6 \quad 7880.50415$ & $0.08-1 \mathrm{j}$ & 7929.98612 & $0.15-1 \mathrm{j}$ & 303 & $0.15-1 \mathrm{j}, \mathrm{a}$ \\
\hline & 2 & 46324 & $0.15-1 \mathrm{j}$ & 7932 & $0.15-1 \mathrm{j}, \mathrm{a}$ & 719 & $0.15-1 \mathrm{j}, \mathrm{a}$ \\
\hline & 2 & $5 \quad 7957.66894$ & $0.06-1 \mathrm{j}, \mathrm{a}$ & 96041 & $0.08-1 \mathrm{j}$ & 745 & $0.19-1 \mathrm{j}, \mathrm{a}$ \\
\hline & 3 & 5798 & $0.09-1 \mathrm{j}$ & 803 & $0.08-1 \mathrm{j}, \mathrm{a}$ & 487 & $0.35-1 \mathrm{j}$ \\
\hline & 3 & 4801 & $0.20-1 \mathrm{j}$ & 806 & $0.06-1 \mathrm{j}$ & 741 & $0.33-1 \mathrm{j}, \mathrm{a}$ \\
\hline & 44 & $4 \quad 8093.58025$ & $0.07-1 \mathrm{j}$ & 313 & $0.07-1 \mathrm{j}, \mathrm{a}$ & 150 & $0.21-1 \mathrm{j}, \mathrm{a}$ \\
\hline & $4 \quad 3$ & 38096 & $0.20-1 \mathrm{j}, \mathrm{a}$ & 135 & $0.05-1 \mathrm{j}$ & 526 & $0.14-1 \mathrm{j}, \mathrm{a}$ \\
\hline & 5 & 174 & $0.23-1 \mathrm{j}$ & 57 & $0.06-1 \mathrm{j}, \mathrm{a}$ & 937 & $0.07-1 \mathrm{j}, \mathrm{a}$ \\
\hline & 5 & 282 & $0.08-1 \mathrm{j}, \mathrm{a}$ & 72 & $0.18-1 \mathrm{j}$ & 069 & $0.10-1 \mathrm{j}, \mathrm{a}$ \\
\hline & 6 & 993 & $0.15-1 \mathrm{j}, \mathrm{a}$ & 644 & $0.08-1 \mathrm{j}, \mathrm{a}$ & 390 & $0.80-1 \mathrm{j}$ \\
\hline & 6 & 1836 & $0.24-1 \mathrm{j}, \mathrm{a}$ & 161 & $0.20-1 \mathrm{j}$ & 353 & $0.15-1 \mathrm{j}$ \\
\hline & 7 & 1853 & 0.40 & 577 & $0.24-1 \mathrm{j}, \mathrm{a}$ & 599 & 1.50 \\
\hline & 7 & 10775 & $0.40-1 \mathrm{j}$ & 85 & $0.25-1 \mathrm{j}$ & 599 & $1.50-1 \mathrm{j}$ \\
\hline & $0 \quad 8$ & 269 & $0.15-1 \mathrm{j}$ & 239 & $0.11-1 \mathrm{j}, \mathrm{a}$ & 895 & $0.15-1 \mathrm{j}, \mathrm{a}$ \\
\hline & 18 & 7917.95666 & $0.20-1 \mathrm{j}$ & 44 & $0.16-1 \mathrm{j}$ & 53 & $0.46-1 \mathrm{j}, \mathrm{a}$ \\
\hline & 17 & 78052.18858 & $0.15-1 \mathrm{j}$ & 876 & $0.12-1 \mathrm{j}, \mathrm{a}$ & 723 & $0.24-1 \mathrm{j}, \mathrm{a}$ \\
\hline & 2 & 78054.11663 & $0.06-1 \mathrm{j}$ & 394 & $0.12-1 \mathrm{j}, \mathrm{a}$ & 49 & $0.10-1 \mathrm{j}, \mathrm{a}$ \\
\hline & 26 & $6 \quad 8150.83307$ & $0.16-1 \mathrm{j}$ & 8202.56374 & $0.08-1 \mathrm{j}, \mathrm{a}$ & 4989 & $0.15-1 \mathrm{j}$ \\
\hline & 36 & $6 \quad 8169.51747$ & $0.06-1 \mathrm{j}, \mathrm{a}$ & 8216.20810 & $0.08-1 \mathrm{j}$ & 8416.57470 & $0.29-1 \mathrm{j}$ \\
\hline & 35 & 5216.20398 & $0.20-1 \mathrm{j}$ & 8261.53081 & $0.08-1 \mathrm{j}, \mathrm{a}$ & 8465.52432 & $0.24-1 \mathrm{j}, \mathrm{a}$ \\
\hline & 5 & 58283.72181 & $0.08-1 \mathrm{j}$ & 8324.19162 & $0.08-1$ & 8520.96603 & $0.15-1 \mathrm{j}, \mathrm{a}$ \\
\hline
\end{tabular}



$\begin{array}{lllllll}8 & 4 & 4 & 8291.54653 & 0.35 & -1 \mathrm{j}\end{array}$
$8334.85257 \quad 0.05-1 \mathrm{j}, \mathrm{a}$
$8533.352530 .35-1 \mathrm{j}$
$\begin{array}{llllllll}8 & 5 & 4 & 8404.50384 & 0.24-1 \mathrm{j}, \mathrm{a} & 8444.04992 & 0.08 & -1 \mathrm{j}\end{array}$
$8638.70344 \quad 0.30-1 \mathrm{j}, \mathrm{a}$
$\begin{array}{llllllll}8 & 5 & 3 & 8406.58207 & 0.15 & -1 \mathrm{j}, \mathrm{a} & 8444.70816 & 0.05-1 \mathrm{j}, \mathrm{a}\end{array}$
$8639.929410 .20-1 \mathrm{j}$
$\begin{array}{lllllll}8 & 6 & 3 & 8552.70901 & 0.10-1 & \mathrm{j}, \mathrm{a}\end{array}$
$8585.41532 \quad 0.35-1 \mathrm{j}$
$8777.54724 \quad 0.45-1 \mathrm{j}, \mathrm{a}$
$\begin{array}{lllllll}8 & 6 & 2 & 8552.99273 & 0.08-1 \mathrm{j}\end{array}$
8585.39269
$0.15-1 \mathrm{j}, \mathrm{a}$
$8777.599550 .40-1 \mathrm{j}$
$\begin{array}{llllll}8 & 7 & 2 & 8721.13771 & 0.35-1\end{array}$
8748.42950
$0.40-1 \mathrm{j}$
$8926.528451 .00-1 \mathrm{j}$
$\begin{array}{llllll}8 & 7 & 1 & 8721.13876 & 0.40-1 j\end{array}$
8748.42936
$0.37-1 \mathrm{j}, \mathrm{a}$
$8926.52845 \quad 1.00-1 \mathrm{j}$
$\begin{array}{lllllll}8 & 8 & 1 & 8912.73417 & 1.01 & 1\end{array}$
8943.39755
$0.30-1 \mathrm{j}$
$9105.96467 \quad 0.45-1 \mathrm{j}, \mathrm{a}$
$\begin{array}{llllll}8 & 8 & 0 & 8912.73417 & 1.01\end{array}$
8943.39755
$0.29-1 \mathrm{j}, \mathrm{a}$
$9105.964880 .50-1 \mathrm{j}$
$\begin{array}{lllllll}9 & 0 & 9 & 8087.39507 & 0.11 & -1 \mathrm{j}\end{array}$
$8137.85565 \quad 0.25-1 \mathrm{j}$
$8335.48808 \quad 0.10-1 \mathrm{j}, \mathrm{a}$
$\begin{array}{llllll}9 & 1 & 9 & 8087.45205 & 0.10-1\end{array}$
8138.00841
$0.15-1 \mathrm{j}, \mathrm{a}$
$8335.539840 .37-1 \mathrm{j}, \mathrm{a}$
$\begin{array}{llllll}9 & 1 & 8 & 8240.86016 & 0.07 & -1 \mathrm{j}, \mathrm{a}\end{array}$
8290.67268
$0.13-1 \mathrm{j}$
$8488.029910 .20-1 \mathrm{j}, \mathrm{a}$
$\begin{array}{llllll}9 & 2 & 8 & 8241.83115 & 0.24 & -1 \mathrm{j}, \mathrm{a}\end{array}$
8291.62793
$0.09-1 \mathrm{j}, a$
$8486.873650 .45-1 \mathrm{j}, \mathrm{a}$
$\begin{array}{lllllll}9 & 2 & 7 & 8361.78497 & 0.32 & -1 \mathrm{j}, \mathrm{a}\end{array}$
8412.63183
$0.10-1 \mathrm{j}$
$8610.00995 \quad 0.35-1 \mathrm{j}, \mathrm{a}$
$\begin{array}{llllllll}9 & 3 & 7 & 8369.43182 & 0.30-1 \mathrm{j} & 8419.79333 & 0.08 & -1 \mathrm{j}, \mathrm{a}\end{array}$
$\begin{array}{lllllllll}9 & 3 & 6 & 8441.58262 & 0.15 & -1 \mathrm{j}, \mathrm{a} & 8503.73297 & 1.00 & 1 \mathrm{a}\end{array}$
$8692.96920 \quad 0.28-1 \mathrm{j}, \mathrm{a}$

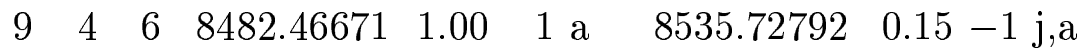
$8734.213030 .25-1 \mathrm{j}$
$\begin{array}{llllll}9 & 4 & 5 & 8513.54626 & 0.10-1 \mathrm{j}, \mathrm{a}\end{array}$
$8558.23387 \quad 0.11-1 \mathrm{j}$
$8757.78227 \quad 0.19-1 \mathrm{j}, \mathrm{a}$

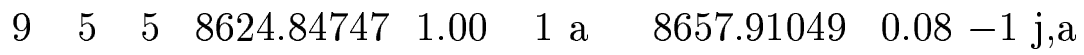
$8854.921620 .25-1 \mathrm{j}$
$\begin{array}{lllllll}9 & 5 & 4 & 8621.78419 & 0.19-1 \mathrm{j}, \mathrm{a} & 8660.38128 & 0.44-1 \mathrm{j}\end{array}$
$8858.59761 \quad 0.19-1 \mathrm{j}, \mathrm{a}$
$\begin{array}{llllllll}9 & 6 & 4 & 8766.62760 & 0.50-1 \mathrm{j} & 8798.64155 & 0.31-1 \mathrm{j}, \mathrm{a}\end{array}$
$8994.46200 \quad 0.40-1 \mathrm{j}$
$\begin{array}{llllll}9 & 6 & 3 & 8768.72906 & 0.19-1 \mathrm{j}, \mathrm{a}\end{array}$
$8798.60388 \quad 0.20-1 \mathrm{j}$
$8994.688780 .32-1 \mathrm{j}, \mathrm{a}$
$\begin{array}{lll}9 & 7 & 3\end{array}$
$8961.05814 \quad 0.37-1 \mathrm{j}, \mathrm{a}$
$9139.67506 \quad 3.00-1 \mathrm{j}$
$\begin{array}{llllll}9 & 7 & 2 & 8935.28681 & 1.00 & 1 \mathrm{a}\end{array}$
8961.01895
$0.30-1 \mathrm{j}$
$9139.684120 .37-1 \mathrm{j}, \mathrm{a}$
$\begin{array}{lllllllll}9 & 8 & 2 & 9128.70157 & 0.71 & \text { d } & 9161.85161 & 0.37 & -1 \mathrm{j}, \mathrm{a}\end{array}$
$\begin{array}{lllllllll}9 & 8 & 1 & 9128.70157 & 0.71 & 3 \mathrm{e}, \mathrm{a} & 9161.84776 & 0.40-1 \mathrm{j}\end{array}$
$\begin{array}{lll}9 & 9 & 1\end{array}$
$9513.41505 \quad 0.50-1 \mathrm{j}$ 
$\begin{array}{lll}9 & 9 & 0\end{array}$

$\begin{array}{lllllll}10 & 0 & 10 & 8274.56469 & 0.06 & -1 \mathrm{j}\end{array}$

$10 \quad 110$

$\begin{array}{lllllll}10 & 1 & 9 & 8446.74640 & 0.06 & -1 \mathrm{j}\end{array}$

$\begin{array}{llllll}10 & 2 & 9 & 8447.62743 & 0.20-1 \mathrm{j}, \mathrm{a}\end{array}$

$\begin{array}{lllllll}10 & 2 & 8 & 8588.50472 & 0.15 & -1 \mathrm{j}, \mathrm{a} & 863\end{array}$

$\begin{array}{llllllll}10 & 3 & 8 & 8593.17871 & 0.24 & -1 \mathrm{j}, \mathrm{a} & 864\end{array}$

$\begin{array}{lllllll}10 & 3 & 7 & 8686.90064 & 0.10-1 \mathrm{j}\end{array}$

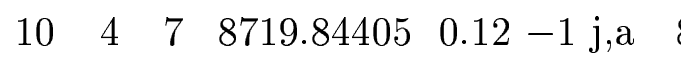

$\begin{array}{llllll}10 & 4 & 6 & 8762.22283 & 0.27 & -1 \mathrm{j}, \mathrm{a}\end{array}$

$\begin{array}{llllll}10 & 5 & 6 & 8859.76147 & 0.23 & -1 \mathrm{j}, \mathrm{a}\end{array}$

$\begin{array}{lll}10 & 5 & 5\end{array}$

$\begin{array}{llllll}10 & 6 & 5 & 9003.96945 & 0.80-1 \mathrm{j}\end{array}$

$\begin{array}{lll}10 & 6 & 4\end{array}$

$\begin{array}{lllllllll}10 & 7 & 4 & 9172.66682 & 1.00 & 1 \mathrm{a} & 9197.45514 & 0.40-1 \mathrm{j}\end{array}$

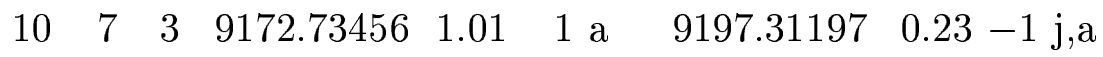

$\begin{array}{llllllll}10 & 8 & 3 & 9367.98192 & 0.99 & 2 \mathrm{e}, \mathrm{a} & 9403.03113 & 0.40-1 \mathrm{j}\end{array}$

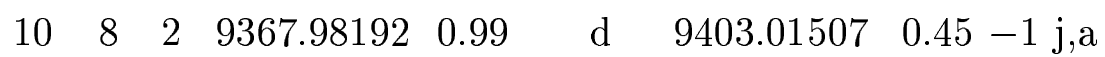

$\begin{array}{lll}10 & 9 & 2\end{array}$

$\begin{array}{lll}10 & 9 & 1\end{array}$

$\begin{array}{lllllllll}10 & 10 & 1 & 9742.80239 & 1.10 & 1 \text { a } & 9783.24165 & 0.72 & \text { d }\end{array}$

$\begin{array}{lllllllll}10 & 10 & 0 & 9742.80239 & 1.10 & \text { d } & 9783.24165 & 0.72 & 2 \text { a }\end{array}$

$\begin{array}{llllllll}11 & 0 & 11 & 8478.78402 & 0.13 & -1 \mathrm{j}, \mathrm{a} & 8530.58419 & 0.40-1 \mathrm{j}\end{array}$

$\begin{array}{llllllll}11 & 1 & 11 & 8478.87945 & 0.13 & -1 \mathrm{j}, \mathrm{a} & 8530.59642 & 0.45-1 \mathrm{j}, \mathrm{a}\end{array}$

$\begin{array}{llllllll}11 & 1 & 10 & 8669.96417 & 0.06 & -1 \mathrm{j}, \mathrm{a} & 8719.46789 & 0.26-1 \mathrm{j}\end{array}$

$\begin{array}{llllllll}11 & 2 & 10 & 8669.12118 & 0.15 & -1 \mathrm{j}, \mathrm{a} & 8720.23627 & 0.16-1 \mathrm{j}, \mathrm{a}\end{array}$

$\begin{array}{llllllll}11 & 2 & 9 & 8831.35058 & 0.10-1 \mathrm{j}, \mathrm{a} & 8882.66345 & 0.15-1 \mathrm{j}, \mathrm{a}\end{array}$
$9513.414680 .45-1 \mathrm{j}, \mathrm{a}$

$8523.78433 \quad 0.20-1 \mathrm{j}$

$8523.793530 .38-1 \mathrm{j}, \mathrm{a}$

$8696.04484 \quad 0.30-1 \mathrm{j}, \mathrm{a}$

$8694.33306 \quad 0.19-1 \mathrm{j}, \mathrm{a}$

$8836.851540 .37-1 \mathrm{j}, \mathrm{a}$

$8840.04549 \quad 0.24-1 \mathrm{j}, \mathrm{a}$

$8940.59265 \quad 0.37-1 \mathrm{j}, \mathrm{a}$

$8968.65415 \quad 0.29-1 \mathrm{j}, \mathrm{a}$

$9016.22840 \quad 0.80-1 \mathrm{j}$

$9094.22390 \quad 0.20-1 \mathrm{j}, \mathrm{a}$

$9234.67413 \quad 0.37-1 \mathrm{j}, \mathrm{a}$

$9235.45197 \quad 0.37-1 \mathrm{j}, \mathrm{a}$

$9377.08612 \quad 1.04 \quad 1 \mathrm{a}$

$\begin{array}{lll}9571.37750 & 1.02 \quad 1 \mathrm{a}\end{array}$

$9751.64756 \quad 1.02 \quad 1 \mathrm{a}$

$9751.64756 \quad 1.02 \quad \mathrm{~d}$

$8729.67158 \quad 0.38-1 \mathrm{j}, \mathrm{a}$

$8729.68222 \quad 0.50-1 \mathrm{j}$

$8918.28009 \quad 1.00 \quad 1 \mathrm{a}$

$8918.51321 \quad 1.03 \quad 1 \mathrm{a}$

$9079.85432 \quad 0.35-1 \mathrm{j}, \mathrm{a}$ 


\begin{tabular}{|c|c|c|c|c|c|c|c|c|c|}
\hline 13 & 9 & & & & 8883.03745 & $0.24-1 \mathrm{j}, \mathrm{a}$ & 9076.57778 & 0.30 & $-1 \mathrm{j}$ \\
\hline 3 & 8 & 8954.07786 & 0.15 & $-1 \mathrm{j}$ & 9007.83016 & $0.33-1 \mathrm{j}, \mathrm{a}$ & 9205.75342 & 0.35 & $-1 \mathrm{j}, \mathrm{a}$ \\
\hline 4 & 8 & & & & 9022.49294 & $0.23-1 \mathrm{j}, \mathrm{a}$ & 9223.38417 & 0.70 & $-1 \mathrm{j}$ \\
\hline 4 & 7 & 9035.75414 & 0.71 & $2 \mathrm{a}$ & 9086.44760 & $0.60-1 \mathrm{j}$ & 9292.69667 & 0.56 & $-1 \mathrm{j}, \mathrm{a}$ \\
\hline 5 & 7 & & & & 9154.44999 & $0.32-1 \mathrm{j}, \mathrm{a}$ & & & \\
\hline 5 & 6 & 9128.79102 & 0.99 & $2 \mathrm{e}, \mathrm{a}$ & 9170.24304 & $0.24-1 \mathrm{j}, \mathrm{a}$ & 9374.78190 & 0.24 & $-1 \mathrm{j}, \mathrm{a}$ \\
\hline 6 & 6 & & & & 9296.35661 & $0.24-1 \mathrm{j}, \mathrm{a}$ & & & \\
\hline 6 & 5 & 9262.70831 & 0.99 & $2 \mathrm{e}, \mathrm{a}$ & 9297.19338 & $0.24-1 \mathrm{j}, \mathrm{a}$ & 1718 & 0.80 & $-1 \mathrm{j}, \mathrm{a}$ \\
\hline 7 & 5 & & & & 9457.53262 & $0.35-1 \mathrm{j}, \mathrm{a}$ & & & \\
\hline 7 & 4 & 9433.41853 & 0.71 & $3 \mathrm{e}, \mathrm{a}$ & 9457.15002 & $0.55-1 \mathrm{j}$ & & & \\
\hline 8 & 4 & 9630.39792 & 1.05 & $\mathrm{~d}$ & & & 9836.46540 & 6.43 & $\mathrm{~d}$ \\
\hline 8 & 3 & 9630.39792 & 1.05 & $1 \mathrm{a}$ & & & 9836.46540 & 6.43 & $1 \mathrm{e}$ \\
\hline 9 & 3 & & & & 9811.33935 & $1.00 \quad 2 \mathrm{e}, \mathrm{a}$ & 10013.04835 & 0.73 & $\mathrm{~d}$ \\
\hline 9 & 2 & & & & 9811.33935 & $1.00 \quad \mathrm{~d}$ & 1001 & 0.73 & $2 \mathrm{a}$ \\
\hline 10 & 2 & 10006.58121 & 1.12 & $\mathrm{~d}$ & 10048.42203 & $0.74 \quad 2 \mathrm{a}$ & & & \\
\hline 10 & 1 & 10006.58121 & 1.12 & $1 \mathrm{a}$ & 10048 & 0.74 & & & \\
\hline 11 & & 10244.80733 & 1.13 & $\mathrm{~d}$ & & & & & \\
\hline 11 & 0 & 10244.80733 & 1.13 & $2 \mathrm{e}, \mathrm{a}$ & & & & & \\
\hline 0 & 12 & 8701.04500 & 1.50 & $-1 \mathrm{j}$ & 8753.24941 & $0.33-1 \mathrm{j}, \mathrm{a}$ & 8953.16005 & 0.90 & $-1 \mathrm{j}$ \\
\hline 1 & 12 & 8701.04648 & 0.37 & $-1 \mathrm{j}, \mathrm{a}$ & 8753.22920 & $0.45-1 \mathrm{j}, \mathrm{a}$ & 8953.15987 & 0.50 & $-1 \mathrm{j}, \mathrm{a}$ \\
\hline 1 & 11 & 8909.05180 & 0.15 & $-1 \mathrm{j}, \mathrm{a}$ & 8960.78803 & $0.23-1 \mathrm{j}, \mathrm{a}$ & 9159.98796 & 0.71 & $2 \mathrm{a}$ \\
\hline 2 & 11 & 8909.81648 & 0.25 & $-1 \mathrm{j}$ & 8961.13575 & $0.25-1 \mathrm{j}$ & 9160.06494 & 1.00 & $2 \mathrm{e}, \mathrm{a}$ \\
\hline 2 & 10 & 9090.59427 & 0.20 & $-1 \mathrm{j}$ & 9141.59622 & $0.27-1 \mathrm{j}, \mathrm{a}$ & 9342.21245 & 1.00 & $1 \mathrm{a}$ \\
\hline 3 & 10 & 9090.88971 & 0.54 & $-1 \mathrm{j}, \mathrm{a}$ & 9141.37257 & $0.20-1 \mathrm{j}, \mathrm{a}$ & 9339.32370 & 1.00 & $1 \mathrm{a}$ \\
\hline 3 & 9 & & & & 9288.39745 & $0.23-1 \mathrm{j}, \mathrm{a}$ & 9486.86189 & 0.98 & $2 \mathrm{e}, \mathrm{a}$ \\
\hline 4 & 9 & 9246.27866 & 1.01 & $1 \mathrm{a}$ & 9295.64684 & $0.51-1 \mathrm{j}, \mathrm{a}$ & 9497.92946 & 0.99 & $2 \mathrm{e}, \mathrm{a}$ \\
\hline 4 & 8 & & & & 9404.10017 & $1.07 \quad 1 \mathrm{a}$ & 9591.49727 & 0.98 & $3 \mathrm{e}, \mathrm{a}$ \\
\hline
\end{tabular}




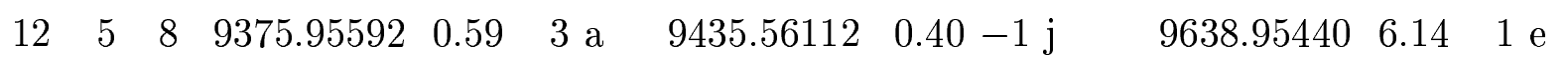

$\begin{array}{lllll}12 & 5 & 7 & 9464.60342 & 0.08-1 \mathrm{j}, \mathrm{a}\end{array}$

$\begin{array}{llllllllllll}12 & 6 & 7 & 9547.81839 & 0.70 & 4 \mathrm{e}, \mathrm{a} & 9580.07650 & 0.50-1 \mathrm{j} & 9784.01486 & 0.70 & 4 \mathrm{e}, \mathrm{a}\end{array}$

$\begin{array}{llllll}12 & 6 & 6 & 9583.27629 & 0.33-1 \mathrm{j}, \mathrm{a}\end{array}$

$\begin{array}{llllllllll}12 & 7 & 6 & 9716.54102 & 0.99 & 2 \mathrm{e}, \mathrm{a} & 9923.20277 & 0.99 & 2 \mathrm{e}, \mathrm{a}\end{array}$

$\begin{array}{lllllllll}12 & 7 & 5 & 9717.42638 & 1.07 & 1 \mathrm{a} & 9740.39797 & 0.46-1 \mathrm{j}, \mathrm{a}\end{array}$

$\begin{array}{llllll}12 & 8 & 5 & 9915.67165 & 1.06 & 1 \mathrm{a}\end{array}$

$\begin{array}{llllll}12 & 8 & 4 & 9915.67165 & 1.06 & \mathrm{~d}\end{array}$

$\begin{array}{llllll}12 & 9 & 4 & 10094.25441 & 5.02 & 1 \mathrm{a}\end{array}$

$\begin{array}{llllll}12 & 9 & 3 & 10094.25441 & 5.02 & \mathrm{~d}\end{array}$

$\begin{array}{llllll}12 & 10 & 3 & 10292.45858 & 1.09 & 1 \mathrm{a}\end{array}$

$\begin{array}{llllll}12 & 10 & 2 & 10292.45858 & 1.09 & \mathrm{~d}\end{array}$

$\begin{array}{llllll}12 & 11 & 1 & 10559.76150 & 0.86 & 2 \mathrm{a}\end{array}$

$\begin{array}{llllll}12 & 12 & 1 & 10772.25795 & 1.26 & 2 \mathrm{e}, \mathrm{a}\end{array}$

$\begin{array}{llllll}12 & 12 & 0 & 10772.25795 & 1.26 & \mathrm{~d}\end{array}$

$\begin{array}{llllllllll}13 & 0 & 13 & 8940.55162 & 0.15 & -1 \mathrm{j}, \mathrm{a} & 8993.46495 & 0.40-1 \mathrm{j} & 9194.17355 & 0.40-1 \mathrm{j}\end{array}$

$\begin{array}{llllllllll}13 & 1 & 13 & 8940.55222 & 0.72 & -1 \mathrm{j}, \mathrm{a} & 8993.46333 & 0.19-1 \mathrm{j}, \mathrm{a} & 9194.17379 & 0.37-1 \mathrm{j}, \mathrm{a}\end{array}$

$\begin{array}{lllllllllll}13 & 1 & 12 & 9167.42019 & 0.24 & -1 \mathrm{j}, \mathrm{a} & 9219.17744 & 0.45-1 \mathrm{j} & 9418.98489 & 0.71 & 3 \mathrm{e}, \mathrm{a}\end{array}$

$\begin{array}{llllllll}13 & 2 & 12 & 9167.50000 & 1.00-1 \mathrm{j} & 9219.10238 & 0.33-1 \mathrm{j}, \mathrm{a}\end{array}$

$\begin{array}{llllllll}13 & 2 & 11 & 9416.30742 & 0.30-1 \mathrm{j} & 9615.06245 & 1.00 & 2 \mathrm{e}, \mathrm{a}\end{array}$

$\begin{array}{lllllllll}13 & 3 & 11 & 9416.95179 & 0.15-1 \mathrm{j}, \mathrm{a} & 9615.89050 & 5.13 & 1 \mathrm{e}\end{array}$

$\begin{array}{lllllllll}13 & 3 & 10 & 9578.39670 & 0.80-1 \mathrm{j} & 9783.75903 & 0.71 & 3 \mathrm{e}, \mathrm{a}\end{array}$

$13410 \quad 9587.30465 \quad 0.32-1 \mathrm{j}$,a

$\begin{array}{llllllllllll}13 & 4 & 9 & 9649.37070 & 6.12 & 1 \mathrm{e} & 9712.55986 & 1.16 & 1 \mathrm{a} & 9909.24095 & 1.01 & 2 \mathrm{e}, \mathrm{a}\end{array}$

$\begin{array}{lllll}13 & 5 & 9 & 9737.39594 & 0.51-1 \mathrm{j}, \mathrm{a}\end{array}$

$\begin{array}{llllll}13 & 5 & 8 & 9740.14906 & 1.00 & 1 \mathrm{a}\end{array}$

$9995.11003 \quad 1.01 \quad 2 \mathrm{e}, \mathrm{a}$

$\begin{array}{llllll}13 & 6 & 8 & 9886.09405 & 0.72 & 2 \mathrm{a}\end{array}$ 
$\begin{array}{llllll}13 & 6 & 7 & 9858.00769 & 0.72 & 3 \mathrm{e}, \mathrm{a}\end{array}$

$\begin{array}{lll}13 & 7 & 7\end{array}$

$\begin{array}{llllll}13 & 7 & 6 & 10025.36732 & 0.60 & 3 \mathrm{a}\end{array}$

$\begin{array}{llllll}13 & 8 & 5 & 10223.64359 & 1.07 & 2 \mathrm{e}, \mathrm{a}\end{array}$

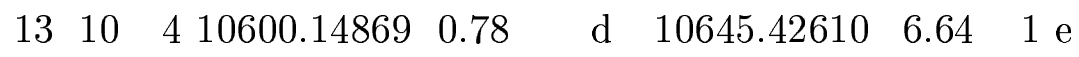

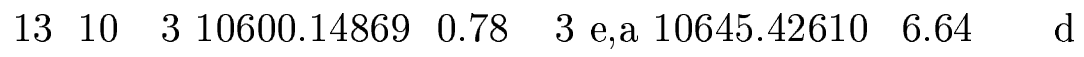

$\begin{array}{llllll}13 & 11 & 3 & 10840.23115 & 1.18 & \mathrm{~d}\end{array}$

$\begin{array}{llllll}13 & 11 & 2 & 10840.23115 & 1.18 & 2 \mathrm{e}, \mathrm{a}\end{array}$

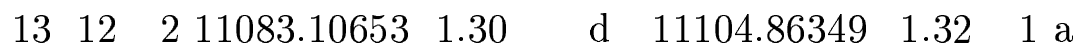

$\begin{array}{llllll}13 & 12 & 1 & 11083.10653 & 1.30 & 1 \mathrm{a}\end{array}$

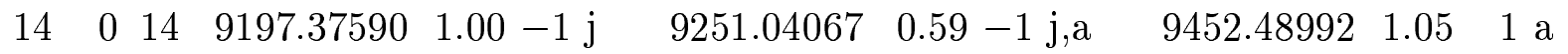

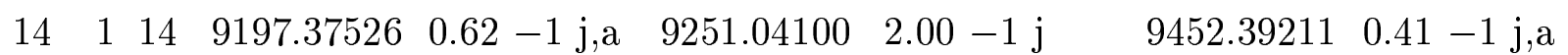

$\begin{array}{lllllllll}14 & 13 & 9494.68677 & 0.42 & -1 \mathrm{j}, \mathrm{a} & 9695.40334 & 1.03 & 1 \mathrm{a}\end{array}$

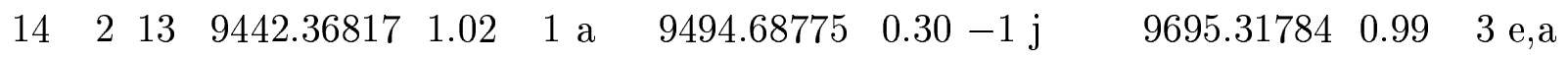

$\begin{array}{lllllllll}14 & 2 & 12 & 9709.39858 & 0.38 & -1 \mathrm{j}, \mathrm{a} & 9909.16120 & 5.22 & 1 \mathrm{e}\end{array}$

$\begin{array}{llllll}14 & 3 & 12 & 9658.22410 & 0.99 & 2 \text { e,a }\end{array}$

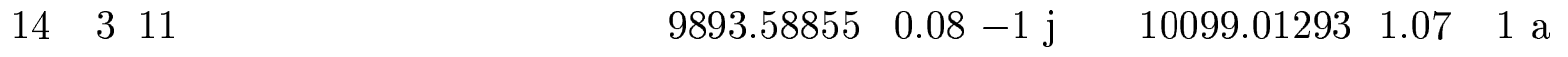

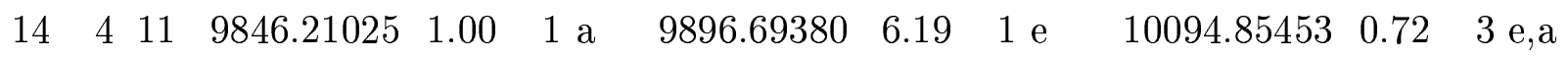

$14 \quad 410 \quad 10042.20096 \quad 1.07 \quad 1 \mathrm{a}$

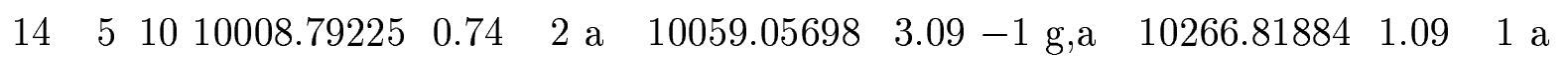

$\begin{array}{lllllllll}14 & 5 & 9 & 10133.01085 & 1.07 & 1 \mathrm{a} & 10337.35586 & 1.12 & 1 \mathrm{a}\end{array}$

$\begin{array}{llllll}14 & 6 & 9 & 10181.87420 & 1.04 & 2 \mathrm{e}, \mathrm{a}\end{array}$

$\begin{array}{lll}14 & 6 & 8\end{array}$

$\begin{array}{lll}14 & 7 & 8\end{array}$

$\begin{array}{lll}14 & 7 & 7\end{array}$

$\begin{array}{lll}14 & 8 & 7\end{array}$

$\begin{array}{lll}14 & 8 & 6\end{array}$ $10422.00088 \quad 0.74 \quad 6$ e,a

$10230.67448 \quad 0.73 \quad 5 \mathrm{e}, \mathrm{a}$

$10594.17602 \quad 0.73 \quad 3$ e,a

$10376.92305 \quad 0.61-1 \mathrm{~g}, \mathrm{a}$

$10763.84200 \quad 6.77 \quad 1 \mathrm{e}$
$10523.52714 \quad 0.76 \quad 2$ a 
$14 \quad 10 \quad 5 \quad 10929.11750 \quad 3.97 \quad 2 \mathrm{e}, \mathrm{a}$

$\begin{array}{lllll}14 & 10 & 4 & 10929.11750 & 3.97 \quad \mathrm{~d}\end{array}$

$14 \quad 11 \quad 4 \quad 11170.62163 \quad 1.16 \quad 1 \mathrm{a}$

$\begin{array}{lllllll}14 & 11 & 3 & 11202.83848 & 6.71 & 1 \mathrm{a}\end{array}$

$\begin{array}{llllll}14 & 13 & 1 & 11682.82493 & 1.66 & 1 \mathrm{a}\end{array}$

$\begin{array}{lllllllllll}15 & 0 & 15 & 9471.45055 & 0.75 & 3 \mathrm{e}, \mathrm{a} & 9525.92583 & 0.40-1 \mathrm{j} & 9728.41594 & 0.60 & 5 \mathrm{e}, \mathrm{a}\end{array}$

$15115 \quad 9525.92599 \quad 0.35-1 \mathrm{j}, \mathrm{a}$

$\begin{array}{llllllllllll}15 & 1 & 14 & 9734.35649 & 1.07 & 1 \mathrm{a} & 9787.38010 & 5.08 & 1 \mathrm{e} & 9988.70356 & 1.07 & 1 \mathrm{a}\end{array}$

$\begin{array}{lllllllll}15 & 2 & 14 & 9787.37670 & 0.36 & -1 \mathrm{j}, \mathrm{a} & 9989.04179 & 1.06 & 2 \mathrm{e}, \mathrm{a}\end{array}$

$\begin{array}{lllllllllll}15 & 2 & 13 & 9967.15843 & 0.78 & 3 \mathrm{e}, \mathrm{a} & 10019.27135 & 0.40-1 \mathrm{j} & 10219.60186 & 1.08 & 2 \mathrm{e}, \mathrm{a}\end{array}$

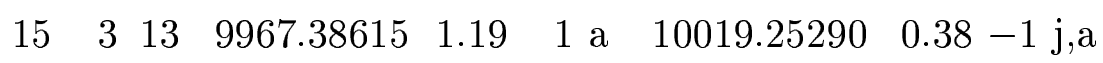

$\begin{array}{llllllll}15 & 3 & 12 & 10168.04277 & 1.03 & 1 \mathrm{a} & 10221.53730 & 3.60-1 \mathrm{~g}\end{array}$

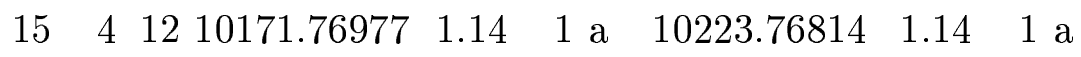

$\begin{array}{lllllllll}15 & 4 & 11 & 10331.64614 & 1.03 & 1 \mathrm{a} & 10593.27472 & 0.73 & 3 \mathrm{e}, \mathrm{a}\end{array}$

$\begin{array}{llllll}15 & 5 & 11 & 10399.77543 & 0.61 & 3 \mathrm{a}\end{array}$

$\begin{array}{lllllllll}15 & 5 & 10 & 10452.68682 & 1.09 & 2 \mathrm{e}, \mathrm{a} & 10720.82315 & 0.60 & 4 \mathrm{e}, \mathrm{a}\end{array}$

$\begin{array}{lllllllll}15 & 6 & 10 & 10561.73258 & 0.76 & 2 \mathrm{a} & 10772.93490 & 7.11 & 1 \mathrm{e}\end{array}$

$\begin{array}{llllllllll}15 & 6 & 9 & 10552.81940 & 6.23 & 1 \mathrm{e} & 10811.02929 & 1.02 & 2 \mathrm{e}, \mathrm{a}\end{array}$

$\begin{array}{lllllllll}15 & 7 & 9 & 10729.71837 & 0.75 & 2 \mathrm{a} & 10951.28087 & 1.19 & 1 \mathrm{a}\end{array}$

$\begin{array}{lllllll}15 & 7 & 8 & 10951.45356 & 1.09 & 1 \mathrm{a}\end{array}$

$\begin{array}{llllll}15 & 8 & 8 & 10875.75901 & 3.93 & 2 \mathrm{e}, \mathrm{a}\end{array}$

$\begin{array}{lllllllll}15 & 8 & 7 & 10875.55240 & 6.28 & 1 \mathrm{e} & 11117.03796 & 1.06 & 1 \mathrm{a}\end{array}$

$\begin{array}{llllll}15 & 9 & 7 & 11078.33527 & 1.12 & 1 \mathrm{a}\end{array}$

$\begin{array}{lllllllll}15 & 10 & 5 & 11279.80928 & 1.18 & 3 \mathrm{e}, \mathrm{a} & 11495.26910 & 6.39 & 1 \mathrm{e}\end{array}$

$\begin{array}{lllll}15 & 11 & 411521.79888 & 5.04 & 1 \mathrm{a}\end{array}$

$\begin{array}{llllllllllll}16 & 0 & 16 & 9762.63607 & 1.05 & 2 \mathrm{a} & 9818.03407 & 0.64-1 \mathrm{j}, \mathrm{a} & 10021.54308 & 1.09 & 1 \mathrm{a}\end{array}$

$\begin{array}{llllllllllll}16 & 1 & 16 & 9762.69542 & 0.76 & 3 \mathrm{e}, \mathrm{a} & 9818.03755 & 2.20-1 \mathrm{j} & 10021.65922 & 1.08 & 3 \mathrm{e}, \mathrm{a}\end{array}$ 


\begin{tabular}{|c|c|c|c|c|c|c|c|c|c|}
\hline 16 & 115 & & & 10097.18225 & 0.77 & $-1 \mathrm{j}, \mathrm{a}$ & & & \\
\hline 16 & $2 \quad 1510043.43172$ & 0.79 & $3 \mathrm{e}, \mathrm{a}$ & 10097.18100 & 3.00 & $-1 \mathrm{j}$ & 10299.49596 & 1.08 & $2 \mathrm{e}, \mathrm{a}$ \\
\hline 16 & 214 & & & 10345.96721 & 0.75 & $4 \mathrm{e}, \mathrm{a}$ & & & \\
\hline 16 & $\begin{array}{lll}3 & 14 & 10293.47767\end{array}$ & 1.10 & $1 \mathrm{a}$ & & & & 10547.31916 & 1.07 & 2 e,a \\
\hline 16 & 313 & & & 10565.32560 & 5.01 & $1 \mathrm{a}$ & & & \\
\hline 16 & 41310514.39392 & 1.02 & $3 \mathrm{a}$ & & & & 10766.65833 & 1.05 & $2 \mathrm{e}, \mathrm{a}$ \\
\hline 16 & 412 & & & 10751.96328 & 0.78 & $2 \mathrm{a}$ & & & \\
\hline 16 & 512 & & & 10754.32460 & 6.34 & $1 \mathrm{e}$ & 10955.31689 & 5.01 & $1 \mathrm{a}$ \\
\hline 16 & 511 & & & 10905.11180 & 2.20 & $-1 \mathrm{~g}$ & & & \\
\hline 16 & $\begin{array}{llll}6 & 11 & 10871.83084\end{array}$ & 1.08 & $2 \mathrm{a}$ & & & & 11144.02350 & 0.78 & $3 \mathrm{e}, \mathrm{a}$ \\
\hline 16 & 610 & & & 10992.93352 & 1.16 & $2 \mathrm{e}, \mathrm{a}$ & & & \\
\hline 16 & 79 & & & 11106.40827 & 1.09 & $1 \mathrm{a}$ & & & \\
\hline 16 & 89 & & & & & & 11490.56960 & 7.38 & $1 \mathrm{e}$ \\
\hline 16 & 107 & & & & & & 11868.78230 & 6.37 & $1 \mathrm{e}$ \\
\hline 16 & 124 & & & 12170.08753 & 4.11 & $2 \mathrm{e}, \mathrm{a}$ & & & \\
\hline 17 & $\begin{array}{lll}0 & 17 & 10071.02632\end{array}$ & 1.26 & $1 \mathrm{a}$ & 10127.28250 & 1.00 & $\mathrm{~d}$ & 10331.80966 & 1.05 & 3 e,a \\
\hline 17 & 11710071.02632 & 1.26 & d & 10127.28250 & 1.00 & $-1 \mathrm{j}$ & & & \\
\hline 17 & 116 & & & 10424.44000 & 3.00 & $-1 \mathrm{j}$ & 10627.23923 & 1.09 & 3 e,a \\
\hline \pm & 216 & & & 10424.01324 & 0.69 & $3 \mathrm{a}$ & & & \\
\hline 17 & $2 \quad 1510636.17846$ & 1.11 & $1 \mathrm{a}$ & 10689.44040 & 14.30 & $-1 \mathrm{~g}$ & 10891.47459 & 1.09 & $2 \mathrm{e}, \mathrm{a}$ \\
\hline $1^{\prime}$ & 315 & & & 10689.44138 & 0.82 & $2 \mathrm{a}$ & & & \\
\hline 17 & $3 \quad 1410872.64309$ & 0.80 & $3 \mathrm{a}$ & 10924.82632 & 1.47 & $-1 \mathrm{~g}, \mathrm{a}$ & & & \\
\hline 16 & 414 & & & 10925.48206 & 1.17 & $2 \mathrm{a}$ & & & \\
\hline$\perp$ & 413 & & & & & & 11326.45323 & 1.05 & $3 \mathrm{e}, \mathrm{a}$ \\
\hline $1^{\prime}$ & 513 & & & 11132.26705 & 1.11 & $3 \mathrm{e}, \mathrm{a}$ & & & \\
\hline 17 & $\begin{array}{lll}5 & 12 & 11235.39007\end{array}$ & 1.11 & $2 \mathrm{a}$ & & & & 11504.31130 & 6.34 & $1 \mathrm{e}$ \\
\hline & 612 & & & 11316.46248 & 1.11 & $2 \mathrm{a}$ & & & \\
\hline
\end{tabular}


$\begin{array}{llllll}17 & 8 & 9 & 11606.64608 & 1.14 & 1 \mathrm{a}\end{array}$

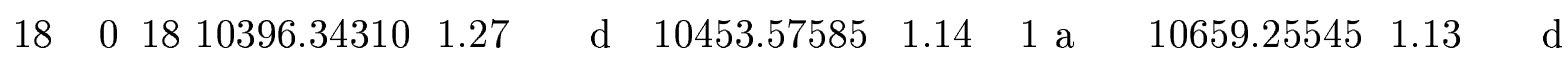

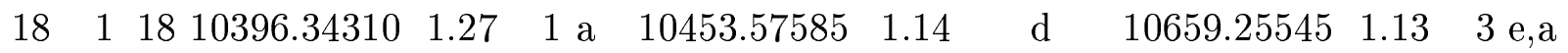

$\begin{array}{llll}18 & 1 & 17\end{array}$

$10767.78050 \quad 1.09-1 \mathrm{~g}, \mathrm{a}$

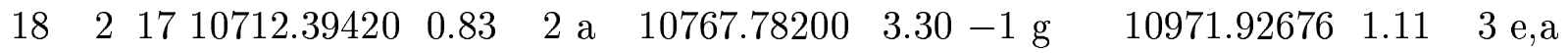

$18 \quad 216$

$11049.60609 \quad 0.81-1 \mathrm{~g}, \mathrm{a}$

$\begin{array}{llllll}18 & 3 & 16 & 10996.01232 & 1.14 & 1 \mathrm{a}\end{array}$

$11049.58119 \quad 1.62-1 \mathrm{~g}, \mathrm{a}$

$\begin{array}{lll}18 & 3 & 15\end{array}$

$11301.82012 \quad 1.06-1 \mathrm{~g}, \mathrm{a}$

$18 \quad 4 \quad 15$

$11503.37432 \quad 1.11 \quad 1 \mathrm{a}$

$18 \quad 4 \quad 14$

$11523.22553 \quad 1.13 \quad 2$ a

$11723.14699 \quad 1.74 \quad 1 \mathrm{a}$

$\begin{array}{lll}18 & 5 & 14\end{array}$

$11725.99604 \quad 1.09 \quad 2 \mathrm{a}$

$\begin{array}{lll}18 & 5 & 13\end{array}$

$11709.59939 \quad 1.20 \quad 1 \mathrm{a}$

$\begin{array}{llllll}18 & 6 & 13 & 11669.79109 & 1.23 & 2 \mathrm{a}\end{array}$

$11916.46575 \quad 1.27 \quad 1 \mathrm{a}$

$\begin{array}{lll}18 & 6 & 12\end{array}$

$12047.27192 \quad 1.20 \quad 1 \mathrm{a}$

$\begin{array}{llllll}19 & 0 & 19 & 10738.57169 & 1.16 & 1 \mathrm{a}\end{array}$

$19 \quad 119$

$\begin{array}{llllll}10796.81431 & 0.88 & 3 \text { e,a } & 11003.71316 & 1.14 & d\end{array}$

$19 \quad 118$

$\begin{array}{lll}11128.39137 & 1.18 \quad \mathrm{~d}\end{array}$

$19 \quad 2 \quad 18$

$11128.39137 \quad 1.18 \quad 1 \mathrm{a}$

$\begin{array}{lll}19 & 2 & 17\end{array}$

$\begin{array}{llllll}11426.30580 & 4.90-1 \mathrm{~g}, ? & 11630.08510 & 1.18 & 1 \mathrm{a}\end{array}$

$\begin{array}{lll}19 & 3 & 17\end{array}$

$11426.32000 \quad 1.08-1 \mathrm{~g}, \mathrm{a}$

$19 \quad 3 \quad 16$

$11897.40367 \quad 1.09 \quad 2$ a

$19 \quad 4 \quad 16$

$\begin{array}{lllll}11694.17123 & 1.10-1 \mathrm{~g}, \mathrm{a} & 11896.59392 & 1.83 & 1 \mathrm{a}\end{array}$

$19 \quad 415$

$12133.12662 \quad 1.10 \quad 1 \mathrm{a}$

$19 \quad 5 \quad 14$

$12328.93352 \quad 1.09 \quad 1 \mathrm{a}$

$19 \quad 7 \quad 13$

$12339.90678 \quad 1.20 \quad 1 \mathrm{a}$

$20 \quad 0 \quad 20$

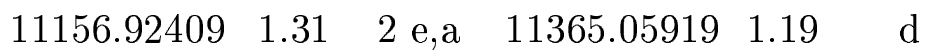




\begin{tabular}{|c|c|c|c|c|c|c|c|}
\hline 20 & 120 & 11156.92409 & 1.31 & $\mathrm{~d}$ & 11365.05919 & 1.19 & $1 \mathrm{a}$ \\
\hline 20 & 119 & 11505.63869 & 1.33 & $1 \mathrm{a}$ & 11712.06830 & 6.66 & d \\
\hline 20 & 219 & 11505.63869 & 1.33 & $\mathrm{~d}$ & 11712.06830 & 6.66 & $1 \mathrm{e}$ \\
\hline 20 & 218 & 11819.34050 & 5.90 & $-1 \mathrm{~g}, ?$ & & & \\
\hline 20 & 318 & 11819.34050 & 5.90 & $-1 \mathrm{~g}$ & 12024.25863 & 1.17 & $1 \mathrm{a}$ \\
\hline 20 & 317 & 12102.66155 & 1.32 & $-1 \mathrm{~g}, \mathrm{a}$ & 12305.73578 & 1.32 & $\mathrm{a}$ \\
\hline 20 & 417 & & & & 12306.00585 & 1.14 & $1 \mathrm{a}$ \\
\hline 20 & 416 & & & & 12558.23288 & 2.28 & $1 \mathrm{a}$ \\
\hline 20 & 516 & & & & 12558.64257 & 1.17 & $1 \mathrm{a}$ \\
\hline 20 & 515 & 12577.64267 & 1.24 & $1 \mathrm{a}$ & & & \\
\hline 20 & 615 & & & & 12782.10004 & 1.17 & $2 \mathrm{a}$ \\
\hline 20 & 713 & 12872.01204 & 1.18 & $1 \mathrm{a}$ & & & \\
\hline 20 & 813 & & & & 13192.49329 & 1.87 & $2 \mathrm{a}$ \\
\hline 21 & 021 & 11533.64536 & 0.85 & $\mathrm{~d}$ & 11743.03943 & 1.19 & $1 \mathrm{a}$ \\
\hline 21 & 121 & 11533.64536 & 0.85 & 3 e,a & 11743.03943 & 1.19 & $d$ \\
\hline 21 & 120 & 11899.60080 & 1.20 & $\mathrm{~d}$ & & & \\
\hline 21 & 220 & 11899.60080 & 1.20 & $-1 \mathrm{~g}, \mathrm{a}$ & & & \\
\hline 21 & 219 & 12228.72193 & 1.39 & $\mathrm{~d}$ & 12434.75588 & 1.14 & $1 \mathrm{a}$ \\
\hline 21 & 319 & 12228.72193 & 1.39 & $-1 \mathrm{~g}, \mathrm{a}$ & & & \\
\hline 21 & 318 & 12527.68510 & 1.03 & $\mathrm{~d}$ & 12731.38508 & 5.03 & $1 \mathrm{a}$ \\
\hline 21 & 418 & 12527.68510 & 1.03 & $-1 \mathrm{~g}, \mathrm{a}$ & 12731.06704 & 1.86 & 1 \\
\hline 21 & 417 & & & & 12997.41769 & 1.23 & 1 \\
\hline 22 & 022 & 11927.07908 & 1.73 & $2 \mathrm{e}, \mathrm{a}$ & 12138.03158 & 1.75 & $d$ \\
\hline 22 & 122 & 11927.07908 & 1.73 & $\mathrm{~d}$ & 12138.03158 & 1.75 & 1 \\
\hline 22 & 121 & 12310.05549 & 1.03 & $2 \mathrm{a}$ & & & \\
\hline 22 & 221 & 12310.05549 & 1.03 & $\mathrm{~d}$ & & & \\
\hline 22 & 220 & 12654.48183 & 1.72 & $-1 \mathrm{~g}, \mathrm{a}$ & & & \\
\hline
\end{tabular}


$22 \quad 3 \quad 20$

$\begin{array}{lll}22 & 3 & 19\end{array}$

$\begin{array}{lll}22 & 5 & 17\end{array}$

$\begin{array}{lll}22 & 6 & 16\end{array}$

$\begin{array}{lll}23 & 0 & 23\end{array}$

$\begin{array}{lll}23 & 1 & 23\end{array}$

$23 \quad 122$

$\begin{array}{lll}23 & 2 & 22\end{array}$

$\begin{array}{lll}23 & 2 & 21\end{array}$

$\begin{array}{lll}23 & 3 & 21\end{array}$

$\begin{array}{lll}23 & 3 & 20\end{array}$

$\begin{array}{lll}23 & 4 & 20\end{array}$

$\begin{array}{lll}23 & 5 & 19\end{array}$

$\begin{array}{lll}24 & 0 & 24\end{array}$

$24 \quad 124$

$\begin{array}{lll}24 & 1 & 23\end{array}$

$\begin{array}{lll}24 & 2 & 23\end{array}$

$24 \quad 2 \quad 22$

$24 \quad 3 \quad 22$

$\begin{array}{lll}24 & 3 & 21\end{array}$

$24 \quad 4 \quad 21$

$\begin{array}{lll}25 & 0 & 25\end{array}$

$25 \quad 125$

$25 \quad 124$

$25 \quad 2 \quad 24$

$25 \quad 2 \quad 23$

$\begin{array}{lll}25 & 3 & 23\end{array}$ $\begin{array}{llllll}12654.48183 & 1.72 & \mathrm{~d} & 12861.46442 & 3.06 & 1 \mathrm{a}\end{array}$

$12967.82402 \quad 1.31-1 \mathrm{~g}, \mathrm{a}$

$\begin{array}{lll}13505.98627 & 1.39 \quad 1 \mathrm{a}\end{array}$

$13724.79183 \quad 1.33 \quad 1 \mathrm{a}$

$\begin{array}{llllll}12337.01814 & 1.21 & \text { d } & 12549.42295 & 1.21 & 2 \mathrm{e}, \mathrm{a}\end{array}$

$\begin{array}{llllll}12337.01814 & 1.21 & 2 \mathrm{e}, \mathrm{a} & 12549.42295 & 1.21 & \mathrm{~d}\end{array}$

$\begin{array}{llllll}12736.89031 & 1.40 & \mathrm{~d} & 12946.95630 & 2.09 & 1 \mathrm{a}\end{array}$

$\begin{array}{llllll}12736.89031 & 1.40 & 2 \mathrm{e}, \mathrm{a} & 12946.95630 & 2.09 & \mathrm{~d}\end{array}$

$13096.12898 \quad 3.05 \quad \mathrm{~d}$

$13096.12898 \quad 3.05-1 \mathrm{~g}, \mathrm{a}$

$\begin{array}{lll}13423.97317 & 1.58 \quad \mathrm{~d}\end{array}$

$13423.97317 \quad 1.58-1 \mathrm{~g}, \mathrm{a}$

$13720.44584 \quad 5.16 \quad 1 \mathrm{a}$

$12763.30375 \quad 1.37-1 \mathrm{~g}, \mathrm{a}$

$\begin{array}{llllll}12763.30375 & 1.37 & \mathrm{~d} & 12977.24281 & 1.90 & 1 \mathrm{a}\end{array}$

$13179.97406 \quad 2.30 \quad 2 \mathrm{e}, \mathrm{a}$

$\begin{array}{llllll}13179.97406 & 2.30 & \mathrm{~d} & 13391.35217 & 1.52 & 1 \mathrm{a}\end{array}$

$13553.61362 \quad 3.04 \quad 1 \mathrm{a}$

$\begin{array}{llllll}13553.61362 & 3.04 & \mathrm{~d} & 13762.29244 & 3.04 & 1 \mathrm{a}\end{array}$

$13895.97773 \quad 2.92 \quad 1 \mathrm{a}$

$14101.99261 \quad 2.05 \quad 1 \mathrm{a}$

$13205.8376013 .72 \quad \mathrm{~d}$

$13205.8376013 .72 \quad 1 \mathrm{e}$

$\begin{array}{lllllll}13639.20965 & 1.68 & \mathrm{~d} & 13851.92932 & 1.41 & 2 \mathrm{e}, \mathrm{a}\end{array}$

$\begin{array}{llllll}13639.20965 & 1.68 & 2 \mathrm{e}, \mathrm{a} & 13851.92932 & 1.41 & \mathrm{~d}\end{array}$

$\begin{array}{lll}14026.67952 & 2.94 \quad \mathrm{~d}\end{array}$

$14026.67952 \quad 2.94-1 \mathrm{~g}, \mathrm{a}$ 


\begin{tabular}{lllllllllll}
25 & 4 & 22 & 14383.30220 & 5.30 & $-1 \mathrm{~g}$ & & & & \\
26 & 0 & 26 & 13664.50267 & 1.58 & $2 \mathrm{e}, \mathrm{a}$ & & & & \\
26 & 1 & 26 & 13664.50267 & 1.58 & $\mathrm{~d}$ & 13881.70520 & 8.19 & $1 \mathrm{e}$ \\
26 & 1 & 25 & 14114.46209 & 1.57 & $1 \mathrm{a}$ & 14328.53779 & 5.06 & $\mathrm{~d}$ \\
26 & 2 & 25 & 14114.46209 & 1.57 & $\mathrm{~d}$ & 14328.53779 & 5.06 & $1 \mathrm{a}$ \\
26 & 2 & 24 & 14515.15560 & 5.60 & $-1 \mathrm{~g}$ & & & \\
26 & 3 & 24 & 14515.15560 & 5.60 & $\mathrm{~d}$ & 14728.38286 & 1.73 & $1 \mathrm{a}$ \\
27 & 0 & 27 & 14139.14751 & 2.24 & $\mathrm{~d}$ & & & & \\
27 & 1 & 27 & 14139.14751 & 2.24 & $1 \mathrm{a}$ & & & \\
27 & 2 & 25 & 15018.80089 & 5.46 & $\mathrm{~d}$ & & & \\
27 & 3 & 25 & 15018.80089 & 5.46 & $1 \mathrm{a}$ & & & \\
28 & 0 & 28 & 14629.33023 & 5.07 & $1 \mathrm{a}$ & & & \\
28 & 2 & 26 & 15537.45460 & 5.45 & $1 \mathrm{a}$ & & \\
\hline
\end{tabular}


TABLE VI.: Term values for the $2 \nu+\delta$ polyad of $\mathrm{H}_{2}{ }^{16} \mathrm{O}$.

\begin{tabular}{|c|c|c|c|c|c|c|c|c|c|c|}
\hline$J I$ & $K_{a}$ & $K_{c}$ & 050 or & 005 & & 130 or & $10^{+} 3$ & & 210 or & $20^{+} 0$ \\
\hline 0 & 0 & 0 & 7542.43660 & 4.88 & $3 \mathrm{e}$ & 8273.97570 & 0.90 & $1 \mathrm{k}$ & 8761.58200 & $0.901 \mathrm{k}$ \\
\hline 1 & 0 & 1 & 7566.01221 & 1.02 & $1 \mathrm{a}$ & 8297.37480 & 0.60 & 2 & 8784.65950 & $0.602 \mathrm{k}$ \\
\hline 1 & 1 & 1 & 7622.73380 & 10.21 & $2 \mathrm{e}$ & 8323.29340 & 0.50 & $3 \mathrm{k}$ & 8799.64110 & $0.603 \mathrm{k}$ \\
\hline 1 & 1 & 0 & 7629.08353 & 0.76 & $2 \mathrm{a}$ & 8329.36750 & 0.90 & $2 \mathrm{k}$ & 8805.15890 & $0.602 \mathrm{k}$ \\
\hline 2 & 0 & 2 & & & & 8343.18160 & 0.50 & $3 \mathrm{k}$ & 8829.53010 & $0.603 \mathrm{k}$ \\
\hline 2 & 1 & 2 & 7663.69870 & 6.28 & $2 \mathrm{e}$ & 8364.05870 & 0.40 & $4 \mathrm{k}$ & 8840.27590 & $0.503 \mathrm{k}$ \\
\hline 2 & 1 & 1 & & & & 8382.24290 & 0.50 & $3 \mathrm{k}$ & 8856.77820 & $0.603 \mathrm{k}$ \\
\hline 2 & 2 & 1 & 7828.73049 & 1.07 & $1 \mathrm{a}$ & 8456.95150 & 0.40 & $4 \mathrm{k}$ & 8899.83650 & $0.404 \mathrm{k}$ \\
\hline 2 & 2 & 0 & 7829.25059 & 1.01 & $1 \mathrm{a}$ & 8457.76620 & 0.60 & $3 \mathrm{k}$ & 8902.69810 & $0.504 \mathrm{k}$ \\
\hline 3 & 0 & 3 & 7681.29313 & 0.73 & $6 \mathrm{e}, \mathrm{a}$ & 8409.60320 & 0.50 & $3 \mathrm{k}$ & 8894.03430 & $0.503 \mathrm{k}$ \\
\hline 3 & 1 & 3 & & & & 8424.60730 & 0.50 & $4 \mathrm{k}$ & 8900.49960 & $0.504 \mathrm{k}$ \\
\hline 3 & 1 & 2 & 7763.11363 & 1.05 & $4 \mathrm{e}, \mathrm{a}$ & 8460.76830 & 0.40 & $4 \mathrm{k}$ & 8933.20540 & $0.604 \mathrm{k}$ \\
\hline 3 & 2 & 2 & & & & 8527.32140 & 0.40 & $5 \mathrm{k}$ & 8969.75930 & $0.405 \mathrm{k}$ \\
\hline 3 & 2 & 1 & 7902.88024 & 1.08 & $1 \mathrm{a}$ & 8532.12310 & 0.40 & $5 \mathrm{k}$ & 8976.54900 & $0.404 \mathrm{k}$ \\
\hline 3 & 3 & 1 & & & & 8660.64910 & 0.60 & $4 \mathrm{k}$ & 9057.76730 & $0.603 \mathrm{k}$ \\
\hline 3 & 3 & 0 & 8122.43917 & 1.04 & $3 \mathrm{e}, \mathrm{a}$ & 8660.73730 & 0.40 & $5 \mathrm{k}$ & 9057.92400 & $0.405 \mathrm{k}$ \\
\hline 4 & 0 & 4 & & & & 8494.66560 & 0.50 & $3 \mathrm{k}$ & 8976.30240 & $0.603 \mathrm{k}$ \\
\hline 4 & 1 & 4 & 7805.63201 & 0.53 & $13 \mathrm{l}, \mathrm{a}$ & 8504.37140 & 0.40 & $4 \mathrm{k}$ & 8979.65800 & $0.504 \mathrm{k}$ \\
\hline 4 & 1 & 3 & & & & 8563.89420 & 1.70 & $3 \mathrm{k}$ & 9033.02700 & $0.603 \mathrm{k}$ \\
\hline 4 & 2 & 3 & 7995.21227 & 0.73 & $2 \mathrm{a}$ & 8620.26550 & 0.40 & $5 \mathrm{k}$ & 9061.15320 & $0.404 \mathrm{k}$ \\
\hline 4 & 2 & 2 & 8002.51464 & 1.03 & $1 \mathrm{a}$ & 8633.09570 & 0.40 & $5 \mathrm{k}$ & 9077.35730 & $0.504 \mathrm{k}$ \\
\hline 4 & 3 & 2 & 8219.44360 & 1.01 & $2 \mathrm{e}, \mathrm{a}$ & 8756.53090 & 0.40 & $6 \mathrm{k}$ & 9152.60240 & $0.406 \mathrm{k}$ \\
\hline 4 & 3 & 1 & 8219.67825 & 1.02 & $1 \mathrm{a}$ & 8757.14080 & 0.40 & $5 \mathrm{k}$ & 9153.64650 & $0.605 \mathrm{l}$ \\
\hline
\end{tabular}




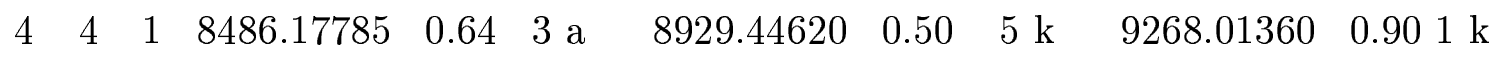
$\begin{array}{llllllllll}4 & 4 & 0 & 8486.17026 & 1.01 & 1 \mathrm{a} & 8929.45380 & 0.80 & 4 \mathrm{k}\end{array}$

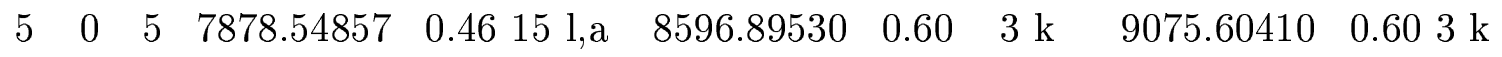
$\begin{array}{llllllllllll}5 & 1 & 5 & 7905.81001 & 1.11 & 1 \mathrm{a} & 8602.79150 & 0.60 & 3 \mathrm{k} & 9077.17750 & 0.60 & 3 \mathrm{k}\end{array}$

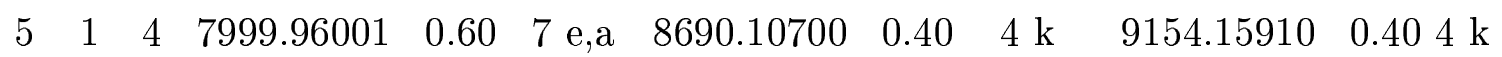
$\begin{array}{llllllll}5 & 2 & 4 & 8735.08010 & 0.40 & 5 \mathrm{k} & 9173.56810 & 0.505 \mathrm{k}\end{array}$

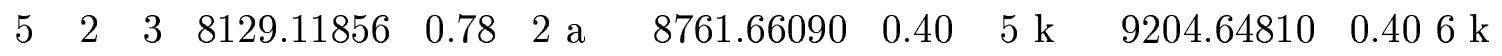

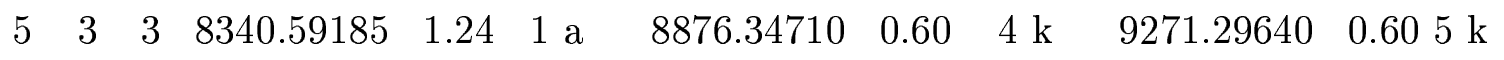
$\begin{array}{llllllllllll}5 & 3 & 2 & 8341.48469 & 0.59 & 3 \mathrm{a} & 8878.58650 & 0.40 & 5 \mathrm{k} & 9275.07950 & 0.40 & 5 \mathrm{k}\end{array}$ $\begin{array}{llllll}5 & 4 & 2 & 8608.18988 & 1.29 & 1 \mathrm{a}\end{array}$ $\begin{array}{lllllll}5 & 4 & 1 & 8608.24408 & 1.19 & 1 \mathrm{a}\end{array}$ $\begin{array}{lllllllll}5 & 5 & 1 & 8906.92402 & 0.56 & \mathrm{~d} & 9257.21960 & 0.90 & 5 \mathrm{k}\end{array}$ $\begin{array}{lllllllll}5 & 5 & 0 & 8906.92402 & 0.56 & 4 \mathrm{a} & 9257.23790 & 0.90 & 4 \mathrm{k}\end{array}$ $\begin{array}{llllllllllll}6 & 0 & 6 & 8004.48535 & 1.03 & 1 \mathrm{a} & 8715.98060 & 0.60 & 3 \mathrm{k} & 9191.65910 & 2.00 & 3 \mathrm{k}\end{array}$

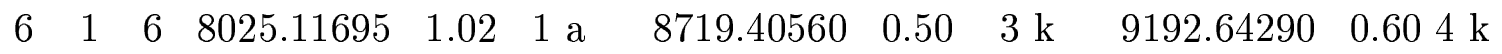
$\begin{array}{lllllll}6 & 1 & 5 & 8837.42730 & 0.60 & 4 \mathrm{k}\end{array}$

$\begin{array}{llllllllllll}6 & 2 & 5 & 8252.83437 & 0.61 & 5 \mathrm{e}, \mathrm{a} & 8870.98700 & 0.50 & 4 \mathrm{k} & 9306.12160 & 0.50 & 4 \mathrm{k}\end{array}$ $\begin{array}{llllll}6 & 2 & 4 & 8917.09310 & 0.90 & 2 \mathrm{k}\end{array}$

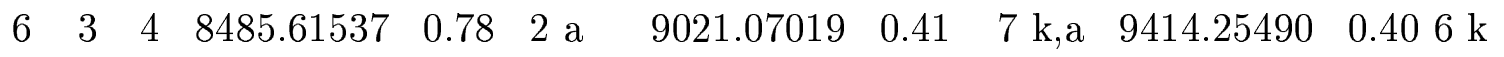
$\begin{array}{llllllll}6 & 3 & 3 & 8488.14622 & 1.19 & 1 \mathrm{a} & 9423.64930 & 0.901 \mathrm{k}\end{array}$

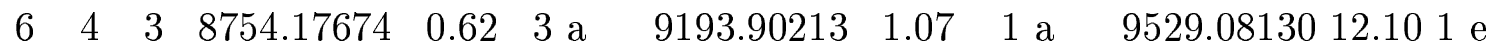
$\begin{array}{lllllll}6 & 4 & 2 & 8754.37891 & 1.40 & 1 \text { a }\end{array}$ $\begin{array}{llllllllll}6 & 5 & 2 & 9054.28188 & 0.90 & 2 & \mathrm{a} & 9402.23440 & 0.40 & 8 \mathrm{k}\end{array}$ $\begin{array}{lllllll}6 & 6 & 1 & 9374.32638 & 0.64 & 3 & \text { a }\end{array}$ $\begin{array}{llllll}6 & 6 & 0 & 9374.32638 & 0.64 & \mathrm{~d}\end{array}$

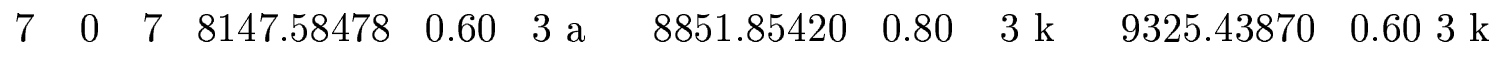

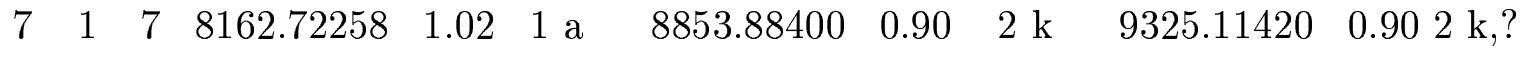


$\begin{array}{llllllllllll}7 & 1 & 6 & 8331.67109 & 0.61 & 3 \mathrm{a} & 9003.74570 & 0.50 & 4 \mathrm{k} & 9451.28030 & 2.00 & 3 \mathrm{k}\end{array}$ $\begin{array}{llllllll}7 & 2 & 6 & 8414.43096 & 1.19 & 1 \mathrm{a} & 9457.95710 & 0.902 \mathrm{k}\end{array}$ $\begin{array}{lllllllll}7 & 2 & 5 & 8463.41894 & 0.47 & 8 \mathrm{e}, \mathrm{a} & 9097.93372 & 0.63 & 6\end{array} \mathrm{k}, \mathrm{a}$

$\begin{array}{llllllll}7 & 3 & 5 & 8654.12309 & 1.19 & 1 \mathrm{a} & 9567.77180 & 0.507 \mathrm{k}\end{array}$ $\begin{array}{lllllll}7 & 3 & 4 & 8660.11387 & 1.08 & 1 & \mathrm{a}\end{array}$ $\begin{array}{llllllllll}7 & 4 & 3 & 8924.61625 & 1.38 & 1 \text { a } & 9363.46259 & 1.06 & 1 \text { a }\end{array}$ $\begin{array}{llllllllll}7 & 5 & 3 & 9225.77959 & 1.72 & 1 \text { a } & 9572.94970 & 0.40 & 7 \mathrm{k}\end{array}$ $\begin{array}{llllllll}7 & 5 & 2 & 9226.17517 & 0.79 & 2 \mathrm{a} & 9842.80820 & 0.506 \mathrm{k}\end{array}$ $\begin{array}{llllll}7 & 6 & 2 & 9546.54997 & 0.78 & \mathrm{~d}\end{array}$ $\begin{array}{lllllll}7 & 6 & 1 & 9546.54997 & 0.78 & 2 & \mathrm{a}\end{array}$ $\begin{array}{llllll}7 & 7 & 1 & 9879.70553 & 0.93 & \mathrm{~d}\end{array}$ $\begin{array}{lllllll}7 & 7 & 0 & 9879.70553 & 0.93 & 2 & \text { a }\end{array}$ $\begin{array}{llllllll}8 & 0 & 8 & 9004.70290 & 0.60 & 2 \mathrm{k} & 9476.36790 & 0.902 \mathrm{k}\end{array}$ $\begin{array}{llllllllllll}8 & 1 & 8 & 8318.67754 & 0.54 & 4 \mathrm{a} & 9006.03610 & 0.90 & 3 \mathrm{k} & 9476.50600 & 0.90 & 2 \mathrm{k}\end{array}$ $\begin{array}{llllllllllll}8 & 2 & 7 & 8597.21463 & 1.05 & 4 \mathrm{e}, \mathrm{a} & 9203.11570 & 0.60 & 4 \mathrm{k} & 9628.38990 & 0.90 & 2 \mathrm{k}\end{array}$ $\begin{array}{lllllllll}8 & 3 & 6 & 8845.55800 & 0.61 & 3 \mathrm{a} & 9753.92820 & 0.60 & 5 \mathrm{k}\end{array}$ $\begin{array}{lllllll}8 & 3 & 5 & 8857.82280 & 1.56 & 1 \mathrm{a}\end{array}$ $\begin{array}{lllllll}8 & 4 & 5 & 9117.28571 & 1.13 & 1 \mathrm{a}\end{array}$

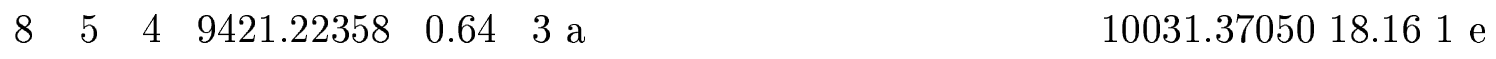
$\begin{array}{lllllll}8 & 6 & 3 & 9742.48438 & 0.84 & 3 & \mathrm{a}\end{array}$ $\begin{array}{llllll}8 & 6 & 2 & 9742.48438 & 0.84 & \mathrm{~d}\end{array}$ $\begin{array}{lllllll}8 & 7 & 2 & 10076.79599 & 1.12 & 1 \text { a }\end{array}$ $\begin{array}{llllll}8 & 7 & 1 & 10076.79599 & 1.12 \quad \mathrm{~d}\end{array}$ $\begin{array}{lllllll}8 & 8 & 1 & 10409.94293 & 1.17 & 1 \mathrm{a}\end{array}$ $\begin{array}{llllll}8 & 8 & 0 & 10409.94293 & 1.17 & \mathrm{~d}\end{array}$ $\begin{array}{llllllllllll}9 & 0 & 9 & 8484.75527 & 0.62 & 3 \mathrm{a} & 9174.68190 & 0.90 & 3 \mathrm{k} & 9644.46850 & 0.90 & 3 \mathrm{k}\end{array}$ $\begin{array}{llllllllllll}9 & 1 & 9 & 8492.84227 & 1.01 & 1 \mathrm{a} & 9175.83240 & 2.80 & 2 \mathrm{k} & 9644.81580 & 0.90 & 1 \mathrm{k}\end{array}$ 


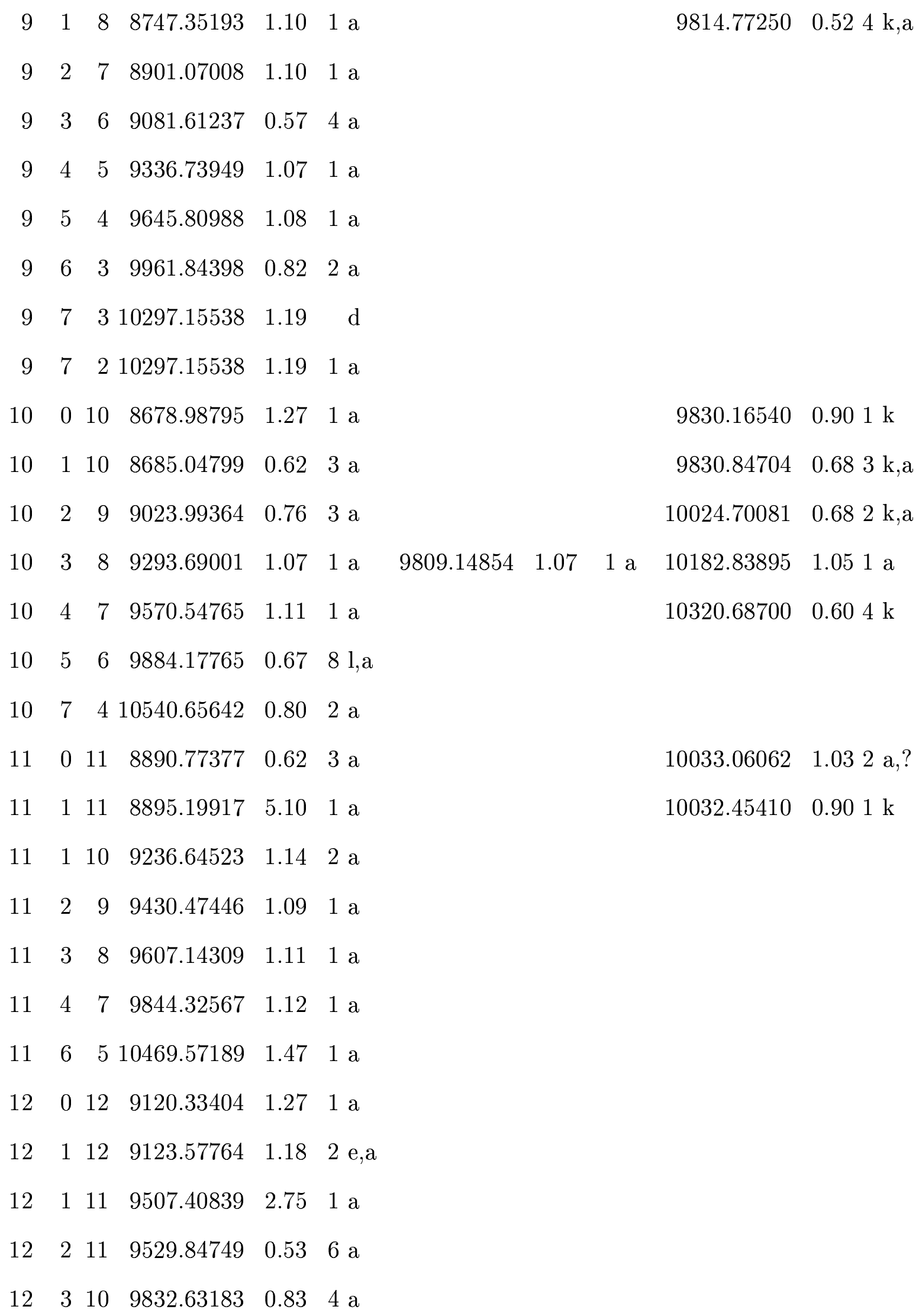




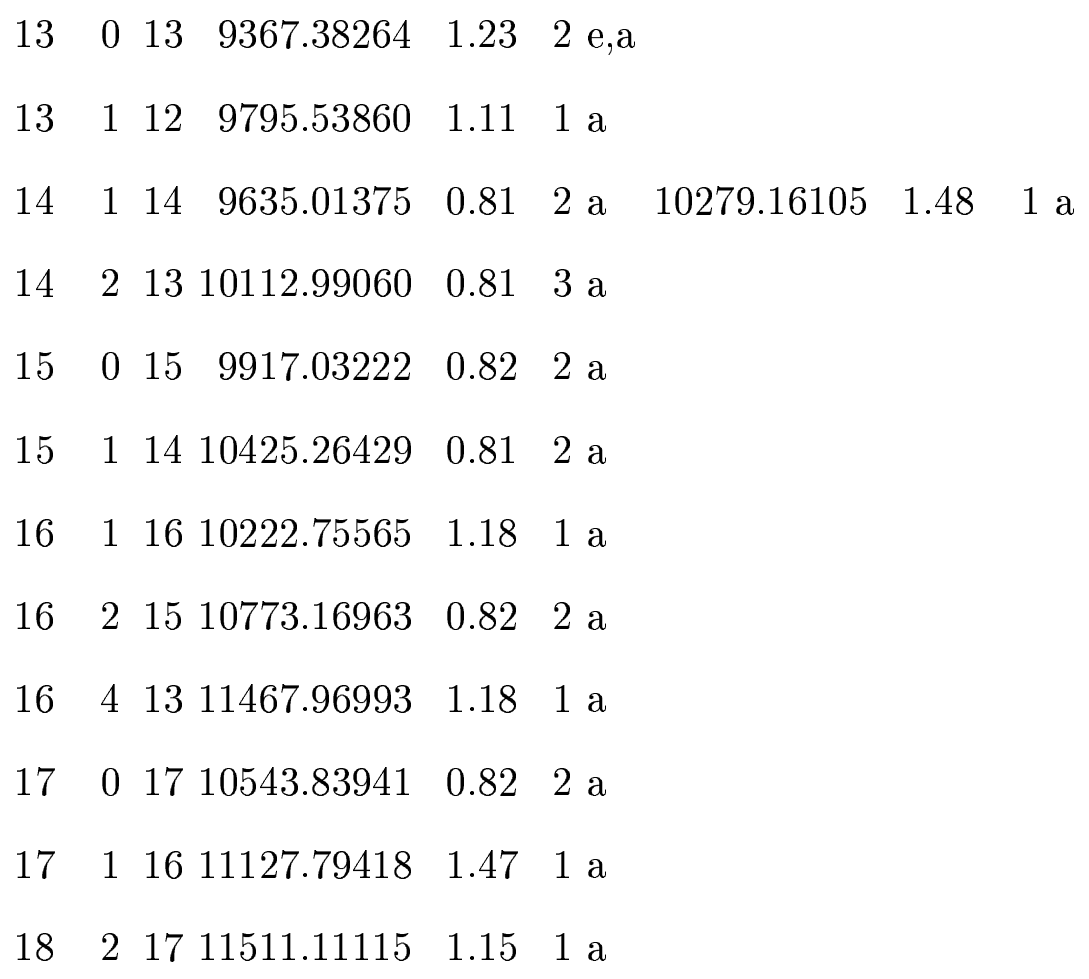

\begin{tabular}{rrrrrrrrrrrr}
\hline$J$ & $K_{a}$ & $K_{c}$ & 031 & or & $10^{-} 3$ & & 111 or & $20^{-} 1$ & & 012 or & 111 \\
\hline 0 & 0 & 0 & 8373.85210 & 0.67 & $2 \mathrm{k}, \mathrm{a}$ & 8807.00058 & 0.67 & $2 \mathrm{k}, \mathrm{a}$ & 9000.13650 & $0.901 \mathrm{k}$ \\
1 & 0 & 1 & 8397.48510 & 0.60 & $3 \mathrm{k}$ & 8830.23180 & 0.50 & $3 \mathrm{k}$ & 9023.49010 & $0.602 \mathrm{k}$ \\
1 & 1 & 1 & 8421.18569 & 0.52 & $3 \mathrm{k}, \mathrm{a}$ & 8844.53720 & 0.60 & $2 \mathrm{k}$ & 9037.19850 & $0.903 \mathrm{k}$ \\
1 & 1 & 0 & 8427.36780 & 0.60 & $2 \mathrm{k}$ & 8850.10680 & 0.90 & $2 \mathrm{k}$ & 9042.83570 & $0.902 \mathrm{k}$ \\
2 & 0 & 2 & 8443.64823 & 0.33 & $7 \mathrm{k}, \mathrm{a}$ & 8875.30880 & 0.40 & $4 \mathrm{k}$ & 9068.75110 & $0.503 \mathrm{k}$ \\
2 & 1 & 2 & 8462.46374 & 0.45 & $4 \mathrm{k}, \mathrm{a}$ & 8885.20710 & 0.50 & $4 \mathrm{k}$ & 9078.28383 & $0.523 \mathrm{k}, \mathrm{a}$ \\
2 & 1 & 1 & 8480.83927 & 0.45 & $5 \mathrm{k}, \mathrm{a}$ & 8903.49710 & 0.50 & $4 \mathrm{k}$ & 9095.16400 & $0.503 \mathrm{k}$ \\
2 & 2 & 1 & 8549.96529 & 0.45 & $4 \mathrm{k}, \mathrm{a}$ & 8944.63260 & 0.60 & $3 \mathrm{k}$ & 9135.84320 & $0.404 \mathrm{k}$ \\
2 & 2 & 0 & 8551.02413 & 0.35 & $6 \mathrm{k}, \mathrm{a}$ & 8945.96440 & 0.40 & $4 \mathrm{k}$ & 9137.25440 & $0.504 \mathrm{k}$ \\
3 & 0 & 3 & 8510.37532 & 0.41 & $6 \mathrm{k}, \mathrm{a}$ & 8939.96360 & 0.40 & $5 \mathrm{k}$ & 9133.58136 & $0.387 \mathrm{k}, \mathrm{a}$ \\
3 & 1 & 3 & 8523.10867 & 0.30 & $9 \mathrm{k}, \mathrm{a}$ & 8945.66310 & 0.40 & $5 \mathrm{k}$ & 9139.06960 & $0.504 \mathrm{k}$ \\
3 & 1 & 2 & 8560.14393 & 0.45 & $5 \mathrm{k}, \mathrm{a}$ & 8979.67480 & 0.40 & $5 \mathrm{k}$ & 9172.50908 & $0.356 \mathrm{k}, \mathrm{a}$ \\
3 & 2 & 2 & 8620.95235 & 0.38 & $8 \mathrm{k}, \mathrm{a}$ & 9014.02145 & 0.37 & $6 \mathrm{k}, \mathrm{a}$ & 9205.96930 & $0.505 \mathrm{k}$ \\
3 & 2 & 1 & 8625.99780 & 0.50 & $4 \mathrm{k}$ & 9020.17450 & 0.40 & $6 \mathrm{k}$ & 9212.46990 & $0.405 \mathrm{k}$
\end{tabular}




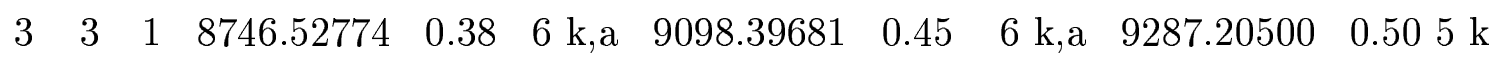

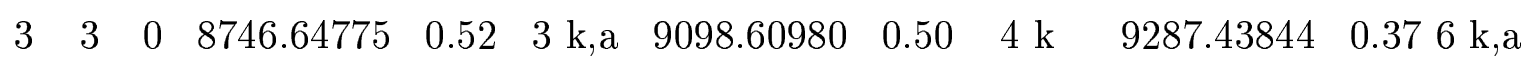

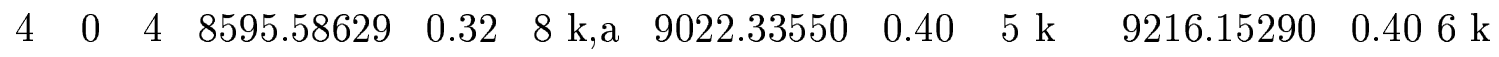

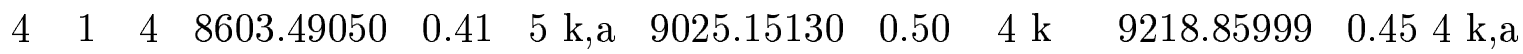
$\begin{array}{llllllllllll}4 & 1 & 3 & 8664.10414 & 0.32 & 8 \mathrm{k}, \mathrm{a} & 9079.71636 & 0.33 & 9 \mathrm{k}, \mathrm{a} & 9273.23620 & 0.50 & 5 \mathrm{k}\end{array}$

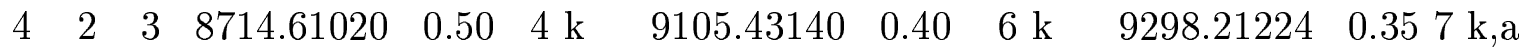

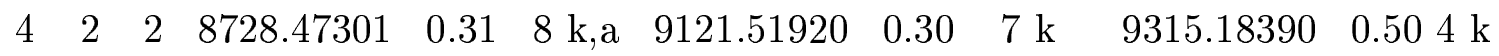

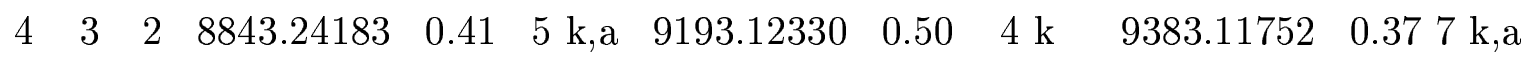

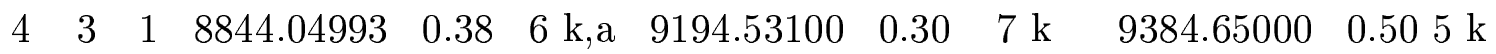

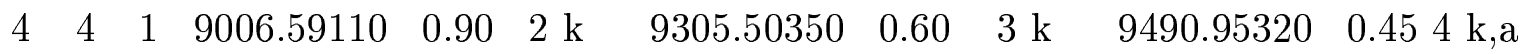

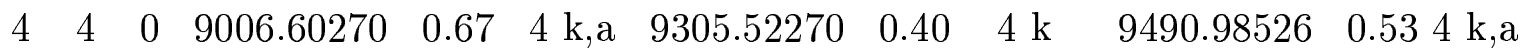

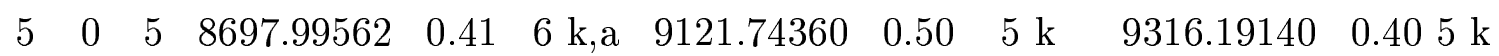

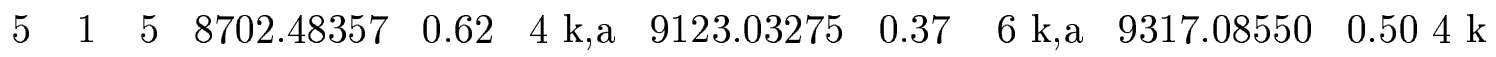

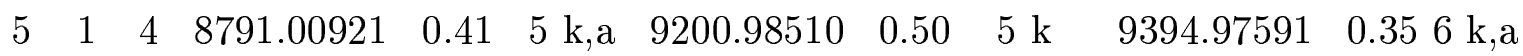

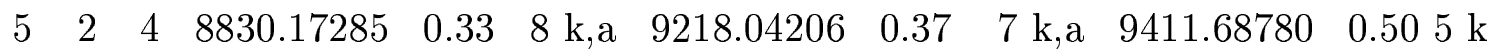
$\begin{array}{lllllllllll}5 & 2 & 3 & 8858.60070 & 0.50 & 3 \mathrm{k} & 9249.24240 & 0.40 & 6 \mathrm{k} & 9444.69030 & 0.405 \mathrm{k}\end{array}$ $\begin{array}{lllllllllll}5 & 3 & 3 & 8964.05460 & 0.40 & 5 \mathrm{k} & 9310.99560 & 0.30 & 8 \mathrm{k} & 9502.70100 & 0.406 \mathrm{k}\end{array}$

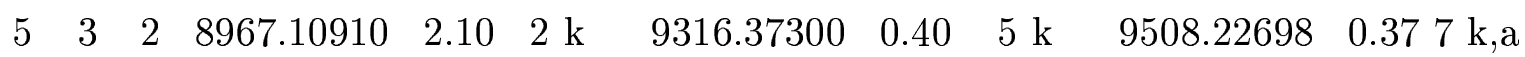

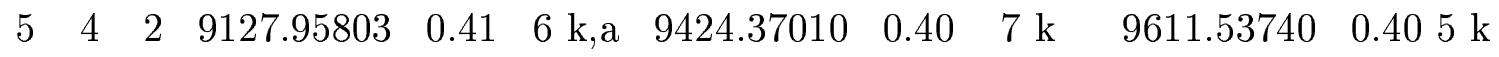

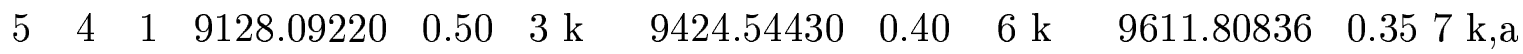
$\begin{array}{llllllllllll}5 & 5 & 1 & 9324.06556 & 0.41 & 5 \mathrm{k}, \mathrm{a} & 9563.99603 & 0.53 & 5 \mathrm{k}, \mathrm{a} & 9745.19930 & 0.903 \mathrm{k}\end{array}$

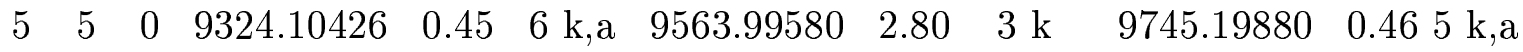

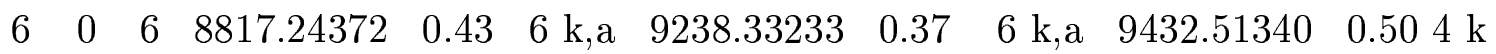

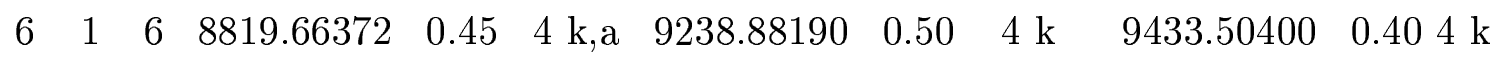

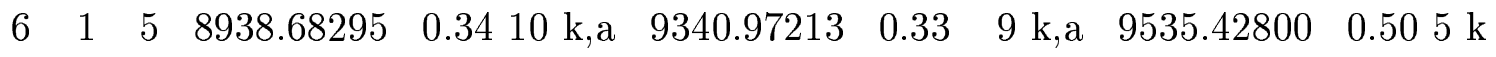
$\begin{array}{llllllllllll}6 & 2 & 5 & 8966.79071 & 0.52 & 4 \mathrm{k}, \mathrm{a} & 9350.94940 & 0.40 & 6 \mathrm{k} & 9545.36911 & 0.338 \mathrm{k}, \mathrm{a}\end{array}$

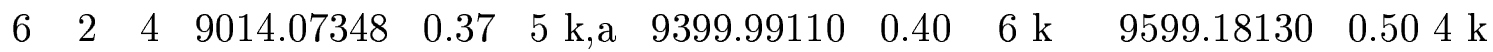




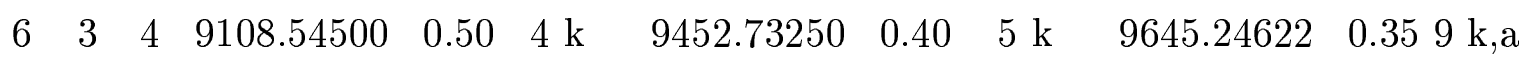
$\begin{array}{lllllllll}6 & 3 & 3 & 9116.88102 & 0.33 & 7 \mathrm{k}, \mathrm{a} & 9465.10660 & 0.30 & 8 \mathrm{k}\end{array}$

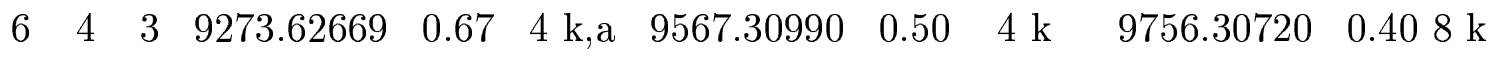

$\begin{array}{llllllllllll}6 & 4 & 2 & 9274.14685 & 0.35 & 6 \mathrm{k}, \mathrm{a} & 9568.20890 & 0.30 & 7 \mathrm{k} & 9757.54980 & 0.50 & 5 \mathrm{k}\end{array}$

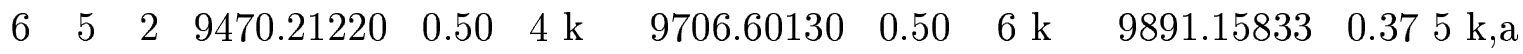

$\begin{array}{llllllllllll}6 & 5 & 1 & 9470.65531 & 0.35 & 10 \mathrm{k}, \mathrm{a} & 9706.60649 & 0.37 & 8 \mathrm{k}, \mathrm{a} & 9891.19680 & 0.60 & 3 \mathrm{k}\end{array}$

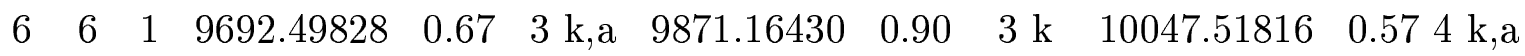

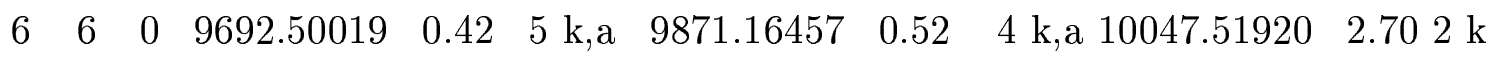

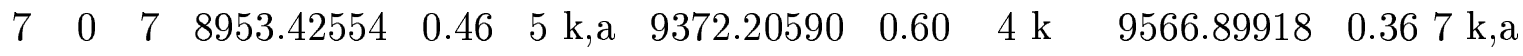

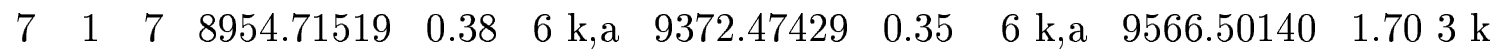

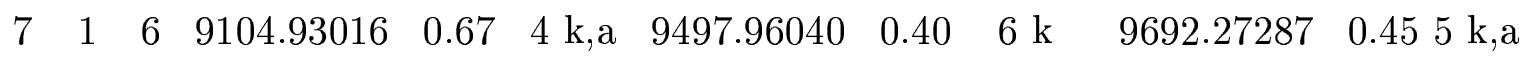

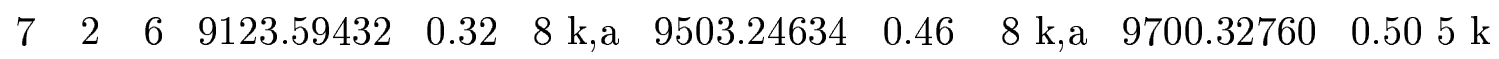

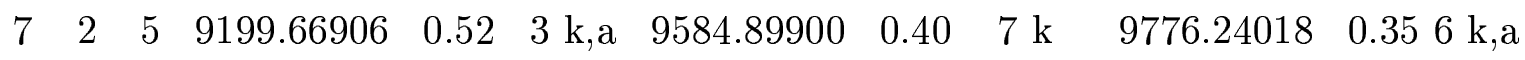

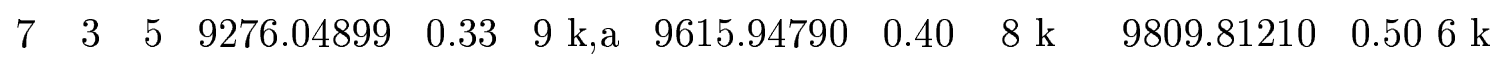

$\begin{array}{llllllllllll}7 & 3 & 4 & 9294.37270 & 6.20 & 2 \mathrm{k} & 9644.30910 & 0.50 & 6 \mathrm{k} & 9836.47750 & 0.60 & 2 \mathrm{k}\end{array}$

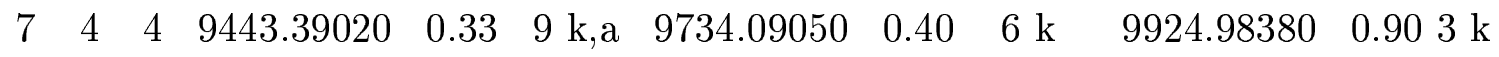

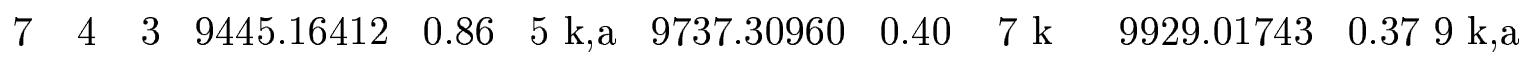

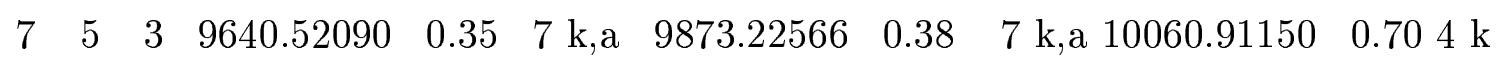

$\begin{array}{llllllllllll}7 & 5 & 2 & 9638.45770 & 0.40 & 6 \mathrm{k} & 9873.28550 & 0.50 & 5 \mathrm{k} & 10061.12070 & 0.40 & 6 \mathrm{k}\end{array}$

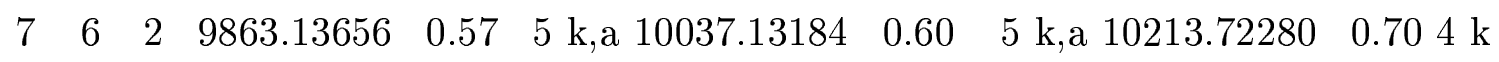

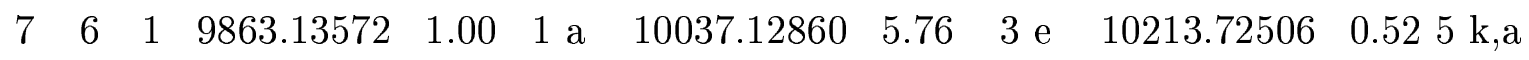

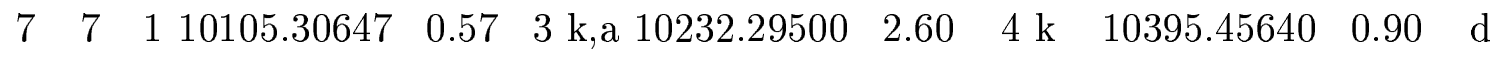

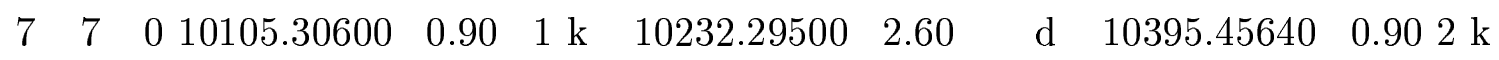

$\begin{array}{llllllllllll}8 & 0 & 8 & 9106.70786 & 0.47 & 5 \mathrm{k}, \mathrm{a} & 9523.47787 & 0.45 & 5 \mathrm{k}, \mathrm{a} & 9718.20360 & 0.90 & 2 \mathrm{k}\end{array}$

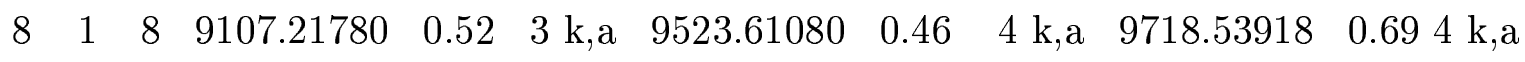

$\begin{array}{llllllllll}8 & 1 & 7 & 9288.18931 & 0.42 & 6 & \mathrm{k}, \mathrm{a} & 9671.44700 & 0.35 & 8 \mathrm{k}, \mathrm{a}\end{array}$

$\begin{array}{lllllllllll}8 & 2 & 7 & 9299.77953 & 0.57 & 3 \mathrm{k}, \mathrm{a} & 9674.22190 & 0.50 & 6 \mathrm{k} & 9868.30222 & 0.418 \mathrm{k}, \mathrm{a}\end{array}$ 


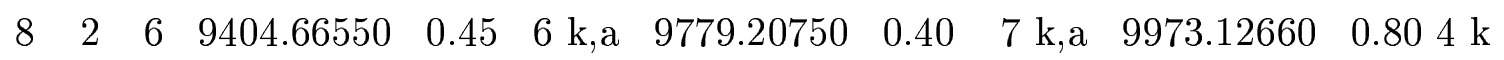

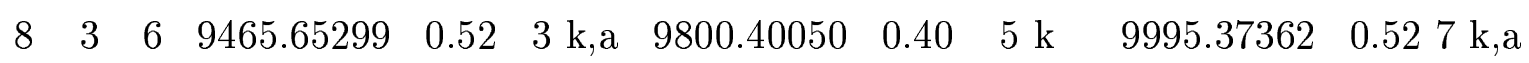

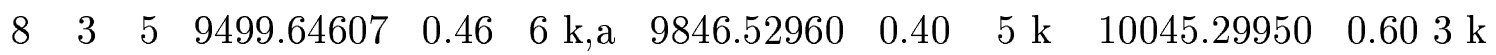

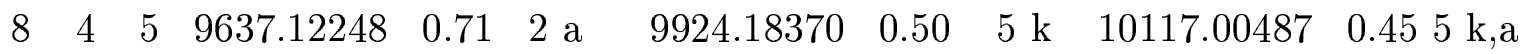

$\begin{array}{llllllllll}8 & 4 & 4 & 9641.68621 & 0.47 & 10 \mathrm{k}, \mathrm{a} & 9932.89188 & 0.35 & 10 \mathrm{k}, \mathrm{a}\end{array}$

$\begin{array}{llllllllllll}8 & 5 & 4 & 9834.83567 & 0.71 & 2 \mathrm{a} & 10063.87950 & 0.50 & 5 \mathrm{k} & 10254.42081 & 0.528 \mathrm{k}, \mathrm{a}\end{array}$

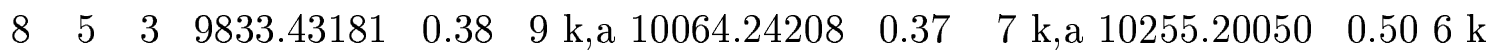

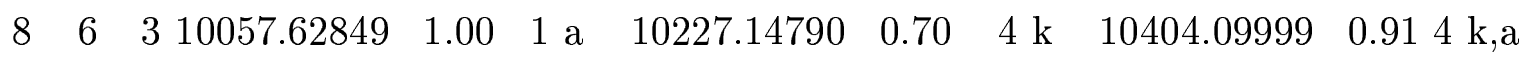

$\begin{array}{llllllll}8 & 6 & 2 & 10057.64592 & 0.52 & 4 \mathrm{k}, \mathrm{a} & 10227.02780 & 0.40 \quad 4 \mathrm{k}\end{array}$

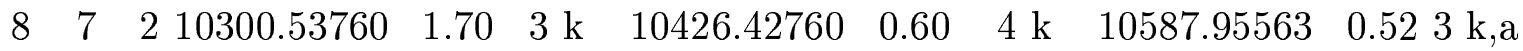

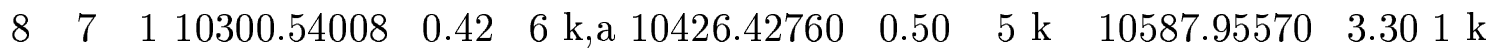

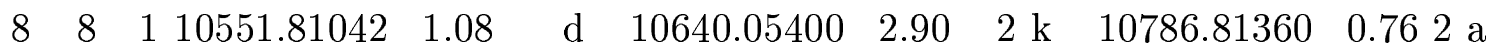

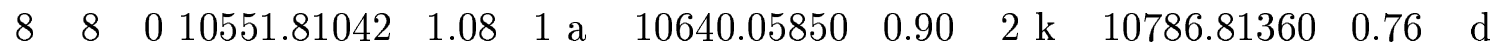

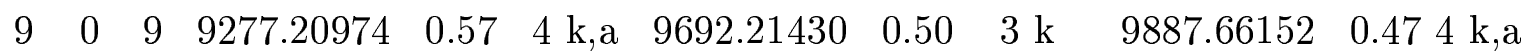

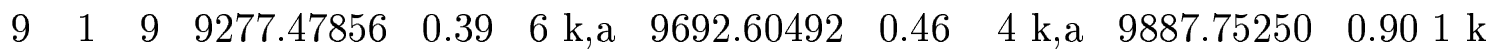

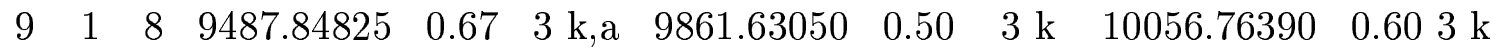

$\begin{array}{llllllllllll}9 & 2 & 8 & 9494.68515 & 0.42 & 6 \mathrm{k}, \mathrm{a} & 9864.45900 & 0.50 & 4 \mathrm{k} & 10056.48780 & 0.70 & 3 \mathrm{k}\end{array}$

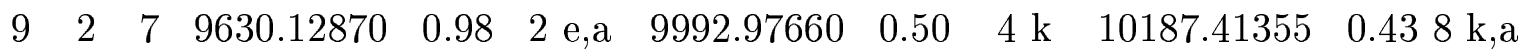

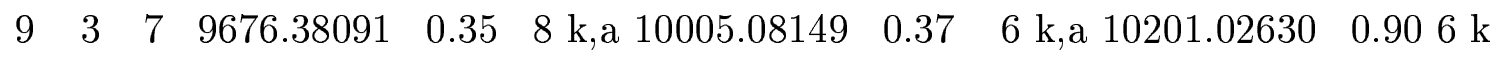

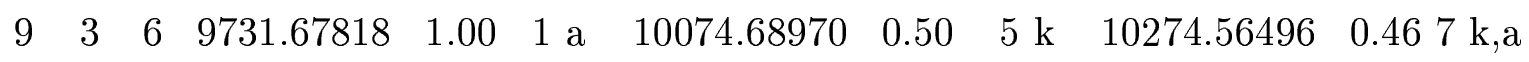

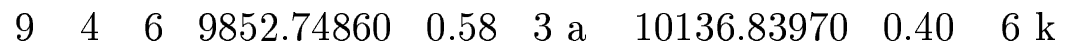

$\begin{array}{llllllll}9 & 4 & 5 & 10155.67762 & 0.50 & 5 \mathrm{k}, \mathrm{a} & 10353.02790 & 0.903 \mathrm{k}\end{array}$

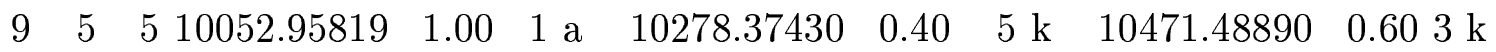

$\begin{array}{lllllllll}9 & 5 & 4 & 10279.85650 & 0.50 & 4 \mathrm{k} & 10473.85790 & 0.60 & 5 \mathrm{k}\end{array}$

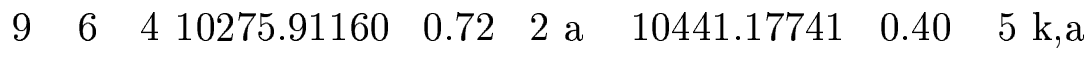

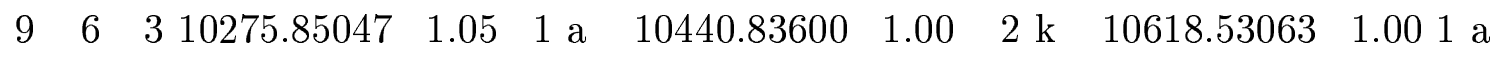

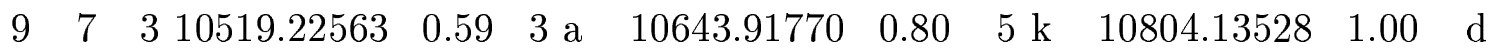




\begin{tabular}{|c|c|c|c|c|c|c|c|c|c|}
\hline 9 & 72 & 2 & & & 10643.91480 & 7.07 & $2 \mathrm{e}$ & 10804.13528 & $1.001 \mathrm{a}$ \\
\hline 9 & 82 & 210770.06619 & 1.01 & $1 \mathrm{a}$ & 10859.12770 & 0.90 & $1 \mathrm{k}$ & 11002.10421 & $1.01 \mathrm{~d}$ \\
\hline 9 & 81 & 110770.06619 & 1.01 & $\mathrm{~d}$ & 10859.12780 & 1.00 & $2 \mathrm{k}$ & 11002.10421 & $1.011 \mathrm{a}$ \\
\hline 9 & $\begin{array}{ll}9 & 1\end{array}$ & 1 & & & & & & 11220.89557 & $1.08 \mathrm{~d}$ \\
\hline 9 & $9 \quad 0$ & 0 & & & & & & 11220.89557 & $1.081 \mathrm{a}$ \\
\hline & 010 & $\begin{array}{ll}0 & 9465.00049\end{array}$ & 0.46 & $4 \mathrm{k}, \mathrm{a}$ & 9878.80370 & 0.50 & $3 \mathrm{k}$ & 10074.34840 & $1.001 \mathrm{k}$ \\
\hline 10 & 110 & $\begin{array}{ll}0 & 9465.08417\end{array}$ & 0.90 & $3 \mathrm{k}, \mathrm{a}$ & 9877.81280 & 0.60 & $3 \mathrm{k}$ & 10074.46200 & $0.901 \mathrm{k}$ \\
\hline 10 & 15 & $9 \quad 9703.96950$ & 0.71 & $2 \mathrm{a}$ & 10068.88028 & 0.46 & $4 \mathrm{k}, \mathrm{a}$ & & \\
\hline 10 & 25 & $9 \quad 9707.81682$ & 1.00 & $1 \mathrm{a}$ & 10068.08380 & 0.70 & $3 \mathrm{k}$ & 10263.84307 & $0.722 \mathrm{a}$ \\
\hline 0 & $2 \varepsilon$ & $8 \quad 9872.59967$ & 0.52 & $5 \mathrm{k}, \mathrm{a}$ & 10224.78957 & 0.40 & $5 \mathrm{k}, \mathrm{a}$ & 10417.85520 & $5.151 \mathrm{e}$ \\
\hline 10 & 38 & $8 \quad 9908.51970$ & 1.01 & $1 \mathrm{a}$ & 10229.02450 & 0.60 & $4 \mathrm{k}$ & 10426.59044 & $1.001 \mathrm{a}$ \\
\hline 10 & 37 & $\begin{array}{ll}7 & 9987.72168\end{array}$ & 0.70 & $4 \mathrm{e}, \mathrm{a}$ & 10338.21443 & 0.50 & $6 \mathrm{k}, \mathrm{a}$ & & \\
\hline 10 & 47 & 710095.31912 & 1.00 & $1 \mathrm{a}$ & 10371.19010 & 0.70 & $5 \mathrm{k}$ & 10567.73565 & $1.022 \mathrm{e}, \mathrm{a}$ \\
\hline 10 & 46 & 6 10114.14161 & 0.71 & $3 \mathrm{e}, \mathrm{a}$ & 10404.67680 & 0.40 & $5 \mathrm{k}$ & & \\
\hline 10 & 5 & 6 & & & 10516.22920 & 0.50 & $4 \mathrm{k}$ & 10711.75415 & $1.001 \mathrm{a}$ \\
\hline 10 & 5 & 510294.34849 & 0.70 & $3 \mathrm{e}, \mathrm{a}$ & 10520.71738 & 0.60 & $4 \mathrm{k}, \mathrm{a}$ & & \\
\hline 10 & 6 & 410517.59247 & 1.03 & $1 \mathrm{a}$ & 10678.43070 & 0.50 & $4 \mathrm{k}, \mathrm{a}$ & & \\
\hline 10 & 7 & 410761.10088 & 0.54 & $4 \mathrm{a}$ & 10884.55620 & 9.97 & $1 \mathrm{e}$ & & \\
\hline 10 & $7:$ & 3 10761.10088 & 0.54 & $\mathrm{~d}$ & 10884.56060 & 0.90 & $2 \mathrm{k}$ & & \\
\hline 10 & 8 & 3 11011.13737 & 5.01 & $\mathrm{~d}$ & 11101.44520 & 10.06 & $1 \mathrm{e}$ & & \\
\hline 10 & 8 & 211011.13737 & 5.01 & $1 \mathrm{a}$ & 11101.44440 & 7.81 & $3 \mathrm{e}$ & & \\
\hline 10 & 9 & 211357.09297 & 1.01 & $\mathrm{~d}$ & & & & & \\
\hline 10 & 9 & 111357.09297 & 1.01 & $1 \mathrm{a}$ & & & & & \\
\hline 11 & $\begin{array}{ll}0 & 1\end{array}$ & 19670.14691 & 0.70 & $3 \mathrm{e}, \mathrm{a}$ & 10081.44740 & 0.90 & $2 \mathrm{k}$ & 10278.41466 & $0.732 \mathrm{a}$ \\
\hline 11 & $\begin{array}{ll}1 & 1\end{array}$ & 19670.07147 & 0.50 & $5 \mathrm{k}, \mathrm{a}$ & 10081.41513 & 0.70 & $3 \mathrm{k}, \mathrm{a}$ & 10278.42470 & $5.222 \mathrm{e}$ \\
\hline 11 & 110 & $0 \quad 9936.88379$ & 0.71 & $3 \mathrm{e}, \mathrm{a}$ & 10294.58020 & 0.70 & $3 \mathrm{k}$ & 10487.96651 & $1.001 \mathrm{a}$ \\
\hline 11 & 210 & $\begin{array}{ll}0 & 9938.86190\end{array}$ & 0.52 & $6 \mathrm{e}, \mathrm{a}$ & 10292.35080 & 0.90 & $3 \mathrm{k}$ & & \\
\hline
\end{tabular}


$11 \quad 2 \quad 9 \quad 10135.07964 \quad 0.98 \quad 2$ e,a $10467.61180 \quad 0.70 \quad 4 \mathrm{k} \quad 10664.66628 \quad 1.001 \mathrm{a}$

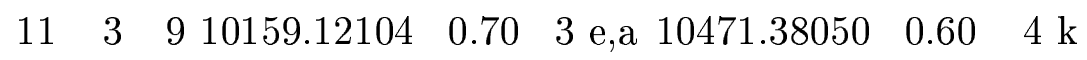

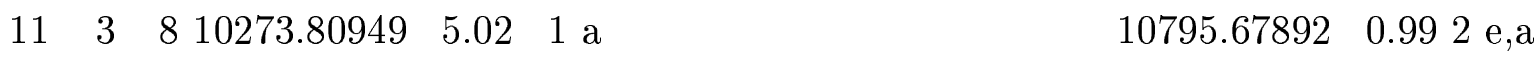

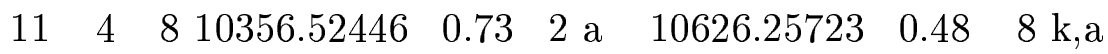

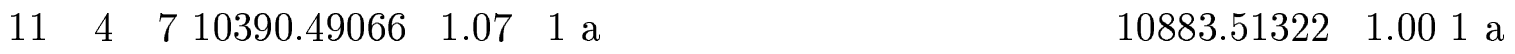

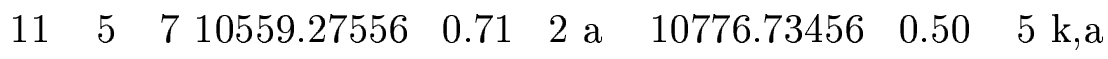

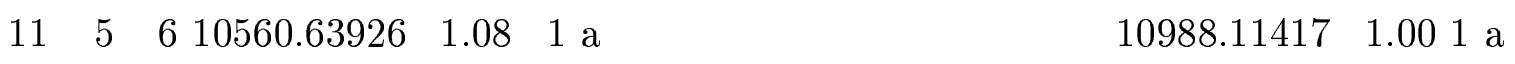

$11 \quad 6 \quad 6 \quad 10782.05465 \quad 1.01 \quad 1$ a $\quad 10940.80730 \quad 0.60 \quad 3 \mathrm{k}$

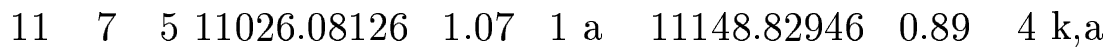

$\begin{array}{llllll}11 & 8 & 4 & 11366.78760 & 2.90 & 1 \mathrm{k}\end{array}$

$\begin{array}{llllll}11 & 8 & 3 & 11366.79040 & 8.37 \quad 2 \mathrm{e}\end{array}$

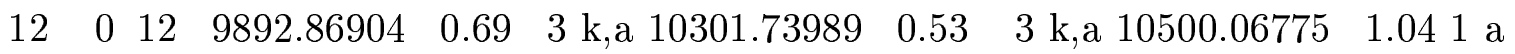

$\begin{array}{llllllllllll}12 & 1 & 12 & 9892.45547 & 1.01 & 1 \mathrm{a} & 10301.45680 & 0.70 & 2 \mathrm{k} & 10499.81409 & 1.02 & 1 \mathrm{a}\end{array}$

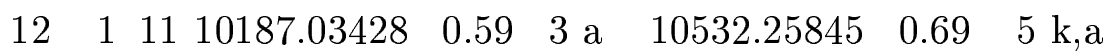

$12 \quad 2 \quad 11 \quad 10187.61713 \quad 0.74 \quad 2$ a $10533.70525 \quad 0.69 \quad 4$ k,a $10729.98714 \quad 1.002$ e,a

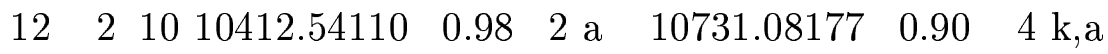

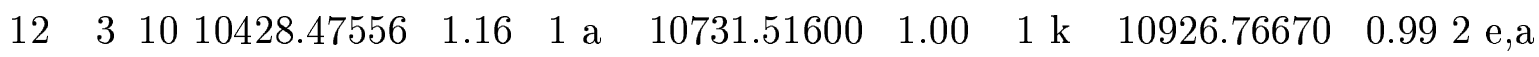

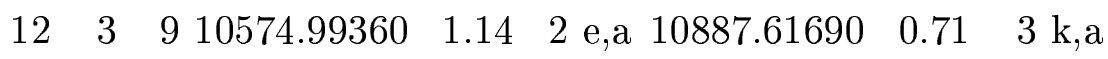

$\begin{array}{lllllll}12 & 4 & 9 & 10638.92957 & 1.08 \quad 1 \mathrm{a}\end{array}$

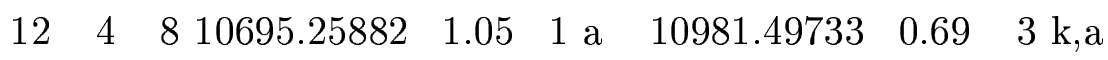

$\begin{array}{llllll}12 & 5 & 7 & 11078.88707 & 0.68 & 4 \mathrm{k}, \mathrm{a}\end{array}$

$\begin{array}{llllll}12 & 6 & 7 & 11069.44092 & 5.02 & 1\end{array}$

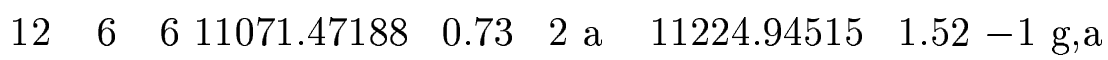

$\begin{array}{llllll}12 & 7 & 5 & 11313.36358 & 1.08 & 1 \mathrm{a}\end{array}$

$\begin{array}{llllllllll}13 & 0 & 13 & 10539.78960 & 1.00 & 2 \mathrm{k} & 10738.73031 & 1.01 & 1 \mathrm{a}\end{array}$

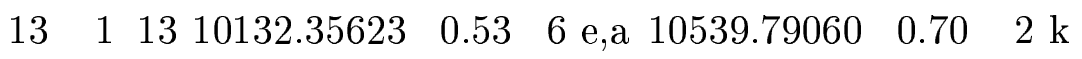

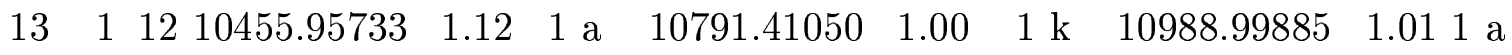




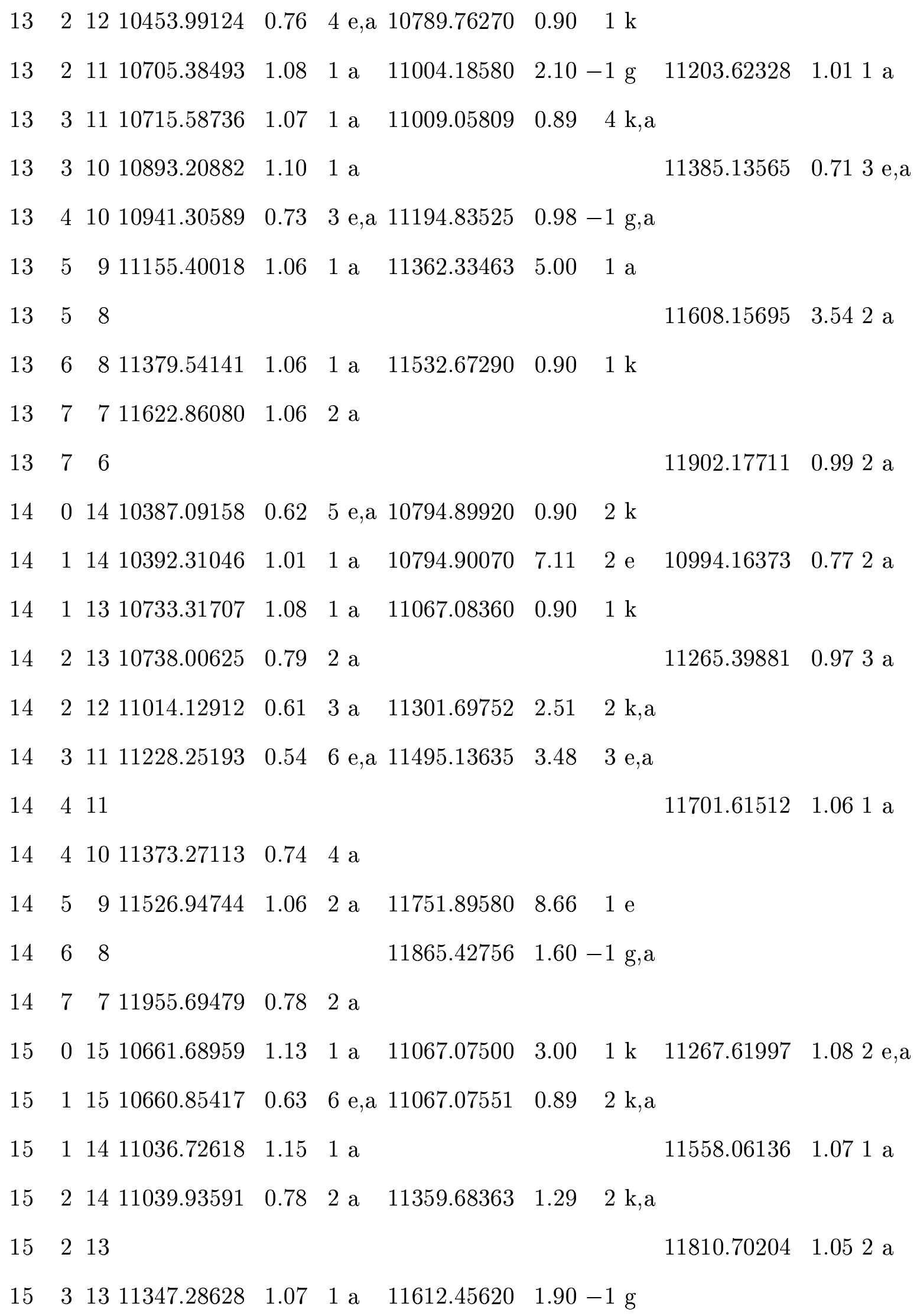




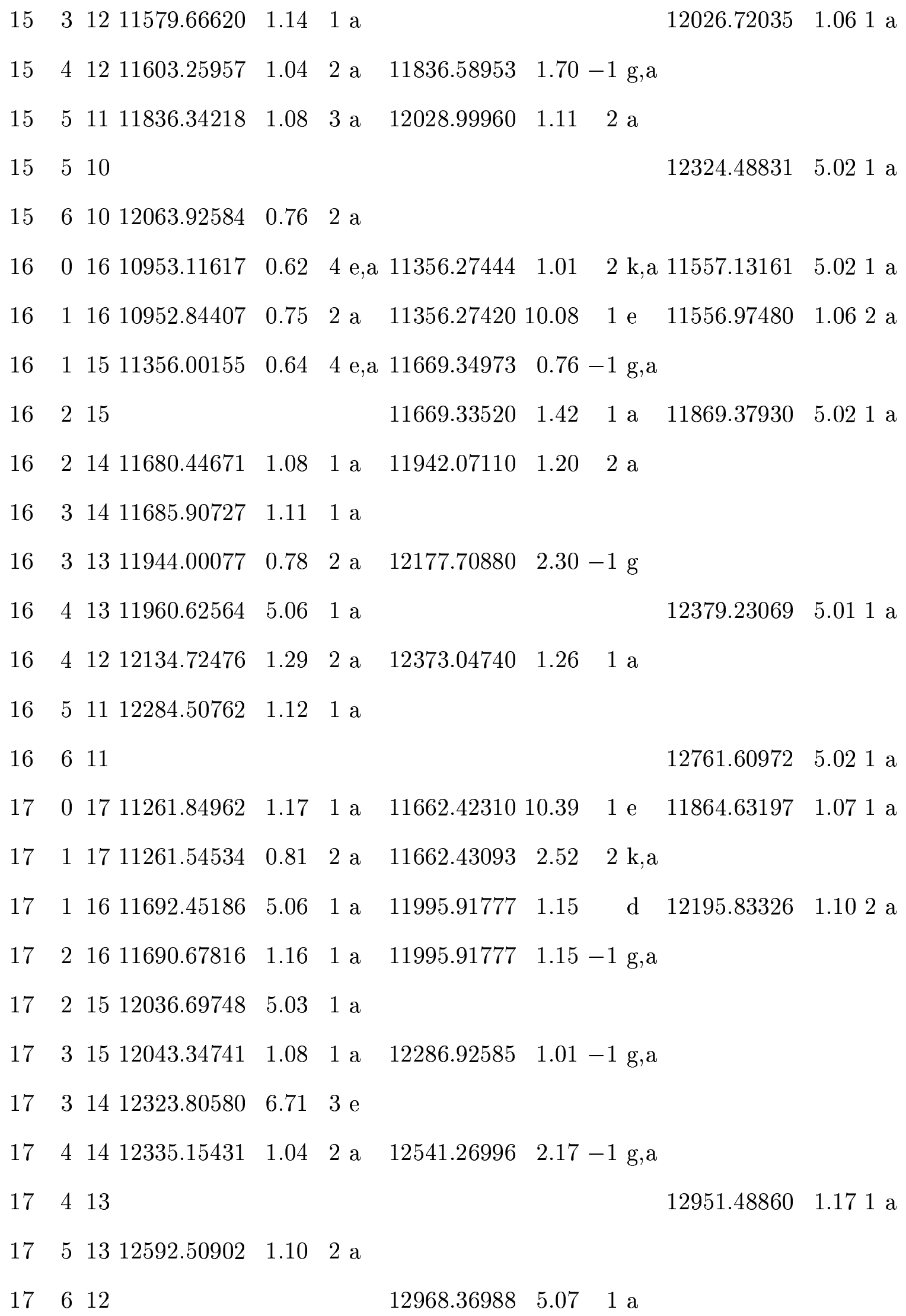




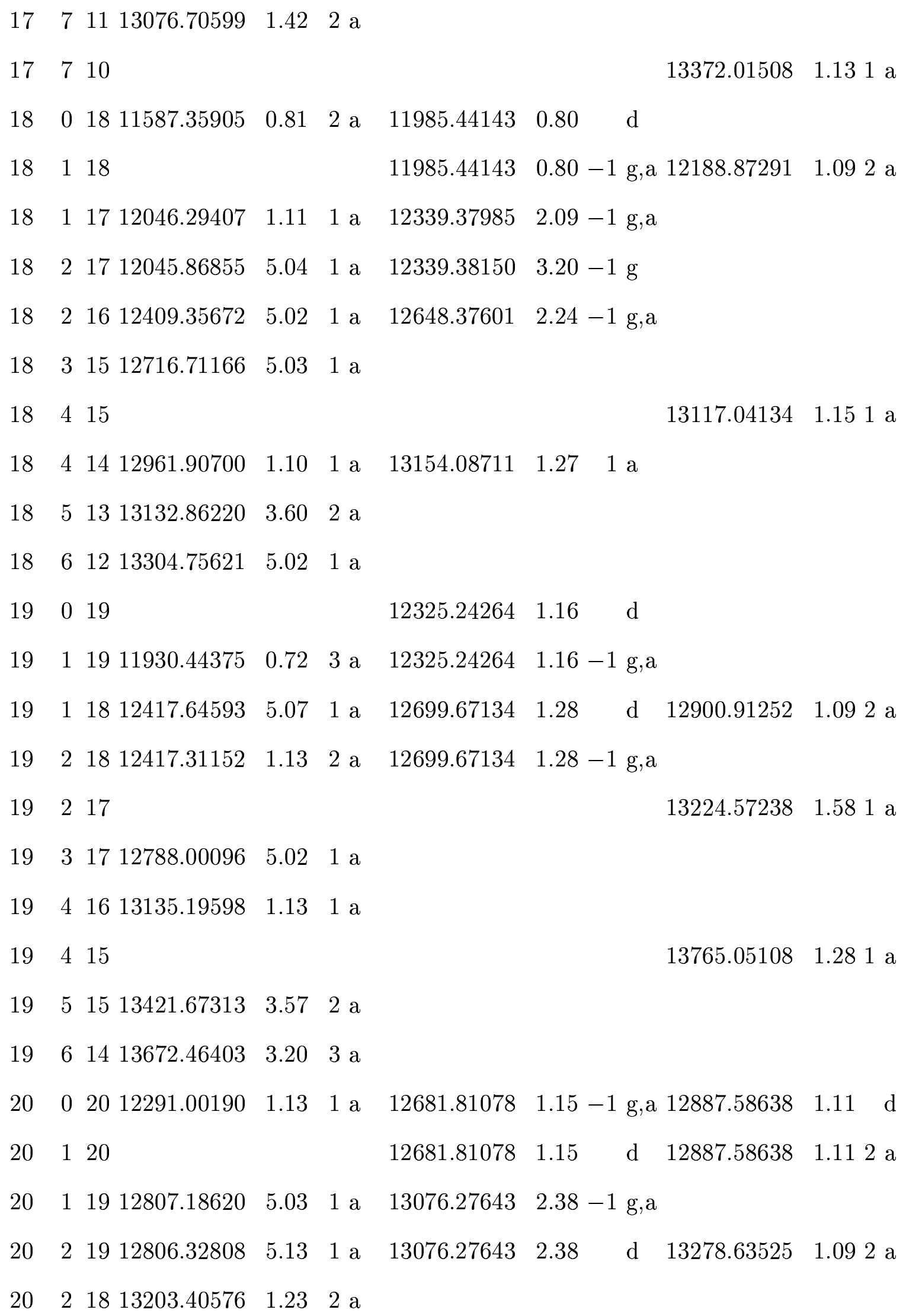




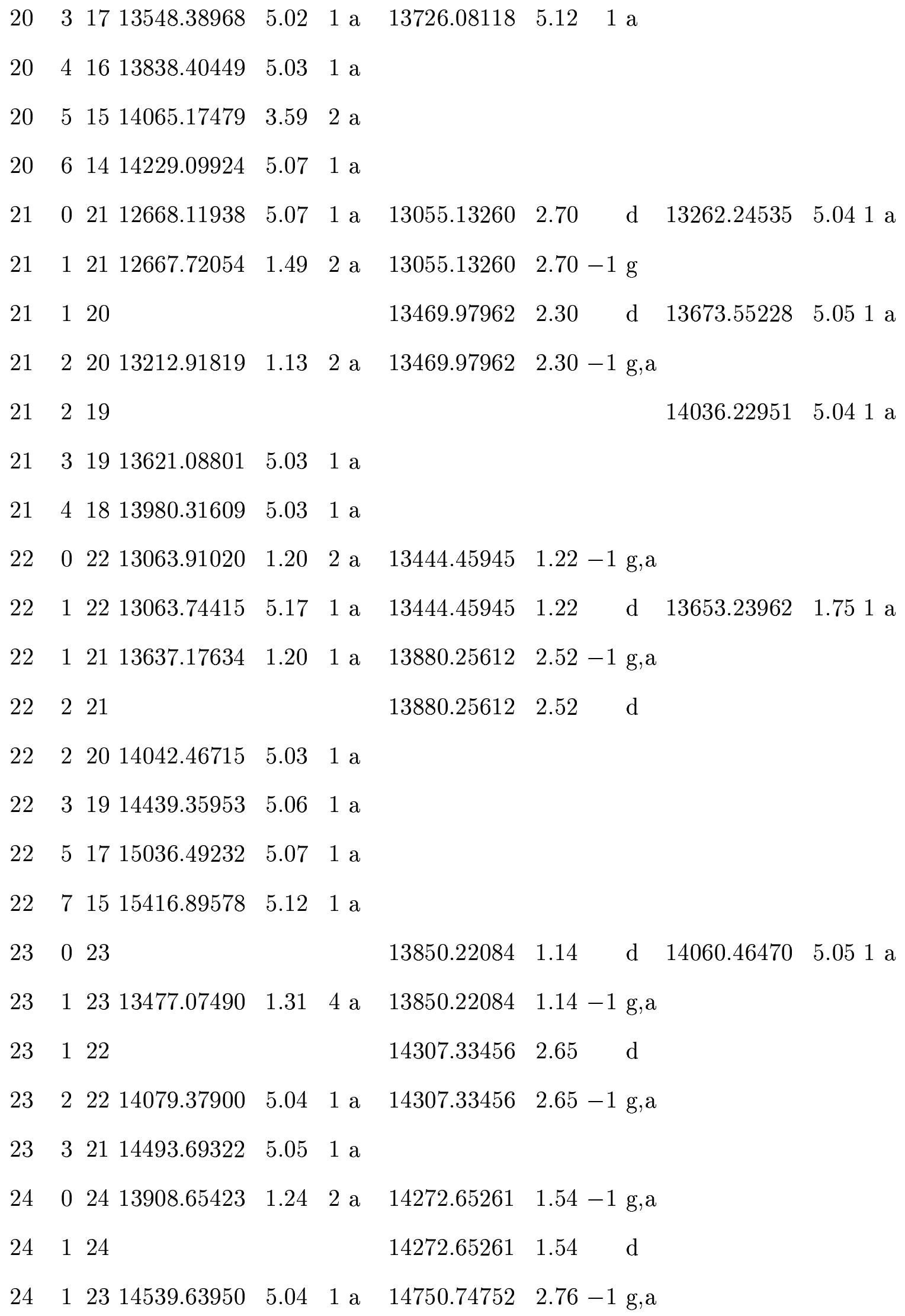




$\begin{array}{llllllllr}24 & 2 & 23 & & & 14750.74752 & 2.76 & \mathrm{~d} \\ 25 & 0 & 25 & & & & 14711.82630 & 3.60 & \mathrm{~d} \\ 25 & 1 & 25 & 14358.71381 & 3.62 & 2 \mathrm{a} & 14711.82630 & 3.60-1 \mathrm{~g} \\ 25 & 1 & 24 & & & 15210.86120 & 3.20 & \mathrm{~d} \\ 25 & 2 & 24 & 15022.56608 & 5.28 & 1 \mathrm{a} & 15210.86120 & 3.20-1 \mathrm{~g} \\ 25 & 3 & 23 & 15431.31706 & 5.14 & 1 \mathrm{a} & & \\ 26 & 0 & 26 & 14830.51263 & 5.06 & 1 \mathrm{a} & & \\ 26 & 1 & 25 & & & & 15687.19180 & 3.50-1 \mathrm{~g} \\ 26 & 2 & 25 & & & & 15687.19180 & 3.50 & \mathrm{~d} \\ 27 & 1 & 27 & 15308.80747 & 5.08 & 1 \mathrm{a} & & & \end{array}$


TABLE VII.: Term values for the $3 \nu$ polyad of $\mathrm{H}_{2}{ }^{16} \mathrm{O}$.

\begin{tabular}{|c|c|c|c|c|c|c|c|c|}
\hline \multicolumn{3}{|c|}{$J K_{a} K_{c}$} & \multirow{2}{*}{$\begin{array}{r}060 \text { or } \\
8869.95380\end{array}$} & \multirow{2}{*}{$\frac{006}{14.291 \mathrm{e}}$} & \multicolumn{2}{|c|}{121 or $20^{-} 2$} & \multicolumn{2}{|c|}{003 or $21^{-} 0$} \\
\hline 0 & 0 & 0 & & & 10328.73090 & $0.901 \mathrm{~m}$ & 11032.40580 & $0.901 \mathrm{~m}$ \\
\hline 1 & 0 & 1 & 8893.46480 & $8.711 \mathrm{e}$ & 10351.99000 & $0.503 \mathrm{~m}$ & 11055.47160 & $0.602 \mathrm{~m}$ \\
\hline 1 & 1 & 1 & 8998.09960 & $11.381 \mathrm{e}$ & 10369.71620 & $0.602 \mathrm{~m}$ & 11065.77450 & $0.602 \mathrm{~m}$ \\
\hline 1 & 1 & 0 & 9004.61700 & $9.252 \mathrm{e}$ & 10375.59420 & $0.602 \mathrm{~m}$ & 11071.11250 & $0.602 \mathrm{~m}$ \\
\hline 2 & 0 & 2 & & & 10397.21510 & $0.404 \mathrm{~m}$ & 11099.97360 & $0.404 \mathrm{~m}$ \\
\hline 2 & 1 & 2 & 9038.46090 & $12.781 \mathrm{e}$ & 10410.23160 & $0.504 \mathrm{~m}$ & 11106.58290 & $0.504 \mathrm{~m}$ \\
\hline 2 & 1 & 1 & & & 10427.68630 & $0.404 \mathrm{~m}$ & 11122.56700 & $0.503 \mathrm{~m}$ \\
\hline 2 & 2 & 1 & & & 10480.32290 & $0.503 \mathrm{~m}$ & 11153.27110 & $0.503 \mathrm{~m}$ \\
\hline 2 & 2 & 0 & & & 10481.55460 & $0.404 \mathrm{~m}$ & 11154.86830 & $0.404 \mathrm{~m}$ \\
\hline 3 & 0 & 3 & 9008.73880 & $7.511 \mathrm{e}$ & 10462.21960 & $0.504 \mathrm{~m}$ & 11163.47670 & $0.405 \mathrm{~m}$ \\
\hline 3 & 1 & 3 & & & 10470.34530 & $0.405 \mathrm{~m}$ & 11166.86320 & $0.405 \mathrm{~m}$ \\
\hline 3 & 1 & 2 & 9139.07571 & $1.471 \mathrm{a}$ & 10504.04540 & $0.505 \mathrm{~m}$ & 11198.42730 & $0.504 \mathrm{~m}$ \\
\hline 3 & 2 & 2 & & & 10549.94380 & $0.405 \mathrm{~m}$ & 11222.53880 & $0.405 \mathrm{~m}$ \\
\hline 3 & 2 & 1 & 9344.38004 & $1.421 \mathrm{a}$ & 10555.72220 & $0.406 \mathrm{~m}$ & 11229.70580 & $0.505 \mathrm{~m}$ \\
\hline 3 & 3 & 1 & & & 10650.03290 & $0.504 \mathrm{~m}$ & 11287.11980 & $0.604 \mathrm{~m}$ \\
\hline 3 & 3 & 0 & 9628.77606 & $1.191 \mathrm{a}$ & 10650.22330 & $0.603 \mathrm{~m}$ & 11287.43830 & $0.504 \mathrm{~m}$ \\
\hline 4 & 0 & 4 & & & 10544.99178 & $0.386 \mathrm{~m}, \mathrm{a}$ & 11244.47150 & $0.406 \mathrm{~m}$ \\
\hline 4 & 1 & 4 & & & 10549.40090 & $0.505 \mathrm{~m}$ & 11245.89620 & $0.405 \mathrm{~m}$ \\
\hline 4 & 1 & 3 & & & 10607.67320 & $0.406 \mathrm{~m}$ & 11296.74570 & $0.406 \mathrm{~m}$ \\
\hline 4 & 2 & 3 & & & 10641.68970 & $0.505 \mathrm{~m}$ & 11313.46230 & $0.505 \mathrm{~m}$ \\
\hline 4 & 2 & 2 & & & 10657.17180 & $0.407 \mathrm{~m}$ & 11331.55920 & $0.406 \mathrm{~m}$ \\
\hline 4 & 3 & 2 & 9725.73788 & $1.561 \mathrm{a}$ & 10745.13950 & $0.406 \mathrm{~m}$ & 11382.13280 & $0.406 \mathrm{~m}$ \\
\hline 4 & 3 & 1 & & & 10746.14750 & $0.405 \mathrm{~m}$ & 11384.18510 & $0.505 \mathrm{~m}$ \\
\hline 4 & 4 & 1 & 10049.28101 & $0.902 \mathrm{a}$ & 10877.34490 & $0.903 \mathrm{~m}$ & 11467.56250 & $0.504 \mathrm{~m}$ \\
\hline
\end{tabular}




\begin{tabular}{|c|c|c|c|c|c|c|c|}
\hline 4 & 4 & 0 & & 10877.36950 & $0.504 \mathrm{~m}$ & 11467.61580 & $0.503 \mathrm{~m}$ \\
\hline 5 & 0 & $5 \quad 9210.16115$ & $0.902 \mathrm{a}$ & 10644.62040 & $0.605 \mathrm{~m}$ & 11343.62460 & $0.406 \mathrm{~m}$ \\
\hline 5 & 1 & 5 & & 10646.78080 & $0.405 \mathrm{~m}$ & 11343.16140 & $0.405 \mathrm{~m}$ \\
\hline 5 & 1 & $4 \quad 9377.22587$ & $1.271 \mathrm{a}$ & 10730.20360 & $0.405 \mathrm{~m}$ & 11414.79490 & $0.406 \mathrm{~m}$ \\
\hline 5 & 2 & 4 & & 10754.75300 & $0.406 \mathrm{~m}$ & 11424.99850 & $0.506 \mathrm{~m}$ \\
\hline 5 & 2 & 39565.52032 & $1.161 \mathrm{a}$ & 10785.65425 & $0.506 \mathrm{~m}, \mathrm{a}$ & 11459.21180 & $0.505 \mathrm{~m}$ \\
\hline 5 & 3 & 3 & & 10863.67100 & $0.406 \mathrm{~m}$ & 11500.44780 & $0.406 \mathrm{~m}$ \\
\hline 5 & 3 & $2 \quad 9847.22887$ & $1.181 \mathrm{a}$ & 10867.74690 & $0.505 \mathrm{~m}$ & 11507.57540 & $0.505 \mathrm{~m}$ \\
\hline 5 & 4 & 2 & & 10996.30880 & $0.408 \mathrm{~m}$ & 11587.40260 & $0.406 \mathrm{~m}$ \\
\hline 5 & 4 & 110171.29165 & $1.341 \mathrm{a}$ & 10996.54780 & $0.504 \mathrm{~m}$ & 11587.83570 & $0.504 \mathrm{~m}$ \\
\hline 5 & 5 & 1 & & 11158.50020 & $0.903 \mathrm{~m}$ & 11693.64300 & $0.603 \mathrm{~m}$ \\
\hline 5 & 5 & 0 & & 11158.49530 & $5.065 \mathrm{e}$ & 11693.64380 & $8.402 \mathrm{~m}$ \\
\hline 6 & 0 & 6 & & 10761.04630 & $0.405 \mathrm{~m}$ & 11457.55740 & $0.602 \mathrm{~m}$ \\
\hline 6 & 1 & $\begin{array}{ll}6 & 9400.64060\end{array}$ & 1.7081 & 10762.04620 & $0.605 \mathrm{~m}$ & 11458.61520 & $0.505 \mathrm{~m}$ \\
\hline 6 & 1 & $5 \quad 9533.58280$ & 6.4051 & 10872.35220 & $0.505 \mathrm{~m}$ & 11550.31070 & $0.405 \mathrm{~m}$ \\
\hline 6 & 2 & 5 & & 10888.31160 & $0.506 \mathrm{~m}$ & 11556.14500 & $0.506 \mathrm{~m}$ \\
\hline 6 & 2 & 4 & & 10939.54810 & $0.407 \mathrm{~m}$ & 11610.45106 & $0.378 \mathrm{~m}, \mathrm{a}$ \\
\hline 6 & 3 & $4 \quad 9991.01874$ & $1.271 \mathrm{a}$ & 11005.25820 & $0.506 \mathrm{~m}$ & 11641.21140 & $0.506 \mathrm{~m}$ \\
\hline 6 & 3 & 3 & & 11016.23570 & $0.407 \mathrm{~m}$ & 11658.49540 & $0.406 \mathrm{~m}$ \\
\hline 6 & 4 & 310316.38200 & $1.281 \mathrm{a}$ & 11140.07560 & $0.406 \mathrm{~m}$ & 11731.15190 & $0.605 \mathrm{~m}$ \\
\hline 6 & 4 & 2 & & 11140.14050 & $0.608 \mathrm{~m}$ & 11733.06220 & $0.406 \mathrm{~m}$ \\
\hline 6 & 5 & 210666.25030 & $1.271 \mathrm{a}$ & 11301.20600 & $0.703 \mathrm{~m}$ & 11840.42310 & $0.604 \mathrm{~m}$ \\
\hline 6 & 5 & 1 & & 11301.15020 & $0.505 \mathrm{~m}$ & 11840.48600 & $0.405 \mathrm{~m}$ \\
\hline 6 & 6 & 1 & & 11492.88140 & $0.505 \mathrm{~m}$ & 11963.85840 & $2.703 \mathrm{~m}$ \\
\hline & 6 & 0 & & 11493.01790 & $0.603 \mathrm{~m}$ & 11963.85810 & $0.503 \mathrm{~m}$ \\
\hline & 0 & 79487.94880 & $1.171 \mathrm{a}$ & 10894.48540 & $0.503 \mathrm{~m}$ & 11590.59270 & $0.504 \mathrm{~m}$ \\
\hline & 1 & 7 & & 10894.82300 & $0.405 \mathrm{~m}$ & 11590.45690 & $0.503 \mathrm{~m}$ \\
\hline
\end{tabular}




\begin{tabular}{|c|c|c|c|c|c|c|c|}
\hline 7 & 1 & $6 \quad 9714.76092$ & $1.111 \mathrm{a}$ & 11031.86410 & $0.505 \mathrm{~m}$ & 11702.55970 & $0.505 \mathrm{~m}$ \\
\hline 7 & 2 & 6 & & 11041.32040 & $0.406 \mathrm{~m}$ & 11706.22580 & $0.406 \mathrm{~m}$ \\
\hline 7 & 2 & 5 & & 11116.49570 & $0.505 \mathrm{~m}$ & 11782.47340 & 0.406 \\
\hline 7 & 3 & 5 & & 11170.31900 & $0.407 \mathrm{~m}$ & 11803.36460 & 0.407 \\
\hline 7 & 3 & 4 & & 11192.12800 & $0.505 \mathrm{~m}$ & 11836.22950 & 0.505 \\
\hline 7 & 4 & 4 & & 11306.78690 & $0.407 \mathrm{~m}$ & 11898.35150 & 0.504 \\
\hline 7 & 4 & 3 & & 11308.69624 & $0.505 \mathrm{~m}, \mathrm{a}$ & 11904.20170 & 0.506 \\
\hline 7 & 5 & 3 & & 11467.97200 & $0.405 \mathrm{~m}$ & 12009.57850 & 0.805 \\
\hline 7 & 5 & 210837.70831 & $1.301 \mathrm{a}$ & 11467.63490 & $0.504 \mathrm{~m}$ & 12009.90050 & 0.504 \\
\hline 7 & 6 & 2 & & 11662.33610 & $0.504 \mathrm{~m}$ & 12127.13280 & 0.503 \\
\hline 8 & 6 & 1 & & 11662.44540 & $0.703 \mathrm{~m}$ & 12127.14300 & 1.703 \\
\hline 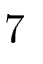 & 7 & 111564.21694 & $1.54 \mathrm{~d}$ & 11914.64590 & $0.703 \mathrm{~m}$ & 12276.49440 & 0.504 \\
\hline 7 & 7 & 011564.21694 & $1.541 \mathrm{a}$ & 11914.64620 & $2.102 \mathrm{~m}$ & 12276.49380 & 2.102 \\
\hline 8 & 0 & 8 & & 11045.39010 & $0.505 \mathrm{~m}$ & 11741.00880 & 0.504 \\
\hline 8 & 1 & $\begin{array}{ll}8 & 9697.22629\end{array}$ & $1.402 \mathrm{a}$ & 11045.14718 & $0.505 \mathrm{~m}, \mathrm{a}$ & 11741.00210 & 0.604 \\
\hline 8 & 1 & 7 & & 11207.74880 & $0.406 \mathrm{~m}$ & 11871.62180 & 0.405 \\
\hline 8 & 2 & 7 & & 11213.90350 & $0.504 \mathrm{~m}$ & 11877.54220 & 0.504 \\
\hline 8 & 2 & 6 & & 11322.45120 & $0.505 \mathrm{~m}$ & 11972.45730 & 0.307 \\
\hline 8 & 3 & 610348.16648 & $1.111 \mathrm{a}$ & 11354.57240 & $0.505 \mathrm{~m}$ & 11985.89550 & 0.504 \\
\hline 8 & 3 & 5 & & & & 12038.67370 & 0.406 \\
\hline 8 & 4 & 5 & & 11497.16260 & $0.605 \mathrm{~m}$ & 12088.28060 & 0.505 \\
\hline 8 & 4 & 4 & & 11502.97190 & $0.407 \mathrm{~m}, \mathrm{a}$ & 12102.73220 & 0.405 \\
\hline 8 & 5 & 4 & & & & 12201.79200 & 0.60 \\
\hline 8 & 5 & 3 & & 11659.29070 & $0.407 \mathrm{~m}$ & & \\
\hline 8 & 6 & 311395.48980 & 0.7041 & & & & \\
\hline 8 & 6 & 2 & & & & 12314.45960 & 0.60 \\
\hline & 0 & 9 & & 11212.03327 & $0.694 \mathrm{~m}, \mathrm{a}$ & 11908.90980 & 0.70 \\
\hline
\end{tabular}




\begin{tabular}{|c|c|c|c|c|c|c|c|}
\hline 9 & 1 & 9 & & 11213.85593 & $0.536 \mathrm{~m}, \mathrm{a}$ & 11908.89500 & $0.603 \mathrm{~m}$ \\
\hline 9 & 1 & 810143.70032 & $1.491 \mathrm{a}$ & 11399.99970 & $0.504 \mathrm{~m}$ & 12057.71130 & $0.505 \mathrm{~m}$ \\
\hline 9 & 2 & 8 & & 11397.13551 & $1.051 \mathrm{a}$ & 12057.47690 & $0.504 \mathrm{~m}$ \\
\hline 9 & 2 & 7 & & 11537.97610 & $0.505 \mathrm{~m}$ & & \\
\hline 9 & 3 & 7 & & 11560.20150 & $0.503 \mathrm{~m}$ & 12188.46840 & $0.903 \mathrm{~m}$ \\
\hline 9 & 3 & 610574.32035 & $1.042 \mathrm{a}$ & & & 12263.00270 & $0.604 \mathrm{~m}$ \\
\hline 9 & 4 & 6 & & 11710.30093 & $0.604 \mathrm{~m}, \mathrm{a}$ & 12300.07350 & $0.405 \mathrm{~m}$ \\
\hline 9 & 5 & 5 & & 11873.48860 & $0.505 \mathrm{~m}$ & 12416.94510 & $0.504 \mathrm{~m}$ \\
\hline 10 & 0 & 10 & & 11398.12530 & $0.603 \mathrm{~m}$ & 12094.21780 & $0.603 \mathrm{~m}$ \\
\hline 10 & 1 & 1010069.20122 & $1.181 \mathrm{a}$ & 11398.16410 & $2.802 \mathrm{~m}$ & 12094.21850 & $2.803 \mathrm{~m}$ \\
\hline 10 & 1 & 9 & & 11609.12759 & $1.111 \mathrm{a}$ & 12260.97780 & $0.903 \mathrm{~m}$ \\
\hline 10 & 2 & 910483.28901 & $1.412 \mathrm{a}$ & & & & \\
\hline 10 & 2 & 8 & & 11772.07017 & $5.021 \mathrm{a}$ & 12400.97300 & $0.902 \mathrm{~m}$ \\
\hline 10 & 3 & 7 & & & & 12506.27910 & $0.605 \mathrm{~m}$ \\
\hline 10 & 4 & 6 & & & & 12579.27530 & $0.603 \mathrm{~m}$ \\
\hline 11 & 0 & 1110259.45464 & $1.082 \mathrm{a}$ & & & 12296.93720 & $2.902 \mathrm{~m}$ \\
\hline 11 & 1 & 11 & & 11598.29460 & $0.902 \mathrm{~m}$ & 12296.94250 & $0.902 \mathrm{~m}$ \\
\hline 11 & 1 & 1010656.51034 & $1.481 \mathrm{a}$ & & & & \\
\hline 11 & 2 & 10 & & & & 12481.40650 & $0.904 \mathrm{~m}$ \\
\hline 12 & 0 & 12 & & & & 12517.01320 & $0.902 \mathrm{~m}$ \\
\hline 12 & 1 & 1210518.07060 & $1.181 \mathrm{a}$ & & & 12517.02270 & $3.001 \mathrm{~m}$ \\
\hline 13 & 0 & 1310756.28972 & $1.281 \mathrm{a}$ & & & & \\
\hline 13 & 1 & 13 & & & & 12754.58910 & $0.901 \mathrm{~m}$ \\
\hline \multicolumn{2}{|c|}{$J K_{a} K_{c}$} & \multicolumn{2}{|c|}{041 or $10^{-} 4$} & \multicolumn{2}{|c|}{022 or 112} & \multicolumn{2}{|c|}{201 or $30^{-} 0$} \\
\hline 0 & 0 & $0 \quad 9833.58450$ & $0.901 \mathrm{~m}$ & & & 10613.35470 & $2.901 \mathrm{~m}$ \\
\hline 1 & 0 & 19857.19780 & $0.602 \mathrm{~m}$ & & & 10636.50070 & $0.503 \mathrm{~m}$ \\
\hline 1 & 1 & 19888.89285 & $0.533 \mathrm{~m}, \mathrm{c}$ & & & 10647.41650 & $0.602 \mathrm{~m}$ \\
\hline
\end{tabular}




\begin{tabular}{|c|c|c|c|c|c|c|c|}
\hline & 1 & $\begin{array}{ll}0 & 9895.32980\end{array}$ & $0.602 \mathrm{~m}$ & & & 10652.60740 & $0.602 \mathrm{~m}$ \\
\hline & 0 & $2 \quad 9903.49144$ & $0.475 \mathrm{~m}, \mathrm{a}$ & & & 10680.54470 & $0.504 \mathrm{~m}$ \\
\hline & 1 & $2 \quad 9929.71270$ & $0.503 \mathrm{~m}$ & & & 10687.62930 & 0.504 \\
\hline & 1 & $1 \quad 9949.07617$ & $0.454 \mathrm{~m}, \mathrm{a}$ & & & 10703.08300 & 0.504 \\
\hline & 2 & 110039.41980 & $0.602 \mathrm{~m}$ & 10670.63280 & $0.603 \mathrm{~m}$ & 10737.01320 & 0.503 \\
\hline & 2 & 010040.30240 & $0.603 \mathrm{~m}$ & & & 10738.43160 & 0.404 \\
\hline & 0 & $3 \quad 9970.75320$ & $0.604 \mathrm{~m}$ & 10655.81360 & $0.503 \mathrm{~m}$ & 10743.62130 & 0.504 \\
\hline & 1 & 39990.39918 & $0.415 \mathrm{~m}, \mathrm{a}$ & & & 10747.26080 & 0.405 \\
\hline & 1 & 210028.81700 & $0.603 \mathrm{~m}$ & 10698.77310 & $0.603 \mathrm{~m}$ & 10777.55870 & 0.405 \\
\hline & 2 & 210110.53981 & $0.425 \mathrm{~m}, \mathrm{a}$ & & & 10805.02430 & 0.405 \\
\hline & 2 & 110114.79890 & $0.505 \mathrm{~m}$ & & & 10811.48330 & 0.505 \\
\hline & 3 & 110265.82060 & $0.603 \mathrm{~m}$ & & & 10874.51600 & 0.40 \\
\hline & 3 & 010265.90240 & $0.602 \mathrm{~m}$ & 10837.23720 & $1.071 \mathrm{a}$ & 10874.77170 & 0.50 \\
\hline & 0 & 410056.98760 & $0.426 \mathrm{~m}, \mathrm{a}$ & & & 10824.12410 & 0.307 \\
\hline & 1 & 410070.39650 & $0.603 \mathrm{~m}$ & 10742.86140 & $0.604 \mathrm{~m}$ & 10825.65930 & 0.40 \\
\hline & 1 & 310133.66021 & $0.425 \mathrm{~m}, \mathrm{a}$ & & & 10874.03870 & 0.40 \\
\hline & 2 & 310204.49140 & $0.504 \mathrm{~m}$ & & & 10894.53590 & 0.406 \\
\hline 4 & 2 & 210216.46164 & $0.455 \mathrm{~m}, \mathrm{a}$ & & & 10911.10120 & 0.406 \\
\hline 4 & 3 & 210362.56920 & $0.503 \mathrm{~m}$ & & & 10967.43820 & 0.406 \\
\hline 4 & 3 & 110363.12513 & $0.427 \mathrm{~m}, \mathrm{a}$ & & & 10969.11660 & 0.407 \\
\hline 4 & 4 & 110559.53870 & $0.902 \mathrm{~m}$ & 11061.27350 & $0.504 \mathrm{~m}$ & 11060.29620 & 0.901 \\
\hline 4 & 4 & 010559.55880 & $0.902 \mathrm{~m}$ & 11061.29920 & $0.603 \mathrm{~m}$ & 11060.33390 & 0.50 \\
\hline 5 & 0 & 510160.65583 & $0.533 \mathrm{~m}, \mathrm{a}$ & 10838.49225 & $1.061 \mathrm{a}$ & 10921.68260 & 0.50 \\
\hline & 1 & 510169.05845 & $0.475 \mathrm{~m}, \mathrm{a}$ & & & 10922.25660 & 0.406 \\
\hline & 1 & 410262.18920 & $0.603 \mathrm{~m}$ & 10924.03998 & $1.061 \mathrm{a}$ & 10996.06460 & 0.503 \\
\hline & 2 & 410320.58955 & $0.356 \mathrm{~m}, \mathrm{a}$ & & & 11004.78310 & 0.40 \\
\hline & 2 & 310345.90780 & $0.603 \mathrm{~m}$ & & & 11036.37650 & 0.407 \\
\hline
\end{tabular}




\begin{tabular}{|c|c|c|c|c|c|c|c|}
\hline 5 & 3 & 310483.42750 & $0.386 \mathrm{~m}, \mathrm{a}$ & & & 11083.35950 & $0.407 \mathrm{~m}$ \\
\hline & 3 & 210485.54440 & $0.503 \mathrm{~m}$ & & & 11089.34680 & $0.405 \mathrm{~m}$ \\
\hline & 4 & 210680.98984 & $0.455 \mathrm{~m}, \mathrm{a}$ & 11182.21660 & $0.505 \mathrm{~m}$ & 11176.88020 & 0.405 \\
\hline & 4 & 110681.10330 & $0.603 \mathrm{~m}$ & 11182.41634 & $0.525 \mathrm{~m}, \mathrm{a}$ & 11177.13150 & 0.407 \\
\hline & 5 & 110911.28990 & $1.704 \mathrm{~m}$ & & & 11293.87120 & 0.307 \\
\hline & 5 & 010911.31900 & $4.431 \mathrm{e}$ & & & 11293.75670 & 0.504 \\
\hline & 0 & 610281.05578 & $0.436 \mathrm{~m}, \mathrm{a}$ & & & 11036.43730 & 0.406 \\
\hline & 1 & 610286.13399 & $0.533 \mathrm{~m}, \mathrm{a}$ & 10955.86203 & $1.061 \mathrm{a}$ & 11036.65430 & 0.506 \\
\hline & 1 & 510412.57340 & $0.475 \mathrm{~m}, \mathrm{a}$ & & & 11129.88240 & 0.406 \\
\hline & 2 & 510458.06280 & $0.903 \mathrm{~m}$ & & & 11134.03070 & 0.605 \\
\hline & 2 & 410502.68673 & $0.524 \mathrm{~m}, \mathrm{a}$ & & & 11185.34000 & 0.308 \\
\hline & 3 & 4 & & 11195.81059 & $1.031 \mathrm{a}$ & 11221.59690 & 0.407 \\
\hline & 3 & 310633.97601 & $0.558 \mathrm{~m}, \mathrm{a}$ & & & 11236.58180 & 0.308 \\
\hline & 4 & 3 & & & & 11317.03820 & 0.506 \\
\hline & 4 & 210827.02657 & $0.455 \mathrm{~m}, \mathrm{a}$ & 11328.27210 & $0.505 \mathrm{~m}$ & 11318.54540 & 0.407 \\
\hline & 5 & 2 & & & & 11433.75210 & 0.505 \\
\hline & 5 & 111057.88191 & $1.191 \mathrm{a}$ & & & 11433.84300 & 0.506 \\
\hline & 6 & 111312.23069 & $1.08 \mathrm{~d}$ & 11663.23884 & $1.081 \mathrm{a}$ & 11575.15040 & 0.902 \\
\hline & 6 & 011312.23069 & $1.081 \mathrm{a}$ & 11663.23884 & $1.08 \mathrm{~d}$ & 11575.15470 & 0.505 \\
\hline & 0 & 7 10418.10133 & $0.703 \mathrm{~m}, \mathrm{a}$ & & & 11168.55350 & 0.505 \\
\hline & 1 & 710421.04385 & $0.475 \mathrm{~m}, \mathrm{a}$ & & & 11167.50520 & 0.405 \\
\hline 7 & 1 & 610582.77280 & $0.902 \mathrm{~m}$ & 11226.25978 & $1.051 \mathrm{a}$ & 11281.49970 & 0.406 \\
\hline 7 & 2 & 610616.12441 & $0.525 \mathrm{~m}, \mathrm{a}$ & & & 11283.14090 & 0.406 \\
\hline 7 & 2 & 510685.39120 & $0.902 \mathrm{~m}$ & & & 11355.19940 & 0.408 \\
\hline 7 & 3 & 510795.88208 & $0.742 \mathrm{a}$ & 11361.00380 & $0.505 \mathrm{~m}$ & 11381.23220 & 0.408 \\
\hline & 3 & 410809.31827 & $1.061 \mathrm{a}$ & 11386.13253 & $0.742 \mathrm{a}$ & 11410.56670 & 0.408 \\
\hline & 4 & & & & & 11480.49870 & 0.40 \\
\hline
\end{tabular}




\begin{tabular}{|c|c|c|c|c|c|c|c|}
\hline 7 & 4 & 3 & & 11499.42300 & $0.606 \mathrm{~m}$ & 11485.34690 & $0.506 \mathrm{~m}$ \\
\hline 7 & 5 & 3 & & & & 11597.34350 & $0.407 \mathrm{~m}$ \\
\hline 7 & 5 & 2 & & & & 11597.70810 & $0.505 \mathrm{~m}$ \\
\hline 7 & 6 & 211483.15112 & $1.101 \mathrm{a}$ & & & 11739.17330 & $0.406 \mathrm{~m}$ \\
\hline 7 & 6 & 111483.15112 & $1.10 \mathrm{~d}$ & & & 11739.17400 & $1.703 \mathrm{~m}$ \\
\hline 7 & 7 & 111752.27925 & $1.071 \mathrm{a}$ & 12035.01037 & $1.07 \mathrm{~d}$ & 11862.10450 & $0.603 \mathrm{~m}$ \\
\hline 7 & 7 & 011752.27925 & $1.07 \mathrm{~d}$ & 12035.01037 & $1.071 \mathrm{a}$ & 11862.10710 & $2.902 \mathrm{~m}$ \\
\hline 8 & 0 & 810571.95558 & $0.484 \mathrm{~m}, \mathrm{a}$ & & & 11317.25620 & $0.505 \mathrm{~m}$ \\
\hline 8 & 1 & 810573.60060 & $0.902 \mathrm{~m}$ & 11239.87759 & $1.061 \mathrm{a}$ & 11317.88020 & $0.503 \mathrm{~m}$ \\
\hline 8 & 1 & 710770.96395 & $0.694 \mathrm{~m}, \mathrm{a}$ & & & 11449.51340 & $0.406 \mathrm{~m}$ \\
\hline 8 & 2 & 710794.02320 & $0.902 \mathrm{~m}$ & 11407.05027 & $1.031 \mathrm{a}$ & 11450.21260 & $0.605 \mathrm{~m}$ \\
\hline 8 & 2 & 6 & & & & 11555.12220 & $0.606 \mathrm{e}$ \\
\hline 8 & 3 & 6 & & 11547.84270 & $0.406 \mathrm{~m}$ & 11561.14070 & $0.408 \mathrm{~m}$ \\
\hline 8 & 3 & 511012.13523 & $1.061 \mathrm{a}$ & & & 11609.56270 & $0.406 \mathrm{~m}$ \\
\hline 8 & 4 & 5 & & & & 11666.66060 & $0.507 \mathrm{~m}$ \\
\hline 8 & 4 & 411192.66532 & $0.762 \mathrm{a}$ & & & 11678.77693 & $0.378 \mathrm{~m}, \mathrm{a}$ \\
\hline 8 & 5 & 4 & & & & 11784.42520 & $0.504 \mathrm{~m}$ \\
\hline 8 & 5 & 3 & & & & 11785.71010 & $0.456 \mathrm{~m}, \mathrm{a}$ \\
\hline 8 & 6 & 3 & & & & 11926.46520 & $0.903 \mathrm{~m}$ \\
\hline 8 & 6 & 2 & & & & 11926.48510 & $0.604 \mathrm{~m}$ \\
\hline 8 & 7 & 2 & & & & 12052.36230 & $5.763 \mathrm{e}$ \\
\hline 8 & 7 & 1 & & & & 12052.36350 & $0.504 \mathrm{~m}$ \\
\hline 9 & 0 & 910742.80912 & $0.693 \mathrm{~m}, \mathrm{a}$ & & & 11484.61120 & $0.605 \mathrm{~m}$ \\
\hline 9 & 1 & 910743.65272 & $0.693 \mathrm{~m}, \mathrm{a}$ & & & 11484.48930 & $0.503 \mathrm{~m}$ \\
\hline 9 & 1 & 810976.01036 & $1.111 \mathrm{a}$ & 11596.70581 & $1.041 \mathrm{a}$ & 11634.98430 & $0.506 \mathrm{~m}$ \\
\hline 9 & 2 & 810991.10229 & $0.762 \mathrm{a}$ & & & 11634.94967 & $0.378 \mathrm{~m}, \mathrm{a}$ \\
\hline 9 & 2 & 7 & & 11732.01787 & $1.051 \mathrm{a}$ & 11755.76710 & $0.705 \mathrm{~m}$ \\
\hline
\end{tabular}




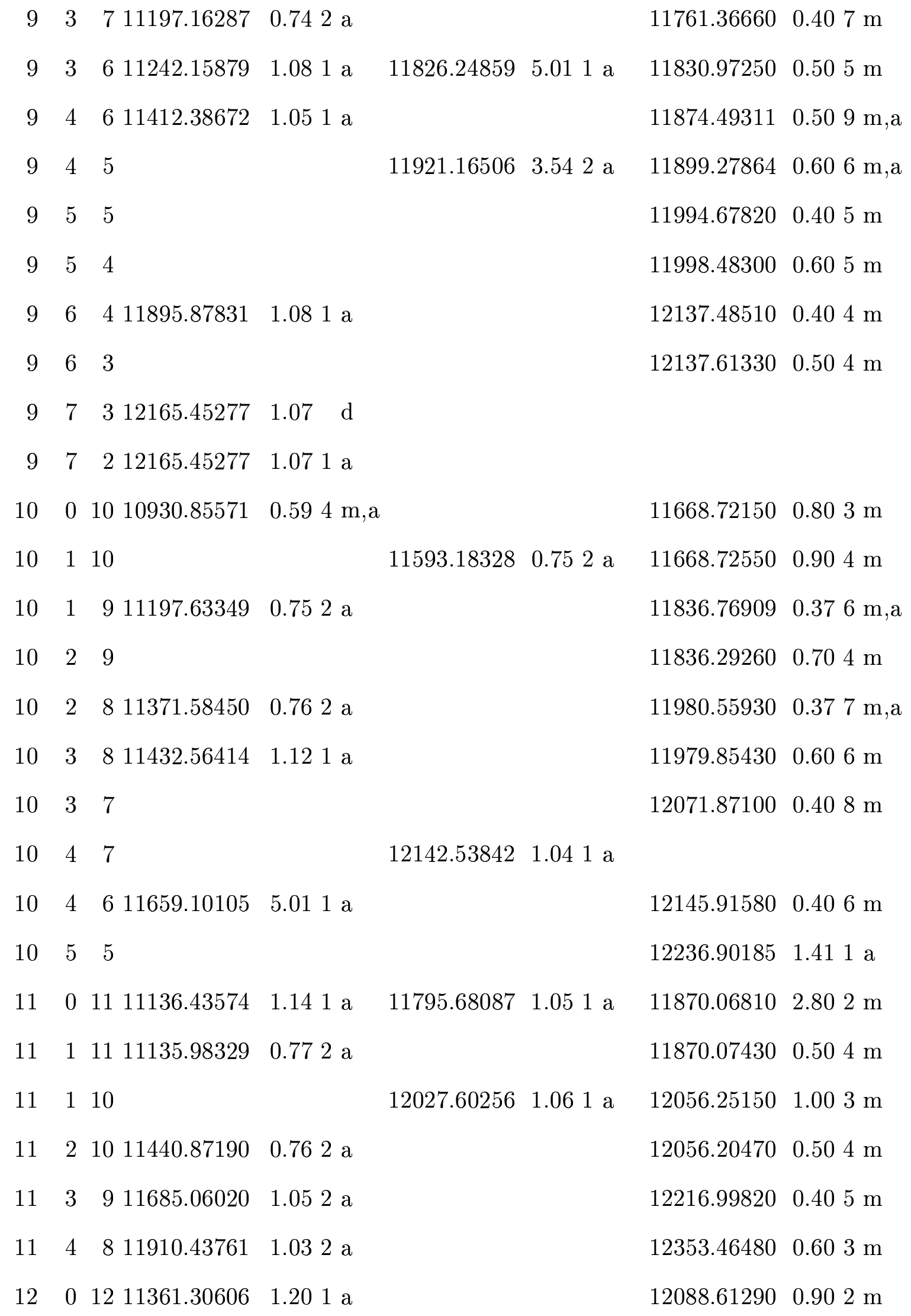




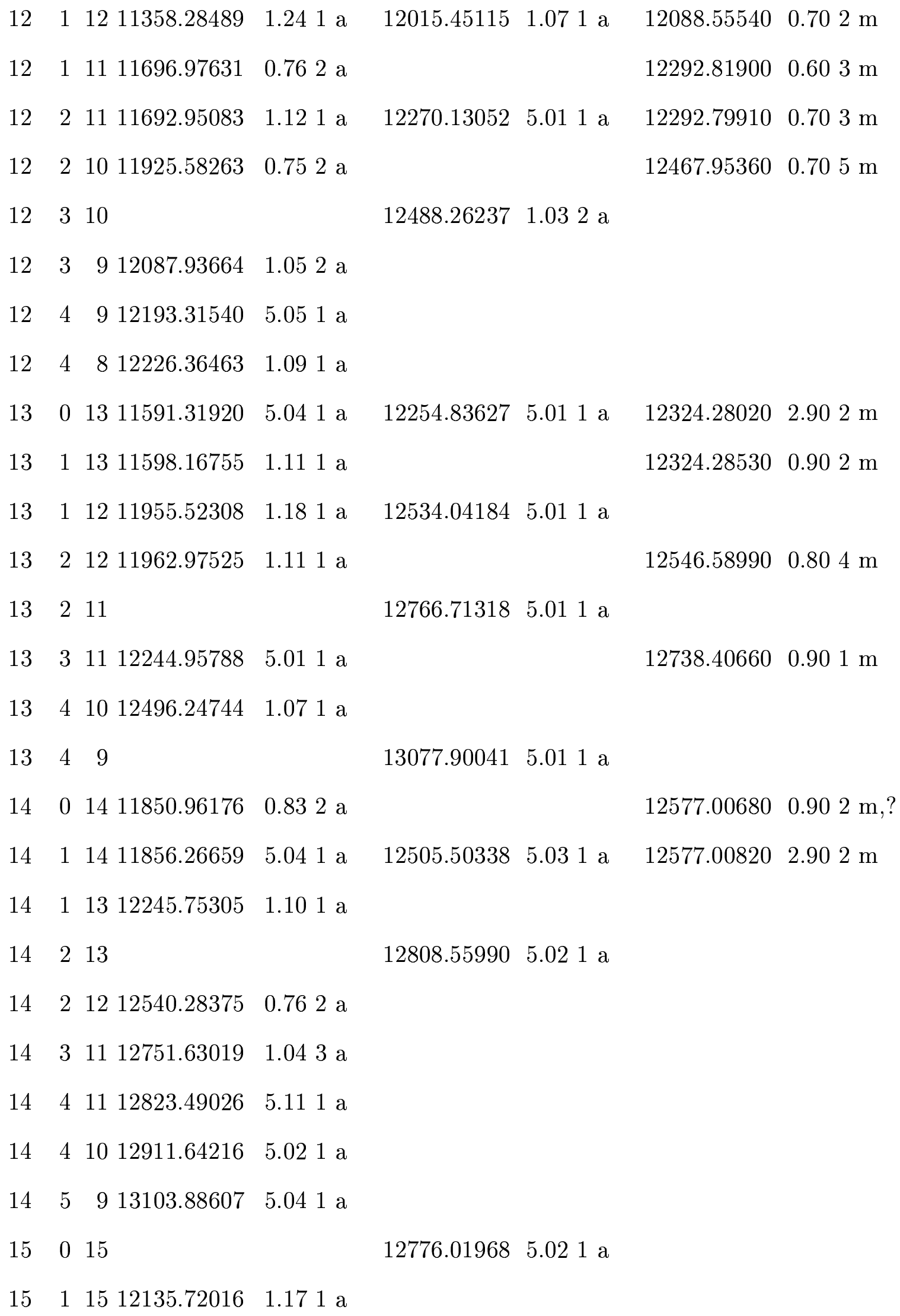




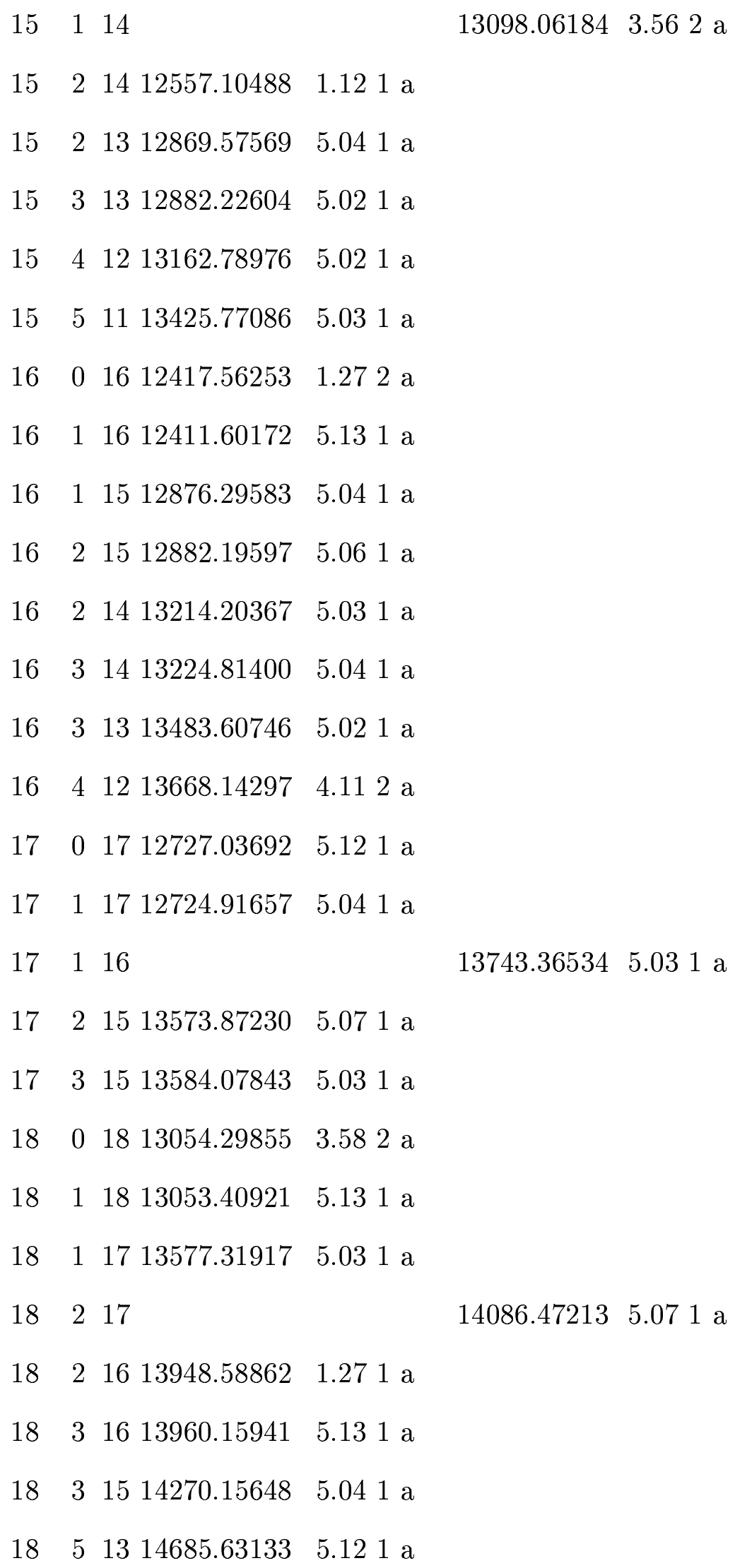




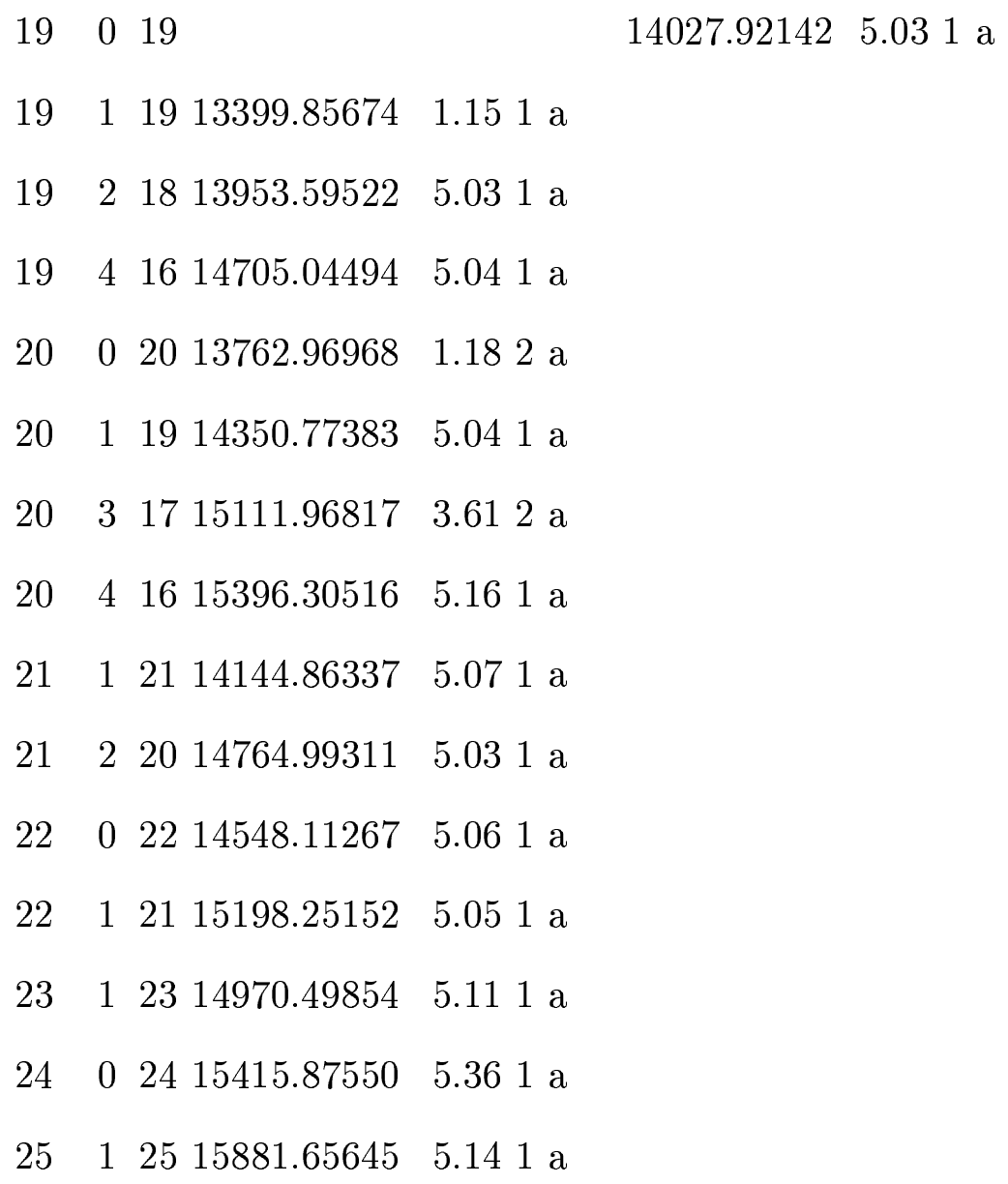

\begin{tabular}{lrrrrrrrr}
\hline$J K_{a}$ & $K_{c}$ & 220 & or & $20^{+} 2$ & 300 or $30^{+} 0$ & 102 or $21^{+} 0$ \\
\hline 0 & 0 & 0 & 10284.36700 & $2.901 \mathrm{~m}$ & 10599.68630 & $8.801 \mathrm{~m}$ & 10868.87570 & $0.901 \mathrm{~m}$ \\
1 & 0 & 1 & 10307.44770 & $0.602 \mathrm{~m}$ & 10622.41720 & $0.602 \mathrm{~m}$ & 10891.67580 & $0.602 \mathrm{~m}$ \\
1 & 1 & 1 & 10324.80550 & $0.803 \mathrm{~m}$ & 10633.55480 & $0.503 \mathrm{~m}$ & 10902.92800 & $0.503 \mathrm{~m}$ \\
1 & 1 & 0 & 10330.73090 & $0.902 \mathrm{~m}$ & 10639.07350 & $0.602 \mathrm{~m}$ & 10908.13480 & $0.602 \mathrm{~m}$ \\
2 & 0 & 2 & 10352.42360 & $0.602 \mathrm{~m}$ & 10666.47790 & $0.504 \mathrm{~m}$ & 10935.82030 & $0.602 \mathrm{~m}$ \\
2 & 1 & 2 & 10365.17500 & $0.604 \mathrm{~m}$ & 10673.87740 & $0.504 \mathrm{~m}$ & 10943.32350 & $0.404 \mathrm{~m}$ \\
2 & 1 & 1 & 10382.84910 & $0.503 \mathrm{~m}$ & 10689.61750 & $0.503 \mathrm{~m}$ & 10958.91890 & $0.603 \mathrm{~m}$ \\
2 & 2 & 1 & 10439.09930 & $0.503 \mathrm{~m}$ & 10724.20810 & $0.404 \mathrm{~m}$ & 10992.45370 & $0.404 \mathrm{~m}$ \\
2 & 2 & 0 & 10440.10560 & $0.602 \mathrm{~m}$ & 10725.48310 & $0.504 \mathrm{~m}$ & 10993.88060 & $0.504 \mathrm{~m}$ \\
3 & 0 & 3 & 10417.22700 & $0.602 \mathrm{~m}$ & 10729.62230 & $0.505 \mathrm{~m}$ & 10999.02170 & $0.603 \mathrm{~m}$ \\
3 & 1 & 3 & 10425.12390 & $0.604 \mathrm{~m}$ & 10733.55630 & $0.504 \mathrm{~m}$ & 11003.08090 & $0.504 \mathrm{~m}$
\end{tabular}




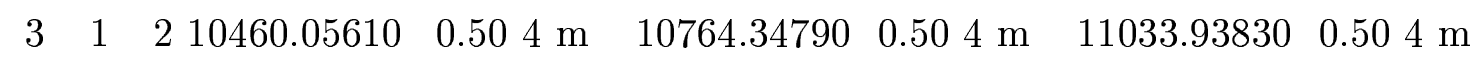

$\begin{array}{lllllllll}3 & 2 & 2 & 10509.53480 & 0.604 \mathrm{~m} & 10792.70220 & 0.902 \mathrm{~m} & 11060.84770 & 0.505 \mathrm{~m}\end{array}$

$\begin{array}{lllllllll}3 & 2 & 1 & 10513.74650 & 0.504 \mathrm{~m} & 10798.52720 & 0.405 \mathrm{~m} & 11067.34600 & 0.405 \mathrm{~m}\end{array}$

$\begin{array}{llllllllll}3 & 3 & 1 & 10612.24550 & 0.504 \mathrm{~m} & 10862.65440 & 0.505 \mathrm{~m} & 11129.71830 & 0.806 \mathrm{~m}\end{array}$

$\begin{array}{llllllllll}3 & 3 & 0 & 10612.38700 & 0.504 \mathrm{~m} & 10862.87290 & 0.505 \mathrm{~m} & 11129.98050 & 0.50 & 4 \mathrm{~m}\end{array}$

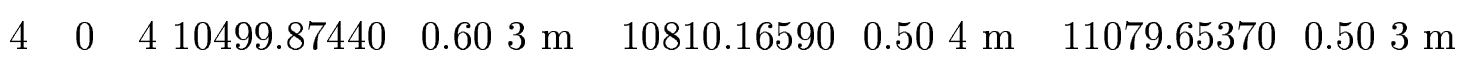

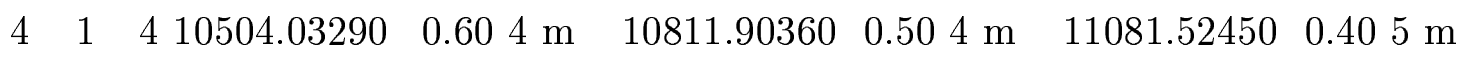

$\begin{array}{llllllllll}4 & 1 & 3 & 10561.03140 & 0.903 \mathrm{~m} & 10861.68800 & 0.605 \mathrm{~m} & 11131.52300 & 0.504 \mathrm{~m}\end{array}$

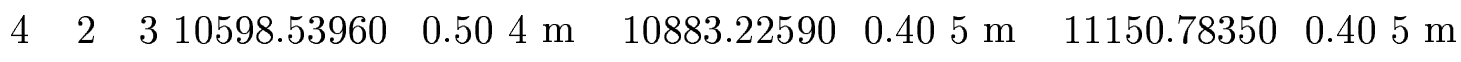

$\begin{array}{llllllllll}4 & 2 & 2 & 10614.40100 & 0.504 \mathrm{~m} & 10898.07710 & 0.504 \mathrm{~m} & 11167.49800 & 0.505 \mathrm{~m}\end{array}$ $\begin{array}{llllllll}4 & 3 & 2 & 10955.97340 & 0.407 \mathrm{~m} & 11223.08670 & 0.406 \mathrm{~m}\end{array}$ $\begin{array}{llllllll}4 & 3 & 1 & 10957.42210 & 0.504 \mathrm{~m} & 11224.82980 & 0.505 \mathrm{~m}\end{array}$ $\begin{array}{lllllllll}4 & 4 & 1 & 11048.38330 & 0.506 \mathrm{~m} & 11314.99390 & 0.405 \mathrm{~m}\end{array}$ $\begin{array}{llllllll}4 & 4 & 0 & 11048.41280 & 0.903 \mathrm{~m} & 11314.99060 & 0.505 \mathrm{~m}\end{array}$

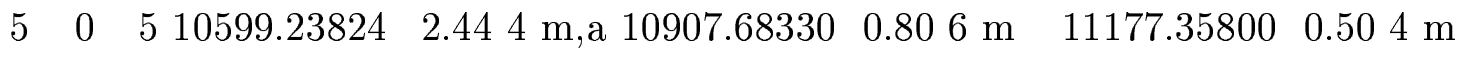

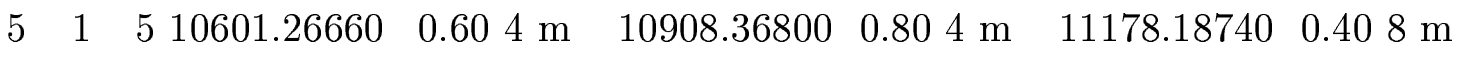
$\begin{array}{llllllll}5 & 1 & 4 & 10979.34040 & 0.505 \mathrm{~m} & 11249.28830 & 0.406 \mathrm{~m}\end{array}$

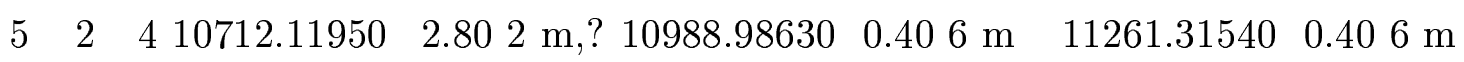
$\begin{array}{llllllllll}5 & 2 & 3 & 10742.04990 & 0.40 & 5 \mathrm{~m} & 11023.39860 & 0.405 \mathrm{~m} & 11293.29660 & 0.406 \mathrm{~m}\end{array}$ $\begin{array}{llllllllll}5 & 3 & 3 & 10824.97460 & 0.604 \mathrm{~m} & 11072.43940 & 0.406 \mathrm{~m} & 11338.71470 & 0.407 \mathrm{~m}\end{array}$ $\begin{array}{lllllllll}5 & 3 & 2 & 11077.67010 & 0.605 \mathrm{~m} & 11345.72340 & 0.40 & 5 \mathrm{~m}\end{array}$ $\begin{array}{llllllll}5 & 4 & 2 & 11165.48540 & 0.607 \mathrm{~m} & 11432.32240 & 0.506 \mathrm{~m}\end{array}$ $\begin{array}{llllllll}5 & 4 & 1 & 11165.71980 & 0.505 \mathrm{~m} & 11432.37530 & 0.406 \mathrm{~m}\end{array}$ $\begin{array}{llllllll}5 & 5 & 1 & 11283.56540 & 0.904 \mathrm{~m} & 11546.93690 & 0.605 \mathrm{~m}\end{array}$ $\begin{array}{llllllll}5 & 5 & 0 & 11283.57680 & 0.505 \mathrm{~m} & 11546.92690 & 0.605 \mathrm{~m}\end{array}$ $\begin{array}{lllllllll}6 & 0 & 6 & 10715.43480 & 0.603 \mathrm{~m} & 11022.34850 & 0.504 \mathrm{~m} & 11292.10460 & 0.603 \mathrm{~m}\end{array}$

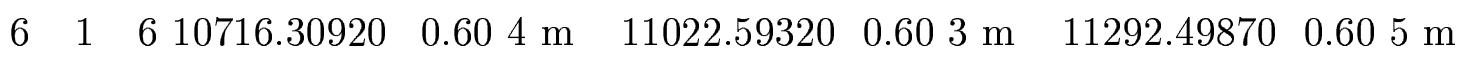




\begin{tabular}{|c|c|c|c|c|c|c|c|}
\hline 6 & 1 & 5 & & 11114.90090 & $2.104 \mathrm{~m}$ & 11384.88280 & $0.503 \mathrm{~m}$ \\
\hline 6 & 2 & 5 & & 11120.51550 & $0.405 \mathrm{~m}$ & 11391.47680 & $0.405 \mathrm{~m}$ \\
\hline 6 & 2 & 4 & & 11172.75430 & $0.405 \mathrm{~m}$ & 11442.95390 & $0.407 \mathrm{~m}$ \\
\hline 6 & 3 & 4 & & 11211.50715 & $0.377 \mathrm{~m}, \mathrm{a}$ & 11478.69340 & $0.406 \mathrm{~m}$ \\
\hline 6 & 3 & 3 & & 11224.77930 & $0.605 \mathrm{~m}$ & 11493.85270 & $0.406 \mathrm{~m}$ \\
\hline 6 & 4 & 3 & & 11306.10480 & $0.407 \mathrm{~m}$ & 11573.95920 & $0.408 \mathrm{~m}$ \\
\hline 6 & 4 & 2 & & 11307.40560 & $0.506 \mathrm{~m}$ & 11574.72200 & $0.507 \mathrm{~m}$ \\
\hline 6 & 5 & 2 & & 11423.92980 & $0.405 \mathrm{~m}$ & 11687.27720 & $0.404 \mathrm{~m}$ \\
\hline 6 & 5 & 1 & & 11424.03170 & $0.604 \mathrm{~m}$ & 11687.20200 & $0.506 \mathrm{~m}$ \\
\hline 6 & 6 & 1 & & 11565.66770 & $0.407 \mathrm{~m}$ & 11822.32080 & $0.604 \mathrm{~m}$ \\
\hline 6 & 6 & 0 & & 11565.66390 & $2.902 \mathrm{~m}$ & 11822.30160 & $8.402 \mathrm{~m}$ \\
\hline 7 & 0 & 710848.45330 & $0.903 \mathrm{~m}$ & 11154.20663 & $0.525 \mathrm{~m}, \mathrm{a}$ & 11424.33600 & $0.902 \mathrm{~m}$ \\
\hline 7 & 1 & 710848.86620 & $0.903 \mathrm{~m}$ & 11154.39830 & $0.504 \mathrm{~m}$ & 11424.53180 & $0.604 \mathrm{~m}$ \\
\hline 7 & 1 & 610985.87060 & $0.504 \mathrm{~m}$ & 11266.98390 & $0.405 \mathrm{~m}$ & 11537.08940 & $0.405 \mathrm{~m}$ \\
\hline 7 & 2 & 6 & & 11268.69430 & $0.506 \mathrm{~m}$ & 11540.44410 & $0.504 \mathrm{~m}$ \\
\hline 7 & 2 & 5 & & 11343.82231 & $0.407 \mathrm{~m}, \mathrm{a}$ & 11615.22410 & $0.408 \mathrm{~m}$ \\
\hline 7 & 3 & 511131.04010 & $0.604 \mathrm{~m}$ & 11372.56720 & $0.405 \mathrm{~m}$ & 11639.41410 & $0.504 \mathrm{~m}$ \\
\hline 7 & 3 & 4 & & 11398.86560 & $0.507 \mathrm{~m}$ & 11668.44730 & $0.406 \mathrm{~m}$ \\
\hline 7 & 4 & 4 & & 11470.16130 & $0.505 \mathrm{~m}$ & & \\
\hline 7 & 4 & 3 & & 11474.05620 & $0.505 \mathrm{~m}$ & 11742.67740 & $0.408 \mathrm{~m}$ \\
\hline 7 & 5 & 3 & & 11587.72050 & $0.706 \mathrm{~m}$ & 11852.31920 & $0.505 \mathrm{~m}$ \\
\hline 7 & 5 & 2 & & 11588.15430 & $0.606 \mathrm{~m}$ & 11852.16350 & $0.507 \mathrm{~m}$ \\
\hline 7 & 6 & 2 & & & & 11986.98050 & $0.703 \mathrm{~m}, ?$ \\
\hline 8 & 0 & 8 & & 11303.52275 & $0.605 \mathrm{~m}, \mathrm{a}$ & 11574.16520 & $0.804 \mathrm{~m}$ \\
\hline 8 & 1 & 8 & & 11302.92449 & $0.456 \mathrm{~m}, \mathrm{a}$ & 11574.36450 & $0.504 \mathrm{~m}$ \\
\hline 8 & 1 & 7 & & 11435.46260 & $0.704 \mathrm{~m}$ & 11705.86460 & $0.504 \mathrm{~m}$ \\
\hline 8 & 2 & 7 & & 11436.44716 & $0.456 \mathrm{~m}, \mathrm{a}$ & 11707.69960 & $0.504 \mathrm{~m}$ \\
\hline
\end{tabular}




\begin{tabular}{|c|c|c|c|c|c|c|c|}
\hline 8 & 2 & 6 & & 11533.97280 & $0.704 \mathrm{~m}$ & 11805.16580 & $0.504 \mathrm{~m}$ \\
\hline 8 & 3 & 611310.07600 & $0.903 \mathrm{~m}$ & 11542.18640 & $0.506 \mathrm{~m}$ & 11820.41810 & $0.404 \mathrm{~m}$ \\
\hline 8 & 3 & 5 & & 11599.22710 & $0.605 \mathrm{~m}$ & 11865.21120 & $0.504 \mathrm{~m}$ \\
\hline 8 & 4 & 5 & & 11655.84540 & $0.407 \mathrm{~m}$ & & \\
\hline 8 & 4 & 4 & & 11666.90370 & $0.506 \mathrm{~m}$ & & \\
\hline 8 & 5 & 4 & & 11774.82890 & $0.406 \mathrm{~m}$ & 12038.16990 & $0.406 \mathrm{~m}$ \\
\hline 8 & 6 & 3 & & 11915.39680 & $0.407 \mathrm{~m}$ & & \\
\hline 8 & 7 & 2 & & 12042.61460 & $0.508 \mathrm{~m}$ & & \\
\hline 9 & 0 & 9 & & 11469.83407 & $0.524 \mathrm{~m}, \mathrm{a}$ & 11743.61630 & $0.404 \mathrm{~m}$ \\
\hline 9 & 1 & 9 & & 11469.91880 & $0.704 \mathrm{~m}$ & 11743.69700 & $2.901 \mathrm{~m}$ \\
\hline 9 & 1 & 8 & & 11620.74440 & $0.504 \mathrm{~m}$ & 11891.75540 & $0.405 \mathrm{~m}$ \\
\hline 9 & 2 & 7 & & 11739.05250 & $0.804 \mathrm{~m}$ & 12012.05680 & $0.407 \mathrm{~m}$ \\
\hline 9 & 3 & 6 & & 11815.13783 & $0.405 \mathrm{~m}, \mathrm{a}$ & & \\
\hline 9 & 4 & 5 & & 11886.57740 & $0.604 \mathrm{~m}$ & & \\
\hline 9 & 5 & 4 & & 11988.67180 & $0.405 \mathrm{~m}$ & & \\
\hline 10 & 0 & 10 & & 11653.77033 & $1.09 \mathrm{~d}$ & & \\
\hline 10 & 1 & 10 & & 11653.77033 & $1.091 \mathrm{a}$ & & \\
\hline 10 & 2 & 9 & & 11822.60990 & $0.504 \mathrm{~m}$ & & \\
\hline 10 & 3 & 8 & & 11963.35251 & $1.012 \mathrm{a}$ & 12241.30770 & $0.506 \mathrm{~m}$ \\
\hline
\end{tabular}


TABLE VIII.: Term values for the $3 \nu+\delta$ polyad of $\mathrm{H}_{2}{ }^{16} \mathrm{O}$.

\begin{tabular}{|c|c|c|c|c|c|c|c|c|c|c|}
\hline \multicolumn{3}{|c|}{$J K_{a} K_{c}$} & \multirow[t]{2}{*}{070 or } & \multirow[t]{2}{*}{007} & \multicolumn{3}{|c|}{230 or $20^{+} 3$} & \multicolumn{3}{|c|}{032 or 113} \\
\hline 0 & 0 & 0 & & & 11767.39000 & 3.00 & $1 \mathrm{n}$ & 12007.77570 & 0.10 & $1 \mathrm{n}$ \\
\hline 1 & 0 & 1 & & & 11790.43340 & 0.50 & $2 \mathrm{n}$ & 12031.20530 & 3.70 & $2 \mathrm{n}$ \\
\hline 1 & 1 & 1 & & & 11814.73300 & 1.80 & $2 \mathrm{n}$ & 12052.65590 & 2.30 & $2 \mathrm{n}$ \\
\hline 1 & 1 & & 10341.05470 & 1.0021 & 11820.83770 & 0.10 & $2 \mathrm{n}$ & 12058.93590 & 0.80 & $2 \mathrm{n}$ \\
\hline 2 & 0 & 2 & & & 11835.48290 & 0.50 & $2 \mathrm{n}$ & 12076.84320 & 0.10 & $2 \mathrm{n}$ \\
\hline 2 & 1 & & 10374.89190 & 1.8031 & 11854.76990 & 0.60 & $3 \mathrm{n}$ & 12093.30450 & 1.30 & $3 n$ \\
\hline 2 & 1 & 1 & . & & 11873.03610 & 0.70 & $3 \mathrm{n}$ & 12112.03690 & 0.50 & $2 \mathrm{n}$ \\
\hline 2 & 2 & 1 & 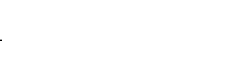 & & 11943.11780 & 0.60 & $3 n$ & 12174.69180 & 3.10 & $3 n$ \\
\hline 2 & 2 & 0 & 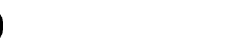 & & 11944.09960 & 3.00 & $3 \mathrm{n}$ & 12175.86140 & 0.90 & $2 \mathrm{n}$ \\
\hline 3 & 0 & 3 & 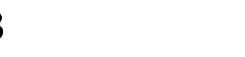 & & 11900.65010 & 0.30 & $3 \mathrm{n}$ & 12142.52910 & 1.20 & $3 n$ \\
\hline 3 & 1 & 3 & 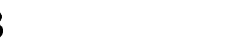 & & 11914.21290 & 1.80 & $3 \mathrm{n}$ & 12153.55020 & 0.10 & $1 \mathrm{n}$ \\
\hline 3 & 1 & 2 & 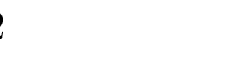 & & 11950.49840 & 0.60 & $3 \mathrm{n}$ & 12190.72690 & 1.30 & $3 n$ \\
\hline 3 & 2 & 2 & 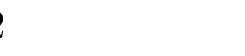 & & 12012.61360 & 1.20 & $3 \mathrm{n}$ & 12245.13390 & 0.10 & $1 \mathrm{n}$ \\
\hline 3 & 2 & 1 & & & 12017.27410 & 0.80 & $5 \mathrm{n}$ & 12250.71220 & 1.30 & $3 \mathrm{n}$ \\
\hline 3 & 3 & 1 & & & 12138.71000 & 3.00 & $1 \mathrm{n}$ & 12360.82990 & 0.10 & $2 \mathrm{n}$ \\
\hline 3 & 3 & 0 & & & 12138.84710 & 1.30 & $3 \mathrm{n}$ & 12360.97060 & 1.70 & $2 \mathrm{n}$ \\
\hline 4 & 0 & 4 & $t$ & & 11983.90200 & 0.80 & $2 \mathrm{n}$ & 12226.15630 & 1.80 & $2 \mathrm{n}$ \\
\hline 4 & 1 & 4 & 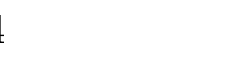 & & 11992.47220 & 1.20 & $3 \mathrm{n}$ & 12231.63830 & 4.00 & $2 n$ \\
\hline 4 & 1 & 3 & & & 12052.14290 & 3.00 & $1 \mathrm{n}$ & 12293.67730 & 0.10 & $1 \mathrm{n}$ \\
\hline 4 & 2 & 3 & & & 12104.80390 & 3.10 & $4 \mathrm{n}$ & 12337.94970 & 0.10 & $1 \mathrm{n}$ \\
\hline 4 & 2 & 2 & 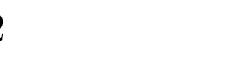 & & 12117.31670 & 2.00 & $3 \mathrm{n}$ & 12353.13350 & 0.40 & $2 n$ \\
\hline 4 & 3 & 2 & 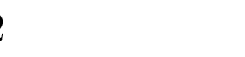 & & 12234.29850 & 1.90 & $2 \mathrm{n}$ & 12457.05840 & 0.40 & $2 \mathrm{n}$ \\
\hline 4 & 3 & 1 & & & 12233.96180 & 3.00 & $1 \mathrm{n}$ & 12458.03570 & 0.10 & $2 n$ \\
\hline
\end{tabular}




\begin{tabular}{|c|c|c|c|c|c|c|c|c|}
\hline 4 & 4 & 1 & 12394.06340 & 3.00 & $1 \mathrm{n}$ & 12607.29530 & 0.10 & $2 \mathrm{n}$ \\
\hline 4 & 4 & 0 & 12394.08480 & 3.00 & $1 \mathrm{n}$ & 12607.30550 & 0.10 & $1 \mathrm{n}$ \\
\hline 5 & 0 & 5 & 12083.94200 & 0.50 & $2 \mathrm{n}$ & 12326.57160 & 0.50 & $2 \mathrm{n}$ \\
\hline 5 & 1 & 5 & 12088.98310 & 0.20 & $2 \mathrm{n}$ & 12329.92780 & 0.10 & $1 \mathrm{n}$ \\
\hline 5 & 1 & 4 & 12176.28090 & 0.20 & $3 \mathrm{n}$ & 12418.98440 & 0.50 & $3 n$ \\
\hline 5 & 2 & 4 & & & & 12452.25920 & 5.00 & $3 \mathrm{n}$ \\
\hline 5 & 2 & 3 & 12244.56430 & 0.80 & $4 \mathrm{n}$ & 12483.00730 & 1.80 & $2 \mathrm{n}$ \\
\hline 5 & 3 & 3 & & & & 12577.12930 & 0.10 & $1 \mathrm{n}$ \\
\hline 5 & 3 & 2 & & & & 12580.70830 & 0.10 & $1 \mathrm{n}$ \\
\hline 5 & 4 & 2 & & & & 12726.45380 & 0.10 & $1 \mathrm{n}$ \\
\hline 5 & 4 & 1 & & & & 12726.61510 & 0.60 & $3 \mathrm{n}$ \\
\hline 6 & 0 & 6 & 12200.38060 & 1.80 & $2 \mathrm{n}$ & 12443.61420 & 0.10 & $1 \mathrm{n}$ \\
\hline 6 & 1 & 6 & 12203.30530 & 0.10 & $2 \mathrm{n}$ & 12446.28130 & 1.60 & $3 n$ \\
\hline 6 & 2 & 5 & 12350.47710 & 0.70 & $2 \mathrm{n}$ & & & \\
\hline 6 & 4 & 3 & & & & 12869.59340 & 0.30 & $3 \mathrm{n}$ \\
\hline 6 & 4 & 2 & & & & 12870.19300 & 2.60 & $3 \mathrm{n}$ \\
\hline 7 & 0 & 7 & 12333.34500 & 0.10 & $2 \mathrm{n}$ & & & \\
\hline 7 & 1 & 7 & & & & 12577.83840 & 0.10 & $1 \mathrm{n}$ \\
\hline 7 & 1 & 6 & 12483.84230 & 0.50 & $2 \mathrm{n}$ & & & \\
\hline 7 & 2 & 5 & 12573.66350 & 3.00 & $1 \mathrm{n}$ & & & \\
\hline 7 & 4 & 4 & 12826.39160 & 1.70 & $4 \mathrm{n}$ & & & \\
\hline 7 & 4 & 3 & & & & 13038.30290 & 0.10 & $2 \mathrm{n}$ \\
\hline 8 & 1 & $\begin{array}{llll}8 & 11039.20050 & 0.80 & 3\end{array}$ & & & & & & \\
\hline 8 & 2 & 7 & & & & 12911.25440 & 0.80 & $2 \mathrm{n}$ \\
\hline 8 & 3 & 6 & 12844.61560 & 0.10 & $2 \mathrm{n}$ & & & \\
\hline 8 & 4 & 5 & & & & 13226.49100 & 3.60 & $3 n$ \\
\hline & $K_{a} I$ & 131 or $10^{-} 3$ & 310 or & $30^{+} 1$ & & 112 or & $21^{+} 1$ & \\
\hline
\end{tabular}




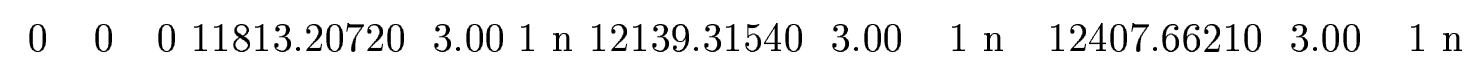

$\begin{array}{llllllllllll}1 & 0 & 1 & 11836.45590 & 0.10 & 2 \mathrm{n} & 12162.10650 & 0.10 & 2 \mathrm{n} & 12430.52290 & 0.40 & 2 \mathrm{n}\end{array}$

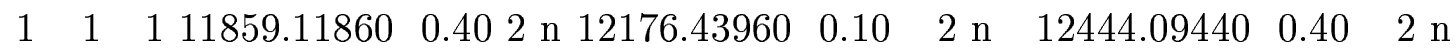

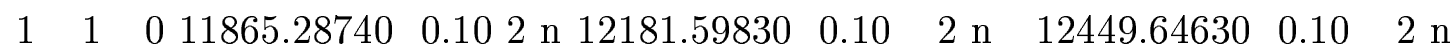

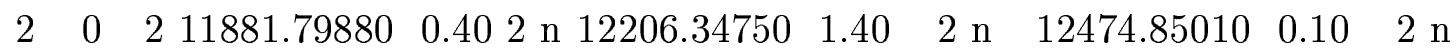

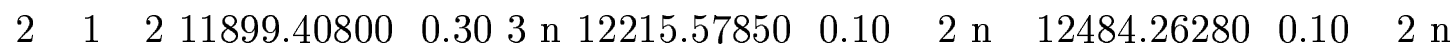

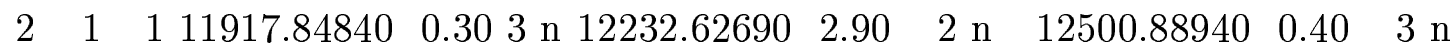

$\begin{array}{llllllllllll}2 & 2 & 1 & 11983.97090 & 0.50 & 2 \mathrm{n} & 12274.40360 & 1.90 & 2 \mathrm{n} & 12541.68490 & 0.10 & 4 \mathrm{n}\end{array}$

$\begin{array}{llllllllllll}2 & 2 & 0 & 11985.06540 & 0.20 & 2 \mathrm{n} & 12275.67610 & 3.00 & 1 \mathrm{n} & 12543.05160 & 0.30 & 3 \mathrm{n}\end{array}$

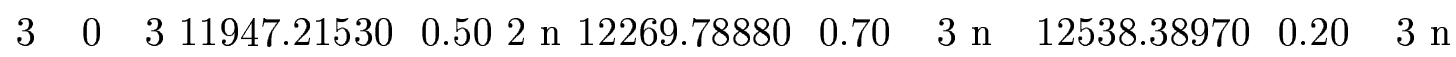

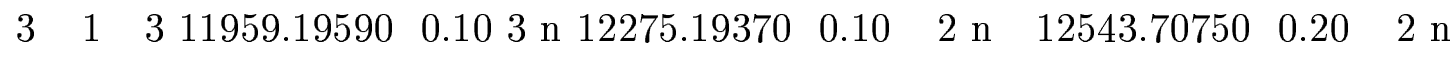

$\begin{array}{llllllllllll}3 & 1 & 2 & 11995.73170 & 0.50 & 2 \mathrm{n} & 12308.11700 & 0.30 & 2 \mathrm{n} & 12576.65200 & 0.10 & 4 \mathrm{n}\end{array}$

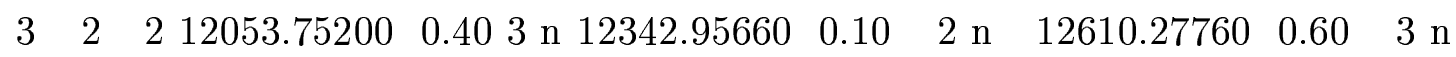

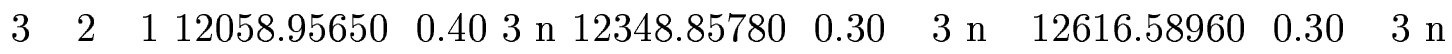

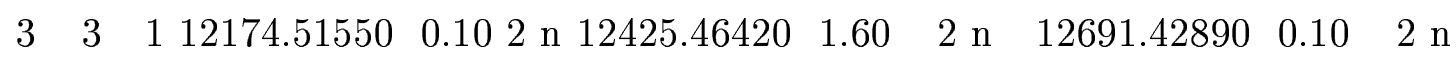

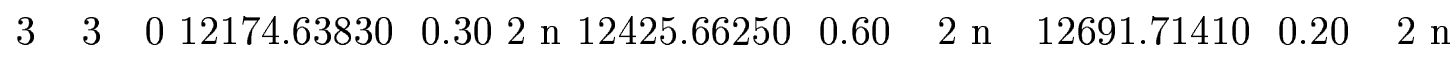

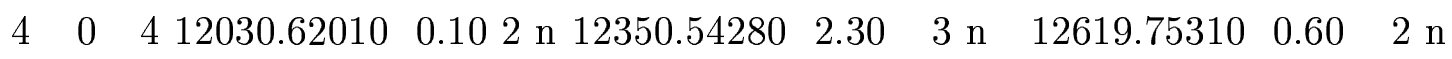

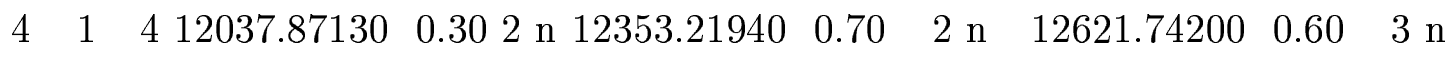

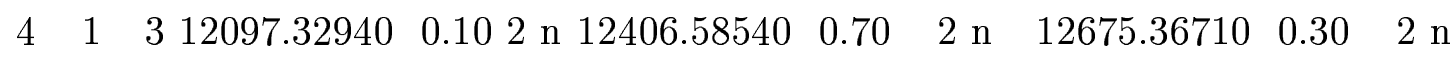

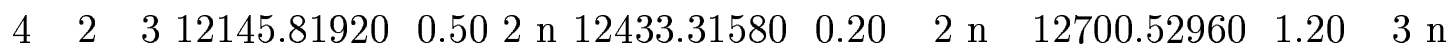

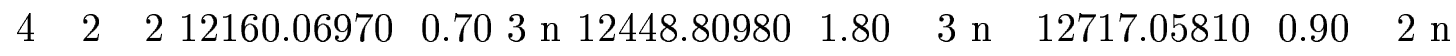

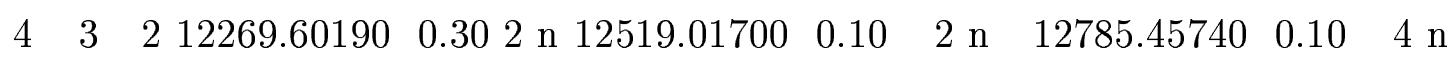

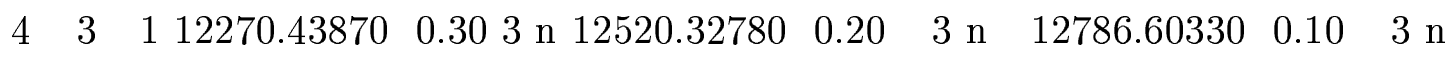

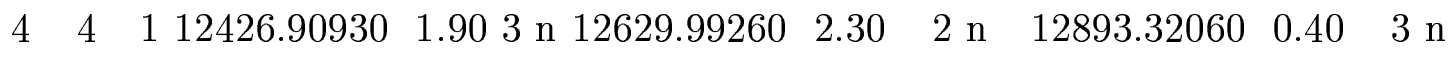

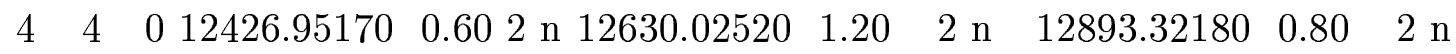

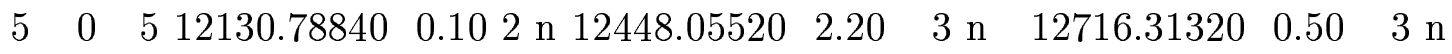

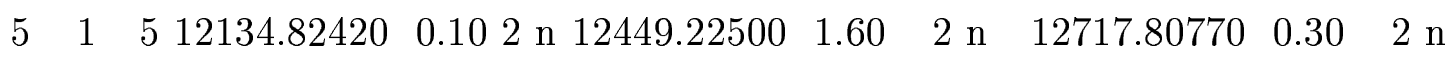




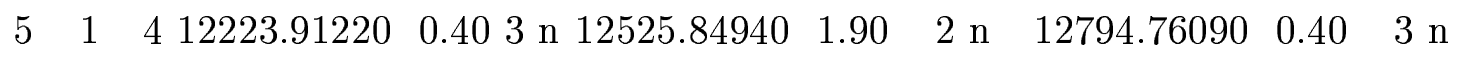

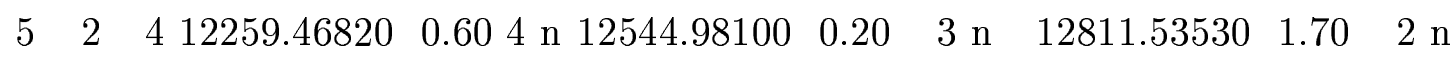

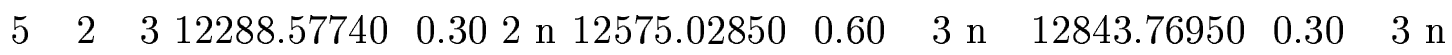

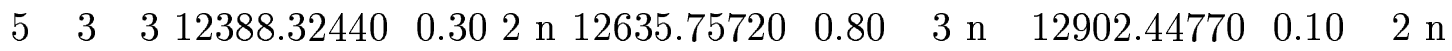

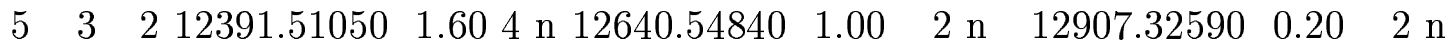

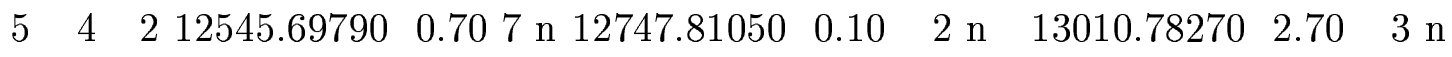

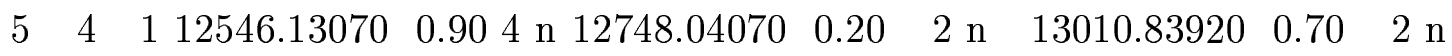

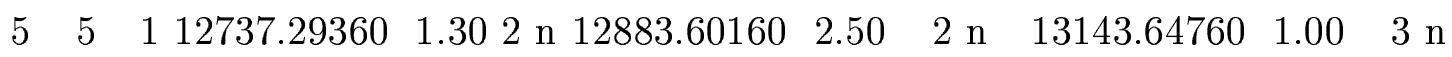
$\begin{array}{llllllllllll}5 & 5 & 0 & 12737.18490 & 1.00 & 2 \mathrm{n} & 12883.63530 & 0.60 & 2 \mathrm{n} & 13143.56620 & 0.30 & 4 \mathrm{n}\end{array}$

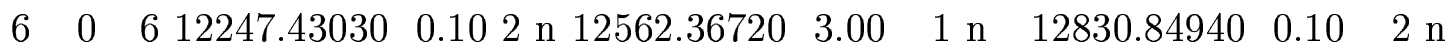

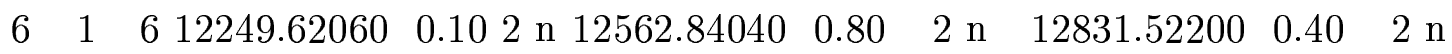
$\begin{array}{llllllllllll}6 & 1 & 5 & 12368.20490 & 0.10 & 2 \mathrm{n} & 12663.43580 & 3.00 & 1 \mathrm{n} & 12932.36990 & 0.40 & 2 \mathrm{n}\end{array}$

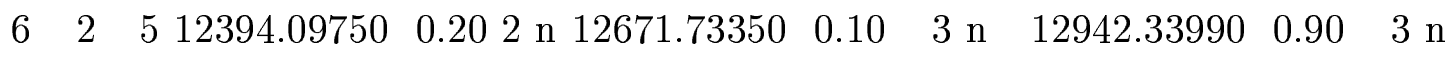

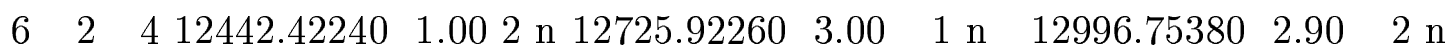

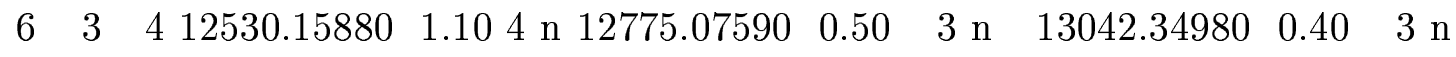

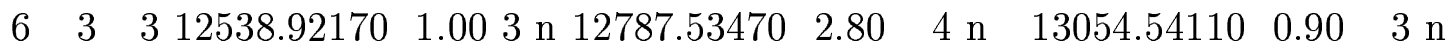

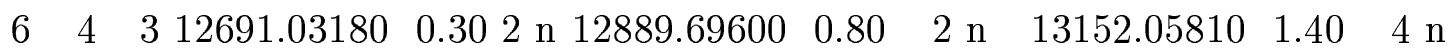

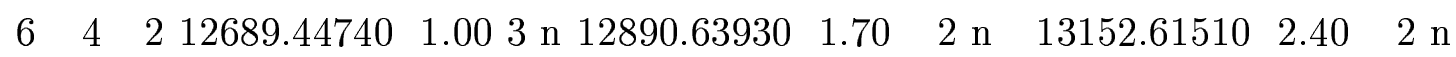

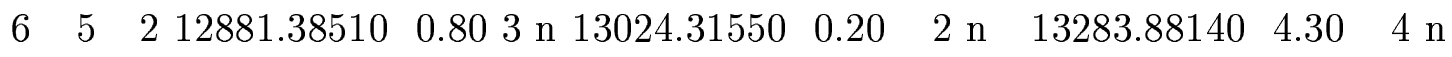

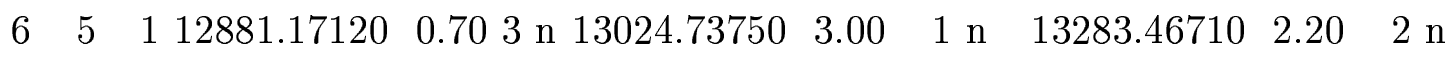
$\begin{array}{lllllllll}6 & 6 & 1 & 13095.68950 & 3.00 & 1 \mathrm{n} & 13450.41870 & 3.70 & 2 \mathrm{n}\end{array}$ $\begin{array}{lllllllll}6 & 6 & 0 & 13095.68910 & 3.00 & 1 \mathrm{n} & 13450.41910 & 3.00 & 1 \mathrm{n}\end{array}$

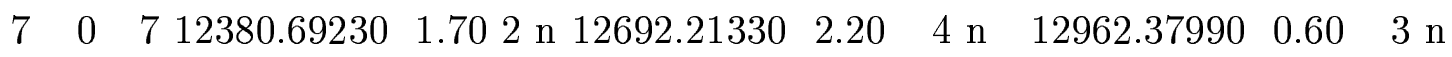

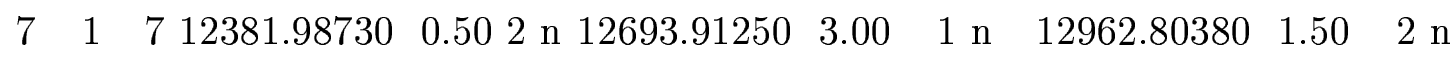
$\begin{array}{llllllllllll}7 & 1 & 6 & 12530.97140 & 0.90 & 2 \mathrm{n} & 12817.63870 & 1.70 & 4 \mathrm{n} & 13086.60060 & 0.90 & 3 \mathrm{n}\end{array}$

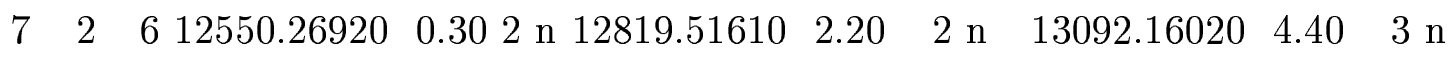

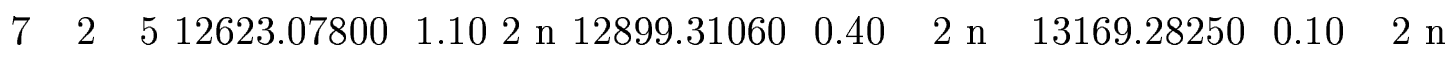




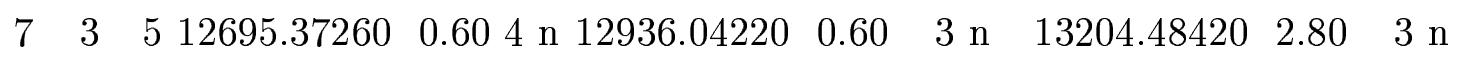

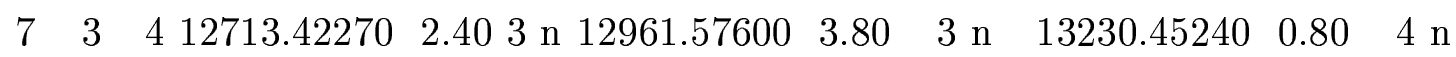

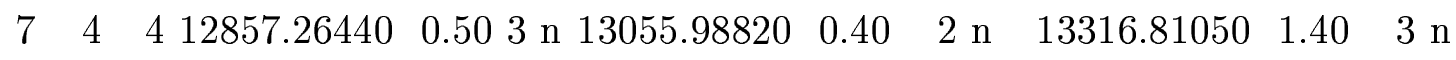

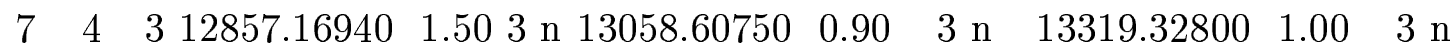

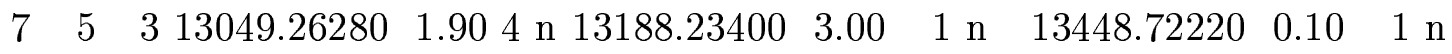

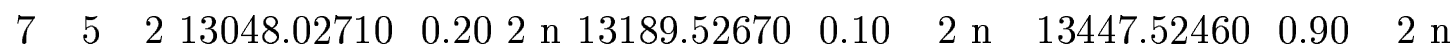
$\begin{array}{llllll}7 & 6 & 2 & 13263.90120 & 2.20 & 4 \mathrm{n}\end{array}$

$\begin{array}{llllll}7 & 6 & 1 & 13263.89640 & 3.00 & 1 \mathrm{n}\end{array}$

$\begin{array}{llllll}7 & 7 & 1 & 13492.33030 & 0.30 & 2 \mathrm{n}\end{array}$

$\begin{array}{llllll}7 & 7 & 0 & 13492.33030 & 0.30 & \mathrm{~d}\end{array}$

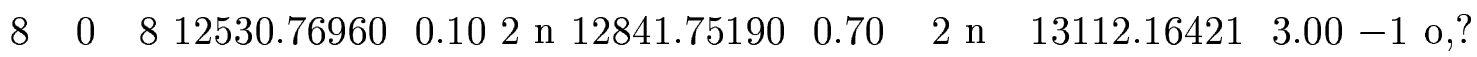

$\begin{array}{lllllllllllll}8 & 1 & 8 & 12532.05840 & 0.20 & 2 \mathrm{n} & 12842.03321 & 0.60 & -1 & \mathrm{o}, ? & 13109.65280 & 0.40 & 2 \mathrm{n}\end{array}$ $\begin{array}{llllllllllll}8 & 1 & 7 & 12710.47460 & 0.10 & 2 \mathrm{n} & 12987.91900 & 5.50 & 2 \mathrm{n} & 13257.36810 & 2.80 & 2 \mathrm{n}\end{array}$

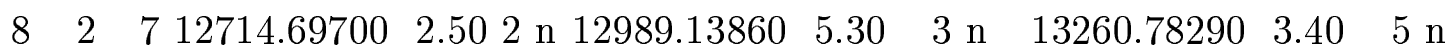

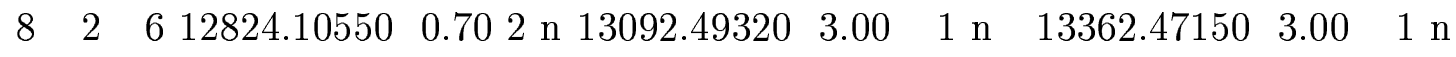

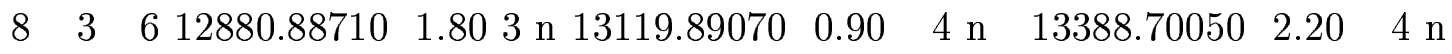
$\begin{array}{lllllllll}8 & 3 & 5 & 13161.54520 & 3.00 & 1 \mathrm{n} & 13435.52350 & 1.70 & 2 \mathrm{n}\end{array}$

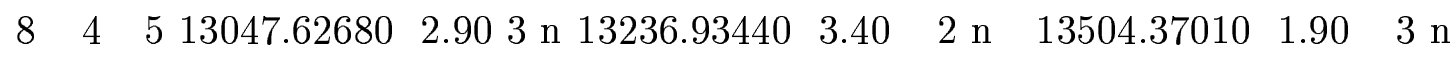
$\begin{array}{lllllllll}8 & 4 & 4 & 13049.64070 & 0.80 & 5 \mathrm{n} & 13252.60760 & 1.80 & 3 \mathrm{n}\end{array}$ $\begin{array}{llllll}8 & 5 & 4 & 13374.04270 & 1.10 & 2 \mathrm{n}\end{array}$ $\begin{array}{llllll}8 & 5 & 3 & 13378.16150 & 3.00 & 1 \mathrm{n}\end{array}$

$\begin{array}{llllll}8 & 6 & 3 & 13455.60890 & 3.001 \mathrm{n}\end{array}$ $\begin{array}{llllll}8 & 6 & 2 & 13455.61290 & 0.70 & 3 \mathrm{n}\end{array}$

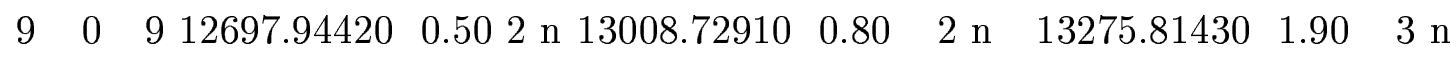
$\begin{array}{lllllllllll}9 & 1 & 9 & 12689.00460 & 1.90 & 2 \mathrm{n} & 13006.37360 & 0.20 & 2 \mathrm{n}\end{array}$ $\begin{array}{lll}9 & 1 & 8\end{array}$ $13443.24200 \quad 1.80 \quad 2 \mathrm{n}$ $\begin{array}{llllll}9 & 2 & 8 & 12907.78530 & 0.50 & 3 \mathrm{n}\end{array}$ 


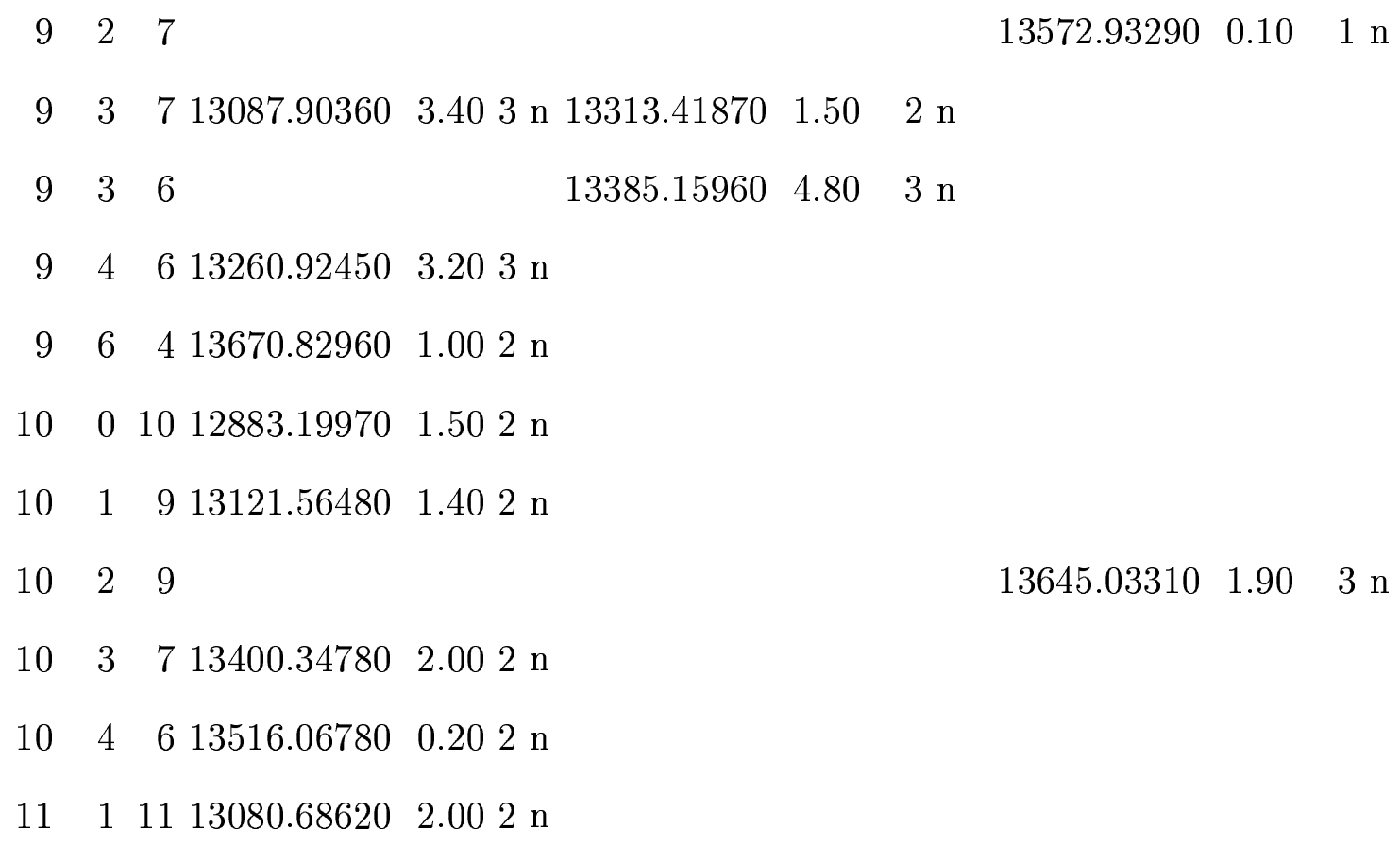

\begin{tabular}{|c|c|c|c|c|c|c|c|c|c|}
\hline \multicolumn{2}{|c|}{$J K_{a} K_{c}$} & \multicolumn{2}{|c|}{051 or $10^{-} 5$} & \multicolumn{3}{|c|}{211 or $30^{-} 1$} & \multicolumn{3}{|c|}{013 or $21^{-1}$} \\
\hline 0 & 0 & 0 & & 12151.25480 & 3.00 & $1 \mathrm{n}$ & 12565.00710 & 3.00 & $1 \mathrm{n}$ \\
\hline 1 & 0 & 1 & & 12173.76450 & 0.10 & $2 \mathrm{n}$ & 12588.15580 & 0.20 & $2 \mathrm{n}$ \\
\hline 1 & 1 & 1 & & 12187.81600 & 0.10 & $2 \mathrm{n}$ & 12600.57510 & 0.20 & $2 \mathrm{n}$ \\
\hline 1 & 1 & 0 & & 12193.34570 & 0.20 & $2 \mathrm{n}$ & 12606.26330 & 0.60 & $2 \mathrm{n}$ \\
\hline 2 & 0 & 211312.79346 & $1.001 \mathrm{a}$ & 12218.98490 & 0.10 & $4 \mathrm{n}$ & 12632.86720 & 0.10 & $2 \mathrm{n}$ \\
\hline 2 & 1 & 2 & & 12227.88070 & 0.20 & $3 \mathrm{n}$ & 12641.20500 & 0.20 & $3 n$ \\
\hline 2 & 1 & 111372.63578 & $1.051 \mathrm{a}$ & 12244.39990 & 0.10 & $4 \mathrm{n}$ & 12658.23710 & 0.50 & $3 \mathrm{n}$ \\
\hline 2 & 2 & 1 & & 12285.36600 & 0.30 & $3 \mathrm{n}$ & 12695.10100 & 0.70 & $2 \mathrm{n}$ \\
\hline 2 & 2 & 0 & & 12286.72510 & 0.10 & $3 \mathrm{n}$ & 12696.65110 & 0.20 & $2 \mathrm{n}$ \\
\hline 3 & 0 & 3 & & 12282.16060 & 0.10 & $4 \mathrm{n}$ & 12696.73700 & 0.20 & $2 \mathrm{n}$ \\
\hline 3 & 1 & 311414.63194 & $1.021 \mathrm{a}$ & 12287.24820 & 0.30 & $4 \mathrm{n}$ & 12701.23420 & 0.20 & $3 \mathrm{n}$ \\
\hline 3 & 1 & 2 & & 12319.85760 & 0.30 & $4 \mathrm{n}$ & 12734.91950 & 0.10 & $3 n$ \\
\hline 3 & 2 & 211567.22510 & $1.021 \mathrm{a}$ & 12353.73120 & 0.30 & $4 \mathrm{n}$ & 12764.66110 & 0.20 & $4 \mathrm{n}$ \\
\hline 3 & 2 & 1 & & 12360.00400 & 0.20 & $4 \mathrm{n}$ & 12771.71020 & 0.20 & $3 \mathrm{n}$ \\
\hline 3 & 3 & 111763.17033 & $1.071 \mathrm{a}$ & 2435.33520 & 0.20 & $4 \mathrm{n}$ & 12839.84820 & 0.20 & $n$ \\
\hline
\end{tabular}




\begin{tabular}{|c|c|c|c|c|c|c|c|c|}
\hline 3 & 3 & 0 & & 12435.55400 & 0.10 & $2 \mathrm{n}$ & 12840.12800 & 0.10 \\
\hline 4 & 0 & 411468.06805 & $1.071 \mathrm{a}$ & 12362.86270 & 0.20 & $4 \mathrm{n}$ & 12777.50160 & 0.20 \\
\hline & 1 & 4 & & 12365.28290 & 0.10 & $3 \mathrm{n}$ & 12779.93420 & 0.30 \\
\hline & 1 & 311558.32919 & $1.011 \mathrm{a}$ & 12418.08450 & 0.40 & $4 \mathrm{n}$ & 12834.47580 & 0.20 \\
\hline & 2 & 3 & & 12443.76730 & 0.40 & $4 \mathrm{n}$ & 12856.00530 & 0.50 \\
\hline & 2 & 211670.88746 & $1.021 \mathrm{a}$ & 12460.17590 & 0.50 & $5 \mathrm{n}$ & 12874.10580 & 0.40 \\
\hline & 3 & 2 & & 12528.75740 & 0.40 & $5 \mathrm{n}$ & 12935.37470 & 1.00 \\
\hline & 3 & 1 & & 12530.20600 & 0.20 & $4 \mathrm{n}$ & 12937.18870 & 0.20 \\
\hline & 4 & 1 & & 12637.86560 & 0.10 & $2 \mathrm{n}$ & 13034.63880 & 0.30 \\
\hline & 4 & 012098.80062 & $1.061 \mathrm{a}$ & 12637.89790 & 0.50 & $2 \mathrm{n}$ & 13034.67900 & 0.60 \\
\hline & 0 & 5 & & 12460.36390 & 1.00 & $3 \mathrm{n}$ & 12875.52780 & 0.40 \\
\hline & 1 & 5 & & 12461.39870 & 0.10 & $3 \mathrm{n}$ & 12876.76970 & 0.60 \\
\hline & 1 & 4 & & 12536.49210 & 0.10 & $3 \mathrm{n}$ & 12954.30670 & 0.40 \\
\hline & 2 & 411778.20167 & $1.021 \mathrm{a}$ & 12554.87980 & 0.40 & $4 \mathrm{n}$ & 12968.11470 & 0.10 \\
\hline & 2 & 3 & & 12586.59330 & 0.10 & $3 \mathrm{n}$ & 13002.84580 & 0.20 \\
\hline & 3 & 3 & & 12645.32980 & 0.30 & $5 \mathrm{n}$ & 13054.37900 & 0.30 \\
\hline & 3 & 2 & & 12650.59070 & 0.20 & $4 \mathrm{n}$ & 13060.74320 & 0.50 \\
\hline & 4 & 2 & & 12755.13600 & 0.40 & $5 \mathrm{n}$ & 13157.07050 & 0.70 \\
\hline & 4 & 1 & & 12755.39310 & 0.10 & $2 \mathrm{n}$ & 13157.34210 & 1.30 \\
\hline & 5 & 112490.17451 & $1.161 \mathrm{a}$ & 12892.32760 & 0.70 & $2 \mathrm{n}$ & 13277.82400 & 3.00 \\
\hline & 5 & 0 & & 12892.32680 & 3.00 & $1 \mathrm{n}$ & 13277.82380 & 3.00 \\
\hline 6 & 0 & 611696.38558 & $1.011 \mathrm{a}$ & 12574.73760 & 0.40 & $3 \mathrm{n}$ & 12990.50770 & 0.20 \\
\hline 6 & 1 & 6 & & 12575.20460 & 0.10 & $3 \mathrm{n}$ & 12989.63060 & 0.60 \\
\hline 6 & 1 & 511841.34029 & $0.732 \mathrm{a}$ & 12678.17750 & 0.40 & $4 \mathrm{n}$ & 13091.86740 & 0.20 \\
\hline 6 & 2 & 5 & & 12683.24730 & 0.50 & $2 \mathrm{n}$ & 13099.99270 & 2.00 \\
\hline & 2 & 411953.58136 & $1.041 \mathrm{a}$ & 12737.55660 & 0.20 & $3 \mathrm{n}$ & 13155.86400 & 0.10 \\
\hline & 3 & 4 & & 12784.40640 & 0.20 & $5 \mathrm{n}$ & 13196.15580 & 0.60 \\
\hline
\end{tabular}




\begin{tabular}{|c|c|c|c|c|c|c|c|c|c|}
\hline & 3 & 3 & & 12797.94390 & 0.20 & $4 \mathrm{n}$ & 13211.91350 & 1.50 & $3 \mathrm{n}$ \\
\hline & 4 & 3 & & 12896.05630 & 0.40 & $4 \mathrm{n}$ & 13302.27680 & 3.40 & $2 \mathrm{n}$ \\
\hline & 4 & 2 & & 12897.23380 & 0.40 & $4 \mathrm{n}$ & 13303.47720 & 0.50 & $2 \mathrm{n}$ \\
\hline & 5 & 2 & & 13033.43300 & 0.10 & $2 \mathrm{n}$ & 13419.59930 & 2.20 & $2 \mathrm{n}$ \\
\hline & 5 & 1 & & 13033.46310 & 0.70 & $3 \mathrm{n}$ & 13419.66100 & 0.60 & $3 n$ \\
\hline & 6 & 1 & & 13200.58220 & 4.10 & $3 \mathrm{n}$ & 13567.21470 & 1.20 & $2 \mathrm{n}$ \\
\hline & 6 & 0 & & 13200.58280 & 1.70 & $3 \mathrm{n}$ & 13567.21310 & 3.00 & $1 \mathrm{n}$ \\
\hline & 0 & 7 & & 12706.17340 & 0.10 & $2 \mathrm{n}$ & 13122.64040 & 0.60 & $2 \mathrm{n}$ \\
\hline & 1 & 711842.17686 & $1.021 \mathrm{a}$ & 12706.85480 & 0.20 & $3 \mathrm{n}$ & 13122.68430 & 0.10 & $2 \mathrm{n}$ \\
\hline & 1 & 612015.56810 & $5.061 \mathrm{a}$ & 12831.97310 & 0.30 & $2 \mathrm{n}$ & 13245.86250 & 0.10 & $3 \mathrm{n}$ \\
\hline & 2 & 612076.44335 & $1.071 \mathrm{a}$ & 12833.57500 & 0.20 & $4 \mathrm{n}$ & 13251.40420 & 1.10 & $2 \mathrm{n}$ \\
\hline & 2 & 5 & & 12910.76240 & 0.30 & $3 \mathrm{n}$ & 13330.48440 & 0.40 & $2 \mathrm{n}$ \\
\hline & 3 & 5 & & 12945.03740 & 0.50 & $4 \mathrm{n}$ & 13358.92800 & 1.40 & $3 \mathrm{n}$ \\
\hline & 3 & 4 & & 12972.43670 & 0.50 & $3 \mathrm{n}$ & 13390.41670 & 3.20 & $2 \mathrm{n}$ \\
\hline & 4 & 412533.13790 & $2.102 \mathrm{n}$ & 13060.37700 & 0.30 & $5 \mathrm{n}$ & 13470.74550 & 0.90 & $2 \mathrm{n}$ \\
\hline & 4 & 3 & & 13064.23230 & 0.60 & $2 \mathrm{n}$ & 13474.63760 & 3.00 & $1 \mathrm{n}$ \\
\hline & 5 & 3 & & 13198.19260 & 0.30 & $3 \mathrm{n}$ & 13585.22200 & 0.90 & $3 \mathrm{n}$ \\
\hline & 5 & 2 & & 13198.34660 & 1.50 & $3 \mathrm{n}$ & 13585.52850 & 3.00 & $1 \mathrm{n}$ \\
\hline & 6 & 2 & & 13366.36840 & 0.20 & $3 \mathrm{n}$ & 13733.83790 & 1.00 & $2 \mathrm{n}$ \\
\hline & 6 & 1 & & 13366.36620 & 2.20 & $2 \mathrm{n}$ & 13733.83580 & 3.60 & $2 \mathrm{n}$ \\
\hline & 7 & 1 & & 13576.56810 & 1.50 & $2 \mathrm{n}$ & & & \\
\hline & 7 & 0 & & 13576.56650 & 3.00 & $1 \mathrm{n}$ & & & \\
\hline & 0 & 811991.75340 & $0.732 \mathrm{a}$ & 12855.24090 & 0.10 & $2 \mathrm{n}$ & 13272.01250 & 0.70 & $2 \mathrm{n}$ \\
\hline & 1 & 8 & & 12854.16030 & 0.10 & $2 \mathrm{n}$ & 13272.01540 & 2.50 & $2 \mathrm{n}$ \\
\hline & 1 & 712209.22840 & $5.031 \mathrm{a}$ & 12999.12110 & 0.10 & -10 & 13416.22540 & 1.70 & $2 \mathrm{n}$ \\
\hline & 2 & 7 & & 13001.51650 & 0.20 & $3 \mathrm{n}$ & 13415.71480 & 1.00 & $2 \mathrm{n}$ \\
\hline & 2 & 612342.39281 & $1.101 \mathrm{a}$ & 13103.48730 & 0.20 & $3 \mathrm{n}$ & 13523.80020 & 3.00 & 1 \\
\hline
\end{tabular}




\begin{tabular}{|c|c|c|c|c|c|c|c|c|c|}
\hline 8 & 3 & 6 & & 13126.70460 & 0.10 & $3 n$ & 13543.45460 & 3.00 & $1 \mathrm{n}$ \\
\hline 8 & 3 & 512501.00734 & $5.021 \mathrm{a}$ & 13172.71490 & 0.30 & $4 \mathrm{n}$ & 13594.52000 & 1.90 & $2 \mathrm{n}$ \\
\hline 8 & 4 & 5 & & 13247.31280 & 0.20 & $2 \mathrm{n}$ & 13662.17160 & 3.00 & $1 \mathrm{n}$ \\
\hline 8 & 4 & 4 & & 13257.44680 & 0.20 & $4 \mathrm{n}$ & 13672.25430 & 2.70 & $2 \mathrm{n}$ \\
\hline 8 & 5 & 4 & & 13386.56720 & 1.60 & $4 \mathrm{n}$ & & & \\
\hline 8 & 5 & 312999.12140 & $0.404 \mathrm{n}$ & 13387.17950 & 0.10 & $3 n$ & 13775.71150 & 0.30 & $\mathrm{n}$ \\
\hline 8 & 6 & 3 & & 13555.69360 & 1.10 & $2 \mathrm{n}$ & & & \\
\hline 8 & 6 & 2 & & 13555.72860 & 1.00 & $4 \mathrm{n}$ & & & \\
\hline 8 & 7 & 2 & & 13683.56990 & 0.20 & $\mathrm{~d}$ & & & \\
\hline 8 & 7 & 1 & & 13683.56990 & 0.20 & $2 \mathrm{n}$ & & & \\
\hline 8 & 8 & 1 & & 13965.53235 & 3.00 & -10 & & & \\
\hline 8 & 8 & 0 & & 13965.53235 & 3.00 & $-1 \mathrm{o}$ & & & \\
\hline 9 & 0 & 9 & & 13019.39410 & 0.40 & $2 \mathrm{n}$ & 13438.55830 & $1.90-$ & $-1 \mathrm{n}$ \\
\hline 9 & 1 & 912167.47511 & $0.722 \mathrm{a}$ & 13020.09800 & 0.10 & $2 \mathrm{n}$ & 13438.57360 & 1.40 & 211 \\
\hline 9 & 1 & 812420.83768 & $5.061 \mathrm{a}$ & 13187.08660 & 1.90 & $4 \mathrm{n}$ & 13603.68760 & 1.10 & \\
\hline 9 & 2 & 812456.70588 & $1.101 \mathrm{a}$ & 13187.12300 & 0.10 & $2 \mathrm{n}$ & 13603.55680 & 2.00 & 3 \\
\hline 9 & 2 & 7 & & 13321.71310 & 1.00 & $3 \mathrm{n}$ & 13733.60580 & 3.00 & $1 \mathrm{n}$ \\
\hline 9 & 3 & 712705.84410 & $0.602 \mathrm{n}$ & 13327.67110 & 1.30 & $3 \mathrm{n}$ & 13749.13350 & 0.80 & \\
\hline 9 & 3 & 6 & & 13396.32980 & 1.30 & $4 \mathrm{n}$ & & & \\
\hline 9 & 4 & 612949.89631 & $5.021 \mathrm{a}$ & 13457.83950 & 0.10 & $3 \mathrm{n}$ & 13876.07220 & 3.00 & \\
\hline 9 & 4 & 5 & & 13477.70810 & 1.30 & $4 \mathrm{n}$ & & & \\
\hline 9 & 5 & 5 & & 13598.33090 & 0.70 & $3 \mathrm{n}$ & & & \\
\hline 9 & 5 & 4 & & 13599.92730 & 0.60 & $2 \mathrm{n}$ & & & \\
\hline 9 & 6 & 4 & & 13768.45560 & 3.00 & $1 \mathrm{n}$ & & & \\
\hline 9 & 6 & 3 & & 13768.57890 & 2.50 & $2 \mathrm{n}$ & & & \\
\hline 9 & 7 & 3 & & 13897.92630 & 0.10 & $2 \mathrm{n}$ & & & \\
\hline$y$ & 7 & 2 & & 13897.92430 & 2.60 & $2 \mathrm{n}$ & & & \\
\hline
\end{tabular}




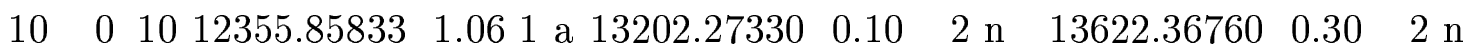

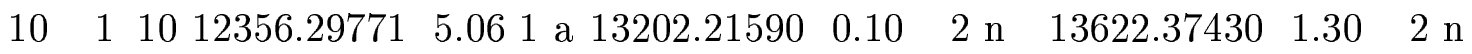

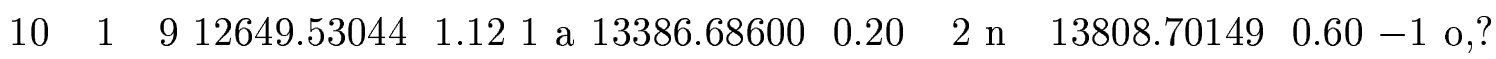

$\begin{array}{lllllllllll}10 & 2 & 9 & 13390.04450 & 0.10 & 2 \mathrm{n} & 13808.29711 & 0.60 & -1 \mathrm{o}, ?\end{array}$

$\begin{array}{llllllllll}10 & 2 & 8 & 12822.74179 & 1.17 & 1 \text { a } & 13545.79900 & 0.40 & 3 \mathrm{n}\end{array}$

$\begin{array}{llllll}10 & 3 & 8 & 13547.73140 & 3.00 & 1 \mathrm{n}\end{array}$

$\begin{array}{llllll}10 & 3 & 7 & 13639.56650 & 1.30 & 3 \mathrm{n}\end{array}$

$\begin{array}{lllllll}10 & 4 & 7 & 13688.45810 & 3.00 & 1 \mathrm{n}\end{array}$

$\begin{array}{llllllll}10 & 4 & 6 & 13724.59670 & 1.20 & 4 \mathrm{n}\end{array}$

$\begin{array}{lllllll}10 & 5 & 6 & 13832.97830 & 3.00 & 1 \mathrm{n}\end{array}$

$\begin{array}{lllllll}10 & 5 & 5 & 13838.71340 & 1.60 & 4 \mathrm{n}\end{array}$

$\begin{array}{lllllllll}11 & 0 & 11 & 13401.91630 & 5.00 & 2 \mathrm{n} & 13822.89600 & 2.00 & -1 \mathrm{o}\end{array}$

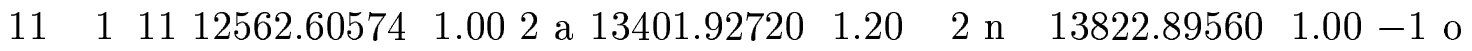

$11 \quad 1 \quad 1012894.94914 \quad 5.061$ a $13609.00610 \quad 1.10 \quad 2 \mathrm{n}$

$\begin{array}{lllllllll}11 & 2 & 10 & 13607.89880 & 0.90 & 3 \mathrm{n} & 14028.06360 & 0.80 & -1\end{array}$

$\begin{array}{lllllllllll}11 & 3 & 9 & 13186.78039 & 5.09 & 1 \text { a } & 13786.81200 & 1.00 & 2 \mathrm{n}\end{array}$

$\begin{array}{llllllll}11 & 4 & 8 & 13939.97100 & 0.60 & 2 \mathrm{n}\end{array}$

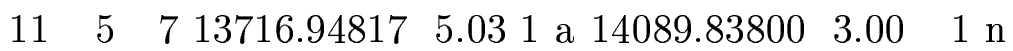

$12 \quad 0 \quad 1212776.73921 \quad 1.131$ a $13618.49016 \quad 3.00-1$ o $14038.99916 \quad 3.00-1$ o,?

1211212786.515405 .111 a $13618.49016 \quad 3.00 \quad \mathrm{~d} 14038.99916 \quad 3.00-1$ o,?

$12 \quad 2 \quad 1013392.91421 \quad 5.021 \mathrm{a}$

$\begin{array}{llllll}12 & 3 & 9 & 13553.94128 & 5.06 & 1 \mathrm{a}\end{array}$

$\begin{array}{llllll}13 & 0 & 13 & 13852.01490 & 3.00-1 \text { o }\end{array}$

$13 \quad 1 \quad 1313028.15903 \quad 1.142$ a $13852.015393 .00-1$ o

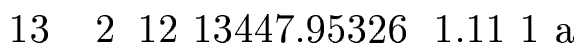

$13 \quad 4 \quad 1014031.83093 \quad 5.031 \mathrm{a}$

$14 \quad 0 \quad 1413280.53329 \quad 5.031$ a $14102.35655 \quad 0.60-1$ o 


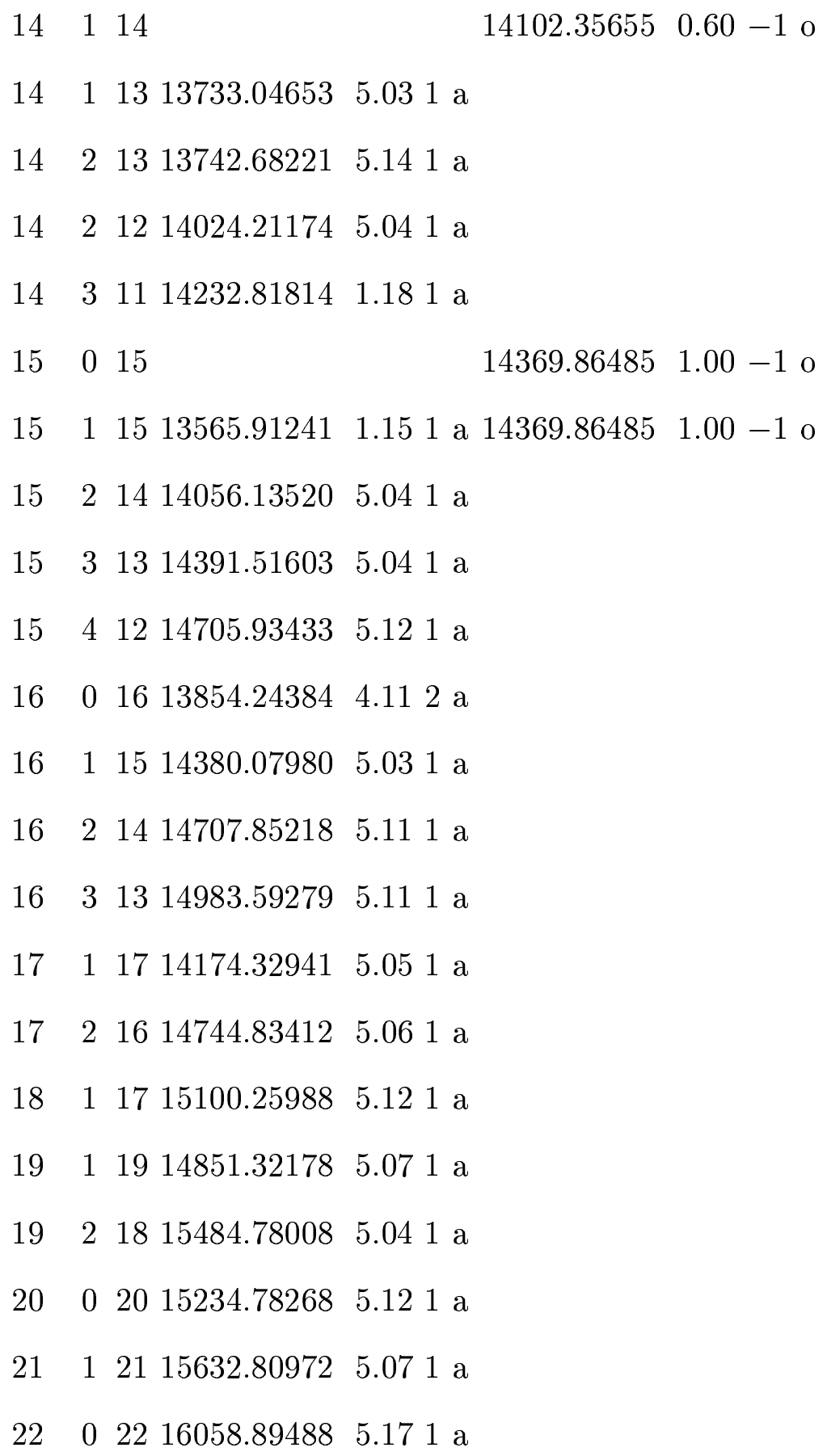


TABLE IX.: Term values for the $4 \nu$ polyad of $\mathrm{H}_{2}{ }^{16} \mathrm{O}$.

\begin{tabular}{|c|c|c|c|c|c|c|c|}
\hline \multicolumn{2}{|c|}{$J K_{a} K_{c}$} & \multicolumn{2}{|c|}{240 or $20^{+} 4$} & \multicolumn{2}{|c|}{141 or $20^{-} 4$} & \multirow[t]{2}{*}{042 or } & \multirow[t]{2}{*}{114} \\
\hline 1 & 0 & 113227.62799 & $4.001 \mathrm{a}$ & & & & \\
\hline 1 & 1 & 113262.55592 & $4.001 \mathrm{a}$ & 13310.10666 & $4.001 \mathrm{a}$ & & \\
\hline 1 & 1 & 013269.26975 & $11.001 \mathrm{a}$ & & & 13513.00782 & $5.401 \mathrm{p}$ \\
\hline 2 & 0 & 2 & & 13324.81350 & $4.001 \mathrm{a}$ & & \\
\hline 2 & 1 & 213302.27163 & $3.581 \mathrm{a}$ & & & 13546.76151 & $3.192 \mathrm{a}$ \\
\hline 2 & 1 & 113321.53467 & $4.001 \mathrm{a}$ & & & & \\
\hline 2 & 2 & 113414.18076 & $2.422 \mathrm{p}, \mathrm{a}$ & 13455.48509 & $4.692 \mathrm{p}, \mathrm{a}$ & 13647.52540 & $4.001 \mathrm{a}$ \\
\hline 2 & 2 & 0 & & 13456.40189 & $3.312 \mathrm{a}$ & 13648.13614 & $0.972 \mathrm{p}, \mathrm{a}$ \\
\hline 3 & 0 & 313338.41586 & $2.832 \mathrm{a}$ & & & 13589.13779 & $3.412 \mathrm{p}$ \\
\hline 3 & 1 & 313361.19154 & $1.001 \mathrm{p}$ & 13409.40278 & $8.001 \mathrm{a}$ & & \\
\hline 3 & 1 & 213399.20712 & $4.001 \mathrm{a}$ & & & 13645.64387 & $1.522 \mathrm{p}, \mathrm{a}$ \\
\hline 3 & 2 & 2 & & 13525.38562 & $1.833 \mathrm{p}, \mathrm{a}$ & 13717.85866 & $2.862 \mathrm{p}, \mathrm{a}$ \\
\hline 3 & 2 & 113487.38463 & $3.111 \mathrm{p}$ & & & 13722.53478 & $8.001 \mathrm{a}$ \\
\hline 3 & 3 & 1 & & 13673.94787 & $2.352 \mathrm{a}$ & & \\
\hline 3 & 3 & 013640.01391 & $4.001 \mathrm{a}$ & & & 13859.74456 & $3.581 \mathrm{a}$ \\
\hline 4 & 0 & 413422.67755 & $4.001 \mathrm{a}$ & & & & \\
\hline 4 & 1 & 413439.11633 & $2.832 \mathrm{a}$ & & & 13685.56792 & $2.391 \mathrm{p}$ \\
\hline 4 & 1 & 3 & & 13551.03590 & $5.201 \mathrm{p}$ & & \\
\hline 4 & 2 & 3 & & 13617.35554 & $0.983 \mathrm{p}$ & & \\
\hline 4 & 2 & 213587.21412 & $4.001 \mathrm{a}$ & & & & \\
\hline 4 & 3 & 2 & & & & 13954.61358 & $2.083 \mathrm{p}, \mathrm{a}$ \\
\hline 4 & 3 & 113734.90013 & $3.471 \mathrm{a}$ & 13769.63362 & $0.924 \mathrm{p}, \mathrm{a}$ & 13954.79342 & $1.235 \mathrm{a}$ \\
\hline 4 & 4 & 113932.56851 & $4.001 \mathrm{a}$ & 13960.35131 & $0.806 \mathrm{p}, \mathrm{a}$ & & \\
\hline 4 & 4 & 0 & & 13959.31048 & $1.185 \mathrm{p}, \mathrm{a}$ & & \\
\hline
\end{tabular}




\begin{tabular}{|c|c|c|c|c|c|c|c|}
\hline 5 & 0 & 513524.05779 & $3.651 \mathrm{a}$ & & & 13775.72505 & $1.241 \mathrm{p}$ \\
\hline 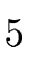 & 1 & 513535.31040 & $4.001 \mathrm{a}$ & & & & \\
\hline 5 & 1 & 4 & & & & 13877.31562 & $4.001 \mathrm{a}$ \\
\hline 5 & 2 & 4 & & 13730.81107 & $1.421 \mathrm{p}$ & & \\
\hline 5 & 3 & 3 & & 13887.64738 & $1.864 \mathrm{p}, \mathrm{a}$ & 14073.45143 & $4.001 \mathrm{a}$ \\
\hline 5 & 3 & 2 & & 13890.02068 & $1.992 \mathrm{p}$ & 14076.12379 & $0.796 \mathrm{p}, \mathrm{a}$ \\
\hline$\zeta$ & 4 & 214050.21544 & $2.144 \mathrm{p}, \mathrm{a}$ & 14080.94414 & $3.322 \mathrm{a}$ & & \\
\hline 5 & 4 & 114051.13433 & $1.232 \mathrm{p}, \mathrm{q}$ & 14079.95481 & $2.952 \mathrm{p}, \mathrm{a}$ & 14256.90613 & $1.442 \mathrm{p}, \mathrm{a}$ \\
\hline 5 & 5 & 114283.43562 & $5.05 \mathrm{~d}$ & 14300.70015 & $1.564 \mathrm{p}, \mathrm{a}$ & 14473.08660 & $1.475 \mathrm{p}, \mathrm{a}$ \\
\hline 5 & 5 & 014283.43562 & $5.051 \mathrm{p}$ & 14300.69062 & $0.873 \mathrm{p}, \mathrm{a}$ & 14473.08247 & $4.00 \mathrm{~d}$ \\
\hline 6 & 0 & 6 & & & & 13894.49408 & $6.041 \mathrm{p}$ \\
\hline 6 & 1 & 513774.96873 & $9.001 \mathrm{a}$ & & & 14025.62734 & $1.551 \mathrm{p}$ \\
\hline 6 & 2 & 513818.41797 & $2.091 \mathrm{p}$ & & & & \\
\hline 6 & 2 & 413865.78698 & $8.571 \mathrm{p}$ & & & & \\
\hline 6 & 3 & 413992.95624 & $2.821 \mathrm{p}$ & & & 14215.89628 & $1.665 \mathrm{p}, \mathrm{a}$ \\
\hline 6 & 3 & 3 & & 14035.92605 & $1.303 \mathrm{p}$ & & \\
\hline 6 & 4 & 314197.51237 & $1.294 \mathrm{p}, \mathrm{a}$ & 14220.48490 & $2.193 \mathrm{p}, \mathrm{a}$ & 14401.14320 & $4.001 \mathrm{a}$ \\
\hline 6 & 4 & 2 & & 14224.05098 & $1.335 \mathrm{p}, \mathrm{q}$ & & \\
\hline 6 & 5 & 214426.46601 & $0.756 \mathrm{p}, \mathrm{a}$ & & & 14617.91627 & $3.002 \mathrm{p}, \mathrm{a}$ \\
\hline 6 & 5 & 1 & & 14444.92409 & $0.747 \mathrm{a}$ & & \\
\hline 6 & 6 & 1 & & 14687.24583 & $1.04 \mathrm{~d}$ & & \\
\hline 6 & 6 & 0 & & 14687.24583 & $1.043 \mathrm{p}, \mathrm{a}$ & & \\
\hline 7 & 0 & 7 & & & & 14026.30487 & $4.001 \mathrm{a}$ \\
\hline 7 & 1 & 7 & & & & 14030.12109 & $3.201 \mathrm{p}$ \\
\hline 7 & 1 & 613941.72862 & $3.581 \mathrm{a}$ & 13991.53329 & $4.091 \mathrm{p}$ & & \\
\hline 7 & 2 & 613971.89433 & $7.551 \mathrm{p}$ & 14019.51449 & $2.701 \mathrm{p}$ & & \\
\hline 7 & 2 & 5 & & & & 14288.10269 & $1.002 \mathrm{p}, \mathrm{q}$ \\
\hline
\end{tabular}




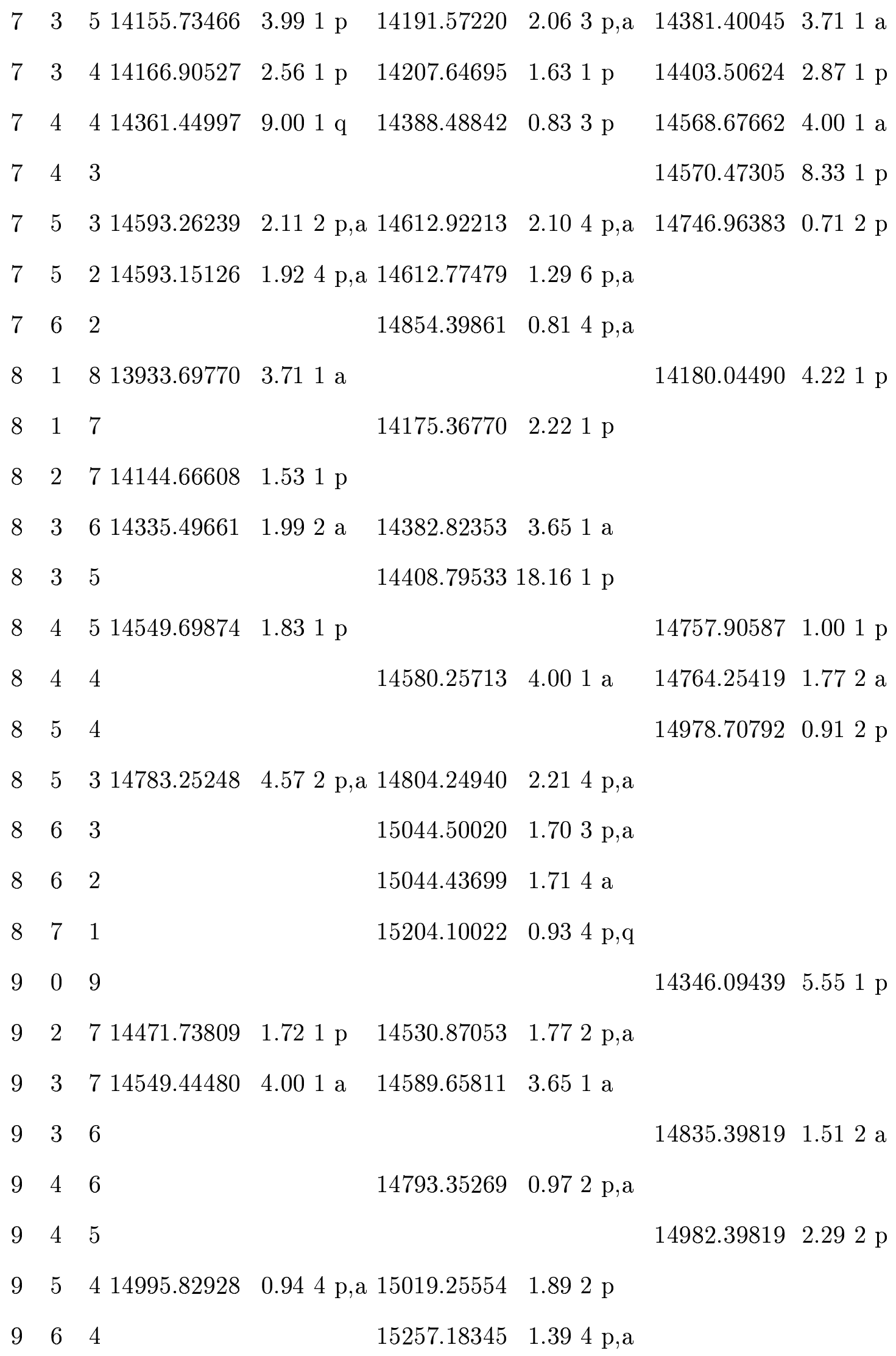




\begin{tabular}{rlllll}
9 & 6 & 3 & 15257.20770 & $1.022 \mathrm{p}$ \\
9 & 7 & 3 & 15416.54364 & $0.962 \mathrm{p}$ \\
11 & 3 & 9 & & 15064.22765 & $1.002 \mathrm{p}, \mathrm{a}$ \\
12 & 3 & 1015283.50594 & $5.001 \mathrm{q}$ & & \\
\hline
\end{tabular}

\begin{tabular}{|c|c|c|c|c|c|c|c|}
\hline \multicolumn{2}{|c|}{$J K_{a} K_{c}$} & \multicolumn{2}{|c|}{320 or $30^{+} 2$} & \multicolumn{2}{|c|}{122 or $21^{+} 2$} & \multicolumn{2}{|c|}{004 or 220} \\
\hline 0 & 0 & 0 & & 13910.89642 & $9.001 \mathrm{a}$ & 14537.50415 & $1.151 \mathrm{p}$ \\
\hline 1 & 0 & 113663.46825 & $0.971 \mathrm{a}$ & 13933.78521 & $1.702 \mathrm{a}$ & 14560.30553 & $2.192 \mathrm{a}$ \\
\hline 1 & 1 & 113681.82730 & $0.301 \mathrm{a}$ & 13950.93171 & $3.651 \mathrm{a}$ & 14569.78841 & $2.832 \mathrm{a}$ \\
\hline 1 & 1 & 0 & & 13956.80063 & $1.791 \mathrm{a}$ & 14575.14088 & $2.392 \mathrm{a}$ \\
\hline 2 & 0 & 213707.90559 & $0.502 \mathrm{a}$ & 13978.26033 & $2.773 \mathrm{a}$ & 14604.18442 & $0.933 \mathrm{p}, \mathrm{a}$ \\
\hline 2 & 1 & 213722.09365 & $0.484 \mathrm{a}$ & 13990.84527 & $1.604 \mathrm{a}$ & 14610.05471 & $1.674 \mathrm{a}$ \\
\hline 2 & 1 & 113738.73390 & $12.001 \mathrm{q}$ & 14008.45630 & $3.192 \mathrm{a}$ & 14626.08190 & $2.163 \mathrm{a}$ \\
\hline 2 & 2 & 113789.80501 & $0.913 \mathrm{p}, \mathrm{a}$ & 14058.21605 & $1.844 \mathrm{a}$ & 14654.33867 & $1.834 \mathrm{a}$ \\
\hline 2 & 2 & 013791.01759 & $3.651 \mathrm{a}$ & 14059.51821 & $2.403 \mathrm{p}, \mathrm{a}$ & 14656.04391 & $0.933 \mathrm{p}, \mathrm{a}$ \\
\hline 3 & 0 & 313771.84974 & $1.463 \mathrm{a}$ & 14042.09282 & $1.363 \mathrm{a}$ & 14666.60035 & $0.894 \mathrm{p}, \mathrm{a}$ \\
\hline 3 & 1 & 313780.01096 & $2.353 \mathrm{a}$ & 14048.22524 & $2.522 \mathrm{p}, \mathrm{a} ?$ & 14669.46747 & $2.034 \mathrm{p}, \mathrm{a}$ \\
\hline 3 & 1 & 213814.97269 & $2.333 \mathrm{a}$ & 14084.96802 & $1.684 \mathrm{a}$ & 14701.08793 & $1.953 \mathrm{a}$ \\
\hline 3 & 2 & 213858.33555 & $2.583 \mathrm{a}$ & 14126.94135 & $0.984 \mathrm{p}, \mathrm{a}$ & 14722.78295 & $1.974 \mathrm{a}$ \\
\hline 3 & 2 & 113864.04904 & $1.144 \mathrm{p}, \mathrm{a}$ & 14132.90607 & $1.445 \mathrm{a}$ & 14730.37646 & $1.174 \mathrm{p}, \mathrm{a}$ \\
\hline 3 & 3 & 113955.34794 & $1.744 \mathrm{I}$ & 14223.54844 & $2.603 \mathrm{a}$ & 14783.47936 & $0.934 \mathrm{p}, \mathrm{a}$ \\
\hline 3 & 3 & 013955.57662 & $1.612 \mathrm{a}$ & 14223.69446 & $2.102 \mathrm{a}$ & 14783.84578 & $1.923 \mathrm{a}$ \\
\hline 4 & 0 & 413853.20109 & $3.502 \mathrm{a}$ & 14123.39816 & $2.523 \mathrm{a}$ & 14746.10290 & $2.123 \mathrm{a}$ \\
\hline 4 & 1 & 413857.67235 & $1.693 \mathrm{p}, \mathrm{a}$ & 14127.63475 & $1.854 \mathrm{p}, \mathrm{a}$ & 14747.56088 & $0.485 \mathrm{a}$ \\
\hline 4 & 1 & 313914.36036 & $3.123 \mathrm{a}$ & 14184.81524 & $1.884 \mathrm{a}$ & 14798.07274 & $2.193 \mathrm{a}$ \\
\hline 4 & 2 & 313948.79041 & $1.114 \mathrm{p}, \mathrm{a}$ & 14217.46517 & $1.854 \mathrm{a}$ & 14812.54513 & $1.755 \mathrm{a}$ \\
\hline 4 & 2 & 213963.98293 & $3.504 \mathrm{p}, \mathrm{a}$ & 14233.35437 & $1.883 \mathrm{p}, \mathrm{a}$ & 14831.45103 & $1.724 \mathrm{p}, \mathrm{a}$ \\
\hline 4 & 3 & 214049.01798 & $1.177 \mathrm{a}$ & 14317.40043 & $1.515 \mathrm{p}, \mathrm{a}$ & 14877.44953 & $2.252 \mathrm{a}$ \\
\hline
\end{tabular}




\begin{tabular}{|c|c|c|c|c|c|c|c|}
\hline & 3 & 114049.98493 & $1.404 \mathrm{p}, \mathrm{a}$ & 14318.47559 & $3.303 \mathrm{p}, \mathrm{a}$ & 14879.78920 & $2.443 \mathrm{p}, \mathrm{a}$ \\
\hline & 1 & 114177.16131 & $0.893 \mathrm{a}$ & 14444.73560 & $1.963 \mathrm{p}, \mathrm{a}$ & 14957.50499 & $2.193 \mathrm{a}$ \\
\hline 4 & 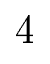 & 014177.18391 & $1.114 \mathrm{a}$ & 14444.67697 & $1.072 \mathrm{p}, \mathrm{a}$ & 14957.57225 & $0.972 \mathrm{p}, \mathrm{a}$ \\
\hline 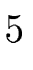 & 0 & 513950.91491 & $1.154 \mathrm{p}, \mathrm{a}$ & 14220.54505 & $1.253 \mathrm{p}, \mathrm{a}$ & 14842.55613 & $1.723 \mathrm{a}$ \\
\hline 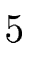 & 1 & 513953.13055 & $9.001 \mathrm{a}$ & 14223.24387 & $3.012 \mathrm{a}$ & 14842.96482 & $0.972 \mathrm{p}, \mathrm{a}$ \\
\hline 5 & 1 & 414035.02901 & $2.232 \mathrm{a}$ & 14306.04962 & $1.794 \mathrm{a}$ & 14914.33561 & $2.104 \mathrm{p}, \mathrm{a}$ \\
\hline 5 & 2 & 414060.90756 & $1.424 \mathrm{p}, \mathrm{a}$ & 14328.94596 & $0.914 \mathrm{p}, \mathrm{a}$ & 14922.48318 & $2.303 \mathrm{p}, \mathrm{a}$ \\
\hline 5 & 2 & 314090.59842 & $1.395 \mathrm{a}$ & 14360.38474 & $1.336 \mathrm{p}, \mathrm{a}$ & 14957.82222 & $1.984 \mathrm{p}, \mathrm{a}$ \\
\hline 5 & 3 & 314165.53805 & $0.894 \mathrm{p}, \mathrm{a}$ & 14434.29172 & $0.944 \mathrm{p}, \mathrm{a}$ & 14994.40826 & $2.702 \mathrm{a}$ \\
\hline 5 & 3 & 214169.56850 & $0.817 \mathrm{a}$ & 14438.25077 & $0.846 \mathrm{p}, \mathrm{a}$ & 15002.42539 & $2.043 \mathrm{a}$ \\
\hline 5 & 4 & 214294.00392 & $1.132 \mathrm{p}, \mathrm{a}$ & 14562.14850 & $3.801 \mathrm{a}$ & 15075.73446 & $4.001 \mathrm{a}$ \\
\hline 5 & 4 & 114294.31012 & $1.106 \mathrm{p}, \mathrm{a}$ & 14561.87623 & $0.914 \mathrm{p}, \mathrm{a}$ & 15076.27815 & $2.243 \mathrm{a}$ \\
\hline & 5 & 114445.31681 & $2.093 \mathrm{p}, \mathrm{a}$ & & & & \\
\hline 5 & 5 & 014445.46924 & $0.915 \mathrm{p}, \mathrm{a}$ & 14722.50420 & $0.953 \mathrm{p}, \mathrm{a}$ & 15175.59420 & $3.331 \mathrm{a}$ \\
\hline 6 & 0 & 614064.77498 & $3.402 \mathrm{a}$ & 14334.96524 & $4.001 \mathrm{a}$ & 14956.21052 & $2.543 \mathrm{p}, \mathrm{a}$ \\
\hline 6 & 1 & 614066.08700 & $1.343 \mathrm{p}, \mathrm{a}$ & 14336.46426 & $1.123 \mathrm{p}, \mathrm{a}$ & 14956.25613 & $2.233 \mathrm{p}, \mathrm{a}$ \\
\hline 6 & 1 & 514174.59754 & $2.513 \mathrm{p}, \mathrm{a}$ & 14446.56870 & $1.601 \mathrm{p}$ & 15047.80459 & $2.683 \mathrm{p}, \mathrm{a}$ \\
\hline 6 & 2 & 514184.23601 & $0.894 \mathrm{a}$ & 14460.57418 & $0.883 \mathrm{p}, \mathrm{a}$ & 15051.59231 & $2.153 \mathrm{a}$ \\
\hline ( & 2 & 414241.11518 & $0.674 \mathrm{a}$ & 14513.54052 & $1.802 \mathrm{p}, \mathrm{a}$ & 15105.97868 & $1.795 \mathrm{p}, \mathrm{a}$ \\
\hline 6 & 3 & 414304.87078 & $1.346 \mathrm{p}, \mathrm{a}$ & 14573.54504 & $2.283 \mathrm{a}$ & 15133.41407 & $3.482 \mathrm{a}$ \\
\hline 6 & 3 & 314315.24360 & $0.886 \mathrm{p}, \mathrm{a}$ & 14582.22007 & $0.933 \mathrm{p}, \mathrm{a}$ & 15152.46558 & $2.832 \mathrm{a}$ \\
\hline 6 & 4 & 314434.53400 & $1.366 \mathrm{p}, \mathrm{a}$ & 14703.56359 & $2.152 \mathrm{a}$ & 15217.77022 & $2.313 \mathrm{a}$ \\
\hline 6 & 4 & 214435.67999 & $11.771 \mathrm{p}$ & & & 15220.15921 & $4.001 \mathrm{a}$ \\
\hline 6 & 5 & 214585.97519 & $0.604 \mathrm{p}$ & 14865.81166 & $2.831 \mathrm{a}$ & 15317.91884 & $2.012 \mathrm{p}, \mathrm{a}$ \\
\hline 6 & 5 & 1 & & 14865.83346 & $3.801 \mathrm{a}$ & 15318.01979 & $2.422 \mathrm{p}, \mathrm{a}$ \\
\hline & 6 & 1 & & 15048.99380 & $2.562 \mathrm{a}$ & 15436.41018 & $0.972 \mathrm{p}, \mathrm{a}$ \\
\hline 6 & 6 & 0 & & & & 15436.41738 & 4.00 \\
\hline
\end{tabular}




$\begin{array}{llllrlrrr}7 & 0 & 714197.05070 & 0.873 \mathrm{a} & 14465.60292 & 2.503 \mathrm{a} & 15085.13795 & 1.106 \mathrm{p}, \mathrm{a} \\ 7 & 1 & 7 & & 14469.48281 & 1.442 \mathrm{p} & 15087.09824 & 1.983 \mathrm{p}, \mathrm{a} \\ 7 & 1 & 614331.01358 & 0.903 \mathrm{p}, \mathrm{a} & 14600.26327 & 1.434 \mathrm{p}, \mathrm{a} & 15199.65486 & 1.65 & 5 \mathrm{p}, \mathrm{a} \\ 7 & 2 & 6 & & 14611.76832 & 1.314 \mathrm{p}, \mathrm{a} & 15199.02991 & 3.542 \mathrm{p}, \mathrm{a} \\ 7 & 2 & 514417.89242 & 0.992 \mathrm{p}, \mathrm{a} & 14690.60777 & 1.196 \mathrm{p}, \mathrm{a} & 15277.54876 & 2.004 \mathrm{a} \\ 7 & 3 & 514463.64833 & 1.304 \mathrm{p}, \mathrm{a} & & & 15293.30610 & 4.001 \mathrm{a} \\ 7 & 3 & 414492.25199 & 0.345 \mathrm{a} & 14758.04268 & 0.972 \mathrm{p}, \mathrm{a} & 15328.81986 & 2.803 \mathrm{p}, \mathrm{a} \\ 7 & 4 & 414598.18552 & 3.471 \mathrm{a} & & & 15383.09353 & 4.001 \mathrm{a} \\ 7 & 4 & 314601.69016 & 1.791 \mathrm{a} & 14868.75538 & 1.214 \mathrm{p}, \mathrm{a} & 15390.40615 & 2.832 \mathrm{a} \\ 7 & 5 & 3 & & & & & & \end{array}$




\begin{tabular}{|c|c|c|c|c|c|c|c|}
\hline . & 1 & 814691.65053 & $3.312 \mathrm{p}, \mathrm{a}$ & & & 15542.39746 & $1.194 \mathrm{p}, \mathrm{a}$ \\
\hline 9 & 2 & 814692.11331 & $3.501 \mathrm{p}$ & & & 15548.23171 & $4.001 \mathrm{a}$ \\
\hline 0 & 2 & 714829.70532 & $2.632 \mathrm{p}, \mathrm{a}$ & & & 15669.81175 & $1.651 \mathrm{p}$ \\
\hline 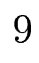 & 3 & 714850.68615 & $2.194 \mathrm{p}, \mathrm{a}$ & & & & \\
\hline 9 & 3 & 614912.97433 & $3.312 \mathrm{p}, \mathrm{a}$ & 15183.16928 & $1.653 \mathrm{p}$ & & \\
\hline & 4 & 614996.55148 & $2.242 \mathrm{p}, \mathrm{a}$ & & & & \\
\hline & 5 & 515207.44945 & $2.371 \mathrm{p}$ & & & & \\
\hline & 5 & 415209.56379 & $2.812 \mathrm{p}, \mathrm{a}$ & & & & \\
\hline & 1 & 1014685.90724 & $3.651 \mathrm{a}$ & & & & \\
\hline & 2 & 914896.99420 & $2.263 \mathrm{p}, \mathrm{a}$ & 15163.47406 & $10.001 \mathrm{q}$ & & \\
\hline & 3 & 815071.40698 & $2.622 \mathrm{p}, \mathrm{a}$ & & & & \\
\hline & 4 & 715228.20333 & $2.702 \mathrm{a}$ & & & & \\
\hline & $K_{a}$ & 221 or & $30^{-} 2$ & 023 or & $21^{-} 2$ & 400 or 4 & $40^{+} 0$ \\
\hline 0 & 0 & 013652.65605 & $7.001 \mathrm{a}$ & 14066.19395 & $6.001 \mathrm{a}$ & 13828.27732 & $6.001 \mathrm{a}$ \\
\hline 1 & 0 & 113675.41815 & $0.302 \mathrm{a}$ & 14089.41055 & $3.581 \mathrm{a}$ & 13850.65498 & $0.492 \mathrm{a}$ \\
\hline 1 & 1 & 113692.39875 & $0.302 \mathrm{a}$ & 14104.56304 & $1.612 \mathrm{a}$ & 13861.66876 & $1.352 \mathrm{p}, \mathrm{a}$ \\
\hline & 1 & 013698.22309 & $0.352 \mathrm{a}$ & 14110.58587 & $2.392 \mathrm{a}$ & 13866.69938 & $2.622 \mathrm{a}$ \\
\hline 2 & 0 & 213719.33225 & $0.214 \mathrm{a}$ & 14134.33534 & $1.234 \mathrm{p}, \mathrm{a}$ & 13893.99551 & $1.173 \mathrm{a}$ \\
\hline 2 & 1 & 213732.38583 & $0.254 \mathrm{p}, \mathrm{a}$ & 14144.99295 & $2.453 \mathrm{a}$ & 13901.43572 & $0.214 \mathrm{a}$ \\
\hline 2 & 1 & 113749.67931 & $0.174 \mathrm{a}$ & 14163.03291 & $1.453 \mathrm{a}$ & 13916.48179 & $1.293 \mathrm{a}$ \\
\hline 2 & 2 & 113799.45413 & $0.283 \mathrm{a}$ & 14207.79060 & $2.372 \mathrm{a}$ & 13949.39621 & $0.654 \mathrm{a}$ \\
\hline 2 & 2 & 013800.72174 & $0.304 \mathrm{a}$ & 14209.25384 & $1.743 \mathrm{p}, \mathrm{a}$ & 13950.76124 & $1.694 \mathrm{a}$ \\
\hline 3 & 0 & 313784.08585 & $0.275 \mathrm{p}, \mathrm{a}$ & 14198.48911 & $2.352 \mathrm{a}$ & 13956.07497 & $0.693 \mathrm{a}$ \\
\hline e & 1 & 313791.08979 & $0.215 \mathrm{p}, \mathrm{a}$ & 14204.77736 & $1.972 \mathrm{a}$ & 13960.49278 & $0.254 \mathrm{a}$ \\
\hline 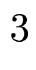 & 1 & 213825.82652 & $0.255 \mathrm{p}, \mathrm{a}$ & 14240.49569 & $1.192 \mathrm{p}, \mathrm{a}$ & 13990.06504 & $0.403 \mathrm{a}$ \\
\hline & 2 & 213867.97307 & $0.174 \mathrm{a}$ & 14277.60165 & $0.864 \mathrm{p}, \mathrm{a}$ & 14016.56624 & $0.785 \mathrm{a}$ \\
\hline 3 & 2 & 113873.90391 & $0.406 \mathrm{p}, \mathrm{a}$ & 14284.36037 & $2.263 \mathrm{a}$ & 14022.80317 & $1.014 \mathrm{a}$ \\
\hline
\end{tabular}




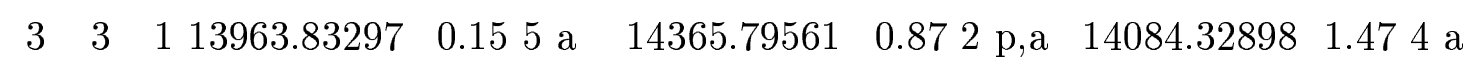

$\begin{array}{lllllllll}3 & 3 & 0 & 13963.79178 & 0.692 \mathrm{a} & 14366.03481 & 2.062 \mathrm{a} & 14084.46768 & 0.775 \mathrm{a}\end{array}$

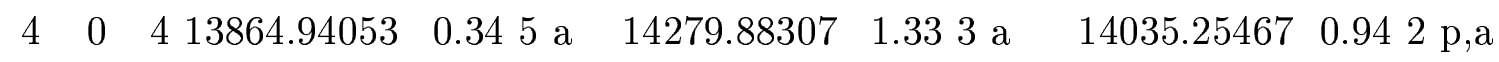

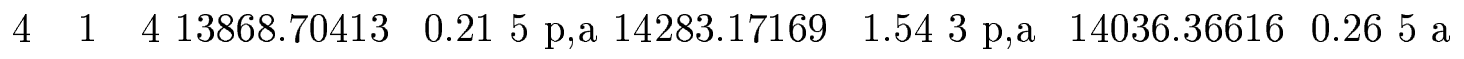

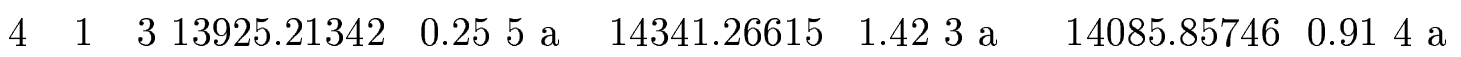

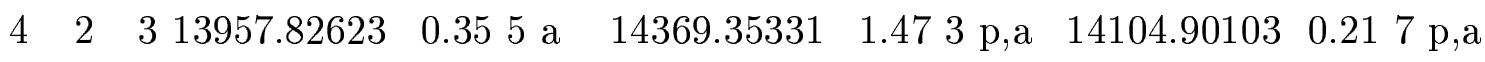

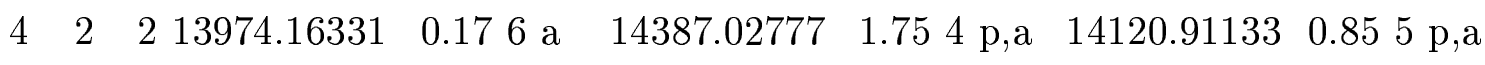

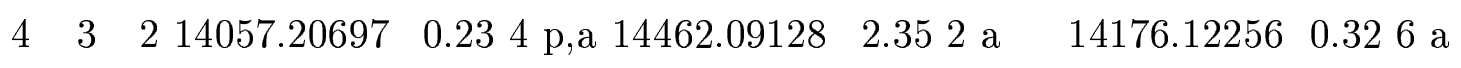

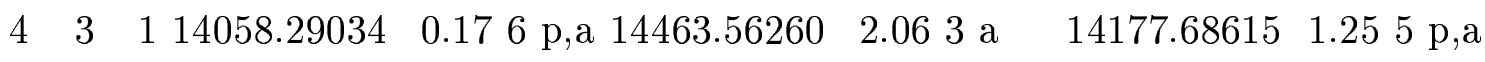

$4 \quad 4 \quad 114183.09284 \quad 0.444 \mathrm{p}, \mathrm{a} \quad 14268.01116 \quad 0.636 \mathrm{p}, \mathrm{a}$

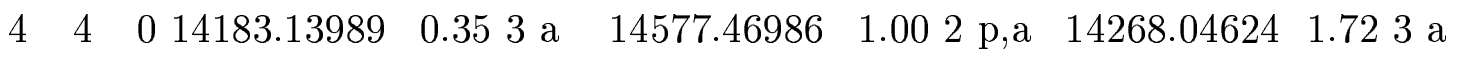

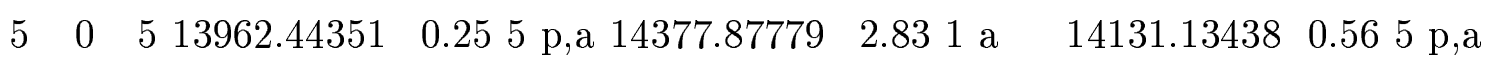

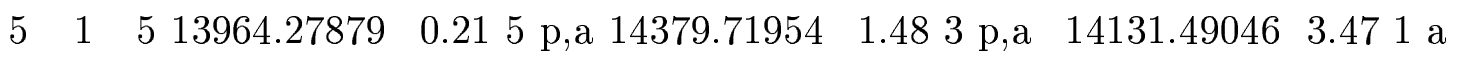

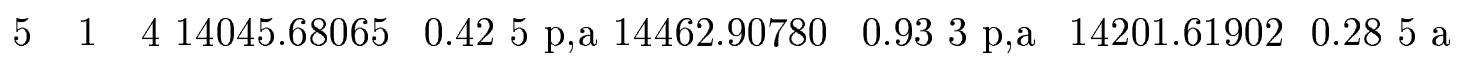

$5 \quad 2 \quad 414067.97123 \quad 0.256$ p,a $14482.07284 \quad 0.894$ p,a $14213.49849 \quad 0.425$ a

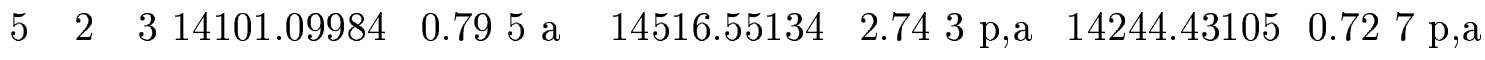

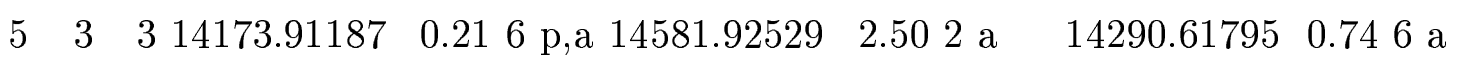

$\begin{array}{lllllll}5 & 3 & 2 & 14178.09225 & 0.535 \mathrm{a} & 14296.26782 & 0.47\end{array} \mathbf{p}, \mathrm{a}$

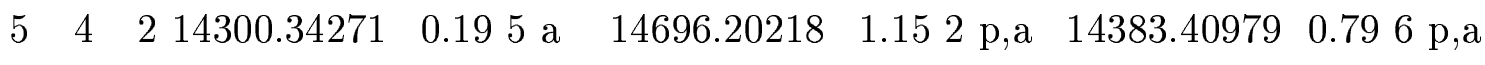

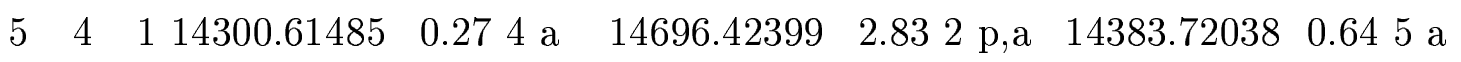

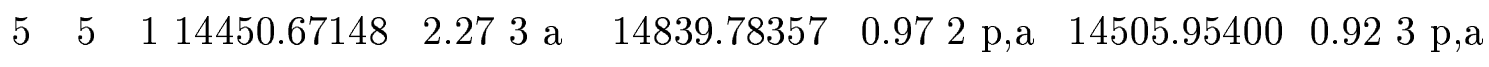

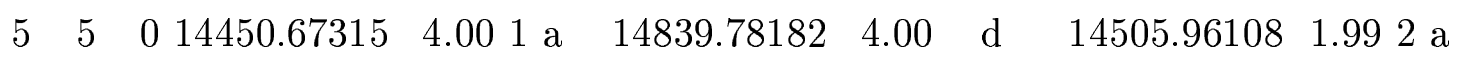

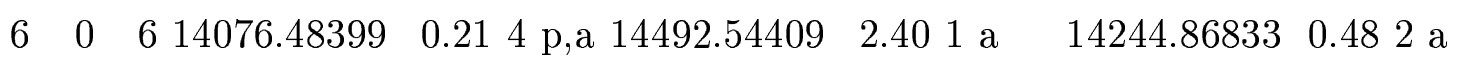

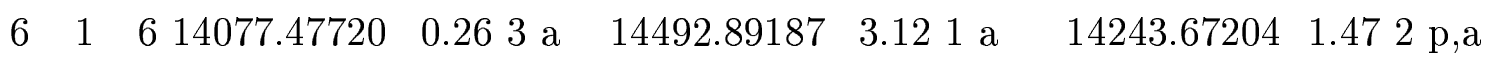

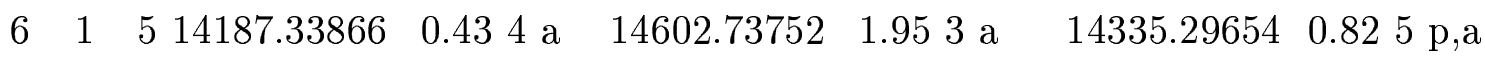

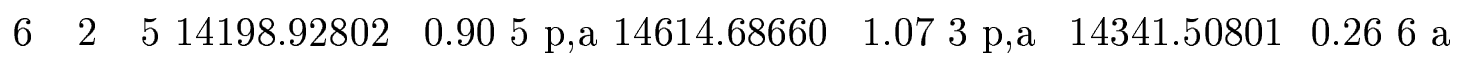

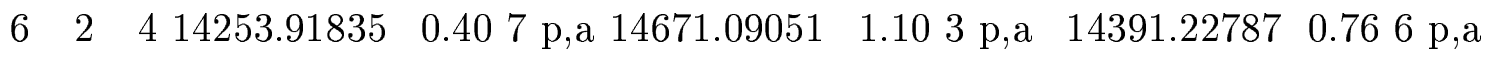




\begin{tabular}{|c|c|c|c|c|c|c|c|}
\hline 6 & 3 & 414313.17338 & $0.765 \mathrm{a}$ & 14724.66563 & $1.273 \mathrm{p}, \mathrm{a}$ & 14427.34450 & $0.417 \mathrm{p}, \mathrm{a}$ \\
\hline 6 & 3 & 314323.93495 & $0.246 \mathrm{a}$ & 14737.98871 & $1.973 \mathrm{a}$ & 14441.02398 & $1.055 \mathrm{p}, \mathrm{a}$ \\
\hline 6 & 4 & 314441.46256 & $0.926 \mathrm{a}$ & 14838.82108 & $3.711 \mathrm{a}$ & 14522.11640 & $1.037 \mathrm{p}, \mathrm{a}$ \\
\hline 6 & 4 & 214442.30461 & $0.217 \mathrm{p}, \mathrm{a}$ & 14839.85021 & $1.262 \mathrm{p}, \mathrm{a}$ & 14523.58909 & $1.276 \mathrm{a}$ \\
\hline 6 & 5 & 214648.42410 & $0.872 \mathrm{p}, \mathrm{a}$ & & & 14645.94101 & $1.644 \mathrm{a}$ \\
\hline 6 & 5 & 114648.45766 & $0.794 \mathrm{a}$ & 14983.01353 & $2.352 \mathrm{p}, \mathrm{a}$ & 14645.96430 & $0.694 \mathrm{p}, \mathrm{a}$ \\
\hline 6 & 6 & 1 & & 15148.55149 & $4.00 \mathrm{~d}$ & 14747.61350 & $1.783 \mathrm{p}, \mathrm{a}$ \\
\hline 6 & 6 & 0 & & 15148.55149 & $4.001 \mathrm{a}$ & 14747.61350 & $1.78 \mathrm{~d}$ \\
\hline 7 & 0 & 714207.31309 & $0.473 \mathrm{a}$ & 14624.10386 & $4.001 \mathrm{a}$ & 14373.32847 & $0.483 \mathrm{a}$ \\
\hline 7 & 1 & 714209.05874 & $0.353 \mathrm{a}$ & 14624.30544 & $2.913 \mathrm{p}, \mathrm{a}$ & 14373.37001 & $0.963 \mathrm{p}, \mathrm{a}$ \\
\hline 7 & 1 & 614342.32143 & $0.834 \mathrm{a}$ & 14759.11979 & $1.001 \mathrm{p}$ & 14481.36816 & $0.427 \mathrm{a}$ \\
\hline 7 & 2 & 614348.37498 & $0.436 \mathrm{p}, \mathrm{a}$ & 14769.04329 & $2.263 \mathrm{p}, \mathrm{a}$ & 14483.96091 & $0.435 \mathrm{p}, \mathrm{a}$ \\
\hline 7 & 2 & 514428.37277 & $0.884 \mathrm{p}, \mathrm{a}$ & 14848.07684 & $4.001 \mathrm{a}$ & 14558.94875 & $0.646 \mathrm{p}, \mathrm{a}$ \\
\hline 7 & 3 & 514474.47130 & $0.436 \mathrm{p}, \mathrm{a}$ & 14889.90760 & $4.001 \mathrm{a}$ & 14584.65834 & $0.814 \mathrm{a}$ \\
\hline 7 & 3 & 414500.21452 & $0.954 \mathrm{a}$ & 14916.45907 & $4.001 \mathrm{a}$ & 14613.07935 & $1.177 \mathrm{p}, \mathrm{a}$ \\
\hline 7 & 4 & 414605.96697 & $0.554 \mathrm{a}$ & 15004.37901 & $1.484 \mathrm{p}, \mathrm{a}$ & 14683.89274 & $1.775 \mathrm{a}$ \\
\hline 7 & 4 & 314608.87436 & $1.154 \mathrm{a}$ & 15008.35300 & $4.001 \mathrm{a}$ & 14686.82531 & $1.216 \mathrm{a}$ \\
\hline 7 & 5 & 314811.90147 & $0.634 \mathrm{a}$ & 15149.95466 & $2.322 \mathrm{p}, \mathrm{a}$ & 14809.53184 & $1.984 \mathrm{p}, \mathrm{a}$ \\
\hline 7 & 5 & 214812.11241 & $1.254 \mathrm{a}$ & & & 14809.74869 & $1.975 \mathrm{a}$ \\
\hline 7 & 6 & 2 & & 15315.84965 & $10.491 \mathrm{p}$ & 14910.12442 & $2.722 \mathrm{a}$ \\
\hline 7 & 6 & 1 & & & & 14910.15709 & $1.644 \mathrm{p}, \mathrm{a}$ \\
\hline 7 & 7 & 1 & & 15496.39643 & $4.001 \mathrm{a}$ & 15075.76534 & $3.08 \mathrm{~d}$ \\
\hline 7 & 7 & 0 & & & & 15075.76534 & $3.082 \mathrm{p}, \mathrm{a}$ \\
\hline 8 & 0 & 814356.07667 & $0.284 \mathrm{a}$ & 14772.51947 & $2.222 \mathrm{a}$ & 14520.09996 & $3.651 \mathrm{a}$ \\
\hline 8 & 1 & 814353.48966 & $0.883 \mathrm{a}$ & & & 14520.09372 & $2.462 \mathrm{a}$ \\
\hline 8 & 1 & 714514.39638 & $0.405 \mathrm{p}, \mathrm{a}$ & 14931.30074 & $4.001 \mathrm{a}$ & 14649.04706 & $1.444 \mathrm{p}, \mathrm{a}$ \\
\hline 8 & 2 & 714516.97973 & $0.993 \mathrm{p}, \mathrm{a}$ & 14932.66158 & $0.912 \mathrm{p}$ & 14649.75273 & $0.874 \mathrm{a}$ \\
\hline
\end{tabular}




\begin{tabular}{|c|c|c|c|c|c|c|c|}
\hline 8 & 2 & 614628.79710 & $1.045 \mathrm{p}, \mathrm{a}$ & 15045.00699 & $1.763 \mathrm{p}, \mathrm{a}$ & 14745.26263 & $0.962 \mathrm{a}$ \\
\hline 8 & 3 & 614656.01791 & $0.985 \mathrm{p}, \mathrm{a}$ & 15077.42106 & $4.001 \mathrm{a}$ & 14762.31125 & $0.745 \mathrm{a}$ \\
\hline 8 & 3 & 514699.42912 & $0.825 \mathrm{p}, \mathrm{a}$ & 15121.24888 & $1.293 \mathrm{p}, \mathrm{a}$ & 14809.25000 & $1.133 \mathrm{p}, \mathrm{a}$ \\
\hline 8 & 4 & 514792.98392 & $1.714 \mathrm{a}$ & 15193.50302 & $4.001 \mathrm{a}$ & 14868.16255 & $0.297 \mathrm{p}, \mathrm{a}$ \\
\hline 8 & 4 & 414801.20585 & $1.084 \mathrm{a}$ & 15202.14081 & $4.001 \mathrm{a}$ & 14878.19256 & $0.904 \mathrm{p}, \mathrm{a}$ \\
\hline 8 & 5 & 414998.92894 & $1.713 \mathrm{p}, \mathrm{a}$ & 15340.86321 & $1.632 \mathrm{p}$ & 14936.65469 & $1.375 \mathrm{p}, \mathrm{a}$ \\
\hline 8 & 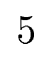 & 3 14999.71105 & $1.423 \mathrm{a}$ & 15341.71328 & $2.222 \mathrm{p}, \mathrm{a}$ & & \\
\hline 8 & o & 3 & & & & 15096.02236 & $1.992 \mathrm{p}, \mathrm{a}$ \\
\hline 8 & 6 & 2 & & 15506.65357 & $2.832 \mathrm{a}$ & 15096.23950 & $1.001 \mathrm{p}$ \\
\hline 8 & 7 & 2 & & 15686.05806 & $2.86 \mathrm{~d}$ & 15224.03374 & $3.692 \mathrm{p}, \mathrm{a}$ \\
\hline 8 & $r$ & 1 & & 15686.05806 & $2.862 \mathrm{p}, \mathrm{a}$ & & \\
\hline 8 & 8 & 1 & & 14957.05068 & $1.001 \mathrm{p}$ & & \\
\hline 9 & 0 & 914515.23742 & $2.522 \mathrm{p}, \mathrm{a}$ & & & 14683.78655 & $1.552 \mathrm{a}$ \\
\hline 9 & 1 & 914518.57846 & $0.473 \mathrm{a}$ & 14938.06174 & $4.001 \mathrm{a}$ & 14683.87120 & $1.271 \mathrm{p}$ \\
\hline 9 & 1 & 814703.07054 & $1.183 \mathrm{p}, \mathrm{a}$ & & & 14831.32663 & $1.493 \mathrm{p}, \mathrm{a}$ \\
\hline 9 & 2 & 814703.04982 & $0.724 \mathrm{p}, \mathrm{a}$ & 15120.89965 & $4.001 \mathrm{a}$ & & \\
\hline 9 & 2 & 714841.40144 & $2.452 \mathrm{p}, \mathrm{a}$ & & & 14948.30083 & $1.334 \mathrm{a}$ \\
\hline 9 & 3 & 714857.30182 & $1.265 \mathrm{a}$ & 15268.23427 & $1.033 \mathrm{p}$ & 14959.07538 & $1.523 \mathrm{a}$ \\
\hline 9 & 3 & 614923.35142 & $2.583 \mathrm{a}$ & & & 15027.90710 & $1.245 \mathrm{p}, \mathrm{a}$ \\
\hline 9 & 4 & 615002.98552 & $1.604 \mathrm{a}$ & 15405.19728 & $4.001 \mathrm{a}$ & 15074.21368 & $1.693 \mathrm{p}, \mathrm{a}$ \\
\hline 9 & 4 & 515019.63040 & $1.603 \mathrm{p}, \mathrm{a}$ & 15447.97999 & $2.672 \mathrm{a}$ & 15094.79490 & $2.163 \mathrm{p}, \mathrm{a}$ \\
\hline 9 & 0 & 515209.32724 & $0.873 \mathrm{p}, \mathrm{q}$ & 15555.42422 & $3.191 \mathrm{p}$ & 15145.87523 & $0.972 \mathrm{p}, \mathrm{a}$ \\
\hline 9 & 5 & 4 & & 15557.96662 & $8.751 \mathrm{p}$ & 15145.10554 & $0.914 \mathrm{p}, \mathrm{a}$ \\
\hline 9 & 6 & 4 & & & & 15304.94409 & $4.001 \mathrm{a}$ \\
\hline 9 & 6 & 3 & & & & 15305.46189 & $1.123 \mathrm{p}, \mathrm{a}$ \\
\hline 9 & 6 & 3 & & & & 15432.52001 & $0.992 \mathrm{p}$ \\
\hline & $y$ & 1 & & 16364.91785 & $0.382 \mathrm{p}$ & & \\
\hline
\end{tabular}




\begin{tabular}{|c|c|c|c|c|c|c|c|}
\hline 9 & 9 & 0 & & 16364.91785 & $0.38 \mathrm{~d}$ & & \\
\hline 10 & 0 & 1014697.15174 & $1.463 \mathrm{p}, \mathrm{a}$ & & & & \\
\hline 10 & 1 & 1014697.97430 & $1.833 \mathrm{p}, \mathrm{a}$ & & & & \\
\hline 10 & 1 & 914911.61032 & $2.253 \mathrm{p}, \mathrm{a}$ & & & 15031.05850 & $8.112 \mathrm{a}$ \\
\hline 10 & 2 & 914906.82909 & $2.473 \mathrm{p}, \mathrm{a}$ & & & & \\
\hline 10 & 2 & 815075.00076 & $2.224 \mathrm{p}, \mathrm{a}$ & & & 15167.60991 & $2.921 \mathrm{p}$ \\
\hline 10 & 3 & 815076.58995 & $1.622 \mathrm{p}, \mathrm{a}$ & & & 15169.22284 & $2.702 \mathrm{a}$ \\
\hline 10 & 3 & 715170.41388 & $1.983 \mathrm{p}, \mathrm{a}$ & & & 15266.43140 & $4.001 \mathrm{a}$ \\
\hline 10 & 4 & 715228.73366 & $5.342 \mathrm{p}, \mathrm{a}$ & & & 15301.08412 & $2.054 \mathrm{p}, \mathrm{a}$ \\
\hline 10 & 4 & 615264.16274 & $1.694 \mathrm{p}, \mathrm{a}$ & & & 15340.93067 & $0.992 \mathrm{p}$ \\
\hline 10 & 5 & 6 & & & & 15377.12925 & $2.502 \mathrm{p}, \mathrm{a}$ \\
\hline 10 & 7 & 4 & & & & 15664.44451 & $2.052 \mathrm{p}$ \\
\hline 11 & 0 & 1114896.42278 & $3.732 \mathrm{p}, \mathrm{a}$ & & & 15063.70809 & $1.582 \mathrm{a}$ \\
\hline 11 & 1 & 1114896.64494 & $0.973 \mathrm{p}, \mathrm{a}$ & 15317.12285 & $2.802 \mathrm{p}, \mathrm{a}$ & & \\
\hline 11 & 1 & 1015122.92401 & $3.581 \mathrm{a}$ & & & & \\
\hline 11 & 2 & 1015131.90379 & $2.462 \mathrm{a}$ & 15545.67533 & $3.602 \mathrm{p}, \mathrm{a}$ & 15247.77628 & $3.651 \mathrm{a}$ \\
\hline 11 & 2 & 915311.19592 & $0.992 \mathrm{p}$ & & & & \\
\hline 12 & 1 & 1215111.09262 & $12.001 \mathrm{a}$ & & & & \\
\hline 12 & 1 & 1115361.57559 & $2.882 \mathrm{p}, \mathrm{a}$ & & & & \\
\hline 12 & 2 & 1015568.94248 & $10.001 \mathrm{a}$ & & & & \\
\hline \multicolumn{2}{|c|}{$J K$} & 301 or & $40^{-} 0$ & 202 or & $31^{+} 0$ & \multicolumn{2}{|c|}{103 or $31^{-} 0$} \\
\hline 0 & 0 & 013830.93785 & $1.001 \mathrm{a}$ & 14221.16122 & $3.001 \mathrm{a}$ & 14318.81265 & $0.501 \mathrm{a}$ \\
\hline 1 & 0 & 113853.27030 & $0.501 \mathrm{a}$ & 14243.51917 & $0.692 \mathrm{a}$ & 14341.42794 & $0.492 \mathrm{a}$ \\
\hline 1 & 1 & 113864.28134 & $0.353 \mathrm{p}, \mathrm{a}$ & 14254.56480 & $2.242 \mathrm{a}$ & 14351.58168 & $0.442 \mathrm{a}$ \\
\hline 1 & 1 & 013869.38787 & $0.212 \mathrm{a}$ & 14259.70385 & $0.442 \mathrm{a}$ & 14356.84479 & $0.352 \mathrm{a}$ \\
\hline 2 & 0 & 213896.47637 & $0.245 \mathrm{a}$ & 14286.80060 & $0.823 \mathrm{a}$ & 14385.04700 & $0.344 \mathrm{a}$ \\
\hline 2 & 1 & 213903.86855 & $0.194 \mathrm{a}$ & 14294.13181 & $0.424 \mathrm{a}$ & 14391.61417 & $0.294 \mathrm{a}$ \\
\hline
\end{tabular}




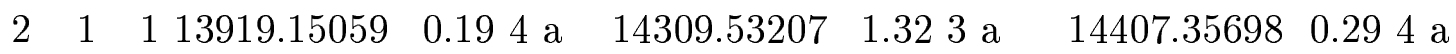

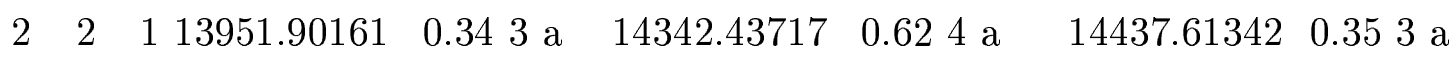

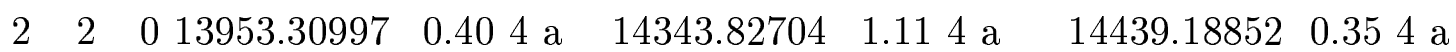

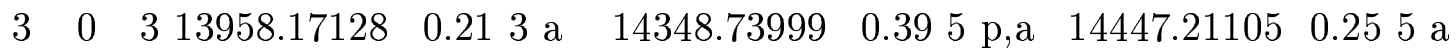

$\begin{array}{llllllllll}3 & 1 & 3 & 13962.25468 & 0.22 & 4 \mathrm{a} & 14352.67260 & 1.085 \mathrm{a} & 14450.77543 & 0.285 \mathrm{a}\end{array}$

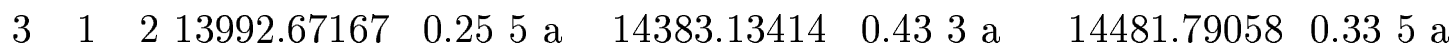

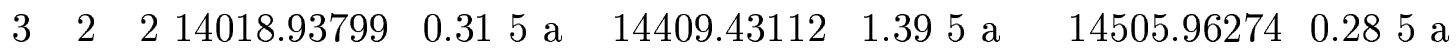

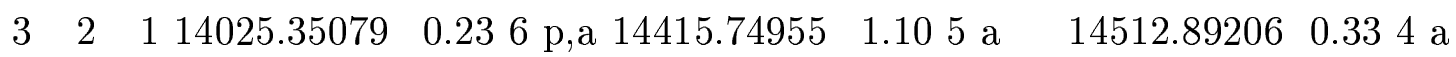

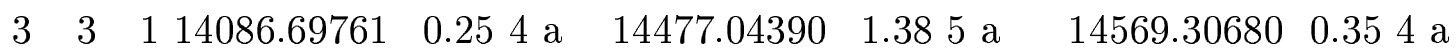

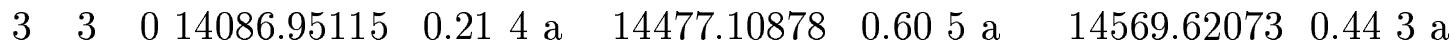

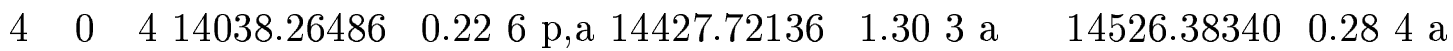

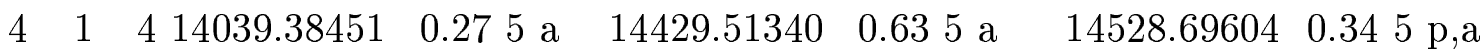

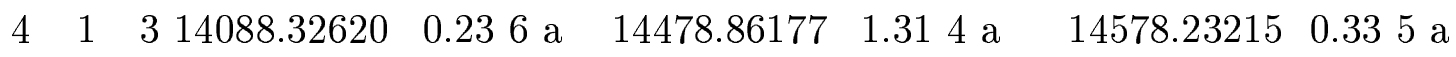

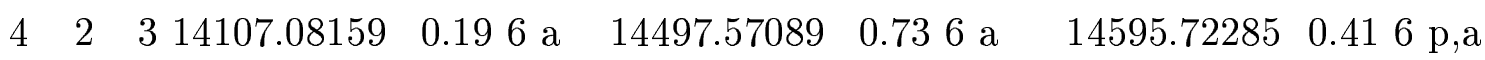

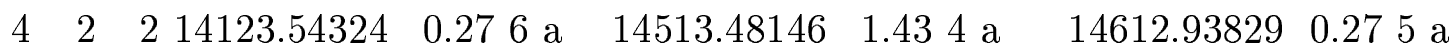

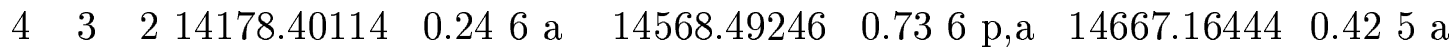

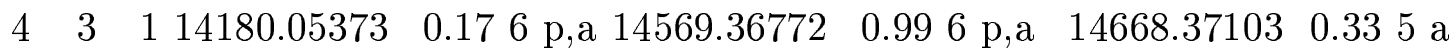

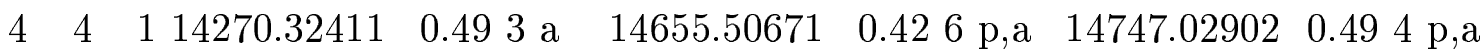

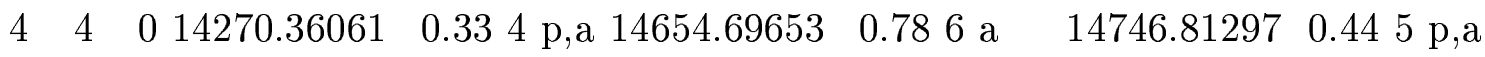

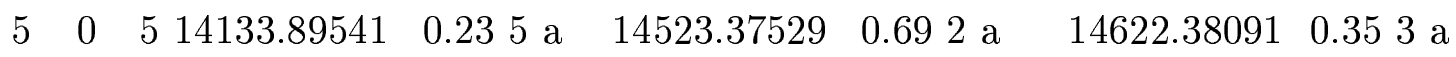

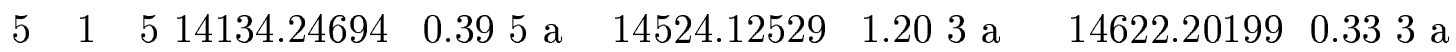

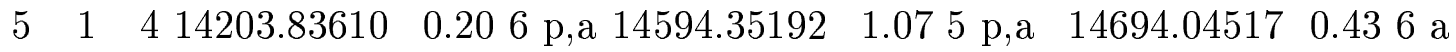

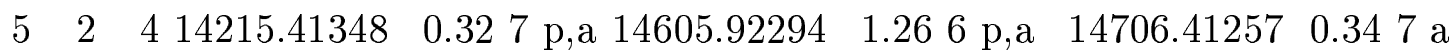

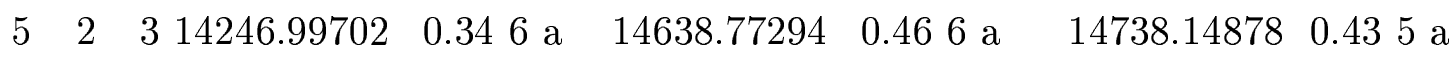

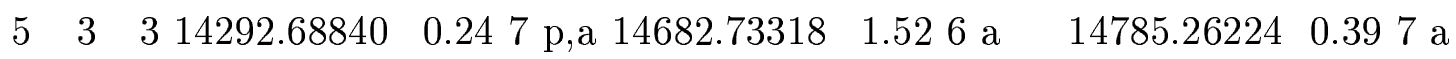

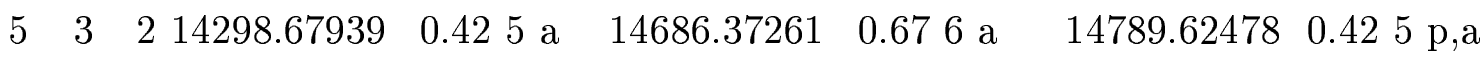

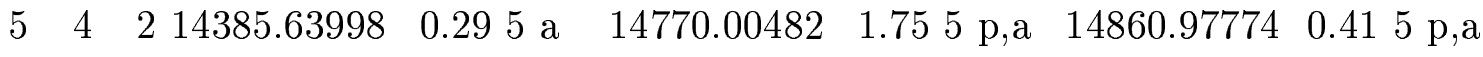




\begin{tabular}{|c|c|c|c|c|c|c|c|}
\hline 5 & 4 & 114385.94945 & $0.344 \mathrm{a}$ & 14767.46178 & $0.586 \mathrm{p}, \mathrm{a}$ & 14861.43662 & $0.864 \mathrm{a}$ \\
\hline 5 & 5 & 114508.53264 & $0.913 \mathrm{a}$ & & & 14970.20628 & $0.903 \mathrm{a}$ \\
\hline 5 & 5 & 014508.53444 & $1.001 \mathrm{p}$ & 14891.28960 & $0.485 \mathrm{a}$ & 14970.18666 & $1.792 \mathrm{p}, \mathrm{a}$ \\
\hline 6 & 0 & 614246.11760 & $0.195 \mathrm{p}, \mathrm{a}$ & 14635.84143 & $2.282 \mathrm{a}$ & 14735.37525 & $0.344 \mathrm{a}$ \\
\hline 6 & 1 & 614246.96226 & $0.254 \mathrm{a}$ & 14636.13645 & $0.833 \mathrm{a}$ & 14735.47433 & $0.443 \mathrm{a}$ \\
\hline 6 & 1 & 514337.32513 & $0.426 \mathrm{a}$ & 14727.27520 & $1.794 \mathrm{a}$ & 14826.93792 & $0.336 \mathrm{p}, \mathrm{a}$ \\
\hline 6 & 2 & 514343.02581 & $0.276 \mathrm{p}, \mathrm{a}$ & 14733.50930 & $1.126 \mathrm{p}, \mathrm{a}$ & 14825.21766 & $0.815 \mathrm{p}, \mathrm{a}$ \\
\hline 6 & 2 & 414393.77202 & $0.327 \mathrm{a}$ & 14785.29808 & $1.695 \mathrm{a}$ & 14886.39855 & $0.406 \mathrm{p}, \mathrm{a}$ \\
\hline 6 & 3 & 414429.07402 & $0.287 \mathrm{p}, \mathrm{a}$ & 14818.98092 & $1.385 \mathrm{p}, \mathrm{a}$ & 14925.54472 & $0.884 \mathrm{a}$ \\
\hline 6 & 3 & 314443.88184 & $0.206 \mathrm{p}, \mathrm{a}$ & 14841.03399 & $0.545 \mathrm{p}, \mathrm{a}$ & 14936.72219 & $0.465 \mathrm{a}$ \\
\hline 6 & 4 & 314524.28875 & $0.436 \mathrm{p}, \mathrm{a}$ & 14909.05366 & $1.274 \mathrm{a}$ & 14998.41585 & $1.345 \mathrm{a}$ \\
\hline 6 & 4 & 214525.69878 & $0.207 \mathrm{p}, \mathrm{a}$ & 14904.04152 & $1.434 \mathrm{a}$ & 15000.27446 & $0.725 \mathrm{p}, \mathrm{a}$ \\
\hline 6 & 5 & 214590.47269 & $1.613 \mathrm{a}$ & 15032.13578 & $1.545 \mathrm{p}, \mathrm{a}$ & 15109.26598 & $1.635 \mathrm{a}$ \\
\hline 6 & 5 & 114590.49972 & $0.434 \mathrm{a}$ & 15031.78118 & $1.791 \mathrm{a}$ & 15108.11757 & $0.854 \mathrm{a}$ \\
\hline 6 & 6 & 114749.59669 & $4.001 \mathrm{a}$ & 15166.37468 & $1.682 \mathrm{p}, \mathrm{a}$ & & \\
\hline 6 & 6 & 014749.59656 & $0.292 \mathrm{a}$ & & & 15238.21113 & $1.503 \mathrm{a}$ \\
\hline 7 & 0 & 714376.04709 & $0.653 \mathrm{p}, \mathrm{a}$ & 14765.26947 & $1.213 \mathrm{a}$ & 14865.52172 & $0.474 \mathrm{p}, \mathrm{a}$ \\
\hline 7 & 1 & 714376.11009 & $0.346 \mathrm{p}, \mathrm{a}$ & 14765.41965 & $1.353 \mathrm{a}$ & 14865.35711 & $0.263 \mathrm{a}$ \\
\hline 7 & 1 & 614487.88950 & $0.254 \mathrm{a}$ & 14876.44298 & $1.474 \mathrm{a}$ & 14976.08433 & $0.654 \mathrm{p}, \mathrm{a}$ \\
\hline 7 & 2 & 614488.75226 & $0.247 \mathrm{a}$ & 14879.83278 & $1.854 \mathrm{a}$ & 14976.20601 & $0.434 \mathrm{p}, \mathrm{a}$ \\
\hline 7 & 2 & 514561.41563 & $0.277 \mathrm{p}, \mathrm{a}$ & 14953.29456 & $1.983 \mathrm{a}$ & 15054.87382 & $0.715 \mathrm{p}, \mathrm{a}$ \\
\hline 7 & 3 & 514586.48379 & $0.288 \mathrm{a}$ & 14976.25269 & $1.066 \mathrm{p}, \mathrm{a}$ & 15090.69368 & $0.557 \mathrm{p}, \mathrm{a}$ \\
\hline 7 & 3 & 414615.70722 & $0.425 \mathrm{p}, \mathrm{a}$ & 15008.86176 & $1.275 \mathrm{a}$ & 15109.89576 & $1.454 \mathrm{a}$ \\
\hline 7 & 4 & 414686.03030 & $0.426 \mathrm{p}, \mathrm{a}$ & 15071.34775 & $0.963 \mathrm{p}, \mathrm{a}$ & 15159.15722 & $1.044 \mathrm{a}$ \\
\hline 7 & 4 & 314690.55494 & $0.734 \mathrm{a}$ & 15063.73181 & $0.795 \mathrm{a}$ & 15164.55430 & $1.694 \mathrm{a}$ \\
\hline 7 & 5 & 314753.47131 & $0.334 \mathrm{p}, \mathrm{a}$ & 15196.61458 & $2.143 \mathrm{p}, \mathrm{a}$ & 15268.47379 & 1.27 \\
\hline & 5 & 214753.61905 & $0.804 \mathrm{a}$ & 15193.53590 & $1.404 \mathrm{p}, \mathrm{a}$ & 15269.86247 & $1.894 \mathrm{a}$ \\
\hline
\end{tabular}




\begin{tabular}{|c|c|c|c|c|c|c|c|}
\hline 7 & 6 & 214911.97920 & $0.474 \mathrm{a}$ & 15329.84931 & $3.121 \mathrm{a}$ & 15399.36145 & $1.734 \mathrm{a}$ \\
\hline 7 & 6 & 114911.98768 & $4.001 \mathrm{a}$ & 15329.94922 & $1.923 \mathrm{p}, \mathrm{a}$ & & \\
\hline & 7 & 115058.35688 & $0.493 \mathrm{a}$ & & & 15553.55287 & $4.001 \mathrm{a}$ \\
\hline & 7 & 0 & & 15423.23860 & $3.121 \mathrm{a}$ & 15553.55287 & $4.00 \mathrm{~d}$ \\
\hline & 0 & 14522.82114 & $0.352 \mathrm{a}$ & 14911.77658 & $2.263 \mathrm{p}, \mathrm{a}$ & 15012.62057 & $0.283 \mathrm{a}$ \\
\hline & 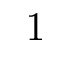 & 814522.85500 & $0.484 \mathrm{p}, \mathrm{a}$ & 14911.15553 & $1.403 \mathrm{p}, \mathrm{a}$ & 15012.65065 & $0.972 \mathrm{a}$ \\
\hline 8 & 1 & 714652.52628 & $0.244 \mathrm{a}$ & 15041.80930 & $2.064 \mathrm{p}, \mathrm{a}$ & 15141.78276 & $0.475 \mathrm{a}$ \\
\hline & 2 & 714653.00396 & $0.485 \mathrm{p}, \mathrm{a}$ & 15042.44480 & $0.904 \mathrm{p}, \mathrm{a}$ & 15141.64398 & $1.523 \mathrm{a}$ \\
\hline & 2 & 614747.43983 & $0.427 \mathrm{a}$ & 15139.70299 & $1.303 \mathrm{p}, \mathrm{a}$ & 15243.18226 & $0.846 \mathrm{a}$ \\
\hline & 3 & 614763.70369 & $0.844 \mathrm{a}$ & 15153.49120 & $0.923 \mathrm{p}, \mathrm{a}$ & 15245.89408 & $1.254 \mathrm{p}$ \\
\hline & 3 & 514811.97650 & $0.276 \mathrm{p}, \mathrm{a}$ & 15204.23602 & $2.722 \mathrm{a}$ & 15307.28995 & $0.845 \mathrm{a}$ \\
\hline & 4 & 514870.32762 & $2.214 \mathrm{a}$ & 15255.78992 & $1.334 \mathrm{p}, \mathrm{a}$ & 15342.34794 & $1.595 \mathrm{p}$ \\
\hline & $T$ & 414881.58893 & $0.415 \mathrm{p}, \mathrm{a}$ & 15276.94562 & $2.572 \mathrm{p}, \mathrm{a}$ & 15354.70377 & $1.814 \mathrm{a}$ \\
\hline & 5 & 41493 & $1.913 \mathrm{a}$ & 1538 & $0.854 \mathrm{p}, \mathrm{a}$ & 15452.68928 & $1.944 \mathrm{a}$ \\
\hline & 5 & 02 & $0.645 \mathrm{a}$ & 803 & $2.912 \mathrm{p}, \mathrm{a}$ & 154 & 2.21 \\
\hline 8 & 6 & 315097.71817 & $2.014 \mathrm{p}, \mathrm{a}$ & 15516.29428 & $3.331 \mathrm{a}$ & & \\
\hline 8 & 6 & 215097.77485 & $0.805 \mathrm{p}, \mathrm{a}$ & 15516.69334 & $3.581 \mathrm{a}$ & 15584.18595 & $1.954 \mathrm{a}$ \\
\hline 0 & 7 & 215243.95350 & $1.962 \mathrm{p}, \mathrm{a}$ & & & & \\
\hline & 7 & 115244.01491 & $1.043 \mathrm{p}, \mathrm{a}$ & 15684.31311 & $4.001 \mathrm{a}$ & & \\
\hline & 8 & 115406.53147 & 0.90 & & & & \\
\hline & 8 & 47 & $0.903 \mathrm{p}, \mathrm{a}$ & & & & \\
\hline 9 & 0 & 914686.61120 & $0.493 \mathrm{a}$ & 15074.83965 & $1.902 \mathrm{a}$ & 1517 & $1.632 \mathrm{a}$ \\
\hline 9 & 1 & 914687.16530 & $0.303 \mathrm{a}$ & 15074.94752 & $2.392 \mathrm{a}$ & 15176 & 0.48 \\
\hline 9 & 1 & 814834.37769 & $0.343 \mathrm{a}$ & 15224.12878 & $0.924 \mathrm{p}, \mathrm{a}$ & 15323.48879 & $1.124 \mathrm{p}$ \\
\hline y & 2 & 814834.41552 & $0.255 \mathrm{p}, \mathrm{a}$ & 15223.68657 & $1.153 \mathrm{p}, \mathrm{a}$ & 15323.64696 & $1.114 \mathrm{l}$ \\
\hline & 2 & 714950.18667 & $0.775 \mathrm{p}, \mathrm{a}$ & 15342.43184 & $1.964 \mathrm{p}, \mathrm{a}$ & 15441.35955 & 2.39 \\
\hline & 3 & 9 & 0. & 1 & $\mathrm{p}, \mathrm{a}$ & 17108 & \\
\hline
\end{tabular}




\begin{tabular}{|c|c|c|c|c|c|c|}
\hline 9 & $\begin{array}{lll}3 & 6 & 15030.71657\end{array}$ & $1.105 \mathrm{p}, \mathrm{a}$ & 15422.45530 & $0.953 \mathrm{p}, \mathrm{a}$ & 15525.07857 & $2.144 \mathrm{p}, \mathrm{a}$ \\
\hline 9 & $\begin{array}{lll}4 & 6 & 15076.39117\end{array}$ & $0.825 \mathrm{p}, \mathrm{a}$ & 15461.52778 & $4.001 \mathrm{a}$ & 15552.25000 & $2.223 \mathrm{a}$ \\
\hline 9 & $\begin{array}{lll}4 & 5 & 15099.25207\end{array}$ & $1.903 \mathrm{a}$ & 15491.69406 & $1.034 \mathrm{p}, \mathrm{a}$ & & \\
\hline 9 & $\begin{array}{lll}5 & 5 & 15148.45623\end{array}$ & $2.752 \mathrm{a}$ & 15600.29207 & $2.413 \mathrm{p}, \mathrm{a}$ & 15658.33633 & $1.001 \mathrm{p}$ \\
\hline 9 & $5 \quad 415151.51216$ & $1.754 \mathrm{a}$ & 15594.15292 & $2.482 \mathrm{p}, \mathrm{a}$ & 15666.88586 & $0.924 \mathrm{p}, \mathrm{a}$ \\
\hline 9 & $\begin{array}{lll}6 & 4 & 15306.86357\end{array}$ & $0.745 \mathrm{p}, \mathrm{a}$ & 15725.70368 & $4.001 \mathrm{a}$ & 15792.88233 & $2.752 \mathrm{a}$ \\
\hline 9 & $6 \quad 315307.08024$ & $3.651 \mathrm{a}$ & & & & \\
\hline 9 & $\begin{array}{lll}7 & 3 & 15453.22017\end{array}$ & $0.725 \mathrm{p}, \mathrm{a}$ & 15827.17082 & $1.141 \mathrm{p}$ & & \\
\hline 9 & $\begin{array}{lll}7 & 2 & 15453.11896\end{array}$ & $0.443 \mathrm{p}, \mathrm{a}$ & & & & \\
\hline 9 & $8 \quad 2 \quad 15612.62331$ & $10.371 \mathrm{p}$ & & & & \\
\hline 9 & $\begin{array}{lll}8 & 1 & 15612.62331\end{array}$ & $10.37 \mathrm{~d}$ & & & & \\
\hline 10 & $\begin{array}{lll}0 & 10 & 14867.39214\end{array}$ & $0.493 \mathrm{a}$ & 15255.30959 & $4.001 \mathrm{a}$ & 15358.40284 & $0.903 \mathrm{a}$ \\
\hline 10 & 11014867.39214 & $0.49 \quad \mathrm{~d}$ & 15255.36090 & $3.581 \mathrm{a}$ & 15358.37995 & $1.002 \mathrm{p}, \mathrm{a}$ \\
\hline 10 & $1 \quad 915033.13048$ & $0.834 \mathrm{a}$ & & & 15522.68541 & $1.404 \mathrm{a}$ \\
\hline 10 & $2 \quad 915033.58030$ & $0.923 \mathrm{p}, \mathrm{a}$ & 15421.76210 & $3.652 \mathrm{a}$ & 15522.70428 & $2.232 \mathrm{p}, \mathrm{a}$ \\
\hline 10 & $\begin{array}{lll}2 & 8 & 15174.17937\end{array}$ & $0.874 \mathrm{a}$ & & & 15660.71646 & $2.153 \mathrm{a}$ \\
\hline 10 & $\begin{array}{llll}3 & 8 & 15175.36471\end{array}$ & $2.223 \mathrm{a}$ & 15564.07812 & $3.471 \mathrm{a}$ & 15661.81249 & $0.953 \mathrm{p}, \mathrm{q}$ \\
\hline 10 & $\begin{array}{lll}3 & 7 & 15269.33032\end{array}$ & $1.443 \mathrm{a}$ & & & 15767.61613 & $2.702 \mathrm{a}$ \\
\hline 10 & $4 \quad 7 \quad 15303.29551$ & $1.833 \mathrm{p}, \mathrm{a}$ & 15688.04746 & $0.894 \mathrm{p}, \mathrm{a}$ & 15777.99576 & $1.001 \mathrm{p}$ \\
\hline 10 & $4 \quad 6 \quad 15343.54020$ & $1.813 \mathrm{a}$ & 15733.28131 & $4.001 \mathrm{a}$ & & \\
\hline 0 & $\begin{array}{lll}5 & 6 & 15379.67407\end{array}$ & $1.963 \mathrm{p}, \mathrm{a}$ & & & & \\
\hline 10 & $5 \quad 515387.32022$ & $0.973 \mathrm{p}, \mathrm{a}$ & 15828.62506 & $4.001 \mathrm{a}$ & 15904.30095 & $1.852 \mathrm{p}$ \\
\hline 0 & 65 & & 15957.79196 & $3.241 \mathrm{p}$ & & \\
\hline 10 & 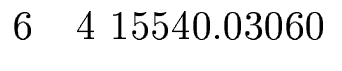 & $2.313 \mathrm{p}, \mathrm{a}$ & 15959.49426 & $3.151 \mathrm{p}$ & & \\
\hline 10 & 74 & & 16063.81392 & $5.781 \mathrm{p}$ & & \\
\hline 10 & 83 & & 16257.58990 & $1.001 \mathrm{p}$ & & \\
\hline 11 & 01115065.15247 & $1.383 \mathrm{p}, \mathrm{a}$ & & & 15556.73871 & $0.94 \mathrm{~d}$ \\
\hline
\end{tabular}




\begin{tabular}{|c|c|c|c|c|c|c|}
\hline 11 & 1 & 1115065.34678 & $0.473 \mathrm{p}, \mathrm{a}$ & & 15556.73857 & $0.904 \mathrm{p}, \mathrm{a}$ \\
\hline 11 & 1 & 1015245.40268 & $4.001 \mathrm{a}$ & $15636.30100 \quad 4.002 \mathrm{p}, \mathrm{a}$ & 15738.46902 & $4.001 \mathrm{a}$ \\
\hline 11 & 2 & 1015248.71253 & $0.843 \mathrm{p}, \mathrm{a}$ & & 15738.65677 & $4.001 \mathrm{a}$ \\
\hline 11 & 2 & 915407.12280 & $4.001 \mathrm{a}$ & & 15895.15273 & $4.001 \mathrm{a}$ \\
\hline 11 & 3 & 915408.13570 & $1.904 \mathrm{p}, \mathrm{a}$ & & 15895.40358 & $4.001 \mathrm{a}$ \\
\hline 11 & 3 & 815527.01755 & $4.001 \mathrm{a}$ & & 16020.55168 & $1.721 \mathrm{p}$ \\
\hline 11 & 4 & 815522.66275 & $1.082 \mathrm{p}, \mathrm{a}$ & & 16025.85291 & $4.001 \mathrm{a}$ \\
\hline 11 & 5 & 6 & & $16088.09053 \quad 3.431 \mathrm{p}$ & & \\
\hline 11 & 6 & 5 & & $16215.79012 \quad 3.991 \mathrm{p}$ & & \\
\hline 12 & 0 & 1215279.02333 & $0.893 \mathrm{p}, \mathrm{a}$ & & 15772.12831 & $1.332 \mathrm{p}, \mathrm{q}$ \\
\hline 12 & 1 & 12 & & & 15772.13341 & $1.33 \mathrm{~d}$ \\
\hline 12 & 1 & 1115481.05772 & $1.612 \mathrm{a}$ & & 15971.19281 & $2.082 \mathrm{p}, \mathrm{a}$ \\
\hline 12 & 2 & 1115481.53213 & $1.001 \mathrm{p}$ & & 15971.23345 & $4.001 \mathrm{a}$ \\
\hline 12 & 2 & 10 & & & 16144.72788 & $4.001 \mathrm{a}$ \\
\hline 12 & 3 & 10 & & & 16144.95387 & $4.001 \mathrm{a}$ \\
\hline 12 & 3 & 9 & & & 16289.91561 & $1.001 \mathrm{p}$ \\
\hline 12 & 4 & 8 & & & 16401.81517 & $4.001 \mathrm{a}$ \\
\hline 13 & 0 & 13 & & & 16004.43287 & $4.00 \mathrm{~d}$ \\
\hline 13 & 1 & 1315510.91991 & $1.493 \mathrm{p}, \mathrm{a}$ & & 16004.43287 & $4.001 \mathrm{a}$ \\
\hline 13 & 1 & 12 & & & 16220.93988 & $4.00 \mathrm{~d}$ \\
\hline 13 & 2 & 12 & & & 16220.93988 & $4.001 \mathrm{a}$ \\
\hline$J$ & $K_{a}$ & 061 or & $10^{-} 6$ & 160 or $10^{+} 6$ & 080 or & 008 \\
\hline 5 & 5 & 114066.85331 & $1.001 \mathrm{p}$ & & & \\
\hline 6 & 0 & 613048.53117 & $1.171 \mathrm{a}$ & & & \\
\hline 6 & 1 & 513220.75337 & $5.041 \mathrm{a}$ & & & \\
\hline 6 & 2 & 413371.63534 & $5.021 \mathrm{a}$ & & & \\
\hline 6 & 3 & 4 & & & 13024.54590 & 0.7021 \\
\hline
\end{tabular}




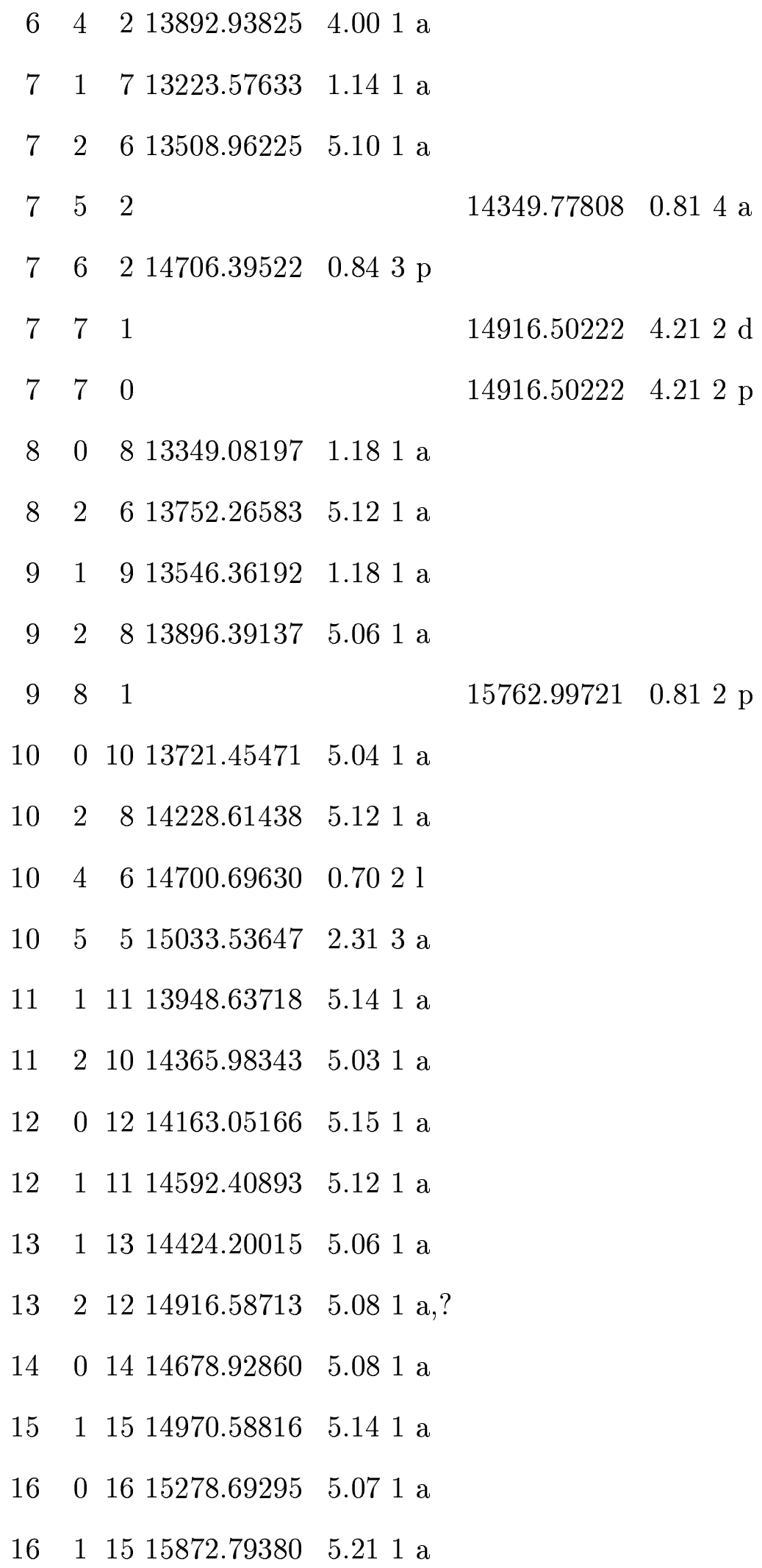


TABLE X.: Term values for the $4 \nu+\delta$ polyad of $\mathrm{H}_{2}{ }^{16} \mathrm{O}$.

\begin{tabular}{|c|c|c|c|c|c|c|c|}
\hline \multicolumn{2}{|c|}{$J K_{a} K_{c}$} & \multicolumn{2}{|c|}{151 or $20^{-} 5$} & \multicolumn{2}{|c|}{330 or $30^{+} 3$} & \multicolumn{2}{|c|}{132 or $21^{+} 3$} \\
\hline 0 & 0 & 0 & & 15108.23862 & $1.001 \mathrm{p}$ & & \\
\hline 1 & 1 & 114719.13198 & $2.012 \mathrm{p}, \mathrm{a}$ & 15152.36471 & $4.001 \mathrm{a}$ & & \\
\hline 2 & 0 & 214716.57835 & $2.752 \mathrm{p}, \mathrm{a}$ & & & & \\
\hline 2 & 1 & 2 & & 15191.73685 & $2.832 \mathrm{a}$ & & \\
\hline 2 & 1 & 114778.91519 & $4.001 \mathrm{a}$ & & & 15480.29062 & $2.832 \mathrm{a}$ \\
\hline 2 & 2 & 114895.73140 & $4.001 \mathrm{a}$ & & & & \\
\hline 2 & 2 & 014896.33058 & $2.832 \mathrm{a}$ & & & & \\
\hline 3 & 0 & 314783.28317 & $4.001 \mathrm{a}$ & 15239.24273 & $2.832 \mathrm{a}$ & & \\
\hline 3 & 1 & 314817.98633 & $2.832 \mathrm{a}$ & 15249.47110 & $2.102 \mathrm{p}, \mathrm{a}$ & & \\
\hline 3 & 1 & 214857.87588 & $4.001 \mathrm{a}$ & & & & \\
\hline 3 & 2 & 214965.73332 & $4.001 \mathrm{a}$ & & & & \\
\hline 3 & 2 & 1 & & 15347.56741 & $1.001 \mathrm{p}$ & & \\
\hline 4 & 0 & 414869.02104 & $3.572 \mathrm{p}, \mathrm{a}$ & 15320.80404 & $1.001 \mathrm{p}$ & & \\
\hline 4 & 1 & 414896.41087 & $1.091 \mathrm{p}$ & 15326.50585 & $3.001 \mathrm{p}$ & & \\
\hline 4 & 1 & 314961.98601 & $4.001 \mathrm{a}$ & & & & \\
\hline 4 & 2 & 3 & & 15432.32911 & $0.972 \mathrm{p}, \mathrm{a}$ & & \\
\hline 4 & 2 & 215066.57724 & $4.001 \mathrm{a}$ & & & 15717.25289 & $2.832 \mathrm{a}$ \\
\hline 4 & 3 & 2 & & 15551.10627 & $0.824 \mathrm{p}, \mathrm{a}$ & & \\
\hline 4 & 3 & 115246.99018 & $4.001 \mathrm{a}$ & & & & \\
\hline 4 & 4 & 0 & & 15703.09934 & $0.972 \mathrm{p}, \mathrm{a}$ & & \\
\hline 5 & 0 & 5 & & & & 15689.17364 & $4.001 \mathrm{a}$ \\
\hline 5 & 1 & 514993.41569 & $3.332 \mathrm{p}, \mathrm{a}$ & & & & \\
\hline 5 & 1 & 4 & & & & 15780.13470 & $1.001 \mathrm{p}$ \\
\hline 5 & 2 & 415172.66620 & $4.001 \mathrm{a}$ & & & & \\
\hline
\end{tabular}




\begin{tabular}{|c|c|c|c|c|c|c|c|}
\hline 5 & 3 & 2 & & 15671.78670 & $3.432 \mathrm{p}, \mathrm{a}$ & 15942.01934 & $2.313 \mathrm{a}$ \\
\hline 5 & 4 & 2 & & 15820.80639 & $3.711 \mathrm{a}$ & 16095.91286 & $4.001 \mathrm{a}$ \\
\hline 5 & 4 & 1 & & 15820.59209 & $3.812 \mathrm{p}, \mathrm{a}$ & 16095.37613 & $2.832 \mathrm{a}$ \\
\hline 5 & 5 & 115856.19065 & $4.001 \mathrm{a}$ & & & 16278.77330 & $4.00 \mathrm{~d}$ \\
\hline 5 & 5 & 0 & & & & 16278.77330 & $4.001 \mathrm{a}$ \\
\hline 6 & 0 & 615093.48361 & $2.832 \mathrm{a}$ & & & & \\
\hline 6 & 1 & 6 & & 15534.72887 & $0.712 \mathrm{p}$ & 15804.35750 & $4.001 \mathrm{a}$ \\
\hline 6 & 2 & 5 & & 15673.97097 & $4.001 \mathrm{a}$ & & \\
\hline 6 & 2 & 4 & & 15729.27160 & $4.001 \mathrm{a}$ & & \\
\hline 6 & 3 & 4 & & 15810.08174 & $4.001 \mathrm{a}$ & 16078.93327 & $0.943 \mathrm{p}, \mathrm{a}$ \\
\hline 6 & 3 & 3 & & 15816.47694 & $4.001 \mathrm{a}$ & & \\
\hline 6 & 4 & 3 & & 15961.40658 & $0.972 \mathrm{p}, \mathrm{a}$ & 16228.19445 & $4.001 \mathrm{a}$ \\
\hline 6 & 4 & 215743.98230 & $0.983 \mathrm{p}, \mathrm{q}$ & 15961.74632 & $0.972 \mathrm{p}, \mathrm{a}$ & 16238.69138 & $4.001 \mathrm{a}$ \\
\hline 6 & 5 & 216137.05673 & $0.992 \mathrm{p}$ & & & 16420.72948 & $4.001 \mathrm{a}$ \\
\hline 6 & 5 & 1 & & & & 16420.72948 & $4.00 \mathrm{~d}$ \\
\hline 7 & 0 & 715230.99482 & $2.231 \mathrm{p}$ & & & & \\
\hline 7 & 2 & 6 & & 15824.29105 & $4.001 \mathrm{a}$ & & \\
\hline 7 & 2 & 5 & & 15907.62497 & $0.962 \mathrm{p}, \mathrm{a}$ & & \\
\hline 7 & 3 & 515667.08066 & $1.001 \mathrm{a}$ & 15970.71247 & $0.712 \mathrm{p}$ & & \\
\hline 7 & 3 & 4 & & 15986.99743 & $0.962 \mathrm{p}, \mathrm{a}$ & 16263.85238 & $4.001 \mathrm{a}$ \\
\hline 7 & 4 & 4 & & 16125.40475 & $4.001 \mathrm{a}$ & & \\
\hline 7 & 4 & 315898.06388 & $0.992 \mathrm{p}, \mathrm{q}$ & 16127.44478 & $0.875 \mathrm{p}, \mathrm{a}$ & 16405.98156 & $4.001 \mathrm{a}$ \\
\hline 7 & 5 & 3 & & & & 16586.72077 & $1.742 \mathrm{p}, \mathrm{a}$ \\
\hline 7 & 5 & 2 & & & & 16586.38767 & $4.001 \mathrm{a}$ \\
\hline 8 & 1 & 8 & & 15800.13549 & $1.001 \mathrm{p}$ & & \\
\hline 8 & 3 & 5 & & 16195.84113 & $3.072 \mathrm{p}, \mathrm{a}$ & & \\
\hline 8 & 4 & 5 & & 16312.26484 & $4.001 \mathrm{a}$ & & \\
\hline
\end{tabular}




\begin{tabular}{|c|c|c|c|c|c|c|c|}
\hline \multicolumn{2}{|c|}{$J K_{a} K_{c}$} & \multicolumn{2}{|c|}{231 or $30^{-} 3$} & \multicolumn{2}{|c|}{033 or $21^{-} 3$} & \multicolumn{2}{|c|}{212 or $31^{+} 1$} \\
\hline 0 & 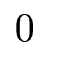 & 015119.02885 & $7.001 \mathrm{a}$ & 15534.70935 & $10.001 \mathrm{a}$ & 15742.79512 & $15.001 \mathrm{a}$ \\
\hline 1 & 0 & 115141.86103 & $3.581 \mathrm{a}$ & 15557.98343 & $4.001 \mathrm{a}$ & 15765.24966 & $2.282 \mathrm{a}$ \\
\hline 1 & 1 & 115162.67370 & $2.213 \mathrm{p}, \mathrm{a}$ & 15576.90894 & $2.492 \mathrm{a}$ & 15778.80541 & $2.732 \mathrm{a}$ \\
\hline 1 & 1 & 015168.82595 & $2.632 \mathrm{a}$ & 15583.25973 & $4.001 \mathrm{a}$ & 15784.29882 & $2.532 \mathrm{a}$ \\
\hline 2 & 0 & 215186.27952 & $1.833 \mathrm{p}, \mathrm{a}$ & 15603.14575 & $2.402 \mathrm{a}$ & 15808.71862 & $0.933 \mathrm{p}, \mathrm{a}$ \\
\hline 2 & 1 & 215202.22169 & $1.654 \mathrm{p}, \mathrm{a}$ & 15617.16327 & $2.004 \mathrm{a}$ & 15818.15211 & $1.804 \mathrm{a}$ \\
\hline 2 & 1 & 115220.62519 & $1.794 \mathrm{p}, \mathrm{a}$ & 15636.14774 & $2.722 \mathrm{a}$ & 15834.62511 & $2.832 \mathrm{a}$ \\
\hline 2 & 2 & 115282.54522 & $2.242 \mathrm{a}$ & 15691.54146 & $2.832 \mathrm{a}$ & 15874.73003 & $1.904 \mathrm{a}$ \\
\hline 2 & 2 & 015283.85782 & $3.212 \mathrm{a}$ & 15692.88763 & $2.462 \mathrm{a}$ & 15876.03988 & $2.313 \mathrm{a}$ \\
\hline 3 & 0 & 315251.11145 & $2.502 \mathrm{a}$ & 15667.80935 & $3.801 \mathrm{a}$ & 15870.96057 & $1.973 \mathrm{a}$ \\
\hline 3 & 1 & 315260.87528 & $1.675 \mathrm{p}, \mathrm{a}$ & 15676.69427 & $2.033 \mathrm{a}$ & 15876.38809 & $0.703 \mathrm{p}, \mathrm{a}$ \\
\hline 3 & 1 & 215297.39650 & $1.643 \mathrm{p}, \mathrm{a}$ & 15714.37823 & $3.711 \mathrm{a}$ & 15909.02558 & $2.133 \mathrm{a}$ \\
\hline 3 & 2 & 215351.26489 & $1.496 \mathrm{p}, \mathrm{a}$ & 15761.61545 & $2.562 \mathrm{a}$ & 15941.97110 & $2.832 \mathrm{a}$ \\
\hline 3 & 2 & 115356.78540 & $1.953 \mathrm{a}$ & 15767.88548 & $2.832 \mathrm{a}$ & 15947.92814 & $2.153 \mathrm{a}$ \\
\hline 3 & 3 & 115465.53525 & $1.394 \mathrm{a}$ & 15866.44711 & $2.562 \mathrm{a}$ & 16021.44265 & $0.973 \mathrm{p}, \mathrm{a}$ \\
\hline 3 & 3 & 015465.54826 & $2.252 \mathrm{a}$ & 15866.63465 & $3.801 \mathrm{a}$ & 16020.77763 & $1.944 \mathrm{a}$ \\
\hline 4 & 0 & 415332.07625 & $1.565 \mathrm{p}, \mathrm{a}$ & 15749.83401 & $2.562 \mathrm{a}$ & 15950.15376 & $2.263 \mathrm{a}$ \\
\hline 4 & 1 & 415338.02925 & $1.624 \mathrm{p}, \mathrm{a}$ & 15754.84502 & $3.471 \mathrm{a}$ & 15952.84728 & $2.123 \mathrm{a}$ \\
\hline 4 & 1 & 315397.86209 & $0.923 \mathrm{p}, \mathrm{a}$ & 15816.37772 & $2.562 \mathrm{a}$ & 16005.94497 & $2.313 \mathrm{a}$ \\
\hline 4 & 2 & 315441.56162 & $2.183 \mathrm{a}$ & 15853.81122 & $2.832 \mathrm{a}$ & 16030.48704 & $0.895 \mathrm{p}, \mathrm{a}$ \\
\hline 4 & 2 & 215456.33300 & $1.474 \mathrm{a}$ & 15870.51496 & $1.954 \mathrm{a}$ & 16049.08728 & $1.943 \mathrm{a}$ \\
\hline 4 & 3 & 215560.63867 & $0.786 \mathrm{a}$ & 15961.22521 & $2.832 \mathrm{a}$ & 16113.26821 & $0.914 \mathrm{p}, \mathrm{a}$ \\
\hline 4 & 3 & 115559.94088 & $1.534 \mathrm{p}, \mathrm{a}$ & 15962.40083 & $2.253 \mathrm{a}$ & 16111.43461 & $1.804 \mathrm{a}$ \\
\hline 4 & 4 & 115707.53893 & $3.502 \mathrm{a}$ & 16098.73528 & $4.001 \mathrm{a}$ & 16223.11247 & $2.622 \mathrm{a}$ \\
\hline 4 & 4 & 015707.55998 & $1.493 \mathrm{a}$ & 16098.77645 & $2.402 \mathrm{a}$ & & \\
\hline 5 & 0 & 515429.90460 & $2.602 \mathrm{a}$ & 15848.25934 & $4.001 \mathrm{a}$ & 16045 & $3 \mathrm{p}, \mathrm{a}$ \\
\hline
\end{tabular}




\begin{tabular}{|c|c|c|c|c|c|c|c|}
\hline & 1 & 515433.18584 & $2.033 \mathrm{a}$ & 15851.56026 & $2.482 \mathrm{a}$ & 16046.94424 & $2.242 \mathrm{p}, \mathrm{c}$ \\
\hline & 1 & 415520.18512 & $2.153 \mathrm{a}$ & 15939.95333 & $4.001 \mathrm{a}$ & 16123.13494 & $2.283 \mathrm{a}$ \\
\hline & 2 & 415552.25513 & $1.824 \mathrm{a}$ & 15967.29624 & $2.672 \mathrm{a}$ & 16139.39450 & $2.832 \mathrm{a}$ \\
\hline & 2 & 315581.80455 & $2.832 \mathrm{a}$ & 16000.17862 & $2.832 \mathrm{a}$ & 16172.02242 & $2.313 \mathrm{a}$ \\
\hline & 3 & 315676.33609 & $1.425 \mathrm{a}$ & 16079.47769 & $2.313 \mathrm{a}$ & 16228.29730 & $4.001 \mathrm{a}$ \\
\hline & 3 & 215679.11826 & $2.223 \mathrm{a}$ & 16083.37974 & $0.972 \mathrm{p}, \mathrm{a}$ & 16238.90829 & $1.783 \mathrm{a}$ \\
\hline & 4 & 215824.59570 & $1.575 \mathrm{a}$ & 16217.88631 & $1.795 \mathrm{a}$ & 16340.50605 & $3.581 \mathrm{a}$ \\
\hline & 4 & 115824.78453 & $1.894 \mathrm{a}$ & 16218.42827 & $4.001 \mathrm{a}$ & 16340.07710 & $3.651 \mathrm{a}$ \\
\hline & 5 & 116000.29808 & $1.453 \mathrm{a}$ & 16383.59705 & $2.702 \mathrm{a}$ & 16473.15255 & 4.001 \\
\hline & 5 & 016000.19588 & $4.001 \mathrm{a}$ & 16383.59705 & $2.70 \mathrm{~d}$ & 16473.15255 & $4.001 \mathrm{a}$ \\
\hline & 0 & 615543.99147 & $1.873 \mathrm{p}, \mathrm{a}$ & 15962.80983 & $2.562 \mathrm{a}$ & 16157.74512 & $4.001 \mathrm{a}$ \\
\hline & 1 & 615546.45308 & $0.933 \mathrm{p}, \mathrm{a}$ & 15964.08597 & $4.001 \mathrm{a}$ & 16158.41336 & $2.702 \mathrm{a}$ \\
\hline & 1 & 515662.01956 & $2.562 \mathrm{a}$ & 16082.49937 & $2.253 \mathrm{a}$ & 16258.14942 & $2.832 \mathrm{a}$ \\
\hline & 2 & 515683.54514 & $2.722 \mathrm{a}$ & 16101.62855 & $4.001 \mathrm{a}$ & 16267.83339 & $0.943 \mathrm{p}, \mathrm{a}$ \\
\hline & 2 & 415729.52706 & $1.964 \mathrm{a}$ & 16156.12034 & $2.562 \mathrm{a}$ & 16320.49884 & $0.712 \mathrm{p}$ \\
\hline & 3 & 415815.99937 & $2.702 \mathrm{a}$ & 16220.76328 & $4.001 \mathrm{a}$ & 16366.29320 & $2.004 \mathrm{a}$ \\
\hline & 3 & 315824.10145 & $1.834 \mathrm{a}$ & 16241.96911 & $2.313 \mathrm{a}$ & 16382.06111 & $4.001 \mathrm{a}$ \\
\hline & 4 & 315965.17216 & $2.243 \mathrm{a}$ & 16362.85769 & $2.832 \mathrm{a}$ & 16482.57477 & $2.622 \mathrm{a}$ \\
\hline & 4 & 215966.23491 & $1.536 \mathrm{a}$ & 16361.63593 & $4.001 \mathrm{a}$ & 16481.20675 & $4.001 \mathrm{a}$ \\
\hline & 5 & 216140.71934 & 2.56 & & & 1661 & $0.972 \mathrm{p}, \mathrm{a}$ \\
\hline & 5 & 11614 & 1.6 & & & 166 & $0.972 \mathrm{p}, \mathrm{a}$ \\
\hline & 6 & 1 & & 16714.34818 & $4.00 \mathrm{~d}$ & 340 & $4.001 \mathrm{a}$ \\
\hline & 6 & 0 & & 16714.35404 & $0.972 \mathrm{p}, \mathrm{a}$ & & \\
\hline & 0 & 715674.75794 & $2.832 \mathrm{a}$ & 16093.95227 & $1.874 \mathrm{a}$ & 16286.44272 & $2.673 \mathrm{a}$ \\
\hline & 1 & 715667.10373 & $3.332 \mathrm{a}$ & 16094.96988 & $2.722 \mathrm{a}$ & 16286.76548 & $4.001 \mathrm{a}$ \\
\hline & 1 & 615820.96593 & $4.001 \mathrm{a}$ & 16241.78709 & $4.001 \mathrm{a}$ & 16409.51606 & $2.832 \mathrm{a}$ \\
\hline & 2 & 615833.72645 & $2.832 \mathrm{a}$ & 16249.876 & 2.313 & & \\
\hline
\end{tabular}




\begin{tabular}{|c|c|c|c|c|c|c|c|}
\hline & 2 & 515920.09181 & $4.001 \mathrm{a}$ & 16333.98935 & $4.001 \mathrm{a}$ & & \\
\hline 7 & 3 & 515977.47103 & $1.765 \mathrm{a}$ & 16385.32208 & $2.223 \mathrm{a}$ & & \\
\hline 7 & 3 & 415994.43915 & $0.972 \mathrm{p}, \mathrm{a}$ & & & 16552.91665 & $0.943 \mathrm{p}, \mathrm{a}$ \\
\hline 7 & 4 & 416129.01446 & $1.914 \mathrm{a}$ & 16529.72361 & $2.832 \mathrm{a}$ & & \\
\hline 7 & 4 & 316133.07452 & $2.832 \mathrm{a}$ & & & 16646.14831 & $4.001 \mathrm{a}$ \\
\hline 7 & 5 & 316304.38186 & $1.813 \mathrm{a}$ & 16695.15535 & $4.001 \mathrm{a}$ & & \\
\hline 7 & 5 & 216304.60291 & $2.253 \mathrm{a}$ & 16695.48299 & $4.001 \mathrm{a}$ & 16776.97901 & $4.001 \mathrm{a}$ \\
\hline 8 & 0 & 815818.40259 & $3.801 \mathrm{a}$ & 16242.43220 & $4.001 \mathrm{a}$ & 16431.51001 & $4.001 \mathrm{a}$ \\
\hline 8 & 1 & 815818.62909 & $4.001 \mathrm{a}$ & & & & \\
\hline 8 & 1 & 715997.27346 & $2.702 \mathrm{a}$ & 16416.98136 & $3.651 \mathrm{a}$ & & \\
\hline 8 & 2 & 716002.45300 & $2.832 \mathrm{a}$ & 16421.70629 & $0.972 \mathrm{p}, \mathrm{a}$ & 16574.90707 & $4.001 \mathrm{a}$ \\
\hline 8 & 2 & 616119.24401 & $2.832 \mathrm{a}$ & 16537.39580 & $2.832 \mathrm{a}$ & & \\
\hline 8 & 3 & 6 & & & & 16701.90270 & $4.001 \mathrm{a}$ \\
\hline 8 & 3 & 516201.71978 & $1.864 \mathrm{p}, \mathrm{a}$ & 16621.17857 & $2.832 \mathrm{a}$ & & \\
\hline 8 & 4 & 5 & & & & 16817.93168 & $4.001 \mathrm{a}$ \\
\hline 8 & 4 & 416324.61514 & $2.213 \mathrm{a}$ & & & & \\
\hline 8 & 5 & 416584.12592 & $4.001 \mathrm{a}$ & & & 16963.13249 & $0.972 \mathrm{p}, \mathrm{a}$ \\
\hline 8 & 5 & 316584.86982 & $2.832 \mathrm{a}$ & & & & \\
\hline 9 & 0 & 915984.11835 & $4.001 \mathrm{a}$ & & & & \\
\hline 9 & 1 & 915982.61915 & $4.001 \mathrm{a}$ & 16407.19440 & $4.001 \mathrm{a}$ & & \\
\hline 9 & 2 & 816189.34011 & $2.832 \mathrm{a}$ & 16610.57977 & $4.001 \mathrm{a}$ & & \\
\hline 9 & 3 & 716361.01813 & $0.972 \mathrm{p}, \mathrm{a}$ & 16776.81005 & $4.001 \mathrm{a}$ & & \\
\hline 9 & 4 & 616524.30483 & $0.972 \mathrm{p}, \mathrm{a}$ & & & & \\
\hline & $K_{a} I$ & 410 or & $40^{+} 1$ & 311 or & $40^{-} 1$ & 113 or & $31^{-1}$ \\
\hline 0 & 0 & 015344.50322 & $8.001 \mathrm{a}$ & 15347.95645 & $0.501 \mathrm{a}$ & 15832.76485 & $2.001 \mathrm{a}$ \\
\hline 1 & 0 & 115366.98790 & $1.432 \mathrm{a}$ & 15370.42150 & $0.501 \mathrm{a}$ & 15855.47597 & $2.831 \mathrm{a}$ \\
\hline 1 & 1 & 115380.62417 & $2.672 \mathrm{a}$ & 15383.69525 & $0.254 \mathrm{p}, \mathrm{a}$ & 15867.77330 & $0.852 \mathrm{a}$ \\
\hline
\end{tabular}


$\begin{array}{lllllllll}1 & 1 & 0 & 15386.05272 & 1.512 \mathrm{a} & 15389.15717 & 0.403 \mathrm{p}, \mathrm{a} & 15873.40115 & 1.832 \mathrm{a}\end{array}$

$\begin{array}{lllllllll}2 & 0 & 2 & 15410.60018 & 2.102 \mathrm{a} & 15413.94821 & 0.214 \mathrm{a} & 15899.31984 & 0.674 \mathrm{a}\end{array}$

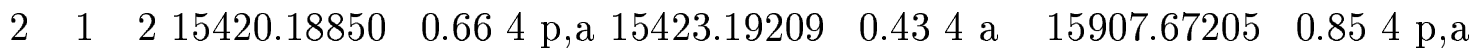

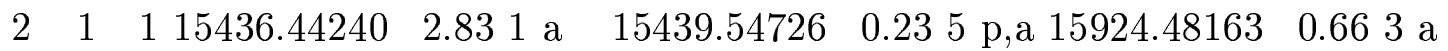

$\begin{array}{lllllllll}2 & 2 & 1 & 15476.57956 & 0.902 \mathrm{a} & 15479.13074 & 0.443 \mathrm{a} & 15961.01160 & 1.773 \mathrm{a}\end{array}$

$\begin{array}{llllllllll}2 & 2 & 0 & 15477.91613 & 1.773 \mathrm{a} & 15480.50011 & 0.263 \mathrm{a} & 15962.53890 & 0.833 \mathrm{a}\end{array}$

$\begin{array}{lllllllll}3 & 0 & 3 & 15473.10388 & 0.833 \mathrm{a} & 15476.22159 & 0.354 \mathrm{a} & 15961.82559 & 1.353 \mathrm{a}\end{array}$

$\begin{array}{lllllllll}3 & 1 & 3 & 15478.85356 & 0.893 \mathrm{a} & 15481.69525 & 0.215 \mathrm{p}, \mathrm{a} & 15966.76100 & 0.814 \mathrm{a}\end{array}$

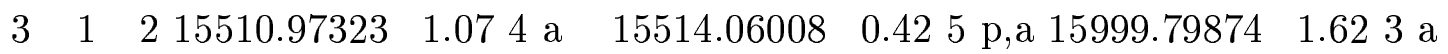

$\begin{array}{lllllllll}3 & 2 & 2 & 15544.11327 & 2.242 \mathrm{a} & 15546.63386 & 0.285 \mathrm{a} & 16030.92361 & 1.045 \mathrm{a}\end{array}$

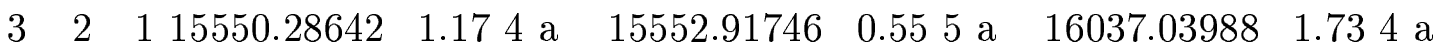

$\begin{array}{llllllllll}3 & 3 & 1 & 15624.32700 & 2.022 \mathrm{a} & 15626.52932 & 0.283 \mathrm{a} & 16103.97257 & 0.902 \mathrm{a}\end{array}$

$\begin{array}{lllllllll}3 & 3 & 0 & 15624.53870 & 0.903 \mathrm{a} & 15626.74667 & 0.484 \mathrm{a} & 16104.25106 & 1.902 \mathrm{a}\end{array}$

$\begin{array}{lllllllll}4 & 0 & 4 & 15552.63973 & 1.632 \mathrm{a} & 15556.89537 & 0.286 \mathrm{p}, \mathrm{a} & 16041.22340 & 0.785 \mathrm{a}\end{array}$

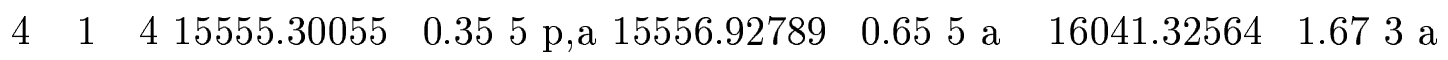

$\begin{array}{lllllllll}4 & 1 & 3 & 15608.16415 & 2.352 \mathrm{a} & 15611.17552 & 0.396 \mathrm{p}, \mathrm{a} & 16097.53662 & 0.795 \mathrm{a}\end{array}$

$\begin{array}{lllllllll}4 & 2 & 3 & 15632.91533 & 0.835 \mathrm{a} & 15635.39023 & 0.644 \mathrm{a} & 16123.00254 & 1.793 \mathrm{a}\end{array}$

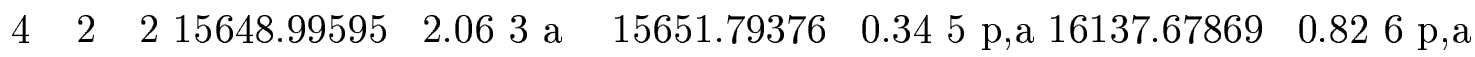

$\begin{array}{lllllllll}4 & 3 & 2 & 15716.74455 & 1.364 \mathrm{a} & 15719.01593 & 0.775 \mathrm{a} & 16195.87052 & 1.953 \mathrm{a}\end{array}$

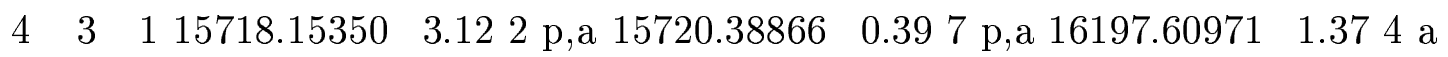

$\begin{array}{llllllllll}4 & 4 & 1 & 15826.08727 & 0.853 & \text { p,a } & 15828.14102 & 2.413 \mathrm{a} & 16296.80927 & 2.562 \mathrm{a}\end{array}$

$\begin{array}{llllllllll}4 & 4 & 0 & 15826.10645 & 2.401 \mathrm{a} & 15828.16709 & 0.814 \mathrm{a} & 16296.87751 & 1.622 \mathrm{a}\end{array}$

$\begin{array}{lllllllll}5 & 0 & 5 & 15648.73232 & 0.893 \mathrm{a} & 15651.84432 & 0.443 \mathrm{a} & 16137.10986 & 1.632 \mathrm{a}\end{array}$

$\begin{array}{llllllllll}5 & 1 & 5 & 15649.33496 & 1.854 \mathrm{a} & 15652.54701 & 0.345 \mathrm{a} & 16137.67441 & 0.82 & 3 \mathrm{a}\end{array}$

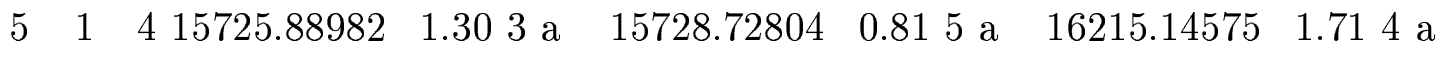

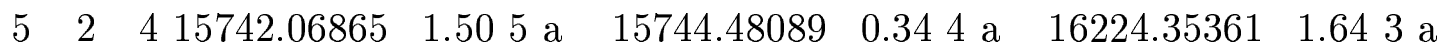

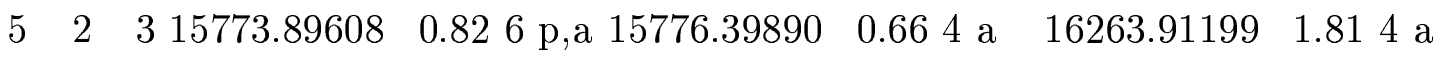




$\begin{array}{llllllllll}5 & 3 & 3 & 15832.06331 & 1.914 \mathrm{a} & 15834.22396 & 0.406 \mathrm{p}, \mathrm{a} & 16310.70316 & 1.455 \mathrm{a} \\ 5 & 3 & 2 & 15837.14989 & 1.324 \mathrm{a} & 15839.47484 & 1.165 \mathrm{a} & 16316.58954 & 1.714 \mathrm{a} \\ 5 & 4 & 2 & 15942.40037 & 2.952 \mathrm{a} & 15944.63546 & 0.785 \mathrm{a} & 16412.72464 & 0.874 \mathrm{p}, \mathrm{a} \\ 5 & 4 & 1 & 15942.61344 & 1.612 \mathrm{a} & 15944.79091 & 1.275 \mathrm{a} & 16413.18056 & 2.243 \mathrm{a} \\ 5 & 5 & 1 & 16088.89301 & 0.93 & \mathrm{~d} & 16090.30022 & 1.723 \mathrm{a} & 16538.73757 & 2.312 \mathrm{a} \\ 5 & 5 & 0 & 16088.89301 & 0.933 \mathrm{p}, \mathrm{a} & 16090.35913 & 2.622 \mathrm{a} & 16538.73757 & 2.31 & \mathrm{~d} \\ 6 & 0 & 6 & & & 15764.62917 & 0.254 \mathrm{p}, \mathrm{a} & 16249.65526 & 0.913 \mathrm{a} \\ 6 & 1 & 6 & 15761.36182 & 0.874 \mathrm{a} & 15764.48003 & 0.863 \mathrm{p}, \mathrm{a} & 16250.00800 & 1.773 \mathrm{a} \\ 6 & 1 & 5 & 15861.90447 & 2.543 \mathrm{a} & 15864.49335 & 0.425 \mathrm{p}, \mathrm{a} & 16350.12340 & 1.295 \mathrm{a} \\ 6 & 2 & 5 & 15870.65325 & 0.865 \mathrm{a} & 15872.97962 & 1.144 \mathrm{a} & 16354.06954 & 1.924 \mathrm{a} \\ 6 & 2 & 415922.23383 & 2.223 \mathrm{a} & 15924.82162 & 0.435 \mathrm{a} & 16413.84951 & 1.394 \mathrm{a} \\ 6 & 3 & 415969.78907 & 1.604 \mathrm{a} & 15971.89864 & 1.175 \mathrm{p}, \mathrm{a} & 16447.90462 & 2.063 \mathrm{a} \\ 6 & 3 & 3 & 15982.68349 & 2.223 \mathrm{a} & 15985.18174 & 0.735 \mathrm{a} & 16461.49156 & 2.113 \mathrm{a} \\ 6 & 4 & 3 & 16082.11500 & 1.833 \mathrm{a} & 16084.03108 & 1.356 \mathrm{a} & 16551.91806 & 2.273 \mathrm{a} \\ 6 & 4 & 2 & 16083.35476 & 4.001 \mathrm{a} & 16085.47168 & 1.055 \mathrm{a} & 16553.91617 & 1.854 \mathrm{a} \\ 6 & 5 & 2 & 16230.20654 & 3.331 \mathrm{a} & 16231.26018 & 0.583 \mathrm{p} & & \\ 6 & 5 & 116230.21274 & 3.581 \mathrm{a} & 16231.88018 & 1.344 \mathrm{a} & 16678.41156 & 2.223 \mathrm{a} \\ 6 & 6 & 1 & 16344.16375 & 4.001 \mathrm{a} & & & & & \\ 6 & 6 & 0 & 16344.16375 & 4.00 & \mathrm{~d} & 16340.74971 & 2.062 \mathrm{a} & 16829.37862 & 3.801 \mathrm{a} \\ 7 & 0 & 715889.11915 & 1.632 \mathrm{a} & 15892.17041 & 0.903 \mathrm{a} & 16379.05680 & 1.943 \mathrm{a} \\ 7 & 1 & 715889.85220 & 3.471 \mathrm{a} & 15893.05950 & 0.443 \mathrm{a} & 16378.40874 & 1.483 \mathrm{a} \\ 7 & 1 & 6 & 16015.34108 & 1.833 \mathrm{a} & 16018.55096 & 0.903 \mathrm{a} & 16501.21005 & 2.702 \mathrm{a} \\ 7 & 2 & 6 & 16017.45937 & 2.832 \mathrm{a} & 16020.19736 & 0.804 \mathrm{a} & 16502.94749 & 0.834 \mathrm{p}, \mathrm{a} \\ 7 & 2 & 5 & 16092.06550 & 0.874 \mathrm{p}, \mathrm{a} & 16094.79460 & 1.753 \mathrm{a} & 16584.09427 & 2.702 \mathrm{a} \\ 7 & 3 & 5 & 16128.00798 & 3.471 \mathrm{a} & 16131.55136 & 1.244 \mathrm{a} & 16608.11151 & 3.322 \mathrm{a} \\ 7 & 3 & 4 & 16154.91101 & 1.904 \mathrm{a} & 16157.65961 & 1.863 \mathrm{a} & 16651.30477 & 4.001 \mathrm{a} \\ 7 & 4 & 4 & 16245.08843 & 3.581 \mathrm{a} & 16246.96172 & 1.415 \mathrm{a} & & \end{array}$




$\begin{array}{llllllllll}7 & 4 & 316248.58190 & 2.062 \mathrm{a} & 16250.90140 & 1.764 \mathrm{a} & 16720.07747 & 2.832 \mathrm{a} \\ 7 & 5 & 316395.03348 & 4.001 \mathrm{a} & 16395.86961 & 1.764 \mathrm{a} & 16841.85156 & 2.313 \mathrm{a} \\ 7 & 5 & 216395.02283 & 4.001 \mathrm{a} & 16398.18539 & 4.001 \mathrm{a} & & \\ 7 & 6 & 2 & & 16506.62219 & 1.774 \mathrm{a} & 16992.72137 & 4.001 \mathrm{a} \\ 7 & 6 & 116606.13546 & 0.943 \mathrm{p}, \mathrm{a} & & & & \\ 8 & 0 & 816035.76925 & 3.121 \mathrm{a} & 16038.79545 & 0.683 \mathrm{a} & 16524.55570 & 1.902 \mathrm{a} \\ 8 & 1 & 816035.87395 & 2.103 \mathrm{a} & 16039.06923 & 2.462 \mathrm{p}, \mathrm{a} & 16525.02324 & 4.001 \mathrm{a} \\ 8 & 1 & 7 & & 16182.97742 & 0.913 \mathrm{a} & 16668.48254 & 2.462 \mathrm{a} \\ 8 & 2 & 716177.54342 & 1.314 \mathrm{a} & 16184.65233 & 1.883 \mathrm{a} & 16668.82732 & 3.121 \mathrm{a} \\ 8 & 2 & 6 & & 16282.99373 & 0.835 \mathrm{p}, \mathrm{a} & 16777.37462 & 0.943 \mathrm{p}, \mathrm{a} \\ 8 & 3 & 616307.36578 & 2.153 \mathrm{a} & 16309.23515 & 2.153 \mathrm{a} & 16787.69670 & 2.832 \mathrm{a} \\ 8 & 3 & 516352.58514 & 4.001 \mathrm{a} & 16355.57987 & 1.485 \mathrm{p}, \mathrm{a} & 16847.24045 & 0.943 \mathrm{p}, \mathrm{a} \\ 8 & 4 & 516430.71191 & 0.943 \mathrm{p}, \mathrm{a} & 16433.25285 & 2.223 \mathrm{a} & 16902.02452 & 2.832 \mathrm{a} \\ 8 & 4 & 416440.06190 & 4.001 \mathrm{a} & 16442.02650 & 1.804 \mathrm{a} & & \\ 8 & 5 & 4 & & & & & & & \\ 8 & 5 & 3 & & & & & & & \end{array}$




\begin{tabular}{|c|c|c|c|c|c|c|}
\hline 10 & 110 & & 16381.10998 & $12.001 \mathrm{a}$ & 16868.05203 & $3.801 \mathrm{a}$ \\
\hline 10 & 19 & & 16566.38991 & $2.062 \mathrm{a}$ & 17051.05094 & $4.001 \mathrm{a}$ \\
\hline 10 & 29 & & 16567.03808 & $2.562 \mathrm{a}$ & 17051.17104 & $4.001 \mathrm{a}$ \\
\hline 10 & 28 & & & & 17205.48321 & $4.001 \mathrm{a}$ \\
\hline 10 & 38 & & 16726.42532 & $3.711 \mathrm{a}$ & & \\
\hline 10 & 37 & & 16819.08195 & $2.832 \mathrm{a}$ & & \\
\hline 10 & 47 & & 16870.07934 & $4.001 \mathrm{a}$ & & \\
\hline 11 & 011 & & 16577.33235 & $3.471 \mathrm{a}$ & & \\
\hline 11 & 111 & & 16577.41018 & $2.032 \mathrm{a}$ & 17064.34703 & $4.001 \mathrm{a}$ \\
\hline 11 & 110 & & 16784.40354 & $4.001 \mathrm{a}$ & 17267.00801 & $1.001 \mathrm{p}$ \\
\hline 11 & 210 & & 16782.97619 & $4.001 \mathrm{a}$ & 17267.71366 & $1.001 \mathrm{p}$ \\
\hline 11 & 38 & & & & 17570.84926 & $1.001 \mathrm{p}$ \\
\hline 12 & 012 & & 16790.30416 & $2.562 \mathrm{a}$ & & \\
\hline 12 & 112 & & 16790.16306 & $4.001 \mathrm{a}$ & & \\
\hline 12 & 111 & & 17013.36855 & $4.001 \mathrm{a}$ & & \\
\hline 12 & 211 & & 17018.46797 & $4.001 \mathrm{a}$ & & \\
\hline & $K_{c}$ & 071 or $10^{-} 7$ & 170 or & $10^{+7}$ & & \\
\hline
\end{tabular}

$\begin{array}{lllllll}0 & 0 & 0 & 13835.37235 & 1.001 \mathrm{a}\end{array}$

$\begin{array}{llllll}2 & 0 & 2 & 13904.45044 & 2.25 & 2 \mathrm{p}, \mathrm{a} \\ 13730.52961 & 1.322 \mathrm{p}, \mathrm{a}\end{array}$

$\begin{array}{lllll}3 & 0 & 3 & 13800.80450 & 1.072 \mathrm{p}\end{array}$

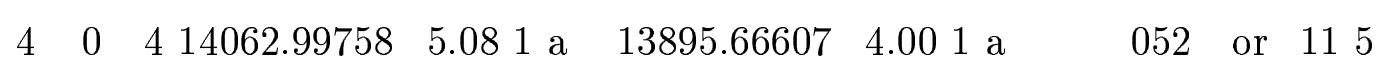

$\begin{array}{lllllll}5 & 2 & 3 & 15771.38705 & 0.712 \mathrm{p}\end{array}$

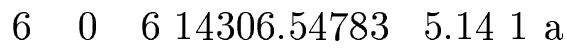

$\begin{array}{lll}6 & 3 & 4\end{array}$

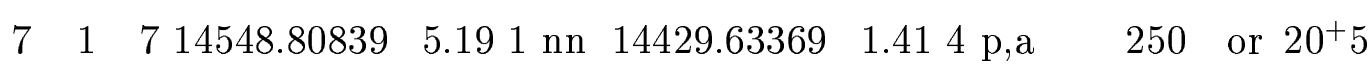
$\begin{array}{lll}7 & 5 & 2\end{array}$
$16301.36654 \quad 0.982 \mathrm{p}$

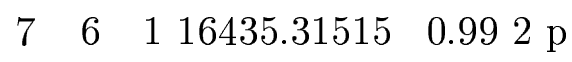




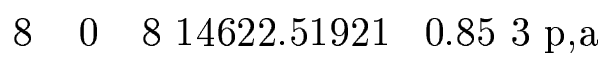

$\begin{array}{llllll}8 & 1 & 7 & 14945.11142 & 5.22 & 1 \mathrm{a}\end{array}$

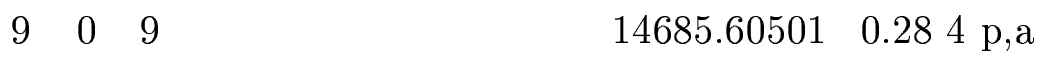

$9 \quad 1 \quad 9 \quad 14888.2005920 .041$ a

$\begin{array}{lllll}9 & 2 & 8 & 15323.81558 \quad 2.832 \text { a }\end{array}$

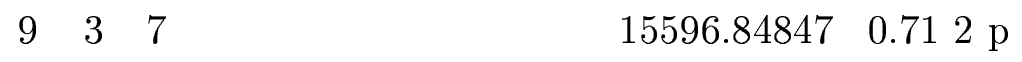

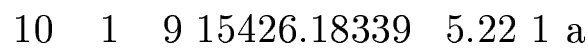

$\begin{array}{lllll}11 & 1 & 11 & 15303.07862 & 3.042 \mathrm{p}, \mathrm{a}\end{array}$ 
TABLE XI.: Term values for the $5 \nu$ polyad of $\mathrm{H}_{2}{ }^{16} \mathrm{O}$.

\begin{tabular}{|c|c|c|c|c|c|c|c|}
\hline \multicolumn{2}{|c|}{$J K_{a} K_{c}$} & \multicolumn{2}{|c|}{340 or $30^{+} 4$} & \multicolumn{2}{|c|}{241 or $30^{-} 4$} & \multicolumn{2}{|c|}{043 or $21^{-4}$} \\
\hline 1 & 0 & 1 & & 16569.09526 & $0.972 \mathrm{p}, \mathrm{a}$ & & \\
\hline 1 & 1 & 1 & & 16597.11831 & $2.832 \mathrm{a}$ & 17015.61174 & $10.001 \mathrm{r}$ \\
\hline 1 & 1 & 0 & & 16603.59042 & $4.001 \mathrm{a}$ & & \\
\hline 2 & 0 & 2 & & 16613.65053 & $0.943 \mathrm{p}, \mathrm{a}$ & & \\
\hline 2 & 1 & 2 & & 16636.53491 & $4.001 \mathrm{a}$ & & \\
\hline 2 & 1 & 1 & & 16655.68503 & $0.934 \mathrm{p}, \mathrm{a}$ & 17075.58937 & $3.712 \mathrm{a}$ \\
\hline 2 & 2 & 0 & & & & 17147.13460 & $4.001 \mathrm{a}$ \\
\hline 3 & 0 & 316665.70385 & $0.943 \mathrm{p}, \mathrm{a}$ & 16677.70615 & $4.001 \mathrm{a}$ & & \\
\hline 3 & 1 & 3 & & 16694.64287 & $0.943 \mathrm{p}, \mathrm{a}$ & 17114.69139 & $10.001 \mathrm{r}$ \\
\hline 3 & 1 & 216721.11141 & $2.832 \mathrm{a}$ & 16732.89302 & $4.001 \mathrm{a}$ & & \\
\hline 3 & 2 & 216795.43846 & $4.001 \mathrm{a}$ & & & & \\
\hline 3 & 2 & 116800.56607 & $4.001 \mathrm{a}$ & & & & \\
\hline 3 & 3 & 116937.39498 & $4.001 \mathrm{a}$ & 16943.37489 & $2.313 \mathrm{a}$ & 17343.26828 & $4.001 \mathrm{a}$ \\
\hline 3 & 3 & 0 & & 16943.58466 & $4.001 \mathrm{a}$ & & \\
\hline 4 & 0 & 416747.98666 & $4.001 \mathrm{a}$ & 16760.07704 & $4.001 \mathrm{a}$ & & \\
\hline 4 & 1 & 4 & & 16771.60172 & $4.001 \mathrm{a}$ & 17193.03469 & $2.832 \mathrm{a}$ \\
\hline 4 & 1 & 3 & & & & 17257.65673 & $2.313 \mathrm{a}$ \\
\hline 4 & 2 & 316885.90557 & $4.001 \mathrm{a}$ & & & & \\
\hline 4 & 2 & 2 & & 16907.96437 & $0.972 \mathrm{p}, \mathrm{a}$ & & \\
\hline 4 & 3 & 217032.09526 & $2.313 \mathrm{a}$ & 17038.41131 & $2.723 \mathrm{a}$ & 17438.46233 & $10.001 \mathrm{r}$ \\
\hline 4 & 3 & 117031.43432 & $4.001 \mathrm{a}$ & 17037.69236 & $2.313 \mathrm{a}$ & & \\
\hline 4 & 4 & 117210.45200 & $2.313 \mathrm{a}$ & 17213.06665 & $4.001 \mathrm{a}$ & & \\
\hline 4 & 4 & 017210.42490 & $4.001 \mathrm{a}$ & 17213.21648 & $4.001 \mathrm{a}$ & 17600.99425 & $4.001 \mathrm{a}$ \\
\hline 5 & 0 & 516846.93449 & $4.001 \mathrm{a}$ & & & & \\
\hline
\end{tabular}




\begin{tabular}{|c|c|c|c|c|c|c|c|}
\hline & 1 & 5 & & & & 17288.39518 & $4.001 \mathrm{a}$ \\
\hline 5 & 1 & 416947.01516 & $4.001 \mathrm{a}$ & 16958.92824 & $4.001 \mathrm{a}$ & 17383.72611 & $3.711 \mathrm{r}$ \\
\hline 5 & 2 & 416997.17102 & $4.001 \mathrm{a}$ & & & & \\
\hline 5 & 2 & 317023.11045 & $4.001 \mathrm{a}$ & & & & \\
\hline 5 & 3 & 317148.69224 & $2.313 \mathrm{a}$ & 17154.76719 & $2.313 \mathrm{a}$ & 17558.32850 & $4.001 \mathrm{a}$ \\
\hline 5 & 3 & 217149.30246 & $2.313 \mathrm{a}$ & & & & \\
\hline 5 & 4 & 2 & & 17330.28404 & $2.723 \mathrm{a}$ & & \\
\hline 5 & 4 & 117327.78235 & $2.313 \mathrm{a}$ & 17330.53108 & $2.313 \mathrm{a}$ & & \\
\hline 5 & 5 & 1 & & 17659.62435 & $10.001 \mathrm{r}$ & 17912.56035 & $10.001 \mathrm{r}$ \\
\hline 5 & 5 & 017657.78373 & $3.711 \mathrm{r}$ & 17659.62435 & $10.00 \mathrm{~d}$ & 17912.56035 & $10.00 \mathrm{~d}$ \\
\hline 6 & 0 & 6 & & & & 17399.11533 & $0.924 \mathrm{p}, \mathrm{a}$ \\
\hline 6 & 1 & 6 & & & & 17402.37546 & $10.001 \mathrm{r}$ \\
\hline 6 & 2 & 417172.63846 & $10.001 \mathrm{r}$ & 17184.49328 & $0.972 \mathrm{p}, \mathrm{a}$ & & \\
\hline 6 & 3 & 417288.06766 & $2.004 \mathrm{a}$ & 17294.26120 & $10.001 \mathrm{r}$ & & \\
\hline 6 & 3 & 317291.68555 & $4.001 \mathrm{a}$ & 17299.28973 & $2.195 \mathrm{a}$ & & \\
\hline 6 & 4 & 317468.33305 & $1.795 \mathrm{a}$ & 17470.74050 & $2.832 \mathrm{a}$ & & \\
\hline 6 & 4 & 217469.48095 & $3.711 \mathrm{r}$ & 17472.11039 & $2.313 \mathrm{a}$ & 17864.72533 & $4.001 \mathrm{a}$ \\
\hline 6 & 5 & 1 & & 17801.58394 & $2.832 \mathrm{a}$ & & \\
\hline 7 & 0 & 717090.64118 & $4.001 \mathrm{a}$ & & & & \\
\hline 7 & 1 & 7 & & 17100.36078 & $4.001 \mathrm{a}$ & & \\
\hline 7 & 1 & 617255.35048 & $4.001 \mathrm{a}$ & & & & \\
\hline 7 & 2 & 6 & & & & 17710.12576 & $4.001 \mathrm{a}$ \\
\hline 7 & 2 & 517340.13528 & $2.752 \mathrm{a}$ & 17368.07404 & $4.001 \mathrm{a}$ & & \\
\hline 7 & 3 & 517450.13759 & $10.001 \mathrm{r}$ & & & & \\
\hline 7 & 3 & 417457.86718 & $2.313 \mathrm{a}$ & 17466.30490 & $4.001 \mathrm{a}$ & & \\
\hline 7 & 4 & 417633.16291 & $0.884 \mathrm{p}, \mathrm{r}$ & 17634.52926 & $2.313 \mathrm{a}$ & & \\
\hline & 4 & 3 & & 17639.19130 & $2.832 \mathrm{a}$ & & \\
\hline
\end{tabular}




\begin{tabular}{|c|c|c|c|c|c|c|c|}
\hline \multicolumn{2}{|c|}{$J K_{a} K_{c}$} & \multicolumn{2}{|c|}{142 or $21^{+} 4$} & \multicolumn{2}{|c|}{222 or $31^{+} 2$} & \multicolumn{2}{|c|}{104 or $32^{+} 0$} \\
\hline 1 & 0 & 1 & & 17249.80712 & $2.832 \mathrm{a}$ & 17770.35365 & $2.672 \mathrm{a}$ \\
\hline 1 & 1 & 116846.48390 & $10.001 \mathrm{r}$ & 17265.33973 & $3.711 \mathrm{r}$ & 17780.07273 & $4.001 \mathrm{a}$ \\
\hline 1 & 1 & 016852.64516 & $2.832 \mathrm{a}$ & 17271.14302 & $4.001 \mathrm{a}$ & 17785.30306 & $0.953 \mathrm{p}, \mathrm{a}$ \\
\hline 2 & 0 & 2 & & 17293.42144 & $2.832 \mathrm{a}$ & 17813.22247 & $2.832 \mathrm{a}$ \\
\hline 2 & 1 & 216885.69123 & $2.832 \mathrm{a}$ & 17304.41002 & $2.243 \mathrm{a}$ & 17819.33882 & $2.004 \mathrm{a}$ \\
\hline 2 & 1 & 116904.11089 & $4.001 \mathrm{a}$ & 17321.85344 & $4.001 \mathrm{a}$ & 17834.99847 & $3.712 \mathrm{r}, \mathrm{a}$ \\
\hline 2 & 2 & 116996.32085 & $4.001 \mathrm{a}$ & 17371.50597 & $2.723 \mathrm{r}, \mathrm{a}$ & 17863.99823 & $2.223 \mathrm{a}$ \\
\hline 2 & 2 & 0 & & 17372.65308 & $2.632 \mathrm{r}$ & 17865.61473 & $2.723 \mathrm{r}, \mathrm{a}$ \\
\hline 3 & 0 & 316927.65208 & $2.313 \mathrm{a}$ & 17355.99155 & $2.313 \mathrm{a}$ & 17874.25555 & $2.313 \mathrm{a}$ \\
\hline 3 & 1 & 3 16944.23825 & $2.223 \mathrm{a}$ & 17362.22181 & $3.552 \mathrm{r}, \mathrm{a}$ & 17877.32125 & $2.832 \mathrm{a}$ \\
\hline 3 & 1 & 216979.99742 & $2.313 \mathrm{a}$ & 17396.87471 & $2.313 \mathrm{a}$ & 17908.25080 & $2.313 \mathrm{a}$ \\
\hline 3 & 2 & 217064.69114 & $3.482 \mathrm{r}$ & 17438.81588 & $4.001 \mathrm{a}$ & 17930.76026 & $2.832 \mathrm{a}$ \\
\hline 3 & 2 & 1 17069.27611 & $2.313 \mathrm{a}$ & 17443.34717 & $2.313 \mathrm{a}$ & 17937.94590 & $2.243 \mathrm{a}$ \\
\hline 3 & 3 & 1 & & 17535.11290 & $10.001 \mathrm{r}$ & 17992.43005 & $4.001 \mathrm{a}$ \\
\hline 3 & 3 & 0 & & 17535.04201 & $4.001 \mathrm{a}$ & 17992.75638 & $2.752 \mathrm{a}$ \\
\hline 4 & 0 & 417009.99809 & $2.313 \mathrm{a}$ & 17435.56970 & $4.001 \mathrm{a}$ & 17952.00714 & $3.712 \mathrm{r}, \mathrm{a}$ \\
\hline 4 & 1 & 417019.28015 & $2.832 \mathrm{a}$ & 17438.24711 & $2.832 \mathrm{a}$ & 17953.31028 & $2.313 \mathrm{a}$ \\
\hline 4 & 1 & 317097.76898 & $2.723 \mathrm{r}, \mathrm{a}$ & 17494.83907 & $7.072 \mathrm{r}$ & 18003.10723 & $2.832 \mathrm{a}$ \\
\hline 4 & 2 & 317153.64155 & $2.004 \mathrm{a}$ & 17527.58876 & $2.313 \mathrm{a}$ & 18018.40446 & $1.965 \mathrm{r}, \mathrm{a}$ \\
\hline 4 & 2 & 217168.86346 & $10.001 \mathrm{r}$ & 17544.18615 & $3.712 \mathrm{r}, \mathrm{a}$ & & \\
\hline 4 & 3 & 217302.22711 & $4.001 \mathrm{a}$ & 17621.78321 & $4.001 \mathrm{a}$ & 18083.92195 & $2.832 \mathrm{a}$ \\
\hline 4 & 3 & 1 & & 17628.79164 & $4.001 \mathrm{a}$ & 18086.04676 & $4.001 \mathrm{a}$ \\
\hline 4 & 4 & 117486.12336 & $3.711 \mathrm{r}$ & 17752.56717 & $2.313 \mathrm{a}$ & 18165.84447 & $4.001 \mathrm{a}$ \\
\hline 4 & 4 & 0 & & & & 18165.92824 & $4.001 \mathrm{a}$ \\
\hline 5 & 0 & 517108.78447 & $2.313 \mathrm{a}$ & 17531.66504 & $2.313 \mathrm{a}$ & 18046.28280 & $2.253 \mathrm{r}, \mathrm{a}$ \\
\hline 5 & 1 & 517113.72309 & $4.001 \mathrm{a}$ & 17532.00959 & $10.001 \mathrm{r}$ & 18046.79464 & $3.712 \mathrm{r}, \mathrm{a}$ \\
\hline
\end{tabular}


$\begin{array}{lllllllll}5 & 1 & 4 & 17198.94000 & 2.253 \mathrm{a} & 17613.63490 & 2.832 \mathrm{a} & 18117.11093 & 1.965 \mathrm{r}, \mathrm{a}\end{array}$ $\begin{array}{lllll}5 & 2 & 4 & 17265.16718 & 2.313 \mathrm{a}\end{array}$

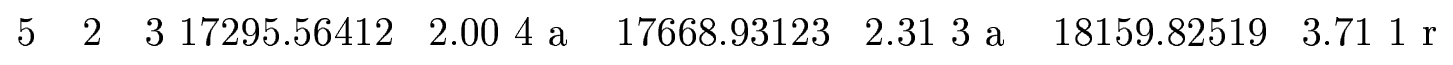

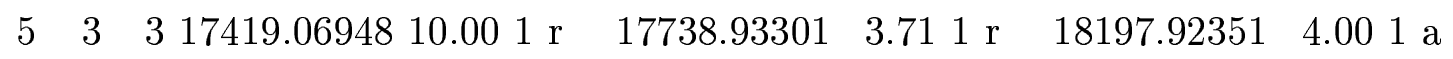

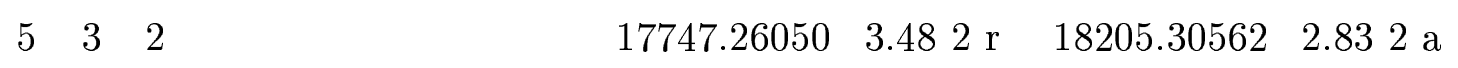

$\begin{array}{llllll}5 & 4 & 2 & 17868.88727 & 3.71 & 1 \mathrm{r}\end{array}$

$\begin{array}{llllllll}5 & 4 & 1 & 17870.39189 & 2.72 & 3 \mathrm{r}, \mathrm{a} & 18281.27431 & 4.001 \mathrm{a}\end{array}$

$5 \quad 5 \quad 1 \quad 18022.2391710 .001 \mathrm{r}$

$\begin{array}{lllllll}5 & 5 & 0 & 18022.41691 & 2.832 \mathrm{a} & 18383.94974 & 4.001 \mathrm{a}\end{array}$

$\begin{array}{lllllll}6 & 0 & 6 & 17223.09722 & 2.313 \mathrm{a} & 18157.36682 \quad 4.001 \mathrm{a}\end{array}$

$\begin{array}{llllllllll}6 & 1 & 6 & 17225.03379 & 2.672 \mathrm{a} & 17642.75144 & 4.001 \mathrm{a} & 18157.29308 & 3.712 \mathrm{r}, \mathrm{a}\end{array}$

$\begin{array}{llllll}6 & 1 & 5 & 17363.87159 & 2.313 \mathrm{a}\end{array}$

$\begin{array}{lllllllll}6 & 2 & 5 & 17396.57868 & 2.832 \mathrm{a} & 17766.68565 & 7.072 \mathrm{r} & 18251.96455 & 2.832 \mathrm{a}\end{array}$

$\begin{array}{lllll}6 & 2 & 4 & 17447.09415 \quad 2.312 \text { a }\end{array}$

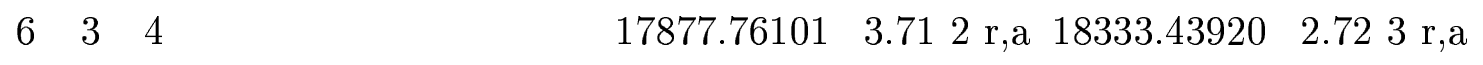

$\begin{array}{llllllll}6 & 4 & 3 & 18008.55729 & 3.71 & 2 \mathrm{r}, \mathrm{a} & 18419.06268 & 4.001 \mathrm{a}\end{array}$

$\begin{array}{llllllll}6 & 5 & 2 & 18160.31830 & 3.71 & 1 \mathrm{r} & 18522.48469 & 4.001 \mathrm{a}\end{array}$

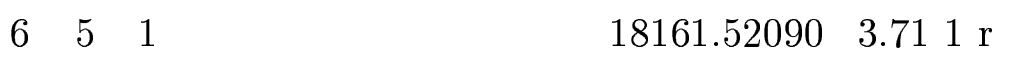

$6 \quad 6 \quad 1 \quad 18400.4193510 .001 \mathrm{r}$

$\begin{array}{lllllll}7 & 0 & 7 & 17370.89011 & 2.053 \mathrm{a} & 18284.67112 \quad 4.001 \mathrm{a}\end{array}$

$\begin{array}{lllllll}7 & 1 & 7 & 17373.49475 & 2.313 \mathrm{a} & 18285.05218 & 4.001 \mathrm{a}\end{array}$

$\begin{array}{lllllll}7 & 1 & 6 & 17526.74031 & 2.832 \mathrm{a} & 18393.77660 & 4.001 \mathrm{a}\end{array}$

$\begin{array}{llllll}7 & 2 & 6 & 17547.29412 & 2.72 & 3 \mathrm{r}, \mathrm{a}\end{array}$

$72517619.633842 .632 \mathrm{r}, ? \quad 18471.65605 \quad 4.001 \mathrm{a}$

$\begin{array}{lllll}7 & 3 & 4 & 18523.23541 & 4.001 \mathrm{a}\end{array}$

$\begin{array}{lllll}7 & 4 & 3 & 18587.34322 & 4.001 \mathrm{a}\end{array}$

$\begin{array}{lllllll}8 & 0 & 8 & 17517.12484 & 2.313 \mathrm{a} & 18430.11664 & 4.001 \mathrm{a}\end{array}$ 
$\begin{array}{lllll}8 & 1 & 8 & 17493.43296 & 2.832 \mathrm{a}\end{array}$

$\begin{array}{lllll}8 & 1 & 7 & 17640.74942 & 2.832 \mathrm{a}\end{array}$

$\begin{array}{lll}8 & 2 & 7\end{array}$

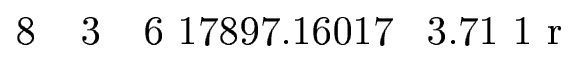

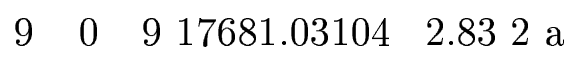

$\begin{array}{lllll}9 & 1 & 9 & 17677.36893 & 2.832 \mathrm{a}\end{array}$

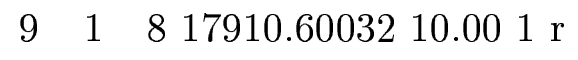

$18429.93024 \quad 4.001 \mathrm{a}$

$18557.97591 \quad 4.001 \mathrm{a}$

\begin{tabular}{|c|c|c|c|c|c|c|c|}
\hline \multicolumn{2}{|c|}{$J K_{a} K$} & \multicolumn{2}{|c|}{420 or $40^{+} 2$} & \multicolumn{2}{|c|}{321 or $40^{-} 2$} & \multicolumn{2}{|c|}{302 or $41^{+} 0$} \\
\hline 0 & 0 & 0 & & 16821.63545 & $1.001 \mathrm{a}$ & 17458.35422 & $12.001 \mathrm{a}$ \\
\hline 1 & 0 & 116846.06843 & $4.001 \mathrm{a}$ & 16844.00637 & $1.791 \mathrm{a}$ & 17480.26671 & $1.552 \mathrm{a}$ \\
\hline 1 & 1 & 116865.11081 & $2.832 \mathrm{a}$ & 16859.33668 & $0.492 \mathrm{a}$ & 17490.72287 & $2.732 \mathrm{a}$ \\
\hline 1 & 1 & 016870.61254 & $2.652 \mathrm{a}$ & 16865.01781 & $1.272 \mathrm{a}$ & 17495.88736 & $1.832 \mathrm{a}$ \\
\hline 2 & 0 & 216889.80571 & $2.313 \mathrm{a}$ & 16887.40081 & $0.674 \mathrm{a}$ & 17522.88466 & $1.933 \mathrm{a}$ \\
\hline 2 & 1 & 216903.83827 & $2.832 \mathrm{a}$ & 16898.38630 & $0.844 \mathrm{a}$ & 17529.62646 & $1.684 \mathrm{a}$ \\
\hline 2 & 1 & 116921.83218 & $4.001 \mathrm{a}$ & 16915.37928 & $0.334 \mathrm{a}$ & 17545.03905 & $1.883 \mathrm{a}$ \\
\hline 2 & 2 & 116956.77560 & $1.483 \mathrm{a}$ & 16959.64955 & $0.924 \mathrm{a}$ & 17575.41705 & $1.863 \mathrm{a}$ \\
\hline 2 & 2 & 016957.90798 & $2.003 \mathrm{a}$ & 16960.65536 & $0.424 \mathrm{a}$ & 17578.51350 & $2.672 \mathrm{a}$ \\
\hline 3 & 0 & 316952.40619 & $1.582 \mathrm{a}$ & 16949.57731 & $1.334 \mathrm{a}$ & 17583.75976 & $1.793 \mathrm{a}$ \\
\hline 3 & 1 & 316961.97039 & $2.064 \mathrm{p}, \mathrm{a}$ & 16956.33144 & $0.354 \mathrm{a}$ & 17587.19609 & $1.974 \mathrm{r}, \mathrm{a}$ \\
\hline 3 & 1 & 216997.88718 & $2.313 \mathrm{a}$ & 16989.86214 & $0.794 \mathrm{a}$ & 17617.62627 & $1.704 \mathrm{a}$ \\
\hline 3 & 2 & 217023.73340 & $1.973 \mathrm{a}$ & 17026.53027 & $0.404 \mathrm{a}$ & 17641.43773 & $1.885 \mathrm{r}, \mathrm{a}$ \\
\hline 3 & 2 & 117030.41810 & $0.994 \mathrm{a}$ & 17033.13962 & $1.104 \mathrm{a}$ & 17649.62360 & $1.405 \mathrm{a}$ \\
\hline 3 & 3 & 117164.05231 & $8.001 \mathrm{a}$ & 17165.48295 & $0.482 \mathrm{a}$ & 17709.00122 & $2.462 \mathrm{a}$ \\
\hline 3 & 3 & 017164.15114 & $1.632 \mathrm{a}$ & 17165.69753 & $0.942 \mathrm{a}$ & 17709.08002 & $1.354 \mathrm{r}, \mathrm{a}$ \\
\hline 4 & 0 & 417030.56157 & $3.331 \mathrm{a}$ & 17028.54808 & $0.814 \mathrm{a}$ & 17661.30525 & $2.123 \mathrm{a}$ \\
\hline 4 & 1 & 417038.44650 & $0.874 \mathrm{a}$ & 17032.48065 & $0.913 \mathrm{a}$ & 17662.79089 & $1.783 \mathrm{a}$ \\
\hline 4 & 1 & 317079.14005 & $0.972 \mathrm{p}, \mathrm{a}$ & 17086.97285 & $0.433 \mathrm{a}$ & 17711.93782 & $2.163 \mathrm{a}$ \\
\hline
\end{tabular}




\begin{tabular}{|c|c|c|c|c|c|c|}
\hline & 317111.71444 & $4.001 \mathrm{a}$ & 17114.41842 & $1.185 \mathrm{a}$ & 17728.36839 & $1.436 \mathrm{r}, \mathrm{a}$ \\
\hline & 217127.77933 & $2.313 \mathrm{a}$ & 17130.82270 & $0.535 \mathrm{a}$ & 17746.39957 & $2.095 \mathrm{r}, \mathrm{a}$ \\
\hline & 217255.89749 & $1.632 \mathrm{a}$ & 17257.53469 & $1.493 \mathrm{a}$ & 17800.91261 & $1.404 \mathrm{a}$ \\
\hline & 117257.24086 & $2.672 \mathrm{a}$ & 17259.07035 & $0.765 \mathrm{a}$ & 17801.49595 & $2.003 \mathrm{a}$ \\
\hline & 117373.42744 & $2.832 \mathrm{a}$ & 17374.86616 & $12.001 \mathrm{a}$ & 17887.29183 & $2.062 \mathrm{a}$ \\
\hline & $\begin{array}{ll}0 & 17373.43827\end{array}$ & $4.001 \mathrm{a}$ & 17374.89123 & $2.352 \mathrm{a}$ & 17887.30632 & $3.471 \mathrm{a}$ \\
\hline & 517128.64705 & $0.473 \mathrm{a}$ & 17123.83753 & $1.714 \mathrm{a}$ & 17755.22870 & $1.943 \mathrm{a}$ \\
\hline & 517132.49431 & $2.004 \mathrm{a}$ & 17124.54974 & $2.033 \mathrm{a}$ & 17755.86009 & $1.944 \mathrm{a}$ \\
\hline & 417220.23444 & $2.832 \mathrm{a}$ & 17204.55330 & $1.284 \mathrm{a}$ & 17825.55273 & $1.724 \mathrm{a}$ \\
\hline & 417219.74766 & $2.032 \mathrm{a}$ & 17222.35578 & $0.635 \mathrm{p}, \mathrm{a}$ & 17835.21393 & $1.903 \mathrm{a}$ \\
\hline & 317250.93702 & $2.313 \mathrm{a}$ & 17254.10175 & $1.824 \mathrm{a}$ & 17866.87136 & $1.614 \mathrm{a}$ \\
\hline & 317369.43474 & $2.462 \mathrm{a}$ & 17372.47710 & $0.904 \mathrm{a}$ & 17916.77623 & $1.764 \mathrm{r}, \mathrm{a}$ \\
\hline & 217375.54021 & $2.134 \mathrm{a}$ & 17377.28833 & $2.143 \mathrm{a}$ & 17918.76600 & $1.754 \mathrm{a}$ \\
\hline & 217490.19102 & $4.001 \mathrm{a}$ & 17491.71871 & $2.183 \mathrm{a}$ & 18001.81074 & $3.711 \mathrm{a}$ \\
\hline & 117490.35649 & $2.253 \mathrm{a}$ & 17491.90018 & $3.801 \mathrm{a}$ & 18001.97518 & 2.103 \\
\hline & 1 & & 1755 & $3.711 \mathrm{r}$ & 5598 & $4.001 \mathrm{a}$ \\
\hline & 017556.45711 & $3.711 \mathrm{r}$ & & & 18113.82246 & $4.001 \mathrm{a}$ \\
\hline & 617241.48661 & $1.503 \mathrm{a}$ & 17235.39137 & $0.765 \mathrm{p}, \mathrm{a}$ & 17865.70584 & $2.832 \mathrm{a}$ \\
\hline & 617244.05052 & $2.143 \mathrm{a}$ & 17234.96706 & $2.173 \mathrm{a}$ & 17865.75330 & $2.193 \mathrm{a}$ \\
\hline & 517336.62791 & $2.832 \mathrm{a}$ & 17340.34778 & $0.646 \mathrm{p}, \mathrm{a}$ & 17956.12365 & 2.254 \\
\hline & 5 & 1.61 & 1734 & 1.61 & 179 & 1.87 \\
\hline & 4 17397.46773 & $2.254 \mathrm{r}, \mathrm{a}$ & 17401.27004 & $0.884 \mathrm{a}$ & 18014.83794 & $7.072 \mathrm{r}$ \\
\hline & 417507.79187 & $4.001 \mathrm{a}$ & 17508.37619 & $3.293 \mathrm{a}$ & 18039.89987 & $1.835 \mathrm{r}, \mathrm{a}$ \\
\hline & 3 & & 17522.09069 & $1.704 \mathrm{a}$ & 18061.99755 & $2.832 \mathrm{a}$ \\
\hline & 317630.91010 & $10.001 \mathrm{r}$ & 17631.79448 & $10.001 \mathrm{r}$ & 18139.42486 & 2.312 \\
\hline & 217631.02517 & $3.711 \mathrm{r}$ & 17632.92197 & $3.711 \mathrm{r}$ & 18140.17202 & $2.722 \mathrm{a}$ \\
\hline & .71411 & $3.711 \mathrm{r}$ & & & 18251.67609 & $2.832 \mathrm{a}$ \\
\hline
\end{tabular}




\begin{tabular}{|c|c|c|c|c|c|c|c|}
\hline 6 & 5 & 117801.02348 & $10.001 \mathrm{r}$ & & & 18252.37546 & $3.712 \mathrm{r}, \mathrm{a}$ \\
\hline 6 & 6 & 1 & & & & 18336.58690 & $10.001 \mathrm{r}$ \\
\hline 6 & 6 & 0 & & & & 18336.58690 & $10.00 \mathrm{~d}$ \\
\hline 7 & 0 & 717358.89693 & $2.832 \mathrm{a}$ & 17354.75096 & $2.832 \mathrm{a}$ & & \\
\hline 7 & 1 & 717353.46089 & $4.001 \mathrm{a}$ & 17362.30386 & $1.972 \mathrm{a}$ & 17992.72185 & $2.832 \mathrm{a}$ \\
\hline 7 & 1 & 617490.87446 & $2.004 \mathrm{a}$ & 17491.93191 & $2.022 \mathrm{a}$ & 18102.64722 & $1.944 \mathrm{a}$ \\
\hline 7 & 2 & 617494.30561 & $2.313 \mathrm{a}$ & 17494.74889 & $1.413 \mathrm{a}$ & 18104.40878 & $7.072 \mathrm{r}$ \\
\hline 7 & 2 & 517637.40498 & $2.004 \mathrm{a}$ & 17568.44581 & $1.944 \mathrm{a}$ & & \\
\hline 7 & 3 & 517666.95213 & $4.001 \mathrm{a}$ & 17668.46050 & $3.711 \mathrm{r}$ & 18204.23971 & $3.711 \mathrm{a}$ \\
\hline 7 & 3 & 417691.03682 & $3.121 \mathrm{a}$ & & & 18231.00318 & $2.632 \mathrm{r}$ \\
\hline 7 & 4 & 4 & & 17795.69461 & $3.711 \mathrm{r}$ & 18300.10572 & $3.711 \mathrm{r}$ \\
\hline 7 & 4 & 3 & & & & 18303.36558 & $2.632 \mathrm{r}$ \\
\hline 7 & 5 & 3 & & 17860.62160 & $10.001 \mathrm{r}$ & 18412.29571 & $3.711 \mathrm{r}$ \\
\hline 7 & 5 & 2 & & & & 18413.14070 & $3.711 \mathrm{r}$ \\
\hline 8 & 0 & 8 & & 17509.28254 & $1.503 \mathrm{a}$ & & \\
\hline 8 & 1 & 8 & & 17495.10434 & $3.711 \mathrm{r}$ & 18136.48454 & $2.632 \mathrm{r}$ \\
\hline 8 & 1 & 7 & & 17654.62756 & $1.612 \mathrm{a}$ & & \\
\hline 8 & 2 & 7 & & 17656.29214 & $2.723 \mathrm{a}$ & & \\
\hline 8 & 2 & 6 & & 17753.74520 & $1.674 \mathrm{a}$ & & \\
\hline 8 & 3 & 6 & & & & 18380.96370 & $2.462 \mathrm{a}$ \\
\hline 8 & 3 & 5 & & 17885.45974 & $1.664 \mathrm{a}$ & & \\
\hline 8 & 4 & 5 & & & & 18457.24243 & $2.632 \mathrm{r}$ \\
\hline 8 & 5 & 4 & & & & 18596.25524 & $10.001 \mathrm{r}$ \\
\hline 8 & 5 & 3 & & & & 18509.21295 & $10.001 \mathrm{r}$ \\
\hline 9 & 1 & 8 & & 17834.41508 & $3.711 \mathrm{r}$ & & \\
\hline 9 & 2 & 8 & & 17836.36141 & $2.832 \mathrm{a}$ & & \\
\hline 0 & 0 & 10 & & 17857.35155 & $2.462 \mathrm{a}$ & & \\
\hline
\end{tabular}




\begin{tabular}{lllllrlrl}
\hline$J$ & $K_{a}$ & $K_{c}$ & 500 or & $50^{+} 0$ & 401 or & $50^{-} 0$ & 203 or & $41^{-} 0$ \\
\hline 0 & 0 & 0 & & 16898.84185 & $0.501 \mathrm{a}$ & 17495.52845 & $1.001 \mathrm{a}$ \\
1 & 0 & 1 & 16920.52715 & $3.711 \mathrm{r}$ & 16920.93915 & $2.352 \mathrm{a}$ & 17517.77224 & $2.062 \mathrm{a}$ \\
1 & 1 & 1 & 16931.91222 & $4.001 \mathrm{a}$ & 16932.30300 & $0.352 \mathrm{a}$ & 17527.68489 & $0.692 \mathrm{a}$ \\
1 & 1 & 0 & 16936.98994 & $2.022 \mathrm{a}$ & 16937.39744 & $0.442 \mathrm{a}$ & 17532.90048 & $1.952 \mathrm{a}$ \\
2 & 0 & 2 & 16963.52392 & $1.623 \mathrm{a}$ & 16964.06050 & $0.352 \mathrm{a}$ & 17560.58935 & $0.444 \mathrm{a}$ \\
2 & 1 & 2 & 16971.11004 & $0.803 \mathrm{a}$ & 16971.45992 & $0.683 \mathrm{a}$ & 17566.22267 & $1.303 \mathrm{a}$ \\
2 & 1 & 1 & 16986.33879 & $2.702 \mathrm{a}$ & 16986.73377 & $0.294 \mathrm{a}$ & 17583.48471 & $0.664 \mathrm{a}$ \\
2 & 2 & 117021.81090 & $1.414 \mathrm{a}$ & 17021.79652 & $0.482 \mathrm{a}$ & 17612.29440 & $1.493 \mathrm{a}$ \\
2 & 2 & 0 & 17023.13184 & $2.722 \mathrm{a}$ & 17023.13986 & $0.352 \mathrm{a}$ & 17613.87557 & $0.833 \mathrm{a}$ \\
3 & 0 & 317024.20378 & $0.473 \mathrm{a}$ & 17024.63421 & $0.434 \mathrm{a}$ & 17621.54498 & $1.344 \mathrm{r}, \mathrm{a}$ \\
3 & 1 & 317029.07998 & $0.873 \mathrm{a}$ & 17029.25846 & $0.345 \mathrm{a}$ & 17624.09125 & $0.663 \mathrm{a}$ \\
3 & 1 & 217059.29290 & $1.244 \mathrm{a}$ & 17059.65620 & $0.425 \mathrm{a}$ & 17656.53345 & $1.274 \mathrm{a}$ \\
3 & 2 & 217088.62954 & $2.253 \mathrm{a}$ & 17088.47278 & $0.335 \mathrm{a}$ & 17677.96014 & $0.995 \mathrm{r}, \mathrm{a}$ \\
3 & 2 & 117094.70227 & $1.514 \mathrm{a}$ & 17094.61332 & $0.933 \mathrm{a}$ & 17684.86714 & $1.374 \mathrm{a}$ \\
3 & 3 & 117107.26259 & $2.062 \mathrm{a}$ & 17108.52145 & $0.444 \mathrm{a}$ & 17742.13480 & $0.864 \mathrm{a}$ \\
3 & 3 & 0 & 17107.49795 & $1.393 \mathrm{a}$ & 17108.76353 & $0.893 \mathrm{a}$ & 17742.45012 & $1.483 \mathrm{a}$ \\
4 & 0 & 417102.88293 & $0.893 \mathrm{a}$ & 17103.10359 & $0.355 \mathrm{a}$ & 17699.13900 & $0.444 \mathrm{a}$ \\
4 & 1 & 417105.22826 & $0.482 \mathrm{a}$ & 17105.42736 & $0.484 \mathrm{a}$ & 17699.25786 & $1.663 \mathrm{a}$ \\
4 & 1 & 3 & 17154.30239 & $1.553 \mathrm{a}$ & 17154.61616 & $0.345 \mathrm{a}$ & 17750.96018 & $0.786 \mathrm{a}$ \\
4 & 2 & 317176.62822 & $0.874 \mathrm{a}$ & 17176.21151 & $0.794 \mathrm{a}$ & 17764.57719 & $1.474 \mathrm{a}$ \\
4 & 2 & 217192.26298 & $1.905 \mathrm{a}$ & 17192.14239 & $0.424 \mathrm{a}$ & 17781.30826 & $0.984 \mathrm{a}$ \\
4 & 3 & 2 & 17197.96565 & $1.453 \mathrm{a}$ & 17199.16103 & $0.784 \mathrm{a}$ & 17832.25170 & $1.485 \mathrm{a}$ \\
4 & 3 & 117199.48386 & $2.013 \mathrm{a}$ & 17200.68132 & $0.424 \mathrm{a}$ & 17834.29554 & $1.205 \mathrm{r}, \mathrm{a}$ \\
4 & 4 & 117297.83278 & $1.513 \mathrm{a}$ & 17298.48120 & $1.983 \mathrm{a}$ & 17918.32833 & $2.562 \mathrm{a}$ \\
4 & 4 & 0 & 17297.88341 & $1.563 \mathrm{a}$ & 17298.51075 & $0.473 \mathrm{a}$ & 17918.37571 & $1.503 \mathrm{a}$
\end{tabular}


$\begin{array}{lllllllll}5 & 0 & 5 & 17197.84651 & 0.493 \mathrm{a} & 17199.02161 & 0.474 \mathrm{a} & 17793.20392 & 1.473 \mathrm{a}\end{array}$

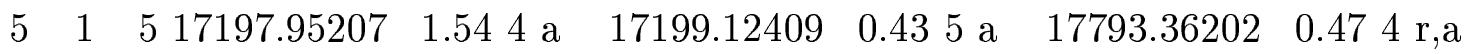

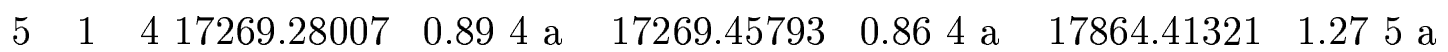

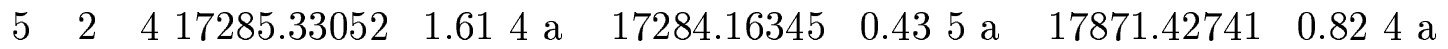

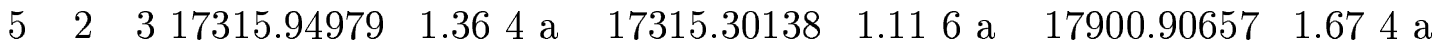

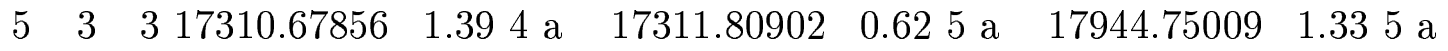

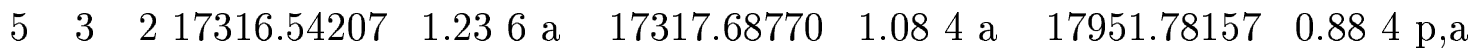

$\begin{array}{llllllllll}5 & 4 & 2 & 17411.52658 & 2.832 \mathrm{a} & 17411.46907 & 0.756 \mathrm{a} & 18031.76899 & 1.78 & 4 \mathrm{a}\end{array}$

$\begin{array}{lllllllll}5 & 4 & 1 & 17411.77159 & 2.832 \mathrm{a} & 17411.78064 & 1.463 \mathrm{a} & 18032.24505 & 1.80\end{array}$

$\begin{array}{llllllllll}5 & 5 & 1 & 17508.84702 & 3.482 \mathrm{r} & 17509.03405 & 0.913 \mathrm{a} & 18143.45827 & 2.003 \mathrm{a}\end{array}$

$\begin{array}{lllllllll}5 & 5 & 0 & 17508.81406 & 2.062 \mathrm{a} & 17509.03405 & 0.91 & \mathrm{~d}\end{array}$

$\begin{array}{llllllllll}6 & 0 & 6 & 17309.42813 & 0.932 \mathrm{a} & 17310.19137 & 0.443 \mathrm{a} & 17903.01414 & 0.85 & 4 \mathrm{a}\end{array}$

$\begin{array}{llllllllll}6 & 1 & 6 & 17309.37376 & 3.121 \mathrm{a} & 17310.22454 & 0.883 \mathrm{a} & 17903.83506 & 1.512 \mathrm{a}\end{array}$

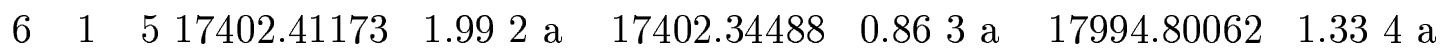

$\begin{array}{lllllllll}6 & 2 & 5 & 17412.79484 & 0.884 \mathrm{a} & 17411.45943 & 1.714 \mathrm{a} & 17997.51501 & 2.043 \mathrm{a}\end{array}$

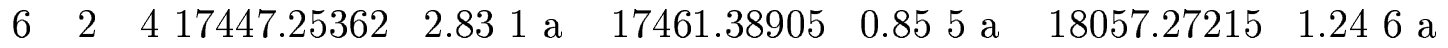

$\begin{array}{lllllllll}6 & 3 & 4 & 17445.45331 & 0.855 \mathrm{a} & 17447.08632 & 2.233 \mathrm{a} & 18078.97700 & 1.84\end{array}$

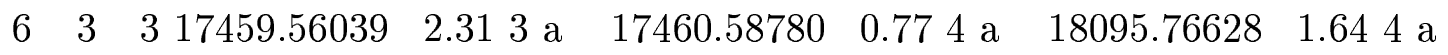

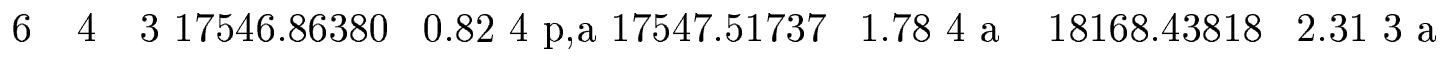

$\begin{array}{lllllllll}6 & 4 & 2 & 17548.31334 & 1.794 \mathrm{a} & 17549.00058 & 1.045 \mathrm{a} & 18170.41757 & 1.814 \mathrm{a}\end{array}$

$\begin{array}{llllllllll}6 & 5 & 2 & 17645.02008 & 2.003 \mathrm{a} & 17645.01414 & 2.064 \mathrm{a} & 18281.98190 & 3.71 & 1 \mathrm{r}\end{array}$

$\begin{array}{llllllllll}6 & 5 & 1 & 17645.07089 & 3.372 \mathrm{a} & 17645.06770 & 1.565 \mathrm{a} & 18281.73831 & 2.00 & 4 \mathrm{a}\end{array}$

$\begin{array}{llllllll}6 & 6 & 1 & 17773.70996 & 2.832 \mathrm{a} & 17778.88630 & 1.62 & \mathrm{~d}\end{array}$

$\begin{array}{lllllllll}6 & 6 & 0 & 17773.70996 & 2.83 & \mathrm{~d} & 17778.88630 & 1.62 & 2 \mathrm{a}\end{array}$

$\begin{array}{lllllllll}7 & 0 & 7 & 17436.58237 & 10.001 \mathrm{r} & 17437.83930 & 1.582 \mathrm{a} & 18030.95690 & 2.352 \mathrm{a}\end{array}$

$\begin{array}{lllllllll}7 & 1 & 7 & 17437.44130 & 1.773 \mathrm{a} & 17438.09252 & 0.442 \mathrm{a} & 18031.21454 & 0.903 \mathrm{a}\end{array}$

$\begin{array}{llllllllll}7 & 1 & 6 & 17552.91130 & 1.675 \mathrm{a} & 17552.24477 & 10.001 \mathrm{r} & 18137.32084 & 3.71 & 1 \mathrm{r}\end{array}$ 


\begin{tabular}{|c|c|c|c|c|c|c|c|}
\hline & 2 & 617560.54324 & $2.003 \mathrm{a}$ & 17557.38602 & $1.453 \mathrm{a}$ & 18141.74329 & $1.644 \mathrm{a}$ \\
\hline & 2 & 517564.79801 & $2.273 \mathrm{a}$ & 17628.02577 & $2.123 \mathrm{a}$ & 18220.05500 & $2.193 \mathrm{a}$ \\
\hline & 3 & 517600.79011 & $1.904 \mathrm{a}$ & 17601.84597 & $1.415 \mathrm{a}$ & 18234.11618 & $1.715 \mathrm{a}$ \\
\hline & 3 & 417628.07301 & $1.974 \mathrm{a}$ & 17628.31373 & $2.113 \mathrm{a}$ & 18265.19471 & $2.313 \mathrm{a}$ \\
\hline & 4 & 417705.66255 & $2.722 \mathrm{a}$ & 17705.97754 & $1.425 \mathrm{a}$ & 18327.93691 & $2.193 \mathrm{a}$ \\
\hline & 4 & 317710.27072 & $1.315 \mathrm{a}$ & 17710.04006 & $1.864 \mathrm{a}$ & 18333.92147 & $2.313 \mathrm{a}$ \\
\hline & 5 & 317803.97126 & $2.622 \mathrm{a}$ & 17803.74931 & $1.994 \mathrm{a}$ & 18440.36894 & $3.711 \mathrm{r}$ \\
\hline & 5 & 217804.22031 & $1.864 \mathrm{a}$ & 17804.04539 & $1.924 \mathrm{a}$ & 18439.60165 & $10.001 \mathrm{r}$ \\
\hline & 6 & 2 & & 17936.79254 & $2.053 \mathrm{a}$ & 18527.15820 & $3.711 \mathrm{r}$ \\
\hline & 6 & 117931.42441 & $2.832 \mathrm{a}$ & 17938.89788 & $10.001 \mathrm{r}$ & & \\
\hline & 7 & 118084.66811 & $10.00 \mathrm{~d}$ & 18083.75779 & $0.914 \mathrm{p}, \mathrm{a}$ & 18644.22013 & $0.971 \mathrm{p}, \mathrm{r}$ \\
\hline & 7 & 018084.66811 & $10.001 \mathrm{r}$ & & & & \\
\hline & 0 & 817582.22918 & $3.711 \mathrm{a}$ & 17582.98420 & $0.942 \mathrm{a}$ & 18175.33611 & $1.413 \mathrm{a}$ \\
\hline & 1 & 817582.63544 & $0.942 \mathrm{a}$ & 17583.15041 & $1.612 \mathrm{a}$ & 18175.22194 & $3.711 \mathrm{r}$ \\
\hline & 1 & 717711.73432 & $2.831 \mathrm{a}$ & 17713.38990 & $1.654 \mathrm{a}$ & 18302.56787 & $2.253 \mathrm{r}, \mathrm{a}$ \\
\hline & 2 & 717725.72853 & $2.103 \mathrm{a}$ & 17720.19677 & $3.651 \mathrm{a}$ & 18303.36173 & $3.711 \mathrm{r}$ \\
\hline & 2 & 6 & & 17813.85574 & $2.153 \mathrm{a}$ & 18401.19284 & $1.586 \mathrm{a}$ \\
\hline & 3 & 617776.68990 & $10.001 \mathrm{r}, ?$ & 17776.77050 & $2.702 \mathrm{a}$ & 18410.71471 & $3.711 \mathrm{r}$ \\
\hline & 3 & 517823.55561 & $3.711 \mathrm{r}$ & 17824.60170 & $1.694 \mathrm{a}$ & & \\
\hline & 4 & 517893.95173 & $3.482 \mathrm{a}$ & 17889.01757 & $2.632 \mathrm{r}$ & & \\
\hline & 4 & 417896.56976 & $10.001 \mathrm{r}$ & 17898.54525 & $1.983 \mathrm{a}$ & 18523.96175 & $3.711 \mathrm{r}$ \\
\hline & 5 & 417983.96324 & $10.001 \mathrm{r}$ & & & 18561.02976 & $10.001 \mathrm{r}$ \\
\hline & 5 & 317984.88731 & $3.711 \mathrm{r}$ & 17986.30860 & $2.184 \mathrm{a}$ & 18562.01860 & $10.001 \mathrm{r}$ \\
\hline & 6 & 3 & & 18117.96643 & $1.002 \mathrm{p}, \mathrm{r}$ & & \\
\hline & 6 & 2 & & 18118.08775 & $3.711 \mathrm{r}$ & 18714.10862 & $3.711 \mathrm{r}$ \\
\hline & 7 & 1.207 & $10.001 \mathrm{r}$ & & & & \\
\hline & 7 & 826 & 00 & & & & \\
\hline
\end{tabular}




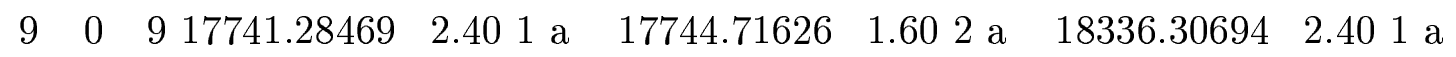
$\begin{array}{lllllll}9 & 1 & 9 & 17743.57411 & 0.932 \mathrm{a} & 18336.29252 & 1.923 \mathrm{a}\end{array}$

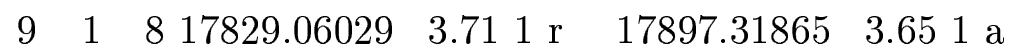
$\begin{array}{lllll}9 & 2 & 8 & 17832.01206 & 10.001 \mathrm{r}\end{array}$

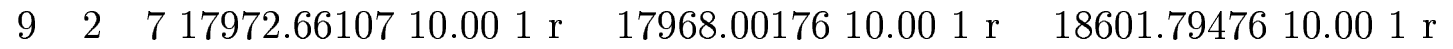
$\begin{array}{lllll}9 & 3 & 7 & 17971.78625 & 3.762\end{array}$

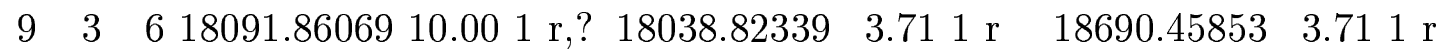
$9 \quad 4 \quad 518111.49654 \quad 3.711 \mathrm{r}$

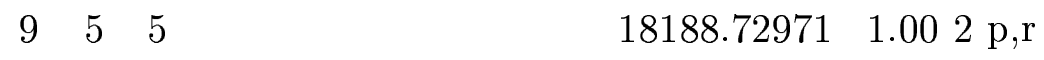

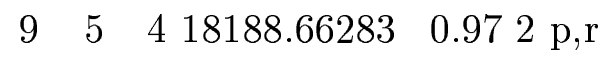

$10 \quad 0 \quad 10$

$17923.20196 \quad 2.032$ a $\quad 18513.95641 \quad 2.742$ a

$10 \quad 110$ $18513.89273 \quad 3.761 \mathrm{a}$

$\begin{array}{lll}10 & 1 & 9\end{array}$ $18098.73979 \quad 2.632$ a $\quad 18676.73526 \quad 3.711 \mathrm{r}$

$10 \quad 2 \quad 9$ $18675.23030 \quad 3.711 \mathrm{r}$

$10 \quad 2 \quad 8$ $18179.0646210 .001 \mathrm{r} \quad 18814.68127 \quad 3.711 \mathrm{r}$

$\begin{array}{lll}10 & 3 & 7\end{array}$ $18915.89779 \quad 3.711 \mathrm{r}$

$11 \quad 111$

$18118.40709 \quad 2.272 \mathrm{a}$

$\begin{array}{lll}11 & 2 & 10\end{array}$ $18318.45673 \quad 3.581 \mathrm{a}$

\begin{tabular}{|c|c|c|c|}
\hline \multicolumn{4}{|c|}{$J K_{a} K_{c}$} \\
\hline 0 & 0 & 017312.53895 & $5.001 \mathrm{a}$ \\
\hline 1 & 0 & 117335.30492 & $3.331 \mathrm{a}$ \\
\hline 1 & 1 & 117349.86615 & $2.632 \mathrm{a}$ \\
\hline 1 & 1 & 017355.82390 & $3.121 \mathrm{a}$ \\
\hline 2 & 0 & 217379.31535 & $2.322 \mathrm{a}$ \\
\hline 2 & 1 & 217389.61106 & $3.622 \mathrm{a}$ \\
\hline 2 & 1 & 117407.34938 & $2.123 \mathrm{a}$ \\
\hline 2 & 2 & 117451.79728 & $2.392 \mathrm{r}, \mathrm{a}$ \\
\hline
\end{tabular}




$$
\begin{aligned}
& 2 \quad 2 \quad 0 \quad 17453.24374 \quad 2.492 \text { a } \\
& \begin{array}{llllll}
3 & 0 & 3 & 17442.09983 & 2.48 & 2 \\
\mathrm{r}, \mathrm{a}
\end{array}
\end{aligned}
$$

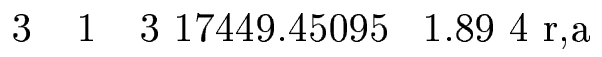

$$
\begin{aligned}
& \begin{array}{lllllll}
3 & 1 & 2 & 17483.38159 & 2.54 & 2 & \text { r,a }
\end{array} \\
& \begin{array}{lllll}
3 & 2 & 2 & 17519.19761 & 2.063 \mathrm{a}
\end{array} \\
& \begin{array}{lllllll}
3 & 2 & 1 & 17525.33628 & 2.68 & 2 & \mathrm{a}
\end{array} \\
& \begin{array}{llllll}
3 & 3 & 1 & 17607.54260 & 2.562 \mathrm{a}
\end{array}
\end{aligned}
$$

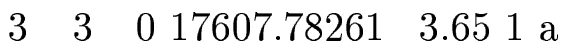

$$
\begin{aligned}
& \begin{array}{lllll}
4 & 0 & 4 & 17521.73620 & 1.943 \mathrm{a}
\end{array}
\end{aligned}
$$

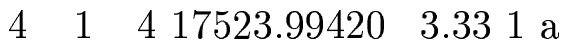

$$
\begin{aligned}
& \begin{array}{llllll}
4 & 1 & 3 & 17582.25497 & 3.76 & 1 \mathrm{a}
\end{array} \\
& \begin{array}{llllll}
4 & 2 & 3 & 17608.95930 & 3.71 & 1 \mathrm{r}
\end{array} \\
& \begin{array}{llllll}
4 & 2 & 2 & 17621.79855 & 2.83 & 2
\end{array}
\end{aligned}
$$

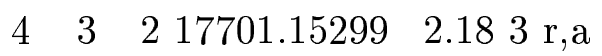

$$
\begin{aligned}
& \begin{array}{llllll}
4 & 3 & 1 & 17701.86949 & 2.02 & 4 \\
\mathrm{r}, \mathrm{a}
\end{array} \\
& \begin{array}{llllll}
4 & 4 & 0 & 17816.19844 & 2.72 & 2 \mathrm{a}
\end{array} \\
& \begin{array}{llllll}
5 & 0 & 5 & 17617.97651 & 3.33 & 1 \mathrm{a}
\end{array} \\
& \begin{array}{lllll}
5 & 1 & 5 & 17618.55695 & 2.252 \\
\mathrm{a}
\end{array}
\end{aligned}
$$

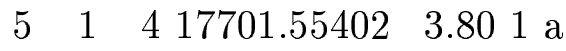

$$
\begin{aligned}
& \begin{array}{lllll}
5 & 2 & 4 & 17721.44879 & 2.562 \mathrm{a}
\end{array} \\
& \begin{array}{lllll}
5 & 2 & 3 & 17756.48327 & 4.001 \mathrm{a}
\end{array} \\
& \begin{array}{lllll}
5 & 3 & 3 & 17816.12683 & 1.894 \mathrm{a}
\end{array}
\end{aligned}
$$

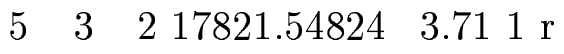

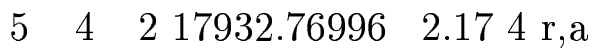

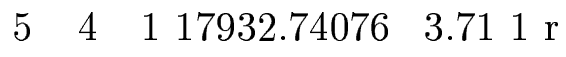

$$
\begin{aligned}
& \begin{array}{lllll}
5 & 5 & 1 & 18073.20450 \quad 4.001 \mathrm{a}
\end{array}
\end{aligned}
$$

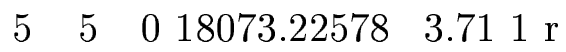


$\begin{array}{lllllll}6 & 0 & 6 & 17730.94397 & 2.39 & 2 & \text { r,a }\end{array}$

$\begin{array}{llllll}6 & 1 & 5 & 17838.71090 & 2.46 & 2 \mathrm{a}\end{array}$

$\begin{array}{llll}6 & 2 & 5 & 17847.1052310 .001 \mathrm{r}\end{array}$

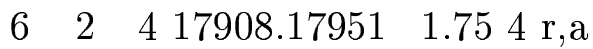

$\begin{array}{llllll}6 & 3 & 3 & 17968.46006 & 2.31 & 3 \mathrm{a}\end{array}$

$\begin{array}{lllll}6 & 4 & 3 & 18073.01734 & 4.001 \mathrm{a}\end{array}$

$\begin{array}{lllll}6 & 4 & 2 & 18074.16205 & 2.313 \mathrm{a}\end{array}$

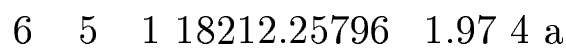

$\begin{array}{llllll}6 & 6 & 1 & 18429.89571 & 3.71 & d\end{array}$

$\begin{array}{lllllll}6 & 6 & 0 & 18429.89571 & 3.71 & 1 & \mathrm{r}\end{array}$ 
TABLE XII.: Term values for the $5 \nu+\delta$ polyad of $\mathrm{H}_{2}{ }^{16} \mathrm{O}$.

\begin{tabular}{|c|c|c|c|c|c|c|}
\hline \multicolumn{2}{|c|}{$J K_{a} K_{c}$} & \multicolumn{2}{|c|}{053 or $21^{-} 5$} & 133 or $31^{-} 3$ & \multicolumn{2}{|c|}{510 or $50^{+} 1$} \\
\hline 0 & 0 & 0 & & & 18392.97402 & $10.001 \mathrm{a}$ \\
\hline 1 & 0 & 1 & & & 18415.04338 & $2.402 \mathrm{a}$ \\
\hline 1 & 1 & 118416.93985 & $4.001 \mathrm{a}$ & 18800.37454 & $4.001 \mathrm{a}$ & \\
\hline 1 & 1 & 0 & & 18806.66356 & 4.001 a 18433.99629 & $2.462 \mathrm{a}$ \\
\hline 2 & 0 & 218418.60581 & $2.313 \mathrm{a}$ & 18825.62981 & 2.832 a 18458.32613 & $2.832 \mathrm{a}$ \\
\hline 2 & 1 & 218456.67346 & $4.001 \mathrm{a}$ & & 18467.74707 & $1.793 \mathrm{a}$ \\
\hline 2 & 1 & 118477.23110 & $2.832 \mathrm{a}$ & 18858.62589 & 2.313 a 18483.98386 & $2.832 \mathrm{a}$ \\
\hline 2 & 2 & 1 & & 18912.98460 & $4.001 \mathrm{a} 18525.51651$ & $2.832 \mathrm{a}$ \\
\hline 2 & 2 & 0 & & 18914.31447 & $2.832 \mathrm{a}$ & \\
\hline 3 & 0 & 318484.93643 & $2.832 \mathrm{a}$ & 18888.91584 & 4.001 a 18520.52575 & $1.833 \mathrm{a}$ \\
\hline 3 & 1 & 318515.71433 & $2.313 \mathrm{a}$ & 18897.41184 & 2.832 a 18525.69241 & $4.001 \mathrm{a}$ \\
\hline 3 & 1 & 218555.42369 & $2.313 \mathrm{a}$ & 18935.38033 & 4.001 a 18557.81979 & $1.943 \mathrm{a}$ \\
\hline 3 & 2 & 2 & & 18981.15272 & 2.313 a 18592.98993 & $2.832 \mathrm{a}$ \\
\hline 3 & 2 & 1 & & 18987.34546 & 4.001 a 18598.81432 & $2.313 \mathrm{a}$ \\
\hline 3 & 3 & 1 & & 19085.28248 & 2.832 a 18681.45815 & $4.001 \mathrm{a}$ \\
\hline 3 & 3 & 0 & & 19085.48929 & 4.001 a 18681.62593 & $4.001 \mathrm{a}$ \\
\hline 4 & 0 & 418570.50097 & $2.832 \mathrm{a}$ & 18969.11869 & $2.832 \mathrm{a}$ & \\
\hline 4 & 1 & 418593.29121 & $4.001 \mathrm{a}$ & 18974.03584 & 4.001 a 18600.88107 & $3.392 \mathrm{a}$ \\
\hline 4 & 1 & 318662.97588 & $2.832 \mathrm{a}$ & 19035.42322 & 2.832 a 18653.77320 & $2.832 \mathrm{a}$ \\
\hline 4 & 2 & 3 & & 19070.72460 & 4.001 a 18679.63226 & $2.313 \mathrm{a}$ \\
\hline 4 & 2 & 2 & & & 18696.98171 & $2.832 \mathrm{a}$ \\
\hline 4 & 3 & 2 & & & 18774.20386 & $4.001 \mathrm{a}$ \\
\hline 4 & 3 & 1 & & 19179.75496 & $2.832 \mathrm{a}$ & \\
\hline 4 & 4 & 1 & & & 18902.23511 & $4.001 \mathrm{a}$ \\
\hline
\end{tabular}




\begin{tabular}{|c|c|c|c|c|c|c|}
\hline 5 & 0 & 5 & & & 18693.43226 & $2.313 \mathrm{a}$ \\
\hline 5 & 1 & $518689.00297 \quad 4.001 \mathrm{a}$ & 19068.11508 & $2.832 \mathrm{a}$ & 18695.32998 & $2.313 \mathrm{a}$ \\
\hline 5 & 1 & 4 & & & 18769.59844 & $2.832 \mathrm{a}$ \\
\hline 5 & 2 & 4 & 19180.90849 & $2.832 \mathrm{a}$ & 18789.38441 & $2.832 \mathrm{a}$ \\
\hline 5 & 2 & 3 & & & 18821.94819 & $4.001 \mathrm{a}$ \\
\hline 6 & 0 & 6 & 19177.56405 & $4.001 \mathrm{a}$ & 18807.90448 & $2.832 \mathrm{a}$ \\
\hline 6 & 1 & 6 & & & 18806.08820 & $2.832 \mathrm{a}$ \\
\hline 6 & 1 & 5 & 19296.49897 & $4.001 \mathrm{a}$ & 18903.84042 & $2.313 \mathrm{a}$ \\
\hline 6 & 2 & 5 & & & 18917.85125 & $2.832 \mathrm{a}$ \\
\hline 6 & 3 & 4 & & & 19027.95523 & $4.001 \mathrm{a}$ \\
\hline 7 & 0 & 7 & & & 18937.40052 & $4.001 \mathrm{a}$ \\
\hline 7 & 1 & 7 & 19306.50506 & $4.001 \mathrm{a}$ & & \\
\hline 7 & 1 & 6 & & & 19064.82546 & $2.313 \mathrm{a}$ \\
\hline 7 & 2 & 6 & & & 19055.90225 & $2.313 \mathrm{a}$ \\
\hline 8 & 2 & 7 & & & 19224.86989 & $2.832 \mathrm{a}$ \\
\hline & & 331 or $40^{-} 3$ & 411 or & $50^{-1}$ & 213 or & $41^{-1}$ \\
\hline
\end{tabular}

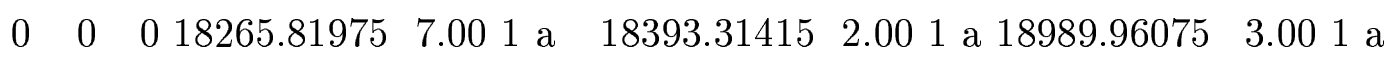

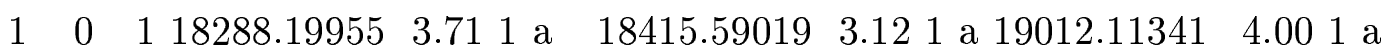

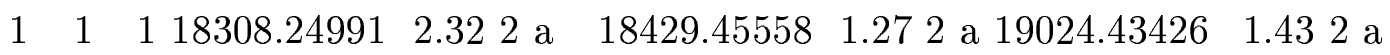

$\begin{array}{lllllllll}1 & 1 & 0 & 18314.32299 & 2.582 \mathrm{a} & 18434.95261 & 2.012 \mathrm{a} & 19030.00665 & 2.832 \mathrm{a}\end{array}$

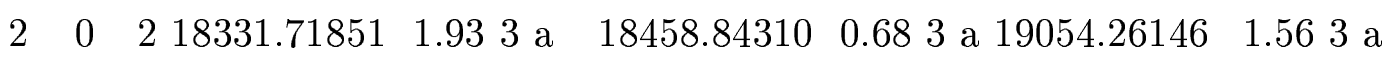

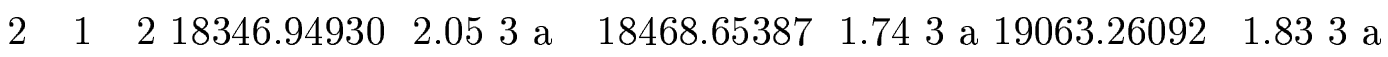

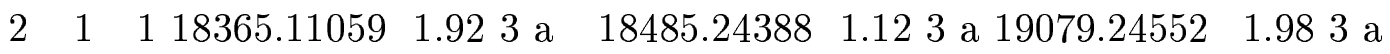

$\begin{array}{lllllllll}2 & 2 & 1 & 18420.56774 & 4.001 \mathrm{a} & 18525.74768 & 2.372 \mathrm{a} & 19116.49055 & 2.832 \mathrm{a}\end{array}$

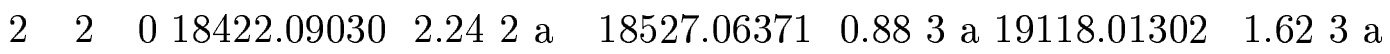

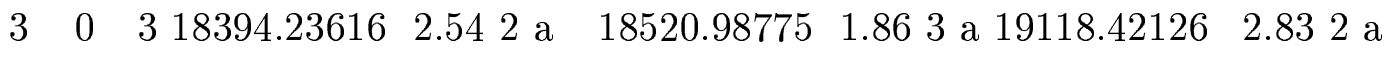

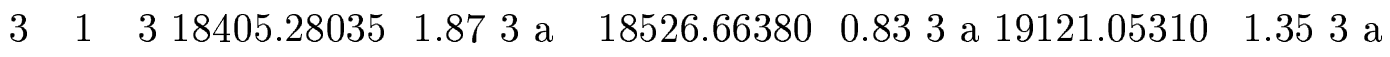




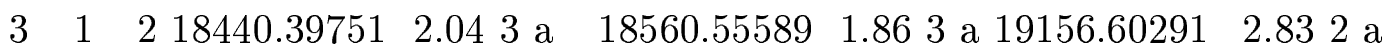

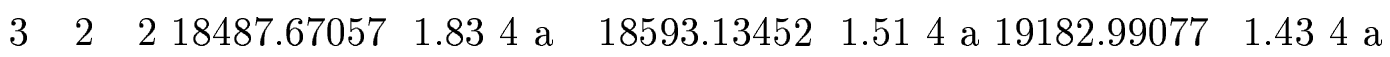

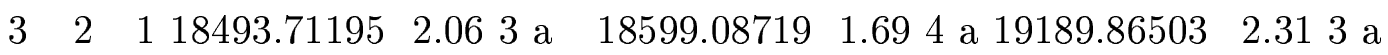

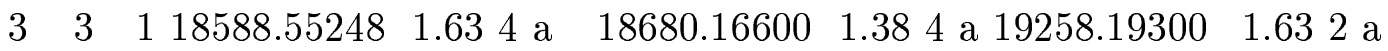

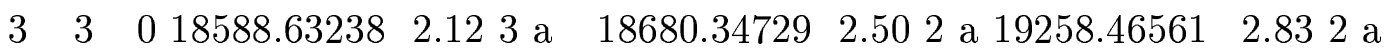

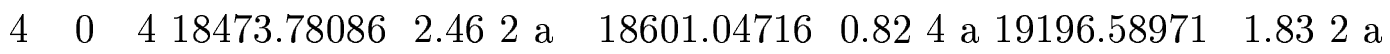

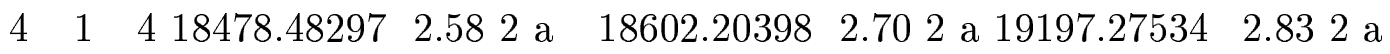

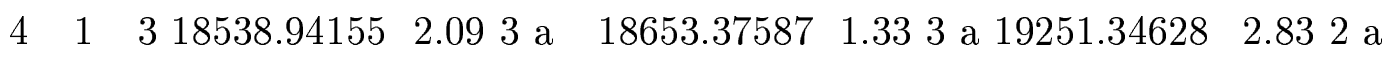

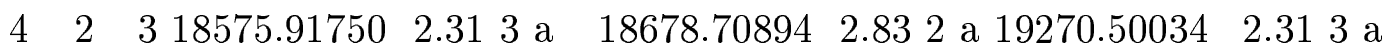

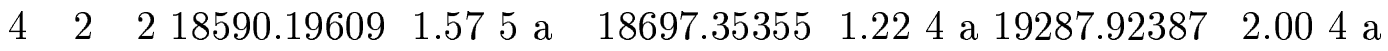

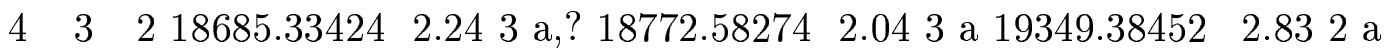

$\begin{array}{llllllll}4 & 3 & 1 & 18682.88158 & 1.78 & 3 \\ \mathrm{a} & 18773.79804 & 1.704 \mathrm{a} & 19351.16940 & 2.31 & 3 \mathrm{a}\end{array}$

$\begin{array}{llllll}4 & 4 & 1 & 18806.52922 & 4.001 \mathrm{a} & 19451.05563 \quad 4.001 \mathrm{a}\end{array}$

$\begin{array}{lllllll}4 & 4 & 0 & 18806.53144 & 1.552 \mathrm{a} & 19451.11615 & 2.832 \mathrm{a}\end{array}$

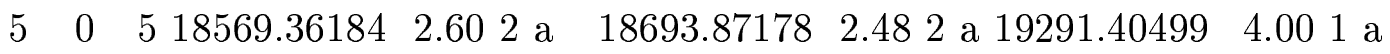

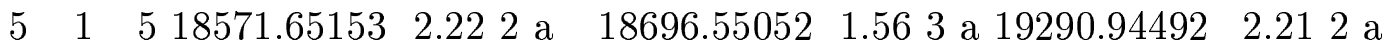

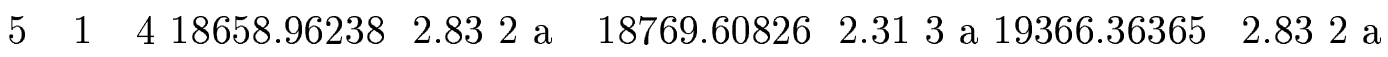

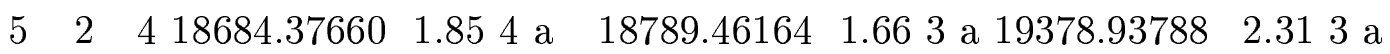

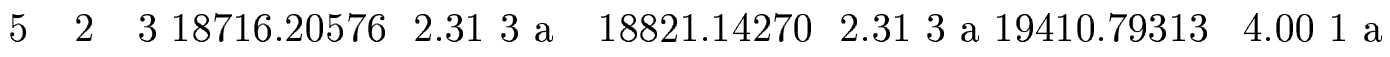

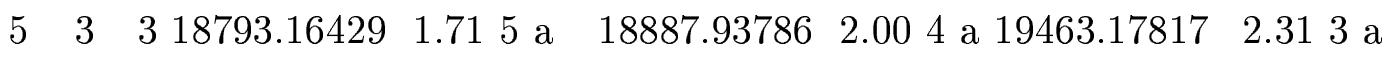

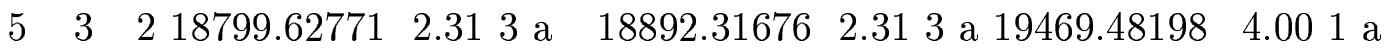

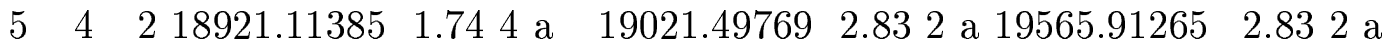

$\begin{array}{lllllll}5 & 4 & 1 & 18921.47744 & 2.313 \mathrm{a} & 19021.64773 & 2.832 \mathrm{a}\end{array}$

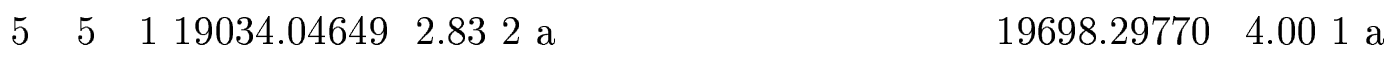

$\begin{array}{llllllll}5 & 5 & 0 & 19034.13657 & 4.001 \mathrm{a} & 19698.29770 & 4.00 & \mathrm{~d}\end{array}$

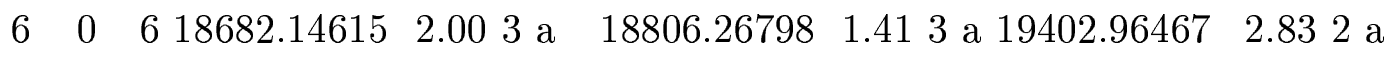

$\begin{array}{lllllllll}6 & 1 & 6 & 18683.28420 & 4.001 \mathrm{a} & 18805.92450 & 2.832 \mathrm{a} & 19402.65211 & 2.832 \mathrm{a}\end{array}$ 


\begin{tabular}{|c|c|c|c|c|c|c|}
\hline 6 & 1 & 518798.43790 & $2.313 \mathrm{a}$ & 18903.95976 & 1.773 a 19499.00636 & $2.832 \mathrm{a}$ \\
\hline 6 & 2 & 518812.17105 & $2.832 \mathrm{a}$ & 18917.91158 & $2.313 \mathrm{a}$ & \\
\hline 6 & 2 & 418862.38996 & $4.001 \mathrm{a}$ & 18969.61151 & 2.313 a 19555.54847 & $2.832 \mathrm{a}$ \\
\hline 6 & 3 & 418928.96939 & $2.313 \mathrm{a}$ & 19025.68242 & $2.313 \mathrm{a}$ & \\
\hline 6 & 3 & 318943.34767 & $2.004 \mathrm{a}$ & 19036.89641 & 2.004 a 19614.52539 & $2.832 \mathrm{a}$ \\
\hline 6 & 4 & 2 & & 19163.11987 & 2.832 a 19705.54970 & $2.832 \mathrm{a}$ \\
\hline 7 & 0 & 718808.76595 & $2.832 \mathrm{a}$ & 18936.58107 & 2.832 a 19531.33477 & $4.001 \mathrm{a}$ \\
\hline 7 & 1 & 718810.85738 & $2.832 \mathrm{a}$ & 18936.89684 & 2.832 a 19531.09151 & $2.832 \mathrm{a}$ \\
\hline 7 & 1 & 618958.24587 & $4.001 \mathrm{a}$ & 19064.82966 & $4.001 \mathrm{a}$ & \\
\hline 7 & 2 & 618959.97437 & $2.313 \mathrm{a}$ & 19055.75273 & 2.832 a 19649.05435 & $2.832 \mathrm{a}$ \\
\hline 7 & 2 & 519041.94055 & $4.001 \mathrm{a}$ & 19139.58322 & 2.832 a 19742.66339 & $4.001 \mathrm{a}$ \\
\hline 7 & 3 & 519084.90244 & $2.004 \mathrm{a}$ & 19185.09500 & 2.832 a 19755.04246 & $4.001 \mathrm{a}$ \\
\hline 7 & 4 & 4 & & 19220.74521 & $2.313 \mathrm{a}$ & \\
\hline 8 & 0 & 818950.68729 & $2.832 \mathrm{a}$ & 19078.11944 & $4.001 \mathrm{a}$ & \\
\hline 8 & 1 & 7 & & 19231.63070 & $2.832 \mathrm{a}$ & \\
\hline 9 & 1 & 9 & & 19240.13636 & $2.832 \mathrm{a}$ & \\
\hline \multicolumn{2}{|c|}{$J K_{a} K_{c}$} & \multicolumn{2}{|c|}{430 or $40^{+} 3$} & 034 or & 223 & \\
\hline 2 & 1 & 218352.43265 & $2.832 \mathrm{a}$ & 19057.74882 & $4.001 \mathrm{a}$ & \\
\hline 3 & 0 & 318400.28330 & $2.542 \mathrm{a}$ & & & \\
\hline 3 & 1 & 3 & & 19113.64259 & $2.832 \mathrm{a}$ & \\
\hline 4 & 1 & 418485.05528 & $4.001 \mathrm{a}$ & 19190.28348 & $2.832 \mathrm{a}$ & \\
\hline 4 & 2 & 218608.07129 & $2.832 \mathrm{a}$ & & & \\
\hline 5 & 0 & 5 & & 19282.35348 & $2.832 \mathrm{a}$ & \\
\hline 5 & 1 & 5 & & 19284.07992 & $2.832 \mathrm{a}$ & \\
\hline 5 & 1 & 4 & & 19373.89752 & $4.001 \mathrm{a}$ & \\
\hline 6 & 0 & 6 & & 19393.60470 & $2.832 \mathrm{a}$ & \\
\hline 6 & 1 & 6 & & 19395.20119 & $4.001 \mathrm{a}$ & \\
\hline
\end{tabular}


TABLE XIII.: Term values for the $6 \nu$ polyad of $\mathrm{H}_{2}{ }^{16} \mathrm{O}$.

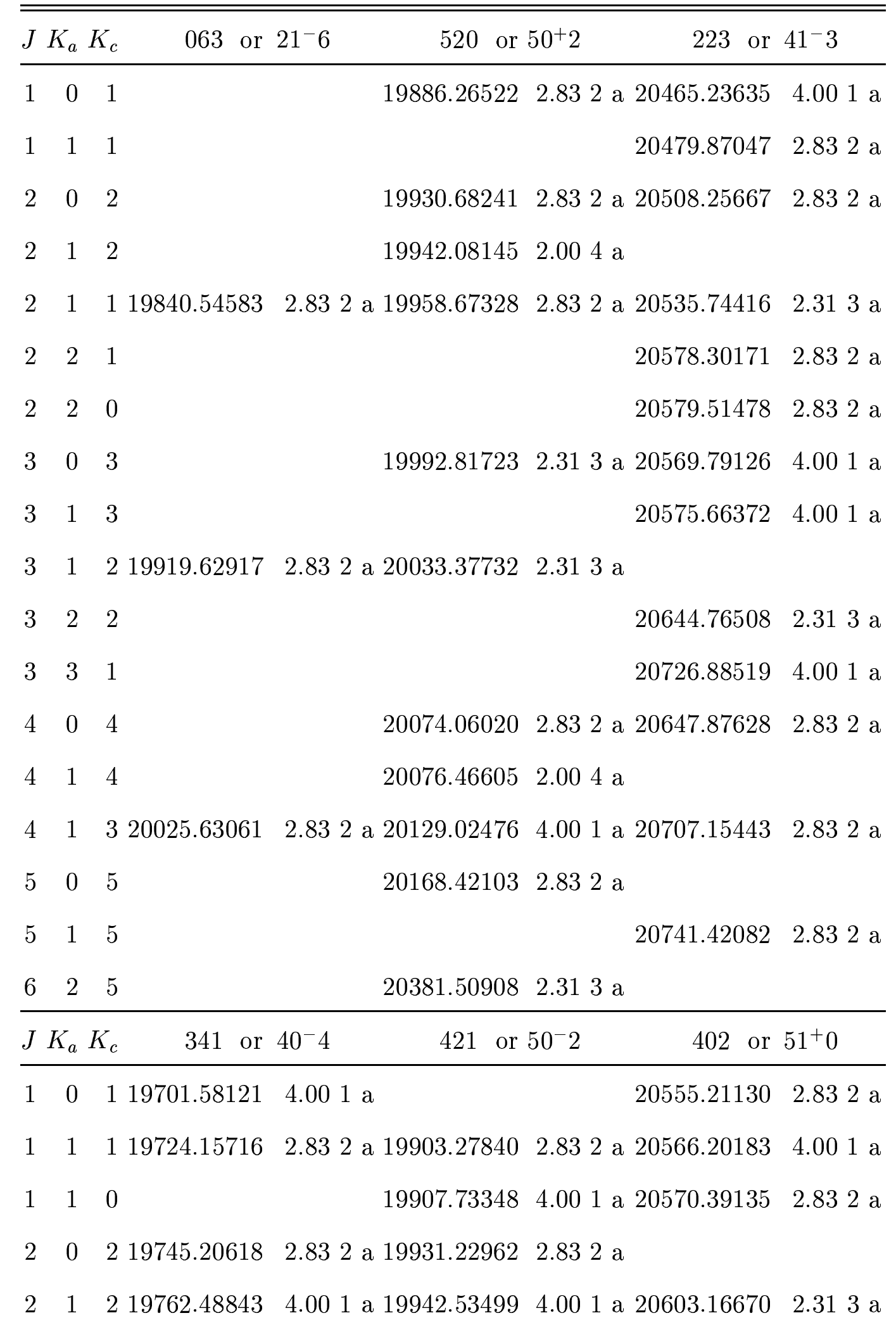




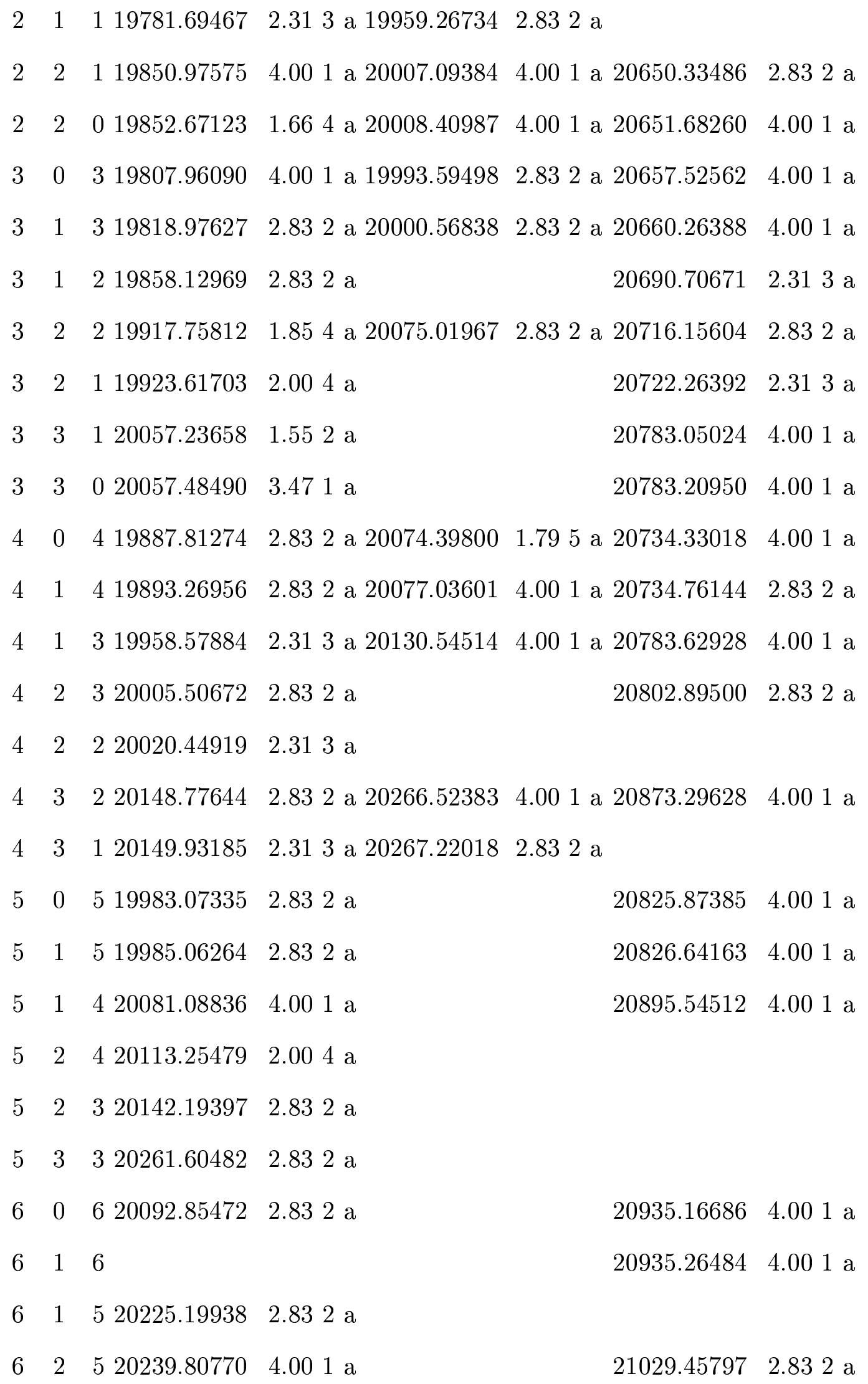


$\begin{array}{lllll}7 & 1 & 720226.57927 & 2.832 \mathrm{a}\end{array}$

\begin{tabular}{|c|c|c|c|c|c|}
\hline \multicolumn{2}{|c|}{$J K_{a} K_{c}$} & \multirow[t]{2}{*}{600 or } & 501 or $60^{-} 0$ & \multicolumn{2}{|c|}{303 or $51^{-} 0$} \\
\hline 0 & 0 & & 19781.10455 & $1.001 \mathrm{a} 20543.13715$ & 10.001 \\
\hline 1 & 0 & 119803.65215 & 2.562 a 19802.58729 & 0.971 a 20566.26157 & $3.921 \mathrm{a}$ \\
\hline 1 & 1 & 119814.27732 & 4.001 a 19813.66805 & 0.692 a 20574.95703 & 2.442 \\
\hline 1 & 1 & 019818.44488 & 2.312 a 19818.65374 & 1.272 a 20580.14635 & 2.832 \\
\hline 2 & 0 & 219843.64302 & 2.152 a 19843.95970 & 0.354 a 20607.72351 & 1.903 \\
\hline 2 & 1 & 219851.14326 & 0.884 a 19852.03095 & 1.723 a 20615.17274 & 3.331 \\
\hline 2 & 1 & 119866.41982 & 3.651 a 19866.66346 & 0.403 a 20628.73543 & 2.073 \\
\hline 2 & 2 & 119900.18997 & 4.001 a 19900.38474 & 1.512 a 20659.94830 & 2.622 \\
\hline 2 & 2 & 0 & 19901.65461 & 0.443 a 20661.48084 & 2.252 \\
\hline 3 & 0 & 319903.12389 & 0.492 a 19903.35244 & 1.272 a 20667.72187 & 4.001 \\
\hline 3 & 1 & 319907.62389 & 1.384 a 19907.75973 & 0.673 a 20670.08288 & 1.923 \\
\hline 3 & 1 & 219937.26291 & 1.364 a 19937.60347 & $1.264 \mathrm{a}$ & \\
\hline 3 & 2 & 219965.25350 & 4.001 a 19965.17229 & 0.634 a 20725.29362 & 1.974 \\
\hline 3 & 2 & 119971.01671 & 2.313 a 19970.94690 & 1.374 a 20732.16243 & 2.313 \\
\hline 3 & 3 & 1 & 20014.73287 & 0.833 a 20793.23090 & 2.532 \\
\hline 3 & 3 & 020014.80535 & 4.001 a 20014.96380 & 2.383 a 20793.48894 & 4.001 \\
\hline 4 & 0 & 419979.09883 & 0.913 a 19979.29554 & 0.833 a 20741.54068 & 2.213 \\
\hline 4 & 1 & 419981.44319 & 0.474 a 19981.63760 & 1.592 a 20744.78728 & 2.832 \\
\hline 4 & 1 & 320032.97357 & 2.312 a 20030.64292 & 0.833 a 20792.68733 & 2.193 \\
\hline 4 & 2 & 320050.62580 & 1.795 a 20050.51278 & 2.033 a 20811.40135 & 4.001 \\
\hline 4 & 2 & 2 & 20064.73667 & 0.775 a 20828.58883 & 2.004 \\
\hline 4 & 3 & 220102.51719 & 2.832 a 20102.81539 & $1.733 \mathrm{a}$ & \\
\hline 4 & 3 & 120104.10579 & 2.313 a 20104.39767 & 1.174 a 20885.13861 & $2.313 \mathrm{a}$ \\
\hline 4 & 4 & 1 & 4915 & 36 & 400 \\
\hline
\end{tabular}




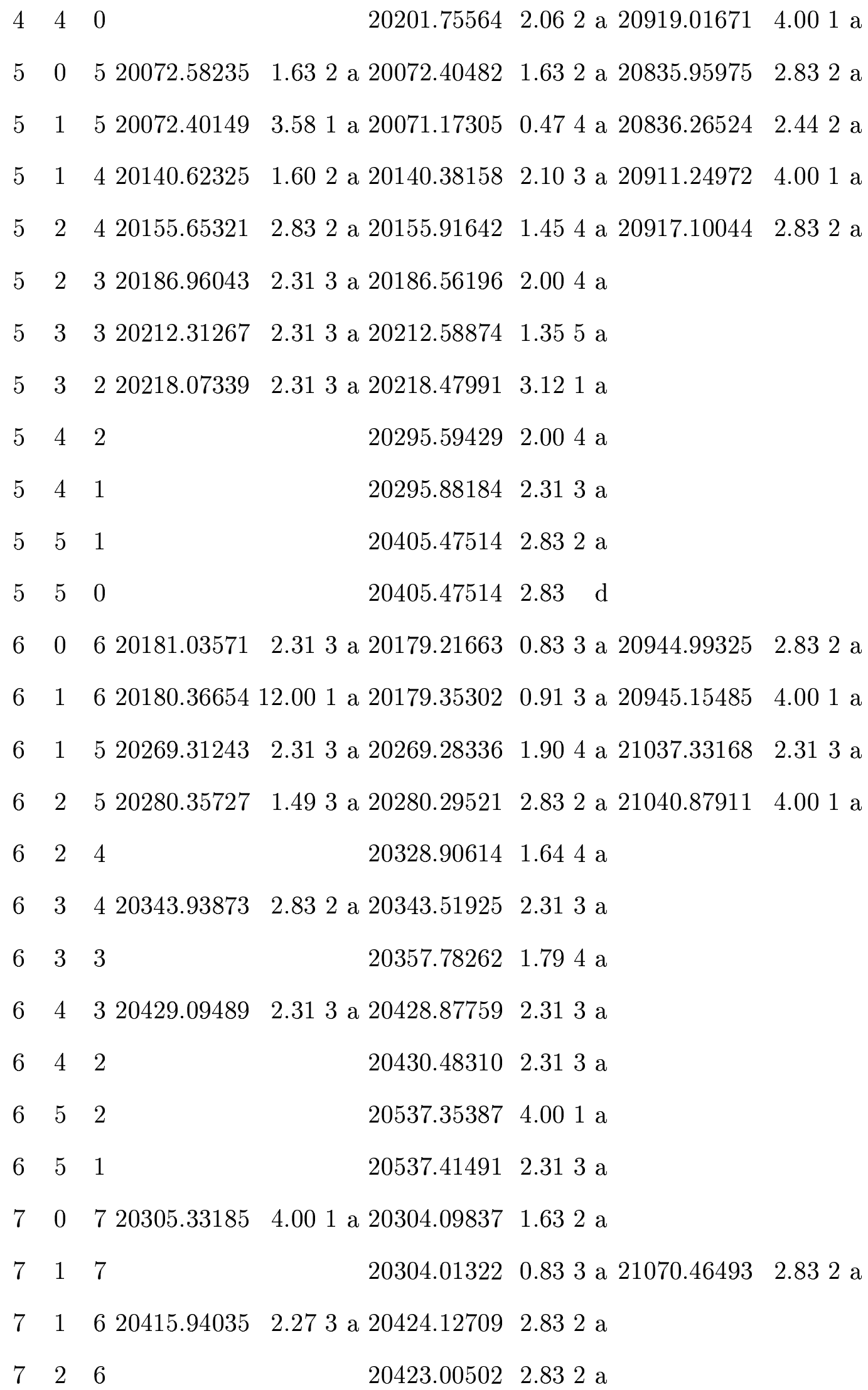


TABLE XIV.: Term values for the $6 \nu+\delta$ polyad of $\mathrm{H}_{2}{ }^{16} \mathrm{O}$.

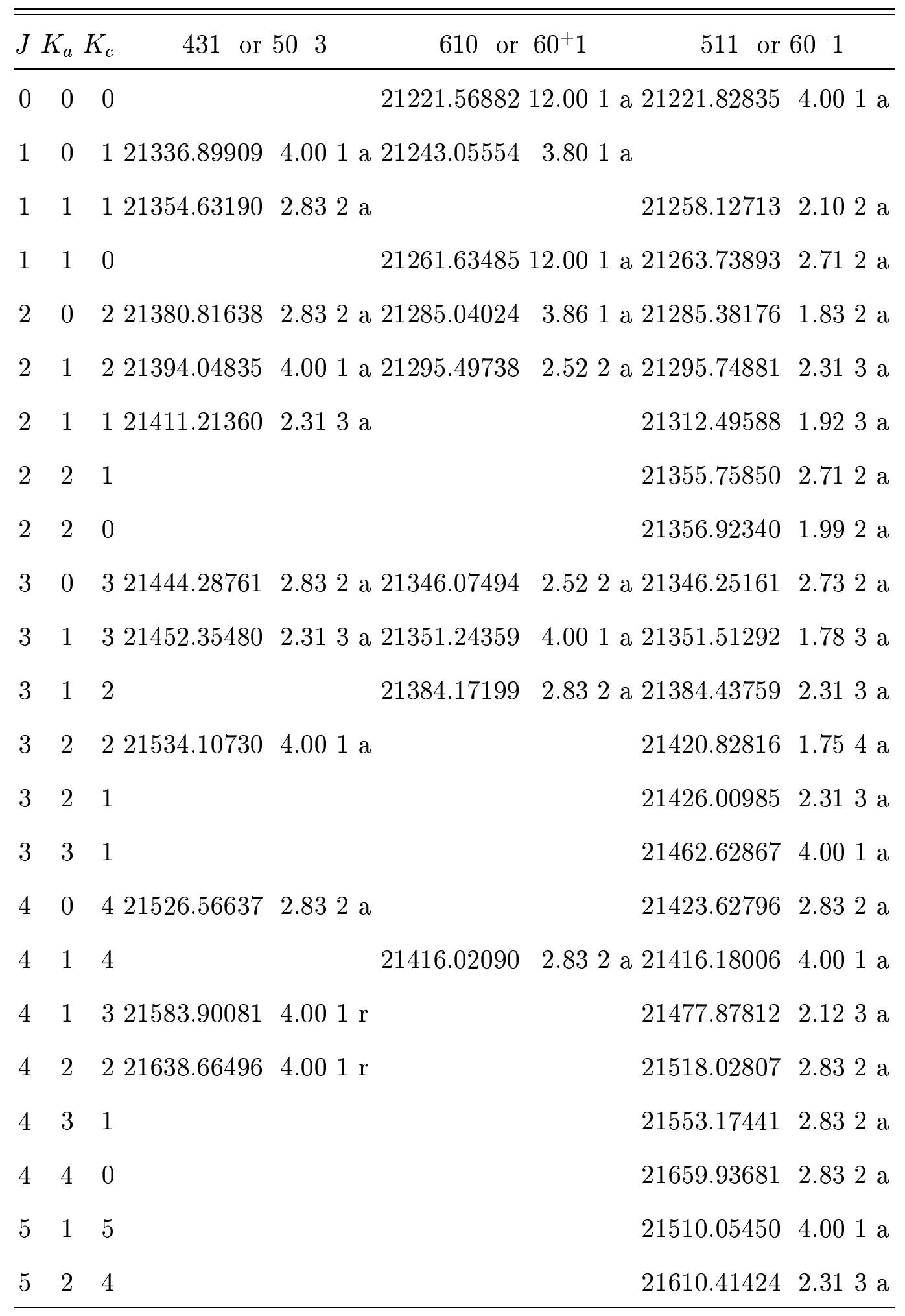




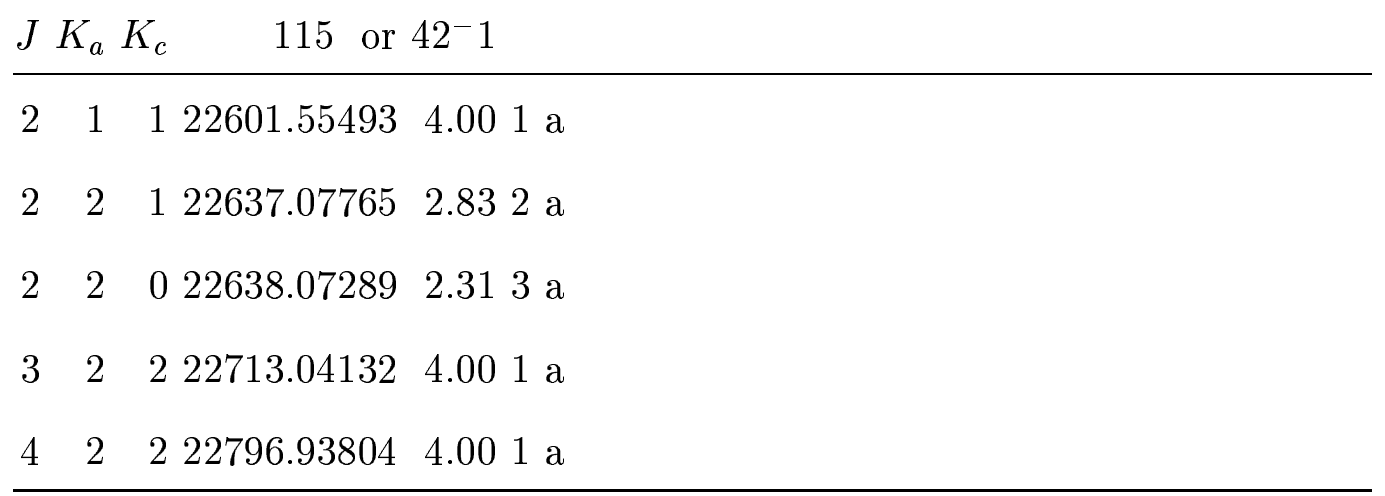


TABLE XV.: Term values for the $7 \nu$ polyad of $\mathrm{H}_{2}{ }^{16} \mathrm{O}$.

\begin{tabular}{|c|c|c|c|c|c|}
\hline \multicolumn{2}{|c|}{$J K_{a} K_{c}$} & 700 or $70^{+} 0$ & 601 or $70^{-} 0$ & \multicolumn{2}{|c|}{521 or $60^{-} 2$} \\
\hline 0 & 0 & 022529.29589 & 4.001 a 22529.44124 & $4.001 \mathrm{a}$ & \\
\hline 1 & 0 & 122550.18384 & 2.832 a 22550.31747 & $2.832 \mathrm{a}$ & \\
\hline 1 & 1 & 1 & 22560.26923 & 2.832 a 22672.81494 & $4.001 \mathrm{a}$ \\
\hline 1 & 1 & 022564.87955 & 4.001 a 22565.03694 & $2.832 \mathrm{a}$ & \\
\hline 2 & 0 & 222589.94696 & 4.001 a 22590.69202 & 2.832 a 22693.59316 & $4.001 \mathrm{a}$ \\
\hline 2 & 1 & 222597.10571 & 4.001 a 22597.25941 & $2.313 \mathrm{a}$ & \\
\hline 2 & 1 & 122611.32639 & 2.832 a 22611.51336 & 2.313 a 22728.51553 & $4.001 \mathrm{a}$ \\
\hline 2 & 2 & 122637.46644 & 2.832 a 22645.80999 & 2.832 a 22787.45757 & $4.001 \mathrm{a}$ \\
\hline 2 & 2 & 022638.52157 & 2.832 a 22646.52208 & 2.313 a 22788.75529 & $2.832 \mathrm{a}$ \\
\hline 3 & 0 & 322648.35749 & 2.313 a 22651.86153 & $2.313 \mathrm{a}$ & \\
\hline 3 & 1 & 322648.43638 & 2.832 a 22651.96076 & 2.313 a 22765.08238 & $4.001 \mathrm{a}$ \\
\hline 3 & 1 & 222679.90779 & 2.313 a 22680.20219 & 2.004 a 22801.93633 & $4.001 \mathrm{a}$ \\
\hline 3 & 2 & 222701.78722 & 4.001 a 22700.58400 & 2.313 a 22852.32854 & $4.001 \mathrm{a}$ \\
\hline 3 & 2 & 122706.70815 & $4.001 \mathrm{a}$ & & \\
\hline 3 & 3 & 1 & 22764.55957 & $2.832 \mathrm{a}$ & \\
\hline 3 & 3 & 022763.71449 & 2.832 a 22764.86354 & $2.832 \mathrm{a}$ & \\
\hline 4 & 0 & 422722.07083 & 2.313 a 22723.72059 & 2.313 a 22835.35519 & $4.001 \mathrm{a}$ \\
\hline 4 & 1 & 422722.10370 & 2.832 a 22723.73573 & $4.001 \mathrm{a}$ & \\
\hline 4 & 1 & 322769.07465 & 4.001 a 22769.73706 & $2.313 \mathrm{a}$ & \\
\hline 4 & 2 & 322783.75838 & $2.004 \mathrm{a}$ & 22937.89704 & $4.001 \mathrm{a}$ \\
\hline 4 & 2 & 2 & 22804.06374 & $2.832 \mathrm{a}$ & \\
\hline 4 & 3 & 222848.92133 & 4.001 a 22849.06973 & $2.832 \mathrm{a}$ & \\
\hline 4 & 3 & 122850.56140 & 4.001 a 22850.61230 & $2.004 \mathrm{a}$ & \\
\hline 4 & 4 & 122929.71765 & 22929.55302 & $4.001 \mathrm{a}$ & \\
\hline
\end{tabular}




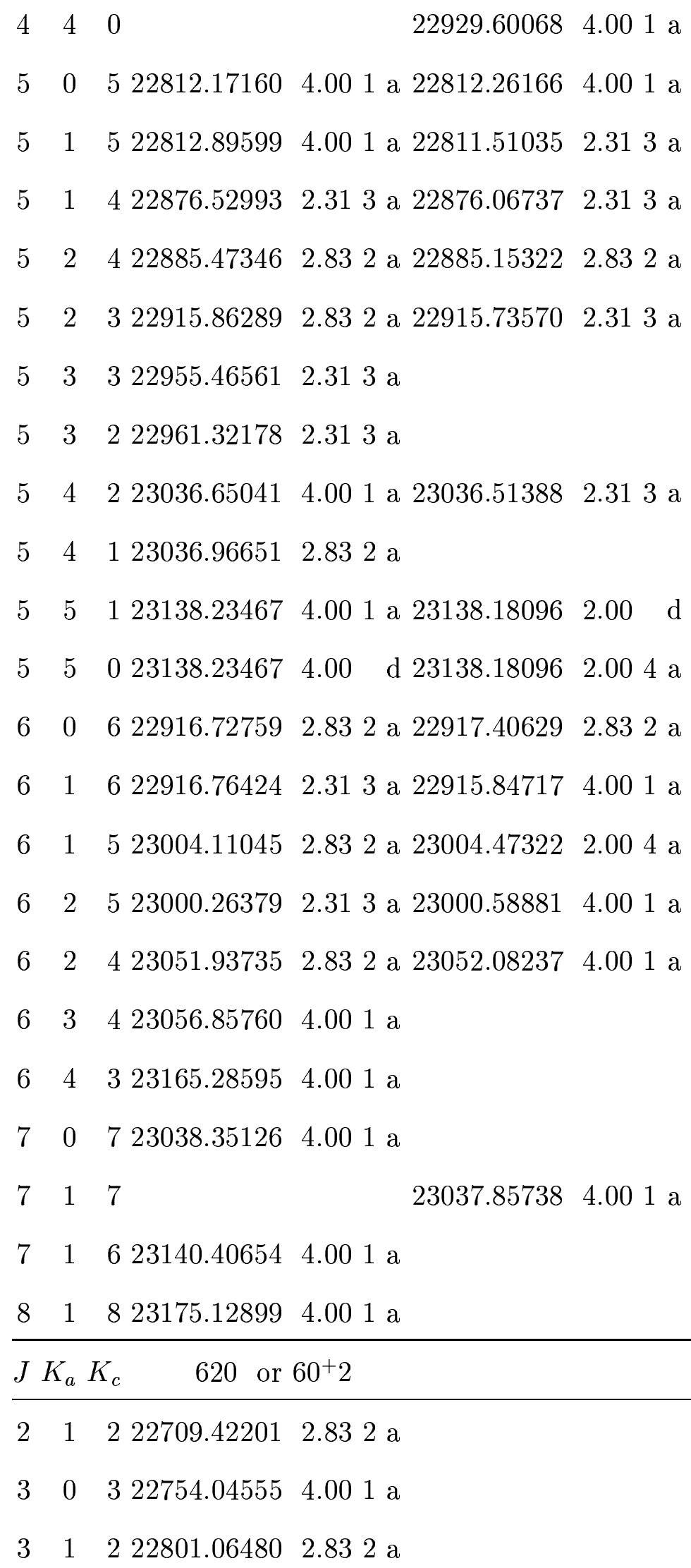


TABLE XVI.: Term values for the $6 \nu+\delta$ polyad of $\mathrm{H}_{2}{ }^{16} \mathrm{O}$.

\begin{tabular}{ccccc}
\hline \hline$J$ & $K_{a}$ & $K_{c}$ & 611 or & $70^{-} 1$ \\
\hline 1 & 1 & 1 & 23981.75174 & $2.832 \mathrm{a}$ \\
2 & 0 & 2 & 24009.95354 & $2.832 \mathrm{a}$ \\
2 & 1 & 2 & 24018.39131 & $4.001 \mathrm{a}$ \\
2 & 1 & 1 & 24034.82294 & $2.832 \mathrm{a}$ \\
2 & 2 & 1 & 24071.34468 & $2.832 \mathrm{a}$ \\
2 & 2 & 0 & 24073.06170 & $2.832 \mathrm{a}$ \\
3 & 1 & 3 & 24075.59799 & $4.001 \mathrm{a}$ \\
3 & 2 & 2 & 24138.21507 & $4.001 \mathrm{a}$ \\
4 & 0 & 4 & 24142.24050 & $4.001 \mathrm{a}$ \\
\hline
\end{tabular}


TABLE XVII.: Term values for the $8 \nu$ polyad of $\mathrm{H}_{2}{ }^{16} \mathrm{O}$.

\begin{tabular}{|c|c|c|c|c|}
\hline \multicolumn{2}{|c|}{$J K_{a} K_{c}$} & \multicolumn{2}{|c|}{800 or $80^{+} 0$} & \multirow{2}{*}{$\frac{701 \text { or } 80^{-} 0}{25120.278454 .001 \mathrm{a}}$} \\
\hline 0 & 0 & 0 & & \\
\hline 1 & 0 & 1 & & $25140.617414 .001 \mathrm{a}$ \\
\hline 1 & 1 & 1 & & $25150.162772 .832 \mathrm{a}$ \\
\hline 1 & 1 & 0 & & $25154.646954 .001 \mathrm{a}$ \\
\hline 2 & 0 & 2 & & $25180.836994 .001 \mathrm{a}$ \\
\hline 2 & 1 & 225180.01252 & $2.832 \mathrm{a}$ & \\
\hline 2 & 1 & 1 & & $25199.789752 .313 \mathrm{a}$ \\
\hline 2 & 2 & 1 & & $25228.302814 .001 \mathrm{a}$ \\
\hline 2 & 2 & 0 & & $25229.542522 .832 \mathrm{a}$ \\
\hline 3 & 0 & 325236.45176 & $4.001 \mathrm{a}$ & $25236.441564 .001 \mathrm{a}$ \\
\hline 3 & 1 & 3 & & $25239.853084 .001 \mathrm{a}$ \\
\hline 3 & 1 & 225266.48383 & $4.001 \mathrm{a}$ & $25266.506394 .001 \mathrm{a}$ \\
\hline 3 & 2 & 225289.24158 & $4.001 \mathrm{a}$ & $25289.226084 .001 \mathrm{a}$ \\
\hline 3 & 2 & 1 & & $25294.902454 .001 \mathrm{a}$ \\
\hline 3 & 3 & 125348.21400 & $4.001 \mathrm{a}$ & $25348.196724 .001 \mathrm{a}$ \\
\hline 3 & 3 & 0 & & $25348.383544 .001 \mathrm{a}$ \\
\hline 4 & 0 & 425312.05177 & $4.001 \mathrm{a}$ & $25310.141312 .832 \mathrm{a}$ \\
\hline 4 & 1 & 425308.65643 & $4.001 \mathrm{a}$ & \\
\hline 4 & 1 & 3 & & $25353.296724 .001 \mathrm{a}$ \\
\hline 4 & 2 & 325369.39978 & $2.832 \mathrm{a}$ & \\
\hline 4 & 2 & 225383.92461 & $4.001 \mathrm{a}$ & \\
\hline 4 & 3 & 225431.25191 & $4.001 \mathrm{a}$ & \\
\hline 4 & 4 & 125510.31492 & $4.001 \mathrm{a}$ & \\
\hline 4 & 4 & 0 & & 25510.345924 .001 \\
\hline
\end{tabular}




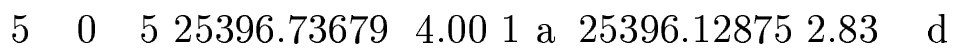

$\begin{array}{lllllll}5 & 1 & 5 & 25396.74729 & 4.001 \text { a } & 25396.12875 & 2.832 \text { a }\end{array}$

$5 \quad 1 \quad 425458.12964 \quad 4.001$ a 25458.201814 .001 a

$5 \quad 2 \quad 425467.99302 \quad 4.001$ a 25468.226554 .001 a

$\begin{array}{lllll}6 & 0 & 6 & 25499.12128 & 4.001 \mathrm{a}\end{array}$

$\begin{array}{llllll}6 & 1 & 6 & 25499.38069 & 2.83 & 2\end{array}$

$\begin{array}{lllllll}6 & 1 & 5 & 25578.73910 & 4.001 \text { a } & 25585.39173 & 4.001 \text { a }\end{array}$

$\begin{array}{llllll}7 & 0 & 7 & 25617.62777 & 4.001 \mathrm{a}\end{array}$

$\begin{array}{llllll}7 & 1 & 6 & 25714.61211 & 4.001 \mathrm{a}\end{array}$

$\begin{array}{llll}7 & 2 & 6 & 25718.363784 .001 \mathrm{a}\end{array}$ 